\title{
Checklist of Vascular Plants of the Southern Rocky Mountain Region
} (VERSION 3)

\author{
NEIL SNOW \\ Herbarium Pacificum \\ Bernice P. Bishop Museum \\ 1525 Bernice Street \\ Honolulu, HI 96817 \\ neil.snow@bishopmuseum.org
}

\section{Suggested citation:}

Snow. N. 2009. Checklist of Vascular Plants of the Southem Rocky Mountain Region (Version 3). $316 \mathrm{pp}$.

Retrievable from the Colorado Native Plant Society (http:/www.conps.orgiplant_lists.html). The author fetains the rights ifrespective of its electronic posting. Please cireulate freely. 


\section{Dedication}

To all who work on behalf of the conservation of species and ecosystems.

\section{Abbreviated Table of Contents}

Fern Allies and Fems.

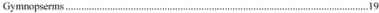

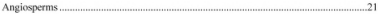

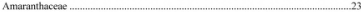

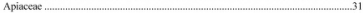

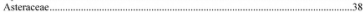

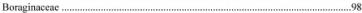

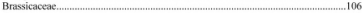

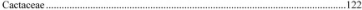

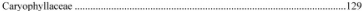

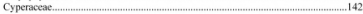

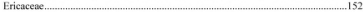

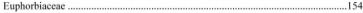

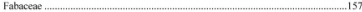

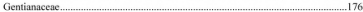

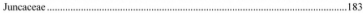

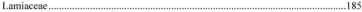

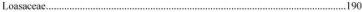

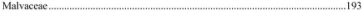

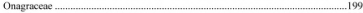

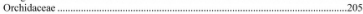

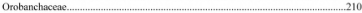

Plantaginaceac .................................................................................................215

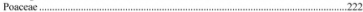

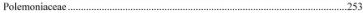

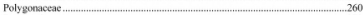

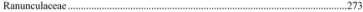

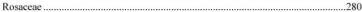

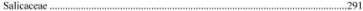

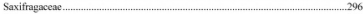

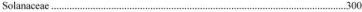

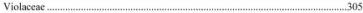

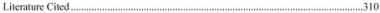




\section{Introduction}

This Checklist provides a summary of vascular plant taxa from the Southern Rocky Mountain Region (SRMR) with selected synonymy. The selected nomenclature is intended to reflect: phylogenetic (evolutionary) relationships as they are currently understood, given that such relationships are the basis for biological classifications, It is understood that nomenclatural changes are not always wamly embraced. Some changes were premature (including in previous versions of the Checklist), and there will be more changes. However, a relative slowdown of changes at the familial and generic levels is likely given the flumy of molecular studies over the past 25 years.

As defined here, the SRMR includes nine counties in southern and eastern Wyoming (Albany, Carbon, Converse, Gosben, Laramie, Niobrara, Natrona, Platte, Sweetwater), all of Colorado, and ten counties from northcentral and northeastern New Mexico (Colfax, Harding, Los Alamos. Mora, Rio Arriba, Sandoval, San Miguel, Santa Fe, Taos, Union). It also includes the northern half of Quay County (north of Interstate 40), New Mexico. The inclusion of three additional New Mexico counties (Harding, Union, part of Quay) compared to the first iteration of the Checklist (Snow04) reflects their high degree of floristic similarity with adjacent high plains counties to the north in Colorado (Schiebout08) and relative lack of taxa more typical of the Chibuabuan Desert flora, which becomes increasingly prominent south of Interstate 40 . The $9,600+$ specimens collected for a recent thesis (Schiebout08) in northeast NM and the ongoing work of Hazlett et al. (in prep.) on the Kiowa National Grassland (specimens mostly at GREE) provided adequate material to incorporate these counties into a slightly expanded concept of the SRMR.

The Checklist relied heavily (see Acknowledgments) on input from numerous specialists and recent publications such as Flora of North America, Intermoumain Flora, Vascular Plants of Wyoming (Dom01), and A Viah Flora (Welsh03). Nomenclature for ferns, fern allies, and gymnosperms generally follows Flora of North America Vol. 2, although more recent classifications exist for ferns (e.g., Goftinet04).

Familial treatments for angiosperms mostly mirror the Angiosperm Phylogeny Group II (APG II) classification. The adoption of APG II by five plant systematics texthooks or something similar (Zomlefer94; Judd07; Sprichiger04; Simpson06; Heywood07) reflects the degree to which APG II has been accepted by professional taxonomists. The retention of clearly outdated familial (e.g., Caprifoliaceac, Chenopodiaceac, Serophulariaceac, Liliaceae s.l.) or generic concepts (e.g., Aster, Seneclo, Sripa) is indefensible. Where data are equivocal or unconvineing regarding the monophyly of certain genera I have taken the broader generic concept (c.g., Elymus). In situations where Bremer, bootstrap or posterior probability support indices of clades are high there has been no hesitation in reducing some genera to synonymy (c.g., Buchioe into Bouteloua).

Widespread adoption of the nomenclature proposed here for the SMRM would align us with other regions and put us ahead of others. None of our larger institutions in the SMRM, towards which people look for seientific leadership, have updated their familial arrangements significantly since the publication of APG II. Further delays in updating familial organization in major herbaria should not be put off indefinitely. Although new or different names may be 
uncomfortable (and remember, taxonomists deal with name changes more than anyone), students pick up new names easily, and it is our obligation to teach what we perceive to represent the best and most recent science and reflect that in our berbaria.

At the specific and infraspecific levels taxonomic opinion often differs between workers assessing variation globally and those having extensive but mostly localized field experience. As one who monographed a medium-sized genus of grasses that occurs on five continents and has hundreds of synonyms I understand keenly some of the underlying reasons for differing taxonomic views. Those with extensive but mostly provincial experience often can recognize ecotypes and populations with some degree of evident difference that may have been ignored or overlooked taxonomically by monographers or those writing broad treatments (e,g. Flora of Norsh Americal. However, if localized variation is not evaluated in the context of variation globally, then a proliferation of beterotypic synonyms typically ensues. The extensive synonymy associated with some taxa in our area reflects this tension, particularly in genera with circumboreal or trans-American distributions (e,g. Fiola, Salix, Sileme). Pronounced manifestations of the local versus global tension is seen with Mimulus gurratis, for which Kartesz and Meacham (KTZ99) recognize nearly 70 synonyms. In the opposite direction, Reveal recognized 41 varieties for Eriogomam umlellatam in Flora North America (Vol. 5 ). In compiling the Checklist close attention was given to the views of those with extensive fieldbased knowledge and overall breadth of taxonomic experience, but wider treatments (FNA. Porter00, Wagner07) also have been considered. I have summarized elsewhere in detail the species concept and criteria I use, which was sometimes used to evaluate the taxonomic treatments of others (Snow97, Snow03).

The degree of synonymy indicated among taxa varies; users hopefully will understand that the primary goal has been to summarize names used (mostly) regionally, either now or previously, and to reflect modern taxonomic concepts. In some cases where the taxonomy of a group is particularly difficult, more extensive synonymy is provided.

Earlier versions (Snow04a, 07a,b) noted its origins in A Checklist of the Vascular Plants of Colorado (http://www.rmh.uwyo.edu). Important differences in the listing of synonyms between these documents is explained below (notes 2-v, 2-vi). In most cases 1 consulted volumes of Flora of North America, A Working Index of New Mexico Vascular Planis (http://web.nmsu.edw/ -kallred/herbweb) by Kelly Allied, A Flora of New Mexico (Martin \& Hutchins 1980-81), the Symhesis of the North American Flora (Kartesz \& Meacham 1999), the plant database at the University of New Mexico Herharium for NM counties in the SRMR. Grasses of Colorado (Shaw08), and the Catalog of the Four Corners Flora of Heil \& O'Kane (http://www.sanjuancollege.edu/Herbarium/catalogtitle.htm). Some state records for Colorado from Heil and O'Kane have not been published or await further confirmation. A number of revisions and monographs that concern taxa from our region have been consulted and cited. Nearly all previous references to the U.S.D.A. PL.ANTS database have been removed since it is a dynamic document, and rechecking its usage would have required more time than that presently available. Likewise, many references to "RMC" (short for Rocky Mountain [Herbarium] Checklist, from 1992) have been removed given its age and other generally reliable sources of printed information since then (e-g, Dom01, FNA volumes). 
Newly reported species and infraspecific taxa from our region in wolumes of Flora of North America generally have been included, but they were incorporated to minimize changes needed at the generic level compared to Snow07b, given the ongoing scoring of characters for taxa in the Southern Rocky Mountain Interactive Flora (Btasher06). In a few cases where species boundaries remain tentative in our region (e.g., Eleocharis, Cirsizm, Grindelia) I have not hesitated to indicate that a doctoral dissertation for that group is needed. Taxa collected only once or twice long ago are in some cases treated as waifs that were subsequently extirpated (e.g. Claytonia umbellata). However, in cases where non-native taxa frequently are planted omamentally (e.g., Limum usifuissimum) and are likely to escape at least occasionally in localized areas, the taxa have been included as "present".

It is unfortunate that the larger herbaria of our region (COLO, RM, CS, UNM, COCO, GREE, KHD) are so unaligned nomenclaturally. Curators and collections managers in the SRMR should consider whether adoption of the nomenclature presented bere (with modifications as necessary) more broadly. The National Science Foundation will support workshops and collaborative initiatives that work towards regional cooperation and standardization. The SRMR lags behind parts of the USA (e.g, southeastern US, California, Intermountain Region, Great Plains), where curators work collaboratively at documenting and disseminating data electronically about their regional flora (e.g., see htp; //www.sernec.org/files/R.A.O.Dec2008.pdf).

"Version 3" reflects six years of updates. While some will groan at changes, it is a good thing that systematics is a vigorous science and enhancing our understandings of species boundaries and evolutionary relationships (and thus generic boundaries). The next version of the Checklist will indicate state-wide distributions for each taxon (WY, CO, NM).

Please send all corrections to me at the Bishop Museum (neil.snowab bishopmuseum.org). 


\section{Notes}

1. Nomenclature at the family level follows Flora of North America for fern allies and gymnosperms and APG II (2003) for flowering plants for see Zomlefer 93; Judd07; Spricher02; Simpson06). Three-letter family acronyms are indicated to the right of each family (Weber82; Snow00; Brasher04; Snow09). Varieties typically are used in lieu of subspecies if the nomenclatural combination is available for variety. Quadrinomials are omitted (for rationale see Snow97), as are forms (formae). Some genera have some species with either subspecies or varieties (e.g., Poa); the allowance of such a mixture rather than to adhere strictly to one or the other (e.g-, Dom01) was to minimize the amount of rank changes from the previous checklist.

2. Names of accepted taxa appear on the far left-hand margin. Synonyms of accepted taxa are placed in square brackets [] and indented below each accepted taxon. The attribution of names and synonyms is based on their use in one or more of the standard floras or checklists. Six important points must be stressed regarding aceepted taxa and their synonyms:

i) The name indicated for a taxon may not be the correct application of the name (i.e., the name being used may not match the type specimen for that name).

ii) A name may correctly match the type specimen but may not be correctly applied to taxa in our region. For example, Carex focma var, foena was misapplied to material in our region that is now properly attributed to Carex siccata.

iii) An indication of a name being an accepted taxon by a particular source (e.g. KTZ) does not necessarily mean that same source credits that taxon to our area.

iv) Taxonomic circumscriptions of many families and genera have changed based on moleculat studies of the past two decades. Oddly enough, our theoretical basis for understanding and interpreting molecular data (e.g., DNA sequencing) is more fully developed than our ability to interpret the overall reliability of morphological of anatomical data. Although some earlier molecular studies were premature in proposing new classifications based on only one gene or limited sampling within a taxon, that tendency is mostly a thing of the past, and the changes herein typically are well supported by more than one source of data.

v) The manner in which this checklist treats the accompanying bracketed synonyms differs from Hartman \& Nelson (2001), even though the visual format is the same. In Hartman \& Nelson (2001) the abbreviations next to a bracketed synonym indicated the accepted names recognized by the various publications. In this document, a literature citation with a synonym can mean the same thing as Hartman \& Nelson (2001). However, it also may indicate that the literature source (e.g. FNA, U13), like this document, recognizes the name only as a synonym, not as an accepted taxon. The expanded usage herein more fully indicates known synonymy irrespective of whether the names have ever been applied to taxa in our region, or whether one of the local sourees (FNA, UT3) recognized it as the correct name for an accepted taxon. This is important because different Floras split taxa at the specific and infraspecific levels in different ways. It has not been possible to exhaustively list all attributions of all sources. The following two examples should help clarify how synonymy is to be intepreted:

\section{Example 1:}


Toxicoscordion paniculatum (Nutt,) Rydb, - W\&W, Zomlefer01, Zomlefer02, SFW

[Helonias paniculata Nuttall] - FNA

[Zigadenus paniculatus (Nutt.) S. Watson] - Dom01, FNA, HDH, IMF, KTZ, UTF, W

Explanation: Toxicoscordion paniculatum is accepted by W\&W (Weber \& Wittmann) and other indicated sources. The two references of Zomlefer indicate where detailed taxonomic data are published to support this combination. FNA recognizes this taxon under the genus Zigadenus, but also lists Helonias as a synonym for that genus. FNA also recognizes the name Toxicoscordion panicularum but only as a synonym. It is important to stress that for full interpretation of the names and bracketed synonyms the user should consult the cited sources to see exactly how the name is treated by each author.

\section{Example 2:}

Several species of Cryprantha (Boraginaceae) have the generic name Krynizzia indicated as a synonym, which are attributed to UT3. In this case, UT3 was the first source we found (although not necessarily the first source published) in which Krynitzkia is recognized as a generic synonym. However, UT3 does not recognize Krynitzkia as a valid genus. Again, users should consult each source to fully interpret our indications of synonymy.

Note: The authoritative confimation of names in synonymy is one of the most timeintensive tasks in systematics, and can be done with confidence only when all type specimens are seen by a single author during a comprehensive revision or monograph of a given genus.

vi) Some author abbreviations have been standardized herein even though different Floras have used them differently. For example, B. L. Robinson has been spelled out in full in our region or abbreviated as B. L. Robin., B. L. Robins., or B. L. Rob. I have not generally made new entries under the synonymy of a given taxon if the only difference was minor variations of abbreviating author(s), although this was done previously (c.g. Snow04a).

3. Braces $\{$ indicate taxa and synonyms credited to the Southern Rockies that remain unsubstantiated, erroneous, or which may be expected given their proximity in adjacent states. The symbols $<>$ enclosing one or more two-letter postal codes (e.g., $\langle\mathrm{KS}, \mathrm{OK}\rangle$ ) indicate states contiguous to the Southern Rockies where a taxon has been documented if not attributed to our region by other sources (primarily Flora of North America [FNA] or the "Symhesis" [KTZ]).

4. Endemic includes taxa that may range beyond the SRMR within the states of NM or WY, but excludes taxa in adjacent states. Designations of endemism herein should not be taken as authoritative; additional confirmation is needed in many instances, and expanded ranges in adjacent states beyond our region are found frequently. 
5. For Colorado species, Harrington (1954) used "in" frequently but "ex" seldomly in the citing of authorities. A semicolon separating authors appears often and may be equivalent to the "ex."

6. Hybrid taxa are indicated by a multiplication sign (X). The hybrid formula follows, then synonyms, if any (e.g. Equisetum $X$ nelsonii).

7. Considerable variation exists between regional herbaria on their recognition of infraspecific taxa, and local Floras regarding their recognition. 'This accounts, in part, for the inclusion of "identification sources", since often only one (e.g., IMF, FNA) source for our region includes keys to infraspecific taxa. 


\section{Abbreviations for literature and reviewers}

APG II - Angiosperni Phylegeny Groupll (2003)

CC - Carolyn Cran ford, Louisville, 00 , pers. comm,

DA = Dirk Albach, Vienna, Austria, pers. comm.

Darn01 - R. Dorn. 2001. Vascular Plants of Wyoming, $3^{\text {rd }}$ Ed. Mountain West Publishing. Cheyenne

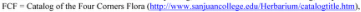

K. D. Heil \& S. L. O'Kane, Jr.

$\mathrm{FL}=$ Friedrich Lauria, pers. comm.

FNA = Flora of North America Editorial Comminee. 1993 and onwards. Flora of North America. Oxford Univ.

Press, New York, NY

FNE = Kaul, R. B.. D. M. Sutherland, S. B. Rolfsmeier. 2006. The Flora of Nebraska. University of Nebraska.

Lincoln.

$\mathrm{GPF}=$ Grear Plains Flora Association. 1986. Flora of the Great Plains. Univ. Press of Kansas, Lawrence. KS

HDH = Harrington, H. D. 1954. Manual of the Plants of Colorado. Sage Books, Denver, CO

HDH2= Harringtoe, H. D. 1964. Manual of the Plants of Colorado. $2^{27}$ Ed. Swallow Press, Chicago, IL

IMF - Cronquist. A., et al. 1972+. Internsountain Flora. Various publishers

IPNI - International Plant Names lndex (www. pni.orgindex.fitml)

JG - Gaskin, John, U.S.D.A (Sydney, MT), pers, comm.

JM - Jane Mygait, pers. comm. 2003; database query from UNM

KT2 - Karlesz, I. T. \&. C. A. Meacham, 1999. Synthesis of the North American Flora Version 1.0. North Carolina

Bot. Garden, Chapel Hill, NC

KTZ2 - Kartesz, J. T. \& C. A. Meacham. Beta (unreleased). Synthesis of the North American Flora. North Carolina

Bot. Garden, Chapel Hill, NC

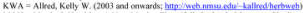

M\&H - Martin \& Hutchins. A New Mexico Flora (full chitation in Literature Cited)

NNL - 1sely, D. 1998. Native and Naturaliasd Leguminosae (Fabosceac) of the United States. Brigham Young

Unir, Provo, UT

NS - Neil Snow, personal comments and observatices

RBS = Shaw, R. B. 2008. Grases of Colorado. University Press of Colorado, Boulder.

RH - Ron Hartman, personal comments (especially API, AST, and CFY)

RMC - Nelson, B. E. \& R. L. Hartman. 1992, Checklist of the Vascalar Plants of Wyoming, unpubl.

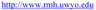

SFE, SFW - Weber, W. A. \& R. C. Wirmann. 1996. Colorado Flora: East Slope, ed. 2. of Colorado Flora: West

Slope. Boch: Univ, Press of Colorado, Niwox, CO

SF! - Documented for both East Slope and West Slope floras (see above)

TK $=$ Tass Kelso, pers. camm.

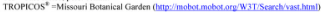

UTF - Welsh, S. L., et al 1993. A Utah Flora, do. 2. Brigham Young Univ. Press, Provo, UT

UT3 = Welsh, S. L., et al. 2003. A Utah Fiora, ed. 3, Brigham Young Univ. Press, Prowo, UT

WAW $=$ William $A$. Weber, pers. comm.

$W J=$ William Jennings, Louisville, $\infty$, pers, comm.

WY Checklist $=$ database guery from RM of plants from WY in SRMR (May 2003)

W\&S = Wooton \& Standley, 1915, Flora of New Mexico, Contr. U.S. Nat, Herb, 19: 1-794

W\&W $=$ Weber, W. A. \& H. C. Witmann. 1002 . Conalog of the Colorado Flora A Biodiversity Baseline. Univ. Press of Colorado. Niwot, $C O$

W\&W' = Weber, W. A. \& R. C. Wittmann. 2000. Catalog of the Colorado Flora, Revised electronic version, II Mar 2000$)$ (herpo/ www ealorado.educumisen)

W\&W'E=Weber, W, A., \& R. C. Wimmann. 1996, Colorado Flora: Eastern Slope, Revised Edition. The University Press of Colorado

W\&W W=Weber, W. A., \& R. C. Wittmann. 1996, Colorado Flona: Westem Slope, Revised Edition. The University Press of Colorado

W\&W E = Weber, W_A. \& R. C. Witmann. 2001. Colorado Flora. Eastern Slope. Thied Edition. The Universiry Press of Colorado 
Herbarium Abbreviations: ARIZ (Univ, Arizona); BRY (Brigham Young Univ.); COCO (Colorado College); COLO (Univ. Colorado - Boulder); CS (Colorado State Univ.); GREE (Univ, Northern Colorado); KHD (Denver Botanic Garden); RM (Univ. Wyoming); UNM (Univ, New Mexico); USFS (National Herbarium, Forest Service [at RM])

\section{Other Abbreviations}

* - invasives in the Southern Rocky Mountains from outside NA

* = invasives considered introduced to the Southern Rockies from elsewhere in NA

- Endemics to the Southem Rocky Mountain Area

$\phi=$ presumed extinct

attrib, = attributed

cult $=$ cultivated

err. $=$ erroneous

$\exp ,=$ expected

ENDEMIC = endemic to SRMR or (at maximum) states of CO, NM, and'or WY

misid, = report based on a misidentified specimen

misapp, $=$ the name was misapplied of used inappropriately

rep. $=$ report, reported

E, W, or E/W - These designations indicate the taxon is documented on the East or West slope ("slope flora East," SFE, "slope flora West," SFW, or both, SF!)

AZ, CO, KS, NE, NM, OK, UT, WY - Two-letter postal codes are used for the states.

$\mathrm{NA}=$ North America

USA = United States of America

\section{Approximate Numerieal Summaries}

Numerical summaries should be taken as no more than approximations of local diversity levels. The reasons in favor of this view are numerous: Familial and generic concepts are always somewhat in flux; some taxa treated at the infraspecific level are treated elsewhere as species; all specimens of locally infrequent species may be misidentified; new taxa are being discovered periodically; new state records are made constantly; whether some species are truly naturalized from ornamental plantings may be hard to discern; taxa that were once established have been extirpated later (e.g., Briza spp.); and compilers can easily miscount longer lists. Clayton09 notes that inclusion of a taxon for which only one or a few specimens were collected many decades ago, and which has not collected or seen thereafter, inflates the real situation. However, I have not re-evaluated records known only from 50 years ago or longer.

Families: 148

Species: 3265

Undescribed taxa: 5

Total: Species + infraspecific taxa: 3748

Presumed extinet: 1 (Cryptantha aperia)

Regional endemics: 134

North American invasive taxa: 113

Extra-American invasive taxa: 419

Invasive taxa total: $\mathbf{5 3 2}$

Invasive taxa in flora by pereent: $14.4 \%$

\section{Genera: 851}

Nothotaxa (species of hybrid origin): 38 Infraspecific taxa: 440 


\section{Acknowledgments}

Earlier incamations of this Checklist (Snow04; Snow07a,b) built from earlier checklists and databases produced or maintained by Ronald Hartman and Burrell "Ernie" Nelson, Kelly Allred, William Weber and Ronald Wittmann, Chris Frazier, Jane Mygatt, and Ken Heil and Steve O'Kane, Jr. My thanks to the above individuals and Ben Legler, John Kartesz, Peter Hoch, Bill Jennings, Monique McHenry, Don Hazlett, Pamela Smith, Bonnie Heidel, Leigh Johnson, Thsan Al-Shebbaz, Dirk Albach, Eric Ribbens, Steve Popovich, Donald Farrar, Peter Root, Barry Johnston, Greg Brown, Andrew Salywon, Michael Schiebout, David Remsen, George Argus, Georgia Doyle, Tim Hogan, Tim Dickinson, Tim Evans, Joe Kirkbride, Mark Egger, Friedrich Lauria, Rick Hammer, Alan Whittemote, and John Gaskin. For annotating specimens dufing my tenure at GREE (and typically other regional herbaria) thanks to Don Hazlett, Pamela Smith, Jeff Brasher, Bob Dom, James Reveal, Andrew Hipp, Andrew Salywon, Stan Welsh, Rob Soreng. Paul Peterson, Kelly Allred, Ron Hartman, Bill Jennings and Ben Legler. My apologies to those whom 1 neglected to record. Thanks also to Bob Williams, Marilyn Orris, Mary Jane Niemann, Pamela Smith, and Marty Arrington for their work at GREE during my tenure at UNC, which liberated me somewhat from the busy-ness of curating an active herbarium during my tenure at UNC and which helped make earlier versions of the Checklist a reality, Georgia Doyle generously volunteered to be curator of photos for three years for the SRMR flora project and developed the database used to document photos for the interactive key project.

My sincere thanks to the U.S. Forest Service, Bureau of Land Management, U.S. National Park: Service, U.S, Fish and Wildlife Service, U, S. Department of Defense, Wyoming Game and Fish, New Mexico Department of Game and Fish, New Mexico State Parks, Colorado Division of Wildlife, and Vermejo Park Ranch for their help and interest in the study of plants. Employees of Natural Heritage Programs in Wyoming, Colorado, and New Mexico have heen important contributors to our knowledge of plants in the SRMR. The Nature Conservaney has facilitated botanical work in our region in numerous ways, including allowing access to privately held properties. Many other individuals have provided assistance in other ways. The landowners and managers of numerous ranches, particularly in New Mexico, have allowed us to collect on their properties. We appreciate their commitment to conservation of our native flora and fauna.

Assembling the Checklist has been facilitated by the Flora of North America project, which has been of considerable value to regional projects such as this one, and whose published volumes have provided insights regarding synonymy and numerous taxonomic problems.

Support for development and maintenance of the Checklist comes from the National Science Foundation (DBI-0237149) to P. M. Simmons, N. Snow, T. Ranker, R. Guralnick, and R. L. Hartman, the Colorado Native Plant Society (https/www conps.org), and Native Plant Society of New Mexico (http $/$ /npsum.unm.edu).

I take full responsibility for all oversights and errors. 


\section{FERN ALLIES}

\section{Equisetacene - EQU}

(Identification sources:

Equisetum arvense L. - Dom01, FNA, GPF, HDH, IMF, KTZ, SF!, UTF, W\&W, EW

[Equisetum arvense L. forma serotinum (C. F. Schutz) Klinge]- HDH

Equisetum X ferrissii Clute - FNA, GPF, IMF, KTZ, UTF

[Equisetum hyemale X E. lavvigatum] - KTZ

Equisetum byemale L. subsp, affine (Engelm.) Calder \& Roy L. Taylor - FNA

[Equisetum hyemale L. var, affine [Engelm.] A.A. Eaton] - Dorn01. GPF, IMF, KTZ, UTF, EW

[Equisetum hycmale L. var. califomicum Milde] - HDH

[Equisetum hyemale L. var. hyemale] - HDH

[Equisetum pracaltum Raf.] - HDH

[Hippochaete hyemalis (L.) Bruhin] - W\&W

[Hippochaete hyemalis (L.) Bruhin subsp. affinis (A. Bram) W. A. Weber] - SF!

Equisetum laevigatum A. Braun - Dom01, FNA, GPF, HDH, IMF, KTZ, UTF, E/W

[Equisetum kansanum Schaffier] - HDH

[Hippochaece laevigata (A. Brain) Farw.]-SF!. W\&W

Equisetum X nelsonii (A. A. Eaton) J. H. Schaffn. - Hartman06, FNA, KTZ

[Equisetum laevigatum X E. variegarum] - KTZ

[Equisetum variegarum Schleich. var, nelsonii A. A. Eaton] - HDH

[Hippochaete variegata (Schleich.) Bruhin] - W\&W

Equisetum pratense Ehrh. - FCF, FNA, HDH rep., KTZ, SF!, W\&W, err. rep., E/W

Equisetum variegatum Schleich. ex F. Weber \& D. Mohr subsp. variegatum - Dom01, FNA

[Equisetam varieganum Schleich. ex F. Weber \& D. Mohr var, variegatum - IMF, KTZ, EW

[Equisetam varieganum Schleich.] - GIF, UTF

[Equisetum varieganum Schleich. var, varieganum] - HDH

[Hippochaete variegata (Schleich) Bruhin] - SF!, W \& W

\section{Hippochacte (see Equisetum)}

\section{Isoëtaceae - ISO}

(Identification sources:

Isoetes bolanderi Engelm. - Dorn01, FNA, HDH, IMF, KTZ, SF!, UTF, W\&W, E/W

[Isoütes bolanderi Engelna, var, bolanderi] - RMC

Isoêtes echinospora Durieu - FNA, IMF, UTF, E

[Isočtes muricata Duriet]] - HDH

[Isoëtes setacea Lam, subsp, muricata (Durieu) Holub] - SFE, W\&W

[lsoëtes tenella Lènan] - KTZ

Isoětes oceidentalis L. F. Hend. - Dorn01, FNA, HDH, KTZ, E/W

[Isoctes lacustris L.] - IMF, SF!, UTF, W\&W

\section{Lycopodiaceae - LYC}

(ldentification sources: 
Diphasiastrum alpinum (L.) Holub - FNA. W\&W ${ }^{1}$

[Lycopodium alpinam L.] - KTZ

Huperzia haleakalae (Brackenridge) Holub - Dorn01, FNA, KTZ, SF!,W\&W ${ }^{1}$, E/W

[Huperzia selago (L.) Bernh.] - W\&W, W\&W', err. rep.

[Huperzia selago (L.) Bernh. ex Mart. \& Schrank] - FNA, misapp.

[Lycopodium selago L.] - HDH

Lycopodium (see also Diphasiastrum, Huperzia)

Lycopodium annotinum L. - Dorn01. FNA, GPF, HDH, KTZ, SF1, W\&W, E/W

[Lycopodium annotinum L. var, annotinum] - RMC

[Lycopodium annotinum L. var. pungens \{La Pylaie) Desv.] - FNA (name invalid)

[Lycopodium dubium Zodiga] - $5 F$ !

\section{Selaginellaceae - SEL.}

(Identification sources:

Selaginella densa Rydb, - Dorn01. FNA, GPF, HDH, SF!, UTF, W\&W, E/W

[Selaginella densa Rydb. var. denisa] - [MF. KTZ

[Selaginella densa Rysb. var, scopulorum (Maxon) R. Tryon] - IMF, KTZ.

[Selaginella densa Rydb. var, standleyi (Maxon) R. Tryon] - KTZ

Selaginella mutica D. C. Eaton ex Underw, var, mutica - Dorn01, FNA, GPF, IMF, KTZ, E/W

[Selaginella mutica D. C. Eaton] - HDH, SF1, UTF, W\& W

[Selaginella pervuiana (J. Milde) Hieronymous - B. Legler [pers, comm. 2009] indicates 3 likely

collections from Colfax $\mathrm{Co}$. NM but these in need of further confirmation

Selaginella scopulorum Maxon - FNA, HDH

[Selaginella densa Rydb, var, scopulorum (Maxon) R. M. Tryon] - FNA

[Selaginella engetmannii Hier, var. scopulorum (Maxon) C. F. Revd] - FNA

Selaginella selaginoides (L.) P. Beauv. ex Mart. \& Schrank - Dom01, FNA, KTZ

[Selaginella selaginoides (L.) Link] - HDH, IMF, SFI, W\&W, EW

Selaginella standleyi Maxon - FNA, HDH

[Sclagiella densa Rydb. var, standleyhi (Maxan) R. M. Tryoa] - FNA

Selaginella underwoodii Hieron, - Dom01, FNA, GPF, HDH, IMF, KTZ, SF!, UTF, W\&W, E/W

- Selaginella weatherbiana R. Tryon - ENDEMIC $<$ CO, NM $>$ FNA, HDH, KTZ, SFE, W\&W, E

\section{Adiantaceae (see PTR)}

\section{FERNS}

\section{Aspidiaceae (sce DRY)}

\section{Aspleniaceae - ASI.}

(Identification sources:

Asplenium adiantum-nigrum L. - FNA, HDH, IMF, KTZ, SFE, UTF, W\&W ${ }^{1}, \mathrm{E}$

[Aspleniam andrewsii A. Nels] - Ws W

Asplenium platyneuron (L.) Britton, Sterns, \& Poggenb. - FNA, SFE, W\&W, E

[Asplenlum platyneuron (L.) Oakes] - HDH

[Asplenium platyneuron (L.) Oakes ex D. C. Eaton]-GPF

[Asplenium plaryneuron (L.) Briton, Sterns, \& Poggenb. var, platyneuren] - KTZ 
Asplenium resiliens Kunze - FNA, GPF, IMF, KTZ, SFE, UTF, W\&W, E

Asplenium septentrionale (L.) Hoffm. - Dorn01, FNA, HDH, IMF, KTZ, SF!, UTF, W\&W. E/W

[Aspleniam septentrionale (L.) Huds.] - GPE

Asplenium trichomanes L. subsp, trichomanes - FNA, KTZ. E/W

[Asplenium trichomanes L.] - GPF, HDH, IMF, SFI, UTF, W\&W

Asplenium trichomanes-ramosum L. - Dorn01, FNA, KTZ, SFW, W\&W, W

[Asplenium viride Hudxan] - GPF, HDH, IMF

[Asplenium viride L.] - UTF, W\& W

\section{Athyriaceat (see DRY)}

\section{Azollaceae $=$ A Z I}

Azolla mexicana C. Presl - FNA, GPF, HDH, exp, IMF, SFE, UTF, W\&W, E

[Azolla mexicana Schltdl. \& Cham. ex C. Presl] - KTZ

\section{Cryptogrammaceae (see PTR)}

Dennstaedtiaceae - DST (including Hypolepidaceae, Polypodiaceae)

\{Pteridium aquilinum (L.) Kuhn var, latiusculum (Desv, Underw, ex A. Heller\} - Dorn01, FNA, GPF, HDH, KTZ, E/W

\{NS - not in our region fide FNA

\{Pieridium aquilinum (L.) Kuhn subsp. lanuginosum (Bong.) Hulten\}. - SF!, W\& W

Pleridium aquilinum (L,) Kuhn var, pubescens Underw, - FNA, GPF, HDH, KTZ, UTF, E/W

[Pheridium aquilinum (L.) Kuhn subsp. lanuginosam (Bong.) Huhén]-SF!, W\&W

\section{Dryopteridaceae - DRY (including Aspidiaceae, Athyriaceae, Onocleaceae, Polypodiaceae, Woodsiaceae)}

(Identification sources:

Athyrium alpestre (Hoppe) Chairv. var. americanum Butters - FNA, E/W

[Athyrium americanum (Butters) Maxon] - KTZ

[Athyrium alpestre (Hoppe) Rylands ex T, Moore subsp. ancricanum (Buners) Leflinger] - W\& W

[Atbyrium alpestre Clairv, var, americinum Butters] - RMC

[Athyrium alpestre (Hoppe) Rylands ex T. Moore var, americanim Butters] - HDH

[Athyrium distentifolium Tausch] - SF]

[Athyrium distentifolium Tausch ex Opiz] - W\& w'

[Athyrium distentifolium Tausch ex Opiz var, americanum (Butters) Crong̨aist] - IMF, UTF, W \& W'

Athyrium filix-femina (L.) Roth ex Mert. var. angustum (Willd.) G. Lawson] - FNA

[Aspidiuns angustum Willd.] - FNA

[Athyrium angustum (Willd.) C. Presl] - FNA

[Atbyrium filix-femina (L) Roth subsp angastum (Willd) Clausea] - KTZ, SFE

[Athyrium filix-femina (L.) Roth ex Mert. subsp. angustum (Willd.) Hultén] - Wa W

[Athyrium filix-femina (L.) Roth var, angustum (Willd.) Moore] - GPF

[Athyrium filix-femina (L.) Roth subsp. angustum (Willd) Clauseo] - FNA

[Athyrium filix-femina (L.) Roth var, michasia (Sprengel) Farwel] - FNA

Athyrium filix-femina (L.) Roth ex Mert. var. califomicum Butters - FNA, E/W

[Athyrium angustum (Willd.) C. Prest var, rubellum (Gilbert) Batters] - $\mathrm{HDH}$

[Atbyrium filis-femina (L.) Roth] - SFW 
[Athyrium filix-femina (L.) Roth ex Mert. var- filix-femina] - HDH

[Athyrium filix-femina (L.) Roth subsp. cyclosocum (Rupr.) C. Christens.] = KTZ SFE

[Athyrium filix-femina (L.) Roth ex Mert. subsp. cyclosorum (Rupe) C. Christens.] -W\&W

[Athyrium filix-femina (L.) Roth var. cyclosorum Rupe.] = GPF

[Athyrium filix-femina (L.) Roth var. cyclosorum (Rupr.) Ledeb.] - IMF, UTF

[Athyrium filix-femina (L.) Roth ex Mert. var. cycloserum Rupr.] - FNA

[Athyrium filix-femina (L.) Roth ex Mert. subsp. filix-femina]- W\& $W^{1}$, err. rep.

\{Cystopteris bulbifera (L.) Bernh.\} - FNA, HDH, exp. IMF, KTZ, UTF, W\&W

Cystopteris fragilis (L.) Bernh. - Dom01, FNA, GPF, HDH, KTZ, SF!, UTF, W\&W, E/W

[Cystopteris fragilis (L.) Berth, var. fragilis] - IMF, RMC

Cystopteris montana (Lam.) Bernh. ex Desv. - FNA. KTZ, W\&W, E/W

[Cystopteris muotanta (Lam.) Bernh.] - HDH, SF!

Cystopteris reevesiana Lellinger - FNA, KTZ, SF!, W\&W, E/W

Cystopteris tenuis (Michx.) Desv, - FNA —NE, UT>, HDH, KTZ, SFW, W\&W

Cystopteris utahensis Windham \& Haufler - FNA, KTZ, SFW, W\&W, W

Dryopteris expans (C. Presl) Frazer-Jenkins \& Jerny - FNA, KTZ, SF! W\&W, EW

[Dryopteris dilatata (Hoffmann) A. Gray]-HDH, W\&W, misapp.

Dryopteris filix-mas (L,) Schott - Dorn01, FNA, GPF, HDH, IMF, KTZ, SF!, UTF, W\&W, E/W

Gymnocarpium X brittonianum (Sarvela) Pryer \& Haufler - KTZ, W\& W'; Pryer93

[Gymnosarpium disjunstum $X \mathrm{G}$. dnopteris] - KT2

Gymnocarpium dryopteris (L.) Newman - Dorn0I, FNA. IMF, KTZ, SF!, UTF, W\&W", E/W

[Gymnosarpium disjunctum (Rupr.) Ching] - FNA, KTZ, W\& $W^{1} \leq W Y$ ?

[Gymnosarpium dryopteris (L.) Newman subsp. disjunctum (Rupr.) Sarvela] - W\&.W, misapp.

[Phegopteris dryopteris (L.) Fee] - HDH

Onoclea sensibilis L,- FNA, GPF, HDH, KTZ, W\&W

[W] - besed on Heber 4272 , in 1948 (COLO), collected slong $\mathrm{CO} 67$, roodside ditch, by fence, on curve near "Papoose Club." A waif in my opinion, this distinctive fem, would have beea documented by early collectors if native, if a remeant popalation, would have ore likely occurred in Boulder Mountain Parks]

\section{Phegopteris (see Gymnocarpium)}

Polystichum lonchitis (L.) Roth - Dorn01, FNA, GPF, HDH, IMF, KTZ, SF!, UTF, W\&W, E/W

Polystichum scopulinum (D. C. Eaton) Maxon - Dorn01, FNA, HDH, KTZ, SFW, W\&W, W

[Polystichum X scopulinum (D. C. Eaton) Maxon] - RMC

Woodsia neomexicana Windham - FNA, KTZ, SF!, W\&W', E/W

[Woodsla mexicana Foe] - FNA, GPF, HDH, exp. W\&W, misapp.

Woodsia orcgana D.C. Eaton subsp. cathcartiana (B. L. Rob.) Windham - Dorn01, FNA. KTZ. $\mathrm{SF}$ !, W\&W $W^{!}$

[Wocdsia oregana D, C, Eaton var, cuthcartiana (B, L. Rob, ) Mortoe] - E/W

[Wocdsia oregana D, C. Eaton] - GPF, HDH, UTF, Ws W

[Wocdsla oregana D. C. Eaton subsp. oregana] - W\&W', er, rep.

[Wocdsia oregana D. C. Eaton var, oregana] - IMF, RMC

[Wocdsia oregana D. C. Eaton forma glandulosa T, M, C. Taylor] - HDH

Woodsia plummerae Lemmon - FNA, KTZ, SFE W\&W, E

Woodsia X schaackii Windham - W\& $W^{\prime}$ 
[Wods a neomexicana $X$ W- oregana] - W\& $W^{\prime}$

Woodsia scopulina D. C. Eaton subsp. laurentiana Windham - FNA, KTZ

Woodsia scopulina D. C. Eaton subsp. scopulina - FNA, KTZ, EN

[Woods ia scopulina D. C. Eaton] - GPF, HDH, SF!. UTF, W\&W

[Wocdsia scopulina D. C. Eaton var. scopulina] - IMF

\section{Hypolepidaceae (see DST)}

\section{Marsileaceac - MSL}

Marsilea vestita Hook, \& Grev, - FNA, GPF, HDH, IMF, KTZ, UTF, E/W

[Marsilea mucronata A. Braun] - SF!, W\& W

[Marsilea vestita Hook. \& Grev. var. vestira] - RMC

\section{Onoclenceae (see DRY)}

\section{Ophieglossaceae - OPH}

(Identification sources:

Botrychium

[NS - Donald Farrar apers, comm., Aug. 2007) indicates the grape ferns will be treated by him in future publicatices as Stepwrifium and the rattesnake fem as Bowyous]

Botrychium campestre W, H. Wagner \& Farrar-Dom01, FNA, KTZ, SFE, W\&W, E

Botrychium echo W, H. Wagner - FNA, KTZ, SF!, W\&W, E/W

Botrychium hesperium (Maxon \& R. T. Clausen) W. H. Wagner \& Lellinger - FNA, KTZ, SF!, W\&W, ENW

[Botrychium lunaria (L.) Sw, subsp. occidentalls A. Löve, D. Lơve. \& B. M. Kapoor] - W\& W

[Botrychium matricarbafolium A. Braun] - GPF

[Botrychium matricariasfolium A. Braun ex K. Koch subsp. hesperium Maxon \& Clausea] - HDH

Botrychium lanceolatum (S. G. Gmel.) Angstr. subsp. lanceolatum - FNA, HDH

[Botrychium lanceolatum (S, G, Gmel, Ángstr, var, lanceolatum] - KTZ, EW

[Botrychium lanceolatam (S, G. Gmel.) Angstr.] - Dom01, GPF, IMF, SFt, UTF, W\&W

Botrychium lanceolatum subsp. nov, "viride" Farrar ined.

[NS - information from S. Popovich and D. Farrar, Aug. 2007]

Botrychium lineare W, H. Wagner - KTZ, SFE, W\&W, E

Botrychium lunaria (L.) Sw. - Dorn01, FNA, GPF, IMF, KTZ, SF!, UTF, E/W

[Botrychium lunaria (Li) Sw. subsp. lunsaria] - $\mathrm{HDH}$

[Borrychium lunaria (L.) Sw. var. lunaria] - RMC

[Botrychium lanaria (L.) Sw, subsp. occidentalis A. Lőve, D. Love \& B. M. Kapoor] - W\&W

[Botrychium lanaria (L.) Sw, var, onondagense (Underw, House] - RMC

Botrychium minganense Vict. FNA, GPF. KTZ, UTF, W\&W, err. rep., W\& W ${ }^{1}$

[Botrychium "colorado" W. H. Wagaer, unpubl.] - W\& W'

[Botrychium hinaria (L.) Sw, var, minganense (Vict.) Dole] - HDH

Botrychium multifidum (S. G. Gmel.) Rupr. - FNA

[Botrychium multifidum (S, G. Gmel.) Trevis.] - Dom01, GPF, KTZ, UTF, E/W

[Botrychium multifidum (S, G, Gmel.) Rupr, subsp, coulteri (Underw, ) Claasen] - HDH, SFI, W \& W

[Secpridium multifidum (S, G, Genel) Nashida ex Tagawa] - Jepson Manual 2 (ined.)

Botrychium paradoxum W. H. Wagner 
[NS - collected 2006 in WY \{D. Farrair, pers. comm., Aug. 2007\} and possibly collected in CO sumumer 2007 but needs genetic confirmation \{S. Popovich, pers. comm.., Aug. 2007\}]

\{Botrychium pallidum W. H. Wagner\} - Snow07b

INS - genetic testing by Farrar indicates these are all sp. nov. "redbank" \{S. Popovich pers. comm., Sep. 2008;

Botrychium pinatum H. H. St. John - FNA, KTZ, SF!, W\&W, E'W

[Botrychium boreale (Fr.) Milde subsp. ubtusilobum (Ruprect) Clausen] - D. Famar, pers. comm.

Botrychiumx simplex E. Hitchc, - Dorn01, FNA, GPF, IMF, KTZ, SF!, UTF, W\&W', EW

[NS -multiple infraspecific assignments masy prove to be present in Colorado based on recent work by Farrar and Popovich (S. Popovich, pers. comm. Oct 2007)]

[Botrychium simplex A. S. Hitche.] - W\&W

[Botrychium simplex E. Hitche, subsp. typicum Clausen] - $\mathrm{HDH}$

[Botrychium simplex E. Hitche. var. tenebrosum (A. A. Eaton) R. Clausen] -May not a valid synonym for

Rocky Mountain material, D. Farrar, pers. comm.. Aug. 2007]

- Botrychium sp. nov. "redbank". Fartar \& Popovich ined. ENDEMIC <CO, NM>

[NS - the species occurs in Colorado and NM, Legler and Popovich, pers. comme.]

Botrychium taxon nov, "furcatum", Farrar ined.

[NS - Steve Popovich, pers, comm.. Feb, 2008]

Botrychium tunux Stenswold \& Farrar

[NS - collected in CO in 2007 and confirmed genetically [S. Popovich, pers. comm.. Oct. 2007\}]

Botrychium virginianum (L.) Sw, - Dorn01, FNA, GPF, KTZ, E/W

[Botrychium virginianum (L.) Sw, subsp. curopacum (Angstr.) Clausen] - HDH

[Botrychium virginianum (L.) Sw. var, virginianum] - RMC

[Borrypus virginianus (L.) Holob] - SF!

[Bolrypus virginianus (L.) Holob subsp. europaeus (Angstr.) Holob] - W\& W

\section{Botrypus (see Botrychium)}

\section{Polypediaceae - PL.P}

(Identification sources:

Polypodium hesperium Maxon - FNA, GPF, HDH, IMF, KTZ, SFW, UTF, W\&W, W

[Polypodium valgare L. subsp, columbianum (Gilbert) Huhtén] - FNA

[Polypodium valgare L. var, columbianum Gilbert] - FNA

[Polypodium valgare L, var, hesperium (Maxon) A. Nelson \& F, I. MacBr,] - FNA

Polypodium saximontanum Windham - Dom01, FNA, KTZ, SF!, W\&W', E/W

[Polypodium artorphum Suksd] - W\&W, W\& $W^{1}$, err rep.

Pteridaceae - PTR (including Adiantaceae, Cryptogrammaceae, Polypodiaceae, Sinopteridaceae)

(Identification sources:

Adiantum aleuticum (Rupr.) Paris - FNA, KTZ, SFW, W\&W', W

[Adiantum pedatum L.]-GPF, IMF, W\&W, W\&W!

[Adiantum pedatum L. var, aleuticum Rupe.] - UTF

Adiantum capillus-veneris L. - FNA, GPF, HDH, IMF, KTZ, SF!, UTF, W\&W, ENW

Argyrochosma fendleri (Kunze) Windham - Dorn01, FNA, KTZ, SF!,W\&W, E/W

[Notholacia fendleri Kunze] - GPF, HDH 
Astrolepis cochisensis (Goodding) Benham \& Windham subsp, cochiensis - FNA. KTZ [Astrolepis cochisensis (Goodding) Benhan \& Windham] - SFE, E

Astrolepis integerrima (Hook.) D. M. Benham \& Windham - FNA. KTZ, W\& W'

[Notholbena sinuata (Sw.) Kauli, var. integerrima Hook.] - GPF

Cheilanthes (see also Notholaena)

[NS - Cheilanthes is polyphyletic [Kirkpatrick07] although our members generally fall into a single clade]

Cheilanthes eatonii Baker - FNA, GPF, HDH, IMF, KTZ, SFE, UTF, W\&W, E

Cheilanthes feei T. Moore - Dorn01, FNA, GPF, HDH, IMF, KTZ, SF!, UTF, W\&W, E/W

Cheilanthes fendleri Hook. - FNA, GPF, HDH, KTZ, SF!, W\&W, E/W

Cheilanthes wootonii Maxon - FNA, GPF, HDH, KTZ, SFE, UTF, W\&W, E

Cryptogramma acrostichoides R. Br, - Dorn01, FNA, HDH, KTZ, SF!, W\&W, EWW

[Coyptogramma crispa (L.) R. Br. var. acrostichoides (R. Br.) C. B. Clarke] - UTF

[Cryptogramma crispa (L.) R. Br. ex Hook. yar, acroslichoides (R. Br.) C. B. Clarke] - IMF

Cryptogramma stelleri (S, G. Gmel.) Prantl - FNA, HDH, IMF, KTZ, SFW,UTF, W\&W, W

Notholaena (see also Argyrochosma, Astrolepis)

Notholaena standleyi Maxon - FNA, GPF, HDH, KTZ, SFE, W\&W', E

[Cheilanthes standloyi (Maxon) Mickel] - W\&W

Pellaea atropurpurea (L.) Link - Dom0I, FNA, GPF, HDH, KTZ, SFE, UTF, W\&W, E

Pellaea breweri D. C. Eaton - Dorn01, FNA, HDH, IMF, KTZ, SFW, UTF, W\&W, W

Pellaea glabella Mett. subsp. simplex (Butters) A. Löve \& D. Lobve - SFW

[Pellaea glabella Mett. ex Kuhn] - UTF, E.W

[Pellaca glabella Metr.] - SFE

[Pellaea glabella Mett. ex Kulan subsp. simplex (Butners) A. LWwe \& D. Love] - FNA. GPF, KTZ, W\& W'

[Pellaea glabella Mett. ex Kuhn var. simplex Butters] - IMF

[Pellaea suksdorfianta Buters] - Dern01, HDH, W\& W

Pellaca glabella Mett. ex Kuhn subsp, occidentalis (E. E. Nelson) Windham - FNA.KTZ. W\&W'

[Pellaea glabella Mett.] - SF:

[Pellaea glabella Mett, ex Kuln] - UTF

[Pellaea glabella Mett. ex Kuba subsp. oscidencalis (E. E. Nelson) Windham]

[Pellaea glabella Mets. ex Kuhn var, occidentalis (E. E. Nelson) Buters] - GPF, HDH

[Pellaes occidental is (E. Nelson) Rydb.] - Dom01. W\&W, E/W

Pellaea truncata Goodding - FNA, IMF, KTZ, SFE, UTF, W\&W, E

[Pellaca longimucronata Hook.] - HDH

Pellaea wrightiana Hook, - FNA, GPF, GPF, KTZ, SFE, UTF, W\&W, E

Salviniaceae ( $\sec$ AZL)

Sinopteridaceae (see PTR)

Thelypteridaceae - THL

Phegopteris connectilis (Michx.) Watt - FNA, KTZ, SFW, W\&W', W

Woodsiaceae (sce DRY)

Snow, N, Junuary 2009. Checklist of Vacculer Plants of the Southom Rodky Momain Region. CVersion $3 x$ 


\section{GYMNOSPERMS}

\section{Cupressaceae = CUP}

(Identification sources: )

Juniperus communis L. var, depressa Pursh - Dom01, FNA. IMF, KTZ, UTF, EWW [Juniperus communis L.] - GPF

[Juniperus communis L. stubsp. alpina (J. E. Sm.) Celak.] - SF!, W\&W, misapo.

[Juniperus communis L. var. montand Aiton] - HDH

[Juniperus commun is L. var. saxatilis Pall.] - HDH

Juniperus horizontalis Moench - Dom01, GPF, HDH rep., KTZ, UTF, W\&W

[Juniperus horizonsalis var. douglasii hort.] - KTZ

[Juniperus horizontalis var, variegata Beissn.] - KTZ

[Juniperus hadsonica Forbes] - KTZ

[Juniperus prostrafa Pers.] - KTZ

[Juniperus repens Nutt.] - KTZ

[Juniperus virginana var. prostrata (Pers.) Tort.] - KTZ

[Sabina horizontalis (Mosech) Rydb.] - KTZ. W\&W'. SFE

[5abina prostrata (Purs.) Antoine] - KTZ

Junipens monosperma (Engelm.) Sarg - FNA, GPF, HDH, IMF, KTZ, UTF, E [Sabina monosperma (Engelm.) Rydb.] - SFE, W\&W

Juniperus osteosperma (Torr.) Little - Dom01, FNA, IMF, KTZ, UTF, W

[Juniperus utahensis (Engelm.) Lemmon] - HDH

[Sabina osteosperna (Tor.) Antoine] - SFW, W\& W

Juniperus scopulorum Sarg. - Dorn0I, FNA, GPF, HDH, IMF, KTZ, UTF, ENW

[Sabina scopulonum (Sarg.) Rydb.] - SF!. W\& W

Juniperus virginiana L. - FNA, GPF, E

[W] - more data are needed to establish the occurrence of this raxon in Colorado]

[Juniperus virginiana L, var, virginikna] - KTZ

[Sabina virginians (L.) Antoine] - SFE. WE W'

Sabina (see Juniperus)

Ephedraceae - EPH

(Identification sources:

Ephedra cutleri Peebles - FNA, IMF, KTZ, SFW, W\&W', W

[Ephedra viridis Coville var, viscida (Cutler) L. D, Benson] - HDH, UTP, W\& W

Ephedra torreyana S, Watson - FNA, GPF, HDH, IMF, SFW, UTF, W\&W, W

[Ephedra tomeyana S. Watson var, torreyana]-KTZ

Ephedra viridis Coville - Dorn01, FNA, IMF, K1Z, SFW, W

[Ephedra viridis Coville var, viridis] - HDH, UTF, W\&W

Pinaceac - PIN

(Identification sources:

Abies bifolia A. Murray var- arizonica (Mirriam) O'Kane \& Heil - FCF

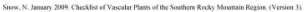


[Abies arizonica Merriam] - SF!, WE W, EWW

[Ábies lasiocarpa (Hook.) Nutt. var. ariznaica (Merriam) Lemmon] - HDH, IMF, KTZ, misapp.

Abies bifolia A. Murray var. bifolia - FNA, SF!, W\&W ${ }^{1}$, E/W

[Ábies lasiocarpa (Houk.) Nutt.] - Dorn01, UTF, W\&W, err. rep.

[A bics lasiocarpa (Hook.) Nutt. var. lasiocarpa] - HDH, IMF, KTZ, RMC, misapp-

Abies concolor (Gord. \& Glend.) Hildebr. - Dorn01, FNA, EN

[Abies concolor (Gord.) LindL] - SF!

[Abies concoloe (Gord.) Coopes] - HDH

[Abies concolor (Gord. \& Glend.) Lindl. ex Hildebr.] - W\&W

[Abies concoloe (Gord. \& Glend.) Lindl.] - UTF

[Abies concolor (Gord. \& Glend.) Lindl. ex Hildebr. var. concolor] - IMF, KTZ, RMC

Picea engelmannii Parry ex Engelm, var. engelmannii - FNA, E/W

[Pioca engelmannii Parry] - Doniol, HDH

[Pioca engelmannii Parry ex Engelm.] - IMF, KTZ, UTF, W\& W

[Pices engelmannii (Parry) Engelm.] - SF!

Picea glauca (Moench) Voss - Dorn01

[Picat cantadensis (P. MilL) B. S. P.] - KTZ

[Picka glauca var. albertina (S. Br.) Sary.] - KTZ

[Picea glauca var. densata Bailcy]- KTZ

[Picka glauca vart, porsildii Raup] - KTZ

Picea pungens Engelm, - Dorn01, FNA, HDH, IMF, KTZ, SF!, UTF, W\&W, EW

Pinus aristata Engelm. - FNA, HDH, IMF, KTZ, SF!, W\&W, E/W

Pinus contorta Douglas ex Loud, var. latifolia Engelm. - Dorn01, FNA. HDH, W\&W, EJW

[Pinus contorta Douglas subsp, latifolia (Engelm, $)$ Cricchf.] - SF!

[Pinus contorta Douglas ex Loud. var. latifolla Engelm. ex S. Wanson] - GPF, IMF, KTZ, UTF

[Pinus murrayana Balf.] - HDH, corr. \& add.

Pinus edulis Engelm - Dorn01, FNA, GPF, HDH, IMF, KTZ, SF!, UTF, W\&W, E/W

Pinus flexilis E. James - Dorn01, FNA, GPF, HDH, IMF, KTZ, SF!, UTF, W\&W, E/W

Pinus ponderosa Douglas ex C. Lawson var, scopulorum Engelm. - FNA, E/W

[FL - any inclusion of $\mathrm{P}$. Lawson in authorship for this species is incerrect]

[Pinus ponderosa P. \& C. Lawsoe] - Donx01, GPF, IMF, UTF

[Pinus ponderosa Douglas ex P. Law son] - HDH

[Pinus ponderosa Douglas subsp. scopulorum (S. Watson) W. A. Wether] - SF:

[Pinus ponderosa Douglas ex P. \& C. Lawson subsp. scopalonum (S. Watson) W. A. Weber] - w\& W

[Pinus ponderosa P. \& C. Lawson var. scopulocum Engelm.] - KTZ

Pinus strobiformis Engelm. - FCF, FNA, HDH, KTZ, SF!, W\&W, E/W

[W] - CO specimens likely hybrids with P. flexilis; more study needed - consult Dkana Tombask, CU-Denwer]

[NS - probsibly cones clase no our area in NM from south fide FNA]

[Pinus ayacahuite Ehrenberg var, brachyptera G, R, Shaw] - FNA

[Pinus ayacahuite var. reflexa (Engelmann) Voss] - FNA

[Pinus ayacahuite var, strobiformis (Engeimann) Lemamon] - FNA

[Pinus flexilis E. James var. Y reflexa Engelmann] - FNA

[Pinus reflexa (Engelmann) Engelmann] - FNA

Pseudotsuga menziesii (Mirb.) Franco var. glauca (Beissn.) Franco - Dorn01, IMF, KTZ E/W

[Psendotsaga menziesii (Mirb, F Fanco] - HDH, corf, \& add, SFI, W\&W

[Pscudotsaga menziesii (Mirb.) Franco var. glauca (Mayr) Franco] - FNA

[Pseudotsaga menziesii (Mirb.) Franco var, glacea (Beísso.) Mayr] - UTF

[Pseadorsaga taxifolia (Podr.) Britton ex Sudworth] - $\mathrm{HDH}$ 


\section{ANGIOSPERMS}

Aceraceae (see SAP)

Acoraceae $=\mathrm{ACO}$

Aconus calamus L. - FNA, HDH, KTZ, SFE, W\&W

Adoxacene - ADX

Adoxa moschatellina L. - Dom01, GPF, HDH, IMF, KTZ, SF!, UTF, W\&W, W

Agavaceae - ASG

Yucca

(Identification sources:

Yucea angustissima Engelm, ex Trel, var- angustissima - FCF, HDH, IMF, KTZ, UTF, WJ, W\&W, misapp.

[NS - FNA does not recognize from CO]

Yucca baccata Torr, var, baccata - FCF, IMF, KTZ, E/W

[Yuces baccata Torr.]-FNA, GPF, HDH, SFl, UTF, WJ, W\& W

[Yucea baccata var, vesportina McKelvey] - FNA

[Yucea vespertina (MeKelvey) S. L. Welsh] ] - FNA

Yueca baileyi Wooton \& Standl, var, baileyi - FCF, IMF, KTZ

[Yucea baileyi Wooton \& Standl.] - FNA, GPF, UTF, WJ

[Yucea baileyi Wooton \& Standl, var. navajoa (J. M. Webber) J. M. Webber] - FNA

[Yuces glauca Nurt.] - HDH. W \& W

[Yucea navajoa J.M. Webber] - FNA

[Yuces standleyi MeKelvey] - HDH, FNA

Yucea glauca Nutt. var. glauca - GPF, KTZ, E/W

[Yucen angesrifolia Pursh] - FNA

[Yucen glauen Nurt.] - Domo1, ENA, SF1, WJ, W\&W

[Yucea glauca Nurt. var. gurneyi McKelvey] - FNA, KTZ

[Yucea glanea Nurt. var. stricta (J. Sims) Trelense] - FNA

[Yucea standkyi McKelvey] - HDH

[Yucea stricta $J$. Sims] - FNA

Yucca harrimaniae Trel. var, harrimaniae - IMF, KTZ, W

[Yueen harrimanise Trel.] - FNA, HDH, SFW, UTF, W], W\&W

Yucca neomexicana Wooton \& Standl. - FNA. WJ

[Yucea harrimanise Trel, var, necenexicana (Wooton \& Standl.) Reveal - GPF, IMF $\angle C O=$, KTZ

[Yucea glasea Nurt] - W\&W

Aizoaceac - AIZ (sce also MOL)

Sesuvium verrucosum Raf, - GPF, HDH, KTZ, SFE, UTF, W\& W, E

* Trianthema portulacastrum L. - GPF, KTZ, UTF

Snou, N, January 2009. Checklist of Vascular Plants of the Southom Rocky Mosmain Region. CVersion 3 . 


\section{Alismaceae (sec ALI)}

\section{Alismatacene - ALI}

(Identification sources:

Alisma gramine um Lej. - Dom01, FNA, IMF, KTZ, SF!, UTF, W\&W, EWW

[Alisma geyeri Torr.]-HDH

[Alisma gramineum J. G. Gmel.] - GPF

Alisma subcordatum Raf, - FNA, GPF, KTZ

Alisma triviale Pursh - Dorn01, FNA, GPF, KTZ, SF!, W\&W, EW

[Alisma plantago-aquatica L.] - HDH, UTF, W\& W'

[Alisma plantago-squatica L. var. americanum Schult. \& Schult. f.] - ]MF

Sagittaria brevirostra Mack, \& Bush - FNA, GPF, KTZ, SF!, W\&W', E/W

Sagittaria cuneata E. Sheld, - FNA, GPF, HDH, IMF, KTZ, SF!, UTF, W\&W, EN

Sagittaria demersa J. G. Smith - FNA

Sagittaria graminea Michx, var-graminea - KTZ, E

[Sagittaria graminea Micho.]-SFE, W\&W

[Sagittaria granirea Mictox. subsp. graminea] - FNA

Sagittaria latifolia Willd. - FNA, GPF, HDH, KTZ, SF!, UTF, W\&W, E/W

[Sagittaria latifolia Willd. var. latifolia]-IMF, RMC

\{Sagittaria longiloba Engelm, ex J. G. Sm.\} - FNA, KTZ

\{Sagittaria longiloba Engelm.; - GPF, HDH, nep.

(Sagittaria longiboba Engelm. ex Tors.\} - w\&W, em. nep.

Sagittaria montevidensis Cham. \& Schltdl. subsp. calycina (Engelm.) Bogin - FNA, SFE, W\&W,E

[Sagittaria calycina Engelm.] - GPF, HDH, exp.

[Sagittaria calycina Eogetm. var. calycina] - KTZ

Alliaceae - ALL (see also THE)

(Identification sources:

Allium acuminatum Hook, - Dorm01, FNA, HDH, IMF, RMC, KTZ, SF!, UTF, W\&W, E/W

[Allium acuminatum var. cuspidarum Fernald] - FNA

[Allium cuspidatum (Fernakd) Ryob.] - FNA

\{Allium bisceptrum S. Watson\}-FNA, UT3

INS - cecurs in San Juan Co, UT, in variety of habitans; to be expectod in COF

Allium brandegeci S. Watson - Dorn01, FNA, HDH, IMF, KTZ, SF!. UTF. W\&W. E/W

[Allium brandegei S. Watson forma viviparum W. A. Weber] - SFW, W\&W

[Allium dichlik (M. E. Jones) M. E. Jones] - FNA

[Allilum minimum M. E. Joses] - FNA

[Allium tribrachteatum Torrey var, diehliii M. E. Jones] - FNA

Allium brevistylum S. Watson - Dorn01, FNA, HDH, IMF, RMC, KTZ, SF!, UTF, W\&W, E/W \{Allium cepa L.\}

[W] - cultivared abundantly in Weld Co, and elsewhere and likely persisting occasionally)

Allium cernuum Roth - Dom01, FNA, GPF, IMF. SF!, UTF. W\&W. E'W

[Allium allegheniense Small] - FNA 
[Allium cerneum Roth var. cernumu] - KTZ

[Allium cerneum Roth var. obtusam Cockerell] - HDH

[Allium cerntum Roth var, obtusum Cockerell ex I. F. Macbr.] - KTZ

[Allium oxyphilum Whemy] - FNA

[Allium recurvatum Rydb.] 0 FNA

\{Allium drummondii Regel\} - GPF. HDH rep., KTZ, W\&W', misid.

(NS - native range comes close to ours fide FNA in WY. CO and NM: to be expected?

\{Allium helleri Small\} - FNA

\{Allium nuttallii S. Watson\} - FNA

(Allium reticulatum Fraser ex G. Don var. nuttallia (S. Watson) M. E. Jones) - FNA

Allium geyeri S. Watson var geyeri - Dom01. FNA, IMF, KTZ, UTF, E/W

[Allium dictyotum Greene] - FNA

[Allium funiculosum A. Nels.] - FNA

[Allium geyeri S. Wasson] - GPF, HDH, SF!, W\&W

[Allium pikeanum Rydb.] - FNA

Allium geyeri S. Watson var. tenerum M. E. Jones - Dorn01, FNA, IMF, KTZ, E/W

[Allium arenicola Osterh.] - FNA

[Allium fibrosum Rydb.] - FNA

[Allium geyeri var-graniferum Henderson] - FNA

[Allium rubrum Osterh.] - HDH, SF!, W\&W

[Allium rydbergii J. F. MacBride] - FNA

[Allium sabulicola Osterh.] - FNA

Allium macropetalum Rydb, - FNA, HDH, IMF, KTZ, SFW, UTF, W\&W, W

[Allium desericola (M. E. Jones) Woot \& Standi.] - FNA

[Allium reticulatum Fraser ex G. Don var, deserticola M. E. Jones] - FNA

Allium nevadense S. Watson - FNA, FCF, HDH, IMF, KTZ, SFW, UTF, W\&W, W

[Allium nevadense var. macropetalum M. Pock] - FNA

* Allium sativum L, - GPF, IMF, KTZ, UTF, W\&W

\{Allium perdulee S. V. Fraser var, perdulce\} - FNA

[NS - range comes close to ours in eastern $\mathrm{CO}$ and NM fide FNA; to be expected]

Allium schoenoprasum L. var. sibiricum (L.) Hartman - KTZ, W\&W, E/W

[Allium schoenoprasum L.] - HDH, IMF, SF!, UTF

Allium textile A. Nelson \& J. F. Macbr - Dom01, FNA, GPF, HDH, IMF, KTZ, SF!, W\&W, $\mathrm{E} / \mathrm{W}$

[Allium aridum Rydb.] - FNA

[Allium reticularum Fraser ex G. Don 1827, non J. Presl \& C. Presi 1817] - FNA

[Allium reticulatum Fraser ex G. Don var, playanum $M$. E. Jones] - FNA

Androstephium (see THE)

Triteleia (see THE)

Alsinaceae (see CRY)

Amaranthaceae - AMA (including much of former Chenopodiaceae)

Acnida (sce Amaranthus)

Amaranthus

(Identification sources: 
* Amaranthus albus L, - Dom01, GPF, HDH, KTZ, SF!, UTF, W\&W, EW

[Amaranthus pabescens (Uline \& Bray) Rydb.] - HDH rep., SEE, W\&W

Amaranthus arenicola L. M. Johnst, - Dom01, GPF, KTZ. SFE, W\&W, E

[Amaranchus torreyi (A. Gray) Benth.] - HDH

Amaranthus blitoides S. Watson - Dorn01, KTZ, SF!, UTF, W\&W', E/W

[Amaranthus saccizans L.] - GPF, HDH

Amaranthus ealifomicus (Moq.) Wats. - Dorn01, FCF, GREE; Smith09?

[Amaranthus albomatginatus Uline \& Bray]-KTZ

[Amaranthus mictophy lles Shinrers] - KTZ

* \{ Amaranthus hybridus L.\} - GPF, HDH, KTZ, UTF, W\&W', doubtful

* Amaranthus palmeri S. Watson - GPF, HDH, KTZ, SFE, UTF, W\&W, E

Amaranthus powellii S, Watson - Dorn01, GPF, KTZ, SFW, UTF, W\&W, W

* Amaranthus retroflexus L. - Dorn01, GPF, HDH, KTZ, SF!, UTF, W\&W, E/W

\{Amaranthus rudis J, D. Sauer\} - GPF, KTZ <CO?

\{Acnida tamarissina (Nutt.) A. W. Wood\} - HDH

\{Amaranthus tamariacinus Nutc.\} - W\&W, misapp.

\{Amaranthus tuberculatus (Mog, J. D. Sauer) - GPF, KTZ $\angle \mathrm{NE}>$, W\&W, misapp.

\{Acnida altissima Riddell] - HDH

Amaranthus wrightii S. Watson - HDH rep, KTZ, SFE, W\&W, E

\section{Atriplex (see also Grayia, Proatriplex)}

(Identification sources:

Atriplex argentea Nutt. var, argentea - E/W

[Atriplex argentea Nutt.] - SFL UTF, W 8 W

[Atriplex argentea Nutt. subsp. argentes] - GPF. HDH, RMC

[Atriplex argentea Nutt. subsp. argentea var. argentea] - KTZ

Atriplex argentea Nutt. var, caput-medusae (Eastw.) Fosberg - KTZ

[Atriplex argentea Nutt, subsp, argentea var, capul-riodusae (Eastw.) Fosbere] - KTZ

[Atriplex sacceria S. Watson var, caput-moducae (Eastw,) S. L. Welsh] - UTF

Atriplex argentea Nutt. subsp. expansa (S. Wats.) H. M. Hall \& Clem. - GPF, HDH. KTZ, E/W

[Atriplex angentea Nutt.]-SFL. W\&W

Atriplex canescens (Pursh) Nutt. var. aptera (A. Nels.) C. L. Hitchc. - Dorn01

[Atriplex apters A. Nelson] - W\&W'

[Atriplex X apsera A. Nelson] - KTZ

[Atriplex cabescens X A. gardneri] - KTZ

[Atriplex canescens (Pursh) Nutt. subsp. aptera (A. Netson) H. M. Hall \& Clem.] - HDH, exp.

[Atriplex gardncti (Moq.) D. Dietr. var. aptera (A. Nelson) S. L. Welsh \& Crompton] -

Atriplex canescens (Pursh) Nutt, var. canescens - Dom01, KTZ, E/W

[Atriplex canescens (Pursh) Nutt.] - GPF, SF

[Atriplex cancscens (Pursh) Nutt, subsp, canescens] - HDH, W\&W

[Atriplex canescens (Pursh) Nutt, var, oecidentalis (Torr. \& Frem. S S.L. Welsh \& Stutz]- UTF

Atriplex confertifolia (Torr. \& Frèm.) S. Wats. - Dorn01, GPF, HDH, KTZ, SF!, W\&W, E/W

Atriplex corrugata S. Watson - HDH, KTZ, SFW, UTF, W\&W, W

Atriplex cuneata A. Nelson var, cuneata

[Atriplex cuncata A. Nelson subsp. cuncata] - KTZ

[Atriplex gardneri (Moq.) Standl.] - W\&W

[Atriplex gardneri (Moq,) D. Dietr, var, cuncata (A. Nelson) S. L. Welsh] - UTF

[Atriplex nutallii S. Watson subsp. cuneata (A. Nelsoa) H. M. Hall \& Clem ] - HDH

Atriplex gardneri (Moq.) D. Dietr. var. gardneri - Dorn01, UTF, E/W 
[Atriplex gardneri (Moq.) D. Dietr.] - SF!

[Atriplex gardneri (Moq.) Standl.] - SF!, W \& W

[Atriplex nutallii S. Watson] - GPF, KTZ

[Atriplex nutallii S. Watson suhsp. gardiseri (Moq-) H. M. Hall \& Clem.] - HDH

[Atriplex nutiallii S. Watson subsp. nuttallii] - HDH

Atriplex gardneri (Moq.) D. Dietr. var. utahensis (M. E. Jones) Dorn - Dorn01. EW

[Atriplex gardneri (Moq. I Standl.] - SF!. W\&W

[Atriplex gardneri (Moq.) D. Dietr. var. tridentata (Kuntre) Macbe_] -UTF

[Atriplex nuttallii S. Watson] - GPF

[Atriplex nuttallii S. Watson subsp. tridentata (Kuntze) H. M. Hall \& Clem.] - HDH

[Atriplex iridentata Kuntze] - KTZ

Atriplex garretti Rydb. - KTZ, UTF

[Atriplex canescens (Pursh) Nutt. subsp. garretti (Rydb.) H. M. Hall \& Clem.] - HDH, W \& W

Atriplex graciliflora M. E. Jones - KTZ, SFW, UTF, W\&W, W

* Atriplex beterosperma Bunge - Dorn01, GPF, SF!, UTF, W\&W, EW

[Atriplex micrantha Ledeb.] - KTZ

* Atriplex hortensis L. - Dorn01, GPF, HDH, KTZ, SF!, UTF, W\&W, E/W

Atriplex obovata Moq. -HDH, KTZ, SFW, UTF, W\&W, W

Atriplex pachypoda Stutz \& Chu - KTZ, W\&W

+ Atriplex patula L. var. patula - UTF, E/W

[Atriplex patala L.] - KTZ, SF!, W\& W'

[Atriplex patula L. subsp. patula] - HDH

Atriplex powellii S. Watson - Dorn01, GPF, HDH, KTZ, SF!, UTF, W\&W, EW

$\div$ \{Atriplex prostrata Bouchér ex DC, \}-KTZ, W\& W', misid

* Atriplex rosea L, - Dom01, GPF, HDH, KTZ, SF!, UTF, W\&W, E/W

Atriplex sacearia S. Watson Dorn01, - HDH, KTZ, SF!, W\&W, E/W

[Alriplex saccaria S. Watson var. stccaria] - UTF, IMF

Atriplex subspicata (Nutt.) Rydb, - Dorn01, KTZ

[Airiplex patula L.] - WNW

[Atriplex patula L subsp. hastata (L.) H. M. Hall \& Clem.] - HDH, KTZ

[Atriplex patula L var, triangularis (Willd.) K. Thome \& S. L. Welsh] - UTF

[Atriplex prostrata Boucher ex DC. subsip. triangularis (Willd.) Rauschert] - W\&W. W\& W', err. rep.

Atriplex suckleyi (Torr.) Rydb, - Dorn01, SFW, W\&W, W

[Atriplex dioica (Nutt.) J. F. Macbr.] - GPF, HDH, exp.

[Endolepis dioica (Nuct.) Stand1.] - KTZ

Atriplex tenuissima A. Nelson -KTZ

[Ariplex tumuissima A. Nelsoe - HDH

[Atriplex woltii S. Watson] - UTF, W\&W

Atriplex truncata (Torr. ex S. Watson) A. Gray - Dom01, KTZ, E/W

[Atriplex trumcata (Tocr.) A. Gray] - HDH, SF!, UTF, W\& W

Atriplex wolfii S. Watson - Dom01, HDH, KTZ, SFW, UTF, W\&W, W

* Axyris amaranthoides L. - GPF, KTZ, SF!, W\&W, E/W

Bassia (sce also Kochia)

Bassia americana (S. Watson) Scott - Dorn0I

Bassia hyssopifolia (Pall.) Kuntze - Dom0I, FCF, GPF, HDH, KTZ, SF!, UTF, W\&W, E/W

Ceratoides (see Kraseheninnikovia)

Chenopodium 
(Identification sources:

* Chenopodium album L. var, album - KTZ, UTF, E/W

[Chenopodium album L.] - Dorn01, GPF, HDH, SF!, W\&W

Chenopodium album L. var, missouriense (Aellen) I. J. Bassett \& C. W. Crompton - W \& W

[Chemopodium berlandieri Moq. var. bushianum (Aellen) Cronquist] - KTZ $<$ KS $>$, W\&W

[Chenopodium paganum Rchb.] - HDH, exp.

* Chenopodium ambrosioides L. var. ambrosioides - KTZ, E/W

[Chenopodium ambrosioides L.] - GPF, HDH. UTF

[Dysphania ambrosioides (L.) Mosyakin \& Clemants] - FNA

[Teloxys ambrosioides (L) W. A. Weber] - SF!. W\&W

Chenopodium atrovirens Rydb, - Dorn01, GPF, HDH rep, KTZ, SF!,UTF, W\&W, E/W

[Chenopodium aridum A. Nelson] - W\& W'

Chenopodium berlandieri Moq, var, berlandieri - KTZ, E/W

[Chenopodium albam L. var. berlandicri (Moq.) Mack. \& Bush] - UTF

[Chenopodium berlandieri Moq.] - HDH, SF!, W\&W

Chenopodium berlandieri Moq, var, boscianum (Moq.) H. A. Wahl-KTZ, E/W

[Chenepodium standiley anum Aellen] - GPF, HDH, exp.

[Chenopodium berlandieri Moq.] - SF!, W\&W

Chenopodium berlandieri Moq, var. zschackei (Murray) Murr ex Asch. - Dorn01, KTZ, E/W

[Chenopodium berlandieri Moq. var. zschackei (Murray) Murray] - GPF

[Chenopodium berlandieri Moq.] - SF!, W\&W

* Chenopodium botrys L. - Dorn01, GPF, HDH, KTZ, UTF, E/W

[Dysphania botrys (Lianaeus) Mosyakin \& Clemanss] - FNA

[Teloxys boxrys (L.) W. A. Weber]-SF!, W\&W

* Chenopodium capitatum (L.) Ambrosi var, capitatum - FNA

[Chenopodium capitatum (L.) Asch. var. capitatum - Dom0I, UTF

[Chenopodium capitafum (L.) Asch.] - GPF, HDH, SF!, W\& W, EWW

[Clecoopodium capicatum (L.) Ambrosi] - KTZ

* Chenopodium capitatum (L.) Ambrosi var. parvicapitatum S. L. Welsh - FNA

[Blicum hastatum Rydb.] - FNA

[Chenopodium oweri A. Helker] - GPF

Chenopodium cycloides A. Nelson - GPF, HDH, KTZ, SFE, W\&W, E

Chenopodium desiccatum A. Nelson - Dorn01, GPF, KTZ, SFE, UTF, W\&W, E

[Chenopodium leptophyllum (Nutr, ex Moq.) S. Watson var, obkongifolium S. Watson] - W\&. W

* Chenopodium foliosum (Moench) Asch. - KTZ, SF!, W\&W, E/W

[Chenopodium capicatum (L.) Asch, var, parvicapitatum S. L. Welsh] - UTF

Chenopodium fremontii S. Watson - Dom01, GPF, HDH, SF!, W\&W, E/W

[Chenopodium fremontii S. Watson var, fremontii] - KTZ, UTF

* Chenopodium glaucum L. var. glaucum-Dorn01, E/W

[Chenopodium gloucum L.] -GPF, KTZ, SFL, W\&W

[Chcoopodium glaseum L subsp. glaseum] - $\mathrm{HDH}$

Chenopodium glaucum L. var. salinum (Standl.) Boivin - Dorn01

[Chenopodium salinum Standl.] - KTZ

[Chenopodiun glaucum L.] - GPF, SF!, W\&W

* Chenopodium graveolens Willd. - HDH, KTZ, E/W

[Dysphania graveolens (Willdesow) Mosyakin \& Clemants] - FNA

[Teloxys graveolens (Willd.) W. A. Weber]-SFI, W\&W

Chenopodium hians Standl. - Dorn01, HDH, KTZ, W\&W, E

[Chenopodium atrovirens Rydh.] - UTF

[Chenopodium album L.] - SFE 
* [Chenopodium humile Hook. $\}-$ KTZ, UTF

\{Chenopodium foliosum (Moench) Asch.\} - W\& W'

Chenopodium incanum (S. Watson) A. Heller var, incanum - GPF, KTZ, EW

[Chenopodium fremontii S. Watson var. incanum S. Watson] - UTF

[Chenupodium incanum (S. Watssa) A. Heller] - Darn01, HDH, SF!, W\&W

Chenopodium leptophyllum (Moq.) Nutt. ex S. Watson - Dom01, KTZ, EW

[Chenopodium leptophyllum Nutt.] - HDH

[Chenopodium leptuphyllum (Moq.) S. Watson] - UTF

[Chenopodium leptophyllum (Nutt.) S. Watson] - SF!

[Chenopodium leptophyilum Nutt. ex Moq.] - GPF

[Chenopodium leptophyllum (Nutt, ex Moq.) S. Watson var. leptophyllum] - W\&W

\{Chenopodium murale L.\} - HDH, exp., KTZ

Chenopodium overi Aellen - HDH, KTZ, EW

[Chenopodium foliosum (Moench) Asch.] - SF

[Chemopodium overi A. Hellsr] - GPF

Chenopodium pallescens Standl. - Dorn01, GPF, HDH, KTZ, W\&W, doubtful

Chenopodium polyspermum L. - FCF

Chenopodium pratericola Rydb, - Dorn01, GPF, HDH, KTZ, SFE, W\&W, E

[Chemopodium albescems Small] - HDH, rep.

[Chemopodium dessicatam A. Nelson] - UTH

Chenopodium rubrum L, var, rubrum - Dom01, E/W

[Chenopodium rubrum L.] - GPF, KTZ, SF!, UTF, WE.W

[Chenopodium rubrum L. forma bumile (Hook.) Asch. \& Graeb] - HDH

[Cbenopodiums rubrum L. forma rubrum] - HDH

Chenopodium rubrum L. var, glomeratum Walle, - Dorn01, E/W

[Chenopodium chenopodioides (L.) Aellen] - HDH rep., KTZ

[Chenopodium rubrum L.] - SF!

Chenopodium simplex (Torr.) Raf. - Dom0I, KTZ, SF!, UTF, W\&W, E/W

[Chenopodium gigantospermum Aelles] - GPF, HDH

Chenopodium strictum Roth var, glaucophyllum (Aellen) Wahl - Dorn0I

[Chenopodium strictum Roth] - W\& W'

[Chenopodium strictum Roth subsp. glancophyllum (Aellen) Aellen \& K. Jast] - GPF

[Clecnopodium albam L.] - W\& W

[Chcnopodium album L. var, striatam (Krasan) comb, nov., ined.] -KTZ

[NS - above variety not published fide IPNI (August 2007)]

Chenopodium subglabrum (S. Watson) A. Nelson - Dorn01, GPF, HDH, exp., KTZ, SFE, W\&W,E

Chenopodium watsonii A. Nelson - Dom01, GPF, HDH, KTZ, SFE, W\&W, E

\section{Cladothrix (see Tidestromia)}

Corispermum

(Identification sources:

Corispermum americanum (Nutt.) Nutt. var. americanum - KTZ. W\&W'; Mosyakin95

[Corispermum americanum (Nutt.) Natt.] - Dom01, W\&W

[Corispenmum orientale Lam.] - GPF, RMC

Corispermum americanum (Nutt.) Nutt. var. rydbergii S. Mosyakin - KTZ, W\& W':

Mosyakin95, E/W

[Corispermum hysscpifolium L.]-GPF, HDH, SF1, W\&W

Snow, N, January 2009. Checklist of Vecculer Plants of the Southum Rodky Momain Regioe. CVersion $3 x$ 
- Corispermum navicula S, Mosyakin - ENDEMIC CCO>, KTZ, W\&W'; Mosyakin95

Corispermum nitidum Kit. ex Schultes - HDH, KTZ, W\&W, W\&W', misapp., W

[Corispermem nitidum Kit.] - GPF, SFW. UTF, W\& W'

Conispermum pacificum S. Mosyakin - KTZ

Corispermum villosum Rydb, - Dorn01. HDH, KTZ, UTF, W\& W'

[Corispermum emarginatum Rydb.] - $\mathrm{HDH}$

Corispermum welshii S. Mosyakin - Dorn01, KTZ

Cycloloma atriplicifolium (Spreng,) J. M. Coult. - Dorn01, GPF, HDH, KTZ, SF1, W\&W, E/W

Dysphania (see Chenopodium)

Endolepis (see Atriplex)

Eurotia (see Krascheninnikovia)

Froelichia floridana (Nutt.) Moq. var, campestris (Small) Fernald - GPF, HDH, KTZ, W\&W, E [Froelichia floridana (Nutt.) MoQ] - SFE

Froelichia gracilis (Hook.) Moq. - GPF, HDH, KTZ, SFE, W\&W, E

Grayia (see also Zuckia)

Grayia spinosa (Hook.) Moq.- Dorn01, HDH. KTZ, UTF, W

[Atriplex grayi Collotzi] - SFW

[Atriplex grayi Collotzi ex W. A. Weber] - WRW

Guilleminea densa (Humb, \& Bonpl, ex Willd,) Moq, var. densa - KTZ, E

[Guillsmiace densa (WIIll.) Moq.] - GPF, SFE

[Guillemines densa (Willd. ex Roem. \& Schult) Mos.] - W\&W

* Halogeton glomeratus (M. Bieb.) C. A. Mey. - Dorn01, HDH, KTZ, SFW, UTF, W\&W,W

[Halogetoe gloneratus (Steph. ex M. Bieb.) C. A. Mey.]

Kochia americana S. Watson - HDH, KTZ, SFW, UTF. W\&W. W

[Bassia americana (S. Watson) Scott]-Dorn01

* \{Kochia prostrata (L.) Schrad.\}-KTZ, UTF

* Kochia scoparia (L.) Schrad. - GPF, HDH, UTF, E/W

[Bassia sieversiana (Pall.) W. A. Weber] - Dorne1, SFI, W\& W

[Bassia seoparia (L.) A. J. Scot] - KTZ

Krascheninnikovia lanata (Pursh) Meeuse \& Smit - Dorn01, KTZ, SF!, W\&W, E/W

[Cerateides lanara (Pursh) Howell] - GPF

[Ceratoides larara (Pursh) Howell var, lanata] - UTF

[Ceratoides lanata (Pursh) Howell var. ruinina S. L. Welsh] - UTF

[Eurxia lanata (Pursh) Moq.] - HDH

[Krascheninnikovia lanata (Pursh) Meeuse \& Smit var. lanata] - UT3

[Kraschenimnikovia ceratoides (L.) Geeldenst, subsp, lanasa (Pursh) H. Hellau] - Helau08

Monolepis nuttalliana (Schult) Greene - Dom01, HDH, KTZ, SF!, UTF, W\&W, E/W

[Monolepis nuttalliana (Roem. \& Schult.) Greene] - GPF

Monolepis pusilla Tor. ex S. Watson - Dom01. KTZ. UTF, W

Snou, N, Janury 2009. Checklist of Vasculer Plants of tho Southom Rocky Moanain Region. CVersion $3 x$ 
[Monokepis pusilla Tarr.]- HDH rep., SFW, W\&W

Proatriplex pleiantha (W. A. Weber) Stutz \& Chu - KTZ, SFW, W\&W, W [Atriplex pleiantha W. A. Weber] - HDH, UTE

\{Salicomia maritima Wolff \& Jefferies\} - KTZ

Salicomia rubra A. Nelson - Dom01, GPE. HDH. KTZ E/W

[Salicomia europaca L.] - UTF

[Salicornia europaca L. sabsp. rubra (A. Nelson) Breitung] - 5F!, W\&W

* Salsola australis R. Br. - SF1, W\&W, ENW

[Salsola iberica Setm. \& Pau] - GPF

[5alsola kali L. var, tenuifolia Tausch] - $\mathrm{HDH}$

[Salsola kali L. subsp. tragus (L.) Celak.] -

[Salsola pestifera A. Nels.] - UTF

[Salsola tragus L. - Dorn01, KTZ

* Salsola collina Pall, - Dom01, GPF, HDH, KTZ, SF!, UTF, W\&W, E/W

Salsola paulsenii Litv, - KTZ, UTF

Sarcobatus (see SRB)

Suaeda

(Identification sources:

Suaeda calceoliformis (Hook.) Moq, - Dorn01, KTZ, SF!, UTF, W\&W, EW

[Suacala depressa (Pursh) 5. Walson] - GPF

[Suacda depressa (Pursh) S. Walson var. depressa] - HDH

[Suacia depressa (Pursh) S. Watson var. erecta S. Warson] - $\mathrm{HDH}$

Suaeda moguinii (Torr.) Greene - GPF, KT/, E/W

[Suscda fruticosa (L.) Forskal] - HDH, w\&W, misapp.

[Suacda moquinil Torr.] - SFW. W\&W

[Suacda moquinii (Torr.) Greene] - GPF, KTZ

[S uncdar nigra (Raf. J. F. Macbr.] - Dorn01, HDH, SF!, W\&W

[Suscde tomeyans \$. Watson] - HDH

[Sanoda tomeyana S. Watson var, torreyana] - UTF

Suaeda nigra (Raf.) Macbr. - Dom01

[Stakds moquinii (Torr.) Greene] - KTZ

Suaeda occidentalis (S. Watson) S. Watson - Dorn01, UTF

[Stunda caleeoliformis (Hook) Moq.] - KTZ

[Subcta occidentalis S. Watson] - HDH, W\&W, no specintens seen

Suckleya suckleyana (Torr.) Rydb, - Dorn01, GPF, HDH, KTZ, SFE, W\&W, E

\section{Teloxys (see Chenopodium)}

Tidestromia lanugionosa (Nutt) Standl. - GPF, HDH, KTZ, UTF, E/W

[Cladothrix laraginosa Nurt.] - SF:

[Cladothrix lanaginosa (Nutt, ex Moq,) Benth. \& Hook.] - W\&W

Zuckia brandegeei (A. Gray) S. L. Welsh \& Stutz ex S. L. Welsh var. brandegeei - KTZ, W 
[Atriplex brandegei (A. Gray) Collotzi] - SF W

[Grayia brandegei A. Giray] - HDH

[Zuckia brandegei (A.Gray) S. L. Welsh \& Stutz var- brandegei] - UTE

[Zuckia brandegei (A. Gray) S. L. Welsh \& Stutz] - W\&W

Zuckia brandegeei (A. Gray) S. L. Welsh \& Stutz ex S. L. Welsh var, plummeri (Stutz \&

Sanders, ) Dorn - Dorn01, KTZ. Stutz87, W

[Atriplex brandogei (A. Gray) Colloczi] - SEW

[Zuckia brandegei (A.Gray) S. L. Welsh \& Stutz var. plummeri (Stutz \& Sanders.) S. L. Welsh] - UTH

\section{Amaryllidaceac (see ALL)}

\section{Anacardiacene - ANA}

\section{Rhus (see also Toxicodendron)}

(Identification sources:

Rhus aromatica Aiton var, simplicifolia (Greene) Cronquist - IMF, UTF. W

[Rhus urilobata Nutt. subsp. trilobata (Nutt. ex Tor. \& A. Gray) W. A. Weber forma simplicifolia (Groeno) W.

A. Weberl - W

[Rhus urilobala Nutl. var. simplicifolia (Greene) F. A. Barkley] - KTZ WJ

[Rhus trilobala Nutl. ex Tor. \& A. Gray var. simplicifolia (Greene) F. A. Barkley] - HDH

Rhus aromatica Aiton var, trilobata (Nutt,) A. Gray ex S. Watson - Dorn01, IMF, E/W

[Rhus aromarica A hion subsp. pilosissima (Engelm.) W. A. Weber] - SFE, W\& W

[W] - subsp. pilosissims is an excepsiooally hairy form of var. vrilobata]

[Rhus aromatica Aiton var, pilasissima (Engelm.) Shinners] - JB, GPF

[Rhus aromatica A sion subsp, trilobata (Nutt.) W. A. Weber] - SF!

[Rhus aromatica Aiton subsp, trilobata (Nutt, ex Torr. \& A. Gray) W. A. Webes] - W\&W

[Rhus aromatica A jien var, trilobata (Nuth, A. Gray] - GPF, UTF

[Rhus trilobata Nutt. vart, trilobata] - KTZ, RMC

[Rhus trilobata Nutt. ex Tort \& A. Gray var, trilobata] - HDH

[Rhus trilobata Nutt, yar, pilcosissima Engelan.] - KT2

Rhus glabra L. - Dorn01, GPF, HDH, IMF, KTZ, SF!, UTF, W\&W, E/W

$\div$ Rhus typhina I. - GPF, IMF, KTZ <KS, UT>, SF!, UTF, WJ (voucher at COLO), E/W

Toxicodendron rydbergii (Small ex Rydb.) Greene - Dorn01, IMF, KTZ, W\&W, E/W

[Rhus radicans L.] - HDH

[Toxicodendroes rydbergi (Small) Geene] - GPF, SFF, UTF

\section{Anthericaceae - ATH}

Echeandia flavescens (Schultes \& Schultes f.) Cruden - FNA, KWA

[Anthericum flavescens Schultes \& Schultes f. in J. J. Roemer et al.] - FNA

[Anthericuan stenccarpum Baker] - FNA

[Anthericum torreyi Baker] - M\&H, FNA

[Anthericum torreyi var, arizonicum Poellnitz] - FNA

[Anthericuan torreyi var, lanceolatum Poellnitz] - FNA

[Anthericuen torreyi vaf, necenexicanum Poellnitz] - FNA

[Echeandia leptophylla Bentham] - FNA

[Phalangiuen flaveseens Kunth] - FNA

Leucocrinum montanum Nutt. ex A. Gray - Dorn01, HDH. MF, TZ. UTF. W\&W. E/W 
[Leucocrinum mantanum Nutt.] - GPF, SF!

Apinceae - API (- Umbelliferae)

[NS - Some traditional generic boundaries are chalknged by molecular data. For example, ITS sequence data indicate that Cymoperus and Lomutimm ane highly polyphyletic. However, traditional boundaries probobly are best recognized until cungruent data sets support the change of existing classifications.]

Aletes (see also Cymopterus, Lomatium, Musineon, Neoparrya)

(Identification sources:

Aletes acaulis (Torr.) J, M. Coult, \& Rose - HDH, KTZ, SFE, W\&W, E

- Aletes humilis J. M, Coult. \& Rose - ENDEMIC $<\mathrm{CO}$, WV>, Dom01, HDH, KTZ, SFE, W\&W, E

* Anethum graveolens L. $\}$ - HDH, exp, KTZ <AZ, KS, NE, OK, UT $>$

Angelica ampla A. Nelson - Dorn01, HDH, KTZ, SF!, W\&W, E/W

Angelica grayi (J. M. Coult. \& Rose) J. M. Coult. \& Rose-Dorn01, KTZ, SF!,W\&W, E/W

[Angelica grayi J. M. Coult. \& Rose] - HDH

Angelica pinnata S. Watson - Dorn01, HDH, IMF, KTZ, SF!, UTF, W\&W, E/W

Angelica roseana L. F. Hend, - Dorn01

[Angelica roseana L. F. Hend] - $\mathrm{HDH}$, IMF $<\mathrm{CO}$, reputedly?, KTZ, UTF, W\& W, err, rep.

* Berula erecta (Huds.) Coville var. incisa (Torr.) Cronquist-Dorn01, GPF, IMF, UTF, E/W

[Berula eresta (Huds,) Coville]- HDH, KTZ, SFt, W\&W

Bupleurum americanum J. M. Coult. \& Rose-Dorn01, KTZ, RMC, W

[Bupleurum triradiatum Adams subsp, arcticum (Regel) Hultén] - SFW, W\&W

* Carum carvi L. - Dorn01, GPF, HDH, IMF, KTZ, SF!, UTF, W\&W, E/W

Cicuta maculata L. var, angustifolia Hook. - Dom() I, GPF, IMF, KTZ, UTF, Mulligan80, E/W

[Cicuta douglasii (DC) ], M. Coult, \& Rose] - HDH, SFI, W\&W, misapp., W\& W'

[Cieuta maculasa L.] - W\& W'

[Cicuta maculata L. var, macalata] - GPF. KTZ

Conioselinum scopulorum (A. Gray) J. M. Coult. \& Rose - Dom01, HDH, SF!, W\&W, E/W

* Conium maculatum L. - Dorn01, GPF, HDH, IMF, KTZ, SF!, UTF, W\&W, E/W

Cymopterus

(Identification sources: )

Cymoptcrus alpinus A. Gray - Dorn01, IMF. RH, E/W

[Oreoxis alpina (A, Gray) J. M. Coult, \& Rose] - HDH. SF!, UTF

[Oreoxis alpina (A, Gray) J, M. Coult, \& Rose subsp alpina] - KTZ, SFt, W\&W

[Oreoxis alpiea (A. Gray) J, M. Cosilt, \& Rose subsp puberulenca W. A. Weber] - KTZ, SF!, W\&W 
[RH - This subsp. endemic to Colorado if recognized.]

$\bullet$ Cymopterus anisatus A. Gray - ENDEMIC $<\mathrm{CO}>$, RH, E/W

[Aletes anisatus (A. Gray) Theobalid \& Treng] - KTZ, SF!, W\&W

[Pleryxis anisata (A. Gray) Mathias \& Constarsed - HDH

Cymoptenus bakeri (J. M. Coult. \& Rose) M. E. Jones - IMF, RH, E/W

[Oreoxis bakeri J. M. Coult. \& Rose] - HDH, KTZ, SF!, UTF, W\&W

Cymopterus bulbosus A. Nelson - Dorn01, HDH, IMF, KTZ, SFW, UTF, W\&W, W

Cymopterus constancei R. L. Hartm. - Dorn01

Cymoptenis duchesnensis M. E. Jones -IMF, KTZ, SFW, UTF, W\&W, W

Cympoterus glomeratus (Nutt.) DC. - Sun05

[C mopterws acaul is (Pursh) Rtal.] - Dorn0I, GPF, HDH, Sun05; SFE, W\&W

[Cymopterus acaulis (Pursh) Ftaf. var, acaulis] - IMF, KTZ, UTF, E

CO mopterus acaulis (Pursh) Ftaf. var. Fendleri (A. Gray) Goodrich] - [MF, KTZ, UTF, W

[Cymopterss fendleri A. Gray]-HDH, SFW, W\& W

\{Cymopterus hendersonii (J. M. Coult. \& Rose) Cronquist\} - IMF, RH, UTF, W\&W, misapp.

\{Pleryxia hendersonit (J. M. Coult. \& Rose) Mathias \& Constance\} - HDH, KTZ

- Cymopterus humilis (Raf.) Tidestr, \& Kittell - ENDEMIC $<$ CO, NM? $>$ RH, E

[Oreoxls bumilis Raf.] - HDH, KTZ SFE, W\&W

Cymopterus lapidosus (Jones) Jones - Dorn01, KTZ.

Cymopterus lemmonii (J. M. Coult. \& Rose) Dom - Dorn01, IMF. UTF. E/W

[Pseadosymopterns mostanus (A. Gray) J. M. Coult \& Rose] - HDH, KTZ, SFI, W\&W

Cymopterus longilobus (Rydb.) W. A. Weber -W\&W, err. rep.

[Pieryxia hendersonii (J. M. Coult. \& Rose) Mothins \& Constanes p.p] - HDH, KTZ

[Aletes anisatus (A. Gray) Theobaid \& Tseng] - W\&W

Cymopterus longipes S. Watson - Dorn01, HDH, KTZ, RH, UTF, W\&W, misapp.

\{Cymoperus longipes S. Warson var, loneipes\}- IMF

Cymopterus macdougalii (J. M. Coult. \& Rose) Tidestr. "var, breviradiatus", combination not available.

[Aletes macdousalii J. M. Coult. \& Rose] - HDH

[Aletes macdougalii J. M. Coult. \& Rose subsp, breviradiatus Theobald \& Tseng] - KTZ, SFW, UTF, W\&W

[Cymopterus masdongalii (J. M. Coult. \& Rose) Tidestr.] - IMF, RH

Cymopterus montanus Torr. \& A. Gray - Dom01. GPF. W\&W.E

[Cymopterns montanus Nutt]-SFE

[Cymopterus montanis (Nutt) Tort, \& A, Gay] - HOH

[Cymopterus motsanus Nutt, ex Torr, \& A. Gray] - KTZ

\{Cymopterus multinervatus (J. M. Coult. \& Rose) Tidestr.\} - HDH, exp., KTZ < AZ, NM,

UT>, RH, SFW, W\&W, misapp. W

\{Cymopterus newberryi (S. Watson) M. E. Jones\} -HDH exp., KTZ $\angle A Z$, UT>, W\&W

Cymopterus petraeus M. E. Jones-IMF. RH. W

[Aletes petraeus (M. E. Jones) W. A. Weber] - SFW, W \& W

[Cymopterus terebinthinus (Hook.) Torr, \& A. Gray var, petroeus (M.E.Jones) Gocdrich]-UTF

[Pteryxia petraea (M. E. Jones) J. M. Coult, \& Rose] - KTZ

- Cymopterus planosus (Osterh.) Mathias - ENDEMIC <CO, WY>. HDH. KTZ. SF!. W\&W.W

[RH - voucher at RM]

Cymopterus purpurascens (A. Gray) M. E. Jones - FCF. HDH. KTZ, RH, misapp.. SFW. UTF.

W\&W. W

Cymopterus purpureus S. Watson - HDH, IMF. KTZ, SFW, W\&W. W

[Cymopterus purpuress $\mathrm{S}$. Watson var, purpureus] - UTF

Cymopterus sessiliflorus (Theobald \& Tseng) comb. nov. ined. - RH. W

[Aletes sessiliflorus Theobald \& Tseng] - KTZ, SFW, W\&W 
Cymoptenus terebinthinus (Hook.) Totr. \& A, Gray var. albiflorus (Torr. \& A, Gray) M. E. Jones - Dorn01, IMF, W

[Cymopterus terebinthinus (Hiock.) Torr. \& A. Gray var. calcareus (M. E. Jones) Cronquist] - SFW. UTF, W\&W

[Pteryxia terebinthina (Hook.) J. M. Coult. \& Rose var. albiflora (Torr. \& A. Gray) Mathias] - KTZ

[Pteryxia terebinthina (Hook.) J. M. Coult. \& Rose var. calcarea (M. E. Jones) Mathias] - HDH

- Cymopterus williamsii R. L. Hartm. \& Const, - ENDEMIC <WV >, Dorn01, KTZ

* Daucus carota L. - Dom01, GPF, HDH, IMF, KTZ, SF1, W\&W, EW

[Daucus carota L subsp. carota] - UTF

\{Daucus pusillus Michx.\} - HDH, exp., KTZ $<$ AZ, KS, NM, OK $>$

* Eryngium planum L. - GPF, KTZ, W\&W

* \{Foeniculum vulgare Mill.\} - $\mathrm{HDH}_{4}$ exp, IMF, KTZ $<\mathrm{AZ}, \mathrm{KS}, \mathrm{NE}, \mathrm{NM}, \mathrm{UT}>$, UTF

Falcaria vulgaris Bernh. - Dorn01

[Falkaria sioides (Wibel) Aschers.] - KTZ

- Harbouria trachypleura (A. Gray) J. M. Coult. \& Rose - ENDEMIC $<$ CO, NM, WY>, Dom0l, HDH, IMF, KTZ, SFE, W\&W, E

Heracleum sphondylium L. var, lanatum (Michx.) Dorn - Dorn01, E/W

[Herackeum Ianaxum Michx.]- HDH, IMF, UTF

[Herackeum moximum W. Bartram] - KT2

[Herackeum sphondylinam L. subsp. montanum (Schleich.) Briq-1-GPE, SF]

[Herackom sphondylinam L. subsp. montanum (Schleich, ex Gasudin) Briq.] - W\&W

* Levisticum officinale W. D. J. Koch - KTZ, W\&W

Ligusticum filicinum S. Watson - Dom01, IMF, KTZ, RH, UTF

[Ligusricum filicinum \$. Wasson var, filisinum] - $\mathrm{HDH}$, exp

Ligusticum porteri J. M. Coult. \& Rose-Dorn0I, IMF, SF!, UTF, W\&W, E/W

[Ligasticum porteri J. M. Coult, \& Rose var, porteri]-HDH, KTZ, RMC

[Ligasticum porteri Coutl, \& Rose var, brevilobum (Rydb.) Mathias \& Constance] - HDH, exp., KTZ <UT?

Ligusticum tenuifolium S. Watson - Dorn01, IMF, KTZ, SF!, UTF, W\&W', E/W

[Ligusticum filicinum S. Watson var. tenuifolium (S. Wasson) Mathiss \& Constance] - HDH, W\&W

Lomatium

(Identification sources:

Lomatium bicolor (Wats.) Coult, \& Rose var. bicolor -WY checklist, KTZ

Lomatium bicolor (Wats.) Coult. \& Rosc var. leptocarpum (T. \& G.) Schlessm. - WY checklist. KTZ

- 1omatium concinnum (Osterh.) Mathias - ENDEMIC $<\mathrm{CO}>$, HDH, KTZ, SFW, W\&W.W

Lomatium cous (Wats.) Coult. \& Rose - Dorn01

[Lomatiun circumdatum (S. Wats.) Coult. \& Rose] - KTZ

[Lomatium montanum Coult. \& Rose] - KTZ

Lomatium dissectum (Nutt.) Mathias \& Constance var. multifidum (Nutt.) Mathias \& Constance

- Dorn01, HDH. IMF. KTZ, W\&W, E/W

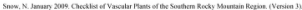


[Lamatium dissectam (Nutt) Mathias \& Constance] - SF!

[Lumatium dissectum (Nutt.) Mathias \& Constance var, eatonii (J. M. J. M. Coult. \& Roxe) Cronquist] - IMF, UTF

[RH - not known whether this variety occurs in Colorado]

- Lomatium eastwoodiae (J. M. Coult. \& Rose) J. F. Macbr. - ENDEMIC <CO>, HDH, KTZ. W

[Aletes esistroodiae (J. M. Coult. \& Rose) W. A. Weber] - SFW. W\&W

[Lomatium parryi(S. Watson) Macbr.] - IMF

Lomatium foeniculaceum (Nutt.) J. M. Coult, \& Rose var, foeniculaceum - GPF, IMF, E/W

[Lomatium foeniculaceum (Nutt.) J. M. Couli. \& Rose] - Dornd1. SFE. HDH

[Lomalium foeniculaceum (Nutt.) J. M. Coult. \& Rose subsp. foeniculaceum] -KTZ. W\&W

[Lomatium foeniculaceum (NuIL.) J. M. Coult. \& Rose subsp. nsacdougalii (J. M. Coult. \&

Rase) Theobald] - KTZ, SFW, W\&W

[Lomatium foeniculaceum (NutL.) J. M. Coult. \& Roso var. macdoagalii (J. M. Coult \& Rose) Cronquist] IMF, UTF

\{Lomatium graveolens (S. Watson) Dorn \& R. L. Hartm, var, graveolens\} - Dorn01, IMF, KTZ $\angle U T, W Y>W \& W$

\{Lomatium muttallii (A. Gray) Macbr.\} - HDH, exp.

Lomatium grayi (J. M. Coult. \& Rose) J, M. Coult. \& Rose var. grayi - Dorn01, IMF, KTZ, W

[Lomatium grayi J. M. Coult. \& Rose]-HDH, SFW, W\&W

Lomatium juniperinum (M. E. Jones) J, M. Coult. \& Rose - Dorn0l, HDH, exp., IMF, KTZ, SFW, UTF, W\&W, W

Lomatium latilobum (Rydb.) Mathias - HDH rep., IMF, KTZ, UTF, W

[Aletes latilobus [Rydlb,) W, A. Weber] - SFW, W\&W

Lomatium leptocarpum (Torr. \& A. Gray) J. M. Coult. \& Rose - Dorn01, HDH, 1MF, W

[Lomatium bicolor (S. Watson) J. M. Coult. \& Rose var, Ieptocarpum (Nutt.) Schlessm.] - SFW

[Lomatium bicolor (S. Watsan) J. M. Coult. \& Rose var. leptocarpum (Torr. \& A. Gray) Schlessm.] - KTZ

[Lomatium bicolor (S. Watson) J. M. Coult. \& Rose var. leptocarpum (Nutt. ex Torr. \& A. Gray) Schlessm.] WEW

Lomatium macrocarpum (Nutt. ex Torr. \& A. Gray) J. M. Coult. \& Rose - Dorn01, KTZ, W

[Lomathum macrocarpum (Hook. \& Am.) J. M. Coult. \& Rose] - GPF, HDH, SFW, UTF, W\&W

Lomatium nuttallii (A. Gray) J. F. Macbr. - Dorn01, GPF, IMF, KTZ, E/W

[Aletes nustallii (A. Gray) W. A. Weber] - SF!, W\&W

[Lomatium megarrhizum (A. Nelson) Mathias]

Lomatium orientale J. M. Coult. \& Rose-Dom01, GPF, HDH, IMF; L. nevadense has priority, possibly conspecific, KTZ, SF!, \&W, ENW

[Lomatium nevadense (S. Watson) J. M. Coult. \& Rose] - UTF

Lomatium triternatum (Pursh) J. M. Coult. \& Rose subsp. platycarpum (Torr.) Cronquist - IMF, SFW, UTF, W\&W, W

[Lomatiun triternatum (Pursh.) Coult, \& Rose) var, platycarpum (Toct.) Boivin] - Dorn0t

[Lomatium simplex (Nutt,) J. F, Macbr.] - HDH

[Lomatium simplex (Nutt.) J. F. Macbr, var, simplex] - KTZ

Musineon divaricatum (Pursh) Nutt. ex Torr. \& A. Gray - Dorn01, IMF, E

[Musineon divaricarum (Pursh) Nutt] - GPF

[Musineon divaricatum (Pursh) Raf.] - SFE

[Musineon divaricatum (Pursh) Raf, var, divaricatum] - KTZ, W\&W'

[Musineon divaricasum (Pursh) Nutt ex Torr. \& A. Gray var, divaricatum] - HDH, W\&W

[Musineon divaricaruen (Pursh) Raf, var, hookeri Torr. \& A, Gray] - W \& W'

[Musineon divaricatum (Pursh) Raf, var, bovkeri (Torr, \& A. Gray) Mathias] - KTZ

[Musineon divaricaum (Pursh) Nutt, ex Torr, \& A. Gray var, hookeri Torr, \& A. Gray] - HDH, W\&W

Musineon tenuifolium Nutt. ex Torr. \& A. Gray - Dom(01, HDH, E.

[Aletes tenuifolius (Nutt.) W. A. Weber] - SFE 
[Aletes tenuifolius (Nutt. ex Torr. \& A. Gray) W. A. Weber] -W\& W

[Musineon tenuifolium Nutt.] - GPF

[Musineon tenuifolium (Nuit.) J. M. Coult. \& Rose

[Musineon tenuifolium (Nutt. ex Torr \& A. Gray) J. M. Coult. \& Rose] - KTZ

- Neoparrya lithophila Mathias - ENDEMIC <CO, NM $>$ KTZ, RH, E

[Aletes lithophilus (Mathias) W. A. Weber] - SFE, W\&W

\section{Oreoxis (see Cymopterus)}

Orogenia linearifolia S. Watson - Dorn01, HDH, IMF, KTZ, SF!, UTF, W\& W, E/W

[Orogenia linearifolia S. Watson var. lata Payson] - SFW

Osmorhiza chilensis Hook. \& Am. - GPF, HDH, IMF, SF!, UTF, W\&W, Lowry84, E/W

[Osmochiza berteroi DC.] - Dom01, KTZ

Osmorhiza depauperata Phil. - Dorn01, GPF, IMF, KT7, SF!, UTF, W\& W, E/W

[Osmorhiza oblusa (J, M. Conlt, \& Rose) Fenrald] - HDH

Osmorhiza longistylis (Torr.) DC. - Dorn01, HDH, KTZ, SFE, W\&W, E

[Osmochiza longistylis (Torr.) DC. var. longistylis] - GPF

Osmorhiza oecidentalis (Nutt. ex Torr. \& A. Gray) Torr. - Dom01, IMF, KTZ, E/W

[Osmortiza oecidentalis (Nutt) Torr.] - HDH, SF!, UTF, WE. W

Oxypolis fendleri (A. Gray) A. Heller - Dom01, HDH, IMF, KTZ, SF!, UTF, W\&W, E/W

* Pastinaca sativa L. - Dom01, GPF, HDH, IMF, KTZ, SF!, W\&W, E/W

[Pastinaca sativa L. subsip. sativa] - UTF

[Pastinsca sativa L. var. sariva]

Perideridia montana (Blank.) Dorn - Dorn01, IMF, E/W

[Perideridia gaindneri (Hook. \& Am.) Mathias] - GPF, HDH

[Perideridia gaindneri (Hook, \& AmL) Mathias subsp, borealis T, I. Cbuang \& Constance] - KTZ, SFt, UTF, WRW

Podistera eastwoodiae (J. M. Coult. \& Rose) Mathias \& Constance - HDH, IMF, KTZ, SF!, UTF, W\&W, E/W

Pseudocymopterus (see Cymopterus)

Pteryxia (see Cymopterus)

\{Sanicula canadensis L.\} - HDH, exp.,

\{Sanicula canadensis L, var, canadensis\} - KTZ $\subset$ KS, NE, OK, WY?

Sanicula marilandica L. - Dorn01, GPF, HDH, KTZ, SF!, W\&W, E/W

Sium suave Walter - Dorn01, GPF, HDH, IMF, KTZ, SF!, UTF, W\&W, E/W

Spermolepis divaricata (Walt.) Raf. ex Ser. - KTZ, M\&H

Zizia aptera (A. Gray) Fernald - Dorn01, GPF, HDH, IMF, KTZ, SFE, UTF, W\&W, E \{Zizia aurea \{L.\} W. D. J. Koch\} - HDH, exp., KTZ<KS, NE, OK 
Apocynaceac - APO (including Asclepiadaceae)

(Identification sources:

Amsonia jonesii Woodson - HDH, IMF, KTZ, SFW, UTF, W\&W, W

Apocynum androsaemifolium L. - Dorn01, GPF, KTZ, SF!, W\&W, E/W

[Âposynum androsaetnifolium L. var. androsaem ifolium] - HDH, IMF, UTF

[Apocynum androsaemifolium L. var. glabrum Macoun] - HDH

[Aposynum androsaemifolium L. var. incanum A. DC.]

Apocynum cannabinum L. - GPF, KTZ, SF!, UTF, W\&W, ENW

[Ápocynum cannabinum L. var. cannabinam] - Dom01, IMF

[Aposymum cannabinum L. var, glaberrimum A. DC.] - HDH, IMF

[Aposynum cannabinum L. var, pubescens (R. Br.) A. DC.]-HDH, exp.

[Apocynum sibericam Jacq.] - UTT, W\& $W^{1}$

[Apocynum sibericum Jaeq- var, salignum [Greene) Fernald] - HDH, IMF

[A posynum sibericam Jacq- var, sibericum] - HDH. exp.

[Aposynum suksdorfii Greene] - $\mathrm{HDH}$

Apocynum X floribundum Greene - GPF, IMF, KTZ, E/W

[Áposynum androstemifolium X A canabinum] - KTZ

[Aposymum medium Greene] - W\& W

[Apocynum X medium Gireene] - SF!, UTF, WSW

[Aposynum medium Greene var. floribundum (Greene) Wondsoo] - HDH

[Apocynum medium Greene var. Iividum (Greene) Woodson] - HDH

Asclepias

Asclepias arenaria Torr. - Dorn01, GPF, HDH, KTZ, SFE, W\&W, E

Asclepias asperula (Decne,) Woodson var, asperula - GPF, IMF, UTF, E/W

[Asclepias asperula Woodson] - SFW

[Asclepias asperula (Dexac.) Woodson] - SFE

[Asclepias asperula (Docue.) Woodson subsp. asperula] - KTZ. W\&W

[Asclepias asperula (Docac.) Woodson subsp. capricornu (Woodson) Woodson] - W\&W, erf, rep.

[Asclepias capricornu Woodson subsp. capricornu] - $\mathrm{HDH}$

[Asclepias capricarnu Woodson subsp. Oocidentals Woodson] - HDH

\{Asclepias brachystephana Engelm.\} - HDH, exp.

[WJ - does not occur north of about latitude $34^{\circ}[\mathrm{eg}$, ca. Socorro, NM]\}

\{Asclepias brachystephana Engelm, ex Torr. - KTZ $\triangle A Z, N M-$ W\&W', misid.

Asclepias cryptoceras S. Watson var. cryptoceras - Dom01, IMF, UTF, W

[Asclepias cryptoceras S. Watson] - $\mathrm{HDH}$, SFW, W\&W

[Asclepias cryptoceras S. Watson subsp. cryptoceras] - KTZ

Asclepias engelmanniana Woodson - Dom01, GPF, HDH, IMF, KTZ, SFE, W\& W, E

INS - Amold Clifford believes this is the same spexies as A. rusbyi (an older name), which has been followed

by UT3, Somebody necds to check type specimens of each name.]

Asclepias hallii A. Gray - Dorn01, GPF, HDH, IMF, KTZ, SF!, UTF, W\&W, E/W

Asclepias incarnata L. var. incarnata - E

[Ásclepias incarnata L.] - Dorn01, GPF, HOH, IMF, SFE, UTF, W\&W

[Asclepias incarnata L. subsp, incarnata] - KT2

Asclepias involucrata Fngelm, ex Torr. - IMF, KTZ, UTF, W\&W, E

[Asclepias involucrata Engelna.] - GPF, HDH, SFE

Asclepias latifolia (Torr.) Raf. - GPF, HDH, IMF, KTZ, SFE, UTF, W\&W, E

Asclepias macrosperma Eastw, - IMF, SFW, UTF, W\&W, W

Snow, N, Janury 2009. Checklast of Vaccular Plants of the Southom Rocky Momain Region. CVersion 3 . 
[Asclepias involucrata Enszelm.] - KTZ

Asclepias macrotis Torr. - GPF, HDH, KTZ, SFE, W\&W, E

Asclepias oenotheroides Cham. \& Schltdl. - GPF, KTZ, SFE, W\&W, E

Asclepias pumila (A. Gray) Vail - GPF, HDH. KTZ, SFE, W\&W, E

Asclepias rusbyi (Vail) Woodson - UT3, CC \& WJ, IMF, KTZ, UTF, W\&W'; COLO, RM

Asclepias speciosa Torr, - Dorn01, KTZ

[Asclepias giffordii Eastw.] - KTZ

Asclepias stenophylla A. Gray - GPF, HDH, KTZ, SFE, W\&W, E

Asclepias subverticillata (A, Gray) Vail-Dom01, GPF, HDH, IMF, KTZ, SF1, UTF. W\&W, ENW

Asclepias tuberosa L, var, interior (Woodson) Shinners - IMF, E/W

[Asclepias tuberosa L. sulsp. imterior Woodson]- GPF, HDH, KTZ, W\& W, err. rep.

[Asclepias tuberosa L. subsp. terminalis Woodson] - GPF, SFE, UTF, W\&W

Asclepias uncialis Greene - Dorn01, GPF, HDH, SFE, W\&W, E

[Asclepias uncialis Greene subsp. uncialis] - KTZ

\{Asclepias verticillata L. ; - HDH rep.. KTZ. W\&W, err. rep.

Asclepias viridiflora Raf, - GPF, HDH, KTZ, SFE, W\&W, E

Funastrum crispum (Benth.) Schhdl, - KTZ, W\&W', Liede96, E

[Sarcostemma crispum Benth.] - GPF, HDH, SFE, W\&W

Sarcostemma (see Funastrum)

Araceae - ARA (see also ACO)

(Identification sources:

Lemna gibba L. - Dorn01 (reported), FNA, GPF, HDH, IMF, KTZ, UTF, W\&W

Lemna minor L. - FNA, GPF, HDH, IMF, KTZ, SF!, UTF, W\&W, E/W

Lemna minuta Kunth - Dorn01. FNA, IMF, KTZ, UTF, E/W

[Lemea minima Phillipi]- $\mathrm{HDH}_{\text {, }} \exp$.

[Leman minof L.] - RMC

[Leman minascula Herter]-SFI, W.SW

Lemna trisulca L. - Dom01, FNA, GPF, HDH, IMF, KTZ, SF!, UTF, W\&W, E/W

Lemna turionifera Landolt - Dorn01, FNA, GPF, KTZ, SF!, W\&W, E/W

[Lemaa minor $\left.\mathrm{L}_{\mathrm{i}}\right]-\mathrm{RMC}$

Lemna valdiviana Phil. - FNA, GPF, HDH, IMF, KTZ, UTF, W\&W

$\leftarrow$ Pistia stratiotes L. - FNA. Snow04b

๘ Spirodela polyrhiza (L.) Schleid. - Dom01, FNA, HDH, IMF, SFE, UTF, W\&W, E/W

[Spirodela polynthiza (L.) Schleid] - GPF. KTZ

[Spirodela polynhiza Schleid.] - SFW

Wolffia borealis (Engelm.) Landolt - FNA, GPF, SFE, W\&W, E

[Wolffia borealis (Engelm. ex Hegelm.) Landolt] -

[Wolffis borealis (Engelm. ex Hegelm, Landolt ex Landolt \& Wildi] - KTZ

[Wolffia punctata Griseb] - IMF, UTF, W\&W!, misapp.

Wolffia columbiana H. Karst. - FNA, GPF, IMF, KTZ, SFE, W\& W, E 


\section{Aruliaceae $=A R L$}

Aralia nudicaulis L. - Dorn01, GPF, HDH, KTZ, SF!, W\&W, E/W

Aralia racemosa L. subsp. bicrenata (Woot, \& Standl.) S. L. Welsh \& Atwood - KTZ, SF!. UTF, WJ, W\&W'; Wen98, ENW

[Aralia racemosa L. - GPF, HDH, exp.. [MF, SF!. W\&W

[Aralia racemosa L. subsp, racemosa]- W\&W'

Asclepiadaceae - (see APO)

Asparagaceat - ASG (including Anthericaceae and parts of LIL)

(Identification sources:

Asparagus officinalis L. - Dorn01, GPF, HDH, IMF, KTZ, SF!, UTF, W\&W, E/W

Echeandia (see ATH)

Leucocrinum (see ATH)

Maianthemum (see RUS)

Nolina (see RUS)

Asteraceae - AST (= Compesitae)

(Identification sources:

Acanthoxanthium (see Xanthium)

* Achillea filipendulina Lam. - KTZ $<$ UT>

[W] - Escaped cultivation and persisting in Idaho Springs CO area, also at Evergecen exit in Jefferson Co, on I70. and seattered along roadsides in Boukder Co.]

Achillea millefolium L. var. lanulosa (Nutt.) Piper - Dorn01, IMF, E/W

[Achillea lamulosa Nut.] - SFL, W\&W

[Achlliea lanulosa Nutt. subsp. alpicola (Rydb.) D. D. Keck] - HDH

[Achillea lanulosa Nutt, subsp، kaulosa] - HDH

[Achilea millefolium L.] - FNA, HDH, SF!, W\&W, W\&W'

[Achillea millefolium L, subsp, lanulosa (Nutr.) Piper] - GPF, UTF

[Achillea millefolium L. var, alpicola (Rydlo.) Garret] - IMF, KTZ

[Achillea millefolium L. var, occidencalis DC.] - KTZ

* Achillea ptarmica L. - FNA

[NS - uncertain whether persisting in our area ousside of cultivatios]

Acosta (sce Centaurea)

Acourtia nana (Gray) Reveal \& King - KWA. KTZ

[Perezia nava Gray]-KTZ, MAH

Snow, N, January 2009. Checklist of Vecculer Plants of the Southom Rodky Momain Regioe. CVersion $3 x$ 
* Acroptilon repens (L.) DC. - FNA, KTZ, SF!, W\&W

[Centaurea pisris Pall.] - HDH

[Centaursa repens L.] - GPF, HDH, IMF, UTF, EW

\{Ageratina havanensis (Kunth), R. M. King \& H. Rob.\} - KTZ <TX

\{Eupatorium havanensis Kunth\}

\{Eupilocium texense (Totr. \& A. Gray) Rydb.\} - HDH, rep.

Ageratina herbacea (A. Gray) R. M. King \& H. Rob.- FNA, KTZ, SF!. W\&W

[Eupatorium herbaceum (A. Gray) Greene] - HDH, IMF, SF!, UTF. E/W

[Ageratina occidentalis (Hook.) R. M. King \& H. Rob.] - W\&W', em.rep.

Agoseris

(Identification sources:

Agoseris aurantiaca (Hook.) Greene var, aurantiaca - FNA, IMF, KTZ, SF!, UTF, E/W

[Agoseris arizonica Greene]- HDH

[A goseris aurantiaca (Hook.) Greene] - GPF, HDH, WA W

[Agoseris rostrata Rydbi] - HDH

Agoseris aurantiaca (Hook.) Greene var, purpurea (A. Gray) Cronquist - FNA, HDH, IMF, KTZ,

SF!, UTF, EJW

[Agoseris aurantiaca (Hook.) Greene] - GPF, W\&W

\{Agoseris elata (Nutt.) Greene\} - HDH, KTZ, W\& W, misapp.

Agoseris glauca (Pursh) Raf. var, dasycephala (Torr. \& A. Gray) Jep8, - Dorn01, FNA, HDH.

IMF, KTZ, SF!, UTF, W\&W, E/W

Agoseris glauca (Pursh) Raf, var, glauea - Dorn01, FNA, IMF, KTZ, UTF, W\&W, E/W

[Agoseris glauca (Pursh) Raf.] - GPF, SF!

[Agoseris glauca (Pursh) Rat, var. agrestis (Osterh.) Q. Jones] - UTF

[Agoseris glauca (Punsh) Rtaf. var. agnestis (Osterh.) Q. Jones ex Cronquist] - IMF, KTZ, WRW

Agoseris graminifolia Greene - KWA, M\&H

[Agoseris aurantiaca (Hooki) Greene var, aurantiasa] - KTZ

Agoseris heterophylla (Nutt.) Greene var, heterophylla - KTZ, W

[Agoser is heterophy lla (Nutt) Greene] - IMF. SFW, UTF, W \& W

Agoseris parviflora (Nutt.) D. Dietrich - FNA

[Agoseris caudata Greenc] - FNA

[Agoseris dens-leonis Greene] - FNA

[Agoser is glauca (Pursh) Raf, var. laciniata (D. C. Eaton) Smilcy] - HDH, 1MF, KTZ, SFI, UTF, W \& W, E.W

[Agoseris glauca (Pursh) Ftar, var, laciniata (D. C. Eaton) Kuntze] - FNA

[Agoseris glauca (Pursh) Raf: var. parviflora (NutL) Rydb.] - HDH

[Agoseris rosea (Nutt) D. Dietrich] - FNA

[Agosris taraxacoides Girene] - FNA

[Agoseris tomentosa Howell] - FNA

[Troximon parviflorum Nutt.] - FNA

Almutaster pauciflorus (Nutt.) A. Lōve \& D. Löve - Dorn01, KTZ, W\&W

[Aster pauciflorus Nutt] - GPF, HDH, IMF, UTF, EN

[Almutaster panaciflorus (Nuti,] A. LBve] - FNA, SF1

Amauriopsis dissecta (A. Gray) Rydb. - FNA

[Amesaria dissecta A. Gray] - FNA

[Bahia dissceta (A. Gray) Briton] - Dorne1, HDH, IMF, KTZ, SF!, UTF, W\& W, EW

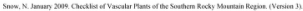




\section{Ambrosia}

(Identification sources:

Ambrosia acanthicarpa Hook, - Dom01, FNA, GPF, IMF, KTZ, SF!. UTF, W\&W, E/W

[Franseria acanthicarpa (Hook_) Coville] - FNA, HDH

* Ambrosia artemisiifolia L. - Dom01, FNA, GPF, IMF, UTF, E [Ambrosia artemisifolia L. var, elatior (L.) Desc.] - KTZ, SFE, W\&W

[Ambrosia elatior L.]- $\mathrm{HDH}$

Ambrosia confertiflora DC. - FNA, GPF, IMF, KTZ, SFE, UTF, W\&W, E

[Franseria confertiflora (DC.) Rydb.] - HDH

Ambrosia grayi (A. Nelson) Shinners - FNA, GPF, KTZ, SFE, W\&W, E

[Franseris tomemtosa A. Gray] - HDH, W\&W, non Nutt.

[Gacrtneria grayi A. Nebon] - FNA

- Ambrosia linearis (Rydb.) W, W, Payne - ENDEMIC <COs, FNA, GPF, KTZ, SFE,

W\&W, E

[Franseria linearis Rydb.] - HDH

[Gacrneria linearis Ryob.] - FNA

Ambrosia psilostachya DC, - Dorn01, FNA, GPF, IMF, KTZ, UTF, E/W

[Ambrosia corconopifolia Torr. \& A. Gray] - $\mathrm{HDH}$

[Ambrosia psilostachya DC. var, coronopifolia (Torr. \& A. Gray) Farw.] - SFI. W \& W

Ambrosia tomentosa Nutt. - Dorn01, FNA, GPF, IMF, KTZ, SF!, UTF, W\& W, E/W

[Franseria discolor Nutt.] - $\mathrm{HDH}$

Ambrosia trifida L. - HDH, SF!, UTF, W\&W

[Ambrosia aptera DC.] -KWA, KTZ, M\&H

[Ambrosia trifida var. aptera (DC.) Kuntze] - KTZ

[Ambrosia trifida var. texana Schoele] - KWA, KTZ

[Ambrosia trifida L. var, trifida] - GPF, IMF, KTZ, E/W

Anaphalis margaritacea (L.) Benth. \& Hook. - Dorn01. FNA, GPF, HDH. IMF, SF!, UTF. W\&W, E/W

[Anaphalis margaritaçea (L.) Benth.] - KTZ

[Anaphalis margaritaceum (L.) Benth. var, occidentalis Greene] - FNA

[Anaphalis margaritaceum (L) Besth. var, subalpina (A. Gray) A. Gray] - FNA

[Geaphaliaum margaritaceum L.] - FNA

\section{Antennaria}

(Identification sources:

Antennaria anaphaloides Rydb. - Dorn01, FNA, GPF. HDH, IMF, KTZ. UTF, EW [Antennaria pulcherrima (Hook,) Greene subsp, anaphaloides (Rydb, W. A. Weber] - SF: WE W [Antennaria pulcherrima (Hook.) Greene var, enaphaloides (Rydb.) G. W. Douglas] - FNA

Antennaria corymbosa E. Nelson - Dorn01, FNA, HDH, IMF, KTZ, SF!, UTF, W\&W, E/W [Antennaria acuta Rydb.] - FNA

[Antennaria dicica (L.) Gaert. var, corymbosa (E., E. Nelson) Jepson] - FNA

[Antenaria hyrgophila Greene] - FNA

[Antennaria nardina Greene] - FNA

Antennaria dimorpha (Nutt.) Torr. \& A. Gray - FNA, Dorn01, HDH, KTZ. SFW, W\& W, W [Gnaphalium dimorphum Nutt.] - FNA

[Anteanaria dimorpha Nutt. var, integra L. F. Hendersoa] - FNA 
[Anteanaria dimorpha Nutt. var. macrocephala D. C. Fat.] - FNA

[Antennaria dimorpha Nutt. var. nuttallii D. C. Eat.] - FNA

[Antennaria Istixqusama Piper] - FNA

[Antennaria macrocephala (D. C. Eat.) Rydh.] - FNA

Antennaria howellii Greene subsp, howellii - FNA, KTZ

[Antennaria callilicpis Greene] - FNA

[Antennaria exima Greene] - FNA

[Antennaria neglecta var. canadensis (Greene) Cronq.] - KTZ

[Antennaria neglecta var. randii (Fern.) Cronq.] - KTZ

[Antennaria neodisica subsp. canadensis (Greene) Bayer \& Stebbins] - KTZ

[Antennaria noodioica var. randii (Fem.) Bojivi] - KTZ

[Antennaria randii Fem. ] - KTZ

[Antennaria spathulata (Fern.) Ferm] - KTZ

Antennaria howellii Greene subsp, neodioica (Greene) R. J. Bayer - FNA

[Anteanaria neodioica Greene] - FNA

[Antennaria alsinoides Greene] - FNA

[Antennaria grandis (Fernald) House] - FNA

[Antennaria neglecta var. necdioica (Greene) Crong̨.] - FNA

[Antennaria neodioica Greene var. attenuata Fernald] - FNA

[Antennaria neodioica Greene var. chlorophyilla Femald] - FNA

[Antemnaria nood doica Green var, grandis Fermald] - FNA

[Antennaria neodioica Green var, inlerjecta Fernald] - FNA

[Antennaria neodioica Green var, rupicola (Fernald) Fernald] - FNA

[Antsmnaria obovata E. E. Nelsen] - FNA

[Antsmnaria rupicola Fernald] - FNA

Antennaria howellii Greene subsp, petaloidea (Fern.) R. Bayer - FNA. KTZ

[Antennaria callilepis Greene] - KTZ

[Anteanaria eximia Greene] - KTZ

[Antennaria neglecta subsp. howellii (Greene) Halken] - KTZ

[Antennaria neglecta var. howellii (Greene) Crong-] - KTZ

[Antemaria neodioica sabsp. howellit [Greene) Bayer] - KTZ

Antennaria lanata (Hook.) Greene - FNA

[Anteanaria carpatica (Wahlenberg) Hook var. lanala Hook. ] - FNA

Antennaria luzuloides Torr. \& A. Gray subsp. luzuloides - FNA, KTZ

[Anteanaria luzuloides Torr, \& A. Gray - Dorn01, HDH, SFI, UTF, W\&W, EN

[Antennaria luzuloides Torr. \& A. Gray var. luzuloides] - IMF

[Anteanaria luzuloids Torf. \& A. Gray var, oblanceolata (Rydb.) M. Peck] - FNA

[Antennaria oblancrolata Rydb.] - $\mathrm{HDH}$

Antennaria marginata Greene - FNA, IMF, KTZ, SF!, W\&W, EW

[Antennaria dioich (L.) Gaert. var, marginata (Greene) Jep5on] - FNA

[Antezanaria fendleri Grectec] - FNA

[Antennaria marginata Greenc var, glandulifera A. Nelson] - FNA

[Anteanaria neglecta Greene] - HOH

|Anteranaria peramoetia Grecenel - FNA

Antennaria media Greene - Dorn01, FNA, HDH, IMF, KT7, SF!, W\& W, EW

[Antennaria alpina (L.) Gaertn.] - HDH, W\& W', misapp.

[Antcenaria alpina (L.) Gaertn, var, media (Greene) Jeps,] - UTF

[Antennaria austromontana E. E. Nelson] - FNA

[Antennaria candida Gresene] - FNA

[Antennaria densa Greene] - FNA

[Antcanaria modesta Groxe] - FNA

[Antennaria mucronata E. E. Nelson] - FNA

Antennaria microphylla Rydb. - Dorn01, FNA, GPF, HDH, IMF, KTZ, SF!,UTF, W\&W, E/W

[Antennaria bracteosa Rydb.] - FNA

[Anteanaria microphylla Lunell var-solstitialis Lunell] - FNA 
[Antennaria nitida Greene] - FNA

[Antennaria rosea Greene var, nitida (Gireere) Breitung] - FNA

[Antennaria solstitialis Lunell] - FNA

Antennaria neglecta Greene-Dorn01, FNA, GPF, HDH, IMF, KTZ, SFE, UTF, W\&W, E

[Antennaria athabascensis Greene] - FNA

[Antennaria campestris Rydb.] - FNA

[Antennaria campestris Rydb. var. athabascensis (Grecne) B. Boivin] - FNA

[Antennaria che lonica Lunell] - FNA

[Antennaria erosa Greene]- FNA

[Antennaria howellii Greene] - SFE

[Antennaria bovellii Greene var. athabascunsis (Greene) B. Boivin] - FNA

[Antennaria bowellii var. campestris (Rydb.) B. Boivin] - FNA

[Antennaria bowellii Greene subsp. neodioica (Greene) R. J. Bayer]-KTZ, W\&W

[Antennaria longifolia Greeto] - FNA

[Antennaria lunstlii Greene] - FNA

[Anteanaria nebrascensis Groene] - FNA

[Antennaria neglecta Greene var. athabascensis (Greene) Roy L. Tay lor \& MacBnde] - FNA

[Antennaria neglecta Greete var. campestris (Rydb.) Steyermark] - FNA

[Antennaria neglecta Greene var. simplex Peck] - FNA

[Antennaria obovata E. Nelson] - HDH

[Antennaria parvula Grexte] - FNA

[Antennaria wilsonii Greene] - FNA

Antennaria parvifolia Nutt, - Dorn01, FNA, GPF, HDH, IMF, KTZ, SF!, UTF, W\&W, E/W

[Antennaria aprica Greene] - FNA

[Antsanaria aprica Greene var. aureola (Lanel1) J. W. Moore] - FNA

[Antennaria apries Greene var, minuscula (B. Boivin) B. Boivin] - FNA

[Antennaria arueola Lunell] - FNA

[Antsnnaria dioicen (L.) Gaert. var, parvifolia (Nutt.) Tort. \& A. Gray] - FNA

[Antennaria latisquamea Greene] - FNA

[Antemnaria minuscula B. Boivin] - FNA

[Antennaria recurva Grocne] - FNA

[Antesnaria doodancha Suksdorf] - FNA

Antennaria pulcherrima (Hook.) Greene subsp. pulcherrima - FNA, W\&W

[Antennaria pulcherrima (Hook.) Greene] - HDH, exp., IMF, KTZ, UTF

Antennaria rosea Greene subsp, arida (E. E. Nelson) R. J, Bayer - FNA, KTZ

[Antconaria arida E. E. Nelsoe]-FNA

[Anteanaria arida E. E. Nelsce subsp, viscidula E. E. Nelson] - FNA

[Antcanaria scariosa E. E. Nelson] - FNA

[Antconaria viscidula (E. E. Nelton) A. Nelson ex Rydb.] - FNA

Antennaria rosea Greene subsp, confinis (Greene) R. J. Bayer - FNA, KTZ

[Antennaria confinis Greene] - FNA

[Antenaria affinis Fenald] - FNA

[Antennaria albicans Femald] - FNA

[Antennaria angustifolia Rydb] - FNA

[Antenonaria arida E. E. Nelsce var, humilis (Rydb.) E. E. Nelson] - FNA

[Antenanaria breitungii A. E. Porsild] - FNA

[Antennaria brevistyla Fensald] - FNA

[Antenanaria concinan E. E. Nelfox)] - FNA

[Antenaria dioica (L.) Gaert var, kemensis Jepson] - FNA

[Antenaria elegans A. E. Porslid] - FNA

[Antennaria foliacea Greene var, humilis Rydo.] - FNA

[Antennaria incarnata A. E. Porsild] - FNA

[Antennaria laingii A. E. Porsild] - FNA

[Antennaria leontopodioides Cody] - FNA

[Antennaria leuchippii Porslid] - FNA

[Antennaria polyphylla Greenc ex C. F. Baker (nom, nud.)] - FNA 
[Antennaria rosea var. angustifolia (Rydb.) E. E. Nelson] - FNA

[Antennaria sedoides Greene] - FNA

[Antennaria sordida Greene] - FNA

[Antennaria subiscosa Fernald] - FNA

[Antennaria tomentella E. E. Nelson] - FNA

Antennaria rosea Greene subsp. pulvinata (Greene) R. J. Bayer - FNA

[Antennaria tomentella Greene] - FNA

[Antennaria alhescnes (E. E. Nelson) Rydb.] - FNA

[Antennaria fusca E. E. Nelson] - FNA

[Antennaria gaspensis (Femald) Fernald] - FNA

[Antennaria bowelii Greene subsp. gaspensis (Fernald Chrnielewski] - FNA

[Antennaria isolepis Greene] - FN $A$

[Antennaria maculata Greene] - FNA

[Antennaria manicouaganda P. Landry] - FNA

[Antennaria medis Greene stubsp. fusca (E. E. Nelson) Chmielewski] - FNA

[Anteanaria noglecta Groente var. gaspensis Fentald] - FNA

[Antennaria neodioica Greene var. gaspensis Fernald] - FNA

[Antennaria peasei Fernald] - FN.A

[Antennaria pulvinata Greene subsp. albescens E. E. Nelson] - FNA

[Anteunaria sansonii Greene] - FNA

[Antennaria straminea Fernald] - FNA

Antennaria rosea Greene subsp, rosea - FNA

[Antennaria acuminala Cireene] - FNA

[Antennaria alboroséa A. E. Pórsild] - FNA

[Antsunaria chlorantha Greene] - FNA

[Anternaria formosa Greene] - FNA

[Antennaria bendersonii Piper] - FNA

[Antsmnaria imbricata E. E. Nelson] - FNA

[Antennaria lanulosa Greene] - FNA

[Antemaria neodioica Greero var. chlorantha (Greene) B. Boivin] - FNA

[Antennaria oxyphylla Greene] - FNA

[Antennaria rosela subsp. divaricara E. E. Nelson] - FNA

[Anteanaria speciosa E. E. Nelson] - FNA

Antennaria rosulata Rydb. - FNA, HDH, IMF, KTZ, SF!, UTF, W\&W, EW

[Antemearia sierrab-blancae Rydb.] - FNA

Antennaria umbrinella Rydb, - Dom01, FNA, HDH, IMF, KTZ, SF!, UTF, W\&W, E/W

[Antennaria aizoides Greene] - FNA

[Antconaria alpisa L.] - HDH, W\& W', misapp.

[Antennaria flavescens Rydb.] - FNA

[Anteanaria reflexa E. Netson] - FNA, HDH

* Anthemis arvensis L, - Dom01, FNA, HDH, KTZ, SF!, W\&W, E/W

[Anthemis arvensis L. var, agrestis (Walli.) DC. - GPF

* Anthemis cotula L. - Dorn01, FNA, GPF, HDH, IMF, KTZ, SF!, UTF, W\& W, E/W

* Anthemis tinctoria L. - Dorn01, GPF, IMF, KTZ, SFW. UTF. W\&W?,W

[Cota tinctoria (L.) J. Gay ex Gussone] - FNA

* Arctium lappa L. - Dorn01, FNA, GPF, IMF, KTZ, SF!, UTF, W\&W, E/W

* Arctium minus Bernh. - Dorn01, FNA, GPF, KTZ, E/W

[Aretium minus (Hill) Bemh.] - HDH, IMF, SFt, UTF, WEW

* Arctium tomentosum Mill. - FNA. GPF, KTZ, SFE. W\&W. E

Arida parviflora (A. Gray) D. R. Morgan \& R. L. Hartman - FNA

[Machaeranthera parviflora A. Gray] - FNA

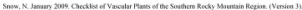


Arnica

(Identification sources: )

Arnica angustifolia Vahl subsp. tomentosa (Macoun) G. W. Douglas \& G. Ruyle-Douglas KTZ. W

[Amica angustifolia Vahl vart. tomentosa (Macoun) Dom] - Domol

[Afnica alpina (L.) Olin subsp. Womentesa (Macoun) Maguire] - SFW

[Arnica alpina (L.) Olin \& Ladau subsp. tometinesa (Macoun) Maguire] - W\& W

Arnica chamissonis Less. - FNA

[Arnica chamissonis Less. var. andina (Nutt.) Ediger \& T. M. Barkley] - UTF

[Amica chamissonis Less. subsp. foliosa (Nutt.) Maguire] - HDH, SF!, WAW

[Amica chamissonis Less. subsp. foliosa (Nutt.) Maguire var, andina (Nutt.) Ediger \& T. M. Barkley] - KTZ

[Arnica chamissonis Less, subsp. foliosa (Nult.) Maguire var, foliosa] - [MF

[Arnica clamissonis Less. var. foliosa (Nutt.) Maguire] - Derno1, UTF, EW

Amica cordifolia Hook, - Dom01, FNA, GPF, HDH, KTZ, SF!, UTF, W\&W, E/W

[Arnica cordifolia Hook, subsp. condifolia] - HDH

[Arnica cordifolia Hook var. cordifolia] - IMF

[Arnica paniculata A. Nels.] - HDH, exp.

Arnica fulgens Pursh - Dorn01, FNA. GPF, HDH, IMF, KTZ, SF!, UTF, W\&W, EW

Arnica gracilis Rydb, - FNA, HDH, exp., KTZ, RMC

[Amica katifolla Bong var, pracilis (Rydb.) Cronegist] - IMF, UTF

Arnica lanceolata Nutt, subsp. prima (Maguire) Strother \& S. J. Wolf - FNA

[Arnica amplexicaulis Nutt.] - Dorno]

[Amica amplexicaulis subsp, genuins Maguire] - KTZ

[Arnica amplexicaulis subsp. prima (Maguire) Maguire] - KTZ

[Amica amplexicaulis var. piperi St. John \& Warren] - KTZ

[Amica amplexicaulis var. prima (Maguire) Beivin] - KTZ

[Arnica amplexifolia Rydb.] - KTZ

[Arnica moltis var, aspera (Greene) Boivia] - KTZ

Arnica latifolia Bong. - FNA, HDH, KTZ, SF!, W\&W, E/W

[Anica istifolia Bong var. latifolia] - Dom01, IMF, UTF

Arnica longifolia D. C. Eaton - Dorn01, FNA, KTZ, SF!, W\&W, EJW

[Arnica longifolia D. C. Eaton subsp. longifolia] - HDH, [MF

[Amica longifolia D. C. Eaton var, longifolia] - UTF

Arnica mollis Hook. - Dom01, FNA, HDH, IMF, KTZ, SF!, UTF, W\&W, E/W

Arnica ovata Greene - FNA

[Amica diversifolia Greenc] - KTZ CUT?, UTF $\angle 00$ -

[Arnica latifolia Bongard var, viscidula A. Gray] - FNA

Arnica parryi A. Gray - Dorn01, FNA, SF!, UTF, W\&W

[Arnica parryi A. Gray var, parryi] - IMF, E/W

[Amica parryi A. Gray subsp parryi]- HDH. KTZ

Arnica rydbergii Greene - Dom01, FNA, GPF, HDH, IMF, KTZ, SF!. UTF. W\& W. E/W

Arnica sororia Greene - FNA, KTZ, Dorn0I

[Arnica fulgens var, sororia (Greene) G.W. Dopglas \& G. Rayle-Douglas] - KTZ

Artemisia

(Identification sources:

* Artemisia abrotanum L. - Dorn(01, FNA, GPF, HDH, IMF, KTZ, SF!, UTF, W\&W, E/W

Snow, N, January 2009. Checklist of Vecculer Plants of the Southom Rocky Momain Region. CVersion $3 x$ 
* Artemisia absinthium L. - FNA, GPF, HDH, IMF, KTZ, SF!, UTF, W\&W, ErW

* Artemisia annua L. - FNA, GPF, IMF, KTZ, SFE, UTF, W\&W, E

[Artemisia chamomilla C. Winkler] - FNA

Artemisia arbuscula Nutt. var, arbuscula - FNA, W

[Artemisia arbuscula Nutt.] - IMF, UTF

[Artemisia arbuscula Nutt. subsp. arbascula] - KTZ

[Artemisia tridentata Nutt. subsp. arbuscula (Nutt.) H. M. Hall \& Clem.] - HDH

[Seriphidium arbusculum (Nutt.) W. A. Weber]-SFW

[Seriphidium arbusculum (Nutt.) W. A. Weber subsp. arbuscelum] - W\& W

Artemisia arbuscula Nutt. var. longiloba (Osterh.) Dorn - E, FNA

[Artemisia arbuscula Nutt.] - IMF

[Artemisia arbuscula Nutt. subsp. longiloba (Osterh.) L. Shultz] - KTZ

[Artemisia longiloba (Osterh.) Beetie] - FNA, UTF

[Artemisia speiformis Osterh, var. lengilobas Osterh.] - FNA

[Seriphidium arbusculum (Nutt.) W. A. Weber subsp. longilobum (Osterh.) W. A. Weber] - FNA, SFE. W\&W

* Artemisia biennis Willd, var. biennis - KTZ, E/W

[Artemisiu biennis Willd.] - FNA, GPF, HDH, IMF, SF!, UTF, W\&W

- Artemisia biennis Willd, var, diffusa Dom - ENDEMIC $<$ WY $>$, Dom01, KTZ

Artemisia bigelovii A. Gray - FNA, GPF, HDH, IMF, KTZ, SF!, UTF, W\&W, ENW

[Artemisia petrophila Wooton \& Standl]. - FNA

[Seriphidiam bigelevii (A. Giray) K. Bremer \& Humphries] - FNA

Artemisia borealis Pallas subsp, borealis - FNA

[Antemisia campestris L. subsp. borcalis (Pallas) H. M. Hall \& Clements] - FNA

[Artemisia campestris L. var. spithamaca Pursh) M. Peck] - FNA

[Artemisia campestris L. var. strutziae S. L. Welsh] - FNA

[Antemisia purshii Besser] - FNA

[Artemisia spithamaca Pursh] - FNA

[Oligosporus groenlandicus (Homemann) A. Löve \& D. Lave] - FNA

Artemisia campestris L. var. caudata (Michx.) Palmer \& Steyerm. - Dorn01, KTZ, E

[Anemisia campestris L. subsp. caudata (Michx.) H. M. Hall \& Clem.] - FNA, GPF, KTZ

[Antemisia caudatus Michx.] - $\mathrm{HDH}$

[Artemisia forwoodii A. Gray] - FNA

[Oligosporus caudanus (Michi.) Polfakov] - SFE, W\& W

Artemisia campestris L. var- pacifica (Nutt.) M. Peck - E/W

[Artemisia campestris L. subsp, borealis (Pall.) H. M. Hall \& Clem. var, scouleriana (Benth.) Cronquist] - UTF

[Antemisia campestris L. subsp. borcatis (Pall.) H. M. Hall \& Ctem. var, scouleriana (Hook.) Crongulst] - KT2

[Artemisia campestris L. subsp. pacificus (Nutt.) H. M. Hall \& Clem.] - FNA

[Anterisia campestris var. petiolata S. L. Welsh] - FNA

[Artemisia campestris L. var. scoukriana (Bessar) Cronquist] - IMF, RMC

[Artersisia desentorum Sprengel var, scculeriana (Besser) Cronquist] - FNA

[Artemisia pacifican Nutt.] - FNA, HDH

[Oligosporus campestris (L.) Cassini sultsp. pacificas(Nutt.) W. A. Weber] - FNA

[Oligosporus pacificus \{Nutt.) Poljakov]-FNA, SF, w\&W

Artemisia campestris L, var. purshii (Hook.) Cronquist - E/W

[Artemisin borealis Pall.] - HDH, fep.

[Artemisia campestris L. subsp, borcelis (Pall.) var, borealis (Pall.) H. M. Hall \& Clem.] - KTZ

[Artemisia campestris L. subsp, borealis Pall. var, purshai (Hook.) Cronguist] - IMF

[Artemisia campestris L. subsp, borealis (Pall.) H. M. Hall \& Clem, var, purshit (Hook.) Cronquist]

[Artemisia spithamaen Pursh] - HDH

[Oligosporus groenlandicus (Homem.) A. Love \& D, Löve] - SFI, W\&W

Artemisia cana Pursh var. cana - Dorn01, E/W

[Artersisia cana Pursh] - GPF, HDH, IMF

[Artemisia cana Pursh subsp, cana] - FNA, KTZ

[Artemisia columbiensis Nutt.] - FNA 
[Seriphidium canum (Pursh) W. A. Weber] - SFt, W\& W

Artemisia cana Pursh var. viscidula Osterh. - Dom01, UTF. E/W

[Artemisia cana Pursh] - GPF, HDH, IMF

[Artemisia cana Pursh sabsp. viscidula (Osterh.) Bectle] - FNA, KTZ

[Artemisia viscidula (Osterh.) Rydb.] - FNA

[Seriphidium canum (Pursh) W. A. Weber]-SF!, W\&W

Artemisia carruthii A. W, Wood ex Carruth - FNA, GPF, HDH, IMF, KTZ, UTF, W\&W, E

[Artemisia bakeri Greene] - FNA

(Artemisia carruthii A. W. Wood] - SFE

[Artemisia coloradensis Osterth.] - FNA

[Antemisisa kansana Britc] - FNA

[Artemisia vulgaris L. subsp, wrightii (A. Gray) H. M. Hall \& Clements] - FNA.

[Arlemisia wrightii A. Gray] - FNA

Artemisia dracunculus L, - Dom01, FNA, GPF, IMF, KTZ, EW

[Artemisia aromatica A. Nelson] - FNA

[Artemisia dracurculina S. Watsen] - FNA

[Artemisia dracunculoides Pursh] - FNA

[Artemisia dracunculoides Pursh subsp. dracunculina (S. Watson) H.M. Hall \& Clements] - FNA

[Artemisia dracunculus L. subsp. dracunculus] - HDH

[Artemisia dracunculus L var. dracunculus]

[Ártemisia dracurculus L. subsp. glauca (Pall.) H. M. Hall \& Clem.] - HDH

[Artemisis dracunculus L. subsp. glawea (Pall. ex Willd.) H. M. Hall \& ClemL]

[Artemisia dracunculus L. var, glauca (Pall.) Besser]-UTF

[Oligosporus dracunculus (L.) Poljakov subsp. dracunculinus (S. Watson) W. A. Weber] - SFE, W.W

[Oligosporus dracunculus (L.) Poljakov subsp. dracumculus] - W\&W', Eurasian.

[Oligosporas dracunculas (L.) Poljakov subsp. glaucus (Pall.) A. Lōve \& D. Löve]] - SF!, W\& W'

[Oligosporus dracunculas (L.) Poljakov subsp. glaucus (Paill. ex Willd.) A. Löre \& D. Löve] - W\& W

[Artemisia glauca Pallas ex willd.] - FNA

[Artemisia glauca Pallas ex willd. var, megacephala B. Boivin] - FNA

Artemisia filifolia Torr, - Dom01, GPF, HDH, IMF, KTZ, UTF, E/W

[Artemisia platersis NutL] - FNA

[Oligosporus filifolius (Torr.) Poljakov] - SF!. W\&W

Artemisia franserioides Greene - FNA, HDH, KTZ, SF!, W\&W, E/W

Artemisia frigida Willd - FNA, GPF, HDH, IMF, KTZ, SF!, UTF, W\&W, E'W

[Artemisia frigida Willd. var, gmeliniana (Besser) Besser] - FNA

[Artemisia frigida Willd, var, williamsiae S. L. Welsh] - FNA

Artemisia laciniata Willd, subsp. parryi (A. Gray) W. A. Weber - FNA

[Artemisia parryi A. Giray] - FNA

[Artemisia saxicola Rydb var. parnyi A. Nélson] - FNA

Artemisia longifolia Nutt. - Dom01, FNA, GPF, HDH rep, KTZ, SFE, W\&W, E

[Artemisia falcata Rydb.] - FNA

[Anemisia ludoviclana Nutr, var, inergifolia A. Nelson] - FNA

[Artemisia natronensis A. Nelson] - FNA

[Anemisia vulgaris L. subsp, longifolia (Nutt.) H.M. Hall \& Clements] - FNA

[Artemisia vulgaris L. var, longifolia (Nutt.) M. Peck] - FNA

Artemisia ludoviciana Nutt. var. albula (Wooton) Shinners - IMF. UTF, W

[Artemisia lodeviciana Nutt, subsp albula (Wooton) D. D, Keck] - FNA, HDH, KTZ, SFW, WEW

[Artemisia microcephala Wooton] - FNA

Artemisia ludoviciana Nutt. var. ineompta (Nutt.) Cronquist - Dorn01, IMF, UTF, EJW

[Artemisia arachnoidea E. Shel\&on] - FNA

[Artemisin atomifena Piper] - FNA

[Artemisia iscompta Nutt.] - FNA

[Artemisia lindleyana Besser] - FNA

[Artemisia ladeviciana Nurt, subsp. incompta (Nunt.) D. D. Keck] - FNA, HDH, KTZ, SFI, W\&W

[Artemisia prescortiana Besser] - FNA 
[Artemisia vulgaris L var, incompta (Nutt.) H. St. John] - FNA

Artemsisa ludoviciana Nutt. var, latiloba NutL - Dorn0]

[Artemisia candicans Rydb.] - KTZ

[Artemisia ludoviciana var. latiloba Nutt.] - KTZ

Artemisia ludoviciana Nutt, var. ludoviciana - Dom01, GPF, IMF, UTF, E/W

[Artemisia ludoviciana Nutt. subsp. ludoviciana] - HDH, KTZ, SF!, W\&W

Artemisia ludoviciana Nut, var. mexicana (Willd, ex Spreng.) A. Gray - IMF, EW

[Artemisia lodeviciana Nutt. subsp, mexicana (Willd.) D. D. Keck]-FNA, HDH, SFI, W\& W

[Artemisia ladoviciana Nutt. subsp. mexicana (Willd. ex Spreng.) D. D. Kedk] - KTZ

[Artemisia ludoviciana Nutt. var. mexicana (Willd.) Fernald] , GPF, UTF

[Artemisia mexicana Willd. ex Spreng.] - FNA

[Artemisia neomexicana Green ex Rydb.] - FNA

[Artemisia revoluta Rydb]- - FNA

Artemisia michauxiana Besser - Dorn01, FNA, HDH rep. IMF, KTZ, SF!, UTF, W\&W, E/W

Artemisia norvegica Fr. var, saxatilis (Besser) Jeps, - IMF, E/W

[Artemisia arctica Less.] - FNA

[Artemisia urctica Less. subsp. aretica] - FNA, KTZ

[Artemisia arctica Less. subsp. comsta (Rydb.) Hultén] - FNA

[Artemisis arctica Less. subsp. chrendorferi Konobkov] - FNA

[Artemisia arctica Lsss. subsp. saxicola (Rydb.) Hulten] - SF!, W\& W

[Artemisia chamissoniana Besser var, sacatilis Besser in W. J. Hook.] - FNA

[Anemisia comala Rydb.] - FNA

[Artemisia norvegica Fr. subsp. saxatilis (Besser) H. M. Hall \& Clem.] - HDH

[Artemisia norvegica Fr, var. piccetorum S. L. Welsh \& Goodrich] - FNA, UTF

Artemisia nova A. Nelson-FNA, TMF, KTZ, UTF, W

[Artemisia arbuscula Nutr. subsp. nova (A. Nelson) G. H. Ward] - FNA

[Artemisia arbuscula Nut, var. nova (A. Netson) Cronguist] - FNA

[Artemisia tridentasa Null subsp. nova (A. Nelson) H. M. Hall \& Clem.] - HDH

[Artemisia tridentata Neatt var, nova (A. Nelson) Cronquist] - FNA

[Seriphidium novum A. Nelson] - SFW

[Seriphidium novum (A. Nelson) W. A. Weber] - W\&W

Artemisia parryi A. Gray - HDH, KTZ, UTF, E/W

[Artemisia laciniata Willd subsp. parryi (A. Gray) W. A. Weber]-SF!, W\&W

Artemisia pattersonii A. Gray - FNA, HDH, KTZ, SF!, W\& W, E/W

[Artemisia monocephala (A. Gray) A. Hetler] - FNA

[Artemisia scopulorum A. Gray var, monosephala A. Gray] - FNA

Artemisia pedatifida Nutt. - Dorn0I, FNA, GPF, HDH, KTZ, E/W

[Oligosporus pedanifidus (Nuat.) Poljakov] - SFI, WeW

- Artemisia porteri Cronq. - ENDEMIC <WY>, Dom01, FNA, KTZ

Artemisia pygmaea A. Gray - FNA, IMF, KTZ, UTF, W

[Seriphidiam pygmacum (A. Gray) W. A. Weber]-FNA, SFW, WQW

Artemisia scopulorum A. Gray - FNA, HDH, IMF, KTZ, SF!, UTF, W\&W, E/W

Artemisia spiciformis Osterh. - FNA, IMF, UTF, E/W

[Antemisia rothrockii] - $\mathrm{HDH}$

[Anemisia X spiciformis Osterh.] - RMC

[Artemisia tridentata Nutt subsp, rothrockil (A. Gray) H. M. Hall \& Clem.] - HDH

[Artersisia tridentara Nuat subsp. spiciforenis (Osterh.) Kartesz \& Gandhi] - FNA, KTZ

[Seriphidium spcifiome (Oslerh.) Y. R. Ling] - FNA

[Seriphidium rothrockii (A. Gray) W. A. Weber] - W\&W, er, rep.

[Serighidiam vaseyanum (Rydb, W W. A. Weber] - SF], WEW

Artemisia spinescens D. C. Eaton - Dorn01, HDH, IMF, UTF, W

[Picrothamnus desertorum Nust.] - FNA, KTZ, SFW, W\&W

Artemisia tridentata Nutt. var. tridentata - IMF, UTF, E/W

[Artemisin nngustifolia (A. Gray) Rydb.] - FNA 
[Artemisia tridentata Nutt. subsp, tridentata] - FNA, HDH, KTZ

[Artemisia tridentata Nutt. subsp, xericensis Winward ex R. Rosentreter \& R. G. Kelscy] - FNA

[Seriphidium tridentatum (Nutt) W. A. Weber] - SF!

[Seriphidium tridentatum (Nutt.) W. A. Weber subsp. tridentatum] - Wa W

Artemisia tridentata Nut, var. vaseyana (Rydb.) B. Boivin - GPF, IMF, UTF, EJW

[Artemisia tridentata Nutt. subsp. vaseyana (Rydb.) Bectle] - FNA, KTZ

[Arlemisia tridentata Nutt. var. pauciflora Winward \& Goodrich] - FNA, UTF

[Artemisia tridentata Nutt. subsp. vaseyanum (Rydb.) W. A. Weber]

[Artemisia vaseyana $R y d b$.$] - FNA$

[Seriphidium vaseyanaum (Rydb.) W. A. Weber] - FNA, W\&W, SF:

Artemisia tridentata Nutt. var, wyomingensis (Beetle \& Young) S. L. Welsh - IMF, UTF, W

[Artemisia tridentata Nutt. subsp. wyomingensis Beetle \& Young] - FNA, KTZ

[Seriphidium tridentatum (Nutt.) W. A. Weber subsp. wyomingense (Beetle \& Young) W. A. Weber] - W\& W

[Seriphidium vaseyanum (Rydb.) W. A. Weber subsp. wyomingensis (Beetle \& Young) W. A. Weber] - SFW

- Artemisia tripartita Rydb, var, rupicola (Beetle) Dom - ENDFMIC $<$ WY $>$, Dorn0I, FNA

[Artemisia tripartita Rybd. subsp. rupicola Bectle] - KTZ

Artemisia tripartita Rydb, var, tripartita - Dorn01. E. FNA

[Artemisia tripantita Rydb.] - HDH, IMF, UTF

[Artemisia tripartita Rydb. subsp. Ifipartita] - KTZ

[Seriphidiam triparticum (Rydb.) W. A. Webor] - SFE. W\&W

\section{Askellia (see Crepis)}

Aster (see also Almutaster, Chaetopappa, Diplactis, Eucephalus, Eurybia, Herrickia, Machaeranthera, Solidago, Symphyotrichum, Xanthisma, Xylorhiza)

[NS - Aster s.1. tas been segregated in FNA Vol. 20 (see also Semple01, Noyes99)]

\{Aster praealtus Poir.\} - HDH, W\&W, err, rep.

\{Aster pracaltus Poir. var. pracaltus; - GPF

\{Symphyotrichum pracaltum (Poir.) G. L. Nesom) - W\& W'. err. rep.

(Symphyotrichum pracaltum (Poir.) G. L. Nesom var, pracalum? $-\mathrm{KTZ}<\mathrm{NE}, \mathrm{OK}>$

\{Aster sibiricus L. var, meritus (A. Nels.) Raup\} - WY checklist

\{Aster behringensis Gandog.\} - KTZ

(Aster meritus A. Nels.) - KTZ

(Aster fichardsonii Spreng.) - KTZ

\{Aster sibiricas subsp richardsonii (Speeng, A\& D. Love\} - KTZ

\{Aster sibirkas var. giganteus (Hook.) Gray\} - KTZ

\{Aster sibiricas var, meritus (A. Nels.) Raup) - KTZ

\{Eurybia merita (A. Nets.) Nesom) - FNA. KTZ

\{Eurybia sibirica (L.) Nesom\} - KTZ

\{Eurybia sibirica (L) subsp. richardsonii\} - KTZ

\{Eurybia sibirica (L-) subsp. giganteus\} - KTZ

\{Eurybia sibirica (L.) var, meritus (A. Nels.)\} - KTZ

\{Baceharis emoryi A. Gray\}- HDH, KTZ $<A Z, N M, U T>$, UTF, W\&W, applies to Baccharis salicina

\{Baccharis glutinosa Pers.\} - GPF, HDH rep.. IMF, UTF, W\&W, applies to B. salicina \{Bacchiaris salicifolia (Ruiz \& Pav.) Pres.) - KTZ

Baccharis salicina Torr. \& A. Gray - FNA, GPF, HDH, IMF, KTZ, SF!, UTF, W\&W, E/W

[Baccharis emoryi A. Gray] - FNA

Baccharis salicifolia (Ruiz \& Pav.) Pers. - FNA

[Baccharis glutincsa Pers.] - FNA 
[Bachlaris viminea DC.] - FNA

[Bachlaris viminesa DC var. atwoodii S. L. Wekh] - FNA

[Molina salicifolia Ruiz \& Pav.] - FNA

Baccharis wrightii A. Gray - FNA, GPF, HDH, IMF, KTZ, SFE, UTF, W\&W, E

Bahia (see Amauriopsis, Picradeniopsis, Platyschkuhria, Sehkuhria)

Balsamita (see Chrysanthemum)

Balsamorhiza hispidula Sharp - Dorn01, FNA, HDH, SFW, W\&W, W

[Balsamorhiza hookeri Nutt. var. hispidula (Shap) Cronquisa] - IMF, UTF

[Balsamortiza hookeri (Hook.) Nutt. var. hispidula (Sharp) Cronguist] - KTZ

Balsamorhiza incana Nutt, - FNA, Dom01, KTZ

Balsamorhiza sagittata (Pursh) Nutt, - Dom01, FNA, GPF, HDH, KTZ, SF!, UTF, W\&W, E/W [Buphthalmum sagittatum Pursh] - FNA

Balsamorhiza x tomentosa Rydb, - WY checklist, KTZ

Berlandiera lyrata Benth. - FNA, GPF, HDH, KTZ, SFE, W\&W, E

[Berlandiera incisa Torr. \& A. Gray] - FNA

Bidens

(Identification sources:

Bidens bigelovii A. Gray - FNA, GPF, HDH, IMF, KTZ, SFE, W\&W, E

[Bidens bipinnata L.] - HDH, doubtful, KTZ, W\&W

* Bidens cernua L, - FNA, Dorn01, GPF, HDH, IMF, KTZ, SFE, UTF, W\&W, E

Bidens frondosa L. - Dorn01, FNA, GPF, HDH, IMF, KTZ, SF!, UTF, E/W

[Bidens frondosa L. var. frondosa] - W\&W

Bidens heterosperma A. Gray - FNA, HDH, KTZ, W\&W

Bidens laevis (L.) Britton, Sterms, \& Poggenb,- FNA, IMF, KTZ, W\& W'

[Bidens laevis Brition, Sterns, \& Poggenb.]-HDH

Bidens polylepis S, F. Blake - FNA, HDH, W\&W

[Bidens aristosa (Michx.) Bricton] - KTZ

Bidens tenuisecta A. Gray - FNA, HDH, IMF, KTZ, SF!, UTF, W\&W, E/W

* Bidens tripartita L, - FNA, KTZ, UTF

[Bidens comosa (A, Gray) Wiegand] - Dorn01, GPF, HDH, IMF, SFE, UTF, W\&W, E

Bidens vulgata Greene - FNA, Dorn01, GPF, HDH, IMF, KTZ

[Bidens frondosa L. var, pubenala Wiegand] - WEW

Bolophyta (sec Parthenium)

Brachyactis (see Aster)

Breea (sec Cirsium)

Brickellia

(Identification sources: 
Brickellia actractyloides A. Gray var, actractyloides - FNA

Brickellia brachyphylla (A. Gray) A. Gray - FNA, GPF, KTZ, E/W

[Brickellia brachyphylla A. Gray]-HDH. SF!. W\&W

Brickellia californica (Torr \& A. Gray) A. Gray - FNA, GPF, HDH, IMF, SF!, UTF, W\&W

[Brickellia californica (Torr. \& A. Gray) A. Gray var. californica] - KTZ, EW

[Bulbostylis califarnica Torr. \& A. Gray] - FNA

Brickellia eupatorioides (L.) Shinners var chlorolepis (Wooton \& Standl.) B. L. Turner - FNA. KTZ, E/W

[Brickellia rosmarinifolia (Ventenat) W. A. Weber subsp. chlorolepis (Wooton \& Standl.) W. A. Weber] - SF!, W\&W

[Kuhnia chorolepis Wooton \& Standl.] - GPF, HDH, UTF

[Kuhnia eupatorioides L. var. chlorolepis (Wooton \& Standl.) Cronquist] - IMF

Brickellia eupatorioides (L.) Shinners var, corymbulosa (Torr, \& A. Gray) Shinners - Dorn01, FNA, KTZ, E

[Brickellia cupatorioides (L.) Shinners] - SFE, W\&W

[Kuhnia eupalorioides I. var. corymbosa Tort. \& A. Gray] - GPF, HDH

Brickellia grandiflora (Hook.) Nutt.] - Dom01, FNA, GPF, HDH, IMF, KTZ, SF!, UTF, W\&W

[Brickellia grandiflora (Hook.) Nutt. var. grandiflora] - E/W

Brickellia longifolia S. Watson var. longifolia - FNA, IMF, KTZ

[Beickellia longifolia S. Wasson] - SFW, UTF, W\&W, W

Brickellia microphylla (Nutt.) A. Gray var, microphylla - FNA

[Brickeltia microphylla (Nutt.) A. Gray var, wassonit (B. L. Rob.) S. L. Welsh] - FNA

[Brickeltia watsonii B. L. Rob.] - FNA

Brickellia microphylla (Nutt.) A. Gray var, scabra A. Gray - Dorn01. IMF, KTZ, UTF, W

[Brickellia microphylla (Nutt.) A. Gray subsp. scabra (A. Gray) W. A. Weber] - SFW, W\& W

[Brickellia scabra (A. Gray) A. Nelson ex B. L. Rob.] - HDH

Brickellia oblongifolia Nutt, vaf, linifolia (D. C. Eaton) B. L. Rob, - FNA, HDH, IMF, KTZ,

UTF, W

[Brickellia lintiolia D. C. Eanon in S. Wats.] - FNA

[Brickellia oblengifolla Nutt.] - SFW, W\&W

Brickelliastrum fendleri (A. Gray) King \& H. Robins. - FNA

[Brickellia fendleri A. Gray] - FNA

* Carduus acanthoides L. - Dom01, GPF, IMF, KTZ, SF!, W\&W, E/W

[Carduus acanthoides L. subsp. acanthoides] - FNA

* Carduus nutans L. - Dorn01, HDH rep., [MF, UTF, E/W

[Cardaus nutuns L., sabsp, leiophy llus (Petrovic) Stoj, \& Stefani] - GPF

[Cardaus numans L. sabsp, macrocephalus (Desf, Nyman] - GPF

[Cardaus nutans L. sabsp, macrolepis (Peterman) Kami] - KTZ, SF!, W.8 W

* Carthamus tinctorius L. - Dorn01, FNA, GPF, HDH, IMF, KTZ, UTF, W\&W

[No vouchers; evidently only in cultivation]

Centaurea

(Identification sources:

* \{Centaurea americana Nutt\} - GPF, HDH, exp., KTZ $<\mathrm{AZ}, \mathrm{KS}, \mathrm{NM}, \mathrm{OK}>$

* Centaurea cyanus L - Dorn01, FNA, GPF, HDH, IMF, KTZ, UTF, E/W

Snow, N, Junury 2009. Checklist of Vaccular Plants of the Southom Rocky Momain Rezion. CVersion $3 x$ 
[W] - used in revegetation but does not appear to persist]

[Leucacantha cyanus (L.) Nieuwl. \& Lanell] - SF!, W\&W

* Centaurea diffusa Lam, - Dom01, FNA, GPF, IMF, KTZ, UTF, E/W

[Acosta diffusa (Lam.) Sojak] - SE!, W\&W

* Centaurea iberica Trev, ex Spreng. - FNA, KTZ

* Centaurea jacea L. - IMF, UTF, KHD (sec note)

[NS - KHD reports Centaurea pratensis as a rare weed in Colorado, which FNA (19:186) treats as "collective name" for intermediates between C. jacea and C. nigral

[Centaurea eriophora L.] - KTZ, EW

[Jaces pratensis Lam.. $5 F$. W\& $\mathrm{W}$

* Centaurea macrocephala Puschk. ex Willd. FNA KTZ, E

[NS - WJ indicates deebtful outside of cultivation in CO, but FNA lists ss present for $\mathrm{CO}$ ]

[Grossheimia macrocephasla (Mussin-Puschkin) Sosnowsky \& Takhtajin] - SFE

[Grossheimia macrocephala (Mussin-Puschkin ex Willd.) Sornowsky \& Takheajan] - W\& W'

* Cenaturea scabiosa L. - Dom01

* Centaurea stoebe L. subsp. micranthos (S. G. Gmelin ex Gugler) Hayek - FNA

[Acosta maculesa (L.) Holub] - SF!, W\&W

[Centaurea biebersteinii DC.] - FNA, KTZ

[Centaurea maculosa Lam. - Dorn01, GPF, RMC. [MF, UTF, E W] - FNA

* Centaurea solstitialis L. - FNA, GPF, HDH, IMF, KTZ, UTF

[Leucantha solstitialis (L.) A. Löve \& D. Lủve] - W\&W

* Centaurea virgata Lam. subsp. squarrosa (Boissier) Gugler - FNA

[Centaurea virgata Lam. var. squarrosa Boissier] - FNA

[Centaurea squarrosa Willd.] - FNA

Chaenactis douglasii (Hook.) Hook. \& Am, var. alpina A. Gray - FNA, KTZ

[Chasenactis alpina (A. Gray) M. E. Jones] - Dom01, IMF, SF!. W\& W, E/W

[Chsonactis alpina (A. Gray) M. E. Jones var. alpina] - HDH, UTF

[Chistactis alpina (A. Gray) M. E. Jones var. leucopsis (Greene) Cockerell ex Stockw.] - W\& W

[Chactactis alpina (A. Gray) M. E. Jones var. leucopsis (Greene) Cockerell] - UTF

[Chsctactis alpina (A. Gray) M. E. Jones var. leusopsis (Greene) Stockw.]-HDH, rep.

[Chsuractis leucopsis Givene] - KTZ

Chacnactis douglasii (Hook.) Hook. \& Am. var. douglasii - HDH, KTZ.

[Clusenactis angustifolia Greene] - $\mathrm{HDH}$

[Chochactis douglasi (Hook.) Hook. \& Am.] - FNA. SF], UTF, W\&W, EW

[Clenenactis doughsi (Hook.) Hook, \& Arn. var, achilleifolia (Hook. \& Am.) A. Nelson] - GPF, HDH, IMF

[Chnocostis douglasili (Hook.) Hook. \& Arn. yar, montana M. E. Jones] - Dorno1, IMF

[Cluscoassis humilis Rydb.] - $\mathrm{HDH}$, rep.

[Hymenopappes douglasii Hook.] - FNA

[Macrocarpus douglasii (Hook.) Nutt.] - FNA

Chaenactis stevioides Hook. \& Am. - FNA, HDH, KTZ, SFW, UTF, W\&W

[Chactactis stevioides Hook. \& Am. var, steviodes] - Dorn01, IMF, W

Chactopappa cricoides (Torr.) G. L. Nesom - Dom01, FNA, IMF, KTZ, UTF, E/W

[Aster arenosus S. F. B lake] - $\mathrm{HDH}$

[Chastopappa ericoides (A. Gray) G. L. Nesom] - W\&Wt

[Leucelene sricoides (Torr.) Gresoe] - SF]

[Leucelene ericoides (Tor, \& A. Gray) Greene] - GPF, W\&W

Chamaechacnactis scaposa (Eastw.) Rydb, - Dorn01, FNA, HDH, IMF, SFW, UTF, W\&W, W

[Chasenactis scaposa Eastw.] - FNA

[Chamaechaenactis scaposa (Eostw.) Rydo, var, scaposa] - KTZ

Snow, N, January 2009. Checklist of Vecculer Plants of the Southum Rodky Momain Region. CVersion $3 x$ 


\section{Chamomilla (see Matricaria)}

Chloracantha spinosa (Benth.) Nesom - KWA, KTZ

[Aster spincsus Benth.] - KWA., M\&H, KTZ

[Erigeron crtegae Blake] - KTZ

[Leucosyris spinosa (Benth.) Greene] - KWA, KTZ

\section{Chlorocrepis (see Hieracium)}

\section{Chrysanthemum}

[NS - generic boundaries unsiable]

* Chrysanthemum coccineum Willd, - KTZ, W\&W

* Chrysanthemum coronarium L. - HDH, KTZ, W\&W

[No knowa voucher at COLO]

* Chrysanthemum leucanthemum L. - Dorn01, GPF, IMF, UTF, E/W

[Chrysanthemum leucanthemum L. var, pinatifidum Lecoq \& Lamotte] - HDH

[Leucanthemum vulgare Lam.] - FNA, KTZ, SF!, W\&W

* Chrysanthemum maximum Ramond - W

[NS - established in parts of Boulfer County]

[Leucanthemum maximum (Ramond) DC.]-FNA, KTZ, SFW, W\& W ${ }^{1}$

\section{Chrysopsis (see Heterotheca)}

\section{Chrysothamnus (see also Erieameria, Lorandersonia)}

(Identification sources:

Chrysothamnus depressus Nutt. - FNA, HDH, IMF, KTZ, SF!, UTF, W\&W, E/W

Chrysothamnus greenei (A. Gray) Greene - Dom01, FNA, IMF, KTZ, SF!, UTF, E/W

[Bigelowia gretoci A. Gray] - FNA

[Chrysorhamnes greetei (A. Gray) Greene subsp. filifolius (Kydb.) H. M. Hall \& Clem. J- HDH, W\&W

[Chrysothammes greenei (A. Gray) Greene subsp. greenei] - HDH, W\& W

Chrysothamnus vaseyi (A. Gray) Greene - Dom01, FNA, HDH, IMF, KTZ, SF!,UTF, W\& W, E/W

[Bigelowia vaseyi A. Gray] - FNA

Chrysothamnus viscidiflorus (Hook.) Nutt. subsp. axillaris (D. D. Keck) L. C. Anderson - FNA

[NS - combination unavailable at varietal level for consistency with other infraspecific taxa]

[Chrysothamnes axillaris D. D. Keck] - FNA

Chrysothamnus viscidiflorus (Hook.) Nutt. var. lanceolatus (Nutt.) Greene - Dom01, IMF, UTF, $\mathrm{F}$.

[Chrysothamnes viscidiflorus (Hook.) Nutt, subsp, elegans (Greene) H. M. Hall \& Clem.]-HDH. W\&W

[Chrysothamnus viscidiflonus (Hook.) Nutt, sabsp, lanceolatus (Nutt.) H. M. Hall \& Clem.] - FNA, GPF, HDH, KTZ, SFE, W:W

Chrysothamnus viscidiflorus (Hook.) Nutt. var. puberulus (D. C. Eaton) Jeps. - IMF, UTF

[Chry sothamnes viscidiflorus (Hook.) Nutt, subsp. puberulus (D. C. Eaton) H. M. Hall \& Clem.] - HDH, KTZ, WEW

Chrysothamnus viscidiflorus (Hook.) Nutt. var. viscidiflorus - Dorn01, 1MF, UTF, E/W

[Chrysotharmnes viscidiflorus (Hook.) Natt.] - SFW

|Chry sothamnes viscidiflonus (Hook.) Nutt, subsp, axillaris (D, D. Keck) L. C. Anderson] - KTZ, W. W

[Chrysothamners viscidiflorus (Hook.) Nutt, subsp, latifolius (D. C. Eaton) H. M. Hall \& Clen.] - W. W

[Clerysothamness viscidiflorus (Hook.) Nutt, sabsp, pumilhs (Nuts.) H. M. Hall \& Clem.] - HDH, W\& W 
[Chry sotharmus viscidiflarus (Hook.) Nutt. sabsp, viscidiflorus] - FNA, GPF, HDH, SFE, W\&W

[Chrysothamnus vixidiflorus (Hook.) Nutt. subsp. viscidiflorus var, latifolius (D. C. Eat.) Greene] = KTZ

[Chrysothamnus viscidiflarus (Hook.) Nutt. sabsp. viscidiflarus var. viscidiflarus] - KTZ.

[Chrysotharmens viscidiflarus (Hook.) Nutt. var. sterophyllus (A. Gray) H. M. Hall] = UTF

[Chrysotharmes viscidiflorus (Hook.) Nutt. sabsp, sienophyllus (A. Gray) H. M. Hall \& Clem.] - HDH, W\&W

[Chrysothamnus viscidiflorus (Hook.) Nutt. subsp. viscidiflorus var, stenophyllus (A. Gray) H. M. Hall] - KTZ

* Cichorium intybus L, - Dorn01, GPF, HDH, IMF, KTZ, SF!, UTF, W\&W, EW

\section{Cirsium}

(Identification sources: )

[NS - The FNA treatment is surely a general improvement for Cirsium but its laxonomy in the SRMR would benefit greatly from a doctoral dissartaion. Our hertaria regionally need a loc of curatorial attention.]

Cirsium arizonicum (A. Gray) Petrak var, bipinnatum (Eastwood) D. J, Keil - FNA

[Chicus arizonicus A. Gray] - FNA

[Cinsus drumondii Tort. \& A. Gray var. bipinnatus Eastuood] - FNA

[Cirsium bipinnatum (Eastwoed) Rydb.] - HDH

[Cirsiam calcaream (M. E. Jones) Woocon \& Standl.] - IMF, KTZ, SFW. W\&W, W

[Cirsiam calcareum (M. E. Jones) Wooton \& Standl, var. bipinnatum (Eastw.) S. L. Welsh - FNA, UTF

[Cirsiam calcancum (M. E. Jones) Wooton \& Standl. var, cakeareum] - UTF

[Cirsiam calcareum (M. E. Jones) Wooton \& Standl. var. pulchellum (Groene) S. L. Welsh) - FNA. UTF

[Cirsiam pukchellum (Greene) Wooton \& StandL] - $\mathrm{HDH}$

[Cirsiam pulchellum (Greene ex Rydb.) Wooton \& Stand I ] FNA

[Cirsium pulchellum (Greene ex Rydb.) Wooton \& Standi. subsp. bipinnarum (Eastwood) Petrak - FNA

[Cirsiam pukchellum (Greene ex Rydb.) Wooton \& Standl. subsp. diftusum (Easrwood) Petrak] - FNA

[Cirsium pukhellum (Greene ex Rydb.) Wooton \& Standl. subsp. glabrescens Petrak] - FNA

* Cirsium arvense (L.) Scop, - Dom01, FNA, GPF, IMF, KTZ, W\&W, E/W

[Breea arvensis (L.) Less.]-SFL, Wd. W'

[Breea incana (Gnelin) W. A. Weber, in ed.] - W\& W'

[Cirsitum arvense (L.) Scop. var, arvense] - HDH

[Cirsium arwense (L.) Scop. var, horridum Wimm, \& Grab.]- UTF

[Cirsium arvense (L.) Scop, var, integrifolium Wimen. \& Grab.] - HDH, RMC

[Cirsium arvense (L.) Ssop, var, mite Wimmer \& Grab.] -UTF

[Cirsiam arvense (L.) Ssop, var, vestifum Wimmer \& Graeboer] - $\mathrm{HDH}$

[Cirsiam incanam (S. G. Gmelin) Fishicr ex Bieberstein] - W\& W

Cirsium barnebyi S, L. Welsh \& Neese - Dorn01 (reported), FNA, IMF, KTZ, SFW, UTF, W\&W, W

Cirsium canescens Nutt. - Dorn01, FNA, GPF. HDH, KTZ, SF!, W\&W, E/W

[Cirsium platiense (Rydb.) Fenald] - $\mathrm{HDH}$

Cirsium clavatum (M. E. Jones) Petrak var, americanum (A. Gray) D. J. Keil - FNA

[Carduus spathulatus Osterhceut.] - KTZ

[Caicus carlinoides Schrank var, anericanus A Gray] - FNA

[Cirsiam ceotaureae (Rydb,) K. Schum.]-Dorn01. FNA, HDH, IMF, KTZ, SF, UTF, W\&W, EW

[Cirsiam griseum (Rydb,) K. Scham.]-FNA, KTZ

[Cirsiam laterifolium [Osterh.) Petrak] - FNA, KTZ

[Cirsiam modestum (Osterh.) Cockerell] - FNA, KTZ

[Cirsium remotifoliam (Hook.) DC. subsp oregonense Petrak]

[Cirsiam scapanolepis Petrak] - FNA, KTZ, WRW

[Cirsiam sparhulifoliam Rydb.] - HDH, FNA

Cirsium clavatum (M. E. Jones) Petrak var. clavatum - FNA, UT3

[Cirsium clavatum (M. E. Jones) Petrak] - JMF, KTZ, UTF 
[Cirsium clavatum (M. E. Jones) Petrak var. markaguntense S. L. Welsh] - FNA

- Cirsium clavatum (M. E. Jones) Petrak var, osterhoutii (Rydb.) D. J, Keil - ENDEMIC $<$ CO . FNA

[Cartuus arencousus Osterthout] - KTZ

[Carúuus osterhoutii Ryđob.] - FNA

[Cirsium araneans Rydb.] - FNA, KTZ, W\&W

[? Cirsium eatonii (A. Gray) B. L. Rob.]- W\& W'

[Cirsium osterhoutii (Rydb.) Petrak] - KTZ, W\&W

Cirsium drummondii Torr. \& A, Gray - FNA, KWA, JM, HDH, KTZ, W\&W, misid.

[Carduas drummondii (Torr. \& A. Gray) Coville] - KWA

[Cirsium coccinarum Ostertheut] - FNA, KTZ

[Cirsium coloradense (Rydberg) Cockerell] - KWA

[Cirsium follosum (Hooker) A.P. de Candolle] - KWA

Cirsium eatonii (A. Gray) B. I. Rob, var, eriocephalum (A. Gray) D. J. Keil - FNA

[Carduus hookerianus Nuttall vat, eriocephales (A. Gray) A. Nelson] - FNA

[Cirsium ratonii (A. Gray) B. L. Rob.] - FNA, HDH, IMF, KTZ, SFI, W\&W, EW

[Cirsiam eatonii (A, Gray) B. L. Rob, var, eatonii] - UTF

[Cisriam ctioceplatum A. Gray] - FNA

[Cirsiam srioceptsalum A. Gray vart leiocephalam D, C. Eat, in S. Wats.] - FNA

[Cirsiam scopulorum (Groene) Cockerell ex Daniels - FNA

[Cirsiam scopulorum (Greene) Cockerel] - HDH, SFI, UTF, W\& W

[Cirsiam ineedyi (Rydb.) Petrak] - W\&W

[Caicus emonii A. Gray] - FNA

[Cuicus ericesphalum A. Gray] - FNA

Cirsium eatonii (A. Gray) B. L. Rob. var. hesperium (Eastwood) D. J. Keil - FNA

[Cirsiam hesperium (Eastw.) Petrak] - SF!, Wo W

[Cirsiam hesperium (Eastw.) Rydb.]-HDH

[Cirsiam hesperiusm (Eastwood) Petrak] - FNA

[Chicus hesperius Esswood] - FNA

Cirsium eatonii (A. Gray) B, L. Rob, var, murdockii S. L. Welsh - FNA

[Cirsium mardockit (S. L. Welsh) Cronq.] - FNA

[Cirsiam polphyllam (Rydb.) Petrak] - FNA

[Cirsiamt twęedyi (Rydb,) Petrak] - FNA

Cirsium flodmanii (Rydb.) Arthur - Dom01, FNA, GPF, IMF, KTZ, SFE, W\&W, E

Cirsium inamoenum (Greene) D. J. Keil var. davisii (Cronq.) D. J.Keil - FNA

[Carduas nevadensis Greene] - KTZ

[Cirsiam davisii Crong.] - KTZ

[Cirsiam humboldtense Rydb.] - KTZ

[Cirsiam subniveun Rydb.] - Dorn01, KTZ

Cirsium neomexicanum A. Gray - FNA, HDH, IMF. KTZ, SFW, W\&W, W

[Cirsiam sercuum A. Nelson] - FNA

[Cirsium humboldtense] - FNA

[Cirsium neomexicanum A. Gray var. utahense (Petrak) S. L. Welsh] - FNA, UTF

[Cisrium undularum (Nurt) Sprenget var, albescens D, C, Eat.] - FNA

[Cirsium utahense Petrak] - FNA

Cirsium ochrocentrum A. Gray - Dorn01, FNA, GPF, HDH, IMF, KTZ, UTF, W\&W. E/W

[Cirsum ochrocentrum (A, Gray) A. Gray] - SF!

[Cirsum ochrocentram A. Gray var, ochrocentram] - FNA

Cirsium ownbeyi S. L. Welsh - Dorn01, FNA, IMF, KTZ, SFW, UTF, W\&W, W

Cirsium partyi (A. Gray) Petrak - FNA, HDH, SF!, UTF, W\&W

[Cirsium inornatum (Wooton $\&$ Standl.) Wooton \& Standi.] - FNA

[Cirsiam gilense (Wooton \& Standl.) Wooton \& Standl.] - FNA

[Cirsium pallidum Wooton \& Standi.] - FNA. HDH, KTZ, W\&W

[Cirsium parryi (A. Gray) Petrak] - FNA, HDH, SF!, UTF, W\&W

Snou, N, Janury 2009. Checklist of Vaccular Plants of tho Southom Rocky Moanain Region. CVersion $3 x$ 
[Cirsium parryi (A. Gray) Petrak subsp. partyi] - KTZ, E/W

[Cirsium parryi (A. Gray) Petrak subsp. mogolionicum Schasck \& G. A. Goodwin] - FNA

[Cnicus parryi A. Giray] - FNA

$\bullet$ Cirsium perplexans (Rydb.) Petrak - ENDEMIC <CO >, FNA, KTZ, SFW, W\&W, W

[Carduus perplesans Rydb.] - FNA

[Cirsium perplexans Rydb.]- $\mathrm{HDH}$

[Cirsium vernale (Ostert_) Cockerell] - FNA, KTZ

$\bullet$ Cirsium pulcherrimum (Rydb.) Schum, var, aridum (Dorn) D. J. Keil - FNA

[Cirsiumn aridum Dom] - Dom01, FNA

Cirsium pukherrimum (Rydb.) K. Sshum, - FNA, GPF, IMF, KTZ, UTF

Cirsium pulcherrimum (Rydb, ) K. Schum, var, pulcherrimum - Dom01, FNA

\{Cirsium remotifolium (Hook.) DC. subsp- remotifolium\} - IMF, KTZ.

[NS - restricted to Pacific states fide FNA [as C. remotifolium (Hook.) Dc, in A. P. DC. \& A. L. P. DC.

Cirsium scariosum Nuttall var, americanum (A. Gray) D. J. Keil - FNA

[Cirsiam americanum (A. Gray) K. SchumL - FNA

[Cirsiam acaule (L.) Scop، var, americantum A. Gray] - FNA

[Cirsium aczalescens (A. Gray) K. Schum.] - FNA

[Cirsiam coloradoense (Rydb.) Cockerell ex Daniels subsp. acaolescers (A. Gray) Petrak] - FNA

[Cirsiam coloradoense (Rydb.) Cockerell ex Daniels subsp. longissimum (A.Heller) Petrak] - FNA

[Cirsiam drummondil Torr, \& A. Gray var, acaulescens (A. Gray) J. F. Macbride] - FNA

[Cirsiam drummondii Tor, \& A, Gray subsp, latisquamuns Petrak] - FNA

[Cirsiam drummondii Torr. \& A. Gny var, onegonense Petrak] - FNA

[Cirsium drummondii Torr, \& A, Gray subsp, vexans Petrak] - FNA

[Cirsium tioganem (J, W. Congd.) Petrak] - FNA

Cirsium scariosum Nuttall var. coloradense (Rydb.) D. J. Keil - FNA

[Carduus soloradensis Rydb.] - FNA

[Cirsium coloradense (Rydb.) Coskerell] - FNA, W\&W

[Cirsiam drummondii Torr, \& A. Gray var, acaulesoens (A. Gray) J. F, Macbr.] - HDH

[Cirsiam erosum (Rydb,) K. Schumane] - FNA

[Cirsium X erosum (Rydb.) K. Schum.] - PLS

[Cirsiam olivensoens (Rydb, ) Petrak] - FNA

[Cirsiam scariosum Nutt.] - IMF, KTZ, SF!, W\&W, W\&W'

[Cirsiam scariosum Nutt, var, scariosam] - UTF

[Cirsium tiganum (Coogd.)] - FNA

[Cirsiam tioganum (Congd.) Petr, var, coloradense (Rydb,) Dorn] - Dorno1, E/W, FNA

[Cirsiam toganum (Congd.) Petr, var, tioganum] - Dom01, E/W, FNA, RMC

[Cirsium scariosum Nutt, var, scariosum Nutt.] - UTF

Cirsium scariosum Nuttall var. scariosum - FNA

[Cirsium butleri (Rydb.) Petrak] - FNA

[Cirsium lacerum (Rydb.) Petrak] - FNA

[Cirsium mangifum (A. Nelson] - FNA

[Cirsium minganense Victorin] - FNA

Cirsium scariosum Nuttall var, thomeae S. L. Welsh - FNA

Cirsium undulatum (Nutt.) Spreng. - Dorn01, FNA, GPF. HDH, IMF, SF?, W\&W

[Cirsium megacephalum (A, Gray) Cockerell] - HDH

[Cirsium tracyi (Rydb.) Petrak] - FNA, IMF, SFW, W\&W

[Cirsium undularum (Nutt.) Spreng, var, trucyi (Rydh.) S. L. Welsh] - KT7, UTF, w

[Cirsium undulatum (Nutt.) Spreng. var. undulatum] - FNA, KTZ, UTF, EN

* Cirsium vulgare (Savi) Ten. - Dorn01. FNA, GPF, IMF, KTZ, SF!, UTF, W\&W, E/W

[Cirsium lancenlatum (L.) Hill] - HDH

Cirsium wheeleri (A. Gray) Petrak - FNA, KTZ. UTF

[Cirsium wheeleri (A. Giray) Petrak var, wheeleri] - UT3 
Conyza (see also Laennecia)

+ Conyza canadensis (L.) Cronquist - FNA, HDH, SF!, W\&W

[Conyza canadensis [L.) Cronquist var. canadensis] - Dom01, GPF, IMF, KTZ, EW

[Conyza canadensis (L.) Cronquist var. slabrata ( $\Omega$. Gray) Cronquist] - GPF, IMF, KTZ, UTF

[Erigeron canadensis L.] - FNA

+ Coreopsis lanceolata L, - FNA, GPF, HDH, KTZ, W\&W, E

[No voucher at $\mathrm{COLO}$ ]

* Coreopsis tinctoria Nutt, - Dom01, FNA, GPF, HDH, SF!, W\&W

[Corcopsis tincterie Nutt. var. tinctoria] - [MF, KTZ, E/W

* Cosmos bipinnatus Cav, - Dorn01, FNA, HDH, IMF, KTZ, UTF, W\&W

[Grows in Denwer]

Cosmos parviflons (Jacq.) Pers, - FNA, KTZ, E/W

[Cosmos parviflorus (Jacy) Kunth]-HDH, SFI, W\&W

Crepis

(Identification sources:

Crepis acuminata Nutt. - Dorn01, FNA, FGP, JMF, UTF

[Crepis actiminata Nust. subsp, acuminata] - HDH, KTZ, EW

[Crepis acuminata Nutt. subsp. pluriflora Babe, \& Stebbins] - FNA, HDH, KTZ, EW

[Crepis angusiata Rydb.] - FNA

[Crepis seselifolia Rydb.]- FNA ${ }^{\circ}$

[Psilochenia acsuminats (Nutt.) W. A. Weber]-SF], W\& W

[Psilochenia acuminata (Nut.) W. A. Weber]-SFI, W\& W

Crepis atribarba A. Heller - Dorn01, FNA, HDH, IMF, UTF, E/W

[Crepis atribarba A. Heller stibsp, atribarba] - KTZ

[Crepis exilis Osterh.] - FNA

[Crepis exilis Osterh. subsp, originalis Babcock \& Stebbins] - FNA

[C. occidestalis Nutt, var. gracilis D. C. Eat.] - FNA

[Psilochenia atriburba (A. Heller) W. A. Weber] - SF!, WA W

* Crepis capillaris Wallr. - FNA, IMF, KTZ, SFE, E

[Crepis capillaris (L.) Wallt.] - GPF, UTF, W\& W

Crepis intermedia A. Gray - Dorn01. FNA. HDH, IMF, KTZ, UTF, W

[Psibochenta indermedia (A. Gray) W.A. Weber] - SFW, W\&W

Crepis modocensis Greene var. modocensis - FNA, IMF. W

[Crepis modocensis Greene subsp, modocensis] - KTZ, RMC

[Crepis modoeensis Giresec] - Doeo1, HDH, UTF

[Psilochenia modocensis (Greene) W, A. Weher]-SFW, WR W

¿Crepis nana Richardson subsp, clivicola Lege? - KTZ, EW

\{A skellia nana (Richardson) W. A. Weber? - SF!, W E W

Crepis nana Richardson var. ramosa (Babc.) Cronquist - Dorn01, FNA, IMF, E/W

[Askellia nana (Richandson) W. A. Weber] - SF, W\&W

[Crepis nana Richardson] - UTF

[Crepis nana Richardson subsp, nana] - HDH, IMF, misapp. KTZ, RMC

Crepis occidentalis Nutt. subsp. conjuncta Babcock \& Stebbins - FNA

Crepis oceidentalis Nutt. var. costata A. Gray - Dorn01, FNA, UTF, E/W

[Crepis occidentalis Nutt. subsp, costata (A. Gray) Babc, \& Stebbins] - GPF, HDH, IMF, KTZ

[Psilochenia occideotal is Nutt.] - SPI

[Psilochenia occidental is (Nuts,) Nutt subsp, costata (A. Gray) W. A. Weber] - WE W

Snow, N, January 2009. Checklist of Vacculer Plants of the Southum Rocky Momain Region. CVersion $3 x$ 
Crepis oceidentalis Nutt. var, occidentalis - Dorn01, FNA, UTF, E/W

[Crepis occidentalis Nutt. subsp. occidentalis] - HDH, IMF, KTZ

[Psilochenia oceidental is Nutt.] - SE!

[Psilochenia occidental is (Nutt.) Nutt. subsp. occidentalis] - W\& W

Crepis runcinata (E. James) Torr. \& A. Gray var, glauca (NutL) B. Boivin - Dorn01. FNA, IMF, UTF, E/W

[Crepis runcinata Torr. \& A. Gray subsp. glsuea (Nutt.) Babc. \& Stebbins] - GPF, HDH

[Crepis runcinata (E. James) Torr. \& A. Gray subsp. glauca (Nutt.) Babc. \& Stebbins] - KTZ

[Psilochenia runcinata (E. Jannes.) A. Löve \& D. Love] - SF]

[Psilochenia runcinata (E. James ex Torr.) A. Lōve \& D. LAve] - W\&W

Crepis runcinata (E. James) Torr. \& A. Gray var. hispidulosa Howell ex Rydb, - Dorn01. FNA, IMF, UTF, EW

[Crepis runcinata Torr. \& A. Gray subsp. hispidulosa (Howell) Babc. \& Stebbins] - HDH

[Crepis runcinata (E. James) Torr, \& A. Gray subsp. hispidulose (Howell ex Rydb.) Babc. \& Stebbins] - KTZ

[Psilochenia runcinala (E. James) A. Löve \& D. Läve] - SF!

[Psilochenia runcinata (E. James ex Torr.) A. Löve \& D. Löve] - W\&W

Crepis runcinata (E. James) Torr. \& A. Gray var, runcinata - Dom01. FNA. IMF. UTF, E/W

[Crepis runcinata Tort. \& A. Gray subsp. runcinata] - HDH

[Crepis runcinata (E. James) Torr. \& A. Gray subsp. runcinata] - GPF, KTZ

[Psilochenia runcinata (E. James) A. Love \& D. Love] - SF!

[Psilochenia runcinata (E James ex Torr.) A. Lơve \& D. LAve] - W\&W

Cyclachaena xanthifolia (Nutt.) Fresen, - SF!, W\&W

[Iva xanthifolis Nutt.] - Dom01, GPF, HDH, IMF, KTZ, UTF, EW

Diaperia prolifera (Nutt, ex DC.) Nutt, var. prolifera - FNA

[Evax prolifera Nutt. ex DC.] - GPF, KTZ, W\& W, E

[Evax prolifera Nuti.] - HDH. SFE

\section{Dieteria}

(Identification sources:

Dieteria bigelovii (A. Gray) D. R. Morgan \& R. L. Hartm. var. bigelovii - FNA

[Aster bigelovii A, Gray]- HDH

[Machaeranthera bigelowii (A. Gray) Greene]-Dorn01, IMF, SF1, W\&W

[Machacranthera bigelovii (A. Gray) Greene var, bigelovii] - KTZ, UTF, Tumes87, EN

[Machacranthera pattersonii (A. Gray) Greene] - SFE, w\& w

Dieteria canescens (Pursh) Nutt. var. ambigua (B. L. Turner) D. R. Morgan \& R. L. Hartmain FNA

[Machacranthera eanescens (Pursh) A. Gray var, ambigua B. L. Turner] - FNA

Dieteria canescens (Pursh) Nutt, var. aristata (Eastwood) D. R. Morgan \& R. L. Hartman - FNA.

[Aster canescens Pursh var, aristatus Eastwocd] - FNA

[Machacranthera canescens (Pursh) A. Gray var, aristata (Festwood) B.L. Tumer] - FNA

[Machacranthera rigida Greese] - FNA

Dieteria canescens (Pursh) Nutt. var. canescens - FNA

[Machacranthern divaricata (Nutt,) Greene] - FNA

[Machacranthera lactevirens Greene] - FNA

[Machacranthera latifolia A. Nelson] - FNA

[Machacranthera pluveralenta (Nutt.) Gireene] - FNA

[Machacranthera viscosa (Nutt) Gireene] - FNA

Dieteria canescens (Pursh) Nutt. var. glabra (A. Gray) D. R. Morgan \& R. L. Hartman - FNA

[Aster canescens Pursh var, viridis A. Gray] - FNA 
[Machacranthera canescens (Pursh) A. Giray var. glabra A. Gray] - FNA

[Machacranthera linearis Greene] - FNA

Dicoria canescens A. Gray - FNA

[Dicoria brandegei A. Gray] - 1[DH rep, SFW, UTF, W\&W

[Dicoria canescens A. Gray subsp. brandegeei ( $\mathcal{A}$. Gray) Kartesz] - KTZ

[Dicoria canescens $A$. Gray var. brandegeei $(A$. Gray) Croaquist] - IMF, W

[Dicoria paniculata Eastw.] - HDH, rep-

[Dicoria canescens A. Gray var, wetherillii (Eastw, Cronquist] - IMF <UT?

\{Dicoria canescens A. Gray subsp. wetherillii (Eastw.) Kartesz, comb. in ed.\} - KTZ

Diplactis alpinus (L.) Semple subsp, vierhapperi (Onno) Semple - FNA

[Aster alpinus L.] - HDH

[Aster alpinus L. var. vierhapperi (Onno) Cronquist - KTZ, SF!, W\& W, E W

[Aster culminis A. Nelsoo] - FNA

[Aster alpinus L. var. vicrhapperi Onbo] - FNA

\section{Dugaldia (see Hymenoxys)}

Dyssodia (see also Thymophylla)

Dyssodia papposa (Vent.) Hitche, - Dorn0I, FNA, GPF, HDH, IMF, KTZ, SF!, W\&W, EW

Echinacea angustifolia DC. - Dorn01, FNA, GPF, HDH, SFE, W\&W

[Echinasea angustifolia DC. var, angustifolia] $-\mathrm{KT}, \mathrm{E}$

Echinacea purpurea (L.) Moench - GPF, KTZ, SFE, W\&W ${ }^{1}$, E

[NS - not native to our region fide FNA]

* Echinops sphaerocephalus L, - HDH, FNA, KTZ, W\&W

\{Eclipta alba (L.) Hassk.\}- HDH, exp.

\{Eclipta prostrata (L.) L.\} - GPF, KTZ

iVerbesina prostrata L.; - FNA

Enceliopsis nudicaulis (A. Gray) A. Nelson var, nudicaulis - IMF, KTZ, W

[Enceliopsis nudicaulis (A. Gray) A. Nelsog] - HDH, exp. SFW. UTF, W\&W

Encelia mutans Eastw, - FNA, KTZ

[Enceliopsis nutans (Entstw.) A. Nelson - HDH, IMF, UTF, WEW, W

[Encel iopsis nutans Eastw.] - SFW

[Enceliopsis nutans (Earsts.) A. Nelson]

Engelmannia peristenia (Raf.) Goodm. \& Lawson - FNA, KTZ, SFE, W\& W', Goodman92

[Engelmannia pinnatifida A. Gray ex Nurt.] - GPF, W\&W, E

[Engelmannia pinnatifida Torr. \& A. Gray]- HDH

[Silphium peristeniam Raf.] - FNA

Ericameria

(Identification sources:

Ericameria discoidea (Nutt.) G. L. Nesom var- discoidea - KTZ, E/W

Snow, N, Junary 2009. Checklist of Vacouler Plants of the Southom Rocky Momain Region. CVersion $3 x$ 
[Ericameria discoidea (Nutt.) G. L. Neswen] - FNA, W\& W'

[Haplopappus macronema A. Gray] - UTF

[Haplopappus macronema A. Gray subsp. macronema] - HDH

[Haplopappus, macronema A. Gray var. macronema] - IMF

[Macronema discoideum Nutt.] - SFt, W\&W

\{Ericameria linearifolia (DC.) Urbatsch \& Wussow\} - KTZ $\angle A Z$. UT $>$. W\&W. ers. rep.

\{Haplopappus linearifolius DC. subsp. interior (Coville) H. M. Hall\} - HDH, rep.

\{Hapkspappus linearifolius DC. var, interior (Coville) M. E. Jones\} - IMF

[Hapkapappus linearifolius A. Gray var, interior (Coville) M. E. Jones\} - UTF

Ericameria nauseosa (Pall, ex Pursh) G. L. Nesom \& G. L. Baird subsp, ammophila L. C. Anderson

[NS - it is disagreeable to mix varieties and subspecies under one species but as the species is recently published no varietial combination is available]

Ericameria nauseosa (Pall. ex Pursh) G. L. Nesom \& G. I. Baird var, bigelovii (A. Gray) G, L. Nesom \& G. I. Baird - E, FNA

[Chry sothamnus neaseosus (Pall.) Britton subsp. bigelovii (A. Gray) H. M. Hall \& Clem.] - HDH, SFE

[Chry sothamnus nasevous (Pall, ex Pursh) Britton subsp. bigelevii (A. Gray) H. M. Hall \& Claml.] - W\&W

[Chry sothamnus neaseosus (Pall.) Britton var. bigelovii (A. Gray) H. M. Hall] - IMF, UTF

[Chry sothamnus natuseosus (Paill. ex Pursh) Britton var. bigelovii (A. Gray) H. M. Hall]

[Ericameria nauseosa (Pall. ex Pursh) G. L. Nesom \& G. I. Baird subsp. nauseosa var. bigelovi (A. Gray) G. L. Nesom \& G. I. Baird] - KTZ

[Linosyris bigelevii A. Gray] - FNA

Ericameria nauseosa (Pall, ex Pursh) G, L. Nesom \& G. I. Baird var, glabrata (A. Gray) G. L. Nesom \& G. I. Baird - E/W

[Chry sothamnus nasuseosus (Pall.) Britton subsp. grawoolens (Nuit.) Piper] - GPF, SF]

Chrysothamimus nasuscosus (Palli) Britton subsp. graveolens (Nuat.) H. M. Hall \& Clkm.f- HDH

[Chrysothamnus nasecosus (Pdill, ex Pursh) Britton subsp. graveolens (Nutt.) Piper] -W\&W

[Chry sothamnus nasasevsus (Pall.) Britton var. glabratus ( $A$. Gray) Cronquist] - UTF

[Chry sothammus nanaseosus (Pall.) Britton var. graveoleas (Nutt.) H. M. Hall] - IMF

[Chry sothammes neaseosus (Pall. ex Pursh) Britton var. graveolens (Nuti.) H. M. Hall] - FNA

[Ericameria nauscosa (Pall. ex. Pursh) G, L. Nesom \& G. I. Baind subsp. nauscosa var, glaterata (A. Gray) G, L. Nesom \& G, I. Baird - KTZ

Ericameria nauseosa (Pall, ex Pursh) G. I. Nesom \& G. I. Baird var, hololeuca (A. Gray) G. L. Nesom \& G. I. Baird - KWA, E

[B belowia graveolens Nutt; var, hololeuca] - FNA

[Chrysothamnus nouseosus (Pajl. ex Pursh), Britu, subsp, gaaphatodes (Greene) Hall] - MAH

[Chry sothamnus nantseosus (Pall.) Bricton subsp, bololeucus (A. Gray) H. M. Hall \& Clem.] - SFE

[Chrysothamnus nouseosus (Pall, ex Pursh) Briton subsp. holokecas (A. Gray) H. M. Hall \& Clem.] - W\&W'

[Ericameria nauseoses (Pall, ex Purshy G. L. Nesom \& G. L. Baird subsp. nauseosa var, hololeuca (A. Gray) G.

L. Nesom \& G. L Baird] - KTZ

Ericameria nauseosa (Pall. ex Pursh) G. L. Nesom \& G. 1. Baird var. juncea (Greene) G. L.

Nesom \& G. I. Baind - PLS

[Chry sothamnes neaseosus (Pall, ex Pursh) Britton var. junceus (Girectic) H. M. Hall \& Clem.]

[Chrysothamnus neuseosus (Pall.) Britton var. junceis (Gireene) H. M. Hall] - UTF, IMF

[Ericameria nauscosa (Pall, ex Pursh) G. L. Nesom \& G. I. Baird subsp. consimilis (Grecne) G. L. Nesom \& G.

1. Baird var, juncen (Greene) G. L. Nesom \& G. I. Baird] - KTZ

Ericameria nauseosa (Pall. ex Pursh) G. L. Nesom \& G. I. Baird var. latisquamea (A. Gray) G. L. Nesom \& G. I. Baird

[Bigelowia graveolens (Nuttall) Gray var, uppendiculara Eastwood] - KWA

[Bigelowia graveolens (Nuttall) Gray var, latisquameus Gray] - KWA

[Chry sothamnuts apgendiculatus (Enstwood) Heller] - KWA

[Chry sotammus latisquameats (Gray) Greene] - KWA

[Chry sothamnes nantseosus subsp. latisguamens (Gray) Hall \& Clements] - KWA, M\&H, KTZ

[Chry sothamnes nonseosus var, latisquameus (Gray) Hall ] - KT2 
[Ericameria nauseosus (Pall. ex Pursh) G. L. Nesom \& G. I. Baind var. latisquameus (Gray) Hall] = FNA. KWA. KTZ

Ericameria nauseosa (Pall. ex Pursh) G. L. Nesom \& G. L. Baird var. leiosperma (A. Gray) G. L. Nesom \& G. I, Baird - FNA, W

[Bigelowia leivoperma A. Gray in A. Gray] - FNA

[Chry sothamnus nouseosus (Pail.) Brition subsp. leivspermus ( $A$. Gray) H. M. Hall \& Clem.] - SFW

[Chrysotharnnus nouseosus (Pall. ex Pursh) Britton subsp. leiospermus (A. Gray) H. M. Hall \& Clem.] - W \&W

[Chrysotharmus naseosus (Pall.) Britton var. leiospermus (A. Gray) H. M. Hall] - IMF, UTF

[Ericameria nauseosa (Pall. ex Pursh) G. L. Neson \& G. I. Baird subsp. consimilis (Greene) G. L. Nesom \& G. I. Baird var. leiospente (A. Gray) G. L. Nesom \& G. L Baird] - KTZ

Ericameria nauseosa (Pall, ex Pursh) G. L. Nesom \& G. I, Baird var, nauseosa - Dorn01, FNA, E

[Chry sothamnus nouseosus (Pall.) Britton subsp. nauseosus] - GPE, HDH, SFE

[Chrysothamnus nouseosus (Pall. ex Pursh) Britton subsp. nauscosus] - W\&W

[Chrysothamnus nauseosus (Pall. ex Pursh) Britton var. nausecasus] - RMC

[Ericameria nauseosa (Pall, ex Pursh) G. L. Nesom \& G. I. Baird subsp. nauseosa var, nausecusa] - KTZ

Ericameria nauseosa (Pall, ex Pursh) G. L. Nesom \& G. I. Baird var, oreophila (A. Nelson) G. L. Nesom \& G. I. Baird - Dom01, E. FNA

[Chry sothamnus neaseosus (Pall.) Bricton subsp. consimilis (Greene) H. M. Hall \& Clem.] - HDH, SFE

[Chry sothamnus neaseosus (Pall. ex Pursh) Britton subsp. consinilis (Greene) H. M. Hall \& Clem.] - W\&W

[Chry sothamners neaseosus (Pall.) Britoon var. consimilis (Ginone) H. M. Haill] - UTF

[Chrysothamnus neasecsus (Pail. ex Pursh) Briton var. oreophilus (A. Nelsoen) H. M. Hail] - RMC

[Chrysodhamnus natuseosus (Pall.) Brition var. oreophilus (A. Nelson) H. M. Hall] - IMF

[Chry sothamnus natueosus (Pall. ex Pursh) Brimon subsp. pinifolius (Greene) H. M. Hall \& Clem.] - HDH. WEW'

[Ericamcria nauseosa (Pall. ex Pursh) G. L. Nesom \& G. I. Baird subsp. consimilis (Grecne) G. L. Nesom \& G. I. Baird var, oreophila (A. Nelson) G. L. Nesom \& G. I. Baird] - KTZ

Ericameria nauseosa (Pall. ex Pursh) G, L. Nesom \& G. I. Baird var. speciosa (Nutt.) G. L. Nesom \& G. I. Baird - FNA

[Chrysothamnus nanecsus (Pail.) Brition subsp. albicaulis (Nutt.) Ftydb.] - GPF

[Clsry sothammus neaseosus (Pall.) Britton var. albicaulis (NutL) Rydb.] - UTF

[Chry sothamnus neaseosus (Pall. ex Pursh) Beimon subsp. speciosus (Nutt) H. M. Hall \& Clem.] - HDH. WeW, erf, rep.

[Clery sothamnus neaseosus (Pall.3 Britton var. speciosus [Nurt.) H. M. Hall] - IMF

[Chrysorhamnus speciosus Nut.] - FNA

[Ericameria nauscosa (Pall, ex. Pursh) G, L. Nesom \& G. I. Baird subsp. nauscosa var. speciosa (Nutt.) G. L. Nesom \& G, I. Baird] - KTZ

[Ericameria nauseosa (Pall. ex Pursh) G. L. Nesom \& G. I. Baird var. turbinata (M. E. Jones) G. L. Nesom \& G. I. Baird;

\{Chrysothaminus nauscosus (Pall.) Briton var. narbinsnus (M. E. Jones) S. F. Blaket - IMF, UTF

\{Chrysothammus nauscosus (Pall. ex Pursh) Britton var, turbiaatus (M. E. Joocs) S. F. Blake)

\{Ericameria nataseosa (Pall. ex Pursh) G. L. Nesom \& G. I. Baird subsp, coersinilis (Greene) G. L. Nesoen \& G L. Baird var, turbinaca (M. E. Jones) G. L. Nesom \& G. L Baird\}-KTZ

Ericameria parryi (A. Gray) G. L. Nesom var- affinis (A. Nelson) G. L. Nesom \& G. I. Baird E/W, FNA

[Clerysothamnus perryi (A. Gray) Greene subsp, affinis (A, Nelson) L. C. Anderson] - SF!, WR W

[Chrysothamnes parryi (A. Gray) Greene var, affinis (A. Nelson) Cronquist] - IMF

[Ericameria parryi (A, Gray) G, L, Nesom \& G, I. Baind var, affinis (A. Nelson) G. L. Neson \& G. I, Baird] KTZ

[Linosyris parryi A. Gray] - FNA

Ericameria parryi (A. Gray) G. L. Nesom var. attenuata (M. E. Jones) G. L. Neson \& G. I. Baird - FNA, KTZ.

[Chrysothamnis parryj (A. Gray) Greene subsp, attentasus (M. E. Joses) H. M. Hall \& Clem.] - HDH, W. W

[Chry sothamnes parryi (A. Gray) Greene var, attenuatus (M. E. Jones) Kittell] - UTF

[Chry sothamnus parryi (A. Gray) Greene var, nevadensis (A. Gray) Kittell] - IMF 
[Ericameria parryi (A. Gray) G. L. Nesom \& G. I. Baird var. attenuata (M. E. Jones) G. L. Nesom \& G. L. Basind] $=$ KTZ

Ericameria parryi (A. Gray) G. L. Nesom var. howardii (Parry ex A. Gray) G. L. Nesom \& G. I. Baird - Dorn01, E/W, FNA

[Chry sothamnus parryi (A. Gray) Greene subsp. howardii (Purry) H. M. Hall \& Clem.] - GPF. 1IDH, SF!

[Chrysothamnus parryi (A. Gray) Greene subsp. howardii (Purry ex A. Gray) H. M. Hall \& Clem.] - W\& W

[Chrysothamnus porry i (A. Gray) Greene var. howardii (Parry ex A. Gray) Kittell] - DMF, RMC

[Ericameria parryi (A. Gray) G. L. Nesom \& G. 1. Baird var. howardii (Parry ex A. Gray) G. L. Nesom \& G. I. Baird] = KTZ

Ericameria parryi (A. Gray) G. L. Nesom var- parryi - Dom01, E/W, FNA

[Chrysothamnus porry i (A. Gray) Greene subsp. parryi]-HDH, SF!, W\&W

[Chrysothamnus parryi (A. Gray) Greene var. partyi] - [MF, UTF

[Ericameria partyi (A. Gray) G. L. Nesom \& G. 1. Baird var. howardii (Parry ex A. Gray) G. L. Nesom \& G. I. Baind] - KTZ

Erigeron

(Identification sources:

\{Erigeron acris L. var, acris\} - TMF (European)

[Erigenon acris L. subsp. acris var. acris;

\{Trimorpha acris (L.) Gray) - Nesomso

\{Erigeron acris L. var, asteroides (Andrz. ex Besser) DC.\} - HDH, GPF

INS - combination nox in FNA Vol. 20

[Erigeron actis L.] - UTF

\{Erigeron actis $\mathrm{L}$. var, asteroides (Andrz) DC.\} - $\mathrm{HDH}$

\{Erigeron acris L. subsp. politus (Fr.) Schinz \& R. Keller\} - KTZ

\{Erigeron elongatus Ledeb.\} - Ws w

\{Trimonpha aeris (L.) G. L. Nesom var. asteroides (Andrz. ex Besser) G. L. Nesom) - Nesom80

'Trimoepha elongata (Ledeb.) Vierhapper) - W\& W'

Erigeron acris L. var. kamschaticus (DC.) Herder - Dom01, FNA

[Erigeron acris L. var. asteroides (Anderz sx Bess.) DC.] - misapp., RMC

[Erigeron acris L.] - UTF

[Erigeron kamtschaticus DC. \{as kamtsctaticum\}] - FNA

[Erigeron yellowstonensis A. Nelson] - FNA

[Erigeron acris L. subsp. kamtscharicus (DC,) H. Hara] - KTZ

[Trimorpha acris (L), Gray var. kamtschatica (DC,) G. L. Nesom] - FNA, Nesom89

[Erigeron annuus (L.) Pers.; - HDH, exp., KTZ $<\mathrm{KS}, \mathrm{NE}, \mathrm{OK}, \mathrm{UT}>$

Erigeron aphinactis (A. Gray) Greene var, aphanactis - FNA

[Erigeron concineus. (Hook, \& AmL) Tor, \& A. Gray var, aphanactis A. Gray] - FNA

Erigeron argentatus A. Gray - FCF. KTZ $<$ CA, NV, UT $>$

Erigeron bellidiastrum Nutt. var, bellidiastrum - FNA, HDH, IMF, KTZ, E

[Erigenon tellidiastrum Nutt.] - Dons01, GPF, SFE, UTF, Wa W

Erigeron bellidiastrum Nutt. var. moustus Cronquist - FNA. HDH, IMF, KTZ, E

[Erigeron bellidiastrum Nutt] - GPF, SFE, UTF, W\&W

Erigeron caespitosus Nutt. - Dorn01, FNA, GPF, HDH, IMF, KTZ, SF!, UTF, W\& W, EW

Erigeron canus A. Gray - Dom01, FNA, GPF, HDH, IMF, KIZ, SF!, UTF, W\&W, E/W

Erigeron compactus Blake var. consimilis (Cronquist) Blake - Dorn01

Erigeron compositus Pursh - FNA, KTZ, SF!, W\&W

[Erigeron compositus Pursh var, discoideus A. Gray] - DornDI, HDH, IMP, RMCENW

[Erigeron compositus Pursh var, glabratus Macous] - GPF, HDH, IMF, UTF

Erigeron concinnus (Hook. \& Aml) Torr. \& A. Gray var. concinnus - FNA. KTZ, W

[Erigeron brandegei A. Gray] - $\mathrm{HDH}$ 
[Erigeron concinıus (Hook. \& Ams) Torr. \& A. Gray] - SFW, W\&W

[Erigeron pum ilus Nutt, subsp. concinnoides Cronquist] - HDH

[Erigeron pumilus Nutt. subsp. concinnoides Crenquist var. concinnus (Hook. \& Am.) Dorn] - IMIF

[Erigeron pumilus Nutt. var. concinnoides Cronquist] - UTF, W\&W

[Erigeron pumilus Nutt, var. concinnus (Hook. \& Arm.) Dorn] - Dorn01

Erigeron concinnus (Hook. \& Am.) Torr. \& A. Gray var. subglaber (Cronquist) G. L. Nesom

- FNA, KTZ, W

[Distasis concinna Hook. \& Am.] - FNA

[Erigeron aphanactis (A. Gray) Greene] - HDH

[Erigeron cencinnus (Hook. \& Ams) Torr. \& A. Gray] - SFW, W \& W'

[Erigeron pumil is Nutc. subsp. coecinnoides Cronqu ist var. subglaber Crottquist] - [MF

Erigeron consimilis Cronquist - FNA, KTZ, W\&W, W

[Erigeron compactus S. F. Blake]-SFW, W\&W

[Erigeron compactus S. F. Blake var. consimilis (Cronquist) S. F. Blake] - [MF, UTF

Erigeron corymbosus Nutt, - Dorn01, FNA, KTZ

Erigeron coulteri Porter - FNA, HDH. IMF, KTZ, SF!, UTF, W\&W, E/W

Erigeron divergens Torr. \& A. Gray var, divergens - HDH, IMF, UTF, EW

[Erigeron divergens Tort. \& A. Gray]-Dom01. GPF, KTZ, SF!, W\&W

Erigeron eatonii A. Gray var. eatonii - FNA, IMF, KTZ, W\&W, E/W

[Erigeron eatonii A. Gray] - Dorn01, SF!, UTF

[Erigeron eatonii A. Gring subsp, eatonia]- HDH

Erigeron elatior (A. Gray) Greene-Dorn01, FNA, HDH, IMF, KTZ, SF!, UTF, W\&W, E/W

[Erigeron grandiflorus Hook. var, elatior A. Gray] - FNA

Erigeron engelmannii A. Nelson var, engelmannii - FNA, IMF, KTZ, E/W

[Erigeron engelmannii A. Nelson] - SFI, UTF, W\&W

[Efigeron engelmannii A. Nelson subsp. engelmannii] - $\mathrm{HDH}$

Erigeron eximius Greene - Dom01, FNA. IMF, KTZ, SF!, W\&W, EW

[Erigeroe superbus Greene ex Rydb.] - FNA. HDH, UTF, W\&W, nomen nudum

[Erigeron formosissimus Greene] - UTF

Erigeron flagellaris A. Gray - Dorn01, FNA, GPF, IMF, KTZ, SF!, UTF, W\&W, E/W

[Erigeron flagellaris A. Gray forma beviligulatus W. A. Webs] - SFE, W\&W

[Erigeron flagellaris A. Gray var. flagellaris] - HDH

Erigeron formosissimus Greene var, formosissimus - Dorn01, FNA, GPF, HDH, W\&W, E/W

[Erigeroe formosissimus Greene] - IMF, KTZ, SFI, UTF

[Erigeron pecosensis StandL.] - FNA

Erigeron formosissimus Greene var. viscidus (Rydb.) Cronquist - FNA, GPF, HDH, W\&W, ENW

[Erigeron viscidus Rydb.] - FNA

[Erigeron formosissimus Greene] - KTZ, SF!

[Erigeron glabellus Nutt, var, viscidus (Rydb.) B. Boivin] - FNA

Erigeron glacialis (Nutt.) A. Nelson var. glacialis - FNA

[Aster glacialis Nun.] - FNA

[Erigeron ealliantheumus Greene] - FNA

[Erigeron peregrinus (Banks ex Pursh) var, angustifolius (A. Gray) Crooquist] - FNA

[Erigeron peregrinus (Banks ex Pursh) Greene var, eallianthemis (Greene) Cronquist] - EJW

[Erigeron peregrinus (Pursh) Greene subsp, callianthemas (Greene) Cronquist] - HDH

[Erigeroo peregrinus (Banks ex Pursh) Greene subsp, callianahemus (Greene) Cronguist var, callianthemus] IMF, KTZ

[Erigeron peregrinus (Banks ex Pursh) Greene subsp, callianchemus (Greene) Cronquisist] - W\&W

[Erigeron peregrinus (Basks) Greene subsp, callianthemas (Greene) Cronq.] - SF!

[Erigeron peregrinus (Pursb) Greene sobsp, callianthemas (Greene) Cronquist var, callianthemus] - UTF

[Erigenon peregrimus (Pursh) Greene var, seaposus (Torr, \& A. Gray) Cronquist] - Dom01, FNA

[Erigeron salsuginosus (Richaed5, ex R. Browb) A. Gray var, glacialis (Nutt.) A Gray] - FNA

Erigeron glabellus Nutt, var, glabellus - Dom0I, FNA, IMF, KTZ, E/W

[Erigeron asper Nutt.] - FNA 
[Erigeron glabellus Nutt.] - SF!, UTF, W\&W

[Erigeron glabellus Nutt. subsp. glabellus] - GPF, HDH

[Erigeron oblanceolatus] - FNA

Erigeron glabellus Nutt var, pubescens Hook, - Dom01, FNA, IMF, KTZ, EW

[Erigeron asper Nutt. var. pubescens (Hook.] Breitung] - FNA

[Erigeron glabellus Nutt.] - SF?, UTF, W\&W

[Erigeron glabellus Nutt. subsp- pubescons (1took.) Cronquist] - FNA, GPF, 1HDH

Erigeron grandiflorus Hook, - FNA, SF! W\&W, E/W

[Erigeron grandiflorus Hook. subsp. grandiflorus] - KTZ

Erigeron humilis Graham - Dorn01, FNA, IMF, KTZ, SFW, UTF, W\&W', W

[Erigeron unalaschkensis DC.] - FNA

[Erigeron uniflorus var. unalaschkensis (DC.) Ostenfeld] - FNA

Erigeron kachinensis S, L. Welsh \& Moore - FNA, IMF, KTZ, SFW, UTF, W\&W, W

[Erigeron katchinensis S. L. Welsh \& Moore] - W\&W

Erigeron lanatus Hook, - Dom01, FNA, KTZ, SF!, W\&W, E/W

Erigeron leiomerus A. Gray - Dorn01, FNA, HDH, IMF, KTZ, SF!, UTF, W\&W, E/W

Erigeron linearis (Hook.) Piper - Dom01, FNA

Erigeron lonchophyllus Hook. - Dorn01, FNA, GPF, HDH, IMF, KTZ, UTF, W\&W, EW

[Trimorpha lonchophylla (Hook.) G. L. Nesom] - SF!, W\& W'; Nesom89

[Erigeron mancus Rydb, - HDH, exp, KTZ $<U T>$, SFW $\angle U T$, possibly $C O>$, W

Erigeron melanocephalus (A. Nelson) A. Nelson - Dom01, FNA, IMF, KTZ, UTF, E/W

[Erigeron melanocephalus A. Nelson] - HDH. SF!, W\&W

Erigeron nanus Nutt. - Dorn01, FNA, KTZ

Erigeron nematophyllus Rydb, - Dom01, FNA, HDH, IMF, KTZ, SF!, UTF, W\&W, E/W

Erigeron nivalis Nutt. - FNA; Hartman06

[Efigeroe actis L.] - UTF

[Erigeron acris L. subsp. actis var. debilis A. Gray]

[Erigeron acris L. subsp. debilis (A. Giay) Piper] - KTZ

[Erigeron acris L. var. debilis A. Gray] - HDH, IMF, EW

[Erigeroen angulosus Gaudin subsp. debilis (A. Gray) Piper)] - FNA

[Erigeton debilis (A. Gray) Rydb.] - FNA

[Erogeton elatus (Hook.) Greene var, bakeri Greene] - FNA

[Erigeron elongatus Ledeb.] - W\&W

[Erigeron jacundus Greme] - FNA

[Erigeroo scotteri B. Boivin] - FNA

[Trimorpha acris (L.) Gray var. debilis (A. Gray) G. L. Nesom] - Nesom89

[Trimorpha eloogata (Ledeb.) Vierhapper] - SFI, W\&W'

Erigeron ochroleucus Nutt. var, ochroleucus - Dom01, FNA, KTZ

[Erigeron partyi Canlys \& Rose] - KT2

[NS - see comment in FNA 20: 293 regarding resurrection to specific status of $E$. parry i outride oxir range]

Erigeron ochroleucus Nutt, var, scribneri (Canby ex Rydb.) Cronq. - Dorn0], KTZ

Erigeron philadelphicus L. var. philadelphicus - FNA, KTZ, E/W

[Erigeron fraternus Greene]

[Erigemon philadelphicus L.] - GPF, HDH, IMF, SFI, W\&W

Erigeron pinnatisectus (A. Gray) A. Nelson - Dom01. FNA, HDH, KTZ, SF!, W\&W, E/W

Erigeron pulcherrimus A. Heller - Dorn01, FNA. IMF, KTZ, SFW, UTF, W\&W. W

[Erigeron bistiensis G. L. Nesom \& Hevron] - FNA

[Erigeron palcherrimus A. Heller var, wyomingia (Rydb.) Cronguist] - FNA, GPF, HDH

Erigeron pumilus Nutt. var, pumilus - FNA, W\&W, E

[Erigeron pamilis Nutt.] - Dorn01, GPF, SFE

[Erigeron pemilus Nutt, subsp, pumilas] - HDH, KTZ

[Erigeron pumilus Nutt, var. pumilus] - IMF

Erigeron radicatus Hook. - FNA 
[Erigeron huberi S. L. Welsh \& N. D. Atwood] - FNA

[Erigeron macounii Greene] - FNA

Erigeron simplex Greene - Dom01, IMF, KTZ, SF!, UTF, W\&W, E/W

Erigeron sparsifolius Eastw, - FNA

[Erigeron utahensis A. Gray var. sparsifolius (Eastw.) Croncquist] - IIDH. IMF, KTZ, UTF, W\&W, W

[Erigeron utahensis A. Gray] - SFW

[Erigeron vivax (A. Nelsun) Rydb.] - FNA

Erigeron speciosus (Lindl.) DC. - Dorn01, FNA, SF!, E/W

[Erigeron conspicuus Rydb.] - FNA

[Erigeron macranthas Nutt.] - FNA

[Erigeron speciosus (LindL.) DC. var, conspicues (Rydb.) Breitung) - FNA

[Erigeron speciosus (Lindl.) DC. var. macranthus (Nutt.) Cronquist] - GPF, HDH, IMF, KTZ, UTE, W\&W

[Erigeron speciosus (LindL) DC. var. speciosus] - GPF, HDH, IMF, KTZ, UTF, W\&W

[Erigeron subtrinervis Rydb. ex Porter subsp. conspicuus (Rydb.) Cronquist] - FNA

[Erigeron subtrinervis Rydb. ex Porter var. conspicuus (Rydb.) Cronquist] - FNA

[Stenactis speciosuis Lind l.] - FNA

† Enigeron strigosus Muhl, ex Willd. var, strigosus - FNA, GPF, IMF, KTZ, E

[Erigeron ramosus (Walter) Britt., Stems \& Poggenburg] - FNA

FNA

[Erigeron ramosus (Walter) B ritt., Stems \& Poggenbarg var. beyrichii (Fischer \& C. A. Meyer) Trelease] -

[Erigeron strigosus Muhl.] - UTF

[Erigeron strigosus Muhl. ex Willd.]-Doen01. HDH

[Erigeron strigosus (Walter) Britt., Stems \& Poggenburg var, beyrichii (Fischer \& C. A. Meyer) Torr. \& A Gray] - FNA

[Stenactis beyrichii Fischer \& C. A. Meyer] - FNA

[Stenactis strigosa (Muhi.) DC.] - SFE

[Stenactis strigosa (Mubl. ex Willd.) DC.] - w\&w

Erigeron subtrinervis Rydb, ex Porter \& Britton - FNA, KTZ, E/W

[Erigeroe conspicuus Rydb.] - KTZ

[Erigeron speciosus (LindL.) DC. var. mollis (A. Gray) S. L. Welsh] - UTF

[Erigeron suberinervis Rydb.] - GPF, SF!, W\& W

[Erigeroo suberinervis Rydb. ex Porter \& Britton var, conspicuus (Rydb.) Crong.] - WYchecklist

[Erigeroe suberinervis Rydb. subsp. suburinervis] - $\mathrm{HDH}$

[Erigeron suberinervis Rydb, var, subtrinervis] - IMF

[Erigeron speciosus var, conspicuus (Rydb.) Breitung] - KTZ

[Erigeroo suberinervis subsp. conspicuus (Rydb.) Crosed] - KTZ

Erigeron tener (A. Gray) A. Gray - Dorn01

[Erigeron eaespitosus Nustall var, tenerus A. Gray] - FNA

Erigeron tracyi Greene - FNA

[Erigeron cinereus A. Gray 1849 \{not Hook. \& Amot 1836\} - FNA

[Erigeron colomexicanus A. Nelson] - KTZ, E

[Erigeroe colo-mexicanus A. Nelson] - SFE, W\&W

[Erigeroe commixtus Greene] - FNA

[Erigeroe divergens Torr. \& A. Gray var, cinereus A. Gray] - HDH, UTF

[Erigeroe divergens Torr. \& A. Gray var, chenereus (A. Gray) A. Gray] - IMF

[Erigeroe modestus A. Grial - GPF

Erigeron uintahensis Cronquist - FNA, HDH, IMF, KTZ, SFW, W\&W, W

[Erigeron speciosus (Lindl.) DC, var, uintahensis (Cronquist) S, L Welsh] - UTF

Erigeron ursinus D. C. Eat. - Dorn01, FNA, HDH, IMF, KTZ, SF!, UTF, W\&W, E/W

[Efigeroe ursinas D. C. Eat, var. meyerae S. L. Welsh] - FNA

[Erigeron ursinas D. C. Eat, var, ursinus] - UT3

Erigeron utahensis A. Gray - Dorn01, FNA, SFW

[Erigeron stenoghyllus A. Gray tas E. stenophyllum!]- FNA

[Erigeron utahensis A. Gray var, terrapleuris (A. Gray) Cronquist] - FNA. HDH. W\&W

[Erigeron utahensis A, Gray var, utahensis] - IMF, KIZ UTF, W 
Erigeron vagus Payson - FNA, HDH, IMF, KTZ, SF!, UTF, W\&W, E/W

Erigeron vetensis Rydb, - Dorn01, FNA, GPF, HDH, KTZ, SF! W\&W, E/W

[Erigeron glandulosus Porter] - FNA

[Erigeron porteri S. F. Blake] - FNA

Erigeron vreelandii Greene - FNA, KTZ, W\&W, E

[Erigeron patens Greenc] - FNA

[Erigeron platyphyllus Greene] - FNA

[Erigeron rudis Wooton \& Stand1.] - FNA

[Erigeron vreelandii Rydb.] - SFE

- Erigeron wilkenii OKane-ENDEMIC $\angle C O$ - FNA, KTZ, SFW, W\&W, W

[Erigeron nematophyllus Rydb.] - IMF

Eucephalus perelegans (A. Nelson \& J. F, Macbr.) W. A. Weber - FNA, SFW, W\&W

[Aster perelegans A. Nelson \& J. F. Makbr.] - IMF, UTF. W

[Eucephalus elegans Nutt. - Donv01, FNA. KTZ, We wi

[Eucephalus perelegans Nutt] - wa W

Eucephalus engelmannii (D. C. Eaton) Greene - Dorn01, FNA, KTZ, SF!, W\&W

[Asser engelmannii (D. C. Eaton) A. Gray] - HDH, IMF, UTF, EW

[Aster elegans (Nuti.) Torr. \& A. Gray var. engelmannii D. C. Eat.] - FNA

[Aster sngelmannii (D. C. Eas.) A. Gray] - FNA

Eupatorium (sce also Ageratina, Eutrochium)

Eurybia integrifolia (Nutt.) G. L. Nesom] - FNA, KTZ <UT, WY>

[Aster integrifolius Nutt.] - FCF, HDH rep, KTZ, IMF, UTF, W\&W, rep.

Euthamia graminifolia (L.) Nutt. - FNA, SFE, W\&W

[Euthamia graninifolia (L.) Nutt, ex Cass, var, major (Michx.) Moldenke] - E

[Euthamia graminifolia (L) Greene var, graminifolia] - KTZ

[Euthamia graminifolia (L.) Nutt, var, major (Michx, Moldenke] - Dornol, GPF

[Solidago graminifotia (L.) Salisb, var, camperam (Greese) Fernald] - HDH

Euthamia gymnospermoides Greene - FNA, GPF, KTZ, SFE, W\&W, E

[Euthamnia camponum Greene] - FNA

[Euthamnia chysothamnoides Greene] - FNA

[Euthamnia glutinosa Rydb.] - FNA

[Euthamnia pulverulenta Greene] - FNA

[Solidago gymnespermoides (Greene) Fernald var. callosa Hamis] - FNA

[Solidago texensis Friesner] - FNA

Euthamia oecidentalis Nutt. - FNA, GPF, IMF, KTZ, SF!, W\&W, E'W

[Euthamnia californica Gandoger] - FNA

[Euthamaia linearifolia Gendoger] - FNA

[Solidago occidectalis (Nutt). Torr. \& A. Gray] - FNA. HDH. UTF

Eutrochium maculatum (L.) E. E. Lamont var. bruneri (A. Gray) E. E. Lamont - FNA

[Eupatoriadelphas maculatus (L.) King \& B. L. Rob. var, bruecri (A. Gray) King \& B. L. Rob, - Dom01

[Eupatoriam maculatum L.] - HDH, SF!, W\&W

[Eupatorium maculatum L. subsp. bruneri (A. Gmy) G. W. Douglas] - FNA, IMF, WEW'

[Eupatorium maculatum L. var, bruneri (A. Gray) Breit.] - GPF, KTZ, UTE, E/W

Flaveria campestris J. R. Johnst. - FNA, GPF, HDH, IMF, KTZ, SF1, UTF, W\& W, E/W

Flaveria trinervia (sprengel) C. Hohr - FNA, KWA, M\&H, W\&S, KTZ

[Flaveria repanda Lagasca] - KWA

[Oedera trinervia Sprengel] - KWA, KTZ

Snow, N, January 2009. Checklist of Vaccular Plants of the Southom Rocky Momain Region. CVersion $3 x$ 


\section{Franseria (see Ambrosia)}

\section{Gaillardia X grandiflora Van Houtte - KTZ}

[W] - A hytrid perennial cultivar in gardens and in revegetation projects; usually identified as G. pulchella, which is an annual. Seen along highways and in Leaisville Open Space (CO) where it disappears after a fena years (usually with Linum perenne)]

[G. aristata X G. pulchella] - KTZ

Gaillardia aristata Pursh - Dom01, FNA, GPF, HDH, IMF, KTZ, SF!, UTF, W\&W, EW

Gaillardia pinnatifida Torr, - FNA. HDH, IMF, SF!. UTF, W\&W, E/W

[Gaillardia gracilis A. Nelson] - FNA

[Gaillardia pinnatifida Torr. var. pinnatifida] - KTZ

Gaillardia pulchella Foug. - FNA, GPF, HDH, SFE, W\&W

[Gaillardia pulchella Foug var. pulchella] - KTZ, E

Gaillardia spathulata A. Gray - FNA. IMF, KTZ, SFW, UTF, W\&W, W

* Galinsoga parviflora Cav, - GPF,HDH, KTZ, SFE, W\&W, E

* Galinsoga quadriadiata Ruiz \& Pav, - Dorn01, FNA, GPF, KTZ, SFE, W\&W, E

[Galinsoga ciliata (RaL.) S. F. Blake] - $\mathrm{HDH}$

\section{Gnaphalium (see also Pseudognaphalium)}

Gnaphalium exilifolium A. Nelson - Dorn01, FNA, HDH, IMF, UTF, E/W

[Gnaphalium uliginosum L. ] - GPF, HDH, IMF, misapp., KTZ, SF! UTF, WR. W

Gnaphalium palustre Nutt, - Dom01, FNA, GPF, HDH, IMF, KTZ, SF!, UTF, W\&W, EW

Ginaphalium uliginosum L, - FNA

[FNA: also in Europe but not clear whether all N. American material is introduced]

Grindelia

(Identification sources: )

[NS - FNA lumps some taxa typically nocognied for our region under either G, seuarrosa or especially under G. birsutula. The latter name has not been wsed for our region lately. While the FN.A treatnent necessarily takes the continencal-w ide perspective and actes - probably correctly - that too many taxa have been recognized, I an hesitant to follow their treatment for our material unsil rigorous, population-based sumpling. has boen carried out in our region (namely, popelation ageregation analysis \{Davis92\} or spocimcan ageregation analysis (Snow97, Snow03\}). To their credit, Strocher and Wetter in FNA 20: 432-434 explain in detais how The lumped taxa have been recognized previously. The taxonomy of Grindelia for the SRMR deserve a dissertation level study.]

Grindelia acutifolia Steyerm. - HDH, KTZ, W\&W

Grindelia aphanactis Rydb. - IMF, M\&H, SFE

Grindelia arizonica A. Gray - FNA

[Grindelia arizonica A. Gray var, dentata Steyerm.] - FNA

[Grindelia arizonica A. Gray var, microphylla Stegerm.] - FNA

[Grindelia arizonica A. Gray var, neomexicana (Wooton \& Standl.] - FNA

[Grindelia arizonika A. Gray var, stenoghylla Steyerm.] - HDH, KTZ, SFW, W\& W, W

[Girindelia laciniata Rydh.] - FNA

[Grindelia neomexicana Wooton \& Standl.] - FNA

[Grindelia scabra Greene var, neonexicana (Wooton \& Standl.) Steyerm.] - FNA

Grindelia ciliata (Nutt.) Spreng. - FNA

[Donia ciliata Nutr,] - FNA 
[Prionopsis ciliata (Nutt.) Nutt. - FNA, SF!, W\&W, EN

[Grindelia papposa G. L. Nesom \& Suh] - FNA, KTZ

[Haplopappus ciliatus (Nutt.) DC.] - FNA, GPF, HDH

$\bullet$ Grindelia decumbens Greene var, decumbens - ENDEMIC <CO>, HDH, KTZ, W\&W

[NS - FNA does not distinguish between varieties]

Grindelia decumbens Greene var, subincisa (Greene) Steyerm. - HDH, KTZ, SFE, W\&W, E

Grindelia fastigiata Greene - HDH, IMF, KTZ, SFW, UTF, W\&W, W

\{Grindelia hirsutula Hook. \& Amott, \} - FNA

INS - see comments above regarding level of lumping!

- Grindelia inornata Greene var, angusta Steyerm. - ENDEMIC $<\mathrm{CO}>$, HDH, KTZ,

[Grindelia inomata Greene] - W\& W'

- Grindelia inomata Greene var. inomata - ENDEMIC $\langle\mathrm{CO}\rangle, \mathrm{HDH}, \mathrm{KTZ}, \mathrm{E}$

[Grindelia inomata Greene] - GPF. SFE, W\&W

Grindelia laciniata Rydb. - FCF, HDH, exp., KTZ <AZ, NM, UT>, W\&W ${ }^{\prime}$, misid.

Grindelia nana Nutt. - FCF

[NS - protsably subsp. integerrima (Rybb.) Steyermark.

Grindelia nuda A. W, Wood var, aphanactis (Rydb,) G. L. Nesom - KTZ, EWW

[Grindelia aphanactis Rydb.]-HDH, IMF, SF], UTF, W\&W

Grindelia nuda A. W. Wood var. nuda - KTZ

[Grindelia squancisa (Pursh) Dunal var, auda (A. W. Wood) A. Gray] - GPF, HDH, W \& W

Grindelia revoluta Steyerm. - GPF, HDH, KTZ, SFE, W\&W, E

Grindelia squarrosa (Pursh) Dunal var, quasiperennis Lunell - Dom01, GPF, IMF, KTZ, SFW, W\&W, W

[Grindelia pereanis A. Nelson] - $\mathrm{HDH}$

Grindelia squarrosa (Pursh) Dunal var. squarrosa - Dorn01, GPF, HDH, IMF, KTZ. UTF, $W \& W^{\prime}, E / W$

[Grindelia squactosa (Pursb) Dunal] - SFE

[Grindelia squarosa (Pursh) Dunal var, serrulata (Rydb.) Steyerm.) - HDH, IMF, KTZ, SFW, UTF, W\&W

Grindelia subalpina Greene-Dorn01, FNA, SFE, W\&W, E

[Grindelia erecta A. Nelsoe] - FNA

[Grindelia subalpina Greene var, erecta (A. Nelsoo) Steyerm, ] - FNA, HDH, KTZ

[Grindelia subalpina Greene var, subalpina] - HDH, KTZ

Grossheimia (see Centaurea)

- Gutierrezia elegans Al Schneider \& P. Lyon - Schneider08

Gutierrezia microcephala (DC.) A. Gray - FNA, IMF, KTZ, SFW, UTF, W\&W, W

[Gutierrezia lucida Greene] - HDH

[Gutierrezia sarothrae (Pursh) Britton \& Rushy var. microcephala (DC.) L. D. Bensin] - HDH

[Xanthocephalum microcephalum (DC.) Shinners] - KTZ

Gutierrezia sarothrae (Pursh) Britton \& Rusby - Dorn01, FNA, GPF, KTZ, SFl, W\&W, E/W

[Gutierrezia lepidota Greene] - HDH

[Gutierrezia sarothrae (Pursh) Britton \& Rusby var, sarothrae] - HDH

[Xanthocephalums sarothrue (Purshi) Shinners] - FNA

Haploęsthes greggii A. Gray var. texana (J. M. Coult.) I. M. Johnst - FNA, GPF, KTZ

[Haplozsthes greggi A. Gray] - HDH rep., W\&W, W\&W', err. rep.

[Haplopappus texanus J, M, Coult. \{ns Aplopappus\}] - FNA

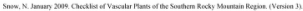


Haplopappus (see Ericameria, Haploësthes, Isocoma, Machaeranthera, Oönopsis, Prionopsis, Pyrrocoma, Rayjacksonia, Solidago, Stenotus, Tonestus; Lane96, FNA Vol. 20)

Helenium aurumnale L. - FNA, GPF. HDH

[Helenium autumnale L. var, montanum (Nutt.) Fernald] - Dom0I, IMF, KTZ, SF!, W\&W, E/W

Helenium microcephalum DC. var, microcephalum - FNA, KTZ

[Helenium microcephalum DC]. -SFE, W\&W

Helianthella microcephala (A, Gray) A. Gray - FNA, HDH, IMF, KTZ, SFW, UTF, W\&W, W

[Encelia microcephala A. Gray]- FNA

Helianthella parryi A. Gray - FNA, HDH, KTZ, SF!, W\&W, EW

Helianthella quinquenervis (Hook.) A, Gray - Dorn01, FNA, GPF, HDH, KTZ, SF!, W\&W, EJW [Helianthus quinquencrevis Hook.] - FNA

Helianthella uniflora (Nutt.) Torr, \& A. Gray - Dorn01. FNA. HDH, SF!, UTF, W\&W

[Helianthella uniflora (Nunt.) Torr. \& A. Gray var. uniflora] - IMF, KTZ. E/W

Helianthus

(Identification sources:

Helianthus annuus L. - Dom0I, FNA, GPF, HDH, IMF, KTZ, SF!, W\&W, E/W

[Helianthus annues L. subsp. lenticularis \{Douglas) Cockerell] - UTF

$\div$ Helianthus ciliaris DC - FNA, GPF, HDH, IMF, KTZ, SFE, UTF, W\&W, E

\{Helianthus grosseserratus M. Martens\} - KTZ $<\mathrm{KS}, \mathrm{NE}, \mathrm{OK}>$, W\&W, misid.

\{Helianthus grosse-serratus M. Martens\} - HDH, exp.

Helianthus maximilianii Schrad. - Dom01, FNA, GPF, HDH, KTZ, SFE, W\&W', F

Helianthus nuttallii Torr, \& A. Gray subsp, nuttallii - FNA, GPF, KTZ

[Helianthes muttallii Torr. \& A Gray] - FNA, HDH, IMF, SF', UTF, W\& W, E/W

[Helianthes mutrallii Tort. \& A. Gray subsp. rydbergii (Briton) R. W. Long] - GPF. KTZ

Helianthus nuttallii Torr. \& A. Gray subsp. rydbergii - Dorn01, FNA

[Helianthus ndbergii Briton] - FNA

\{Helianthus X orgyaloides Cockerell\} - KTZ

\{Helianthus maximiliani X H. salicifolius\} - KTZ

Helianthus pauciflorus Nutt. subsp. subrhomboideus (Rydb.) O. Spring \& E. Schilling - FNA. KTZ

[Helianthos pauciflorus Nutr. subsp. subrhomboideus (Rydo.) Cronquist] - KTZ

[Helianthus rigidus (Cass.) Desf.] - $\mathrm{HDH}$

[Helianthus rigidus (Cass,) Desf, subsp, subihomboideus (Rydb.) Heiser] - GPF, W\& W

[Helianthus rigidus (Cass.) Desf, var, subrhomboideus (Rydlo, Crongaist] - Dom01, EW

[Helianthes rigidus (Cass.) Desf, var, subrhomboideus (Rydlo, Heiser] - SF!

Helianthus petiolaris Nutt. var. fallax (Heiser) B. L. Turner

[Heliantius petiolaris Nurt. subsp. fallax Heiser] - FNA. IMF, KTZ. UTF

Helianthus petiolaris Nutt. var. petiolaris - E/W

[Helianthes petiolaris Nutt] - Dorno1, GPF, HDH, SFI, WE W

[Helianthes petiolaris Nutt. subsp. petiolaris] - FNA, IMF, KTZ

Helianthus pumilus Nutt, - FNA, GPF, HDH, KTZ, SFE, W\&W, E

\{Helianthus salicifolius A. Dietr.\} - HDH rep., KTZ $<\mathrm{KS}, \mathrm{OK}>$, W\&W, err. rep.

* Helianthus tuberosus L. - FNA, GPF, HDH, IMF, KTZ, SF!, W\&W, EW

[Helianthus tomentoses Michx.] - FNA 
[Helianthus tuberosus Nutt.] - UTH

[Helianthus tuberosus Nutt. var, subcanescens A. Gray] - FNA

Heliomeris longifolia (B, L. Rob. \& Greenman) Cockerell var. longifolia - FNA

[Gymnolomia annua B.L. Rob. \& Greenman]

[Gymnolomia longifolia B. L. Rob.\& Greenman] - M\&H

[Gymnolonia multifloes (Nuttall) Bentham \& Hooker var- annuss M.E. Jones]

[Heliomeris longifolia Robirs. \& Greenman] - KWA

[Heliomeris longifolia Ceckerell] - 1PNI

[Viguiera longifolia (B.L. Rob. \& J.L. Greenman) Blake var. annua (M.E. Jones) Welsh] - FNA, KWA

Heliomeris multiflora Nutt. var. multiflora - FNA, KTZ, SF!

[Heliomeris multiflora Nutt.] - SF!. W\&W

[Viguiera multiflera (Nutt) S. F. Blake var. multiflora] - IMF, UTT, EW

[Viguiera multiflera (Nutt) S. F. Blake] - Dorn01, HDH

Heliomeris multiflora Nutt. var, nevadensis (A. Nelson) Yates - FNA, KTZ. SFW

[Heliomeris multiflora Nutt.] - SFW, W\&W

[Viguiera multiflora (Nutt.) S. F. Blake] - HDH

[Viguiera mulhiflora (Nutt) S. F. Blake var, nevadensis (A. Nelson) S. F. Blake] - IMF, UTF, W

Heliopsis helianthoides (L.) Sweet var, occidentalis (T. R. Fisher) Steyerm. - KTZ

[NS - present in San Mizuel Co, NM]

Heliopsis helianthoides (L.) Sweet var, seabra (Dunal) Femald - FNA, GPF, SFE, W\&W, E

[Heliopsis scabra Dumal] - HDH

Herrickia glauca (Nutt.) Brouillet var, glauca - FNA

[Aster glaucodes S. F. Blake] - IMF

[Aster glaucodes S. F. Blake var. formosus (Greene) Kittell] - HDH rop.,

[Aster glaucodes S. F. Blake var. glaucodes] - HDH, UTF, EW

[Eucephalus glascus Nutt.] - SF!, W\& W

[Eurybia glauca (NuIL) G. L. Nesom] - KTZ, W\&W ${ }^{3}$

Herrickia horrida Wooton \& Standl, - FNA, SFE, W\&W, E

[Aster horridus (Wooton \& Standi.) S. F, B lake]-HDH, exp.

[Eurybia horrida (Wooton \& Standi.) G. L. Nesom] - KTZ, W\& W'

๘ Heterosperma pinnatum Cav, - FNA, KTZ, SFE, W\&W', E

Heterotheca

(Identification sources:

Heterotheca canescens (DC.) Shinners - FNA, KTZ $<$ KS, NM, OK $>, S F E$, W\&W, W\& W'. Semple96, E

[Chry sopsis canescens (DC.) Tor, \& Gray] - GPF

Heterotheca fulerata (Greene) Shinners var. amplifolia (Rydb.) Semple - FNA, KTZ, W\&W

Semple96

[Chrysopsis amplifolia Rydh.] - FNA

[Chry sopsis foliosa Nurt.]- $\mathrm{HDH}$

[Chrysopsis foliosa Nutt. var, amplifolia (Rydb.) A. Nelson] - FNA

[Chrysopsis nitidula Wooton \& Standl.] - FNA

[Heterothece fulcrata (Greene) Shinners] - WaW

Heterotheca fulcrata (Greene) Shinners var. fulcrata - ENA, KTZ, W\& W', Semple96

[Chrysopsis cryptocephala Wooton \& Standl.] - FNA 
[Chrysopsis elata Osterh.] - FNA

[Chrysopsis fulcrata Greene] - Dorn01, GPF, HDH

[Chrysopsis resinolens A. Nelson] - FNA

[Chrysopsis resinolens A. Nelson var. ciliata A. Nelson] - FNA

[Chrysopsis resinolens A. Nelson subsp. ciliata (A. Nelson) S. F. Blake] - FNA

[Heterotheca fulcrata (Gireene) Shinners] - W\&W

Heterotheca latifolia Buckley - E

Heterotheca pumila (Greene) Semple - Dorn01, FNA, KTZ, SF!, W\&W, E/W, Semple96

Heterotheca stenophylla (A. Gray) Shinners var, angustifolia (Rydb,) Semple - FNA, KTZ, Semple96

[Chry sopsis angustifolia Rydb] - FNA

[Chrysopsis villosa (Parsh) Nutt. ex DC. var. angustifolia ( $\mathrm{fydb}$ ) Cronquix] - FNA

[Heterotheca villosa (Pursh) Shinners var. angustifolia (Rydlb.) V. L. Harms] - FNA

Heterotheca stenophylla (A. Gray) Shinners var, stenophylla - FNA, KTZ, Semple96

[Chrysopsis stenophylla (A. Gray) Shinners] - GPF

Heterotheca subaxillaris (Lam.) Britton \& Rusby - IMF, KTZ, E

[Heterotheca latifolia Buckley] - GPF, SFE. W\& W

[Heterotheca latifolia Buckley var. macgregoris B. Wagenkn.]

[Helerotheca subaxillaris (Lam.) Britton \& Rusby subsp. latifolia (Buckley) Semple] - FNA

Heterotheca villosa (Pursh) Shinners var, ballardii (Rydb.) Semple - FNA

[Chrysopsis ballardii Rydb.] - FNA

Heterotheca villosa (Pursh) Shinners var, foliosa (Nutt.) Harns - FNA, KTZ, UTF, W\&W', Semple96, E

[Chry sopsts foliosa Nurt.] - HDH

[Chry sopsis villosa (Pursh) Nutt, var. foliosa (Nutt.) DC.] - GPF

[Heterothee: foliosa (Nutt.) Shinners] - SFE, W\& W'

Heterotheca villosa (Pursh) Shinners var, minor (Hook.) Semple-Dorn01, FNA, KTZ, W\&W ${ }^{1}$, Semple96, E/W

[NS - additional synoeymy FNA 20: 252]

[Chrysopsis hispida (Hook.) DC.] - HDH

[Chry sopsis villosa (Pursh) Nutt, ex DC.] - HDH

[Chrysopsis villosa (Pursh) Nutt. var. hispida (Hook.) A. Gray] - GPF

[Chrysopsis villosa (Pursh) Nut. ex. DC. var, hispida (Hook.) A. Gray] - IMF

[Helerotheck villosa (Pursh) Nuft] - SF]

[Heterothech villosa (Pursh) Shinners var, hispida (Hook.) Harms] - UTF, W\&W

Heterotheca villosa (Pursh) Shinners var, nana (A. Gray) Semple - Dorn01, FNA, KTZ, Semple96, E/W

[Chrysopsis canesewn (DC.) Tor, \& A> Gray var, nana A. Gray] - FNA

[Chrysopsts horrida Rydb.] - FNA

[Heterotheen horrida (Rydb.) Harms] - RMC

[Heterotheca villosa (Pursh) Nurt] - SF1

[Heterotheca villosa (Pursh) Shinners var. horrida (Hook.) Semple] - KT2

Heterotheca villosa (Pursh) Shinners var, pedunculata (Greene) Harms ex Semple - FNA, KTZ. W\&W, Semple96, E/W

[Chrysopsis pedusculata Greete] - FNA

[Heterotheca villosa (Pursh) Nurt.] - SF1

Heterotheca villosa (Pursh) Shinners var, scabra (Eastw.) Semple - KTZ, Semple96, E/W

[Heterotheca villoso (Pursh) Nurt] - SF:

Heterotheca villosa (Pursh) Shinners var. villosa - Dorn01, FNA, KTZ, W\&W, Semple96, E/W

[Chrysopsis mellis Nuit.] - FNA

[Chrysopsts villosa (Parsh) Nutt, ex DC.] - HDH

[Chry sopsis villosa (Pursh) Nut, var, villosa] - GPF

[Chrysopsis viscidu (A. Gray) Greene] - HDH, W\& W' 
[Heterotheca villosa (Pursh) Nurt] - SF!

Heterotheca zionensis Semple - FNA, KTZ, UTF, Semple96

Hieracium albiflorum Hook, - Dom01. FNA, GPF, HDH. IMF, KTZ, UTF, E/W

[Chlorocrepis albiflora (Hook.) W. A. Weber] - SF!, W\&W

* Hieracium aurantiacum L, - Dom01, FNA, KTZ, SFE, W\&W, E

Hieracium fendleri Sch. Bip. var, fendleri - FNA, KTZ, E/W

[Chlonocrepis fendleri (Sch. Bip.) W. A. Weber]-SF!, W\&W

[Hieracium fendleri Sch. Bip.]-HDH, IMF, UTF

Hieracium triste Willd ex Spreng, - Dom01, FNA

[Chlurosrepis tristis (Willd.) A. Love \& D. Lôve subsp. gracilis (Hook.) W. A. Weber] - SF!

[Chlorocrepis tristis (Willd. ex Spreng.) A. Lơve \& D. Love subsp. gracilis (Hoek.) W. A. Weber] - W\&W

[Hieracium grasile Hook.] - HDH, UTF

[Hieracium gracile Hook. var. dosonsum (A. Gray) A. Gray] - KTZ

[Hieracium gracile Hook. var. gracile] - KTZ, EW

[Hieracium triste Willd. ex Spreng. var. gracile (Hook.) A. Gray] - IMF

Hieracium umbellatum L. - Dorn01, FNA, GPF, KTZ

[NS - see FNA Vol. 19:287 for additional synenymy]

[Hieracium umbellatum L. yar. scabriusculum Farw.]-RMC

Hymenopappus

(Identification sources:

Hymenopappus filifolius Hook. var, cinereus (Rydb,) I, M. Johnst, - FNA, GPF, IMF, KTZ, SF!, UTF, W\&W, E/W

[Hymenopappos arenosus A. Heller] - HDH

[Hymenopappes cinervas Rydb.] - HDH

[Hymenopappus filifolius Hook.] - $\mathrm{HDH}$

\{Hymenopappus filifolius Hook, var, lugens (Greene) Jeps, \} - FNA, IMF, KTZ, W\&W, err, rep. $\{$ Hymenopapous filifolius Hook. $-\mathrm{HDH}$

\{Hymenopappus filifolias Hook. var. cinereus (Rydb.) L. M. JohnsLi - UTF, W\& W'

'Hymenopappus lugens Greene? - $\mathrm{HDH}$

¿Hymenopappus filifolius Hook. var. lugens (Grevee) B. L. Turmer;

Hymenopappus filifolius Hook. var, luteus (Nutt.) B. L. Turner - Dom01, FNA, IMF, KTZ,

SFW, UTF, W\&W, W

[Hymenopappes luteus Nutt.] - HDH

[Hymenopsppus filifolias Hook.]- $\mathrm{HDH}$

Hymenopappus filifolius Hook. var, megacephalus B. L. Turner - Dom01, FNA, IMF, KTZ, SFW, W\&W, W

[Hymenopappos filifolius Hook.] - HDH

[Hymenogappus filifolius Hook, var, cinereus (Rydb.) I. M. Johnst.] - UTF

Hymenopappus filifolius Hook. var. nudipes (Maguire) B. L. Turner - Dorn01, FNA

[Hymenopappus filifolius Hock, var, alpestris (Maguire) Shimners] - KTZ

- Hymenopappus filifolius Hook. var. parvulus (Greene) B. L. Turner - ENDFMIC $<\mathrm{CO}>$, FNA, KTZ. SF!, W\&W, EW

[Hymenopappers filifolias Hook.]- HDH

Hymenopappus filifolius Hook. var. pauciflorus (L. M. Johnst.) B. L. Turner - FNA, IMF, KTZ W\&W

[Hymenopappus filifolias Hook.] - HDH

[Hymenopappus filifolius Hook, var, cinereus (Rydb.) L. M. Johnst.] - UTF

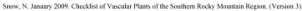


Hymenopappus filifolius Hook, var, polycephalus (Osterh.) B. L. Turner - FNA, GPF, KTZ, SFE, W\&W

[Hymenepappus polycephalus Osterh.] - Dom01, E

[Hymenopappus cinereus Rydb.] - HDH

Hymenopappus flavescens A. Gray var, flavescens - FNA, GPF, KTZ, E

[Hymenupappus flavescens A. Gray] - SFE, W\&W

- Hymenopappus newberryi (A. Gray) I. M. Johnst, - ENDEMIC $<C O$, NM $>$, FNA, KTZ, SF!, W\&W, ENW

[Leucampyx newberryi A. Gray] - HDH

\{Hymenopappus scabiosaeus L'Hér.\} - W\&W, err. rep.

$\{$ Hymenopappus corymbesus Torr. \& A. Gray\}-HDH, rep.

;Hymenopappus scabiosacus L'Her, var. corymbosus (Torr. \& A. Gray) B. L. Tumer; - KTZ ×KS, NE, OK?

Hymenopappus tenuifolius Pursh - FNA, GPF, HDH, KTZ, SFE, W\&W, E

Hymenoxys (see also Tetraneuris)

Hymenoxys brandegeei (Porter ex A. Gray) K. F. Parker - FNA, HDH, M\&H

[Aclines brandezei (Porter) Rydb.] - M\&H

[Actinella brandegei Porter] - M\&H

[Rydbergia brandegei (Porter) Rodb.] - SF!, WR W

[Tetraneuris brandegeci (Ponter ex A. Gray) Parker] - JM, KWA. KTZ, E/W

Hymenoxys grandiflora (Torr. \& A. Gray) Parker - Dorn01, FNA, HDH, UTF

[Tetraneuris grandiflora (Tort. \& A. Gray ex A. Gray) Parker] - KTZ, EW

[Hymenoxys grandifloea (Torr. \& A. Gray ex A, Gray) Parker] - IMF

[Rydbergia grandiflora (Tor, \& A. Gray) Gireene]-SF!, W\&:W

Hymenoxys helenioides (Rydb.) Cockerell - FNA, HDH, IMF, KTZ UTF

[Hymenoxys X belenioides (Rydb.) Cockerell] - Anderson96. E/W

[Hymenoxys richardsonii X H. hoopesii] - Anderson96

[Picradenia belenioides Rydb.] - SFL, W\&W

Hymenoxys hoopesii (A. Gray) Bierner-Dorn01, FNA, KTZ, E/W

[Dugaldia hoopesii (A. Gray) Rydb.] - SF, w. W

[Helenium hoopesii A, Gray]- HDH, IMF, UTF

Hymenoxys odorata DC. - FNA, GPF, HDH, KTZ, E.

[Picradenia odorata (DC) Brition] - SFE, W\&W

Hymenoxys richardsonii (Hook.) Cockerell var. floribunda (A. Gray) Parker - Dom01. FNA.

GPF. HDH. IMF, KTZ, UTF, E/W

[Actinella richardsonii (Hook.) var, floribanda A, Gray] - FNA

[Picradenia richardsonii Hook] - W\&W

[Picradenia richardsonii Hook. subsp. floribunda (A. Gray) W. A. Weber] - W \&W', SFW

[Picradenia richardsonii Hook. var, floribunda (A, Gray) W, A. Weber] - SFE

Hymenoxys richardsonii (Hook.) Cockenell var. richardsonii - Dorn01, FNA, GPF, HDH. IMF,

KTZ, UTF, E/W

[Picradenia richardsonii Hook.] - WEW

[Picradenia richardsoni Hook subsp, richardsonii] - SF?, W\& W'

[Picradenia richardsonii Hook. var, richardsonii] - KT2

* Hypochacris radicata L. - FNA. HDH, IMF, KTZ, SFE, UTF, W\&W, E

Isocoma rusbyi Greene - KTZ, Nesom91, W

[Haplopappus drummondii (Torr, \& A. Gray) S. F, Blake] - HDH rep, IMF, UTF

[Haplopappus heterophyllus (A, Gray) S. F. Blake] - GPF

[Haplopappus plariflonus (A. Gray) H. M. Hall] - HDH, rep

[Haplopappus pluriflorus (Tor, \& A. Gray) H. M. Hall] 
[Haplopappus rusbyi (Cireene) Cronquist] - IMF

[Isocema drammondi (Tarr. \& A. Gray) Greene]-SFW, W \& W

[lsocoma pluriflura (Torr. \& A. Gray) Greene] - W\&W. W\& W

Iva (see also Cyclachaena. Oxytenia)

Iva annua L. - FNA, GPF, W\&W, err. rep.

[Iva annua L. var. annua] - KTZ

Iva axillaris Pursh - Dom01, FNA, GPF, HDH, IMF, KTZ, SF!, W\&W, E/W

[Iva axillaris L.] - UTF

[lva axillaris Pursh var. axillaris] - Dorn01

[lva axillaris Pursh var. robustior Hook.] - Dom 01

Jacea (see Centauren)

Krigia biflora (Walter) S, F, Blake - FNA, GPF, HDH, SFE, W\&W

[Krigia billora (Walter) S. F. Blake var. biflora] - KTZ, E

\section{Kuhnia (see Brickellia)}

\% Lactuca biennis (Moench) Fernald - Dom01, Hartman06, FCF, FNA, GPF, IMF, KTZ, SF!, W\&W, E/W

[Laktuca spicata (LamL) Hitchc.] - HDH

[Sonchus bientis Moench] - FNA

Lactuca eanadensis L.] - Dom01, FNA, GPF, HDH, IMF, KTZ, SF!, UTF, W\&W

[Lactuea canadensis L. var. longifolia (Michx.) Farw.] - E.W

Lactuca graminifolia Michx.] - FNA, HDH, SFW, W\&W

[Lactuca graminifolia Michx. var, graminifolia] - W

[Lictuca graminifolia Mictox, vir, arizonica McV mugb] - KTZ

Lactuca ludoviciana (Nutt.) Riddelt - Dorn01, FNA, GPF, IMF, KTZ, SFE, UTF, W\&W, E

[Lictuca ladoviciana (Nurt) DC.] - HDH

[Sonchus ladoviciana Nust.] - FNA

* L actuca serriola I.. - Dorn01, FNA, GPF, IMF, KT7, SF!, UTF, W\&W, E/W

[Lactuea scariola L. var, integrata Girenier \& Godron] - HDH, orthograpby

[Lictuca scariola L. var. scariola] - HDH, orthography

Lacnnecia coulteri (A. Gray) G. L. Nesom - FNA, KTZ, E/W

[Conyza coulteri A. Gray]-HDH, IMF, SF!, UTF, W\&W

Lacnnceia schiedeana (Less.) G. L. Nesom - FNA, KTZ, E/W

[Conyza schiedearia (Less.) Cronquiss] - HDH, IMF, SF!. WR W

[Erigerooschiedeanus Less.] - FNA

* Lapsana communis L. - FNA, GPF, HDH, IMF, KTZ, SFE, UTF, W\&W, E

Lepidotheca (see Matricaria)

Leucancantha (see Centaurea)

Leucanthemum (see Chrysanthemum)

Leucelene (see Chaetopappa)

Snow, N, January 2009. Checklist of Vacculer Plants of the Southom Rocky Momain Regioe. CVersion $3 x$ 


\section{Leucampyx (see Hymenopappus)}

\{Liatris aspera Michx.\} W\&W, err, rep.

(Liatris aspera Michx. var. aspera\} - KTZ <KS, NE, OK:

Liatris lancifolia (Greene) Kittell - Dom01, FNA, GPF, HDH, KTZ, SFE, W\&W, E

[Lacinaria lancifolia Groenc] - FNA

[Liatris kansansa (Britt.) Rydb.] - FNA

Liatris ligulistylis (A. Nelson) K. Schum, - Dom01, FNA, GPF, HDH, KTZ, SF!. W\&W, E/W

[Lacinaria Ifigulistylis A. Nelson] - FNA

Liatris punctata Hook, var, punctata - FNA, KTZ

[Liatris punctata Hook.] - Dom01, GPF, HDH, SF!, W\& W, E/W

[Liarris punctata Hook. var, nebraskana Gaiser]- KTZ

Liatris squarrosa (L.) Michx, var, glabrata (Rydb,) Gaiser - FNA, GPF, KTZ, SFE, W\&W, E

[Liatris squartusa (L.) Michx, var, glabrata (Rydlo.) F. C. Gates]

[Liatris squarrosa (L.) Michx.] - HDH

Leibnitzia lyrata (Schultz-Bipontinus) G. L. Nesom - Kartese (pers, comm. 2008)

[NS - Legler \{pers, comm 2009 \} reports 2 collections from Colfax Co., NM]

\section{Ligularia (sce Packera, Senecio)}

Logfia arvensis (L.) Holub - Dom01, FNA

[Filago arvensis L.] - Dom01, KTZ

Lotandersonia baileyi (Wootonm \& Standl.) Urbatsch, R. P. Roberts \& Neubig - FNA

[Chry sothammes baileyi Wooton \& Standl.] - KTZ

[Chrysothamnus pulchellus (A. Gray) Greene subsp. taileyi (Wooton \& Standt.) H. M. Hall \& Clem.] - KWA, GPF, HDH, SFE, WEW

[Chrysothamnus pulchellus (A. Gray) Greene var, haileyi (Woston \& StandL) S. F. Blake - IMF, UTE, E

Lotandersonia linifolia (Greene) Urbatsch, R. P. Roberts \& Neubig - FNA

[Chry sothamnus linifolius Greene] - Dom01, IMF, KTZ, SFW, UTF, W\&W, W

[Chrysothamnus viscidiflorus (Hook.) Natt, sabsp, linifolius (Grecne) H. M. Hall \& Clem.] - HDH

-Lorandersonia microcephala (Cronquist) Urhatsch, R. P. Roherts \& Neubig - ENDEMIC $<\mathrm{CO}, \mathrm{NM}>$. FNA

[Haplopappus microosphalus Cronquist] - KTZ

[Tonestus microcephalus (Cronguist) G.L. Nesom \& D. Morgan] - KTZ

Lygodesmia (see also Prenanthella, Shinnersoseris)

(ldentification sources: )

Lygodesnia grandiflora (Nutt.) Torr. \& A. Gray var. arizonica (Tomb) S. L. Welsh - FNA, IMF. UTF

[Lygodesmiz arizonica S. Tomb] - KTZ, SFW, W\&W, W

Lygodesnia grandiflora (Nutt.) Torr. \& A. Gray var. dianthopsis (D. C. Eat.) S. L. Welsh - FNA, IMF

[Lygodesmis dianthopsis D, C, Ear) Tomb] - FNA

[Lygodesmia juncea (Pursh) D. Don ex Hook, var, dianchopsis D. C. Eat. in S. Wats.] - FNA

- Lygodesmia grandiflora (Nutt.) Torr. \& A. Gray var. doloresensis (Tomb) S. L. Welsh - FNA. 
UTF

[Lygodesmis doloresensis Tomb.] - FNA

Lygodesmia grandiflora (Nutt.) Torr, \& A. Gray var, grandiflora - FNA, IMF, UTF

[Lygodesmia grandiflora (Nutt.) Torr. \& A. Gray var. stricta Maguire] - FNA

Lygodesmia juncea (Pursh) D. Don ex Hook, - Dom01. FNA. IMF, KTZ, E/W

[Lygodesmia junces (Pursh) Hook.] - GPF

[Ly godesmia junces (Pursh) D. Dori] - HDH, SF!, UTF, W\&W

\section{Machaeranthera (see also Arida, Dieteria, Rayjacksonia, Xanthisma, Xylorhiza)}

(Identification sources:

Machaeranthera canescens (Pursh) A. Gray var. ambigua B. L. Tumer - IMF, W\&W, EW

[Machaeranthera canescens (Pursh) A. Gray] - GPF. SF!

[Machaeranthera canescens (Pursh) A. Gray subsp. cancscens var. ambigua B. L. Turner] - KTZ

Machaeranthera canescens (Pursh) A. Gray var, aristata (Eastw.) B. L. Tumer - UTF, W\&W, E/W

[Machaeranthera canescens (Pursh) A. Gray] - GPF, SF!

[Machaeranthera canescens (Pursh) A. Gray subsp. glabra (A. Gray) B. L. Turner var. aristata (Eastw.) B. L. Tumer]-KTZ

[Machaeranthersa rigida Greene] - IMF

Machactanthera canescens (Pursh) A. Gray var. canescens - Dom01, IMF, UTF, W\&W, E/W

[Aster cankscens Pursh] - HDH

[Aster rubrotinctus S, E, Blake]- $\mathrm{HDH}$

[Machaeranthera cancscens (Pursh) A. Gray] - GPF, SF!

[Machaeranthera canescens (Pursh) A. Gray subsp, cancscens var, canescens] - KTZ

[Machaeranthera canescens (Pursh) A. Gray var. latifolia (A. Nels.) S. L Welsh] - UTF

[Machaeranthera linearis Greenc] - GPF

Machaeranthera canescens (Pursh) A. Gray var. glabra A, Gray - Dorn01, W\&W, E/W

[Machaeranthera canescens (Pursh) A. Gray] - GPF, SF!

[Machaeranthera canescens (Pursh) A. Gray subsp. glabra (A. Gray) B. L. Turner var. glabra

A. Grayl-KTZ

[Machaeranthera linearis Greans] - IMF

Machaeranthera gracilis (Nutt.) Shinners - KTZ, SFW, W\&W, W

[Haplopappus gracilis (Nutt.) A. Gray] - HDH, IMF, UTF

\{Machacranthera leucanthemifolia (Greene) Greene\}-HDH, W\&W, err. rep.

\{Aster leucanhemifolius Greene? - $\mathrm{HDH}$

'Machacranthera canescens (Pursh) A. Gray var, canescens; - W\&.W'

iMachacranchera canescens (Puish) A, Gray subsp, conescens var, leucanthemifolia (Greene) S, L. Welsh!

- KTZ $\leq$ UT $>$

Macheeranthera parviflora A. Gray - IMF, KTZ, SF!, UTF, W\&W, E/W

[Aster parvulus S.F. Blake] - $\mathrm{HDH}$

Machacranthera tanacetifolia (Kunth) Nees - Dorn01, FNA, GPF, KTZ, SF!, W\&W, E/W

[Aster tanacetifolius Kunth] - FNA, HDH

[Macheranthera coronopifolia (Nutt). A. Nelson] - KTZ

\section{Macronema (sce Ericameria)}

* Madia glomerata Hook. - Dorn01, FNA, GPF, HDH, IMF, KTZ, SF!, UTF, W\&W, E/W

Malacothrix sonchoides (Nutt.) Torr. \& A. Gray - Dorn01, FNA. IMF, KTZ, SFW, W\&W. W

[Malacothrix sonchoides (Nutt.) Torr, \& A. Gray var, scochoides] - HDH 
Malacothrix torreyi A. Gray - Dom01, FNA, IMF, KTZ, SFW, UTF, W\&W, W

[Malacothrix sonchoides (Nutt) Torr. \& A. Griy var. torreyi A. Gray] - HDH

Matricaria

[NS - generic boundaries have been unstable]

Matricaria maritima L. - Dorn01

* Matricaria matricarioides (Less.) Porter - Dorn01, GPF, HDH. IMF, E/W

[Chamomilla suaveolens (Pursh) Rydb.]

[Lepidothecs suaveolens Nutt.] - SF!. W\& W

[Lepidotheca suaveolens (Pursh) Nutt.] - W\& W'

[Matricaria discoidea DC.] - FNA, KTZ

* Matricaria perforata Merat - KTZ, SF!, W\&W, E/W

[Matricaria inodora L.] - $\mathrm{HDH}$

[Matricaria maritima L.] - GPF, UTF

[Matricartia maritima L. var. inodora (L.) Soó] - IMF

[Tripleurospermum inodorum (L.) Sch. Bip.] - FNA

[Tripleurospermum perforata (Merat) M. L.ainz] - KTZ

Matricaria recutita I, - Dom0!

[Chamomills chamomilla (L.) Rydb.] - KTZ

[Chamomilla reticulata (L.) Rauschert]- KTZ

[Matricaria chanomilla L. 1755 \& 1763, non 1753] - KT2

[Matricaria chansomilla L. 1753] - FNA

[Matricaria chanomilla var, coronata (J, Gay) Coss. \& Germ.] - KTZ

[Matricartia susweolens L.]

\{Melampodium cinereum DC.\} - HDH, KTZ, W\&W

Melampodium leucanthum Tort. \& A. Gray - FNA, GPF, HDH, KTZ, SFE, W\&W, E

[Melampodium strigosum Stuessy] - W\&W

Melampodium strigosum Stuessy - FNA

Microseris nutans (Hook.) Sch. Bip, - Dom0I, FNA, GPF, IMF, KTZ, E/W

[Microseris nutans (Gicyer) Sch. Bip.] - SF!, UTF

[Microseris nutans (Hook.) Sch. Bip. subsp. nutans]

[Microseris nutans (Geyer ex Hook.) Sch. Bip.]-W\& W

[PTilocalais nutans (Geyer) Greene] - HDH

Mulgedium pulchellum (Pursh) G. Don in R. Sweet - FNA

[Listuca oblongifolia Nuit - GPE, EW] - DornD]

[Lictuca pulchella (Pursh) DC.] - HDH, IMF

[Lactuea tatarica (L.) C. A. Mey, var, pulchella (Pursh) Breitung] - KTZ

[Lactuca tatarica (L.) C, A. Mey, subsp, pulchella (Pursh) Stebbins] - SFI, UTF, W\&W

[Sonchus paichellus Puish] - FNA

Nothocalais cuspidata (Pursh) Greene - Dorn01, FNA, HDH, KTZ, SFE, W\&W, E [Microseris cuspidara (Pursh) Sch, Bip] - GPF

Nothocalais nigreseens (Henderson) Heller - Dom0I

Oligoneuron (see Solidago)

Oligosporus (see Artenisia)

* Onopordum acanthium L. - Dom01, GPF, HDH, IMF, KTZ, SF!, UTF, W\& W, E/W

Snow, N. Junury 2009. Checklist of Vascular Plants of the Southom Rocky Moumain Region. CVersion $3 x$ 
[Onopordum acanthium L. subsp. acanthium] - FNA

* Onopordum tauricum Willd, - GPF, FNA, HDH, KTZ, W\&W, E

[Onopordam tauricum L..] - SFE

Oõnopsis engelmannii (A, Gray) Greene - KTZ, SFE, W\&W, E

[Bigelowia engelmannii A. Gray] - FNA

[Haplopappus engelmannii (A. Gray) H. M. Hall] - FNA, GPF, HDH

Oōnopsis foliosa (A. Gray) Greene var. foliosa - FNA, KTZ, E

[Haplopappus fremontii A. Gray] = FNA

[Haplopappus fremontii A. Gray subsp. fremontii] - GPF, HDH

[Oünopsis folisa (A. Gray) Greene] - SFE, W\& W

- Oönopsis foliosa (A. Gray) Greene var. monocephala (A. Nelson) Kartesz \& Gandhi

ENDEMIC $<\mathrm{CO}>$, FNA, KTZ, E

[Haplopappus fremosti A. Gray subsp. mooocephalus fA. Nelsom) H. M. Hall] - HDH

[Ö̈nopsis foliosa (A. Gray) Girete] - SFE. W\&W

[Pyrrocoma fotiosa A. Gray ] - FNA

Oönopsis multicaulis (Nutt.) Greene-Dom01, FNA, KTZ

[Oönopsis argillacea A. Nelson] - FNA

[Haplopappus multicaulis (Nutt.) Gray] - FNA

[Stonosus multicaulis Nutr.] - FNA

- Oŏnopsis sp. nov., G. K. Brown \& T. Evans incd, - ENDEMIC $\langle\mathrm{CO}>$

Oonnopsis wardii (Gray) Greene - Dom01FNA

[Huplopappus fremontil subsp, wardii (Grag) Hall] - FNA. KTZ

[Haplopappus fremootii var, wardi A. Gray in A. Gray et al, fas Aplopappus fremonti var, wardił] - FNA

[Haplopappus wardii (Gray) Dorn] - KTZ

[Oanopsis condersata (A. Nelson) A. Nelson] - FNA

[Obnopsis wardii (A. Gray) Greene var, condensata A. Nelson] - FNA

Oreochrysum parryi (A. Gray) Rydb.] - Dorn01, FNA, KTZ, SF!, W\&W

[Haplopappus parryi A, Gray]- HDH

[Solidego parryi $(A$. Groy) Greene] - IMF, UTF, EW

Oxytenia acerosa Nutt. - FNA. HDH, SFW, UTF, w\&W

[iva acerosa (Nuit) R. C. Jackson] - IMF, KTZ, W

Packera (see also Senecio)

(Identification sources:

Packera cana (Hook.) W. A. Weber \& A. Love - Dom01, FNA, KTZ, SF! W\&W, EN

[Senceio canes Hook.] - GPF, HDH, IMF, UTF

[Senecio hallii var. discoides W. A. Weber] - FNA

[Senecio harbourii Rydh.] - HDH

[Senceio lanamiensis A. Nelson] - FNA

[Senecio purshianus Nutt.] - HDH

Packera crocata (Rydb.) W. A. Weber \& A. Löve - Dorn01, FNA. KTZ, SF!, W\&W. E/W

[Senceio aureus L- var. croceus A, Gray] - FNA

[Senecio crocatus Rydb.] - HDH, IMF, UTF

[Senecio pyrrhochrous Greene] - FNA

[Senecio tracyi Rydb.] - FNA

Packera debilis (Nutt.) W. A. Weber \& A. Löve-Dom01, FNA, KTZ, SFE. W\&W, Barkley99. E 
[Senecico dehilis Nutt.] - HDH, IMF

[Senecio helerodoxus Green ex Rydb.] - FNA

Packera dimorphophylla (Greene) W. A. Weber \& A. Löve var. dimorphophylla - Dorn01, FNA. KTZ, Barkley99, E/W

[Packera dimarphophylla (Greene) W. A. Weber \& A. Lowe] - SF!, W\&W

[Packera dimorphophylla (Greene) W. A. Weber \& A. Lóve subsp. dimorphaphylla] - W\&W

[Senecio dimorphophyllus Greene] - HDH

[Senecio dimorphophyllus Greene var. dimorphophyllus] - IMF. UTF

Packera dimorphophylla (Greene) W, A. Weber \& A. Löve var, intermedia (T. M. Barkley) D.

K. Trock \& T. M. Barkley - KTZ; Trock98, E/W

[Packera dimorphophylla (Greene) W. A. Weber \& A. Lơve] - SF!

[Packera đimorphophylla (Greene) W. A. Weber \& A. Lówe subsp. intermedia (T. M.

Barkley) W. A. Weber \& A. Live] - W\&W

[Senocico dimorphophyllus Greene var, intermedius T. M. Barkkcy] - IMF, UTF

Packera fendleri (A. Gray) W. A. Weber \& A. Löve-Dom01, FNA, KTZ, SF!, W\&W, Barkley 99. E/W

[Senecio fendleri A. Gray] - RMC

[Senecio fendleri A. Gray var. fendleri] - $\mathrm{HDH}$

[Setiecio fendleri A. Gray var. lanstus Osterh.] - $\mathrm{HDH}$

\{Packera hartiana (A. Heller\} W. A. Weber \& A. Löve\} - KTZ <AZ, NM, UT>; Barkley99

\{Sonecio bartianus A, Heller\}-IMF, W\&W, misid.

Packera multilobata (Torr, \& A. Gray ex A. Gray) W. A. Weber \& A. Löve- Dorn01, FNA.

KTZ, W\&W, Barkley 99, W

[Packera multilobata (Tor. \& A. Gray) W. A. Weber \& A. Löve] - SFW

[Senceio kecoreus Grectiman] - FNA

[Serectio multilobanus Torr. \& A. Gray] - UTF

[Senceio multilobatus Torr. \& A. Gray ex A. Gray] - HDH, IMF, RMC

[Setictio nelsonil Rydo, var uintahensis A. Nelson] - FNA

[Senocio prolixus Greenman] - FNA

[Senocio slygius Greene] - FNA

[Sencoio thomberi Girenman] - FNA

[Setvetio uintahensis (A. Nelson) Groenman] - HDH, FNA

Packera neomexicana (A. Gray) W. A. Weber \& A. Lõve var. mutabilis (Greene) W. A. Weber

\& A. Löve-FNA, KTZ; Barkley99, E/W

[Packera neomexicana (A. Gray) W. A. Weber \& A. Lave]-SF, W\&W

[Senecio mutabilis Greene] - HDH

[Serecio necmexicantes A. Gray]- HDH

[Senceio tecomexicanus A. Gray var. metcalfei (Greene ex Wooten \& Stand1.) T. M. Barkley] - FNA

[Setcocio Beomexicanus A. Gray var. mutabilis (Greene) T. M. Barkley]- IMF, UTF

\{Packera obovata (Muhl. ex Willd.) W. A. Weber \& A. Löve\}-KTZ $<$ KS, OK>, W\&W, Barkley99

\{Senecio obovatus (Muhl. ex Willd.\}

Packera oödes (Rydb.) W. A. Weber - SF!, W\&W, E/W

[Senecio codes Rydb.] - $\mathrm{HDH}$

[Senecio streptanthifolius Greene var, oüdes (Rydlb,) J. F. Bain] - KTZ, Bain8s

Packera pauciflora (Pursh) W. A. Weber \& A. Ldve - Dorn01, FNA, KTZ, W\&W, Barkley99, E [Packera panciflonus A, Lóve \& D. Löve] - SFE, W\& W

[Senecio aureus L var, discoideus Hook.] - FNA

[Seriocio discoideus (Hook.) Britton]- FNA

[Senecio fedifolius Rydb.] - HDH, rep.

[Senecio kembertii Greene]. FNA

[Senecio panciflonus Pursh] - FNA

Packera paupercula (Michx.) W. A. Weber \& A. Love-Dorn01, FNA, KTZ, W\&W, Barkley99, 
E

[Packera paupercula (Michx.) A. Löve] - SFE, W\& $W^{\prime}$

[Senccio flavoriens Rydb.] - FNA

[Senocio pauperculas Michx.] - GPF, IMF, UTF

[Senccio tweedyi Rydb.] - FNA

Packera plattensis (Nuth.) W. A. Weber \& A. Lõve - Dorn01, FNA, KTZ, SFE, W\&W, Barkley99, E

[Senscio plattensis Nutt.] - GPF, HDH, IMF, RMC

[Senecio pseudotomentosus Mackenzie \& Bush] - FNA

Packera porteri (Greene) C. Jeffrey - FNA, KTZ; Barkley99, EW

[Ligularia porteri (Greene) W. A. Weber] - SF!, W\&W

[Senccio porteri Grecne]- HDH, IMF

Packera pseudaurea (Rydb.) W. A. Weber \& A. Love var, flavula (Greene) D. K. Trock \& T, M.

Barkley - FNA, KTZ, Trock98, E/W

[Packera pseucaurea (Rydb.) W. A. Weber \& A. Love] - SF]

[Packera pseudaurea (Rydb.) W. A. Weber \& A. Löve subsp. flavula (Greene) W. A. Weber \& A. Love] . W\&W

[Senecio flavulas Girene] - HDH

[Setecio pseudaureas Rydb. var. flavulus (Greene) Greenm.] - RMC

Packera pseudaurea (Rydb,) W. A. Weber \& A. Love var. pseudaurea - Dorn01, FNA, KTZ, Barkley 99 , EW

[Packera pseudaurea (Rydb.) W. A. Weber \&. A. Löve]-SF!

[Packera pseudaurea (Ryub.) W. A. Weber \&. A. Löve subsp. pecudaurea] - W\& W

[Senccio poeudaureus Rydb.] - HDH

[Servocio pseudaureas $\mathbb{R} y d b$. var. psecudaureus] - GPF, IMF, RMC

Packera quaerens (Greene) W. A. Weber \& A. L.öve - KTZ

[Senceio quacrens Gireno] - M\&H. KWA

Packera sanguisorboides (Rydb.) W. A. Weber \& A. Löve - FNA

[Senceio sangisortooides Rydb.] - FNA

Packera streptanthifolia (Greene) W. A. Weber \& A. Love-Dom0I, FNA, KTZ, SF!, W\&W, Barkley 99, E/W, Veldkamp08

[Senceio cymbalarioides Nunt.] - HDH

[Sencilo sereptanthifolius Greene] - HDH, IMF, UTF

[Senceio streptanthifolius Greene var. borealis (Torf. \& A. Gray) J. F. Bain] - RMC: Bain88

[Serscio streptanthifotius Greene var. nubicaulis (Greene) J. F. Bain] - Dom01: Bain8s

[Senccio streptanthifolius Greene var. streptanthifolius] - Dorn01; Bain88

Packera subnuda (DC.) Trock \& Barkley - Dom01 (see also Veldkamp08 re nomenclature)

Packera tridenticulata (Rydb.) W. A. Weber \& A. Love - Dorn01, FNA, KTZ, SF!, W\& W. Barkley99, E/W

[Senecio acutidens Rydb.] - HDH

[Senecio compactus (A,Gray) Rydb.] - FNA

[Senecio dersus Greene] - FNA

[Senecio mutabilis Greene] - HDH

[Senecio oblanceolatus Rydb.] - FNA

[Senecio tridersticulatus Rydb.] - GPF, RMC

Packera werneriifolia (A. Gray) W. A. Weber \& A. Löve-Dorn01, FNA, KTZ, SF!, W\&W, Barkley99, E/W

[Packera wernemifolia (A, Gray) W. A. Weber \& A. Love var, wemeriifolia] - Dorn0]

[Packera wernerifolia (A. Gray) W. A. Weber \& A. Lowe var, alpita (A. Gray) Dom] - Domol

[Sencelo alpicola Rydb.] - FNA

[Sencilo molinarius Groenm.] - HDH, rep.

[Senecio perennans A, Nelson] - FNA

[Senecio sexposs Klan] - HDH 
[Senecio scaposuis $A$. Nelson] - FNA

[Senecio werneriifolius (A. Gray) A. Gray] - IMF, UTF

[Senecio werneriacfolius A. Gray var. werneriac folius] - HDH

[Senecio werneriifislius A. Gray var. incertus Greenm.] - HDH, rep.

[Senecio werneriifolius (A. Gray) A. Gray var- wernerififolius] - UT3

Palafoxia rosea (Bush) Cory - FNA

[Palafoxia macrolepis (Rydb.) Cory] - HDH

[Palafoxia rosea (Bush) Cory var, macrolepis (Rydb.) B. L. Turner] - SFE, W \& W

[Palafoxia rosea (Bush) Cory var. macrolepis (Rydb.) B. L. Turner \& M. L. Morris] - Dorn01, GPF, KTZ, E

Palafoxia sphacelata (Nutt. ex Torr.) Cory - FNA, KTZ, W\&W, E

[Palafoxia splascelata (Nutt.) Cory] , GPF, HDH, SFE

[Stevia sphscelata Nutt.] - FNA

\{Parthenice mollis A. Gray\} - HDH rep., KTZ $<A Z>$, W\&W, err, rep.

- Parthenium alpinum (Nutt) Torr. \& A. Gray - ENDEMIC $<$ CO, NM, WV> Dorn01. FNA. KTZ, E

[Bolophyta alpina Nutt.] - FNA, SFE, W\&W

[Bolophyta tetrancuris (Barneby) W. A. Weber] - FNA, SFE, W\&W

[Parthenium alpinum (Nutt.) Torr. \& A. Gray var, telraneuris (Barneby) Rollins) - HDH

[Parthenium tetransuris Bamxby] - KTZ

[RH - a tetraploid raee of Parthenium alpinum, morphologically not distinct. Endemic if recognized.]

Parthenium ligulatum (M. E. Jones) Barneby - FNA, IMF, KTZ, UTF, W

[Bolophyta ligulata (M. E. Jones) W. A. Weber] - SFW, WEW

Pectis angustifolia Torr, var, angustifolia - FNA, IMF, KTZ, E/W

[Pectis angustifolia Tor.] - Dom01, GPF, HDH, SFI, UTF, W\&W

Pericome caudata A. Gray - FNA, GPF, IMF, KTZ, SFW, EW

[Pericome caudata A. Gray var, caudata] - HDH. SFE, W\& W

[Pericome caudata A. Gray var. glandulosa (Goodnsan) Harrington] - HDH, SFE, WEW

Petasites sagittatus (Banks ex Pursh) A. Gray - GPF, W\&W, E/W

[Petasites frigidus (L.) Fries var. sagitasus (Banks ex Pursh) Cherniansky] - Dorn01, FNA, KTZ

[Petasites sagituatus (Bonks) A. Gray] - SF!

[Pecasites sagituala (Pursh) A. Gray]-HDH

Petradoria pumila (Nutt.) Greene var. pumila - FNA, IMF, UTF, W

[Petradoria pumila (Nunt) Greenc] - Dom01, SFW, WNW

[Petradoria pumila (Nutt.) Greenc subsp. pumila] - KTZ

[Solidago petradoria S. F. Btake] - HDH

\section{Picradenia (sec Hymenoxys)}

Picradeniopsis oppositifolia (Nutt.) Rydb. ex Britton - Dorn01, FNA, KTZ, E

[Bahia cppositifolia (Nuat.) DC.] - $\mathrm{HDH}$

[Picradcaiopsis oppositifolia (Nutt.) Rydo.] - GPF, SFE, WEW

Picradeniopsis woodhousei (A. Gray) Rydb. - FNA. GPF, KTZ, SFE. W\&W, E

[Bahia woodhousei A, Gray] - $\mathrm{HDH}$

Picrothamnus (see Artemisia)

Snow, N, January 2009. Checklist of Vecculer Plants of the Southum Rodky Momain Regioe. CVersion $3 x$ 
Platyschkuhria integrifolia (A. Gray) Rydb, - Dorn01, FNA

[Bahia nudicaulis Gray] - HDH, KTZ

[Bahia nudicaulis A. Gray var. desertonum (M. E. Jones) Cronquist] - IMF

[Bahia nedicaulis A. Gray var. oblongifolia (A. Gray) Cronquist] - IMF

[Platyschkuhria integrifolia (A. Gray) Rydb.var. desertorum (M. E. Jones) W. Ellison] - KTZ, UTF

[Platyschkuhria integrifolia ( $A$ - Gray) Rydb. var. integrifolia] - KTZ

[Platyschkuhria integrifolia (A. Gray) Rydb, var, oblongifolia ( $\Omega$. Gray) W. Elison - FCF. KTZ, SFW, UTF, W\&W, w

[Bahia oblongifolia A. Gray] - HDH, rep.

\{Pluchea sericea (Nutt.) Coville\}- KTZ $\langle A Z, N M, U T\rangle$, W\&W, err. rep.

\section{Podospernum (see Scorzonera)}

Prenanthes racemosa Michx. - FNA. HDH,SF!, W\&W

[Prenanthes racemusa Michx. var. multiflora (Cronquist) Dom] - Dornol fas taxon distinct from L. junceał. KTZ, EW

[Prenanthes racemosa Michx. subsp. multiflora Cronquist] - GPF

[Prenanthes racemosa Michx. var, racemosa] - KTZ <WY $>$, E/W

Prenanthella exigua (A. Gray) Rydb, - FNA, KTZ, SFW, UTF, W\&W, W

[Lygodesmia exigua (A. Gray) A. Gray] - IMF

[Lygodesmia exigua A. Gray] - HDH

\section{Prionopsis (see Grindelia)}

Pseudognaphalium canescens (DC,) W. A. Weber subsp. canescens - FNA, KTZ, E

[Gnaphalium canescens DC.] IMF, name applies to Mexican material

[Gnaphaliam wrightii A. Gray] - GPF. HDH, IMF, UTF

[Pscudognaphalium canescens (DC.) W. A. Webor] - SFE, WEW

Pseudognaphalium jaliscense (Greenm.) Anderberg - FNA

[Gnaphalium jaliscense Grecom.] - FN.A

Pseudognaphalium macounii (Greene) Kartesz - Dorn01. FNA

[Gnaphaliam macoanii Gerenc] - FNA

Pseudognaphalium microcephalum (Nutt.) Anderberg - FNA

[Gnaphalium cancscens DC.] IMF, name applies to Mexican msteria]

[Gnaphalium microcephalum Nutt.] - UTF

[Gnaphaliam microcephalum Nutt var, microcephalum] - IMF, misapp.

[Gnaphalium microcephalum Nutt. var, thermale (E. E. Nelson) Cronquise] - Dorn01. IMF

[Gnaphaliam wrightii A. Gray] - GPF, HDH. IMF, UTF

[Pseudognaphalium canescens (DC,) W. A. Weber] - SFE, W E W

[Pseudognaphal ium canescens (DC,) W. A. Weber subsp. microcephalum (Nut.) Kanesz] - KTZ

[Psendognaphaliam canescens (DC,) W. A. Weber subsp. microcephalam (Nutt.) Stebbins \& Keil] - E

[Psendognaphalium canescens (DC.) W. A. Weber subsp. thermale (E. Nels,) Kartes] - KTZ

$\div$ Pseudognaphalium stramincum (Kunth) W. A. Weber - Dom0I, KTZ, SF!, W\&W, E/W

[NS - it is usclear whether this name is the same taxon used by Nesom in FNA Vol, 19:418]

[Gnaphalium chilense Spreng.] - GPF, HDH. UTF

[Gnaphaliam chilense Spreng, var, chilense] - RMC

[Gnaphalium stramineum Kuntb] - IMF

[Psuedognaphalium stramineum (Kunth) Anderberg fand synonyms therein?? FNA Vol. 19: 418] - FNA

\&Pseudognaphalium viscosum (Kunth) W. A. Weber - KTZ, SF!, W\&W ${ }^{1}$, EW

[Geaphalium macounii Greenc] - HDH, IMF 
[Gnaphalium viscosum Kunth] - GPF, IMF, misapp., UTF

[Pseudognaphalium macounii (Greene) Kartesz] - KTZ

Psilocarphus brevissimus Nutt, var, brevissimus - FNA, KTZ

\section{Psilochenia (see Crepis)}

Psilostrophe bakeri Greene - FNA, FCF, HDH, IMF, KTZ, SFW, W\&W, W

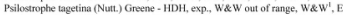

[WJ-roedside waif. Several vists back to original collection site yielded no additional collections]

[Psilostrophe lagetinae (Nutt.) Greene] - SFE

[Psilostrophe tagetina (Nutt.) Greene var. tagetina] - KTZ

Psilostrophe villosa Rydb, - FNA, SFE, exp.

[Psilostropho tagetina (Nutt.) Greene var, serifera (A. Nelson) B. L. Turner] - FNA

Plilocalais (see Microseris)

Ptiloria (see Stephanomeria)

\{Pyrthopappus grandiflorus (Nutt) Nutt\} - GPF, KTZ $<$ KS, NM, OK>, W\&W, err.

Pyrnocoma clementis Rydb, var, clementis - Dorn01, FNA, KTZ, E/W

[Haplopappus slemaris (Rydb.) S. F. Blake] - HDH, LMF, UTF

[Pyrocona clementis Rydb.] - SFI, W\&W

Pyrrocoma crocea (A. Gray) Greene var. crocea - FNA, KTZ, E/W

[Haplopappus croceus A. Gray] - HDH

[Haplopappus croceus A. Gray subsp. croceus] - UTF

[Haplopappus croceus A. Gray var. eroceus] - IMF

[Pymocoma crocea (A. Gray) Greene] - Dom01, SF!, W\& W

\{Pyrrocoma integrifolia (Porter ex A. Gray) Greene\} KTZ $<W Y>$, W\&W, misid.

\{Haplopappus inregrifolias A. Gray) - UTF

\{Haplopappus integrifolias A. Gr subsp. incegrifolitus\} - $\mathrm{HDH}$

Pyrrocoma lanceolata (Hook.) Greene var. lanceolata - FNA, KTZ, E/W

[Donia lascsolata Hook.] - FNA

[Haplopappus lanceolasus (Hook, Torr. \& A. Gray] - FNA. HDH, IMF, UTF

[Haplopappus lanceolatus (Hook,) Torr. \& A. Gray subsp, lanceolatas] - GPF

[Haplopappus lanceolatus (Hook.) Torr. \& A. Gray subsp. solidagineus (Greene) H. M. Hall] - FNA

[Haplopappus lanceolatus (Hook.) Torr. \& A. Gray var. sublanatus. Cody]-FNA

[Haplopappus lanceolatus (Hook.) Torr. \& A. Gray subsp. terminalis (D. C. Eat) A. Gray] - FNA

[Haplopappus lanceolatus (Hook.) Torr. \& A. Gray subsp. vasseyi (Parry ex D. C. Eat.) H. M. Hall] - FNA

[Haplopappus lanceotatus (Hook.) Torr. \& A. Gray var. vaseyi Parry ex D. C. Eas.] - FNA

[Pyrrocona lanceolata (Hook.) Greene]-Dom01, SFI, W\&.W

Pyrrocoma uniflora (Hook.) Greene var, uniflora - FNA, KTZ, E/W

[Haplopappus contractus H. M. Hall] - FNA

[Haplopappus uniflons (Hook, ) Torr. \& A. Gray var. usiflorus] - HDH, IMF

[Pyroconsh unifloes (Hook.) Greene] - Dorn01, SFt, W\& W

Rafinesquia neomexicana Gray - FNA, KWA, M\&H, KTZ

[Nemoseris necmexicana (Gray) Greene] - KWA. M\&H

Ratibida columnifera (Nutt.) Wooton \& Standl. - Dorn01, FNA, GPF, HDH, IMF, KTZ, SF!, UTF, W\&W, E/W 
Ratibida tagetes (E, James) Barnhart - Dom01, FNA, GPF, HDH, KTZ, SFE, W\&W, E

Rayjacksonia annua (Rydb.) R. L. Hartm, \& M. A. Lane-Dorn01, FNA, KTZ, W\&W', Lane96. E.

[Haplopappus annuas (Rydh.) Conj] - FNA. GPF

[Haplopappus, phylocephalus DC. subsp. annuus (Rydb.) H. M. Hall] - HDH

[Machaeranthera annum (Rydb.) Shinners] - FNA

[Machaeranthera phyllocephala (DC.) Shinners] - SFE, W\&W

[Sideranthus annutus Rydb.] - FNA

\{Rayjacksonia phyllocephala (DC.) R. L. Hartm. \& M. A. Lane\}-KTZ, W\&W'; Lane96

Rudbeckia hirta L, var pulcherrima Farw, - Dorn01, FNA. GPF, KTZ. E/W

[Rudbeckia hirta L.] - HDH. SF!, UTF, W\&W

Rudbeckia laciniata L, var, ampla (A. Nelson) Cronquist - Dorn01, FNA, IMF, KTZ, UTF, EN

[Rudbeckia ampla A. Nelson]-SF1, Ws.W

[Rudbeckia laciniata L.] - HDH

Rudbeckia laciniata L. var, hortensia Bailey - Dorn0]

Rudbeckia laciniata L, var. laciniata - Hartman06

Rudbeckia montana A. Gray - FNA, HDH, KTZ, UTF

[Rudbeckia occidetitalis Nut. var. montana (A. Gray) Perdue] - IMF, SFW, W\& W. W

Rudbeckia occidentalis Nutt, - FNA, KTZ, UTF

[Rudbeckia oceidentslis Nutt. var. oceidentalis] - IMF

Rudbeckia triloba L, var, trilobe-GPF, KTZ, E

[Rudbeckia beadlei Small] - FNA

[Rudbeckia triloba L.] - SFE, UTF, w\&W

[Rubbeckia trilobal L, var, beadki (Small) Fernald] - FNA

Rydbergia (see Tetraneuris)

\{Saussurea alpina DC \} - HDH, KTZ, W\&W, misapp.

Saussurea weberi Hult. - Dorn01, KTZ, SF!, W\&W, E/W

[Saussurea densa (Hook.) Rydb.]

Schkuhria multiflora Hook. \& Arn. - FNA, KTZ, SFE, E

[Bahia neomexicana A. Gray] - HDH, W\&W

Scabrethia seabra (Hook.) W. A. Weber sulosp. scabra - FNA, W\&W'

[Seabrethia seabra (Hook.) W.A. Weber subsip. eanescens (W. A. Weber) W. A. Weber] - W\& W'

[Wyethia scabra Hook.] - IMF, UTF, W

[Wyethia seabra Hook, subsp, scabra] - SFW

[Wyethia scabra Hook, var, scabra]- HDH, KTZ, W\&W

Scabrethia seabra (Hook.) W. A. Weber subsp. canescens (W. A. Weber) W. A. Weber - FNA

[Wyethia seabra Hook, subsp، caneseses W. A. Weber] - SFW

[Wyethia scabra Hook, var, canescens W. A. Weber]- HDH, KTZ, W\&W

* Scorzonera laciniata L. - Dorn01, FNA, GPF, KTZ, E

[Podospermum laciniatum (L.) DC.] - SFE, W\&W

Senecio (see also Packera, Ligularia)

Snow, N, Junury 2009. Checklist of Vaccular Plants of the Southom Rocky Momain Region. CVersion $3 x$ 
[NS - The generic boundaries of Senceio s.l. are under sludy [e.g., Pelser0?] and additional changes can be expected. The treatment here follows FNA]

(Identification sources:

Senecio amplectens A. Gray var. amplectens - FNA, HDH, IMF, KTZ, UTF, EN

[Ligularia amplectens (A. Gray) W. A. Weber]-SE!, W\&W

Senecio amplectens A. Gray var, holmii (Greene) Harrington - FNA, HDH, IMF, KTZ, UTF, E/W

[Ligularia amplectens (A. Gray)W. A. Weber var. hallii (Greene) Dom] - Dornol

[Ligularia holmii (Greenc) W. A. Wober] - SF!. W\&W

Senecio atratus Greene - Dorn01. FNA. HDH, IMF, KTZ, SF!, UTF, W\&W, E/W

\{Senecio bigelovii A. Gray var, bigelovii\} - HDH, IMF, KTZ <AZ, NM >

\{Ligularia bigelovii (A. Gray) W. A. Weber var, bigeloviii\} - W\&W, misspp.

Senecio bigelovii A. Gray var, hallii A. Gray - FNA, HDH, IMF, KTZ, UTF, EWW

[Ligularia bigelovii (A. Gray) W.A. Weber]-SFW

[Ligularia bigelovii (A. Gray) W. A. Weber var. hallii (A. Gray) W. A. Weber]- SFE, W\&W

[Ligularia bigelovii (A. Gray) W. A. Weber var. hallii (A. Gray) Dorn] - Domol

Senecio crassulus A. Gray - FNA, GPF, HDH, IMF, KTZ, SF!, UTF, W\&W, ENW

Senecio eremophilus Richardson var, eremophilus - FNA, GPF, IMF, KTZ

[Senecio eremophilas Rictardson]- Dom01. HDH, rep.

[Settecio glaucitilolius Rydb.] - FNA

Senecio eremophilus Richardson var. kingii (Rydb.) Greenm. - FNA, IMF, KTZ, RMC,UTF, E/W

[Senceio ambrosioides Rydb.] - FN. H. HD

[Senccio eremophilus Richardson subsp. kingi (Rydb) G. W. Douglas \& G. Ruyle-Douglas] - W\& W

[Sercolio eremophilas Rydb. subsp. kingii (Rydo.) G. W. Douglas \& G. Ruyle-Douglas] - SF!

[Senecio kingii Rydb.] - FNA

Senecio flaccidus Less, var. flaccidus - FNA

[Senocio douglasii DC. var. jamesii (Torr. \& A. Gray) Eidiget ex Correll \& M. C. Joluston] - FNA

[Sertextio douglasii DC. var. kongilobus (Benth.) L. D. Benson] - FNA

[Senocio kngilobus Benth.] - FNA

\{Senecio flaccidus Less, var. douglasii (DC.) Turner \& T. M. Barkley\} - KTZ, W\&W', E/W

\{Senecio douglasii DC. - IMF

\{Senecio douglasii DC, subsp, longilobus (Benth.) W. A. Webor\} - WeW', erf, rep.

\{Senecio douglasii DC, var, kngilobus (Benth.) L. D. Benson\}-GPF, UTF

\{Senecio flaceidus Less.\} - IMF

\{Senecio flaceidus Less, subsp. douglasii (DC.) Turner \& T. M. Barclay\} - SFE

¿Senecio flaceidus Less, subsp, douglasii (DC.) Turuer \& T, M, Barkley) - W.E W

iSenecio flaceidus Less, var, docuglasii (DC, Turner \& T, M. Barclay)-SFW

\{Senecio flaceidus Less, var, flaccidus\} - KTZ

[Senecio loegilobas Benth.] - HDH

Senecio fremontii Torr. \& A. Gray var. blitoides (Greene) Cronquist - Dom01, FNA, IMF, KTZ, $\mathrm{SF}$ !, UTF, E $/ \mathrm{W}$

[Sencio carthamoides Grece] - $\mathrm{HDH}$

[Senseio fremontii Tor, \& - FNAA. Gray subsp, blitoides (Ginene) W. A. Weber] - W\&W

[Senocio inversastas Greene] - FNA

Senecio fremontii Torr. \& A. Gray var. fremontii - Dom0I

Senecio hydrophilus Nutt. - Dorn01, FNA, GPF, HDH, IMF, KTZ, SF!, UTF, W\&W, E/W

Senecio integerrimus Nutt. var, exaltatus (Nutt) Cronquist - Dorn01, FNA, GPF, IMF, KTZ

UTF. E/W

[Senecio bookeri Tor. $\&$ A. Gray] - HDH

[Sencio integervimus Nutt.]-HDH, SF!, W\&W

Snow, N. Janury 2009. Checklast of Vaccular Plants of tho Southom Rodky Momain Region. CVersion 3 . 
Senecio integerrimus Nut, var, integerrimus - Dom01, FNA, GPF, IMF, KTZ, EN

[Senccio integertimus Nutt.] - HDH, SF!, W\&W

Senecio lugens Richardson - Dom01

Senecio pudicus Greene - FNA, HDH, IMF, KTZ, UTF, EW

[Ligularia pudica (Greene) W. A. Weber] - SF], W\&W

Senecio rapifolius Nutt - Dorn01, FNA, GPF, HDH, KTZ, SFE, W\&W, E

Senecio riddellii Torr. \& A. Gray - FNA, GPF, HDH, KTZ, SFE, W\&W

[Senecio spartioides Torr. \& A. Gray var, fremontii (Torr. \& A. Gray) Greenm. ex L. D. Williams] - E

Senecio serra Hook. var, admirabilis (Greene) A. Nelson - Dom01, FNA. IMF, KTZ, SF!, UTF, W\&W, EN

[Senceio admirabilis Greene] - FNA

[Setiecio serra Hook.] - HDH

Senecio serra Hook. var, serra - Dom01, FNA, KTZ, W\&W, IMF

[Senecio andinas Nutt.] - FNA

[Sencxio lanceolatus Torr. \& A. Gray] - FNA

[Senccio millikenii Eastwood] - FNA

[Sericcio serra var-altior Jepson] - FNA, KTZ

[Senccio scrra var. integriusculus Gray] - KTZ

[Senccior solidago Rydb.] - FNA

- Senecio soldanella A. Gray - ENDEMIC $<C O, N M>$, FNA, HDH, KTZ, E/W

[Ligularia soldanetla (A. Gray) W. A. Weber]-SF!, W\& W

Senecio spartioides Torr. \& A. Gray var, fremontii (Torr. \& A. Gray) Greenm, ex Wms. Dorn01

Senecio spartioides Torr. \& A. Gray var. multicapitatus (Greenm. ex Rydb.) S. L. Welsh Dorn01, KTZ, E/W

[Sencelio multicapitacus Greene] - W: W"

[Senceio multicapitacus Greenm.] - SF !

[Senexio multicapitatus Greenm. ex Rydb.] - HDH, W. W

[Senceio spartioides Torr. \&. A. Gray var, multicapitarus (Greenm.) S. L. Welsh - ]MF, UTF

Senecio spartioides Torr. \& A. Gray var, spartioides - Dom01, IMF, KTZ, UTF, E/W

[Setictio spartioides Torr. \& A. Gray] - GPF, HDH, SFt, W\& W

Senecio sphacrocephahus Greene - Dorn01, FNA, HDH rep., KTZ, W\& W, misid.

- Senecio taraxacoides (A. Gray) Greene - ENDEMIC $<\mathbf{C O}, \mathbf{N M}>$, FNA, HDH, KTZ, E/W

[Ligalaria taracacoides (A. Gray) W. A. Weber] - SF!, W\&W

[Senecio atoplestens A, Gray var, taraxacoides A. Gray] - FNA

Senecio triangularis Hook. - FNA, HDH, IMF, KTZ, SF!, UTF, W\&W, ENW

[Sencio gibbonsii Greene] - FNA

[Senceio triangalaris var, angustifolius G. N. Jenes] - FNA

* Senecio vulgaris L, - Dom01, FNA, GPF, HDH rep., IMF, KTZ, SF!, UTF, W\&W, E/W

Senecio wootonii Greene - FNA, HDH, KTZ, SF! W\&W, E/W

Seriphidium (see Artemisia)

Shinnersoseris rostrata (A. Gray) Tomb - Dorn01, FNA, GPF, KTZ, SFE, W\&W, E

[Lygdoesmia juncea (Pursh) Hook, var, rostrata A. Gray] - FNA

[Lygodesmia rostrota A. Gray] - FNA. HDH

Silphium integrifolium Michx. var, laeve Torr. \& A. Gray - Dorn01, FNA, GPF, KTZ, E

[Silphium integrifolium Michx.] - HDH, SFE, W\&W

[Silphium speciosum Nutt.] - FNA

Silphium laciniatum L. - FNA, GPF. HDH, SFE. W\&W

[Silphium laciniatum L, var, laciniatum] - KTZ, E 


\section{Solidago (see also Euthamia, Oreochrysum, Petradoria)}

[NS - Quadrinomials noc recognized [Snow97\} or listed here, e.y. S. simplex complex]

(Identification sources:

Solidago canadensis L. var, gilvocanescens Rydb. - Dorn01, GPF, HDH, IMF, KTZ

[Doria canescens (Rydb.) Lunell] - FNA

[Solidago altissima L.] - HDH

[Solidage allissima L. subsp. gilvocanescens (Rydb.) Semple] - FNA

[Solidage canadensis L.] - SF!, UTF, W\&W

[Solidago canadensis L. var, canadensis] - HDH

[Solidago gilvocantescens (Rydb.) Smyth] - FNA

[Solidago lepida DC. var. elongata (Nutn.) Fernald] - HDH

[Solidago lepida DC. var. Fallax Femald] - HDH

Solidago canadensis L. var, salebrosa (Piper) M. E. Jones - Dom01, GPF, IMF, KTZ

[Solidago canadensis L.] - W\&W

[Solidago polyphylla Rydb.] - IMF

[Solidago serra Rydb.] - IMF

Solidago eanadensis L. var, seabra Torr. \& A. Gray - GPF, IMF, SF!, E/W

[Solidago altissima L.] - KTZ

[Solidago altissima L. var. procera (Aiton) Femald] - $\mathrm{HDH}$

[Solidago cansdensis L.] - UTF. WR W

[Solidago canadensis L. var. eansdensis] - HDH

[Solidago caradensis L. var. scabra (MahL. ex willd.) Torr. \& A. Gray] -

[Solidago lepida DC. var. elongata (Nutt.) Fernald] - HDH

[Solidage lepida DC. var. fallax Fomald] - HDH

Solidago gigantea Aiton - Dom01, FNA, IMF, KTZ, SF!, W\& W', E/W

[NS - additional synonymy in FNA 20: 156]

[Solidago gigantea Aiton var. leiophylla Fernald] - HDH

[Solidago gigantea Aiton var. serotina (Kuntee) Cronquist] - GPF

[Solidago serotinoides A. Lơve \& D. Lőve] - W\&W

Solidago lepida DC. - FNA

[NS - probably overly subdivided; not recognizing subspecies and varieties as per FNA]

[Aster lepidus (DC,) Kuntze] - FNA

[Solidago canadensis L, var. lepida (DC, ) Cronquist] - FNA

[Solidago canadensis L. subsp. salebrosa (Piper) D. D. Keck] - FNA

[Solidago gigantea Ait. var. sakbrosa (Piper) Friesner] - FNA

[Solidago lepida (DC.) var, salebrosa (Piper)Senple] - FNA

[Solidago salebrosa (piper) Rydo.] - FNA

[Solidago serotina (Ait.) var. salcbrosa Piper] - FNA

[Solidago sera Rydb.] - FNA

Solidago missouriensis Nutt. var, fasciculata Holz. - Dorn01, KWA. JM, M\&H

[NS - FNA does not subdivide this species following resent treatment of Cronquist but points out that further study of sposies is resded]

[Solidago glabcrima Martens] - KTZ, M\&H

[Solidago glaberrima Martens var, moritura (Steele) Palmer \& Steyermark] - KTZ

[Solidngo missouriensis var, glaberrima (Martens) Rosendahl \& Cronq] - KTZ

[Solidaeo tenuissima Woot. \& Standl.] - M\&H

Solidago missouriensis Nutt. var. missouriensis - Dorn01, GPF, IMF, KTZ, E/W

[Solidnow miseouriensis Nutr.] - HDH, SFI, UTF, WEW

Solidago missouriensis Nutt. var. tenuissima (Wooton \& Standl.) C. \& J. Taylor-KTZ, E/W

[Solidago missouriensis Niatt.] - HDH, SFI, UTF, WEW 
[Solidage missouriensis Nutt. var. extraria A. Gray]

Solidago mollis Bartl, - Dom01, FNA, GPF, HDH, SFE, UTF, W\&W

[Doria incana (Torr. \& A. Gray) LunelI] - FN.A

[Doria mollis (Bartling) LuneII] - FNA

[Solidago incana Torr. \& A.Gray] - FNA

[Solidago mollis var. angustata Shinners] - FNA

[Solidago mollis var, nemoralis (Bartling) A. Gray] - FNA

[Solidago mollis Bartl. var. mollis] - KTZ E

Solidago multiradiata Aiton] - FNA, SF!. IMF, W\&W

[Aster multiradiatus (Ait.) Kuntze] - FNA

[Solidago algida Piper] - FNA

[Solidago cusickii Piper] - FNA

[Solidage dilatala $A$. Nelson] - FNA

[Solidago ciliosa Greene] - HDH

[Solidago multiradiaca var. arclica (DC.) Fenvald] - FNA

[Solidage multiradiaca Aiton var. scopulorum A. Gray] - Dorn01. KTZ UTF, W\&W. E/W

[Solidago scopalorum (A. Gray) A. Nelson] - FNA

[Solidago virgaurea L. var. arctica DC.] - FNA

[Solisdago virgaurea L. var. multiradiata (Ait.) Torr. \& A. Gray] - FNA

Solidago nana Nutt, - FNA. HDH, IMF, KTZ, SF!, UTF, W\&W, E/W

[Aster nanus (Nutt.) Kuntre] - FNA

[Solidage nivea Rydb.] - FNA

Solidago nemoralis Aiton var. Iongipetiolata (Mack. \& Bush) Palmer \& Stey, - Dorn01, GPF, KTZ, W\&W, E/W

[Doria pulcherrima (A. Nelson) Lunell] - FNA

[Solidago canadensis L. var. eaboseens A. Gray] - FNA

[Solidage decemflora DC.] - FNA

[Solidago longipetiolata Mackenzie] - FNA

[Solidago nemoralis Aitoe] - SFI, UTF

[Solidago nemoralis Airce subsp. kngipetiolata (Mackenzie \& Bush) G. W. Douglas] - FNA

[Solidago nemoralis Aiton var. longipstiolata (Mackenzie \& Bush) E. J. Palnuer \& Stcyerm.] - FNA

[Solidago nemoralis Aitoe subsp. decenfloea (DC) Brammall ex Semple] - FNA

[Solidago pulcherrima A. Nelson] - FNA

[Solidago petiolaris Aiton\} - see FNA

\{Solidago petiolaris Aiton vart. angusta(Torr. \& A. Gray) A. Gray\} - KTZ

Solidago ptarmicoides (Nees) B. Boivin - FNA, GPF, E

[NS - additional synowymy in FNA 20: 164]

[Aster ptarmicoides (Ness) Torr. \& A. Gray]-HDH

[Oligoncuron album (Nutt.) G. L. Nesom] - KTZ

[Unamia alloa (Nurt.) Rydb.] - SFE, W\&W

Solidago rigida L. subsp, humilis (Porter) S. B. Heard \& Semple - FNA

[Solidago rigida L. var, rigida] - GPF, E/W

[Oligoesuron bombycinum Lunell] - FNA

[Oligoesuron rigidum (L.) Small - SF, W W W

[Oligosstron rigidum (L.) Small var, humile (Porter) G. L. Nesom] - KTZ

[Oligonesuron rigidum (L.) Small vas, rigidum] - KTZ - KS, NE, OK

[Solidago boembycinam (Lunnell) Friesner] - FNA

[Solidago canescens (Rydb.) Friksner] - FNA

[Solidago jacksonii (Kuntze) Fernald var, humilis (Porter) Beaudry] - FNA

[Solidago parvirieida Beandry] - FNA

[Solidago rigida L I - $\mathrm{HDH}$

[Solidago rigida L var, canescens (Rydb,) Breitang] - FNA

[Soliditgo rigida L subsp, hamilis (Porter) S. B. Heard \& Semple] - Dornho1

[Solidago rigida L var, hamilis Porter] - Donot, GPF

Solidago simplex Kunth var. nana (A. Gray) G. S. Ringius - SF!, W\& W ${ }^{1}, E / W$

Snow, N, Junuary 2009. Checklist of Vacculer Plants of the Southom Rocky Momain Region. CVersion $3 x$ 
[Solidago bellidiflora Gireene] - FNA

[Solidago decumbens Greene var. decumbens] - HDH

[Solidage glutinusa Nutt.] - HDH

[Solidago glutinusa Nutt. var. nana (A.Gray) Croquist] - FNA

[Solidage humilis Pursh var. mana A. Gray in A. Gray] - FNA

[Solidago simplex Kanth] - RMC, Domoi

[Solidago simplex Kunth subsp. simplex var. nana (A. Gray) G. S. Ringius] - FNA, IMF, KTZ

[Solidage spatulata DC. var. nana (A. Gray) Cronquist] - UTF, W\&W

[Solidago spathulata Kunth var, nana (A. Gray) Cronquist] - W\& W'

Solidago simplex Kunth var, simplex - SF!, W\&W $W^{1}$, EW

[Aster bonplandii Kuntze] - FNA

[Aster confertiflurus (DC.) Kuntre] - FNA

[Solidago aursola Greene] - FNA

[Solidago decumbens Greene]-FNA

[Solidage decumbens Greene var. oreophila (Rydb) Fernald] - FNA, HDH

[Solidago multiradiata Ait. var. neomexicansa (A. Gray) Wooton \& Stand.] - FNA.

[Solidage oreophila Rydb.] - FN.A

[Solidage simplex Kunth] - RMC

[Solidago simplex Kunth subsp. simplex var. simplex] - FNA, IMF, KTZ

[Solidage spathulata DC. var. glutinosa (NutL) D. D. Keck] - FNA

[Solidage spathulata DC. vart. neomexicana (A. Gray) Croneuist] - UTF, W\&W

[Solidage vespertina Piper] - FNA

[Solidago virgasurea L. var- confertitlera (DC.) Kurla] - FNA

Solidago speciosa Nutt, var, pallida Porter - Dorn01, KTZ, SFE, W\&W, E

[Solidago pallida (Porter) Rydb.] - HDH

[Solixago speciosa Nutt, var, rigidiuscula Torr. \& A, Gray] - GPF

[Solichage speciosa Nutt subsp. pallida (Porter) Semple] - FNA

Solidago velutina DC, subsp, sparsiflora (A. Gray) Semple - FNA

[NS - more extensive synonymy in FNA 20: 160]

[Solidago velutina DC.] - Doen01, FNA, IMF, KTZ SF!, W\& W, EW

[Aster velutinus (DC.) Kuntze] - FNA

[Solidago sparsiflona A. Gray]-GPF, HDH, UTF

Solidago wrightii A. Gray - FNA, SFE

[Aster britonii Kuntoe] - FNA

[Solidago bigelovil A. Gray] - FNA

[Solidago bigelovii A, Gray var, wrightii (A, Gray) A. Gray] - FNA

[Solidago wrightil A. Gray var. adenophora S. F. Blake] - KTZ, WAW, E

* Sonchus arvensis L.- Dorn01, SF!, UTF, W\&W, EW

[Sonchus arvensis L. subsp. anensis] - FNA, GPF, KTZ

[Senchus arvensis L. var, arvensis] - HDH, IMF

* Sonchus asper (L.) Hill - Dorn01, FNA, GPF, HDH, IMF, KTZ, SF!, UTF, W\&W, E/W

* Sonchus oleraceus L. - Dom01, FNA, GPF, HDH, IMF, KTZ, SF!, UTF, W\&W, E/W

* Sonchus uliginosus M. Bicb. - Dorn01, SF!, UTF, W\&W, E/W

[Sonchus arvensis L. var, glabrescens Gunther, Grab, \& Wimm.] - HDH, IMF

[Sonchus arvensis L. subsp, uliginosus (M. Bieb,) Nyman] - FNA, GPF, KTZ

[Sonchus uliginosus M, Bieb, subsp, glabrescens Guenther]

Sphaeromeria argentea Nutt. - Dorn01, FNA, IMF, KTZ, SFW, W\&W, W

[Tanacetum nuttallii Torr. \& A. Gray]- $\mathrm{HDH}$

Sphacromeria capitata Nutt. - Dorn01, FNA, IMF, KTZ, SFW, UTF, W\&W, W

[Tanacetum capitatum (Nun.) Torr. \& A. Gray] - FNA

- Sphacromeria simplex (A. Nels.) Heller - Dorn01, FNA. KTZ

[Tanscetum simplex A. Nels.] = KTZ

Snow, N, Junary 2009. Checklist of Vacouler Plants of the Southom Rocky Mounain Region. CVersion $3 x$ 


\section{Stenactis (see Erigeron)}

Stenotus acaulis (Nutt.) Nutt.] - Dorn01, FNA, KTZ

[Chrysopsis acaulis Nutt.] - FNA

[Haplopappus acaulis (Nutt.) A. Gray] = FNA, IMF

[Haplopappus acaulis (Nutt.) A, Gray subsp. acaulis] - HDH

[Haplopappus, zcaulis (Nutt) A. Gray var. acaulis] - UTF

[Haplopappus acaulis (Nutt.) A. Gray var. glabratus D. C. Eaton] - UTF

[Stenotus acaulis Nutt] - SFW, WRW

[Stenotus acaulis (Nuti.) Nutt. var-acaulis] - W

Stenotus armerioides Nutt. var, armerioides - FNA, KTZ, E/W

[Haplopappus amerioides (Nutt.) A. Gray] - Dor01, GPF, HDH, RMC

[Haplopappus amerioides (Nutt.) A. Gray var. armerioides] - IMF, UTF

[Stonotus armerioides Nutt.] - SF!, W\&W

\{Stenotus amerioides Nutt, var, gramineus (S. L. Welsh \& F. J. Sm.) Kartesc \& Gandhi\} - KTZ $\langle\mathrm{UT}>$

\{Haplopappus armerioides (Nutt) A. Gray var, gramineus S. L. Welsh \& F. J.Sm.\} - IMF, UTF

Stephanomeria exigua Nutt, subsp, exigua - FNA, KTZ, W

[5tephanonseria exigua Nutt.]- HDH, IMF, SFW, UTF, W\&W

Stephanomeria pauciflora (Torr.) A. Nelson] - FNA, GPF, HDH, IMF, KTZ, SF!, UTF, W\&W

[Stephanomoria pauciflora (Torr.) A. Nelson var. pauciflora] - E/W

Stephanomeria runcinata Nutt, - FNA, GPF, IMF, KTZ, SF!, UTF, W\&W, EW

Stephanomeria tenuifolia (Raf.) H. M. Hall] - FNA, SFW, W\&W'

[Ptiloria ramosa Rydb.] - HDH

[Stephanomoria minor (Hook.) Nutt. var. minor] - KTZ

[Stephanomeria tenaifolia (Torr.) H. M. Hall]-GPE, HDH

[Stephanomeria tenaifolia (Torr.) H. M. Hall var. tenuifolia]-UTF

[Stephanonkeria tenuifolia (Raf.) H. M. Hall var. tenuifolia]-IMF, KTZ W

[Stephanomerin wrightii A. Gray]-W\&W

\{Stephanomeria virgata Bentham\} - HDH, exp., W\&W, err, rep.

[Stephanomeria virgata Bentham subsp. pleurocarpa (Greene) Goutisb) - KTZ

Symphyotrichum

(Identification sources:

Symphyotrichum $X$ amethystinum (Nutt.) G. L. Nesom] - FNA, KTZ

[Aster $X$ amethystinus Nuit.] - E

[Aster ericoides L. var, ericoides X A novac-anglinae] - KTZ

[Symphyotrichum amethystinus (Nuat.) G. L. Nesom] - W \& W'

[Virgulus X amethystimus (Nuts,) Reveal \& Keener] - SFE, W\& W

Symphyotrichum aseendens (Lindl.) G. L. Nesom - Dorn01, FNA. KTZ. W\& W'

[Aster adseendens Lindl.] - HDH, IMF, EW

[Aster chilensis Nees subsp, adscendens (LindL) Cronquise] - GPF, UTF

[Aster orthophyllus Greene] - SFE

[Virgalaster ascendens (Lindl.j Semple] - SF:

[Virgalaster ascendens (Lindl, ex Hook.) Semple] - W\&W

Symphyotrichum boreale (Torr. \& A. Gray) A. LBve \& D. Love-Dom01, FNA. KTZ, W\&W'

[Aster borealis (Torr. \& A. Gray) Prov]-E/W

[Aster junciformis Rydb.] - WY checklist

[Aster borealis Prov.] - IMF 
[Aster junciformis Rydb.] - GPF, HDH, SF!, UTF, W\&W

[Symphyotrichum breale (Prov.) Löve \& L.Bve] - Dorma!

Symphyotrichum campestre (Nutt.) G. L. Nesom - FNA, W\&W'

[Aster campestris Nutt.] - HDH, IMF, UTF, EW

[Aster bloomeri A. Gray] - FNA

[Aster campestris Nutt.] - FNA

[Aster campestris Nutt. var. bloomeri ( $A$. Gray) A. Gray)] - FNA

[Aster campestris Nutt. var. campestris] - RMC

[Symphyotrichum campestre (Nuts.) G. L. Nesom var, bleameri (A. Gray) G. L. Nesom] - FNA

[Symphyotrichum campestre (Nutt.) G. L. Nesom var. campestre] - KTZ, W\&W'

[Virgulus cantpestris (Nutt.) Reveal \& Keener] - FNA, SF!. W\& W

Symphyotrichum ciliatum (Ledeb.) G. L. Nesom - Dom01, FNA, KTZ

[Aster bractyactis S. F. Blake] - GPF, HDH, IMF, UTF, EW

[Brachysetis ciliala (Ledeb.) Ledeb. sabsp. angusia (Lindl.) A. G. Jones]

[Brachyactis ciliata Ledeb. subsp. angusta (L.indi.) A. G. Jones] - SF!. W\& W

[Erigeron cilatus Ledeb.] - FNA

Symphyotrichuym ciliolatum (Lindl.) A. Love - FNA

[Aster ciliolatus Lindl. - FNA]

[Aster ciliolatus Lindl- var. comatus (Fernald) A. G. Jones] - FNA

[Aster lindleyanus Tort. \& A. Gray] - FNA

[Aster wilsonii Rydb] - FNA

Symphyotrichum eatonii (A. Gray) G. L. Nesom - Dorn01, FNA, KTZ

[Aster bracteolanus Nutr] - SFW, W\&W, w

[Aster eatonii (A. Gray) Howell]- HDH, as synonym of A. oregonus, GPF, IMF, UTF

[Aster ofegonus (Nutr.) Tort. \& A. Gray - MM. M\&H, HDH, W\&W, eft. Rep.

[Symphyotrichum bracteolatum (Nutt.) G. L. Nesom] - W\& W'

Symphyotrichum ericoides (L.) G. L. Nesom var. ericoides - FNA, KTZ

[Aster ericoides L. var. ericoides] - E/W

[Aster ericoides L.] - FNA, GPF, HDH, IMF

[Symphyotrichum ericoides (L.) G. L. Nesom] - FNA. WE W'

[Virgulus ericoides (L.) Reveal \& Kexner] - FNA, SFL, W\&W

Symphyotrichum ericoides (L.) G. L. Nesom var. pansum (S. F. Blake) G. L. Nesom - FNA,

KTZ

[Aster ericoides L. var. pansus (S. F. Blake) B. Boivin] - E/W

[Aster ericoides L. subsp. pansus (S. F. Blake) A. G. Jones var. pansus]

[Aster ericoides L. subsp. partsus (S. F, Blake) A. G. Jotics var. Stricticaulis (Torr. \& A. Giray) F. C. Gates]

[Aster ericoides L. var. strictichulis (Torr. \& A. Gray) F. C. Gates] - RMC

[Aster pansus (S. F. Blake) Cronguist] - GPF, IMF, UTF

[Symphyotrichum ericoides (L.) G. L. Nesom] - W\& W'

[Symphyotrichum ericoides (L.) G. L. Nesom var, stricticaule (Tor, \& A. Gray) G. L. Nesom] - KTZ

[Virgalus ericoides (L.) Reveal \& Keener] - SF!, W\&W

Symphyotrichum ericoides (L.) G. L. Nesom var. stricticaule (Torr. \& A. Gray) G. L. Nesom -

Dorn01. FNA

Symphyotrichum falcatum (Lindl.) G. L. Nesom var. crassulum (Rydb.) G. L. Nesom - Dorn01, KTZ

[Aster commutatus (Tor, \& A. Gray) A. Gray] - GPf

[Aster commutatus (Torr, \& A, Gray) A, Gray var, commutatus] - HDH

[Aster commutatus (Torr. \& A. Gray) A. Gray var, crassulas (Rydb.) S. F. Blake] - HDH

[Aster commutatus (Tor, \& A. Gray) A, Gray var, polycephalas (Hydb,) S. F, Blake] - HDH

[Aster falcatus Lindl.] - UTF

[Aster falcatus Lindli subsp, commutarus (Torr, \& A. Gray) A. G. Joncs var, commutatus] - FNA

[Aster falcatus Lindt, subsp, commutarus (Tor, \& A. Gray) A. G. Joess var, crassulus (Rydb, ) Cronquist] -

FNA

[Aster falcatus Lindl, var, commutatus (Torr, \& A, Gray) A, G. Jones] - IMF, EW

[Aster falcanus Lindl, var, crassulas (Rydb, ) Cronquist] - IMF, RMC

Snow, N, Janury 2009. Checklist of Vaccular Plants of tho Southom Rocky Momain Region. CVersion 3 . 
[Symphyotrichum falcatum (LindL) G. L Nesom] - W\& W

[Symphyotrichum falcatum (Lindl.) G. L. Nesom var. commutatum (Tor.\& A. Gray) G. L Nesom] - KTZ

[Virgulus falcatus (Lindl.) Reveal \& Keener] - SF!, W\&W

Symphyotrichum falcatum (Lindl.) G. L. Nesom - Dom01, FNA, W\&W

[Aster falcatus Lindl.] - GPF, HDH, UTF

[Aster falcatus Lindl. var. fakcatus] - IMF, E.W

[Symphyotrichum falcatum (LindL) G. L Nesom var. falcatum] - KTZ

[Virgulus falcatus (Lind.) Reveal \& Keener] - SF!, W\& W

Symphyotrichum fendleti (A, Gray) G. L. Nesom - FNA, KTZ

[Aster fendleri A. Gray] - FNA, GPF, HDH, E

[Virgulus fendleri (A. Gray) Reveal \& Keeter] - FNA, SFE, W\&W

Symphyotrichum foliaceum (DC.) G. L. Nesom var apricum (A. Gray) G. L. Nesom - Dom01, FNA, KTZ

[Aster foliaceus Lindi.]-SF

[Aster foliaceus LindL, ex DC, var, apricus A. Gray] - EW

[Aster foliaceus Lindl. var. apricus A. Gray] - IDH, IMF, UTF

[Aster foliaceus Lindl. ex DC. var. foliaceus] - W\&W

[Symphyotrichum foliaceum (DC.) G. L. Nesom] - W\& W'

Symphyotrichum foliaceum (DC, ) G. L. Nesom var. canbyi (A. Gray) G, L. Nesom - Dorn01.

FNA, KTZ

[Aster foliaceus Lindl.] - SF!

[Aster folisceus Lindl. ex DC, var, canbyi A. Gray] - EWW

[Aster folizeceus Lindl, var. sanbyi A. Gray] - HDH, IMF, UTF

[Aster folisceus Lindl, ex DC. var, foliaceus] - W\&W

[Symphyotrichum foliaceum (DC.) G. L. Nesom] - W\&W'

Symphyotrichum foliaceum (DC,) G, L, Nesom var. parryi (D, C. Eat.) G. L. Nesom - Dorn01, KTZ

[Aster foliaceus Lindl.] - SF:

[Aster foliaceus Lindl. ex DC. var. parni (D. C. Eaton) A. Gray] - E/W

[Aster foliaceus Lindl. var. parryi (D. C. Eason) A. Gray] - FNA. IMF, UTF

[Asser foliaceus Lindl. var, frondeus A. Gray] - HDH

[Aster foliaseus Lindl. ex DC. var. foliaceus] - W\&W

[Symphyotrichum follaceum (DC,) G. L. Nesom] - Ws W'

Symphyotrichum frondosum (Nutt.) G. L. Nesom - Dorn01, FNA, KTZ

[Aster frondosus (Nutt.) Torr. \& A. Gray] - HDH, IMF, UTF, E. W

[Brachystis trondosa (Nuit.) A. Gray]-SFI. W\&W

Symphyotrichum laeve (L.) A. Love \& D. Love var. laeve - FNA, W\&W', err. rep.

[Aster lacvis L.] - UTF

[Aster laevis L. var, lacvis] - FCF

Symphyotrichum laeve (L.) A. Love \& D. I.ove var. geyeri (A. Gray) G. L. Nesom - FNA, KTZ. W\& $W^{\prime}$

[Aster laevis L.] - UTF

[Aster hevis L, var, geseri A. Gray ]- GPF, HDH. IMF, SF1, W\&W, EW

[Symphyotrichum laves (L.) A. Lowe \& D. Love]

Symphyotrichum lanceolatum (Willd) G. L. Nesom var. hesperium (A. Gray) G. L. Nesom -

Dorn01. FNA

[Aster hesperias A, Gray] - IMF, UTF

[Aster laneeolatus Willd, subsp, hesperius (A. Gray) Semple \& Chemiel] - SFI, W\& W, EW

[Aster hespertus A. Gray yar, hesperius] - HDH

[Aster hesperius A. Gray var, lastevirens (Greene) Cronguist] - GPF, HDH

[Symphyotrichum lancelolatum (Willd.) G, L. Nesom] - We W'

[Symphyotrichum lanceolatum (Willd.) G, L. Nesom subsp. hesperium (A. Gray) G. L. Nesom var, hesperium

(A. Gray) G. L. Nesom] - KTZ

Symphyotrichum molle (Rydb.) G. L. Nesom - Dorn01, FNA

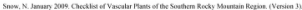


[Aster mollis Rydb.] - FNA

Symphyotrichum novae-angliae (L.) G. L. Nesom - Dorn01, FNA, KTZ, W\&W'

[Aster novac-angliae L.] - FNA, GPF, HDH, IMF, UTF, E

[Symphyotrichum foliaceum (DC.) G. L. Nesom] - W\& W'

[Virgulus novae-angliae (L.) Reveal \& Keener] - FNA, SFE, W\&W

Symphyotrichum oblongifolium (Nutt.) G. L. Nesom - Dom01, FNA, KTZ, W\&W'

[Nster kumleini Fr.] - HDH

[Aster oblongifolius Nutt.] - E

[Aster oblongifolius NutL, var. oblongitolius] - GPF

[Virgalus oblongifolius (Nutt.) Reveal \& Keener] - FNA, SFE, W\&W

- Symphyotrichum porteri (A. Gray) G. L. Nesom - ENDEMIC $<$ CO, NM, WV>, Dom01,

FNA, KTZ, W\&W

[Aster ericoides L. var. strictus Porter] - $\mathrm{HDH}$, W\&W, misapp.

[Aster porteri A. Gray - HDH, SFE, W\&W, E

Symphyotrichum spathulatum (Lindl.) G, L. Nesom var, spathulatum - KTZ

[Aster occidentalis (Nutt.) Torr. \& A. Gray] - UTH

[Aster oxcidentalis (Nutt.) Torr. \& A. Gray var. fremontii (Torr. \& A. Gray) A. G. Jones] - E/W

[Aster occidentalis (Nurt.) Torr. \& A. Gray var. occidentalis] - HDH. E/W

[Aswer spathulatus LindL] - SF!

[Aster spathulasus Lindt. ex DC.] - WEW

[Aswer spathulatus Lindl. var. spathulatus] - IMF

[Symphyotrichum spathulatum (LindL) G. L. Nesom] - W\&W

Tagetes micrantha Cavanilles - FNA, KWA, KTZ, M\&H

[Tagetes fragraatissima Sessé \& Moc.]-KTZ

Tagetes patula L. - Dorn01

\section{Tanacetum (sce also Chrysanthemum, Sphaeromeria)}

* Tanacetum balsamita L. - Dorn01, FNA, IMF

[Balsamita major Desf]] - FNA, KTZ, W\&W

[Chrysanthemum batsamita L.] - GPF, HDH

[Chrysanthemum balsamita (L. ) Baillon] - FNA

[Chrysanthemum batsamita (L. ) Baillon var. tanacetoids Boisser] - FNA

[Pyrethrum majus (Disf.) Terelev] - FNA

* Tanacetum parthenium (L.) Sch. Bip. - Dom01, FNA, IMF, KTZ, W\&W

[Chrysanchemum parthenium (L.) Bernh.] - GPF, UTF

* Tanacetum vulgare L. - Dom0I, FNA, GPF, HDH, IMF, KTZ, SF!, UTF, W\&W, E/W

Taraxacum ceratophorum (Ledeb.) DC. - Dorn01, FNA, HDH, IMF, UTF, W\& W', misid., E/W

[NS - see FNA V0. 19:247 for addicional syronymy]

[Taraciacum dumetorim Greene] - W:W

[Tarzxacum officinale Weber ex F. H. Wige subsp. ceratophorum (Ledeb, Schinz ex Thell]- KTZ

[Teraciasum ovinum Greetne] - WR W

[Taraciacsm ovinum Rydb.] - SF!

Taraxacum eriophorum Rydb, - Dorn01, HDH, KTZ, SF!, W\&W, EJW

Taraxacum erythrospermum Andrz. ex Besser - FNA, HDH

[Taraxneum laevigatum (Willd.) DC, var, erythrospernum (Andrz, ex Besser) J. Weiss] - FNA

[Taraxacum officinale F. H. Wiggers var, eryhtrospermam(Andrz ex Besser) Babington] - FNA

[Taracucum scanisum Dahlstodt] - FNA

* Taraxacum lacvigatum (Willd.) DC - Dorn01, GPF, IMF, KTZ, UTF, E/W

[Taraxacum officinale Weber] - SF

Taraxacum lyratum (Ledeb.) DC.] - HDH, IMF, KTZ, UTF, W\&W, misapp., E/W

[Taracacum plymatocarpum J, Vahi] - W\&W, W\&W', misid. 
* Taraxacum officinale Weber ex F. H, Wigg- Dorn01, FNA, IMF, UTF, W\&W, E/W

[Taraxacum officinale Weber] - GPF, SF!

[Taraxacum officinale F. H. Wigg - - HDH

[Taraxacum officinale Weber ex F.H. Wigg. subsp. officinale] - KTZ

Taraxacum scopulorum (A. Gray) Rydb, - Dom01, SF!, W\&W

[Taraxactum offiinale F. H. Wiggers var, scopulorum $A$. Gray in $A$. Gray] - FNA

[Leontadon scopulorum (A. Gray) Rydb.] - FNA

* Taraxacum vulgare Lam.

[Taraxacum officinale Weber ex F. H. Wigg. subsp. vulgare (Lam.) Schinz \& R. Keller] -

[Taraxacum officinale Weber ex F. H. Wigg. subsp, officinale] - KTZ

Tetradymia canescens DC, - Dorn01, FNA, HDH, IMF, KTZ, SF!, UTF, W\&W, EJW

\{Tetradymia glabrata Torr. \& A. Gray\} - KTZ $<\mathrm{UT}>$, \&W, err. rep.

Tetradymia nuttallii Torr. \& A. Gray - Dom01, FNA, HDH, IMF, KTZ, SFW, UTF, W\&W, w

Tetradymia spinosa Hook, \& Am,-Dorn01, FNA, HDH, KTZ, SFW, UTF, W\&W, W

[Tetradymia spinosa Hook. \& Am. var. spinosa] - IMF

\section{Tetraneuris}

(Identification sources:

Tetraneuris acaulis (Pursh) Greene vat, acaulis - Dorn01, FNA, KTZ, E/W

[Hymenoxys acaulis (Pursb) Parker var, acmulis] - GPF, HDH, IMF

[Tetraneurbs acaulis (Pursh) Greese] - SFI, W.EW

Tetraneuris acaulis (Pursh) Greene var. arizonica (Greene) Parker - Dorn01, FNA, KTZ, W\&W, err. rep. E/W

[Hymenoxys acaulis (Pursh) Parker var. arizonica (Greene) Parker] - HDH, IMF, UTF

[Tetraneuris acaulis (Pursh) Greene] - SF!, W\&W

Tetraneuris acaulis (Pursh) Greene var. caespitosa A. Nelson - Dom01, FNA, KTZ, ENW

[Hymenoxys acaulis (Pursh) Parker var. cacspitosa (A. Nolson) Parker] - HDH, IMF

[Hymenoxys acaulis (Pursh) Parker var. lanoua (Greene) Cronquist] - UTF

[Tetrancuris bevifolia Greene] - SF1, W\&W

Tetrancuris acaulis (Pursh) Greene var. epunctata (A. Nelson) Kartesz \& Gandhi - FNA. KTZ.

[Hymenoxys acauls (Pursh) Parker var, epunetata (A, Nelson) Cronquis] - IMFoCOs, UTF

[Tetraneuris acaulis (Pursh) Greone] - SFI, WRW

Tetraneuris argentea (A. Gray) Greene-KWA, JM, KTZ $\angle A Z, N M>$, W\&W $<A Z, N M>$

[Actinella angentea Gray] - KiZ, MS.H

[Actinella formosa A. Nels.] - M\&H

[Actinella leptoclada Giray]-MEH

[Hymenoxys argentea (Gray) Parket] - M\&H KTZ

Tetrancuris ivesiana Greene - FNA, KTZ, SF!, W\&W, E/W

[Hymenoxys acaulis (Pursh) Parker var, ivesiana (Groene) Parker] - HDH, UTF

[Hymenoxys argentea (A. Gray) Parker var. ivesiana (Greene) Cronquist] - IMF

Tetrancuris scaposa (DC.) Greene var. scaposa - FNA, KTZ, E

[Hymenoxys seaposa (DC.) Parker var glabrata (Nuts.) Parker] - GPF

[Hymenoxys scaposa (DC.) Parker var, lisearis (Nutt) Parker] - HDH

[Hymenoxys scaposa (DC,) Parker var, scaposa] - GPF, HDH

[Tetraneuris scaposa (DC) Greene] - W\&W

[Tetraneuris sesposa (DC.) Greene var. linearis Parker] - SFE

[Tetraneuris seaposa (DC) Greene var. linearis (Neit) Parker] - KT7

Tetraneuris torreyana (Nutt.) Greene - Dom01, FNA. K1Z, SF!, W\&W, E/W

[Hymenoxys toreyarua (Nutt.) Parker]-IMF, UTF 
\{Thelesperma ambiguum A. Gray) - GPF, KTZ, W\&W

Thelespernta caespitosum Dom - Dorn01

Thelesperma filifolium (Hook.) A. Gray - FNA. SFE

[Thelesperma filifolium (Hook.) A. Gray var. intermedium (Rydb.) Shimers] - Dorn01, GPF, KTZ W \& W, E

[Thelesperma trifidum (Poir.) Britson] - HDH

Thelesperma megapotamicum (Spreng, Kuntze - Dom01, FNA, HDH, KTZ, SFE, W\&W, E

- Thelesperma pubescens Dorn - ENDEMIIC $<$ WV $\geq$, Dorn01, KTZ

[NS - as recognized by Darn01 evidently endemic to WY (see UT3:236)]

[Thelesperma subnudum var. alpinum Welsh] - KTZ, UT3

[Thelesperma windhamii C. J. Hansen] - UT3

Thelesperma subnudum A. Gray - FNA, GPF, HDH, SF!, W\&W

[Thelesperma caespitosam Dorn] - KTZ

[Thelesperma subnudum Gray var. caespilosum (Dom) Welsh] - KTZ

[Thelesperma subnudumt A. Gray var. subnudum] - IMF, KTZ, UTF, E/W

[Thelesperma marginatum Rydb.] - Dom01, KTZ

[Thelesperma subnudum A. Gray var marginatum (Rydb.) T. E. Mekhert ex Cronq.] - KTZ

Thymophylla aurea (A. Gray) Greene ex Britton var, aurea - KTZ, E

[Dyssodia aurea (A. Gray) A. Nelson] - GPF, HDH, SFE, WzW

[Lowellia aureas A. Gray] - FNA

[Thymophylla aurea (A. Gray) Greene] - W\& W'

[Thymophylla aurea (A. Gray) Greene var. aurea] - KTZ

Tonestus lyallii (A. Gray) A. Nelson - FNA, KTZ, SF!, W\&W, EW

[Haplopappus lyalli A. Gray]-HDH, IMF

Tonestus pygmaeus (Torr, \& A. Gray) A. Nelson - FNA, KTZ, SF!, W\&W, ENW

[Haplopappus pygmaeus [Torr. \&. A. Gray) A. Giray] - HDH

[Stenotus pyzmacus Torr. \& A. Gray] - FNA

Townsendia

(Identification sources:

Townsendia annua Beaman - FNA, IMF, KTZ, SFW, UTF, W\&W, W

Townsendia eximia A. Gray - FNA, HDH, KTZ, SFE, W\&W, E

Townsendia exscapa (Richards.) Porter - Dom01, FNA, GPF, HDH, KTZ, SF!, W\& W, E/W

[Aster evscapa Ricahind, in J. Franklin et al.] - FNA

[Towasendia sericea Hook.] - FNA, HDH

Townsendia fendleri A. Gray - FNA, KTZ, SFE, W\&W, E

Townsendia formosa Greene - FNA, GREE (Hazlent I/, /55; Colfax Co., NM)

- Townsendia glabella A. Gray - ENDEMIC $<\mathrm{CO}>$, FNA, HDH, KTZ, SEW, W\&W, W

Townsendia grandiflora Nutt. - Dom01, FNA, GPF, HDH, KTZ, SFE, W\&W, E

Townsendia hookeri Beaman - Dorn01, FNA, GPF, IMF, KTZ. SF!, UTF, W\&W, E/W

Townsendia incana Nutt. - Dorn01. FNA, HDH, IMF, KTZ, SFW, UTF, W\&W, W

Townsendia leptotes (A. Gray) Osterh. - Dorn01, FNA, HDH, KTZ, SF!, UTF, W\&W, E/W

- Townsendia microcephala Dom - ENDEMIC <WY>. Dom01, FNA, KTZ

Townsendia montana M. E. Jones - FNA. W\&W

[Towasendia alpigena Piper var, alpigena] - KTZ

[Towasendia alpigena Piper var, caelitinensis (S. L. Welsh) Kartess \& Gandhi] - FNA

[Towasendia movtana M. E. Jones var, montana] - IMF, UTF 
[Townsendia moniana M. E. Jones var, caelilinensis S. L. Welsh] - FNA

Townsendia nuttallii Dom - Dorn01, KTZ

[Townsendia hookeri Beaman\} - IMF

Townsendia parryi D. C. Eaton - Dorn01, FNA, KTZ

- Townsendia rothrockii A. Gray ex Rothrock- ENDEMIC <CO $>$, FNA, KTZ, W\&W, EWW

[Towasendia rothrockii A. Gray]-HDH, SF!

Townsendia spathulata Nutt-Dorn01, FNA, KTZ

Townsendia strigosa Nutt, - FNA, IMF, KTZ, SFW, UTF, W\&W, W

[Townsendia strigosa Nutt. var. strigosa] - UT3

[Townsendia strigosa var- prolixa S. L. Welsh] - FN.A

* Tragopogon dubius Scop, - Dorn01, FNA, GPF, IMF, KTZ, UTF. E/W

[Tragopogon dubius Scop. subsp. major (Jacq.) Vollman] - HDH, SF!, W\&W

Tragopogon lamottei Rouy - Dom01

* Tragopogon porrifolius L. - Dorn01, FNA, GPF, HDH, IMF, KTZ, SF!, UTF, W\&W, E/W

* Tragopogon pratensis L. - Dorn01, FNA, GPF, HDH, IMF, KTZ. SF!, UTF, W\&W, E/W

Trimorpha (see Erigeron)

Tripleurospermum (sce Matricaria)

Unamia (see Solidago)

Verbesina encelioides (Cav.) Benth. \& Hook. - FNA

[Verbesina encelioides (Cav.) Benth. \& Hook. f. ex A. Gray var. exauriculaca B. L. Rob. \& Greenm-] - IMIF. E/W

[Verbesina encelioides (Cav.) Benth, \& Hook. sabsp. exauriculata (B. L. Rob. \& Greenm.) J. R. Coleman] GPF

[Verbesina encelioides (Cav.) Bench. \& Hook. f. ex A. Gray subsp. exauriculata (B. L. Rob. \& Greenm.) J. R. Coleman] - KTZ

[Verbesina encelioides (Cav.) Benth. \& Hook, var, exmuriculana B. L. Rob. \& Greenm.] - Dorn01. HDH

[Verbesina enceliokdes (Cav,) Benth. var, exaurisulata B. L. Rob. \& Greenm.] - UTF

[Ximeneska encelioides Cav.] - SF!, W\&W

Vernonia baldwinii Torr. var. interior (Small) B. G. Schub. - E, FNA

[Vemonia baldwinii Torr. subsp. interior (Small) W. Z. Faust] - GPF, KTZ, SFE, W\&W

Vemonia fasciculata Michx. - FNA, HDH

[Vemonia fasciculata Michx, var. corymbosa (Schwein. ex Keating) Daniels] - E.

[Vemonia fasciculata Michx, subsp, corymbosn (Schwein.) A.Love \& D.LEve] - SFE, W\&W

[Vemonis fasciculata Michx, subsp, corymbosa (Schwein, ex Kearing) S, B, Jones] - GPF, KTZ

Vernonia marginata (Torr.) Raf. - FNA, GPF, HDH, IMF, KTZ, SFE, UTF, W\&W, E

Viguiera (see Heliomeris)

Virgulus (see Aster)

Virgulaster (see Aster)

Wyethia amplexicaulis (Nutt.) Nutt. - Dorn01, FNA, HDH, IMF, KTZ, SF!, UTF, W\&W. E/W Wyethia arizonica A. Gray - FNA, HDH, IMF, KTZ, SFW, UTF, W\&W, W

Snow, N, Junury 2009. Checklist of Vaccular Plants of the Southom Rocky Momain Region. CVersion 32 
- Wyethia X magna A, Nelson - ENDEMIC <CO, NM>, FCF, KTZ, SFW, W

[Wyethia amplexicaulis $X$ W. arizonica] - KTZ

[Wyethia magna A. Nelson ex W. A. Weber] - W\&W

Xanthisma

(Identification sources:

- Xanthisma coloradense (A. Gray) D. R. Morgan \& R. L. Hartm. - ENDEMIC CCO, WY>

[RH - with numerous new collecticens during the past 25 years, the two varieties appear to have merged]

[Aster coloeadoensis A. Gray] - FNA, HDH

[Haplopappus coloradoënsis (A. Gray) R. L. Hartm. ex Dorn] - RMC

[Machaefanthera coloradensis (A. Gray) Osterh.] - Domo1, IMF, SF!, EJ

[Machaefanthera coloradoensis (A. Gray) Ostorh. var: brandegoei (Rydb.) T. J. Watsont ex R. L. Hartm.] - K.TZ

[Machaeranthera coloradoẽnsis (A. Gray) Osterh. var. brandegei (Rydb.) T. J. Watson ex R. L. Hartm.] WE $W^{\prime}$

[Machaeranthera coloradoensis (A. Gray) Osverh. var, coloradoensis] - KTZ

[Machaeranthera coloradolnnsis (A. Gray) Ostorh, var. coloradoênsis] - WEW

Xanthisma gracile (Nutt.) D. R. Morgan \& R. L. Hartman - FNA

[Dieteria gracilis Nuts.] - FNA

[Haplopappus gracilis (Nutt.) A. Gray] - HDH, IMF, UTF

[Happlopappus ravenii R. C. Jackson] - FNA

[Machaeranthera gracilis (Nutt.) Shimers - KTZ. SFW, W\&W, W

Xanthisma grindelioides (Nutt.) D. R. Morgan \& R. L. Hartman var. grindelioides - FNA

[Eriocarpam grindelioides Nutt.] - FNA

[Haplopappus nuttallii (Torr.) A. Gray] - HDH

[Haplopappus nuttallii Torr. \& A. Gray var, nattallii] - FMC

[Machacranthera griedelioides (Nent) Shinners] - Dorn01, GPF, SFW, W\&W

[Machacrantherat griedelioides (Nunt.) Shinners var, grindel ioides] - IMF, KTZ, UTF, W

Xanthisma spinulosum (Pursh) D. R. Morgan \& R. L. Hartm, var. glaberrimum (Rydb.) D.R. Morgan \& R. L. Hartm. - FNA

[Sideranthus glaberrimus Rydb.] - FNA

[Huplopappus spinulosus (Pursh) DC: subsp. glabenrimus (Fydb.) H. M. Hall] - FNA

[Haplopappus spinulosus (Pursh) DC. var, glaberrimus (Rydb.) S. F. Blake] - FNA

[Macheranthera pinnatifida (Hook.) Shinners var, glaberrima (Rydb,) B, L- Tumer \& R. L. Hartm.] - Dorno1. FNA

[Xanthisma glaberrimum (Rydb.)] G. L. Nesom \& O'Kennon] - FNA

Xanthisma spinulosum (Pursh) D. R. Morgan \& R. L. Hurtm. var. spinulosum

[Haplopappus spinulosus (Pursh) DC, sulrap. eotala (Small) H. M. Hall] - FNA

[Haplopappus texensis R. C. Jachson] - FNA

[Machaeranthera 1exensis (R. C. Jackson) Shinners] - FNA

[Sideranthus turbinellus Rydb] - FNA

Xanthisma spinulosum (Pursh) D. R. Morgan \& R. L. Hartm. var. paradoxum (B. L. Turner \& R.

L. Hartm.) D. R. Morgan \& R. L. Hartm. - FNA

[Machaeranthera pinnatifida (Hook.) Shinners var. paradoxa B, L. Turner \& R. L. Hartm.] - FNA

[Haplopappus spinulosus (Pursh) DC. var, paradoxus (B, L. Tumer \& R. L. Hartm.) Cronq.] - FNA

* Xanthium spinosum L. - FNA, GPF, HDH, IMF, KTZ, UTF, E

[Acnnthoxanthium spinosum (L.) Fourr.] - SFE, WRW

* Xanthium strumarium L. - FNA. SF!. W\&W

[NS - numeroes additional synonymy in FNA 21: 19]

[Xanthium strumarium L., var, canadense (Mill.) Tom, \& A. Gray] - Dom01, GPF, IMF, KTZ, UTF, EW

Snow, N, Janury 2009. Checklist of Vaccular Plants of the Southom Rocky Momain Region. CVersion $3 x$ 
[Xanthium chilense Mill.] - $\mathrm{HDH}$

[Xanthium italicum Moretti] - HDH

[Xanthium pensylvanicum Wallr.] - HDH

Xanthocephalum (see Gutierrezia)

Ximenesia (see Verbesina)

Xylorhiza glabriuscula Nutt, - Dorn01, FNA, SFW, W\&W

[Xylorhiza glabriuscula Nutt. var. glabriuscula] - GPF, KTZ, UTF, W

[Aster xy lorrhiza Torr. \& A. Gray] - HDH

[Machaerunthera glabriuscula (Nutt.) Croncuist \& D. D. Kosk] - IMF

\{Xylorhiza glabriuscula Nutt, var, villosa (Nutt.) A. Nelson\} - KTZ

\{Machaseranhera glabriuscula (Nutt) Cronquist \& D. D. Keck\} - IMF

\{Xylorhiza glabriuscula Nutt.\} - W\&W

Xylorhiza venusta (M. E. Jones) A. Heller - FNA, KTZ, SFW, UTF, W\&W, W

[Aster venustus M. E. Jones] - HDH

[Machaeranthera venusta (M. E. Jones) Cronquist \& D. D. Keck] - [MF

Zinnia grandiflora Nutt, - FNA, GPF, HDH, IMF, KTZ, SFE, W\&W, E

\section{Balsaminaceac - BLS}

Impatiens capensis Meerb. - GPF, HDH, KTZ, SFE, W\&W, E

\section{Berheridaceae - BER}

Berberis fendleri A. Gray - FNA, HDH, KTZ, SF!, UTF, W\&W, E/W

Berberis fremontii Torr. - FNA, HDH

[Mahonia fremonii (Torr.) Fedde] - KTZ, SFW, UTR, W\& W. W

Berberis haematocarpa Wooton - FNA

[Mahonia haemarocarpa (Wooton) Fedde] - KTZ, SFE, W.EW, E

Berberis repens Lindl, - FNA, GPF, HDH

[Mahonia repens (LindL.) G. Don] - Dorn01, KTZ, SF!, UTF, W\&W, E/W

* Berberis vulgaris L. - FNA, HDH, KTZ,SFE, UTF, W\&W, E

Mahonia (see Berberis)

Betulaceae - BET (including Cotylaceae)

Alnus incana (L.) Moench var. occidentalis (Dippel) C. L. Hitche. - Dom01, E/W

[Alous incana (L.) Moench subsp, rugosa (Du Roi) R. T. Clausen var, occidentalis (Dippel) C. L. Hitche.]-UTF

[Alnus incana (L.) Moench subsp, tenuifolia (Nutt) Breitung] - FNA, KTZ, SFI, W\&W

[Alnus incana (L.) Moench subsp. tenuifolia (Nutt.) Breitung forma incisa (W. A. Weber) W. A. Weber]-SFW, WRW

[Alnas renu ifolia Nust.] - HDH

Betula X eastwoodiae Sarg. - FNA. KTZ

[Berala fontinalis X B. glandulosa]- WRW

Snow, N, January 2009. Checklist of Vacculer Plants of the Southom Rodky Momain Region. CVersion $3 x$ 
[Betula glandulosa X B. occidentalis] - FNA

[Betula mana X B. occidentalis] - KTZ

Betula glandulosa Michx. - Dom01, Hartman06, FNA, HDH, SF1, UTF, W\&W, E/W

[Betula nana L.] - KTZ

Betula oceidentalis Hook, - Dom01, FNA. GFC, GPF, HDH, KTZ, UTF, E/W

[Betula fontinalis Sarg.] - SF!, W\&W

Betula papyrifera Marshall var, papyrifera - GPF, KTZ, E

[Betula papyrifera Marshall] - Dom01, FNA, HDH, SFE, UTF, W\&W

* Betula pendula Roth - FNA, KTZ. UTF

[W] - an escape in Jefferson Ca.]

(Betula pumila L.) - FNA, err, in ed.; Dornot (also dismisses its occurrence in WY)

$\{$ WJ - not in Colorado\}

[Betula glandulosa Michx. var. glandulifera (Regel) Gleasoo) - GPF

[Betula pumila L. var, glandulifera Regel\} - KTZ

Betula X utahensis Britton - FNA. KTZ, SFE, UTF, W\&W fide formula, E

[Betula occidentalis X B. papyrifera] - KTZ

Corylus comuta Marshall var, comuta - KTZ, E

[Coryles comuta Marshall] - Dern01, GPF, HDH, SFE, WE. W

[Coryless comuta Marshall subsp. cornuta] - FNA

\section{Bignoniacene - BIG}

\% Campsis radicans (L.) Seem. ex Bureau - IMF, KTZ, E/W

[W] - widely cultivated but evidently never truly "escaping"]

[Campsis radicans (L.) Seem.] - GPF, SF!. UTF, W\& W

Catalpa speciosa (Warder) Warder ex Engelm, - KTZ $<\mathrm{KS}, \mathrm{NE}$, OK, UT>

[W] - probably onty rarely persisting as an escapos in our region]

[Caralpa speciosa Warder]-GPF, UTF

[Caralpa speciosa Warder ex Engeim.] - IMF

Boraginaceae - BOR (including Ehretiaceae and HYD)

\{Amsinckia douglasiana A. DC.\} - HDH, KTZ, W\&W, misid.

\{Amsinckia intermedia Fisch. \& C. A. Mey.\}-GPF, HDH, IMF, UTF, W\&W, misid.

(Amsinckla menziesii (Lehm.) A. Nelson \& Macbr, var, intermedia (Fisch. \& C. A. Mey.) Ganders - KTZ $\angle A Z$. NE, UT, Wr?

4 Amsinckia lycopsoides Lehm. Dorn01, GPF, KTZ, SFE, W\&W, E

4 Amsinckia menzicsii (Lehm.) A. Nelson \& J. F. Macbe. var. menziesii - KTZ, E

[Amsinckia menziesii (Lehm.) A. Nelson \& J, F. Macbe.] - IMF, SFE, UTF, WRW

[Amsinckia retrorsa Suksd.] - SFE, W\&W

[Amsinckia rugoss Rydb] - $\mathrm{HDH}$

* Anchusa azurea Mill. - KTZ, SFE, UTF, W\&W, E

* Anchusa officinalis L. - Dom01, HDH, IMF, KTZ, SFE, UTF, W\& W, E

* Asperugo procumbens L. - Dorn01, GPF, HDH, IMF, KTZ, SF!, UTF, W\&W, E/W

Buglossoides (see Lithospermum) 
Cryptantha

(Identification sources:

Cryptantha affinis (A. Gray) Greene - Dorn01, KTZ, HDH, W\&W'

[Krynitzkia affinis A. Gray] - UT3

Cryptantha ambigua (A. Gray) Greene - Dom01, HDH,IMF, KTZ, SFW. UTF, W\&W, W

$\uparrow \bullet$ Cryptantha aperta (Eastw.) Payson - ENDEMIC $<\mathrm{CO}>$, HDH, KTZ, W

[EXTINCT fide KTZ]

[Oreocarya aperta Eastw.] - SFW, W\&W

Cryptantha bakeri (Greene) Payson - HDH, IMF, KTZ, UTF, W

[Oreocarya bakeri Greene] - SFW, Ws W

Cryptantha breviflora (Osterh.) Payson -IMF, KTZ, UTF, W

[Oreocarya beviflord Ostert.] - SFW, W\&W

Cryptantha caespitosa (A, Nelson) Payson - Dorn01, HDH, exp., IMF, KTZ, UTF, W

[Oreocarya cacsphosa A. Nelson] - SFW, W\& W

Cryptantha cana (A. Nelson) Payson - Dorn01, GPF, HDH, KTZ, E

[Oreocarya cana A. Nelson]-SFE. W\&W

Cryptantha celosioides (Eastw) Payson - Dom01, GPF, IMF, KTZ, E

[Cryptantha bradburiana Payson] - $\mathrm{HDH}$

[Oreocarya celosioides Eastw.]-SFE, WRW

Cryptantha cinerea (Greene) Cronquist var, cinerea - IMF, KTZ, W

[Cryptantha cinerea (Torr, Cronquist var, cinerea] - UTF

[Croptantha jamesii (Torr, Payson var, jamesii] - HDH

[Krynitzkia mutiteaulis var. sctosa M. E. Jones] - UT3

[Oreocarya suffruticosa (Torr.) Greene] - SFW, W\& W'

Cryptantha cinerea (Greene) Cronquist var, jamesii Cronquist - Dorn01, IMF, KTZ, E/W

[Cryptantha cinerea (Torr.) Cronquist var, jamesii (Payson) Crooquist] - UTF

[Croptantha jamesii (A. Gray) Payson var, disticha (Eastwood) Paysco] - UT3

[Cryptantha jamesii (Torr, Payson] - GPF

[Cryptantha jamesii (Torr.) Pryson var, jamesii] - HDH

[Krynitzkia jamesii A. Gray] - UT3

[Oreocarya disticha Fastwood] -1/T3

[Oreocarya suffhuticosa (Torr.) Greene] - SFI, W\&W

Cryptantha cinerea (Greene) Cronquist var. pustulosa (Rydb.) L. C. Higgins - KTZ, E/W

[Cryptantha cinerea (Torr,) Cronguist var pustulosa (Rydb.) L. C. Higgins] - UTF

[Cryptantha jamesii (Torr,) Payson var, pustulosa (Rydb,) Harrington] - HDH

[Cryptantha pustulosa (Rydb,) Pryson] - IMF

[Oreocarya pestulosa Rydb,] - SF!, W\&W

Cryptantha circumscissa (Hook. \& Am.) I. M. Johnst. var circumscissa - HDH, KTZ, W

[Cryptantha circumsciss (Hook. \& Am.) 1. M. Johnst.] - Dora01, IMF, SFW, UTF, W\& W

\{Cryptantha confertifolia (Greene) Payson\}-IMF, KTZ, UTF <UT?

\{Cryptantha crassisepala (Torr. \& A. Gray) Greene var. crassisepala\}-KTZ,W\& W', E

\{Cryptantha crassisepala (Torr. \& A. Gray) Greene\} - GPF, HDH, SFE.

\{Cryptantha kelseyana Greene\} - W\&W

Cryptantha crassisepala (Torr. \& A. Gray) Greene var. elachantha 1. M. Johnst. - IMF, KIZ,

SFW, UTF, W\&W, W

[Cryptantha crassisepala (Torr. \& A. Gray) Greene] - HDH

Cryptantha elata (Eastw.) Payson - HDH, IMF, KTZ, UTF, W

[Oreocarya clata Eastw.]-SFW, W\&W

Cryptantha fendleri (A. Gray) Greene - Dorn01, GPF, HDH, IMF. KTZ, SF!, UTF. W\&W. E/W

Snow, N, Junury 2009. Checklist of Vaccular Plants of the Southom Rocky Momain Region. CVersion $3 x$ 
[Cryptantha pattersonii (A. Gray) Greene] - HDH

[Krynitzkia fendleri A. Gray] - UT3

Cryptantha flava (A. Nelson) Payson - Dorn01, HDH, IMF, KTZ, UTF, W

[Oreocarya flava A. Nelson] - SFW, W\&W

Cryptantha flavoculata (A. Nelson) Payson-Dom01, HDH, IMF, KTZ. UTF, W

[Oreocarya tlavoculata A. Nelson]-SFW, Wew

Cryptantha fulvocanescens (S. Watson) Payson var. fulvocanescens - KTZ <AZ, NM, UT>

[Cryptantha fulvocanescens (S. Watson) Paysan] - DMF, UTF

[Cryptantha fulvocanescens (A. Gray) Payson] - HDH

[Krynitzkia fulvocanescens A. Gray] - UT3

[Oreocarya fulwocanescens (A. Gray) Greene] - SFW, W\& W'

Cryptantha fulvocanescens (S. Watson) Payson var, nitida (Greene) Sivinski - KTZ, W

[Cryplantha fulvosancscens (A. Gray) Payson] - HDH

[Oreocarya fuhocanescens (A. Gray) Payson] - SFW

[Oreocarya nitida Greene] - W\& W

Cryptantha gracilis Ostert. - Dom01, HDH, IMF, KTZ, SFW, UTF, W\&W, W

- Cryptantha gypsophila Reveal \& C. R. Broome - Reveal06

Cryptantha humilis (A. Gray) Payson - KTZ, W

[Cryptantha bumilis (Grocte) Paysoe] - IMF

[Cryptantha humilis (Growe) Paysce var. conmixta (Macbride) L. C. Higgins] - UT3

[Cryplancha bumilis (Growe) Paysoe var, nsma (Easrw.) Payson] - UTF

[Cryptantha bumilis (Grove) var. shantzii (Tidestrome) L. C. Higgins - UT3

[Coyptantha nana (Eastw.) Payson var, nans] - $\mathrm{HDH}$

[Cryptancha nana (Easrw.) Payson var, ovina Payson] - UT3

[Oreocarya conmikta Macbride] - UT3

[Oreocarya dolocosa Macboride] - UT3

[Oreocarya humilis Greene subsp. nana (Eastw.) W. A. Weber]-SFW, W\& W

[Oreocarya shantzii Tidestrom] - UT3

Cryptantha kelseyana Greene - Dom01, HDH, IMF, KTZ, UTF

[Copptantha crassiscpala (Tor. \& A. Gray) Greene var, crassiscpala] - HDH, W\& W'

Cryptantha longiflora (A. Nelson) Payson - HDH, IMF, KTZ, UTF, W

[Oreocerna longiflora A. Nelson] - SFW, W\&W

Cryptantha mensana (M. E. Jones) Payson - IMF, KTZ, UTF, W

[Krynitzkia monsana M. E. Jones] - UT3

[Oreocarya mensana M. E. Jones] - SFW, WaW

Cryptantha minima Rydb. - Dom01, GPF, HDH, IMF, KTZ, SF!, W\&W, EW

Cryptantha osterhoutii (Payson) Payson - HDH, IMF, KTZ, UTF, W

[Oreocarya osterhouti Payson] - SFW, WRW

Crypsantha paradoxa (A. Nelson) Payson - HDH, IMF, KTZ, UTF, W

[Oreocarya paradosa A. Nelson] - SFW, W\&W

Cryptantha pterocarya (Torr.) Greene var. cycloptera (Greene) J. F. Macbe- - HDH, KTZ

Cryptantha pterocarya (Torr.) Greene var. pterocarya - HDH, IMF, KTZ, UTF, W

[Cryptantha pterocarya (Torr.) Greene] - SFW, W\&W

Cryptantha recurvata Coville - HDH, IMF, KTZ, SFW, UTF, W\&W, W

Cryptantha rollinsii I. M. Johnst. - Dorn01, IMF, KTZ, UTF, W

[Oreocarya rollinsii (I. M. Johnst) W. A. Weber] - SFW, W\&W

Cryptantha scoparia A. Nelson - Dom01, IMF, KTZ, SFW, UTF, W\&W, W

Cryptantha sericea (A. Gray) Payson - Dom01, IMF, KTZ, UTF, W

[Cryptantha sericea (A, Gray) Payson var, sericea] - HDH

[Krynitzkia sericea A. Gray] - UT3

[Oreocarya sericea (A. Gray) Greene] - SFW, WEW

Cryptantha stricta (Osterh.) Payson - Dorn01, HDH, IMF, KTZ, UTF, W 
[Oreocarya stricta Osterh_] - SFW, W\&W

Cryptantha thyrsiflora (Greene) Payson - Dom01, GPF, HDH, KTZ, E

[Oreocary a thyrsiflona Gineeno] - SFE, W\&W

Cryptantha tenuis (Eastw.) Payson - FCF

Cryptantha torreyana (A. Gray) Greene - Dorn01. W\&W', misid.

[Cryptantha calycosa (Gray) Rydb.] - KTZ

[Cryplantha eastwoodise St. John] - KTZ

[Copptantha flexuosa (A. Nels.) A. Nels.] - KTZ

[Cryptantha torreyana var. calycosa (Gray) Greene] - KTZ

[Cryptantha torreyena var. pumila (Heller) L. M. Johnst.] - KTZ

[Krynitzkia torreyana A. Grasy]- UT3

- Cryptantha virgata (Porter) Payson - ENDEMIC $\propto \mathrm{CO}, \mathrm{WY}>$, Dorn01, HDH, KTZ, E [Oreocarya virgata (Porter) Greene] - SFE, W\&W

Cryptantha watsonii (A. Gray) Greene - Dorn01, HDH, IMF, KTZ, SFW, UTF, W\&W, W

[Krynitzkia watsonii A. Gray]-UT3

- Cryptantha weberi I. M. Johnst. ENDEMIC $<\mathrm{CO}>$, HDH, KTZ, E/W

[Oreocarya weberi (L. M. Johnst.) W. A. Weber] - SF! W W W

*Cynoglossum officinale L, - Dom01, GPF, HDH, IMF, KTZ, SF!, UTF, W\&W, EN

* Echium vulgare L, - Dom01, GPF, HDH, IMF, KTZ, UTF, W\&W

Ellisia nyctelea (L.) L. - Dorn0I, IMF, KTZ, SF!, W\&W, E/W

[Ellisia nyctelea L.] - GPF, HDH

Eritrichium nanum (Vill.) Schrad, var, elongatum (Rydb,) Cronquist - Dorn01, IMF, UTF, E/W

[Eritrichiam nanum (Vili.) Schrad. var, argenteum (Wight) L. M. Johnst.] - IMF, not distinct

[Eritrichium naniam (Vill.) Schrad ex Gaudin yar, elongatum (Rydb.) Cronguist] - KTZ

[Eritrichium elongatum (Rydb.) W. F. Wight var. angenteum (Wighn I. M. Johnst.] - HDH

[Efiorichium elongatem (Rydb.) W. F. Wight var. elongatum] - HDH

[Eritrichiams nanum (Vill.) Schrad. ex Gaudin var. aretioides (Cham.) Herder] - KTZ

[Eritrichium nanum (Viil.) Schrad ex Gaudin var, argenteum (Wright) L. M. Johnston] - KTZ

[Eritrichum aretioides (Cham.) A. DC.] - SFt, W\& W

[Eritrichum nanum (Vill.) Schrad, ex Gaudin var, elongatum (Rydb.) Cronquist] - RMC

\section{Euploca (sec Heliotropium)}

Hackelia

(Identification sources:

Hackelia besseyi (Rydb.) J. L. Gentry - KTZ, SFE, W\&W, E

Hackelia deflexa (Wahlenb.) Opiz var. americana (A. Gray) Fernald \& I. M. Johnst. - Dorn01, GPF. KTZ

[Hackelia deflexa (Wahlenb.) Opiz var, americana (A. Gray) Fernald \& I. M. Johnst. ex Fernald] - RMC

[Hackelia deflexa (Wahlenb,) Opiz] - GPF, HDH, exp.

Hackelia floribunda (Lehm.) I. M. Johnst. - Dorn01, GPF, HDH, KTZ, SF!, UTF, W\&W, ENW

[Hackelia leptoplaylla (Rydb.) L. M. Johnst] - HOH, W\& W, et, rep.

- Hackelia gracilenta (Eastw.) L M. Johnst. - ENDEMIC $<\mathrm{CO}>, \mathrm{HDH}, \mathrm{KTZ}$, SFW, W \&W, W

Heckelia micrantha (Eastw.) J. L. Gentry - Dorn01, IMF, KTZ, SFW. UTF, W\&W. W

Hackelia patens (Nutt) I. M. Johnst. var. patens - Dom01, KTZ

Snow, N. Junury 2009. Checklist of Vascular Plants of the Southom Rocky Mounain Region. CVersion $3 x$ 
[Hackelia diffiasa var. caerulesoens (Rydb.) 1. M. Johnst.] - KTZ

Hackelia pinetorum (Greene ex Gray) I.M. Johnston - KWA, M\&H, KTZ

Hackelia ursina (Greene ex Gray) I.M. Johnston - M\&H, KWA, KTZ

Heliotropium convolvulaceum (Nutt) A. Gray var convolvulaceum - IMF, KTZ, E/W

[Euploca convolvulacea Nutt.] - SFE.

[Euploca coevolvulacea Nutt. subsp. californica (Greene) Abrams] - 5FW, W E W

[Euploca convolvulacea Nutt. subsp. convolvalacea] - W\&W

[Heliotropium convolvulaceum (Nutt) A. Gray] - GPF, HDH, UTF

Heliotropium curassavicum L. var. curassavicum - Dom01, M\&H, KWA

[Heliotropium xerophilum CockereI] - M\&H

\% Heliotropium curassavicum L, var. oculatum (A. Heller) I, M. Jolanst. - IMF, KTZ $<$ AZ, UT>, UTF, E/W

[Heliotropium curasavicum L. subsp. oculatum (A. Heller) Thome] - SF!, WEW

Heliotropium curassavicum L. var, obovatum DC, - GPF, IMF, KTZ, UTF

[Heliotropium spatulatum Rydb.] - HDH

Hesperochiron pumilus (Griseb.) Porter - Dorn01, IMF, UTF, W

[Hesperochiren pumilus (Douglas) Porter] - SFW, WAW

[Hesperochiron pumilus (Douglas ex Griseb.) Porter] - KTZ

Hydrophyllum capitatum Douglas ex Benth. var. capitatum - IMF, KTZ, UTF, E/W

[Hydrophyllum capitatum Douglas] - SF!

[Hydrophyllum capitatum Douglas ex Benth.] - Dorm01, HDH, W\&W

Hydrophyllum fendleri (A. Gray) A. Heller var, fendleri - IMF, KTZ, EN

[Hydrophyllum fendkri (A. Gray) A. Helikr) - Dorno1, HDH, SF!, UTF, W\& W

Lappula cenchrusoides A. Nelson - WY checklist, HDH, exp, KTZ <NE, WY>

* Lappula occidentalis (S. Watson) Greene var. cupulata (A. Gray) L. C. Higgins - KTZ, UTF,

$\mathrm{E} / \mathrm{W}$

[Lappola marginata (M. Bieb.) Gürke] - KTZ, SF!, W\&W

[Lappula redowskii (Hornem.) Girene var. cupalata (A. Gray) M. E. Jones] - Donn01, IMF

[Lappola redowskii (Hornem.) Greene var. desertorum (Greene) 1. M. Johnst.] - HDH, IMF

[Lappula texana (Scheele) Britton] - GPF, RMC

[Lappula texana (Scheele) Britron var. coronata (Girene) A. Nelson \& J. F. Macbs.] - HDH

[Lappola texana (Scheele) Britton var. texana] - HDH

Lappula occidentalis (S. Watson) Greene var. occidentalis - KTZ, UTF, EW

[Lappula redowskif (Hornem.) Gesene] - GPF. SF!, WEW

[Lippula redowskii (Hornem.) Greene var. redowskii] - Dorne1, HDH, IMF

* Lappula squarrosa (Retz.) Dumort. - IMF, KTZ, SF!, UTF, W\&W, E/W

[Lappula ech inata Gilib.] - GPF. HDH

[Lappula fremontii (Torr, Greene] - $\mathrm{HDH}$, exp

[Lappula squarrosa (Retz) Dum, var, erecta (A. Nelson) Dorn] - Dornt?

[Lappola squarrosan (Rete) Dum, var, sqgaarosa] - Dorne]

* Lithospermum arvense L. - Dorn01, GPF, HDH, IMF, UTF, E

[Buglossoides arvensis (L.) I. M. Johnst] - KTZ, SFE, W\&W

[Buglossoides arvense (L.) I. M. Johnst.] - W\&W'

Lithospermum caroliniense (Walter ex J. F. Gmel.) MacMill. var. croceum (Fernald) Cronquist KTZ, E

[Lithospermam canescens (Michx.) Lehm.] - HDH, exp, W\& W', misap.

[Lithospermum carolinense (Watter) MacMill.] - GPF

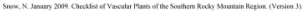


[Lithospennum caroliniense (Walter) MacMill.] - HDH

[Lithespermum croceums Femald] - SFE, W:W

Lithospermum cobrense Greene- M\&H, KWA, KTZ

Lithospermum incisum Lehm, - Dorn01, GPF, HDH. IMF, KTZ, SF!, UTF, W\&W, E/W

Lithospermum multiflorum Torr. ex A. Gray - Dom01, HDH, IMF, KTZ, UTF, W\&W. EN

[Lithespermum multiflorum Torr.] - SF!, UTF, W\& W'

Lithospermum ruderale Douglas ex Lehm. - Dorn01, HDH, IMF, KTZ, UTF, W\&W, W

[Lithospermum ruderale Douglas] - SFW

\{Lycopsis arvensis L. \} - HDH rep., W\&W, err. rep.

\{Anchusa arvensis (L.) M. Bieb.\} - KTZ $\approx$ WY

Mertensia alpina (Tort,) G. Don - HDH, IMF, KTZ, SFE, W\&W, E

Mertensia arizonica Greene - Dorn01, IMF, KTZ, UTF, W

[Mertensia arinonica Greene var. grahamii L. O. Williams] - SFW, W \& W

Mertensia brevistyla S. Watson - Dom01, HDH, IMF, KTZ, SF!,UTF, W\&W, EW

Mertensia ciliata (E, James ex Torr.) G. Don var. ciliata - KTZ, E/W

[Mertensia ciliata (E. James) G. Don] - HDH, SF], UTF

[Mertensia ciliasa (E. James ex Torr.) G. Don] - Domo1, W\&W

[Mertensia ciliata (Torr) G. Don] - GPF

[Mertensia ciliata (Torr.) G. Don var. ciliasa] - IMF

Mertensia franciseana A. Heller - HDH, IMF, KTZ, SF!, UTF, W\&W, E/W

Mertensia fusiformis Greene-Dom0I, HDH, IMF, UTF, W\&W, E/W

[Mertensia brevistyla S. Wats.] - SF!, W\& w $^{1}$

[Mertensia oblongifotia (Nutt) G. Don] - KTZ

Mertensia humilis Rydb, - Dorn01, HDH, KTZ, SFE, W\&W, E

Mertensia lanceolata (Pursh) A. DC - Dorn01, SF!, EN

[Mertensia bakeri Greene var, bakeri] - $\mathrm{HDH}$

[Mertensia bakeri Greene var, osterhoutii L. O. Will lams] - HDH

[Mertensia lanceolaca (Pursh) A. DC. var, beachyloba (Greene) A. Nelson] - GPF, HDH. KTZ

[Mertensia lanceolaca (Pursh) A. DC. var, corisces (A. Nelson) L. C. Higgins \& S. L. Welsh] - KTZ, UTF

[Mertensia lanceolata (Pursh) DC, var, fendleri A. Gray] - $\mathrm{HDH}$

[Mertensia lanceolaca (Pursh) DC, var, lanceolata] - GPF, HDH, KTZ, W\&W

[Mertensia lanceolaca (Pursh) DC, yar, pubeas (L.F. Mactor, L.O. Williams] - HDH

[Mertensia lanceolaca (Pursh) DC. var. secundorum Cockerell] - HDH, KTZ

[Mertensia viridis A. Nelson var, cynoglossoides (Greene) J. F. Mactor.] - HDH

Mertensia oblongifolia (Nutt.) G. Don - GPF, IMF, KTZ, SFW, UTF, W\&W, W

[Mertensia oblongifolia (Nutt.) G. Don]- KTZ.

[Mertensia oblongifolia (Nutt.) G. Don var. nevadensis (A. Nelson) L. O. Williams] - HDH

Mertensia viridis (A. Nelson) A. Nelson - Dorn01, E/W

[Mertensia lanceolasa (Pursh) DC.] - SF!

[Mertensia lanceolara (Pursh) DC, var, viridis A. Nelson] - WoW

[Mertensia lanceolara (Pursh) DC, var, nivalis (S. Watson) L. C. Higgins] - UTF

[Mertensia oblongifolia (Nunt.) G. Don] - KTZ

[Mertensia viridis A. Nelscon] - IMF

[Mertensia viridis A. Nelson var, cava (Rydb.) L. O, Williams] - HDH

[Mertensia viridis A. Nelson vae, dilatata (A. Nelson) L, O. Williams] - HDH

[Mertensia viridis A. Nelson var, parvifolia L. O, Wiltiams] - HDH

[Mertensia viridis A. Nelson var, viridis] - HDH

Myosotis alpestris F. W. Schmidt - Dorn01, HDH, W

[Myosotis asiatica (Vesterg.) Schischk, \& Serg.] - KTZ, SFW, W\&W

Snow, N, January 2009. Checklist of Vacculer Plants of the Southom Rocky Momain Region. CVersion $3 x$ 
* Myosotis scorpioides L, - Dorn01, GPF, IMF, KTZ, SF!, UTF, W\&W, E/W

Nama densum Lemmon var, parviflorum (Greenm,) C. L. Hitchc, - Dorn01, IMF, KTZ, UTF, W [Nama densam Lemmon] - SFW, W\& W

Nama dichotomum (Ruiz \& Pay.) Choisy - HDH rep., KTZ, SFW, W\&W, W

Nama hispidum A. Gray -FCF, GPF, HDH rep., IMF, KTZ, SFW, UTF, W\&W, W\&W', err. rep., W

Nama retrorsum J. T. Howell - IMF, KTZ <AZ, NM, UT>, UTF, W\&W'

Nemophila breviflora A. Gray - Dorn01, HDH, TMF, KTZ, SF!, UTF, W\&W, E/W

Neomphila menziesii H. \& A. - Dorn01

Onosmodium molle Michx, var, occidentale (Mack.) I. M. Johnst. - Dorn01, GPF, HDH, E [Onusmodium bejariense A. DC. var. oceidentale (Mack.) B. L. Turner] - KTZ

[Onosmodium molle Michx. sabsp, occidentale (Mack.) Cochrane]- KTZ, SFE, W\&W

\section{Oreocarya (see Cryptantha)}

Pectocarya penicillata (H, \& A.), A. DC, - Dorn01, KTZ

$[$ C. noglossum penicillarum $\mathrm{H}$ \& \& A.] $-\mathrm{KTZ}$

[Pectocarya linearis var. penicillatum (H. \& A.) M. E. Jorss] - KTZ

Phacelia

(Identification sources:

Phacelia alba Rydb, - Dorn01, IMF, KTZ, SF!, UTF, W\&W, EW

[Phacelia ncomexicana Thurb. ex. Torr.] - W\& $w^{1}$, misapp.

[Phacelia ncomexicana Thurb. ex Torf, yar, alba (Rydb.) Brand] - HDH

Phacelia bakeri (Brand) J. F. Macbr, - KTZ, SF!, W\&W, E/W

Phacelia caerulea Greene - KWA

[Phacelia intermedla Woot, p.p.]-KTZ

Phacelia constancei N. D. Atwood - IMF, KTZ, SFW, UTF, W\&W, W

Phacelia crenulata Torr, ex S. Watson - IMF, W

[Phacelia corrugata A. Nelson] - HDH

[Phacelia crenuiata Tort.] - SFW, W\&W

[Phacelia crenulata Torr, var, corngata (A. Nels.) Brand] - UTF

[Phacelia crenulata Torf, ex S. Wasson var, corrugata (A. Nets.) Brand] - KTZ

Phacelia demissa A. Gray var. demissa - HDH, KTZ, UTF, W

[Phacelia demissa A. Gray] - Dom01, IMF, SFW, W\&W

Phacelia denticulata Osterh. - Dorn01, HDH, KTZ, SFE, W\&W, E

- Phacelia formosula Osterh, - ENDEMIC $<C O>$, HDH, KTZ, SFE, W\&W, E

Phacelia glandulifera Piper - Dom01, KTZ

[Phacelia ivesiana var, glandulifera (Piger) A. Nelson \& J. F, Machr,] - KTZ

Phacelia glandulosa Nutt. var. glandulosa - KTZ

[Phscelia glandulosa Nutt. var. deserta (A. Nels.) Brand] - KTZ

[NS - var, deserta Endemic to WY if recognized]

Phacelia hastata Dougles ex Lehm. var. hastata - UTF, IMF, KTZ, E/W

[Phacelia hastata Douglas] - SF!

[Phacelia hastata Douglas ex Lehm.] - Dornol, WEW 
[Phacelia hastata Douglas ex L.ehm, var, alpina (Rydh.) Crooq.] - IMF

[Phacelia hastata Douglas ex Lehm. var. leocophylla (Torr.) Crenquist] - GPF

[Phacelia leucaphylla Torr.] - HDH, syn. of P. heterophylla var. heterophylla

Phacelia heterophylla Pursh var, heterophylla - HDH, IME, E/W

[Phacelia heterophylla Pursh] - GPF, SF!, UTF, W\&W

[Phacelia heterophylla Pursh subsp. heterophy lla] - KTZ

Phacelia heterophylla Pursh var, virgata (Greene) Dorn - Dorn01

[Phacelia heterophylla Pursh subsp. virgata (Greene) Heckard] - KTZ

Phacelia incana Brand - Dorn01, IMF, KTZ, SFW, UTF, W\&W, W

Phacelia integrifolia Torr, var, integrifolia - GPF, HDH rep., KTZ, W

[Phacelia integrifolia Torr.] - IMF, SFW, UTF, W\&W

Phacelia jvesiana Torr, var, ivesiana - HDH, KTZ, UTF, W

[Phacelia ivesiana Torr.] - Dum01, IMF, SFW, W\&W

Phacelia linearis (Pursh) Holz. - Dorn01, KTZ

\{Phacelia Jutea (Hook. \& Am.) J. T. Howell var. scopulina (A. Nelson) Crong. ? - IMF,

UTF $<\mathrm{CO}$.

Phacelia neomexicana Thurb, ex Torr, var, alba (Rydb.) Brand - WY checklist

[Phacelia neomexicana Thurb. var. pseudoarizonica (Brand) J. Voss] - KTZ

Phacelia salina (A. Nels, J. T. Howell - Dorn01, KTZ

Phacelia scopulina (A. Nelson) J. T. Howell var- scopulina - KTZ

Phacelia scopulina (A. Nelson) J. T. Howell var. submutica (J. T. Howell) Halse - KTZ, W

[Phasclia lutea (Hook, \& Arn.) ). T. Howell var, sabnutica (J. T. Howell) Croneuist] - IMF

[Phacelia subnutica J. T, Howell] - HDH, SFW, W\&W

Phacelia sericea (Graham ex Hook.) A. Gray var, ciliosa Rydb. - Dorn01, E/W

[Phacelia sericca (Giraham) A. Gray]-SFE

[Phacelia sericed (Graham) A. Gay subsp. ciliosa (Rydb.) J. M. Gillem] - HDH, KTZ, SFW, WE W

[Phacelia sericea (Grahtam) A. Gray var, ciliosa Rydb.] - IMF, UTF

[Phacelia kdahoensis L. F. Hend.]-HDH, W\& W, misapp.

Phacelia sericea (Graham ex Hook.) A. Gray var, sericea - Dorn0I, E/W

[Phacolia scricea (Grahtsm) A. Gray] - HDH, SF!

[Phacelia stricea (Grahtam) A. Gray subsp. sericea] - KTZ, W\& W

[Phacelia serisca (Grahum) A. Gray var, serioes] - ]MF, UTF

- Phacelia splendens Eastw. - ENDEMIC <CO, NM>, HDH, KTZ, SFW, UTF, W\&W, W

Phacelia tetramera Howell - Dorn01

Plagiobothrys leptocladus (Greene) I. M. Johnst. - Dorn01, K'TZ.

[Allocarya leptocladus Greene Greene] - KT2

[Plagiobochrys orthocarpus (Greene) I. M. Johnst.] - KTZ

Plagiobothrys scouleri (Hook. \& Am.) I. M. Johnst. var. hispidulus (Greene) Dorn - Dom01, KTZ, E/W

[Plagiobochrys nelsonit (Greene) I, M. Johnst] - HDH

[Plagiobochrys secpulorum (Greene) I. M. Johnst.] - HDH

[Plagiobochrys scculeri (Hook, \& Am.) L. M. Johnst.] - GPF

[Plagiobochrys sccoleri (Hook, \& Am.) L. M. Johnst subsp penicillata (Greene) A. Love) - SFI, W. W

[Plagiobochrys scculeri (Hook, \& Am.) I. M. Johest. var, peaicillatus (Greene) Cronquist] - IMF, UTF

* Symphytum oflicinale L. - KTZ, SF!, UTF, W\&W, E/W

Tiquilia nuttallii (Hook.) A. T. Richardson - Dorn01, IMF, KTZ, SFW, UTF, W

[Tiquilia nuttallii (Benth.) A. T. Richardson] - W\&W

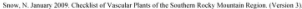




\section{Brassicaceae - BRA}

(Identification sources:

Aethionema (see Aurinia)

*Alliaria petiolata (M, Bieb.) Cavara \& Grande - GPF, FCF, KTZ, SFE, UTF, W\&W, E

Alyssum (see also Aurinia)

* Alyssum alyssoides (L.) L, - Dorn01, KTZ, FCF, SF!, UTF, W\&W', ENW

[Alyssam alyssioides L.] - GPF, HDH, w\&W

* Alyssum desertorum Stapf - Dom01, GPF, SF!, UTF, W\&W, EW

[Alyssum desertorum Stapf var. desertorum] - KTZ, FCF

* Alyssum murale Waldst, \& Kit. - KTZ, FCF, SFE, W\&W, E

Alyssum parviflorum M. Bieb, var. micranthum (C. A. Mey.) Dorn - Dom01, W\&W , E/W

[Alyssam minus (L.) Rothm.] - UTF

[Alyssam mines (L.) Rothm, var. micranthum (C. A. Mey.) Dudley] - GPF, KTZ, FCF

[Alyssum parviflorum M. Bicb.] - SF!, W\&W

[Alyssam parviflorum Fisch. ex M. Bieb, yar, micranthum (C. A. Mey.) Dom]-Dornol

Arabidopsis (see Thellungiella)

Arabis (see also Boechera, Turritis)

(Identification sources:

Arabis hirsuta (L.) Scop. var. glabrata Torr. \& A. Gray - HDH Dom01, exp., KTZ FCF, UTF, W\&W, W\&W $W^{\prime}, E / W$

[Arabis hirsuta (1.) Scop.] - SF!

Arabis hirsuta (I.) Scop. var, hirsuta - KTZ, FCF, UTF, W\&W', err, rep.

[Arabis hirsuta (L.) Scop.] - SF!

Arabis hirsuta (L.) Scop, var, pyenocarpa (M. Hopkins) Rollins - Dom01, GPF, HDH, KTZ, FCF, W\&W, E/W

[Arabis hirsuta (L.) Scop.] - SF!

[Arabis hirsuta (L.) Scop. var, glabrata Torr. \& A. Gray] - UTF

\{Arabis laevigata (Muhl. ex Willd.) Poir, var. laevigata\}-KTZ, FCF

\{Arabis hastata Eaton\} - Rollins 93

\{Arabis heteropbylla Nutt ex Torrey \& A. Gray\} - Rollins93

\{Arabis lacvigata (Muht.) Poif.\} - GPF

(Arabis laevigata var. heteroplyylla (Nutt. ex Torry \& A. Giray) Farw.\} - Rollias93

\{Arabis lyracfolia DC.; - Rollins93

\{Turnitis lacvigara Muhlenb. ex Willd.) - Rollins93

* Armoracia rusticana P. Gaertn. B. Mey, \& Scherb. - Dorn01, GPF, KTZ, FCF, W\&W

[Amoracia rusticana Gaertn.] - UTF

[Rorippa anmoracia (L.) Hitchc.] - HDH

* Aurinia saxatilis (L.) Desy, - KTZ <UT>, FCF, E

[Aethionema sacatile (L.) R. Br.] - SFE, W\&W

[Alyssum saxatile L] - UTF 
* Barbarea orthoceras Ledeb, - Dom01, GPF, HDH, KTZ, FCF, SF!, UTF, W\&W, E/W

\{Barbarea verna (Mill.) Asch.; - M\&H, exp.. HDH rep., KTZ. W\&W

* Barbarea vulgaris R. Br.-Dorn01, GPF, FCF, SF!, UTF, W\&W, E/W

[Bartarea vulgaris W.T. Aiton] - KTZ

* Berteroa incana (L.) DC, - Dom01, GPF, KTZ, FCF, SFI, UTF, W\&W, E/W

Boechera

(Identification sources:

Bochera brachycarpa (Torr, \& A. Gray) Dom - Dom01

Boechera cobrensis (M.E. Jones) Dorn - Dorn01

[Arabis cobrensis M. E. Jones] - KTZ

Boechera consanguinea (Greene) Windham \& Al-Shehbaz - Windham07a

[Arabis holboclii Horemann yar. consanguines (Greene) G. A. Mulligan] - Windham07a

- Boechera ctandallii (B. L. Rob.) W. A. Weber - ENDEMIC <CO, WY>, Dom01, SFW, W\&W

[Arabis crandallii B. L. Rob.] - HDH, KTZ, FCF, RMC, W

Boechera demissa (Greene) W. A. Weber

[NS - Follose ing UT3 we do not recognize infraspecific taxa for our negion]

[Arabis demissa Greene] - UTF, UT3

[Arabis demissa Greene va, demissa] - $\mathrm{HDH}, \mathrm{KTZ}, \mathrm{FCF}, \mathrm{EW}$

[Arabis demissa Greene var. languida Rollins] - $\mathrm{HDH}$, exp.. KTZ, FCF, EW

[Bocchera fendleri (Wats.) W. A. Weber var., languida (Rollins) Dorn] - Dom01

[Bocchera axylobula (Givene) W. A. Weber]-SFI, W\& W

Boechera divaricarpa (A. Nelson) A. Löve \& D. Löve - SF!, W\&W, Windham07b

IArabis X divaricarpa A. Nelson - KTZ, FCF, EW

[Arabis drummondis $X$ A. holbocilii] - KTZ

[Arabis divaricarpa A. Nelson] - GPF

[Arabis divaricarpa A. Nelson var. divaricarpa] - HDH

[Arabis holboellii Homem.] - UTF

[Arabis stokesiac Rydb.] - Windham07b

Boechera drepanoloba (Greene) Windham \& Al-Shehbaz - Windham07a

[Arabis lemmonii S. Watson sar. drepanoloba (Greeno) Rollins] - Windham07a

Bocchera drummondii (A. Gray) A. Lôve \& D. Löve - SF!, W\&W

[Arabis confinis S. Watsen var, continis] - EW

[Arabis drummondil A. Gray] - GPF, HDH, KTZ, FCF, UTF, EW

Boechera duchesnensis (Rollins) Windham \& Al-Shehbaz \& Allphin - Windham07b

[Arabis pulchra M. E. Jones ex S. Watson var, pallens M. E. Joeses] - HDH, KTZ, FCF, W

[Arabis pulchra M. E. Jones var. pallens M. E. Jones] - UTF

[Boechera pulchra (M. E. Jones) W. A, Weber] - SFW

[Boechera pulchra (M. E. Jones ex S, Watson) W. A. Weber sulosp. pallens (M. E. Jones) W. A. Weher] WRW

[Bocebera pukchra (M. E, Jones ex S, Watson) var, duchesencens (fRoll ins) Dorn - Windhaen07b

[Boechera pulchra (M. E. Jones ex S, Wans.) W, A. Weber var, pallens (M. E. Jones) Dorn]- Dorn0]

Boechera fendleri (S. Watson) W. A. Weber var. fendleri - Dorn01

[Bocehera fendleri (S, Watson) W. A. Weber subsp, fendleri] - W\&W

[Arabis fendleri (S, Warson) Greene var, fendleri]-GPF, HDH, KTZ, FCF, EW

[Arabis helboellii Hornem, var, fendleri S, Watson] - UTF

[Bocchera fendleri (S. Watson) W, A. Weber] - SFI

Bocchera fernaldiana (Rollins) W. A. Weber var. fendleriana - W\&W

Snow, N, Junury 2009. Checklist of Vaccular Plants of the Southom Rocky Momain Region. CVersion $3 x$ 
[Arabis fernalutiana Rellins var. femaldiana] - HDH, KTZ, FCF, W

[Arahis fernaldiana Rollins] - UTF

[Boechera fermaldiana (Rollins) W. A. Weber] - SFW. W\&W'

Boechera fernaldiana (Rollins) W. A. Weber subsp. vivariensis (S. L. Welsh) Windham \& AlShehbaz - Windham06

[Boechera vivariensis (S. L. Welsh) W. A. Weber] - Windham06

Boechera formosa (Greene) Windham \& Al-Shehbaz - Windham06

[Arabis formosa Greene] - Windham06

[Arabis pulchra M. E. Jones ex S. Watson var. pallens M. E. Jones] - HDH, KTZ, FCF, W, Windham06

[Boeshera pulchra (M. E. Jones ex S. Watson) W. A. Weber subsp. pallens (M. E. Jones) W. A. Weber] WRW

[Boechera pulchra (M. E. Jones ex S. Watson) W. A. Weber var. pallens (M. E. Jones) Dorn] - Windham06

Boechera glareosa Dom - Dom03

Boechera gracilenta (Greene) Windham \& Al-Shehbaz - Windham07b

[Arabis gracilenta Greene] - Windhamo7b

[Arabis selhyi]-HDH, KTZ, FCE, UTF, W, Windhan07b

[Bocchera selbyi (Rydb.) W. A. Weber] - Dom01, SFW, W\&W, Windhan07b

Boechera grahamii (Lehmann) Windham \& Al-Shehbaz - Windham07b

Boechera gunnisoniana (Rollins) W. A. Weber - SFW, W\&W

Arsobis gunnisoniana Rollins - HDH, KTZ, FCF, W

[Bocethera glareosa Dom] - Al-Shebbaz03

Boechera holboellii (Hornem.) Löve \& Love var, collinsii (Fern.) Dorn - Dom01

[Arabis holboellii Hornem. var. collinsii (Fem.) Roll.] - WY checklist.

[Arabis bourgovii Rydb.]-KTZ

[Arabis collinsii Fern.] - KTZ

[Arabis dacotica Greene] - KTZ

[Arabis divaricarpa var. dacotica (Greene) Boivin] - KTZ

[Arabis retrofracta sar. eollinsii (Fem.) Boivin] - KTZ

[Boechera collinsii (Fem.) A. \& D. Lowe] - KTZ

Boechera holboellii (Hornem.) var, pinetorum (Tidestr.) Dorn - Dorn01

[Arabis holboellii Hornem, var, pinctorum (Tidestr.) Rollins] - GPF, HDH, KTZ, FCF, UTF, E'W

[Arabis pinetorum Tideste.] - Dom01

[Bocshera retrofracsa (Grahan) A. Lōve \& D. Love] - SFI, W\&W

Bocchera holboellii (Hornem.) var. secunda (Howell) Dorn - Dorn01

[Arabis holboellii Hornem. var. secunda (Howell) Jeps.] - UTF, E.W

[Arabis holboellii Homem, vac, retroffacta (Graham) Rydb.] - HDH, KTZ, FCF

[Arabis secunda Howel]] - Dorno1

[Boeshera retrotracta (Graham) A. Löve \& D. Lôve]-SFI, W\& W

Boechera lemmonii (S. Watson) Weber var, drepanoloba (Greene) Dorn - Dorn0I

[Arabis lemmonii S. Watson var. drepanoloba (Greene) Rollins] - KTZ, FCF, EW

[Bocchera lemmoni (S, Watson) W. A. Weber] - SF!

Boechera lemmonii (S. Watson) Weber var- lemmonii - Dornd)!

[Arabis lemmonii S. Watson vac. Iemmonii] - HDH, KTZ, FCF, UTF, EW

[Boechera lemmonii (S, Watson) W, A. Weber]-SF, W\&W

Bocchera lignifera (A. Nelson) W. A. Weber-Dorn01. SF!, W\&W, Windham06

[Arabis lignifera A, Nelson] - HDH, KTZ, FCF, EW

[Arabis holboellii Hornem, var, pinetorum (Tidestr, Rollins] - UTF

Bocchera lyallii (S. Watson) Dorn - A1-Shehbez03, Dorn01, Windham06

[Arabis Iyallii Wats, vat, lyallii] - KTZ

Bocchera microphylla (Nutt.) Dorn var, macounii (Wats.) Dorn - Dorn01

[Arabis microphylla Nutt, var, macounii (Wats.) Roll] - WY checklist

[Arabis macounii Wats.] - KTZ

Boechera microphylla (Nutt.) Dorn var. microphylla - Dom01

[Arabis microphyila Nutt var, microphylla] - WY checklist 
[Arabis tenuicula Greene] - KTZ

Bochera nuttallii (Robins.) Dorn - Dorn01

Arabis nuttallii Robins.] - WY cheeklist

[Arabis bridgeri M.E. Jones] - KTZ

[Arabis macella Piper] - KTZ

- Boechera oxylobula (Greene) W. A. Weber- ENDEMIC $<C O>$, SFl, W\&W, Al-Shehbaz03,

Windham06

[Arabis aprica Osteth. ex A. Nels.] - Windham06

[Arabis oxylobula Greene]-HDH, KTZ, FCF, E/W

[Arabis demissa Greene] - Windhamo6

[Arabis rugocarpa Oster.] - Windliamos

- Boechera pallidifolia (Rollins) W. A. Weber-ENDFMIC $<C O>$, SFW, W\&W'.

A]-Shehbaz03

[Arabis pallidifolia Rollins]- KTZ, FCF, W, Windhamo6

[Arabis thompsonii S, L. Welsh] - Windham06

[Booshera thompsoaii (S. L. Welsh) N. H. Holmgen] - Windhans06

Boechera pendulina (Greene) W. A. Weber - Windham06

[Arabis demissa Greene]-UTF

[Arabis demissa Greene var, fusseola Rollins] - HDH

[Arabis pendulina Greene var, pendulina] - KTZ, FCF, EW

[Arabis pendulina Greene var, nusseola (Rollins) Rollins] - KTZ, FCF, EN

[Bocebera oxylobula (Greete) W. A. Weber]-SFI, W\& W

[Boechera pendulina (Greene) Weber var, russeola (Rollins) Dort] - Don01

Boechera pendulocarpa (A. Nels.) Windham \& AL-Shehbaz - Windham(66

[Arabis pendulocarpa A. Nels.]-RMC, Windham06

[Arabis holboellii Hornem, var, pendulocarpa (A. Nels, Rollins] - Windhan06, HDH, KTZ, FCF, UTF, EAW

[Bocehera pendulocarpa (A. Nels.) A. Love \& D. Love var, pendulocarpa (A. Nels.) N. Snow] - Windhamos

Bocchera perennans (S. Watson) W. A. Weber - Dom01, SFW, W\&W

[Arabis perennans S, Watson] - HDH, KTZ, FCF, UTF, W

[Arabis perennans S. Watson var, perennans] - UT3

Bocchera retrofracta (Graham) A. Love \& D. Löve - SF!, W\&W, Windham06

[Arabis exilis (A. Nels.)] - Windhame6

[Arabis retrofracta Graham] - Windhamo6

[Arabis helboellii Hornem, var retrofracta (Graham) Rydb.] - HDH, KTZ, FCF Windham06

[Boechera exilis (A. Nels.) Dorn] - Dom01

[Turritis retrfracta (Graham) Hook.] - Windhamole

- Boechera spatifolia (Rydb.) Windham \& Al-Shehbaz - ENDEMIC $<$ NM, CO $>$ - Windham06

[Arahis fendleri (S. Watson) Greene var. spatifolia (Rydb.) Rollins] - GPF, HDH, KTZ, FCF, E/W

[Arahis holboellii Homem. var. Fendleri S, Watson] - UTF

[Boechera fendleri (S. Watson) W. A. Weher] - SF!

[Boechera fendleri (S. Watson) W. A. Weher suhsp. spatifolia (Rydb.) W. A. Weber]- W\& W

[Boechera fendleri (S. Watson) W. A. Weber var, spatifolia (Rydb.) Doon] - Dorn0!

- Boechera villosa Windham \& Al-Shehbaz - ENDEMIIC $<$ NM $>$ - Windham06

Boechera williamsii (Roll.) Dom var. saximontana (Rollins) Dorn - Dorn01

[Árahis williamsii Rall. var. saximontana (Roll.) Roll.] - WY checklist, KTZ

[Arabis microphylla var. saximontara (Roll.) Roll.] - KTZ

[Arabis pendulocarpa var. saximentana (Rodl_) Dorm] - KTZ

Brassica (see also Sinapis)

* Brassica elongata Ehrh, var, integrifolia (Boiss.) Breistr, - SFW, W\&W ${ }^{1}$, W

[Brassica elongata Ehrh.] - KTZ

[Brassica elongata Ehrh. subsp. integrifolia (Bloiss.) Breistr.] - FCF

* Brassica juncea (L.) Carn, - Dom01, GPF, KTZ, FCF, UTF, E 
[Brassica juncea (L.) Cosson] - HDH, SFE, W\&W

* Brassica napus L. - SF!, W\&W , E/W

* Brassica nigra (L.) W. D. J, Koch - GPF, HDH, KTZ, FCF, SFE, UTF,W\&W, E

* Brassica rapa L. - Dorn01, FCF, W\&W, W\&W', misapp.

[Brassica campestris L.] - GPF, HDH, UTF

[Brassica rapa L. var. rapa] - KTZ

Braya glabella Richardson - Dorn01, KTZ, FCF, E/W

[Braya glabella R. Br.]-SF!

[Braya glabella (Richardson) S. Watson var- glabella] - W \& W

Braya humilis (C. A. Mey.) B. L. Rob, - Dorn01, HDH (mentioned without name, p. 256),

KTZ. FCF, SFE. W\&W, E/W

[Braya humilis (C. A. Mey.) B. L. Rob. subsp. wentosa Rollins] - SFW

* Camelina microcarpa Andrz, ex DC, - Dorn01, GPF, FCF, UTF, W\&W, E/W

[Camelina microcarpa DC.] - KTZ

[Camelina microcarpa Andrz.] - HDH, SF!

* Camelina rumelica Velen, - KTZ, FCF, SF1, W\&W, EW

* Camelina sativa (L.) Crantz - Dorn01, GPF, HDH, exp, KTZ, FCF, W\&W, er, rep.

* Capsella bursa-pastoris (L.) Medik, - Dorn01, GPF, HDH, KTZ, FCF, SF!, UTF, W\&W, E/W

Cardamine

(Identification sources:

Cardamine breweri S. Watson var, breweri - KTZ, FCF, UTF, E/W

[Cardamine breweri S. Walson] - Dorno1, SFI, WeW

Cardamine breweri S. Watson var. leibergii (Holz.) C. L. Hitche. - KTZ, FCF, E/W

[Cardamine breweri S. Walson var bewweri]-RMC

[Cardamine breweri S. Watson]-SF!, W\&W

[Cardatnine vallicola Greene]- HDH

Cardamine cordifolia A. Gray var. cordifolia - KTZ, FCF, UTF, E/W

[Cardamine cordifolia A. Gray]- HDH, SFI, W\& W

Cardamine cordifolia A. Gray var, incana A. Gray ex M. E. Jones - KTZ, Hartman06, FCF, E/W

[Cardamine cordifolia A. Gray]-HDH. SF!, W\&W

[Cardamine cordifolia A. Gray var, incana M. E. Jones] - W\&W'

Cardamine oligosperma Nutt, var. oligosperma - KTZ

[Cardamine oligosperma Nutt] - Donw01. W\&W. err. rep.

[Cardamine oligosperma Nutt. ex Torr. \& A. Gray] - UTF

[Candamine oligosperma Nutt, ex Torr, \& A, Gray var, oligosperma] - FCF, W\& W'

[Cardamine umbellata Greene] - $\mathrm{HDH}$

[Cardanine unijuga Rydb.] - HDH, exp.

Cardamine pensylvaniea Muhl. ex Willd. - Dom01, HDH, KTZ, FCF, UTF, W\&W, E/W

[Cardanine pensylvanica Muhl.] - GPF, SF!

\section{Cardaria (sce also Lepidium)}

[NS - All of our species in Cardarla may ultimately be transfered to Lepidium]

s. Cardaria chalepensis (L.) Hand.-Mazz. - Dom01, GPF, KTZ. FCF, SF!, UTF, W\&W. E/W

* Cardaria draba (L.) Desv. - Dom01, GPF, HDH, KTZ, FCF, SF!, UTF, W\&W, E/W

Snow, N, Janury 2009. Checklist of Vaccular Plants of the Southom Rocky Momain Region. CVersion $3 x$ 
* Cardaria pubeseens (C, A. Mey, Jarm, - Dom01, GPF, KTZ, FCF, SF!, W\&W, E/W

[Cardaria pubesecins (C. A. Mey.) Janm. var- elongata Rollins] - UTF

[Cartaria pubeseens (C. A. Mey.) Rollins var. elongata Rollins] - HDH

Caulanthus crassicaulis (Torr.) S. Watson var, crassicaulis - KTZ, FCF, UTF, W

[Caulanthus crassicaulis (Torr.) S. Watson] - Dorn01, HDH, SFW, WEW

* Chorispora tenella (Pall.) DC, - Dorn01, GPF, KTZ, FCF, SF!, UTF, W\&W, E/W

[Chorispora tenella DC.] - HDH

* Conringia orientalis (L.) Dumort, - Dorn01, GPF, HDH, KTZ, FCF, SF!, UTF, W\&W, EW

Descurainia

(Identification sources: )

Descurainia brevisliqua (Detling) Al-Shehbaz \& Goodson - Goodson07

[Descurainia obtusa [Greene) O. E. Shulz var, brevisiliqua Detling] - Goodsen07

Descurainia californica (A. Gray) O, E, Schulz-Dorn01, HDH, KTZ, FCF, SF!, UTF, W\&W, E/W

- Descurainia kenbeilii Al-Shehbaz - Al-Shebaz07a, ENDEMIC <CO $>$

Descurainia incana (Bemh, ex Fisch, \& C. A. Mey,) Dorn var, incana - Dom01, E/W

[Descuraiaia incana (Bemah.) Dorn] - SF:

[Descuraiaia incuna (Bemh. ex Fisct. \& C. A. Mey.) Dom] - FCF, W \&W

[Descuraiaia incana (Berah. ex Fisch. \& C. A. Mey.) Dom subsp, incenna] - KTZ

[Descurainia tichandsonili (Sweet) O. E. Schulz] - GPF

Descurainia incana (Bernh. ex Fisch. \& C. A. Mey.) Dorn var, incisa - E/W

[Descuraiaia incana (Benth.) Dorn] - SF!

[Descurainia incana (Bernh, ex Fiseh, \& C, A. Mey,) Dom] - W\&W

[Descurainia incana (Bernh, ex Fisch, \& C, A. Mey.) Dom subsp, incisa (Engelm, Kartesz \& Gandhi] - HDH, KTZ

[Descuraiaia incisa (Engelm.) Beitton subsp. incisa] - SF!

[Descuraiaia incisa (Engelm. ex A. Gray) Britton subsp, incisa] - FCF, WR W'

[Descurainia richandsoenii (Sweet) O. E. Schulz] - GPF

[Descuraiaia richardscenii (Sweet) O. E. Schulz subsp, incisa (Engelm.) Detling] - HDH

[Descuraiaia richard sonii (Sweet) O. E. Schulz var-sonnei (B. L. Rob.) C. L. Hitche,] - UTF

Descurainia incana (Bemh. ex Fisch. \& C. A. Mey.) Dorn var. macrosperma (O. E. Schulz) Dom

- Dorn01, EWW

[Descuraiaia incana (Benah.) Dorn] - SF1

[Descuraiaia incisa (Bemb, ex Fisch, \& C. A. Mey.t Dom] - FCF, SFW, W\&W

[Descuraiaia incana (Bertan, ex Fisch. \& C. A. Mey.) Dom var, brevipes (Nutt) S. Welsh - UT3

[Descurainia incana (Bernh, ex Fisch, \& C. A. Mey.) Dom subsp, procera (Greene) Kartesz \& Gandhi] - KTZ

[Descurainia richandsonii (Sweet) O, E. Schulz] - GPF

[Descuraiaia richardscoii (Sweet) O. E. Schulz subsp, richardsonii] - HDH

[Descuraiaia richardsonii (Sweet) O. E. Schulz subsp, procera (Greene) Detling] - HDH

[Descurainia richardsonii (Sweet) O, E. Schulz var, brevipes (Nutt) S. L. Welsh \& Reveal] - UTF

Descurainia incana (Bemh. ex Fisch. \& C. A. Mey.) Dorn var. major (Hook.) Dorn - Dorn01, EN

[Descurainia incana (Bernh.) Dorn] - SF?

[Descuraiaia incana (Benah, ex Fisch, \& C., A. Mey.) Dom] - FCF, W\&W

[Descuraiaia incana (Benah, ex Fisch, \& C. A. Mey, Dom subsp, incana] -KTZ

[Descurainia richandsenii (Sweet) O. E. Schulz] - GPF 
Descurainia incana (Bemh, ex Fisch. \& C. A, Mey,) Dorn var, viseosa (Rydb,) Dom - Dorn01, EN

[Descurainia incana (Benah. ex Fisch. \& C. A. Mey.) Dom] - W\&W

[Descurainia incana (Bemh. ex Fisch, \& C. A. Mey.) Dom subsp. viscesa (Rydb.) Kartesz \& Gondhi] - KTZ, FCF

[Descurainia incisa (Engelm.) Britton subsp. viscosa (Rydb.) Rollins - SF!

[Descurainia incisa (Engelm. ex $\lambda$. Gray) Britton subsp. viscosa (Rydb.) Rollins - FCF, W\&W

[Descurainia richandsonii (Sweet) O. E. Schulz] - GPF

[Descurainia richardsenii (5weet) O. E. Schul2 subsp. viscosa (Rydb.) Detling] - HDH

[Descurainia richardsonii (Sweet) O. E. Schulz var. viseosa (Rydb.) M. Peck] - UTF

Descurainia nelsonii (Rydb,) Al-Shehbaz \& Goodson - Goodson0?

[Descurainia brachycarpa (Richardson) O. E. Shulz var, nelsonii (Rydb.) O. E. Schulz in Engler] - Goudson07

[Descurainia pinnata (Walter) Britton] - W\&W

[Descurainia pinnata (Walter) Britton subsp, nelsonii (Ryob.) Detling] - HDH, KTZ, FCF, SF!, W\& W'

[Descurainia pinata (Walter) Britton var. nelsonii (Rydb.) M. Peck] - Dom01, UTF, EW

[Sophis nelsonii Rydb.] - Goodson07

Descurainia obtusa (Greene) O.E.Schulz subsp, adenophora (Woot. \& Standl.) Detling - KWA. M\&H, KTZ

[Sophia adenophora Wooc. \& Standl.] - M\&H

Descurainia obtusa (Greene) O.E, Schulz subsp, obtusa - KWA, M\&H, FCF

[Descurainia obtusa (Greene) O. E. Schul2] - HDH, rep.

[Descurainia obtusa stubsp. Lypica Detling] - KTZ

[Sophia obrusa Greene]-M\&H

Descurainia pinnata (Walter) Britton var. beachycarpa (Richardson) Fernald - Dom01

[Descurainia pinnata (Walter) Britton] - W\&W

[Descuraiaia pinnata (Walter) Britton subsp. brakchycarpa (Richardson) Detling] - GPF, HDH, KTZ, FCF. W\&W

Descurainia pinnata (Walter) Britton var, filipes (A. Gray) M. Peck - Dom01, UTF, E/W

[Descurainia incisa (Engelm.) Britton subsp. filipes (A. Gray) Rollins] - SF!

[Descurainia incisa (Engelm. ex A. Gray) Britton subsp. filipes (A. Gray) Rollins] -FCF, W\& W ${ }^{3}$

[Descurainia pinnata (Walter) Britton] - W\&W

[Descurainia pineata (Walter) Britton subsp. filipes (A. Gray) Detling] - HDH. KTZ

Descurainia pinnata (Walter) Btitton var, intermedia (Rydb.) C. L. Hitchc. - Dorn0I, UTF, E/W [Descurainia pinnata (Walter) Britton] - W\&W

[Descurainia pineasa (Walcer) Beitton subsp, interened la (Rydb, Detlinel - GPF, HDH, KTZ, FCF, SFI, W\&W'

Descurainia pinnata var. ochroleuca (Woot.) Shinners - KWA, M\&H

[Descurainia pinnata subsp, ochroleusa (Woot.) Detling] - KTZ, M\&H

[Sophia ochroleuca Woot.]-KTZ

Descurainia pinnata (Walter) Britton var, osmiarum (Cockerell) Shinners - Dorn0l, UTF, E/W

[Descurainia pinnata (Walter) Britton] - W\& W

[Descuraiaia pinnata (Walter) Britton subsp. halictonum (Cockerell) Detling] - GPF, HDH, KTZ, FCF, SF!. WR $W^{\prime}$

Descurainia pinnata (Walter) Britton var, paysonii (Detling) S. L. Welsh \& Reveal - Dom01, UTF

[Descuraiaia hecisa (Engelm. ex A. Gray) Briton subsp، paysonii (Detling) Rollins] - FCF

[Descuraiaia pinnata (Walter) Britton] - W\&W

[Descuraiaia pinnana (Walter) Beitton subsp, paysonii Detling] - HDH, exp.. KTZ

- Descurainia ramosissima Rollins - ENDEMIC $<C O>, K T Z$. FCF, SFE, W\&W. E

- Descurainia torulosa Rollins - ENDEMIC <WY $>$, KTZ, Dorn01

* Descurainia sophia (L.) Webb ex Prant] - Dom01, HDH, KTZ, UTF, W\&W, E/W

[Descurainia sophia (L.) Webb] - GPF, FCT, SF!, W\&W'

- Dimorphocarpa wislizeni (Engelm.) Rollins - KTZ, FCF, SFW, W\&W, W

Snow, N, January 2009. Checklist of Vecculer Plants of the Southum Rocky Momain Regioe. CVersion $3 x$ 
[Dithyrea wislizenii Engelm.] - HDH rep., UTF

* Diplotaxis muralis (L.) A, DC - KTZ, FCF, SFW, W\&W, W

[Diplotaxis muralis A. DC.] - UTF

\section{Dithyrea (see Dimorphocarpa)}

Draba

(Identification sources:

Draba albertina Greene - KTZ, FCF, SF!, W\&W, EW

[Draba crassifolia R. Grah.] - HDH

[Draba steneloba Ledeb. var, naria (O. E. Schulz) C. L. Hitche.] - HDH

Draba aurea Vahl ex Homem. - Dorn01, KTZ, FCF, W\&W, E/W

[Draba aurea Vahl] - GPF. HDH. SF!, UTF

Draba borealis DC, - Dom01, KTZ, FCF, SF!, W\&W, EW

Draba cana Rydb, - Dom01, W\&W, E/W

[Draba breweri S. Watson var. cama [Rydb.) Rollins] - KTZ, FCF, SF!, W\&W'

[Draba lanceolata Foyle]-GPF, HDH, UTF

Draba crassa Rydb, - HDH, KTZ, FCF, SF!, UTF, W\&W, E/W

Draba crassifolia Graham var, crassifolia - Dorn01, HDH, E/W

[Draba crassifolia Graham] - KTZ, FCF, SF!, UTF, WEW

[Draba sctnoloba Ledeb.] - $\mathrm{HDH}$

Draba cuneifolia Nutt. ex Torr. \& A. Gray var, cuneifolia - HDH, KTZ, FCF, UTF, E/W

[Draba cuncifolia Nutt.] - GPF, SF1

[Draba cunsifotia Nutt ex Torr. \& A. Gray] - W\&W.

[Draba cuncifolia NutL var. cuncifolia] - GPF

- Draba exunguiculata (O. E. Schulz) C. L. Hitche, - ENDF.MIC $<$ CO $>$, HDH, KTZ, FCF, SF!, W\&W, E/W

\{Draba fladnizensis Wulf. var, fladnizensis\} - KTZ, FCF, out of range

Draba fladnizensis Wulf. var. pattersonit (O. E. Schulz) Rollins - KTZ, FCF, SF!, W\& W', E/W [Draha fladnizensis Wulf] - HDH, UTF, W\&W

Draba globosa Payson - Dom01, KTZ, FCF, SF!, W\&W, E/W

[Draba apiculata C. L. Hitchc.] - KTZ, RMC

[Draba densifolia Nutt, ex Torr, \& A. Gray var, apiculata (C. L. Hitche,) S. L. Welsb] - UTF

[Draba densifolia Nutt, ex Torr. \&. A. Gray var, globosa (Payson) S. Welsh] - UT3

- Draba graminea Greene - ENDEMIC $<\mathrm{CO}>$, HDH, KTZ, FCF, SF!, W\&W, E/W

- Draba grayana (Rydb.) C. L. Hitche. - ENDEMIC $<$ CO, NM $>$. Hartman06, HDH, KTZ, FCF, SF!, W\&W, E/W

Draba helleriana Greene - Al-Shehbaz07b. HDH. SF!, W\&W

[Draba aurea Vahl ex Hornemann var. stylosa $A$. Gray] - Al-Shehbaz07b

[Draba helleriana Gireene var. helleriana] - KTZ, FCF, EJW, Snow07b

[Draba helleriana Gireene var. leiocarpa O. E. Schulz in Engler] - Al-Shehhazo7b

[Draha helleriana Gireene var. patens (Heller) O. E. Schalz] - Snow07b, KWA. MM, M\&H, KTZ

[Draba helleriana Gireene var. neomexicana (Greene) O. E. Schulz in Fingler] - Al-Shehban07b

[Draha helleriana Greene var. patens (A. Heller) O. E. Schulz in Engler] - A 1-Shehbaz07b

[Draha helleriana Gircene var. pinetorum (Greene) $O$. E. Schulz in Eingler] - Al-Shehbarojb

[Draba neomexicana Greene] - Al-Shehbaz07b

[Draha neomexicana Greene var, rohusta A. Heller] - Al-Sbehbaz07h

[Draha pallida A. Heller] - Al-Shehbaro?h 
[Draba patens $A$. Heller] = Al-Shehhaz07b

[Draba pinetorum Greene] - A]-Shehbsal0? b

Draba incerta Payson - Dorn01, SFW, UTF, W\&W, W

[Draba incerta Payson var, incerta] - KTZ, FCF

Draba juniperina Dorn - Dorn01

Draba lonchocarpa Rydb, var lonchocarpa - KTZ, FCF, UTF, E/W

[Draba lonchocarpa Rydb.| - Dom01, SF!, W\&W

[Draba lonchosarpa. Rydb. var, exigua Scbulz] - UTF

[Draba nivalis Liljeblad var, exigua (O. E. Schule) C. L. Hitchc.] - HDH

Draba macounii O. E. Schulz - KTZ, FCF

- Draba malpighiacea Windham \& Al-Shebaz - Al-Shehbaz07b

$\div$ Draba nemorosa L, var, nemorosa - E/W

[Draba nemonosa L.] - Dom01, GPF, HDH, KTZ, FCF, SF!, UTF, W\& W

Draha oligosperma Hook. var. juniperina (Dorn) Welsh - WY checklist, KTZ

Draba oligosperma Hook, var, oligosperma - UTF, E/W

[Draba olligosperna Hook] - Donx1, HDH, KTZ, FCF, SFL, wa w

Draba porsildii Mulligan var- porsildii - Dorn01, KTZ, FCF, E/W

[Draba porsildii Mulligan] - SFL, W\&W

Draba rectifructa C. L. Hitche, - HDH, KTZ, FCF, SF!, UTF, W\&W, E/W

Draba reptans (Lam.) Fernald - Dorn01, KTZ, FCF, SF!, W\&W, E/W

[Draba reptans (Lam.) Fernald var. reptans] - GPF, HDH, UTF

[Draba reptans (Lam.) Fernald var. micrantha (Nutr.) Fermald] - GPF

[Draba foptans (Lam.) Fernald var. stellifera $(\mathrm{O}$. E. Schulz) C. L. Hitche.] - UTF

[Draba reptans (L.am.) Fernald forma micrantha (Nurt. C. L. Hitche.] - HDH

[Draha reptans (Lam.) Fernald forma stellifera (O, E. Schulz) C. L. Hitehe] - HDH

- Draba smithii Gilg ex O. E. Schultz - ENDEMIC <CO>, HDH, KTZ. FCF, W\&W, E [Drabn smithii Gilg] - SFE

Draba spectabilis Greenc - SF!, W\&W. Al-Shehbaz07b

[Draba oxyloba Greene] - Al-Shehbazozb

[Draba spectabilis Greene var, bella O, E. Schulz in Engler] - Al-Shehbaz07b

[Draba spectabilis Greene var, oxylebe (Greene) Gilg \& O. E. Schulz] - Dorne1, HDH, FCF, EN, AIShehbazi) $7 \mathrm{~b}$

[Draba spectabilis Greene var, oxyleba (Greene) Gilg \& O. E. Schulz ex O. E. Schulz] - KTZ

[Draba spectpbilis Greene var, purpesii Gilg \& O. E. Schulz in Engler] - Al-Shehbozi)?

[Draba spectabilis Greene var, spectabilis] - HDH, KTZ. FCF, UTF, EW

\{Draba stenoloba Ledeb_\} - GPF <CO>, KTZ, FCF, UTF

\{GPE likely referring to Draha albertina;

- Draba streptobrachia Price - ENDEMIC $<\mathrm{CO}>$, KTZ, FCF, SF!, W\&W. EW

[Draha spectahilis Greene var, dasycarpa (O. E. Schulz) C. L. Hitchc-] - HDH

Draba streptocarpa A. Gray - Dorn01, KTZ, FCF, SF!, W\&W, E/W

[Draha streptosarpa A. Gray var, streptocarpa] - HDH

[Draha streptocarpa A. Grily var. tonsa (Wooton \& Standl.) O. E. Schulz] - HDH

Draba ventosa A. Gray - Dorn01, HDH, exp.. KTZ. FCF, SFW, W\&W, W

[Draba ventosa A. Gray var, ventosa] - UTF

- Draba weberi Price \& Rollins - ENDEMIC $<C O>$, KTZ, FCF, SFW, W\&W, W

Enucastrum gallicum (Willd.) O. E. Schulz - Dorn01

[Brassica erucastrum L.] = KTZ

* [Enca vesicaria (L.) Cav, subsp. sativa (Mill.) Thell.\} - KTZ <AZ, KS, NE, NM, UT>, FCF

\{Eruca sativa Mill.\} - GPF, MDH, rcp.

\{Eruca vesicaria (L) Cav. var. sativa (Mill.) Thell.; - UTF

Snow, N. Janury 2009. Checklist of Vascular Plants of tho Southom Rocky Momain Region. CVersion 31 
Erysimum asperum (Nutt.) DC, - GPF, HDH, FCF, SF!, UTF, W\&W, E/W

[Erysimum asperum (Nutt.) DC. var. asperum] - Durn01

[Erysimum asperum (Nutt.) DC. var. arkansanum (Nutt) A. Gray] - Dorn01

[Erysimum capitatum (Douglas ex Heok.) Greene var. capitatum]-KTZ

Erysimum capitatum (Douglas ex Hook.) Greene var. argillosum (Greene) R.J. Davis - E/W

[Erysimum asperum (Nutt.) DC.] - UTF

[Erysimum capitatum (Douglas) Greene] - SFl, W\& W

[Erysimum capitatum (Douglas ex Hook.) Greene var. purshii (T. Durand) Rollia] - FCF, KTZ, W\& W ${ }^{1}$

Erysimum capitatum (Douglas ex Hook.) Greene var, capitatum - KTZ, FCF, E/W

[Erysimum asperum (Nutt) DC.] - UTF

[Erysimum capitatum (Douglas) Greene] - GPF, SF!, W\& W

[Erysimum nivale (Greene) Rydb.] - HDH

[Erysimtum capitatum (Douglas) Greene var, capitatum] - W\& $W^{\prime}$

[Erysimum wheckri Rothr.] - HDH

* Erysimum cheiranthoides L. subsp, altum Ahti - Dom01. SF!. W\&W, E W

[Erysimum cheiranthoides L.] - GPF, HDH, KTZ, FCF, UTF

Erysimum inconspicuum (S. Watson) MacMill - Dom01, GPF, HDH, SF!, UTF, W\&W, E/W

[Erysimum inconspicuum (S. Watson) MacMill, var, inconspicuum] - KTZ, FCF

*Erysimum repandum L. - Dom01, GPF, HDH, KTZ, FCF, SF!, UTF, W\&W, EN

* Euclidium syriacum (L.) R. Br,-Dorn01, FCF, SFW, UTF, W\&W, W

[Euclidium syriacum (L.) W. T. Aiton] - KTZ

- Eutrema penlandii Rollins - ENDFMIC $<\mathrm{CO}>$, FCF, HDH2, KTZ, E/W

[NS - Al-Shehbaz fpers, comm, 2004! indicates this taxon moy nod transfer to Thellangiella: however, the combinsaion is not yet available in that genas]

[Eutrema edwardsii R. Br. subsp. penlandii (Rollins) W. A. Webcr] - SFE, W\&W

[Eutrema edwardsii R, Br, subsp. penlandil Rollins] - SFW

Halimolobos virgata (Nutt.) O. E. Schulz - Dorn01, HDH, KTZ, SF!, UTF, W\& W, E/W

[Halimolobos virgata (Nutt, ex Torr. \& A. Gray) O. E. Schulz] - FCF

Hesperidanthus (see Thelypodiopsis)

* Hesperis matronalis L. - Dorn01, GPF, HDH, KTZ, FCF, SF!, UTF, W\&W, E/W

Hutchinsia procumbens (L.) Desv, - Dom01, HDH, KTZ, FCF, UTF, W

[Hymenolobus procumbens (L.) Nutt.] - SFW

[Hymenolobus procumbens (L.) Nutt, ex Torr. \& A. Gray] - W\&W

Hymenolobus (see Hutchinsia)

* Iberis amara L. - KTZ, FCF, SFE, W\&W,E

* Isatis tinctoria L. - Dom01, KTZ, FCF, SFW, UTF, W\&W, W

Koniga (see Lobularia)

Lepidium

Snow, N. Junury 2009. Checklist of Vascular Plants of the Southom Rocky Mounain Region. CVersion $3 x$ 
(Identification sources:

Lepidium alyssoides A. Gray var, alyssoides - KTZ, FCF, SFW, EW

[Lepidium alyssoides A. Gray]-SFE, W\& W

[Lepidium montanum Nutr.] - $\mathrm{HDH}$

[Lepidium montanum Nutt. subsp. alyssoides (A. Gray) C. L. Hitche.] - W \& W

[Lepidium montanum Nutt. var. alyssoides (A. Gray) M. E. Jones] - Dorn01

Lepidium alyssoides A. Gray var, eastwoodiae (Wooton) Rollins - KTZ, FCF, SFW, W

[Lepidium alyssuides A. Gray]

[Lepidium montanum Nutt.] - HDH

[Lepidium montanum Nutt. var. eastwoodiae (Wooton) C. L. Hitche.] - W \& W'

* Lepidium campestre (L.) R. Br, - Dorm01, GPF, HDH, FCF, UTF, E/W

[Lepidium campestre (L.) W. T. Aiton] - KTZ

[Neolepia campestre (L.) W. A. Weber] - SF?, W\&W

Lepidium crenatum (Greene) Rydb, - KTZ, FCF, SFW, W\&W', W

[Lepidium alyssoides A. Gray var. alyssoides] - KTZ, FCF

[Lepidium alyssoides A. Gray var. eastwoodiae (Wooton) Rollins] - KTZ FCF

[Lepidium montanum Nuti.] - $\mathrm{HDH}$

[Lepidium montanum Nutr. var. spatulatum (B. L. Rob.) C. L. Hicchc.] - UTF. sensu UT material, w\& W $^{1}$

千 Lepidium densiflorum Schrad, var. densiflorum - Dorn01, HDH, KTZ, FCF, UTF, E/W

[Lepidium densiflorum Schrad.] - GPF, SF!, W\&W

[Lepidium montanum Nutt. var, spatulatum (B. L. Rob.) C. L. Hitchc.] - W\&W

Lepidium densiflorum Schrad, var. macrocarpum Mulligan - Dorn01, KTZ, FCF, E/W

[Lepidium densiflorum Schrad.]-SF!, W\&W

[Lepidium densiflorum Schrad. var. densiflorum] - UTF

Lepidium densiflorum Schrad. var. ramosum (A. Nels.) Thell. - Dom01, HDH, KTZ, FCF, UTF, EJW

[Lepidium densiflorum Schrad.] - SFI, W\&W

Lepidium integrifolium Nutt. var. heterophyllum S. Watson - KTZ, W

[Lepidium integrifolium Nutt, ex Torr. \& A. Gray var. heterophyllum S. Watson] - FCF

[Lepidium montanum Nutt.] - Dom01, HDH, SFW

[Lepidium montanum Nutt. var, heterophyllum (S. Watson) C. L. Hitebe.] - UTF

[Lepidium montanum Nut1. var, spatulatum (B. L. Rob.) C. L. Hitchc.] - W\&W

Lepidium lasiocarpum Nutt, var, lasiocarpum - HDH, KTZ, W

[Lepidium lasiocarpum Nutt.] - SFW, W\& W

[Lepidium lasiocarpum Nurt. ex Torr. \& A. Gray var. lasiocarpum] - FCF, UTF

* Lepidium latifolium L. - Dom01, GPF, HDH, KTZ, FCF, UTF, E/W

[Cardaria latifolia (L.) Spach] - SF!, WEW

$\bullet$ Lepidium montanum Nutt. var. coloradense Rollins - ENDEMIC $<\mathrm{CO}>$, KTZ, SFW, $W \& W^{\prime}$.

W

[Lepidium montanum Nutt, ex Torr, \& A. Gray var, coloradense Rollins] - FCF

Lepidium montanum Nutt. var. jonesit (Rydb.) C. L. Hitche. - KTZ, SFW, UTF, W\&W, W

[Lepidium montanum Nutt.] - HDH

[Lepidium montanum Nutt. ex Torr. \& A. Gray var, jonesii (Rydb.) C. L. Hitcho.] - FCF

Lepidium montanum Nutt. var. montanum - Dorn01, KTZ. SFW. UTF, W\&W'. W

[Lepidium montanam Nurt.] - HDH

[Lepidium montanum Nurt. subsp, montanum] - Wis W

[Lepidium montanum Nutt. ex Torr. \& A. Gray var, montanum] - FCF

- Lepidium montanum Nutt. var. tenellum (L. O. Williams) C. L. Hitche. - ENDEMIC $<\mathrm{CO}>$.

KTZ. SFW, W\&W. W

[Lepidium montanum Nutt. ex Torr. \& A. Gray var. tenellum (L. O. Williams) C. L Hitche.] - FCF

Snow, N. Junury 2009. Checklist of Vascular Plants of the Southom Rocky Mounain Region. CVersion $3 x$ 
Lepidium montanum Nutt, var, wyomingense (C. L. Hitche, ) C. L. Hitchc, - KTZ, SFW, W\&W', W

[Lepidium montanum Nutt. ex Torr. \& A. Giray var. myoungensis (C. L. Hitche) C. L. Hitche.] - FCF

\{Lepidium paysonii Rollins\} - KTZ $<W Y>$. FCF, W\&W', label err?

* Lepidium perfoliatum L. - Dorn01, GPF, HDH, KTZ, FCF, SF!, UTF, W\&W, E/W

* Lepidium ramosissimum A. Nelson var. bourgeauanum (Thell.) Rollins - Dom01, FCF.

Hartman 06, SFW. EJW

[Lepidium bourgeasanum Thell.] - W\&W

[Lepidium densiflorum Schrad.] - GPF

[Lepidium densillorum Schrad. var. beurgeauanum (Thell.) C. L. Hitche.] - HDH

[Lepidium densiflonum Schrad, var. densiflorum] - UTF

[Lepidium ramosissimum $A$. Nelson] - SFE, W\&W

- Lepidium ramosissimum A. Nelson var, divergens (Osterh.) Rollins - ENDEMIC $<\mathrm{CO}>$.

KTZ, FCF, SFW, E/W

Lepidium ramosissimum A. Nelson var, ramosissimum - Dorn01, KTZ, FCF, E/W

[Lepidium densiflorum Sctsad. var. ramosum (A. Nelson) Thell.] - WaW

[Lepidium ramosissimum A. Nelson] - GPF, HDH, SFt, W\& W

* Lepidium sativum L, - Dom01, GPF, KTZ, FCF, UTF, W\&W

$\div$ Lepidium strietum (S. Watson) Rattan - KTZ, FCF, SFW, UTF, W\&W, W

$\div$ Lepidium virginicum L. var, medium (Greene) C. L. Hitchc. - Dotn01, HDH, KTZ, E/W

[Lepidlum virginisum L.] - GPF. SFI, W\&W

[Lepidium virginisum L. var, virginicum] - W\& W'

\% Lepidium virginicum L. var, pubeseens (Greene) Thell. - Dorn0I, KTZ, FCF, UTF, E/W

[Lepidium virginicum L.] - GPF, SF!, WEW

[Lepidium virzinicum L. var, pubescens (Greene) C. L. Hitchc.]- HDH

[Lepidium virginicam L. var, virginicum] - W\& W'

Lesquerella

(Identification sources:

\section{)}

[NS - OKane and Al-Shehbaz placed all Lesqwerelio isto Phovoria apart from the auriculate-leaved species of the southenst, which will go into Prgsania. I prefer seeing published phylogenies before making the transfer.]

Lesquerella alpina (Nutt.) S. Watson var. alpina - KTZ, UTF, E/W

[Lesquerella alpina (Nutt.) S. Wasson] - Dorn01, GPF. HDH

[Lesquerella alpira (Nutt.) S, Watson subsp, alpina] - SF]

[Lesquenella alpina (Nutt, ex Torr. \& A. Gray) S. Watson subsp. alpina] - W \& W

[Lesquenella alpina (Nutt, ex Tort, \&. A. Gray) var, alpina] - FCF

Lesquerella arenosa (Richardson) Rydb. var. arenosa - Dorn01

[Lesquerella ludoviciana var, arencesa (Richandson) S. Wats.] - KTZ

Lesquerella arenosa (Richardson) Rydb. var. argillosa Rollins \& E. A. Shaw - Dorn01. GPF.

KTZ, FCF, W\&W, E

[Lesquerella arencsa (Richardson) Rydb.] - SFE

- Lesquerella calcicola Rollins - ENDEMIC $<C O, N M>, H D H, K T Z, F C F, S F E, W \& W, E$

Lesquerella condensata A. Nelson-Dorn01, KTZ, FCF

[Lesquerella alpina (Nutt,) S. Wasson var, condensata (A. Nelson) C. L. Hitche.] - UTF

- Lesquerella congesta Rollins - ENDEMIC $\langle$ CO $>$, KTZ, FCF. SFW, W\&W, W

Lesquerella fendleri (A. Gray) S. Watson-GPF, HDH, KTZ, FCF. SFI, UTF, W\&W, E/W

\{Lesquerella intermedia (S. Watson) A. Heller\} - HDH, KTZ, FCF. UTF, W\&W

Lesquerella ludoviciana (Nutt.) S.Wats.-Dorn01. GPF, HDH, KTZ, FCF, SF!, W\&W, E/W

Snow, N. Junury 2009. Checklist of Vascular Plants of the Southom Rocky Mounain Region. CVersion $3 x$ 
- Lesquerella macrocarpa A. Nelson - ENDEMIC $<W Y>$, Dom01, KTZ

Lesquerella montana (A. Gray) S, Wats, - Dom01, GPF, HDH, KTZ, FCF, SFE, W\&W, E

Lesquerella ovalifolia Rydb. ex Britton var. ovalifolia - E

[Lesquerella ovalifolis Rydb.] - HDH, SFE, W\&W

[Lesquerella ovalifolis Rydb, subsp. ovalifolia] - GPF, FCF

[Lesquerella ovalifolis Rydb. ex Britoon sabsp, ovalifolia] - KTZ

- Lesquerella parviflora Rollins - ENDEMIC $<C O>$, KTZ, FCF, SFW, W\&W, W

Lesquerella parvula Greene - Dorn01, KTZ, FCF, W\&W', E/W

[NS - see O'Kane06 for distriubcion details]

[Lesquerella alpira (Nutt.) S. Wanson] - HDH

[Lesquerella alpira (Nutt.) S. Wasson sabsp. parvula (Greene) Rollins \& E. A. Shaw] - SF1.

[Lesquerella alpina (Nutt, ex Tor, \&. A, Gray) S. Watson subsp. parvula (Greene) Rollins \&

E. A. Shaw] - W\&W

[Lesquerella alpina (Nutt.) S. Wanson var. parvula (S. Watson) S. L. Welsh \& Reveall - UTF

Lesquerella rectipes Wooton \& Standl. - HDH, KTZ, FCF, SFW, UTF, W\&W, W

Lesquerella subumbellata Rollins - HDH, KTZ. FCF, UTF, W\&W', W

[Lesquerelta alpina (Nutt.) S. Wasson sabsp. alpina] - SFW

[Lesquerella alpina (Nutt. ex Torr. \& A. Gray) S. Watson subsp. alpina] - W \& W

- Lesquerella vicina Anderson, Reveal, \& Rollins - ENDEMIC $<C O>, K T Z$, W\& W', Anderson 97

* Lobularia maritima (L.) Desv, - Dorn01, KTZ, FCF, UTF, W\&W, E

[Koniga maritima (L.) R. Br.] - HDH

[Lobularia maritima Desv,] - SFE

* Lunaria annua L - KTZ

* Malcolmia africana (L.) R. Br, - Dorn01, HDH, SFW, W\&W. W

[Malcolmia africana R. Be.] - GPF, FCF, UTF

[Malcolmia africana (L,) W, T, Aiton] - KTZ

Nusturtium microphyllum Boenn. ex Richenb. Castilleja 23(4): 6 (2004)

Nusturtium officinale R. Br.-Dorn01, GPF, SF!, UTF, W\&W. EW

[Rorippa nasturtiurn-aquaticum (L) Hayek] - KTZ, FCF

[Rorippa nasturtium-aquaticum (L) Schinz \& Thell.] - HDH

Neolepia (see Lepidium)

Noccaea montana (L.) F. K. Mey. - Dorn01SF!. W\&W. E/W. Koch04

[Nocesea montana (L.) F, K. Mey, var, montana]

[Thlaspi alpestre L.] - HDH

[Thlaspi montanum L- var, montanum] - KTZ, FCF, UTF

Pennellia longifolia (Benth.) Rollins - KTZ, FCF

Pennellia micrantha (A. Gray) Nieuwl. - HDH, KTZ, FCF, SF!, W\&W, EJW

Physaria

(Identification sources: ) 
Physaria acutifolia Rydb, var, acutifolia - KTZ, FCF, UTF, W

[Physaria acutifolia Rydb.] - Dom01HDH, SFW, W\&W

[Physaria australis (Paysen) Rollins] - HDH

- Physaria alpina Rollins - ENDEMIC <COs, KTZ. FCF, SF!, W\&W, E/W

- Physaria bellii Mulligan - ENDEMIC $<C O>$, KTZ, FCF, SFE, W\&W, E

Physaria brassicoides Rydb. - Dorn01. Snow04b

\{Physaria curvipes (A. Nels.) Grady \& O'Kane - Grady07)

INS - closely approsches the northern end of S. Rockies in Johnson Co., WY, juss north of Natronoa Co.)

Physaria didymocarpa (Hook.) Gray var. didymocarpa - Dorn01

[Physaria didymocarpa var, nocmalis Kuntze] - KTZ

- Physaria eburniflora Rollins - ENDEMIC $<$ WV $>$ Dornol

Physaria floribunda Rydb. suhsp, floribunda

[Physaria floribunda Rydo.] - HDH, SF?, UTF, W\& w

[Physaria floribunda Rydlb, var, floribundo] - KTZ, FCF, E/W

- Physaria floribunda Rydb, subsp, osterhoutii (Payson) OKane - ENDEMIC <CO> O'Kane07

[Physaria floribunda Rydo.] - SFW

[Physarla floribunda Rydo, var, osterhoutii (Payson) Rollins] - KTZ, FCF, SFE, WQW', EN

[Physaria osterhoutii Payson] - HDH, W\&W

- Physaria obcordata Rollins - ENDEMIC $<C O>$ KTZ, FCF, SFW, W\&W, W

- Physaria pruinosa (Greene) O'Kane \& Al-Shehbaz - ENDEMIC <CO, NM>

[Lesquerella pruinosa Girene] - HDH, KTZ, FCF, SFW, W\&W

- Physaria pulvinata $O^{+}$Kane \& Reveal - O'Kane06

- Physaria rollinsii Mulligan - ENDFMIC <CO>, KTZ, FCF, SFW, W\&W, W

- Physaria scrotiformis O'Kane - ENDEMIC $<\mathrm{CO}>\mathrm{O}^{\prime} \mathrm{Kane} 07$

Physaria spatulata (Rydb.) Grady \& O'Kane - Grady07

[Lescourella alpima (Nuth.) S. Wats, var. spmulata (Rydb.) Payson] - KTZ

[Lesquerella soatulata Rydb] - Grady0?

[Lesquerella reediana subsp, sputhulata (Rydb, ) OKane \& Al-Shehbaz] - Al-Sbehbaz02

- Physaria vitulifera Rydb, - ENDEMIC $<$ CO, WY>, Dom01, HDH, KTZ, FCF, SFE,W\&W, E, Jennings04

* Raphanus raphanistrum L. - HDH, KTZ, FCF, UTF, W\&W

* Raphanus sativus L. - Dorn01GPF, HDH, KTZ, FCF, UTF, W\&W

Rorippa (see also Armoracia, Nasturtium)

(Identification sources:

Rorippa calycina (Engelm.) Rydb. - Dom01, KTZ

[Nasturtium calycinum Engelm.] - KTZ

Ronippa curvipes Greene var. alpina (S. Watson) Stuckey - Dorn01, SFW, UTF, W\&W, EW

[Rorippa alpina (S. Watson) Rydb,] - HDH, KTZ, FCF, SFE, W\&W'

Rorippa curvipes Greene var, curvipes - Dom01, KTZ. FCF. UTF, W\&W. E

[Rorippa curvipes Greene] - GPF, HDH, SFE, W\& W'

Rorippa curvipes Greene var. integra (Rydb.) Stuckey - Dorn01

Rorippa curvipes Greene var. truncata (Jeps.) Rollins - KTZ, FCF

[Rorippa truncata (Jeps,) R, Stuckey] - Dornol

\{Rorippa curvisiliqua (Hook.) Bessey ex Britton var. Iyrata (Nutt.) Stuckey\} - W\&W, err. rep. \{Rorippa curvisiliqua (Hook-) Bessey ex Britton var. lyrata (Nutt. ex Torr. \& A. Gray) Stuckey\} - FCF

[Rorippa curvisiliqua (Honk.) Besser ex Britton var. lyrata (Nutt.) M. Peck ex C. L. Hitche.\} - KTZ 
\{Rorippa lyrata (Nutt.) Rydb.\} - HDH

(Rorippa islandica (Oeder ex J. A. Murray) Borbás\} - HDH, W\&W, err. rep.

\{Rorippa islandica (Oeder) Bortás\} - KTZ

Rorippa palustris (L.) Besser var, fernaldiana (Butters \& Abbe) Stuckey - Dorn01, FCF

[Rorippo islandica (Oeder) Barbuis var. glahra (O. E. Schulz) S. L. Welsh \& Reveal] - UTF

[Rorippa palustris (L.) Besser subsp. fermaldiana (Butters \& Abbe) Jonsell] -KTZ

[Rorippa palustris (L.) Besser subsp. glabra (O. E. Schulz) R. Stuckey var. femaldiana (Butters \& Abbe) Stucky] - GPF

[Rorippa palustris (L) Besser var. glabra (O. E. Schul2) R. Stuckey] - W\& W

Rorippa palustris (L.) Besser var, hispida (Desv, Rydh. - Dorn01, FCF, E/W

[Rorippa hispida (Dess.) Britton] - HDH

[Rorippa islandica (Oeder) Borbís var. hispida (Desv.) Butters \& Abbel - UTF

[Rorippa palustris (L) Besser subsp. hispida (Desv.) Jonsell] - KTZ, SF!, W\& W

[Rorippa palustris (L) Besser subsp. hispida (Desv.) Jonseli var. hispida] - GPF aCOO.

Rorippa palustris (L.) Besser var, palustris - FCF

Rorippa sinuata (Nutt.) Hitche, - Dorn01, GPF, HDH, KTZ, SF!, UTF, W\&W, E/W

[Roripps sinuata (Nutt. ex Torr. \& A. Gray) Hitche.] - FCF

Rorippa sphaerocarpa (A. Gray) Britton - Dorn01, HDH, KTZ, FCF, SF!, UTF, W\&W, E/W

* Rorippa sylvestris (L.) Besser - GPF, Hartman06, KTZ, FCF, SFE, UTF, W\&W, E

Rorippa tenerrima Greene - Dom01, GPF, KTZ, FCF, SFE, UTF, W\&W, E

Rorippa teres (Michx.) Stuckey - KTZ, SF!, W\&W, E/W

[Rorippes obcusa (Nutt.) Britton) - HDH

[Roripps teres (Mictox.) Stuckey var, teres] - FCF

Schoenocrambe (see Sisymbrium, Thelypodiopsis)

* Sinapis alba L- $-\mathrm{KTZ}, \mathrm{FCF}$, W\&W

[Brassica hirta Moersth] - GIP, HDH, UTF

[Rorippen coloradens is R. Stuekey] -Al-Shehbaz, pers. comm, 2007. KTZ, FCF, SFE, W\&W, E

* Sinapis arvensis L. - Dorn01, KTZ, FCF, SF!, W\&W, ENW

[Brassica kaber (DC.) Wheeler] - GPF, HDH, UTF

Sisymbrium (see also Arabidepsis, Thelypodiopsis)

(Identification sources:

* Sisymbrium altissimum L. - GPF, HDH, KTZ, FCF, SF!, UTF, W\&W, E/W

* Sisymbrium austriacum Jacq, - KTZ, SFE, W\&W, E

* Sisymbrium irio I, - FCF, GPF $<$ CO $>, \mathrm{KTZ}<\mathrm{AZ}, \mathrm{NM}, \mathrm{UT}>, \mathrm{FCF}$

Sisymbrium linifolium Nutt. - $\mathrm{HDH}$

[Schoenocrambe linifolla (Nurt) Greene] - Dom01, KTZ, FCF, SF1, UTF, W\&W, EW

* Sisymbrium loeselii L. - GPF, KTZ, FCF, SF!, W\&W, E/W

* Sisymbrium officinale (L.) Scop. - HDH, KTZ, FCF, SFE, UTF, W\&W, E

[S isymbrium officinale $\{$ L.) Seop, var, kiocarpum DC.] - GPF

[Sisymberium officinale $\{\mathrm{L}$, Seop. var, officinale] - GPF

Smelowskia calycina (Steph. ex Willd.) C. A. Mey. var. americana (Regel \& Herd.) Drury \& Rollins - Dorn01, KTZ. E/W

[Smeiowskia calycina ('Steph.) C., A. Mey.]-SF!

[Smelowskia calycina (Steph, ex Willd.) C. A. Mey.] - WR W

[Smelowskia calycina C. A. Mey, var, americana Drury \& Rollins] - $\mathrm{HDH}$

Snow, N, January 2009. Checklist of Vecculer Plants of the Southom Rocky Momain Regioe. CVersion $3 x$ 
[Smelowskia calycina C. A. Mey. var. americana (Regel \& Hend.) Drury \& Rollins] - UTF

[Smelowskia calycina (Steph.) C. A. Mey, var- americana (Regel \& Herd.) Drury \& Rollins] - FCF

Stanleya albescens M. E. Jones - HDH, KTZ. FCF, SFW, UTF, W\&W, W

Stanleya pinnata (Pursh) Britton var, integrifolia (E. James) Rollins - GPF, HDH, FCF, W\&W, W

[Stanleya integrifolia E. James] - UTF

[Stanleya pinnata (Pursh) Brittoa] - Dornol

[Stanleya pinnata (Pursh) Britwo var. integrifolia (E. James) Robins.] - SFW

[Stanleya pinnata (Pursh) Brition var. integrifolia (E. James ex Torr.) Rollins] - KTZ

Stanleya pinnata (Pursh) Britton var, pinnata - GPF, HDH, KTZ, FCF, UTF, W\&W, E/W

[Stanleya pinnata (Pursh) Brittoa] - SF!

[Stanleya pinnata (Pursh) Brition var. bipinnaka (Greene) Rollins] - HDH, KTZ, FCF

Stanleya viridiflora Nut1. - Dorn01, HDH, KTZ, SFW, W\&W, W

[Stanleya viridiflora Nute. ex Torr. \& A. Gray] - FCF, UTF

\section{Stanleyella (see Thelypodium)}

Streptanthella longirostris (S. Watson) Rydb, var, longirostris - RMC. W

[Streptanthella longirostris (S. Walson) Rydb.] - Dorn01. HDH, KTZ. FCF, SFW, UTF, W\& W

Streptanthus cordatus Nutt, - Dom01, GPF, SFW, W

[Streptanthus cordahus Nutt. es Torr. \& A. Gray]- HDH, UTF, W\&W

[Streptanthus cordahus Nuti. var, cordatus] - KT2

[Streptanthus cordatus Nult. \&x Torr. \& A. Gray var, corckatus] - FCF

Subularia aquatica L. var americana (Mulligan \& Calder) Bovin - KTZ, FCF, E

[Subularia aquatica L.] - Dorn01. SFE, Ws' ' $^{\prime}$

Thellungiella salsuginea (Pall.) O. E. Schulz] - SFE, W\&W

[Arsbidopsis salsuginea (Pall, N, Busch] - KTZ, FCF, E

[Sisymbrium salsugincum Pall.] - $\mathrm{HDH}_{4}$ rep.

Thelypodiopsis

(Identification sources:

Thelypodiopsis aurea (Eastw,) Rydb, - KTZ, FCF, SFW, UTF, W\&W, W

[Sisymbrium auncum (Eastw) Payson] - $\mathrm{HDH}$

Thelypodiopsis elegans (M. E. Jones) Rydb. - Dom01, KTZ, FCF, SFW, UTF, W\&W, W

[S isymbrium elcgans (M. E. Jones) Payson var, elegans] - HDH

- Thelypodiopsis juniperorum (Payson) Rydb. - ENDEMIC $<$ CO $>$, KTZ, FCF, SFW, W\&W. W

[Sisymbriurn elegans (M. E. Jones) Payson var, juniperorum (Payson) Harrington] - HDH

Thelpyodiopsis linearifolius (Gray) Al-Shehbaz

[Schoenocrambe linearifolia (A. Gray) Rollins] - KTZ, FCF, SF], W\& W, EW

[Hesperidanthus linearifolius (A. Gray) Rydb.] - HDH

Thelypodium

(Identification sources: 
Thely podium integrifolium (Nutt.) Endl. ex Walp, var. gracilipes B. L. Rob, - ENW

[Thelypodium integrifolium (Nutt.) EndL] - Dom01, SF!, W\&W

[Thelypodium integrifolium (Nutt.) EndL. var. gracilipes B. L. Rob.] - UTF

[Thelypodium integrifolium (Nutt. ex Torr. \& A. Gray) Endil, subsp. gracilipes (B. L. Rob.) AL-Shehbaz] - FCF

[Thelypodium integrifolium (Nutt.) EndL. ex Walp. subsp. gracilipes (B. L. Rob.) Al-Shehbaz] - KTZ

[Thelypedium rhomboideum Greene var. gracilipes (B. L. Rob.) Payson] - HDH

Thelypodium integrifolium (Nutt,) Endl. ex Walp, var, integrifolium - E/W

[Thelypodium integrifolium (Nutt.) EndL] - GPF, SF!, W\& W

[Thelypodium integrifolium (Nutt.) EndL. var. integrifolium] - UTF

[Thelypodium integrifolium (Nutt. ex Torr. \& A. Gray) Endl. subsp. integrifolium]- FCF

[Thelypodium integrifolium (Nutt.) EndL ex Walp. subsp. integrifolium] - KTZ

[Thelypodium lilacinum Greene] - HDH

[Thelypodium rhomboideum Greene var. rhomboideum] - HDH

Thelypodium laxiflorum AI-Shehbaz - KTZ, FCF, SFW, UTF, W\&W, W

Thelypodium paniculatum A. Nelson - Dorn01, KTZ, FCF, SFE, W\&W, E

[Thelypodium sagittatum (NutL) Endl.] - HDH

Thelypodium sagittatum (Nutt.) Endl, ex Walp, var, sagittatum - E

[Thelypodiops is sagittata (Nut.) O. E. Schul2 var. sagitata] - UTF

[Thelypodium sagitatum (NumL) Endl.] - Dorn01, HDH, SFE

[Thely podium sagittatum (NumL) Engl. subsp. sagittatum] - FCF. WEW

[Thelypodium sagintatum (NutL ex Torr. \& A. Gray) Endl. ex Walp. stubsp. sagittatum] - KTZ

Thelypodium wrightii A. Gray subsp. oklahomensis Al-Shehbaz - KTZ, FCF, SFE, W\&W, E

\{Thelypodium wrightii $\mathrm{A}$. Gray subsp, wrightii) - KTZ $<A Z, N M>F C F$, W\&W, err. rep.

\{Stankyclla wrighti (A. Gray) Rydb.\} - HDH

Thelypodium wrightii A. Grays - GPF, UTF

Thlaspi (see also Noccaea)

* Thlaspi arvense L. - Dorn01, GPF, HDH, KTZ, FCF, SF!, UTF, W\&W, E/W

† Turritis glabra I. var, glabra-SF!, W\&W, Al-Shehbaz03, O'Kane03

[Arabis glabra (L.) Bernh. var. glabra] - FCF, UTF, EW, Rollins93

[Arablis glabra (L.) Bernh.] - GPF, HDH, KTZ

[Arabis mactocarpa (Nutt. ex Tort. \& Gray) Tortey]-Rollins93

[Arabis perfoliata Lism.] - Rollinso3

[Turritis glabra var, lilacina 0 . E, Schulz] - Rollins 93

[Turritis macrosarpa Nutt, ex. Torr. \& Gray] - Rolling93

Wislizenia refracta Engelm. subsp. refracta - KWA, M\&H, W\&S, KTZ

[Wislizenia refracta var. melilotoides (Greene) I.M. Johnston] - KTZ

\section{Cactaceae - C.AC}

(Identification sources:

)

Coryphantha missouriensis (Sweet) Britton \& Rose var. missouriensis - GPF, UTF, E/W

[Coryphantha missouriensis (Sweet) Britton \& Rose] - Dom01, SFI, W\& W

[Escobaria missoeriensis (Sucet) D. R. Hant var, missouriensis] - KTZ

[Manmillaria missouriensis Sweet] - HDH

\{Coryphantha vivipara (Nutt.) Britton \& Rose var. arizonica (Engelm.) W. T. Marshall\} - UTF.

Snow, N, Junury 2009. Checklist of Vaccular Plants of the Southom Rocky Momain Region. CVersion $3 x$ 


\section{W\&W}

\{Escobaria vivipara (Nut1.) Bexbaum var. arizonica (Engelm.) D. R. Hunt\}-KTZ

Coryphantha vivipara (Nutt.) Britton \& Rose var. radiosa (Engelm.) Backeb.) - M\&H. GPF $\langle C O>, K T Z, S F W, W \& W, W$

[Escobaria vivipara (Nutt.) Buxbaum kar, radiosa (Engelm.) D. R. Humt] - KWA. KTZ

[Mammillaria vivipara (Nutt.) Haw.] - HDH

Coryphantha vivipara (Nutt.) Britton \& Rose var. vivipara - GPF, SFE, UTF, W\&W, E

[Coryphantha vixpara (Nutt.) Britton \& Rowe] - Dorno1

[Escobaria vivipara (Nutt.) Buxbaum var, vivipara] - KTZ

[Mammillaria vivipara (Nutt.) Haw.] - HDH

\section{Cylindropuntia (see Opuntia)}

\section{Echinocactus (see Pediocactus, Selerocactus)}

Echinocerus

(Identification sources:

\section{)}

Echinocereus coccineus Engelm, var, coccineus - HDH, KTZ, W

[Echinocereus coccineus Engelm, var. inermis (K. Schum.) Boiss.] - $\mathrm{HDH}$

[Echinocereus triglochid inus Engelm, var, melanacanthus (Engelm.) L. D. Benson] - UTF, W\& W

[Echinocereus triglochid tanus Engelm, var, triglochidiatus] - W\&W

Echinocereus fendleri (Engelm.) F. Seitz var, fendleri - KTZ, W

[Echinocereus fendleri (Engelm.) F. Seitz] - KTZ

[Echinocereus fendleri (Engelm.) Rumpler] - HDH. SFW, W\&W

Echinocereus reichenbachii (Terscheck ex Walp.) F. Haage var. perbellus (Britton \& Rose) L. D.

Benson-GPF, KTZ, E

[Echinocereus reichenbachii (Terscheck) F. Haage] - HDH

[Echinocereus reichenbachii (Terscheck) F. Haage var, perbellus (Brinton \& Rose) L. D. Benson] - SFE, W\& W

Echinocereus triglochidiatus Engelm. var. mojavensis (Engelm. \& Bigel.) Benson - FCF

Echinocereus triglochidiatus Engelm, var. neomexicanus (Standl.) Standl. ex W,T. Marshall -

KWA. M\&H

[Some (see KWA\} feel this may be a sterile hybrid between E: coccineus $x$ E. chlocanthus]

[Echinocereus coccineus var, arizonicus (Rose ex Orcutt) Ferguson] - KTZ $\triangle A Z$ ?

[Echinocereus neomexicanus Standl.] - MKH

[Echinocereus rosei Woot. \& Standl.] - M\&H

[Echisocereas polyacantbus Engelm.] - M\&H

[Cereus necenexicasus (Stand1.) Tidestr.] - IPNI

Echinocereus triglochidiatus Engelm. var, triglochidiatus - KTZ, W\&W, E/W

[Echinocereus triglochidiatus Engelm.] - HDH, SF!

[Echinocereus triglochidiatus Engelm, vas. gonacanthus (Engelm. \& Bigelow) Boisscv. \& C. Davidson] WEW

Echinocereus viridiflorus Engelm. var. viridiflorus - KTZ, E

[Echinocereas viridiflorus Engelm.]-Dorn01, GPF, HDH, SFE. WEW

\section{Escobaria (sce Coryphantha)}

Mammillaria (see also Coryphantha)

Mammillaria heyderi Muchlenpf. - KTZ 
[NS - B. Legler \{pers. comm. 2009\} also confirms 2 collections from Colfax $\mathrm{Co}_{4}, \mathrm{NM}$ : mostly likely the typical variety]

Opuntia

(Identification sources:

Opuntia cymochila Engelm. \& Bigelow - KTZ, E

[Opuntia macrorhiza Engelm, var- macrorhiza] L. Berson - GPF, KTZ

[Opuntia macrorhiza Engelm.] - Dorn0I, SFE, UTF, W\&W

fOpuntia davisii Engelm. \& Bigelow; - HDH

\{Cylindropuntia davisii (Engelm. \& Bigelow) Kunth\} - W\&W, err. rep.

$\mathrm{OK}$.

\{Opantia tunicata (Lehm.) Link \& Otto var. davisii (E.ngelm. \& Bigelow) L. D. Benson\}-GPF, KTZ \&NM,

Opuntia engelmannii Salm-Dyck var. engelmannii - KWA, KTZ

[Opuntia discata Griffiths] - KWA

[Opunisa dillei Griffichs] - KWA, KTZ

[Opuntis megakarpa Griffiths] - KIZ

[Opuntia microcarpa Englem. ex B. D. Jackson] - KWA

[Opunta oecidsetalis var. megacarpa (Griffiths) Munz| - KTZ

[Opuntia phaeacantha Englem. var. discasa (Grifliths) Bcnson] - KWA, M\&H

[Opuntis lardospina Griffiths] - KWA

Opuntia erinacea Engelm. \& Bigelow ex Engelm. var, erinacea - KTZ, W

[Opunta erinacea Engelm.] - SFW

[Opuntia crinacea Engelna, var, erinacea] - UTF

[Opunta crinacea Engelns. \& Bigelow var, erinacva] - W\& w

[Opuntia lystricina Engelm. \& Bigelow] - HDH

Opuntia erinacea Engelm. \& Bigelow ex Engelm. var, utahensis (Engelm.) L. D, Benson -

Dorn01, KTZ

[Opunta erinacea Engelm, var. utahensis (Engelm.) L. D. Bensos]-UTF

[Opuntis erinacea Engelms. \& Bigelew var, utahensis (Engelm.) L. D. Benson] - GPF, W\& W

[Opunta fhodantla K. Schum.]-HDH

Opuntia fragilis (Nutt.) Haw, var, brachyarthra (Engelm. \& Bigelow) J, M. Coult. - KTZ, SFW, W\&W, W

[Opunta fragilis (Nutt.] Haw.] - Down1, UTF

[Opuntas schwerinians K. Scham.]- $\mathrm{HDH}$

Opuntia fragilis (Nutt.) Haw, var, fragilis - KTZ, W\&W, E

[Opuntia fragilis (Nutr.) Haw]] - GPF, HDH, SFE, UTF

- Opuntia heacockiae Arp - ENDEMIC $<\mathrm{CO}>$, KTZ, SFE, W\&W, E

\{Opuntia humifusa (Raf.) Raf.\} - GPF, W\&W, err, rep.

\{Opantia humifusa Raf)\}- $\mathrm{HOH}$

[Opeantis humifusa (Raf, Raf, var, humifusa] - KTZ

Opuntia imbricata (Haw.) DC. var. imbricata - KTZ, E/W

[Cylindropuntia imbricath (Haw,) Knuth] - SF!, W\&W

[Opuntia arborescens Engelm.] - HDH

[Opuntia imbricatz (Haw.) DC.] - GPF

Opuntia macrocentra Engelm. - KWA

[Opuntia violacea Engelm.] - KTZ

[Opuntia violacea var,castetteri L. Benson ] - KT2

[Opuntia violacea var, macrocentra (Enuelm-) L. Benson ] - KTZ, M\&H

Opuntia phacacantha Engelm. var. camanchica (Engelm. \& Bigelow) L. D. Benson - GPF, KTZ, W\&W, EN 
[Opuntia phaeacantha Engelm.] - HDH, SF:

Opuntia phaeacantha Engeim. var, major Engelm. - GPF, KTZ, UTF, W\&W

3Opuntia phacacantha Engelm. var, phaeacintha\} - GPF, KTZ, UTF, cCO fide L. D. Benson:-

Opuntia polyacantha Haw, var, juniperina (Britton \& Rose) L. D. Benson - Dorn01, KTZ, W

[Opuntia polyacantha Haw.] - W

[Opuntia polyacantha Haw. var. polyzcantha] - UTF, W\& W

Opuntia polyacantha Haw, var, polyacantha-Dorn01, GPF, KTZ, UTF, W\&W, EW

[Opuntis polyacantha Haw.]-HDH, SF!

[Opuntia rutila Nutt.] - HDH

Opuntia polyacantha Haw. var, rufispina (Engelm. \& Bigelow ex Engelm.) L. D. Benson Dorn01, KTZ, W

[Opuntia polyacantha Haw.] - HDH, SFW

[Opuntia polyacantha Haw. var. rufispina (Engelm. \& Bigelow) L. D. Benson] - UTF

[Opuntis polyacantha Haw, var. rufispina (Engeim.) L. D. Benson] - W \& W

Opuntia polyacantha Haw. var, trichophora (Engelm. \& Bigelow) J. M. Coult. = GPF, UTF, KTZ

[Opuntia polyacantha Haw. var. polyacantha] - W\&W

[Opuntia trichophora (Engelm.) Brition \& Rose] - HDH

Opuntia X viridiflora Britton \& Rose $-\mathrm{KTZ}$

[Cy lindropuntia viridiflora (Britton \& Rese) F. M. Kanth] - KWA

[Opuntia imbricats X O. whipplei]-KTZ (pro sp.) - XTZ

[Opuntis whipplei Engelm. var. viridiflora (Britton \& Rose) L. Benson] - KWA

Opuntia whipplei Engelm. \& Bigelow var, whipplei - UTF, W

[Cylindropuntia whipplei (Engelm. \& Bigelow) Knuth]-SFW. W \& W

[Opuntas whipplei Engelm. \& Bigelow] - KTZ

- Opuntia wootonii Girffiths - ENDEMIC $<$ NM $>$

[Opuntis engelmannii var. wookonii (Griffiths) Fosberg] - KTZ

[Opuntia phaegcantha var, wootonii (Griftiths) L. Benson]- KWA, M\&H, KTZ

\{Pediocactus knowltonii L. D, Benson\} - KTZ, SFW, W\&W, W

Pediocactus simpsonii (Engelm.) Britton \& Rose var. simpsonii - Dorn01, KTZ, W\&W, EJW

[Echinocactus simpsonet Esgelm.] - HDH

[Pediocactus simpsonii (Engelm.) Briton \& Rose] - GPF, SFW, UTF

[Pediocactus simpsonii (Engelm.) Brition \& Rose var, minor (Engelm,) Cockerell] - KT, SFE, W\&W

Sclerocactus cloveriac K. D. Heil \& I. M. Porter subsp, cloveriac - KTZ

[Sclerocactus cloweriae K. D. Hell \& J. M. Porter] - KTZ

Selerocactus glaucus (J, A. Purpus ex K. Schum.) L. D. Benson - KTZ, W

[Echinocactus whipplei Engelm. \& Bigelow] - HDH

[Sckerocactus glaucus (K. Schum.) L. D. Benson] - SFW, W\& W

[Sckerocactus whipplei (Eagelm.) Britton \& Rose var, roscus (Clover) L. D. Benson] - UTF

[Selerocactus whipplei (Engelm.) Britton \& Rose var. glascus (Purpus) S. Welsh] - UT.

- Sclerccactus mesae-verdae (Boissev, ex Boissev. \& C. Davidson) L. D. Benson

- ENDEMIC $<\mathrm{CO}, \mathrm{NM}>, \mathrm{KTZ}, \mathrm{W}$

[Echinocactus inesae-verdae (Boisser. \& C. Davidson) L. D. Benson] - HDH

[Scleroeactus mesae-kerdae Boissev, \& C. Davidson] - SFW

[Sclerocactus mesac-verdse (Boissey.) L. D. Benson] - UTT

[Sclerocactus mesae-verdse (Boissev, \& C. Davidson) L. D, Benson] - W\&W

Sclerocactus parviflorus Clover \& Jotter var. intermedius (Peebles) Woodruff \& L. D. Benson W

[Echinocactus whipplei Engelm. \& Bigelow] - $\mathrm{HDH}$

[Sclerocactus parviflorus Clover \& Jocter] - W\&W

[Sclenocactus parviflorus Clover \& Jocter subsp intermedias (Pecbles) K. D. Heil \& J, M. Porter] - KTZ 
[Sclerocactus whipplei (Engelm.) Britton \& Roxe var. roseus (Clover) L. D. Bensan] - UTT

[Sclerocactus whipplei (Engelm. \& Bigelow) Britton \& Rose var. intermedius (Peebles) Benson] - SFW

Selerocactus parviflonus Clover \& Jotter var, parviflorus

[Sclorosactus parviflorus Clover \& Jotter] - W\&W

[Sclerocactus parviflorus Clower \& Jotter subsp. parviflorus] - KTZ

[Sclerocactus whipplei (Engelm.) Britton \& Rose var. rosous (Clover) L. D. Benson] - UTF

\section{Caesalpiniaceac (see FAB)}

\section{Calochortaceac (see LIL)}

\section{Callitrichaceac - CLL}

Callitriche hermaphroditica L. - Dorn01, GPF, HDH, IMF, KTZ, SF!, UTF, W\&W, E/W

Callitriche heterophylla Pursh var, heterophylla - KWA, KTZ, M\&H, EW

[Callieriche anceps Fern.] - KTZ

[Callitriche heterophylla Pursh]-GPF, HDH, IMF, SF! UTF, W\& W, doublful, W\& W'

[Callitriche beterophylla Pursh subsp. heterophylla] - KTZ

Callitriche palustris L. - Dom01, HDH, IMF, KTZ, E/W

[Callitriche vema L.] - GPF, KIZ, SF!, UTF, W\&W

Campanulaceae - CAM (including Lobeliaceae)

(Identification sources: )

Campanula aparinoides Pursh - Dorn01, GPF, HDH rep., KTZ, SFE, W\&W, E

* Campanula glomerata L. - KTZ<UT>, UTF

[W] - rapidly spresding through vacant kots in Marble, Gumnison Co.]

Campanula parryi A. Gray var. parryi - IMF, KTZ, E/W

[Campanala peirsy A. Gray] - Dorne1, HDH, SF!, UTF, W\&W

* Campanula rapunculoides L. - Dorn01, GPF, HDH, corr. \& add. IMF, KTZ, SFE, W\&W, E

Campanula rotundifolia L. - Dom01, GPF, HDH, IMF, KTZ, SF!, UTF, W\&W, ENW

[Campanula gieseckiana Vesi] - SFE

[Camapanala groenlandica Berlin] - SFE

[Campanula rotundifolia L. subsp, groenlandica (Berlin) A. LWe \& D. Love] - SFW, possibly, alpine raee

Campanula uniflora L, - Dorn01, HDH, IMF, KTZ, SF!, UTF, W\&W, E/W

Downingia laeta (Greene) Greene - Dorn01, KTZ

Heterocodon rariflorum Nutt. - HDH, IMF, KTZ, SFW, W\&W, W

[Specularia rariflora (NutL.) McVaugh] - KTZ

\section{Legousia (sce Triodanis)}

Lobelia cardinalis L. - GPF, KTZ, E

[Lobelia cardinalis L. subsp, yranainea (Lam.) McVaugh] - HDH, IMF, SFE, UTF, W EW

\{Lobelia kalmii L.\} - HDH rep., GPF $<\mathrm{CO}>\mathrm{KT}$, W\&W, doubtful

Lobelia siphilitica L. var. ludoviciana A. DC - Dorn01, GPF, HDH, KTZ, SFE, W\&W, E

[Lobelia X speciosa Sweet var. occidentalis Bowden\} - KTZ 
\{Lobelia spicata Lam.\} - HDH, rep, <NE>, KTZ <KS, NE, OK>

\{Porterella carnosula (Hook, \& Arn.) Torr. $-\mathrm{HDH}$, exp., IMF $<\mathrm{CO}>$, KTZ $\angle A Z$, UT, WY $>$, UTF

Specularia (see Heterocodon, Triodanis)

Triodanis holzingeri McVaugh - Dorn01, GPF, HDH, KTZ, SFE, possible hybrid, W\&W, E [Specularia holzingeri (McVaugh) Fern.] - KTZ

Triodanis leptocarpa (Nutt.) Nieuwl. - Dorn01, GPF, HDH, KTZ, SFE, W\&W, E [Specularia keptocarpa (Nutt.) Giray]- KTZ

* Triodanis perfoliata (L.) Nieuwl, - Dom01, GPF, HDH, IMF, KTZ, SFE, UTF, W\&W, E

[Legousia perfoliata (L.) Britt.] - KTZ

[Specularia perfoliata (L.) A DC.] - KTZ

Cannabaceae - CAN (including some fomerly in ULM)

*Cannabis sativa L, - Dom01, FNA, HDH, SF!, W\&W, E/W

[Cannabis sativa L. subsp. indica (LamL) Small \& Cronquist] - UTF

[Cannabis sariva L. subsp. soniva] - UTF

[Cannabis sariva L. sabsp. sativas vat, sativa] - GPF, KTZ

[Cannabis sariva L. sabsp. sativa var. spontanea Vavilov] - GiPF

\% Celtis occidentalis L. - Dorn01, GPF, HDH, KTZ, UTF, W\&W, cult.

Celtis reticulata Torr. - GPF, SF!, UTF, W\&W, E/W

[NS - A lan Whittemore, National Arbonctum, indicates \{pers. comm., 2007) that this is the correct name for plants in our region, but not the correct one for planis farther south and east]

[Cellis laevigata Willd. var. reticulata (Torr.) L. D. Benson] - KTZ

[Cehis occidentalis L.] - HDH

\{Humulus lupulus L. var. lupuloides E. Small\} - FNA, GPF, KTZ $<$ KS,NE,UT, WY $>$, E/W $\{$ Humulus americanus Nuen.\} - HDH, UTF

¿Humulus tepulus L. subsp. americanus (NutL) A. Love \& D. Lơve) - SFE, W\& W'

\{Humulus lepulus L. var, smericanus (Nurn.) A. Lowe \& D. Love) - W\&W

Humulus lupulus L. var. neomexicanus A. Nelson \& Cockerell-Dorn01, FNA, GPF, KTZ

\section{Cleomaceae - CMC}

\section{Cristatella (see Polanisia)}

Cleome lutea Hook. var. lutea - Dorn01, K IZ, W

[Clooms lusea Hook.] - GPF, HDH, SFW, UTF, W\&W

Cleome multicaulis Sessé \& Moç. ex DC. - Dom01, W\&W, E

[Cleome multicaulis DC] - KTZ.

[Cleone multicaulis Sesse \& Moc.] -SFE

[Cleome sonorac A. Gray]- HDH

Cleome serrulata Pursh - Dorn01, GPF, KTZ, SF!, W\&W, E/W

[Cloome serrulata Pursh forma inornata (Ginene) W. A. Weber]-SF!

[Cleome serrulata Pursh var, angusta (M. E. Joses) Tidestr.] - HDH, UTF

[Cleome serrulata Pursh var, semulata] - HDH, UTH 
Cleomella angustifolia Torr - GPF, HDH, KTZ, SFE, W\&W, E

Cleomella palmeriana M. E. Jones - HDH rep., KTZ, SFW, W\&W, W

[Cleomella montrosae Payson] - HDH

[Cleomella nana Eastw.] - HDH

[Cloomella palmeriana M. E. Jones var. palmerians] - UTF

Cleomella plocasperma - FCF

\{Polanisia dodecandra (L.) DC. var, dodecandra\}

\{Polanisia dodectandra (L., DC. subsp. dodecandra\} - GPF. KTZ

\{Polanisia graveolens Raf.\} - HDH, rep.

Polanisia dodecandra (L.) DC, var, trachysperma (Torr, \& A. Gray) H. H. Iltis - UTF, EW

[Polanisia dodecandra (L.) DC.] - SF!, W\&W

[Polanisia dodecandra (L.) DC, subsp. Irachysperma (Torr. \& A. Gray.) H. H. Ittis] - GPF, KTZ

[Polanisia trachysperma Torr. \& A. Gray]-Dorn01. HDH

Polanisia jamesii (Torr. \& A. Gray) H. H. Iltis - GPF, KTZ, SFE, W\&W, E

[Cristatella jamesii Torr. \& A. Gray]-HDH

\section{Capparaceae (see CMC)}

\section{Caprifoliacene - (CPR)}

(Identification sources: )

Distegia (see Lonicera)

Lonicera dioica L, var- glaucescens (Rydb.) Butters - Dorn01, KTZ

[Lonicera dioica var. desygyna (Rehd.) Gleason] - KTZ

[Lonicera dioica var. glaucessens (Ryob.) Butters] - KTZ

[Lonicera dioica var, orientalis Gkeson] - KTZ

[Lonicera glausessens (Rydlb.) Rydb.] - KTZ

[Lonicera glausescerts var, dasygyna Reldd.] - KTZ

Lonicera involucrata (Richardson) Banks ex Spreng. var. involucrata - IMF, E/W

[Disiegis involacrsa (Bsaks) Cockerell] - SF]

[Disiegia involacraca (Bsolks es Speeng.) Cockereli] - W\&W

[Lonicera imoluerata (Richardson) Banks] - HDH

[Lonicera imolscrara (Richardson) Banks ex Spreng] - UTF

[Loeicera imolucrater Baaks ex Spreng.] - Dorn01

[Lonicera imolucrata Banks ex Spreng, var, imwolocrata] - KTZ

* Lonicera morrowii A. Gray - Dorn01, KTZ, SF!, W\&W, E/W

* Lonicera tatarica L. - Dom01, GPF, KTZ, SFE, UTF, W\&W, E

\{Lonicera utahensis S. Watson\}- HDH, exp., IMF, KTZ<AZ,NM,UT,WY>, UTF, W\&W. misid.

Lonicera $x$ bella Zabel - WY checklist

[Lonicera morrowii $x$ L. tatarica] - KTZ

Lonicera $\mathrm{x}$ minutiflora Zabel - WY checklist

[Lonicera morrowil $x$ L. xylosteoides] - KTZ

[Lonicera x muendeniensis] - KTZ

Sambucus (see SAM)

Snow, N, Junury 2009. Checklist of Vaccular Plants of the Southom Rocky Momain Region. CVersion $3 x$ 
Symphoricarpos albus (L.) S. F. Blake var, albus - Dorn01, IMF, KTZ, E

[5ymphoricarpos albus (L.) S. F. Blake] - GPF, HDH, SFE, UTF, W\&W

[Symphoricarpas tivularis Suksd.] - HDH

\{Symphoricarpos albus (L.) S. F. Blake var, laevigatus (Fernald) S. F. Blake\} - IMF, KTZ

Symphoricarpos longiflorus A. Gray - HDH, IMF, KTZ. SFW, UTF, W\&W, W

Symphoricarpos occidentalis Hook, - Dorn01, GPF, HDH, IMF, KTZ, SF!, UTF, W\&W, ENW

[Symphoricarpos orbiculatus Moench] - GPF, KTZ ×KS, NE, OK, UT>, UTF, W\&W, cult.

[Symphoricarpos crbiculatus A. Gray] - HDH, rep.

Symphoricarpos oreophilus A. Gray var. oreophilus - IMF, KTZ, UTF, EW

[Symphoricarpos oreophilus A. Gray]- HDH

[Symphericarpos rotundifolius A. Gray]-SF!, W\& W"

Symphoricarpos oreophilus A. Gray var, utahensis (Rydb,) A. Nelson - Dom01, KTZ, UTF, E/W

[Symphoricarpos rotundifolius A. Gary] - SF!, W\& W ${ }^{1}$

[Symphoricinpos tetonensis A, Nelsob]- HDH

[Symphericarpos utahensis Rydb.] - HDH

[Symphericarpos vaccinioides Rydb.] - HDH

Symphoricarpos rotundifolius A. Gray var, rotundifolius - KTZ, E/W

[Symphoricarpos palmeri G. N. Jooes] - GPF, HDH, KTZ

[Symphoricarpos rotundifolius A. Gray] - HDH, SF!, W\&W

Viburnum (see VIB)

Caryophyllaceae - CRY (including Alsinaceae)

(Identification sources:

* Agrostemma brachyloba (Fenzl) Hammer - KTZ, W\&W ${ }^{1}$, rep.?

\{NS - a garden waif from Boukder; not considered establishod by FNA 5: 215:

\{Agrostemma gracilis Boiss.\} - W\&W

Agrostemma githago L. var, githago - FNA

[Agrostemama gichago L.] - Dorno1, GPF, HDH rep., KTZ?, RMC. W \& W", rep.

Alsinanthe (sec Minuartia)

Alsine (sce Stellaria)

Anotites (see Silene)

Arenaria

(Identification sources:

Arenaria (see also Eremogone, Minuartia, Mochringia)

Arenaria lanuginosa (Michx.) Rohrbach var. saxosa (A. Gray) Zarucchi, R. L. Hartm. \& Rabeler - FNA

[Arenaria confusa Rydb.] - FNA, HDH

[Avenaria lanuginosa (Michx.) Rohıb.] - UTF

[Arenaria lanuginasa (Michx.) Fohrh. var. cinerascens (B. L Rob.) Shinners] - FNA. EWW

Snow, N. Junury 2009. Checklist of Vaccular Plants of the Southom Rocky Momain Region. CVersion $3 x$ 
[Arenaria lasuginosa (Michx.) Rohrb subsp. saxusa (A. Gray) Maguire - FNA, KTZ

[Arenaria stoosa A. Gray] - FNA, HDH

[Spergulastrum lanuginosum Michx. subsp. saxosum (A. Gray) W. A. Weber] - FNA. SFt, W\&W

*Arenaria serpyllifolia L. var, serpyllifolia - FNA

[Anenaria serpyllifolia L]- Dom01, GPF, HDH, KTZ?, SFE, UTF, W\&W

[Arenaria serpyllifolia L. subsp. serpyllifolia] - E

\section{Cerastrium}

(Identification sources:

Cerastium arvense L. subsp, strictum Gaudin - FNA

[NS - see FNA 5: 80 for lengthy synceymy, includ ing many combinations by either Greene or A. Nels.]

[Cerastium arvense L.]-Dom01, GPF, HDH, UTF, E/W

[Cerastium arvense L. subsp. fuegianum (Hook f) Ugbor.] - KTZ

[Cerastium arvense L. subsp. strictum (L.) Ugbor.] - KTZ

[Cerastium strictum L.] - SF!, W\&W

Cerastium beeringianum Cham. \& Schltdl. FNA, HDH, UTF

[NS - see FNA S:81 for additional symonymy]

[Cerastium becringianum Cham. \& Schltel. var- capillare Femald \& Wiegand] - Dom01, E/W

[Cerastium beeringianum Cham. \& Schltul. subsp. earlei [Rydb.) Hultén] - KTZ, SF!, W\&W

Cerastium brachypodum (Engelm, ex A. Gray) B. L. Rob, - Dorn01. FNA, GPF, KTZ, E/W

[Cerastium adsurgens Raf.] - FNA

[Cerastium adsurgens Rat, var. brachypodum Engelm. ex A. Gray] - FNA

[Cerastium brachypodum (Engelm.) B. L. Rob.] - HDH

[Cerastium brachypodum (Engelm.) B. L. Rob, var compactum B. L. Rob.] - FNA

[Cerastium nutans Raf. var, brachypodum Engelm.]-SF!

[Cerastium nutans Raf. var, brachypodum Engelm. ex A. Gray] - WEW

[Cerastium nutans Raf.] - UTF

Cetastium fastigiatum Legler, pers. comm.

[NS - FNA (Vol. 5: 85) discusses the taxonomy of this and related species. Legler reports several collections from Colfax $\mathrm{Co}$, $\mathrm{NM}$ but confirms the taxonomy of the species complex may need more attention]

* Cerastium fontanum Baumg. subsp. vulgare (Hartm,) Greuter \& Burdet - FNA, KTZ, E/W

[Cerastium caespitosum Gillbert] - FNA

[Cerastium saespitosum Baumg, subsp, triviale (Link) Jalas] - FNA

[Cerastium triviale Link] - FNA

[Cerastium vulgare Hartman] - FNA

[Cerastium vulgatum L.] - GPF

[Cerastium vulgatum L. var, hirsitum Fries] - FNA. HDH

* Cerastium glomeratum Thuill. - GPF, KTZ $<\mathrm{KS}, \mathrm{NM}, \mathrm{OK}>$

[NS - FNA report for NM may not apply yet, but taxon widespread and likely to be collected in our area soon] [Cerastium viscosum L.] - HDH, exp.

Cerastium nutans Raf. var. nutans - FNA, KTZ

[Cerastium longipedunculatum Muht, ex Britr.] - FNA

[Cerastium nutans Raf.] - GPF, HDH, UTF

[Cerastium nutans Raf, var, occidentale B. Boivin] - FNA

\section{Conosilene (sce Silene)}

Coronaria (see Silene) 
* Dianthus armeria L. - Dom01, FNA, GPF, KTZ, SF!, UTF, W\&W

[Dianthus armeria L. subsp. ammeria] - EW

* Dianthus barbatus L. - Dorn01, KTZ, UTF

[NS - FNA does not indicate subsp, barbatus in our region]

[Dianthus barbatus L. subsp. barbatus] - RMC

* Dianthus deltoides L. subsp. deltoides - FNA, E/W

[Dianchus deltoides L.] - Dorn01, KTZ, SFI, UTF, w\& W

Drymaria depressa Greene - FNA

[Drymaria effusa A. Gray var. depressa (Greene) Duke] - KTZ, SFE, W\&W, E.

Drymaria glandulosa Bartlin in C. Prest var. glandulosa - B. Legler, pers. comm. 2009

[NS - Legler reports multiple collections from Colfax Co., NM]

Drymaria leptophylla (Cham. \& Schlid.) Fenzl ex Rohrb, var, leptophylla-FNA, KTZ, W\&W. err. rep.

Drymaria molluginea (Seringe) Didrichsen - B. Legler, pers, comm, 2009

[NS - Legler reports multiple collections from Colfax $\mathrm{Con}_{n} \mathrm{NM}$ ]

Eremogone

(Identification sources:

Eremogone congesta (Nutt.) Ikonn, var. congesta - Dorn01, FNA

[Arstaria congesta Nutt. ex Torr. \& A, Gray]- HDH

[Arenaria congesta Nutt, var, congesta] - KTZ, UTF, E,W

[Eremogone congesta (Nutt.) Ikonn.] - SF]

[Eremogone congesta (Nutt. ex Torr. \& A Gray) tkonn] - W \& W

Eremogone congesta (Nutt.) Ikonn, var, lithophila (Rydb.) Dorn] - Dom01, FNA

[Arenaria congesta Nutt. var. congesta] - UTF

[Arenaria congesta Nutt. var, lithophila (Rydb.) Maguire] - KTZ, EW

[Eremogone congesta (Nult.) Ikont.] - SF?

Eremogone eastwoodiae (Rydb.) Ikonn. - FNA

[Arenaria eastwoodiae Rydb. var, adenophora Kearncy \& Pecbles] - KTZ, W

Eremogone eastwoodiae (Rydb.) Ikonn. var. adenophora (Keamey \& Peebles) R. L. Hartman \&

Rabelet - FNA

[Arenaria eastwoodicac Rydb. var. adenophora Keancy \& Pecbles] - FNA

[Arenaria fendleri A. Gray var. eastabodiae (Rydb) Harrington] - HDH

[Arenaria fendleri A. Gray var. esstwoodiae (Rydb.) S. L. Welsh] - UTF

[Eremogone kingii (S. Wacsen) Ikeen, subsp. uimtahensis (A. Nelson) W. A. Webef] - SFW, W: W

Eremogone fendleri (A. Gray) Ikonn. - Dom01, FNA. SF!

[Arenaria fendleri A. Gray]-GPF

[Arenaria fendleri A. Gray var, brevifolia (Maguine) Maguire] - KTZ, EN

[Arenaria fendleri A. Gray var, diffusa Poner \& Coulter] - FNA

[Arenaria fendleri A. Gray var. fendieri] - UTF, HDH, KTZ, UTF, EW

[Arenaria fendleri A. Gray var. porteri Rydb.] - FNA

[Arenaria fendleri A. Gray var. Twcedyi (Rydb.) Maguire] - HDH, KTZ

Eremogone hookeri (Nutt.) W. A. Weber var. hookeri - Dorn01. FNA

[Arenaria hookeri Nutt. ex Torr. \& A. Gray] - HDH

[Arenaria hookeri Nutt, subsp. desertonum (Maguire) W. A. Weber] - EN.A, KTZ

[Arezaria hookeri Nutn. var, desertorum Maguire] - FNA, UTF

[Arenaria hookeri Nutt. subsp, hookeri] - KTZ

[Arenaria hookeri Nutt. ex Torr. \& A. Gray var, bookeri]-GPF

[Eremogone hookeri (Nutt.) W. A. Weber] - SF- 
[Eremogone hookeri (Nutt. ex Tort. \& A. Gray) W. A. Weber subsp. desertorum (Maguire) W. A. Weber] . WEW

[Eremogone hookeri (Nutt. ex Torr. \& A. Gray) W. A. Weber subsp, hookeri] - W \& W

Eremogone hookeri (Nutt.) W. A. Weber var. pinetorum (A. Nels.) Dorn - Dorn01, FNA

[Arenaria hookeri Nutt. ex Torr. \& A. Gray] - HDH

[Arenaria hookeri Nutt. ex Torr. \& A. Gray var, pinctorum (A. Nelson) Maguire] - GPF

[Arenaria honkeri Nuti. subsp-pinetorum (A. Nelson) W. A. Weber] - KTZ

[Arenaria hookeri Nutl. var. pinetorum (A. Nelson) Maguire] - E/W

[Eremogene hookeri (Nutt.) W. A. Webcr] - SF!

[Eremogone hookeri (Nutt. ex Torr. \& A. Gray) W. A. Weber subsp. pinetorum (A. Nelson) W. A. Weber] WSW

Eremogone kingii (S. Watson) Ikomn, var. glabrescens (S. Watson) Dorn - Dom01

[Arenaria fendleri A. Gray var. glabrescens $\$$. Warson] - UTF

[Arenaria kingii (S. Walson) M. E. Jones var. glsbrescens (S. Walson) Maguire] - RMC. W

[Arenaria kingii (S. Watson) M. E. Jones subsp. uintahensis (A. Nelson) Maguire] - KTZ

[Arenaria uincahensis A. Nelson]- $\mathrm{HDH}$

[Eremogone kingii (S. Watson) Ikonn. subsp. uintahensis (A. Nelson) W. A. Weber]-SFW, W\&W

\section{Gastrolychnis (see Silene)}

* Gypsophila elegans M. Bieb, - FNA, GPF, KTZ, SF!, UTF, W\&W, EW

* Gypsophila paniculata L. var, paniculata - FNA, E/W

[Gypsophila paniculara L.] - Dorne], GPF, KTZ SF!, UTF, W\&W

* Gypsophila scorzonerifolia Ser.-Dorn01, FNA, KTZ, SFE, UTF, W\&W, E

* Holosetum umbellatum L. subsp, umbellatum - FNA

[Holosceum umbellatum L.] - Dorn01, GPF, KTZ, SF!, W\&W, EWW

[Holosteum umbellatum L. var. umbeliatum] - UTF

Loeflingia squarrosa Nutt. - FNA

[Loeflingia squarrosa Nutt.var, artemisiarum (Barneby \& Twiss.) Dom] - Domut, KTZ

\section{Lidia (see Minuartia)}

Lychnis (sec Silene)

Melandrium (see Silene)

\section{Minuratia}

(Identification sources:

\{Minuartia elegans (Cham. \& Schltdl.) Schischk. - KTZ

Minuartia macrantha (Rydb.) House - FNA, KTZ, E/W, Hartman08

[Alsinanhe macrantha (Rydb,) W, A. Weber] - SFt, W\&W

[Alsinopsis macrantha Rydb.] - FNA

[Arenaria filicran Maguire] - HDH. W\&W, misapp.

[Arenaria macrastha (Rydb.) A. Netson ex J, M. Coult. \&. A. Nelson]-HDH

[Aremaria rubella (Walsi.) Smith var. filifolium (Maguire) S. L. Welsh] - FNA

[Minuartia filicrum (Maguire) MeNeill) - FNA

Minuratia nutallii (Pax) Briq. var, nuttallii - FNA

[Arenaria nutrallii Pax] - Dome1, HDH reps, UTF 
[Arenaria pengens Nuttall] - FNA

[Minuartia nuttallii (Pax) Briq. subsp. nuttallii] - KTZ, W

[Minuopsis nuttallii ( ( Fax) W. A. Weber]-SFW, WaW

Minuartia obtusiloba (Rydb.) House - Dorn01, FNA, KTZ, EW

[Alsinopsis obtusiloba Rydb.] - FNA

[Arenaria obtusa Torr_non Allioni 1785] - FNA

[Arenaria obtusilaba (Rydb.) Fermald] - HDH, UTF

[Arenaria sajanensis Willd.] - HDH

[Lidia biflora (L.) A. Love \& D. Love] - W\&W, emr. rep.

[Lidia obtusilaba (Rydh.) A. L.ove \& D. Live] - SF!, W\&W W

Minuartia rubella (Wahlenb.) Hiem - Dom01. FNA, KTZ, E/W

[Alsine rubella Wahblenb.] - FNA

[Arenaria rubella (Wahlenb.) J. E. Sm.] - GPF. HDH

[Arenaria rubella (Wahlenb.) J. E. Sm. var. rubella] - UTF

[Tryptane rubella (Wahlenb.) Rcab.] - SF!, W\&W

Minuartia stricta (Sw,) Hiern - FNA, KTZ, Woln79, E

[Alsinamhe stricta (5w.) Rchb_] - SFE, W\&W

[Arenaria rossii Richardsen] - HDH, W\&W, misapp.

[Minuartia russii (R. Br. ex Richardson) Graebe.] - KTZ

[Spergula stricta Sw.] - FNA

\section{Minuopsis (sce Minuartia)}

Moehringia lateriflora (L.) Fenzl - Dorn01, FNA, KTZ, SF!, W\&W, E/W

[Asenaria laseriflora L.]-GPF, HDH, UTF

Moehringia macrophylla (Hook.) Fend - FNA, KTZ, W

[Moctiringia macrophyllia (Hook.] Torr.]-SFW, w\&W

[Arenaria macrophylla Hook.] - HDH, UTF

Paronychia

(Identification sources:

Paronychia depressa (Tort. \& A. Gray) Nutt. ex A. Nelson - FNA, KTZ

[Parcenychia depressa Nuat. ex Torr. \& A. Gray]- Dorn01, GPF, HDH

[Paroeychia depressa Nutt ex Torr. \& A. Gray var, brevicuspis (A. Nels.) Chaudhri] - FNA

[Paronychia depersan Nutt ex Torf. \& A. Gray var. diffusa (A. Nets.) Chaudhri]-FNA

[Parceychia jamesii Torr, \& A. Gray] - W\&W

Paronychia jamesii Torr. \& A. Gray - Dorn01, FNA, GPF, HDH, KTZ, SF!,W\&W, E/W

[Parocychia janesii Torr. \& A. Gray var, birsuta Chatadhri] - FNA

[Parceychia jamesii Torr. \& A. Gray var. parviflora Chaudhri] - FNA

[Paroeychia jamesii Torr, \& A. Gray var, praclongifolia Chatdhri] - FNA

Paronychia pulvinata A. Gray - Dorn01, FNA, HDH, KTZ, SF!, W\&W, EJW

[Paroeychia pulvinata A. Gray var. longiaristata Chaudhri] - UTF

Paronychia sessiliflora Nutt. - Dorn01. FNA, GPF. HDH. KTZ, SF!, UTF. W\&W. E/W

Pseudostellaria jamesiana (Torr.) W. A. Weber \& R. L. Hartm. - Dorn01, FNA, KTZ, SF!, W\&W, E/W

[Stellaria jomesiana Torr.] -HDH, UTF

* \{Sagina apetala Ard. \} - KTZ, W\&W'

* Sagina procumbens L. - FNA, KTZ, UTF; Crow78 
Sagina saginoides (L.) H. Karst. - Dorn01, FNA, KTZ, SF!, W\&W, E/W

[Sagina saginoides (L.) Britt.] - HDH, UTF

Saponaria (see also Vaccaria)

* Saponaria officinalis L. = Dom01, FNA, GPF, HDH, KTZ, SF!, UTF, W\&W, E/W

Silene

(Identification sources:

Silene acaulis (L,) Jacq, - FNA

[Silene acaulis (L.) Jacq. var, subacaulescens (F. N. Wms.) Femald \& H. St John] - KTZ, UTF, EW

[Silene acaulis L. subsp. subacaulescens (F. N. Wms.) C. L. Hitchc. \& Maguire] - HDH, SF!

[Silene acaulis (L.) Jace. subsp. sabactulessens (F. N. Wms.) Ferrald \& H. St. John] - W\&W

[Silene acaulis (I.) L. subsp. subactulesoens (F. N. Wms.) C. L. Hitche. \& Maguire] - W\&W'

Silene antirnhina L. - Dom01, FNA, GPF, HDH. KTZ, SE!. UTF, W\&W, EN

$\{*$ Silene conoidea L. $\}$ - KTZ

* Silene csertii Baumg. - Dorn01, FNA, GPF, KTZ, SF!, W\&W, EW

* Silene dichotoma Ehrh, subsp. dichotoma - FNA

[Silene dichotoma Ehrh.] - Dorno1, GPF, KTZ, SF!, W\&W, E/W

Silene drummondii Hook. subsp. drummondii - FNA

[Gastrolychnis drummondii (Hook.) A. Love \& D. LOWe]-SF!. W\&W

[Lychnis drummondii (Hook.) S. Watson] - HDH, UTF

[5 ilene drummondit Hook.] - GPF

[Silene drummondii Hook. var. kruckebcrgii Bocquet] - KTZ

[Silene drummondii Hook. var. Arummondii - KTZ, EW

Silene drummondii Hook. subsp. striata (Rydb.) J. K. Morton - FNA

[Gastrolychnis drumenondi (Hook.) A. Lõve \& D. LB̉ve]-SF!. W\&W

[Lychnis drummondir (Hook.) S. Wasson] - HDH, SF, UTF

[Silene drummondii Hook. var. striata (Rydb.) Bocy.-KTZ, E/W

\{* Silene gallica L, - KTZ, SFE, W\&W, E\}

Silene hitchguirei Bocq. - Dorn0I, FNA, E/W

[Gastrolyctonis apetala (L.] Tolm. \& Kozhanch. subsp. uralensis A. Love \& D. Love] - SF!

[Gastrolychnis apetala (L.) Tolm. \& Kazhanch. subsp. uralensis (Rupr.) A. Love \& D. Love] - W\& W

[Lychnis apetala L. subsp. mostana (S. Wacson) Maguife] - HDH

[Lychnis apetala L, var, montana (S. Watsan) C, L. Hitche] - UTF

[Silene uralensis (Rupr,) Bocquet subsp, montana (S. Wasson) MeNeill] - KTZ

[Silene uralensis (Rugr,) Bocquet subsp, uralensis] - KTZ

Silene kingii (S. Watson) Bocq. - Dom01, FNA, KTZ, E/W

[Gastrolyctonis kingil (S. Wasson) W. A. Weber] - SF], WRW

[Lychnis apetala L. var. kingi (S. Watson) S. L. Welsh] - UTF

[Lychnis kingii S. Wascon] - HDH

* Silene latifolia Poir. - Dorn01, FNA

[Lychnis alba Mill.] - HDH. UTF

[Melandriam dioicum (L) Cosson \& Germain] - SFt, W.\&W

[Silene latifolia Poir, subsp, alba (Mill.) Greater \& Burdet] - KTZ, EW

[Silene pratensis (Raft) Godr. \& Gern.] - GPF

Silene menziesii Hook, - FNA, HDH, UTF

[Anotites alsinoides Greene] - FNA

[Anotites bakeri Greene] - FNA

[Anotites costata Gresne] - FNA

[Anotites dobilis Ginesse] - FNA

[Anotites diffusa Gresne] - FNA 
[Anotites dorrii (Kellogg) Greene] - FNA

[Anotites ellipticat Greene] - FNA.

[Anotites halaphila Greene] - FNA

[Anotites latifolia Greene] - FNA

[Anotites macilenta Greene] - FNA

[Anotites menziesii (Hook.) Greene] - SF!, W\&W

[Anotitos nodosa Greene] - FNA

[Anotites picta Greene] - FNA

[Anotives tenerrima Greeme] - FNA

[Anotites tereticaulis Groene] - FNA

[Anotites villosula Greente] - FNA

[Anotites viscosa Greene]- FNA

[Silene darii Kelloge] - FNA

[Silene menziosii Hook. subsp. dorii (Kellogg) C. L. Hitche. \& Magaire] - FNA

[Silene menziosii Hook. subsp. menziesii var-menciesii] - KTZ

[Silene menziesii Hook. var, menziesii] - Docn01.GPF, E/W

[Silene menziesii Hook. subsp. menziesii var. viscosa (Greene) C. L. Hitchc. \& Maguire] - KTZ

[Silene menzicsï Hook. var. visesesa (Grecne) C. L. Hitehe. \& Maguire] - Dern01, GPF. EW

[Silene obovata A. E. Porsild] - FNA

[Silene stellarioides Nutt, ex Torr. \& A. Gray] - FNA

* Silene noctiflora L, - Dom01, FNA, GPF, HDH, KTZ, SFE, UTF, W\&W, E

Silene parryi (S. Wats, C. L. Hitche, \& Maguire - Dorn01, FNA

[Lychnis parryi 5 : Wats.] - FNA

[Silene douglasii (S. Wats.) C. L. Hitske, \& Maguine var macounii (S. Wats.) Hooker - FNA

[Silene douglasii Hooker var, macounii (S, Wais.) B. L. Robins.] - KTL

[Silene macovani S, Wass.] - KTZ

[Silene scoukeri (S. Wats.) var, macounis Bojvin] - KTZ

[Silene tetoenens is A. Nels.] - KTZ

[Silene teiragyna Suksdorf] - FNA

[Wahlbergella parryi (Wats.) Rydb,] - KTZ

Silene plankii C.L. Hitchoock \& Macguire - KWA, M\&H, KTZ, (not rep. by JM)

Silene seaposa B. L. Rob, - FNA

[S ilene scaposa var. lobata C. L. Hitche. \& Maguire] - FNA

Silene scouleri Hook. subsp. hallii (S. Watson) C. L. Hitchc. - HDH, KTZ, UTF, E/W

[Lychnis elara S. Wass.] - FNA

[Silene hallii S. Wats. - FNA

[Silene concolor Greene] - FNA

[Silene scouleri Hook, var, concolor (Greene) C. L. Hitche. \& Maguire] - FNA

[Silene scouleri Hook, subsp. hallii (S. Watson) C. L. Hitctic. \& Maguire]-SFI, W\&W

Silene uralensis (Ruprecht) Bocquet subsp, uralensis - FNA

[Gastrolychinis uralensis (Rups.) Bocxuet ] - FNA

* Silene vulgaris (Moench) Garcke - Dorn01, GPF, KTZ, SF!, W\&W, E/W

[Behen vulgaris Moench] - FNA

[Silene cucubalus Wibel]- $\mathrm{HOH}$

[Silene inflase Smith] - FNA

[Silene latifolia (Milice) Britten \& Rendle var, pubescens (DC.) Farwell] - FNA

* Spergula arvensis L. - Dorn01, FNA, GPF, HDH, KTZ, SFE, W\&W, E

† [Spergularia echinosperma (Celak.) Aschers. \& Gracbn.; - Dorn01

\{Spergularia salsuginea var bracteata B, L. Robins.\} - KTZ

4 Spergularia salina J. Presl \& C. Presl - FNA, KTZ

[Spergularia marina (L.) Griseb.] - Dorn01, GPF, HDH, SFE, UTF, W\&W, E

[Spergularia tenuis Greene] - FNA 
[Tissa marina (L.) Britton] - FNA

* Spergularia media (L.) C. Prest ex Griseb. - Dom01, E, FNA

[Spergularia maritima (AlL) Chiov.] - KTZ

[Spergularia media (L.) C. Presi] - HDH, SFE, UTF, W\&W

* Spergularia rubra (L.) J. Presl \& C. Presl - Dorn01, FNA, GPF, KTZ, UTF, W \&W, EW

[Spergularia rubra (L) Presl] - HDH, SF!

Spergulastrum (see Arenaria)

Stellaria

(Identification sources:

Stellaria (see also Pseudostellaria)

Stellaria borealis Bigelow subsp. borealis - FNA, HDH, KTZ, W\&W, Morton89

[NS - see FNA S:101 for additional synonymy]

[Svellaria boreal is Bigelow] - Dom01, HDH

Stellaria calycantha (Ledeb,) Bong. - Dorn01, FNA, GPF, KTZ, SF!, W\&W, Morton89, E/W

[Arenaria calycantha Ledeb.] - FNA

[Alsine calyeinthin (Ledeb.) Rydb.] - FNA

[Alsine simeoei Howell] - FNA

[Stellaria borealis Bigelons var, simeoei (Howeil) Fernald] - FNA

[Sterllaria simecei (Hoxell) C. L. Hitche.] - FNA

Stellaria crassifolia Ehrh, - Dom01, FNA, GPF, HDH, SF!, W\&W, E/W

[Stellaria crassifolia Ehth. var, crassifolia] - KTZ

[Stellaria crassifolia Ehth. var, eriocalycina Schischkin] - FNA

[5tellaria crassifolia Ehcth, var, linearis Fenzl] - FNA

[Stellaria gracilis Rictardson] - FNA

* Stellaria graminea L, - FNA, GPF, HDH, KTZ, SFE, W\&W, E

[Alsine graminea (L.) Brition] - FNA

Stellaria irrigua Bunge - FNA, KTZ, SF!, W\&W, E/W

[Alsine polygonoides Greeve ex Rydb،, 1913, non Moeluringia polygonoides Mert. \& W. D. J.

Koch, 1813] - FNA

[NS - see coenteents in FNA Vol. 5, p. 107 reganding uncertainty of distinctinxss of Colorado material]

Stellaria longifolia Muhl. ex Willd. - Dorn01, GPF, HDH, W\&W, Morton89, E/W

[Alsine longifolia (Mubl. ex Willd.) Brition] - ENA

[Stellariatatrata (J. W. Moore) B. Boivin] - FNA

[Stellaria atrata (J. W. Moore) B. Boivin var, eciliata B. Boivin] - FNA

[Stellaria diffusa willd. ex Schlect.] - FNA

[Stellaria longifolia Torr.] - UTF

[Stellaria longifolia MuhL.] - SF!

[Stellaria longifolla Muhl. ex Willd. var. eciliana (B. Boivin) B. Boivin] - FNA

[Stellaria longifolia MuhL. ex Willd. var, loegifolia] - KTZ

Stellaria longipes Goldie subsp. longipes - FNA

[NS - For many additional synonyms see KTZ or FNA 5; 108]

[Stellaria longipes Goldie var, longipes - GPF, HDH, UTF, EW

[Stellaria longipes Goldie] - Dorn01, KTZ, SFI, W\&W

[Stellaria monantha Hultén] - Dom01. Chinappas4 \& 91, E/W

* Stellaria media (L.) Vill. - Dom01, FNA, GPF, UTF, E/W

[Alsine media L.] - SFt, W\&W

[Stellaria apetala Ucria ex Roem.] - FNA

[Stellaria media (L.) Cyrill.] - GPF. $\mathrm{HDH}$

[Stellaria media (L.) Vill, subsp, media] - KTZ

Snow, N, January 2009. Checklist of Vaccular Plants of the Southom Rocky Momain Region. CVersion 3 . 
[Stellaria media (L.) Vill. var. procera Klatt \& Richter] - FNA

Siellaria obtusa Engelm. - Dorn01, FNA, HDH, KTZ, SF1, UTF, W\&W, Morton89, EW

[Alsine obtusa (Englem.) Rose]-FNA

[Alsine washingtoniana (B. L. Rob. A A. Heller] - FNA

[Stellaria viridula (Piper) St. John] - FNA

[Stellaria washingteniana B. L. Rob.] - FNA

Stellaria pallida (Dumortier) Crépin - FNA

[Alsine pallida Dumortier] - FNA

[Stellaria boreana Jordan] - FNA

[Svellaria media (L.) Vill. subsp. pallida (Damortior) Ascherson \& Graebner] - FNA

Stellaria umbellata Turcz, - FNA, HDH, SF!

[Stellaria umbellara Turcz, ex Karel. \& Kir.]-Dorn01, KTZ, UTF, W\&W, E/W

Tryphane (see Minuartia)

* Vaccaria hispanica (Mill) Rauschert - Dorn01, FNA, KTZ, EW

[Saponaria vaccaria L.] - HDH

[Vaccaria pyramidata Medicus] - GPF, SF!, UTF, W\&W

Celastraceate - CEL (see also CRO)

Celastrus seandens L. - Dorn01, KTZ

Paxistima myrsinites (Pursh) Raf, - Dom01, IMF, KTZ, SF!, W\&W, E/W

[Pachystima myrsinites (Pursh) Raf.] - HDH, UTF

Celtidaceae (see CAN and ULM)

Ceratophyllaceae - CTP

Ceratophyllum demersum L. - Dorn01, FNA, GPF, HDH, KTZ, SF!, UTF, W\&W, E/W

Chenopodiaceae (see AMA)

Cistaceae - CIS

Crocanthemum (see Helianthemum)

Helianthemum bieknellii Fernald - GPF, HDH, KTZ, E.

[Crocanthemum bickenellii (Femald) Janch.] - SFE, W\& W

$\{$ Lechea intermedia Legg, $\}$ - GPF, $\mathrm{HDH}<\mathrm{NE}>$

(Lechea istermedia Lege ex Britton). Domit

\{Lechea istermedia Legg ex Britton var, intermedia\} - KTZ $\langle$ WY $>$

\section{Clusiaceae - CLU}

Hypericum formosum Kunth var. scouleri (Hook.) J. M. Coult. - Dorn01

[Hypericum formosum Kunth] - HDH, SF, UTF, W\&W 
[Hypericum scouleri Hook. subsp. nortoniax (M. E. Jones) J. M. Gillett] - KTZ

[Hypericum formosum Kunth var. nortoniae (M. E. Jones) C. L. Hitche.] - E/W

Hypericum formosum Kunth var, formosum - Dorn01

Hypericum majus (A. Gray) Britton - GPF, HDH, KTZ, SFE, W\&W, E

*Hypericum perforatum L, - Dom01, GPF, HDH, KTZ, SF!, W\&W, E/W

\section{Colchicacene- CHC}

Disporum trachycarpum (S. Watson) Benth. \& Hook, - GPF, HDH, IMF, UTF, W\&W, EW [Prosartes trachycarpa S. Watson] - Dorn01, FNA., KTZ, SF!, W\& W'

Streptopus amplexifolius (L.) DC, - Dom01, FNA, GPF, W\&W', misapp., E/W

[NS - Vimersten07 refers Streptopus back to a narrowly defined Liliaceac]

[Strcptopus amplexifolius (L.) DC. \& Lam.] - HDH

[Streptopus amplexifolius (L.) DC. var. chalazatus Fassett] - IMF, KTZ, UTF

[Streptopus farsettii A. Löve \& D. Löve]-SF!, W\&W

\section{Commelinaceae - CMM}

Commelina dianthifolia Delile - FNA, HDH, SF!, W\&W, EN

[Commelina đianthifolia Delile var. Longispatha (Torr.) Brashier] - KTZ

Commelina erecta L. - FNA. E

[Commelina erecta L. var, anguslifolis (Michx, ) Fernald] - GPF, KTZ, SFE, W \& W

[Commetina crispa Wooton] - HDH

Tradescantia bracteata Small - FNA, HDH, exp.

[NS - repertedly collected in Chero County is 2008 from reliable source]

[Tradescantia bracteata Small ex Brition] - KTZ <KS, NE. OK, WY?

\{Tradescantia canaliculata Raf, \} - HDH, exp.

\{Tradescantia ohiensis Raf? - FNA, KTZ KS, NE, OK>

Tradescantia occidentalis (Britton) Smyth var. occidentalis - FNA. HDH, IMF, KTZ, E/W

[Tradescantia oxcidentalis (Britton) Smyth] - Dorn01, GPF, SF1, UTF

[Tradescantia oceidentalis (Beitton) Smyth var, scopulorum (Rose) E. S. Asderson \& Woodson] - HDH rep. IMF, KTZ, W\&W

\section{Compositae (see AST)}

Convallariaceae-CVL (see LIL)

Convolvulaceae - CNV (including Cuscutaceae)

(Identification sources:

Calystegia macounii (Greene) Brummitt - Dorn01, GPF, KTZ, SFE, W\&W, E

Calystegia sepium (L.) R. Br. var. angulata (Brummitt) N. H. Holmgren - Dorn01. IMF, E/W

[Calystegia sepium (L) R. Br, subsp. americana (Sims) Brummitt] - KTZ, SFW

[Calystegia sepium (L.) R, Br, subsp. angulata Brummitt] - GPF, KTZ, SFE, W\&W

[Convolvulus sepium L.]-HDH, UTF

[Convolvulus sepium L, var, americanes Sins] - HDH, rep.

\{Calystegia silvatica (Kit.) Griscb. subsp. fraterniflora (Mack. \& Bush) Brummitt\} - KTZ 
\{Calystegia sylvatica (Kit.) Griseb. subsp. fraterniflora (Mack. \& Bush) Brumenitt\} - GPF

Convolvulus (see also Calystegia)

* Convolvulus arvensis L. - Dorn01, GPF, HDH, IMF, KTZ, SF1, UTF, W\&W, EW

Convolvulus equitans Benth، - GPF, KTZ, SFE, UTF, W\&W, E

[Convolvulus incanus Vah]] - $\mathrm{HDH}$

\{Convolvulus hermannioides A. Gray\} - HDH, rep.

\{Convolvulus equitans Benth.\} - KTZ, W\&W

Cuscuta

(Identification sources:

* Cuscuta approximata Bab, var, urceolata (Kuntze) Yunck, - HDH. E/W

[Cuscuta anthemi A. Nelson] - HDH

[Cuscuta spproximata Bab.]-Derno1, KTZ UTF

[Cuscuta approximata Bab. var. approximata] - IMF

[Cuscuta tpithymum (L.) L. subsp. approximata (Bab.) Rouy] - SF!, W\& W

Cuscuta californica Hook. \& Am. var, breviflora Engelm. - KTZ, W

[Cuscuta occidentalis Millspaugh] - Dom01, HDH, IMF, UTF

[Grammica ocxidentalis (Millspraugh) Hadac \& Chrtek] - W\& W

[Grammica occidentalis (Millspraugh) W.A. Weber]-SFW

\{Cuscuta cephalanthi Engelm, - GPF, HDH, exp, IMF, KTZ $\angle A Z, K S, N E, N M, O K, U T>$, UTF

\{Grammica oephalanthi (Engelm.) W. A. Webar\} - W\& W

\{Cuscuta coryli Engelm.\} - GPF, HDH, exp., KTZ<AZ, KS, NE, NM, OK>

\{Grammica coryli (Engelm.) W. A. Weber\} - W\&W

Cuscuta cuspidata Engelm. - GPF, HDH, IMF, KTZ, UTF, E/W

[Grammica cuspidata (Engelm.) Hadac \& Chrtek] - SH], W\&W

Cuscuta denticulata Engelm. var. denticulata - KTZ, W

[Cuscuta denticulaa Engelm. - HDH, IMF, UTF

[Grammica denticulata (Engelm.) W. A. Weber] -SFW, W\&W

\{Cuscuta gronovii Willd. ex Schult, var, gronovii\} - KTZ

\{Cascuta gronovii willd - GPF, HDH, rep.

\{Grammica grosovii (Wils. ex Roem. \& Schult.) Hadec \& Chntek\} - WS W, misapp.

(Grammica umbrosa (Hook.) W. A. Weber) - W\&W

Cuscuta indecora Choisy var. indecora - KTZ, E/W

[Cuscuta indecora Chossy]-GPF, IMF, UTF

[Grammica indecora (Choisy) W. A. Weber]-SF], W\&W

Cuscuta indecora Choisy var. neuropetala (Engelm.) Hitchc. - HDH, KTZ, E

[Cuscuta indecora Chobsy] - Dom01, IMF, UTF

[Grammica indecora (Choisy) W. A. Weber var, neuropetala (Engelm.) W. A. Weber]-SFE. Wo W

- Cuscuta fasciculata Yunker - KWA, KTZ

Cuscuta megalocarpa Rydb. - Dorn01, HDH, IMF, KT7, UTF, W

[Cuscuta curta (Engeim.) Rydb] - GPF

[Grammica umbrosa (Hook.) W. A. Weber] - SFW, W\&W

Cuscuta pentagona Engelm. var. pentagona - FCF, IMF, KTZ, W

[Cuscuta campestris Yuncker] - HDH

[Cuscuta pentazona Engelm.]-GPF, HDH rep_UTF

[Cuscuta pentugona Engelm. var, calycina Engelm.] - IMF

[Grammica campestris (Yuncker) Hadac \& Chrtek] - SFW, W\&W

[Grammica pentagona (Engelm.) W. A. Weber]- W\&W, err, rep.

Cuscuta plattensis A. Nelson - Dorn01 
Cuscuta umbellata Kunth - GPF, HDH, KTZ, E/W

[Grammica umbellata Kunth] - SFE

[Grammica umbellata (Kunth) Hadaç \& Chrtek] - W\&W

[Grammica umbellata (Kunh) W. A. Weber] - SFW

Evolvulus nuttallianus Schult - Dorn01, KTZ, UTF, W\&W. E/W

[Evolvulus nuttallianus Roem. \& Schult.] - GPF, HDH, IMF, SF!

Evolvulus sericeus Sw, var. sericeous -KWA, KTZ

[Evolvulus sericeus var, discolor (Benth.) Gray] - KTZ.

[Evolvulus wilcoxianes House] - KWA

Grammica (see Cuscuta)

Ipomoea capillacea (Kunth) G. Don - KWA, KTZ

[Convolvulus capillaceus Kunth ] - KTZ

[Iponoea muricata Cav., bon (L.) Jacq. ] - KTZ, KWA

[lpomoea patens (Giray) House] - KWA

Ipomea cristulata $\mathrm{H}$, Hallier $\mathrm{f}$ - $\mathrm{KWA}, \mathrm{KTZ}$

[Iponaiea hederacea (L.) Jacq.] - HDH, exp.

[lpomoea hederacea Jacq.] - GPF, KTZ <AZ, KS, NE, NM, OK ?

Ipomoea leptophylla Torr, - Dom01, GPF, HDH, KTZ, SFE, W\&W, E

\{ Iponoea longifolia Benth.\} - M\&H, err. rep., HDH, exp., KTZ $\triangle A Z$ ?

* Ipomoea purpurea (L.) Lam, - KWA, JM, KTZ, HDH, rep.

[lpomoea parpurea L.] - W\&W', rep.

[lpomoea purpurea (L) Roth] - GPF, KTZ CAZ, KS, NE, NM, OK, UT?

\section{Coptaceae (see RAN)}

\section{Cornaceae - COR}

\section{Chamaepericlymenum (sec Cornus)}

Comus canadensis I. var, canadensis - E/W

[Clumacpericlymenum canadense (L) Asch \& Grachner] - SF, W\&W

[Comus canadensis L.] - Dorn01, GPF, HDH, KTZ

Comus sericea L, var, sericea - Dorn01, IMF, UTF, E/W

[Comus stolonifera Michx.] - GPF, HDH

[Comus sericea L- subsp. sericea] - KTZ

[Swida sericea (L.) Holub.] - SFt, W\&W

\section{Swida (see Cornus)}

\section{Corylaceate (sce BET)}

\section{Crassulaceac - CRS}

\section{Amerosedum (see Sedum)}

Clementsia (sec Sedum) 


\section{Cockerellia (see Sedum)}

Crassula drummondii (Torr \& A, Gray) Fedde - KTZ, E

[Crassula aquarica (L.) Schōnland] - IMF. SFE, W\& W

[Tillaea aquatica L.] - HDH. UTF

\section{Rhodiola (see Sedum)}

* Sedum acre L. - Dorn01, HDH, IMF, KTZ, SFW, W\&W, W

Sedum cockerellii Britton - Legler (pers, comm. 2009), HDH rep., KTZ <AZ, NM >

[NS - Legler reports one collection from Colfax Co., NM]

[Cockerellia cockerellii (Britton) A. LËve \& D. Live] - W\&W, id. not possible

Sedum integrifolium (Raf.) A. Nelson - Dom01, HDH, E/W

[Rhodiolat integrifolia Raf.] - SF!, W\& W

[Rhodiola integrifolia Raf. subsp. integrifolia] - KTZ

[Rhodiola integrifolia Raf. subsp. procera (Clausen) Kartesz] - KTZ

[Sedum integrifolium (Raf.) A. Nelson sabsp. inlegrifolium] - RMC

[Sedum rosea (L.) Scop. var. integrifolium (Raf.) A. Berger] - IMF, UTF

[Tolmachevia integrifolia (Raf) A. Lồve \& D. Löve] - W\&W'

Sedum lanceolatum Torr, - Dorn01. GPF, IMF, UTF, E/W

[Amcrosedum lanceolatum (Torr.) A. Love \& D. Love]-SF!. W\&W

[Sedun lanceolatum Torr. subsp. Ianceolarum] - KTZ

[Scdum lanscolanum Torr. var. lanceolatum] - RMC

[Sedum lanceolalum Torr. subsp. subalpinum (Blank.) Clausen] - KTZ

[Sedum stenopetalum Pursh] - $\mathrm{HDH}$

Sedum thodanthum A. Gray - Dorn01, HDH, IMF, KTZ, UTF, EJW

[Ckmensia rodantha (A. Girsy) Rose] - SFI, W\&W

[Rhodiolat rhodantha (A. Gray) Jacobsen] - KTZ

* Sedum spurium M. Bieb, - IMF, KTZ, UTF, W

[W] - A posulation also established on East Slope in $\mathrm{CO}$ in Boulder canyon]

[Spathelata spuria (M Bieb. ) A. LDve \& D. LOSe]-SFW, W\&W

\{Sedum stenopetalum Pursh\} - IMF, KTZ <WY>

\{Amerosedum sconopecalum (Pursh) A. Lowe \& D. Love\} - WNW, err, rep.

\{Sodurb stenogetalum Pursh var. stenopetalum\} - RMC

Spathulata (sce Sedum)

Tillaea (see Crassula)

Crossosomataceae - CRO (see also CEL)

Forsellesia meionandra (Koehne) A. Heller - HDH, FCF, SFW, UTF, W\&W, W

[Glossopetalon spinescens A. Gray var, meionandram (Koeline) TreL.] - IMF, KTZ

Forsellesia planitierum Ensign - HDH, exp.. SFE, W\&W. E

[Glossopetakon planitienum (Ensign) H. St. John] - GPF, KT2

\{Forsellesia spincscens (A. Gray) Greene\} - HDH rep., W\&W, err. rep.

\{Glossopetalon spinescens A. Gray var, spinescens\} - KTZ $<A Z, N M, O K>$

Glossopetalon (see also Forsellesia)

Glossopetalon spinescens Gray var. meionandrum (Koehne) Trel. - Dorn01

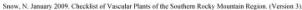




\section{Cruciferae (see BRA)}

\section{Cucurbitacene - CUC}

Cucurbita foetidissima Kunth - Dom01, GPF, HDH, KTZ, SF!, UTF, W\&W, E/W

Cyclanthera dissecta (Torr. \& A. Gray) Arn. - GPF, HDH, KTZ, SFE, W\&W, E

Echinocystis lobata (Michx.) Torr. \& A. Gray - Dorn01, GPF, HDH, KTZ, SF!, W\&W, E/W

Cuscutaceae (see CNV)

Cyperacene - CVP

(Identification sources:

Amphiscirpus nevadensis (S. Watson) Oteng-Yeb, - Dorn01, SF!, W\&W, E/W, FNA

[Scirpus nevadensis S. Watsoe] - GPF, HDH, IMF, KTZ, UTF

Bolboschoenus fluviatilis (Torr.) Sojak - FNA

[Bolbaschoenus maritimes (L.) Palla subsp. fluviatilis [Tort.) A. Lõve \& D. Lōve] - W\& W]

[Schoenoplechus floviatilis (Torr.) M. T. Strong] - KTZ

[Scirpus fluviatilis (Torf.) A. Gray]-GPF, IMF, UTF

Bolboschoenus maritimus (L.) Palla subsp, paludosus (A. Nelson) A. Löve \& D. Löve - SF!, W\&W, E/W

[Bulboschoenus maritimus (L.) Palla subsp. paludosus (A. Nelson) T. Koyama] - FNA

[Bulboschoenus maritimes (L.) Palla var. paludosus (A. Netson) Doen] - Dom01

[Schoenoplectus maritimus (L.) Lye] - KTZ

[Scirpus maritimus L.] - UTF

[Scirpus maritimus L. var. psludosus (A. Nelson) Kiik.] - GPF, IMF

[Scirpus paladosus A. Nelson] - HDH

Carex

(Identification sources:

Carex albonigra Mack. - Dom01, IMF, KTZ, SF!, UTF, FNA, E/W

[Carex albo-nigra Mack.] - HDH, W\& w

Carex aquatilis Wahlenb, var. aquatilis - KTZ, FNA, E/W

[Carex aguatilis Wahlenb.] - Dom01, HDH, IMF, SF1, UTF

[Carex aquatilis Wahlenb, var, altior (Rydb.) Ferrald] - GPF

[Carex aquatilis Wahlenb. subsp, aquatilis] - W\&W

Carex aquatilis Wahlenb, var. stans (Drejer) Boott - KTZ, E

[Carex aquatilis Wahlenb, subsp, stans (Drejer) Hultén] - SFE, W\& W

[Carex aquatilis Wahlenb, var, minor Boott] - FNA

Carex arapahoensis Clokey - HDH, IMF, KTZ, FNA, SF!, UTF, W\&W, E/W

Carex atherodes Spreng, - Dorn01, GPF, HDH, IMF, KTZ, SF!, UTF, W\&W, E/W

Carex athrostachya Olney - Dorn01, GPF, HDH, IMF, K1Z, SF!, UTF, W\& W, E/W 
Carex atrata L. var, chalciolepis (Holm) Kok, - Dom01, IMF, E/W

[Carex chalciolepis Holm] - HDH, SF!, W\&W

[Carex heteroneura Boott var. chalciolepis (Holm) F. J_ Herm.] - KTZ, UTF

Carex atrata L. var, erecta Boot - Dorn01, IMF, E/W

[Carex atrata L.] - HDH, SF!, W\&W

[Carex epapillosa Mack.] - SF!, W\&W, FNA

[Carex heteruneura Bosti] - FNA

[Carex heteroneura Boott var, epapillosa F. J. Herm.] - UTF

[Carex beteroneura Boott var. epapillosa (Mack.) F. J. Herm.] - KTZ

Carex atrosquama Mack. - KTZ, SF!, UTF, FNA, W\&W, E/W

Carex aurea Nutt, - Dom01, GPF, HDH, IMF, KTZ, FNA, SF!, UTF, W\&W, E/W

Carex backii Boott var, backii - E, Saarela0]

[Carex backii Boott] - Dorn01, GPF, IMF, KTZ, SFE, UTF, W\&W, FNA

Carex bebbii (L. H. Bailey) Fernald - GPF, SFE, W\&W, EW

[Carex bebbii Olney] - SFW

[Carex bebbii (L. H. Bailey) Olney ex Fernald] - Domo1. FNA

[Carex bebbii Olney ex Fernald] - HDH, IMF. KTZ, UTF

Carex bella L. H. Bailey - GPF, HDH, IMF, KTZ, FNA,SF!, UTF, W\&W, EW

Carex bipartita All. - Dom01, TMF, UTF, E/W

[Carex lachenalii Schkuht] - KTZ, SF!, W\& W, FNA

Carex brevior (Dewey) Mack, ex Lunell - Dorn01, GPF, HDH, FNA, IMF, E

[Carex breviof (Dewey) Mack.] - KTZ, SFE, w\& W

Carex breweri Boott var, paddoensis (Suksd.) Cronquist - Dom01, IMF, UTF, E/W

[Carex engelmannii L. H. Bailey] - HDH, KTZ, SF!, W\&W, FNA

Carex brunnescens (Pers.) Poir. subsp. brunnescens - FNA

[Carex brunsescens (Pers,) Poir, subsp. sphakrostachya (Tuck,) Kalela] - FNA, KTZ, E/W

[Carex brundescens (Pers.) Poir.] - Domol, GPF, HDH, IMF, SF!, UTF, W\&W

Carex buxbaumii Wahlenb, - GPF, HDH, IMF, KTZ, FNA, SF!, UTF, W\&W, E/W

Carex canescens L. var. canescens - E/W

[Carex cantescins L.] - Dom01, HDH, IMF, SF!. UTF, W\& W

[Carex cantescens L. subsp. canescens] - KTZ. FNA

Carex capillaris L. - Dorn01, HDH, IMF, KTZ, SF!, UTF, FNA, W\&W, E/W

[Carex capillaris L. var. elongata Olney] - GPF

Carex capitata L. var. arctogena (H. Sm.) Hultén - E/W

[Carex capitata L. sulsop, arctogena (H. Sm.) Hitonen] - KTZ

[Carex capitata L.] - Dorn01, HDH, exp. IMF, UTF, FNA

[Carex capitata L. subsp. aretogena (H. Sm.) Bscher] - SF!, W \& W

Carex concinna R. Br. - Dom01, GPF, HDH, KTZ, FNA, SF!, W\&W, E/W

Carex conoidea Willd.

[NS - confirmed from S. Poporich basod on det, by T, Reznicok; wouchers at COLO, CS, KHD, MICH. RM]

[Carex katahdinensis Femald] - FNA

Carex cordillerana Saarela and B. A. Ford - Saarela01

Carex crawei Dewey - Dorn01, GPF, IMF, KTZ, FNA, SFE, UTF, W\&W, E

Carex deweyana Schwein. var, bolanderi (Olney) Boott - IMF, UTF

[NS - needs cofimation from SRMF region in NM]

[Carex bolanderi Olney] - KTZ $\angle A Z, N M, U T, W Y>$ FNA

Carex deweyana Schwein. var, deweyana - Hartman06, IMF, KTZ, FNA, E/W

[Carex deweyana Schwein.] - GPF, HDH, SFt, W\&W

Carex diandra Schrank - Dorn01, GPF, HDH, IMF, KTZ, SF!. UTF, W\&W, E/W

Carex disperma Dewey - Dom01, GPF, HDH, IMF, KTZ, SF!, UTF, W\& W. E/W

Carex douglasii Boott - Dorn01, FNA, GPF, HDH, IMF, KTZ, SF!, UTF, W\&W, E/W

Carex ebenea Rydb. - HDH. IMF. FNA. KTZ, SF!. UTF, W\&W. E/W 
Carex echinata Murray subsp, echinata - FNA, KTZ, ENW

[Carex angustior Mack.] - HDH, SF!, W\& W

[Carex echinata Murray] - Domv01, W\&W

[Carex muricata L.] - IMF, UTF

Carex egglestonii Mack, - Dorn01. HDH, IMF, KTZ, FNA, SF!, UTF, W\&W, EWW

Carex elynoides Holm - Dom01, HDH, IMF, KTZ, FNA, SF!, UTF, W\&W, E/W

Carex emoryi Dewey - Dorn01, GPF, HDH, KTZ, FNA, SF!, W\&W, EW

Carex exsiccata L. H. Bailey - KTZ, SF1, W\&W, FNA, EN

[Carex vesicaria L. var. vesicaria] - IMF, UTH

\{Carex festucacea Schkuhr ex Willd. - KTZ $<$ KS, OK> FNA, W\&W', misid.

fCarex festucacea Schkuhr\} - GPF. W\&W

Carex filifolia Nutt - Dorn01, GPF, HDH, IMF, KTZ. FNA. SF!, UTF, W\&W, EW

\{Carex foenes Willd var, foenea\} - E/W

\{NS - name has been misapplied to our material: see FNA p. 307 for this and var. tuberculata\}

\{Carex siccata Dewey\} - HDH, IMF, KTZ, UTF

\{Carex foenca Willd.; - Dornol, GPF, SF!, Wow

\{Carex foenea Willd, var, tuberculata F, J, Herm.\} - PLS

\{Carex foenea Willd.\} - GPF, W \&W

\{Carex siccata Dewey\}-IMF, KTZ, UTF

Carex foetida All, var, vernacula (L. H. Bailey) Kük, - Dorn01. IMF, UTF, E/W

[Carex vernacula L. H. Bailey] - HDH, KTZ, SF!. W\& W, FNA

\{Carex garberi Fernald\} - KTZ $\langle$ WY $>$, FNA

\{Carex aurea Nuth. - IMF, UTF

Carex geyeri Boott - Dorn01, HDH, IMF, KTZ, FNA, SF!, UTF, W\&W, E/W

\{Carex gravida L. H. Bailey var, gravida\} - GPF, KTZ

\{Carex gravida L. H. Bailky\} - FNA

Carex gravida L. H. Bailey var. lunelliana (Mack.) F. J. Herm. - GPF, HDH, KTZ, SFE, W\&W, FNA, E

[Carex gravida L. H. Bailey] - FNA

Carex gynocrates Wormsk. ex Drejer - Dorn01, FNA, HDH, KTZ, E/W

[Carex dioica L. subsp. gynocrates (Wormskiold) Hultén] - SF], W\&W

[Carex dioica L. var, gynocrates (Wornskiold) Ostenf] - IMF, UTF

Carex hassei L. H. Bailey - FNA $<C O ?>, \mathrm{HDH}, \mathrm{KTZ}<\mathrm{NM}, \mathrm{UT}>, \mathrm{SF}$ !, W\&W, E/W

[Carex aurea Nutt.] - IMF, UTF

Carex haydeniana Olney - Dom01, HDH, IMF, FNA, KTZ, SF!, UTF, W\&W, E/W

\{Carex heteroneura W. Boott var, brevisquama F. J. Herm., pubL. without Latin dese. or typification; - KTZ

\{Carex atrata L. - IMF, SF

¿Carex atrata L var, erecta Bootr\} - RMC

Carex hoodii Boott - Dom01, GPF, HDH, IMF, KT7, FNA, SF!, UTF, W\&W, E/W

Carex hystricina Muhl. ex Willd. - Dom01, GPF, FNA, HDH, IMF, KTZ, UTF, W\&W, E/W [Carex hystricina Muhl.] - SF!

Carex illota L. H. Bailey - Dom01, HDH, IMF, KTZ, FNA, SF!, UTF, W\&W, E/W

Carex incurviformis Mack. var. danaensis (Stacey) F. J. Herm - Dorn01, KTZ

[Carex incurviformis Mack.] - FNA

Carex interior L. H. Bailey - Dorn01, GPF, FNA, HDH, IMF, KTZ, SF!, UTF, W\&W, E/W

[Carex seirpoides Schkuhr ex Willdenow] - FNA

Carex jonesii L. H. Bailey - Dorn01, HDH, IMF, KTZ, FNA, SF!, UTF, W\& W, E/W

Carex lacviculmis Meinsh. - KTZ, FNA, SF!, W\&W, E/W

Carex lanuginosa Michx. - GPF, HDH, IMF, SF!, UTF, W\&W, E/W

[Carex pellita Mubl. ex Willd.] - Dorn01, KTZ 
[Carex lasiocarpa Ehrh.] - FNA

Carex lasiocarpa Ehrh. - Doen01, FNA, SFE, UTF, W\&W, E

[Carex lasiocirps Ehrh. var. americana Fernald] - GPF, KTZ

Carex lenticularis Michx. var, pallida (Boott) Dorn - Dorn01, EN

[Carex kelloggii Boctt] - HDH

[Carex lenticularis Michx.] - UTF

[Carex lenticularis Michx. var. lenticularis] - DMF

[Carex lenticularis Michx. var. lipocarpa (Holm) L. A. StandL] - FNA, KTZ SF!, W\&W

Carex leporinella Mack, - FCF, FNA

Carex leptalea Wahlenb, subsp, leptalea - KTZ, E/W

[Carex leptalea Wahlenb.] - DornD1, GPF, FNA, HDH, IMF, SF!, UTF, W'SW

Carex leptopoda Mack, - KTZ, FNA

[Carex deweyana Schwein. yar. leptopoda (Mack.) Boivin] - PLS

[Carex deweyana Sehwein.] - SF!

[Carex deweyana Schwein. var. bolanderi (Olney) W. Bocet] - UTF

[Carex deweyana Schwein. subsp. leptopoda (Mack.) Calder \& Taylor] - IMF

Carex limosa L. - Dom01. FNA. GPF, HDH rep.. IMF, KTZ. SF!, UTF, W\&W, EW

Carex livida (Wahlenb, Willd, var, radicaulis Paine - KTZ, FNA, E

[Carex livida (Wahlenb.) Willd.] - SFE, W\&W

Carex macloviana D'Urville - Dorm01, FNA, SF!, W\&W, E/W

[Carex subfusca W, Boott] - KTZ

Carex maritima Gunn, - KTZ, SF!, W\&W, FNA <Canada>, ENW

Carex microglochin Wahlenb, - Dorn01, HDH, KTZ, SF!, UTF, W\&W, E/W

[Carex mikroglochin Wahlenb, subsp. microglochin] - FNA

Carex microptera Mack, var. limnophila (F. J. Herm.) Dorn - Dorn01, E/W

[Carex limnophila F. J. Herm.] - IMF, SFL, UTF, W\&W

[Carex microptera Mack.] - KTZ. FNA

Carex microptera Mack, var, microptera - E/W

[Carex festivella Mack.] - HDH, SF!, W\& W

[Carex microptera Mack.]-GPF, IMF, FNA, KTZ, SFI, UTF, W\&W

Carex misandra R. Br. - Dom01, HDH, IMF, KTZ, SF!, UTF, W\&W, E/W

[Carex fuliginosa Schkuhe] - FNA

Carex molesta Mack, ex Bright - KTZ, E

[Carex molesta Mack.] - GPF, SFE, W\&W

Carex nardina Fr. var. hepbumii (W. Boott) Kok. - KTZ, E/W

[Carex hepbarnii Boost] - HDH, W\& W'

[Carex nardina Fr.] - Domol, IMF, UTF, FNA

[Carex nardina Fr. subrsp. hepburni (Bootr) A. Löve \& D. Lóve] - SF!, W\& W

Carex nebrascensis Dewey - Dorn01, FNA, IMF, KTZ, SF!, UTF, W\&W, E/W

[Carex nebraskensis Dewey] - GPF, HDH

Carex nelsonii Mack. - Dorn01, HDH, KTZ, FNA, SF!, UTF, W\&W, E/W

[Carex estesiana Kelso] - FNA

[Carex nova L. H. Bailey] - Dorno1 (as distinet form C. nelsonii), IMF

Carex neurophora Mack. - Dom0I, HDH, FNA, IMF, KTZ, SF!, UTF, W\&W, E/W

Carex nigricans C. A. Mey. - Dorn01, HDH, FNA, IMF, KTZ, SF!, UTF, W\&W, E/W

\{Carex norvegica Retz, var, norvegica\} - KTZ, FNA <eastern Canada?, W

\{Carex nonegica Hetz subsp. nonegica\} - SFW, W\&W

Carex norvegica Retz. var. stevenii (Holm) Dorn - Dorn01, E/W

[Carex media R. Br, var, steveail (Holm) Fernald] - HDH

[Carex norvegica Retz.] - IMF, UTF

[Carex norvegica Retz, subsp, stevenii (Holm) E. Murray] - KTZ

[Carex norvegica Retz, subsp, stevenii (Holm) D. Murray]-SF!, W\&W

Snow, N. Junury 2009. Checklist of Vascular Plants of the Southom Rocky Mounain Region. CVersion $3 x$ 
[Carex stevenii (Holm) Kalela] - W\&W ${ }^{1}$, FNA

Carex nova L. H. Bailey var. nova - E/W

[Carex nova L. H. Bailey] - Dorn0I. HDH, IMF, KTZ, FNA, SF!, UTF, W\& W'

[Carex nova $A$. Nelson] - W\&W

Carex nova L. H. Bailey var. pelocarpa (F, J. Herm.) Dom - Dom01, E/W

[Carex pelocarpa F. J. Herm.]-KTZ, SF!, W\& W, FNA

[Carex nova L. H. Bailey] - [MF, UTF

Carex obtusata Lilj. - Dorn01, FNA, GPF, HDH, IMF, KTZ, SF!, UTF, W\&W, EW

Carex occidentalis L. H. Bailey - Dorn01, FNA, HDH, IMF, KTZ, SF!, UTF, WEW, E/W

Carex oederi Retz, var. recterostrata (L. H. Bailey) Dorn - E/W

[Carex oederi Retz] - IMF

[Carex ooderi Retz var, viridula (Michx.) Kak.] - see IMF, UTF

[Carex viridula Michix_] - Dorn01, GPF, HDH, SF!, W\&W

[Carex viridula Michx. subsp. viridula] - KTZ, FNA

Carex orecharis Holm - Dorn01, HDH, FNA, KTZ, SFE, W\&W, E

[NS - a 'regional endemic' of $\mathrm{CO}$ and $\mathrm{WY}$, including parts of UT]

Carex pachystachya Cham. ex Steud. - Dorn01, FNA,HDH, IMF, KTZ, UTF, W\&W, EWW

[Carex pachystachya Cham.] - SF!

Carex parryana Dewey var, paryana - Dom01, KTZ

[Carex parryana Dewey] - GPF, IMF, UTF, FNA

[Carex parryana Dewey subsp. parryana] - W\&W

Carex parryana Dewey var, unica L. H. Bailey - Dorn01, E/W

[Carex ballii Oltsoy]-GPF, HDH, KTZ, FNA

[Carex parryana Dewsy] - IMF. UTF

[Carex parryana Dewey subsp. halliti D. F. Murray] - SFW

[Carex parryana Dewey subsp. hallii [Olncy) D. F. Murray] - SFL, W\& W

Carex paupercula Michx, - Dom01, HDH, IMF, UTF, E/W

[Carex magellanica Lam, subsp, irrigua (Sm,) Hultén] - W\&W

[Carex magellanica Lam, subsp, irrigua (Wahlenb.) Hitonen] - FNA

[Carex magellanica Lam. subsp. irrigua (Wahlenb.) Hultén] - KTZ, SF!

Carex paysonis Clokey - Dom01, FNA

[Carex podocarpa var, peysonis (Clokey) Boivin] - KTZ

[Carex tolmei auct nos Boort] - KTZ

Carex peckii Howe - FNA, GPF, KTZ, SFE, W\&W, E

Carex pensylvanica Lam. var. digyna Boeck. - Dorn01, E/W

[Carex beliophila Mack.] - GPF. HDH

[Carex inops L. H. Bailey subsp. heliophila (Mack.) Crins] - KTZ FNA

[Carex pensylvanica Lam. subsp, heliophila (Mack.) W. A. Weber] - SFI, W\&W

Carex perglobosa Mack. - HDH, IMF, FNA, KTZ, SF!, UTF, W\&W, E/W

Carex petasata Dewey - Dorn01, FNA, HDH, IMF, KTZ, SF!, UTF, W\&W, E/W

Carex phaeocephala Piper - Dorn01, HDH, FNA, IMF, KTZ, SF!, UTF, W\&W, E/W

Carex pityophila Mack. - HDH, FNA, KTZ, E/W

[Carex geophila Mack.] - IMF, KTZ, SFI, UTF, W\&W

Carex praceptorum Mack. - Dom01, IMF, FNA, SF!, UTF, W\&W, E/W

[Carex pracceptorium Mack.] - HDH, KTZ

Carex pracgracilis Boott - Dom01, FNA GPF. HDH, IMF, KTZ, SF!, UTF, W\&W, E/W

Carex praticola Rydb. - Dom01, GPF, FNA, HDH, IMF, KTZ, SF!, UTF, W\&W, E/W

Carex pyrenaica Wahlenb. subsp. pyrenaica - KTZ, E/W

[Carex standallii Gand.] - SF. W\&W

[Carex pyrenaks Wahlenb.] - Dorn01, HDH, IMF, UTF, W\& W', misapp.

[Carex micropoda C. A. Meyer] - FNA

Carex raynoldsii Dewey - Dorn01, HDH, IMF, FNA, KTZ. SF!, UTF. W\& W, E/W

Snow, N, Junury 2009. Checklist of Vaccular Plants of the Southom Rocky Momain Region. CVersion $3 x$ 
Carex retrorsa Schwein. - GPF, FNA, HDH, IMF, KTZ, SFW, UTF, W\&W, W

Carex rosea Schkuhr ex Willd. - Hartman06

Carex rossii Boot - Dom01, HDH, IMF, FNA, KTZ, SF!, UTF, W\&W, E/W

[Carex brevipes Boot] - SF!, W\& W

[Carex rossii Bootl ex Hook.] - GPF

\{Carex rostrata Stokes\} - see C. utriculata

Carex rupestris All. - Dom01. FNA, IMF, UTF, E/W

[Carex drummondiana Dewey] - HDH

[Carex rupestris All. subsp. drummondiana (Dewey) Holab] - SF?, W \& W

[Carex rupestris All. var. drummondiana (Dewey) L. H. Bailey] - KTZ.

Carex sartwellii Dewey - Dorn01, FNA, GPF, HDH, SF!, W\&W, E/W

[Carex sartwellii Dewey var, sartwellii] - KTZ

Carex saxatilis L. - Dorn01, IMF, FNA, KTZ, UTF, E/W

[Carex physocarpa C. Presi] - $\mathrm{HDH}$

[Carex X playsocarpioides Lepage] - KTZ

[Carex saxatilis XC, utriculata] - KTZ

[Carex saxatilis L. subsp. laxa (Trautv.) Kalela| - SF!, W\&W

[Carex saxatilis L. var. major Olncy] - see IMF

Carex saximontana Mack. - Dorn01, Sarrela01, FNA

[Carex baskii Boot var. subrostrata (Bates) Dorn] - E

[Carex backii Boott] - IMF, KTZ, UTF, FNA

[Carex saximontana Mack.] - GPF, HDH, KTZ, SFE, WEW, FNA

Carex scirpoidea Michx, var. pseudoscirpoidea (Rydb.) Cronquist - Dom01, IMF, UTF, E/W

[Carex preudoscirpoidea Rydb.] - HDH, wsw

[Carex scirpoidea Michx, subsp. pseudoscirpoidea (Rydb.) Dunlop] - FNA, KTZ, SF!, W\& W'

Carex scirpoidea Michx, var. scirpiformis (Mack.) O'Neill \& Duman - Dorn01, FNA

[Carex scirpoidea Michx.] - GPF

[Carex seirpoidea Michx, subsp. scirpoidea] - KTZ

Carex scirpoidea Michx. var. scirpoidea - Dorn01, E/W

[Carex seirpoidea Micha.] - SFI, W\&W

[Carex seirpoidea Mishx, subsp. seirpoidea] - KTZ, FNA

Carex scoparia Schkuhr ex Willd, var. scoparia - KTZ, FNA, E

[Carex scopariar Schkuhr] - SFE, UTF

[Carex scoparia Schikuh ex Willd.] - GPF, HDH, W\&W

Carex scopulorum Holm - Dorn01, HDH, FNA, IMF, SF!, UTF, W\&W, E/W

[Carex bigelowii Tort ex Schwein.]- HDH, KTZ, w\& W, misapp.

[Carex chimaphila Holm] - HDH

[Carex gymnoclada Holm] - HDH

[Carex seopalonum Holm var, bractecas (L. H. Bailey) F. J. Herm.] - KTZ

[Carex seopolonum Holm var, scopulorum - KT2

Carex siccata Dewey - FNA, HDH, IMF, KTZ, UTF

[Carex foenes Willd, var. foenea] - E/W

[Carex foenea Willd.]-GPF, SFt, W\&W

[Carex foenea Willd.]-GPF, W\& W

[Carex siccata Dewey] - IMF, KTZ, UTF

Carex simulata Mack. - Dom(1) FNA, HDH, IMF, KTZ, SF!, UTF, W\&W, E/W

Carex sprengelii Dewey ex Spreng, - Dorn01, FNA, GPF, HDH, KTZ, W\&W, E/W

[Carex sprengelii Dewey] - SH

Carex stenophylla Wahlenb. - Dorn01. IMF. UTF. E/W

[Carex duriuscula C. A. Mey] - KTZ, FNA

[Carex eleocharis L. H. Bailey] - GPF, HDH

[Carex stenophylla Wahlenb, subsp. eleocharis (L. H. Bailey) Hultén] - SF!, W\&W

Carex stenoptila F. J. Herm. - Dorn01, HDH, FNA, KTZ. SF!, W\&W, E/W 
Carex stipata Muhl, ex Willd, var. stipata - FNA, IMF, KTZ, EW

[Carex stipata Muhl.] - GPF, SF!

[Carex stipata Muhl. ex Willd.]- Dorm01, HDH, W\&W

[Carex stipata Muhl. var. stipata] - UTI

Carex stricta - Dorn01

* Carex sychnocephala J. Curey - FNA, GPF, KTZ, SFE, W\&W, E

[W] - possibly a disjunct vs. adventive]

Carex tahoensis Smiley - KTZ, FNA

Carex tenera Dewey - WY Checklist

[Carex tenera Dewey var, echinodes (Fem.) Wicg.] - KTZ

[Carex tenera Dewey var. tencra] - FNA

Carex tenuiflora Wahlenb, - KTZ, FNA, SFE, W\&W, E

Carex torreyi Tuck, - FNA, GPF, HDH, KTZ, SFE, W\&W, E

Carex utriculata Boott - Dom01, FNA, KTZ, SF!, W\&W, ENW

[Carex rostrata Stokes] - HDH, W\&W, misapp.

[Carex rostrata Stokes var, rostrata] - RMC

[Carex rostrata Stokes ex With.] - IMF, UTF

[Carex rostrata Seokes ex Will.] - GPF

Carex vallicola Dewey var, vallicola - K'TZ, E/W

[Carex vallicola Dewey] - Done01, FNA, GPF, HDH, IMF, SF!, UTF, W\&W

* Carex vesicaria L. var. vesicaria - IMF, KTZ, UTF, E/W

[Carex vesicaria L.] - Dorn01, HDH. SFt, W\& W, FNA

[Carex vesicaria L. monile (Tuck.) Femald] - GPF

Carex vulpinoidea Michx. - Dorn01. FNA, GPF, HDH, IMF, SF!, W\&W, E/W

[Carex vulpinoidea Michx, vat, vulpinoidea] - KTZ

Carex xerantica L. H. Bailey - Dom01, FNA,GPF, HDH, IMF, SF!,UTF, W\&W, E/W

Cyperus acuminatus Torr. \& Hook. ex Torr, - Dom01, KTZ, E

[Cyperus acuminatus Tort. \& Hook.] - GPF, HDH, IMF, SFE, UTF, W\& W, FNA

Cyperus aristatus Rottb, var, aristatus - E/W

[Cyperus aristatus Rotrb.] - GPF. HDH. IMF. SF], UTF, W\&W

[Cyperus squarrosus L.] - Domi01, KTZ FNA

\{Cyperus diandrus Torr.\} - GPF, HDH rep., KTZ $\angle \mathrm{NE}>$, FNA $\angle \mathrm{NE}>$, W\& $W^{\prime}$, no specimens

Cyperus engelmannii Steud, - GPF, Castilleja 23(4): 6 (2004)

* Cyperus erythrorhizos Muhl. - Dorn01, FNA, GPF, HDH, exp., IMF, KTZ, SF!, UTF, W\&W, E/W

4 \{Cyperus esculentus L. var. esculentus\} - KTZ, FNA, E

\{Cyperus esculentus Li - GPF, HDH, IMF, SFE, UTF, W\&W

4 Cyperus esculentus L. var, leptostachys Boeck. - KTZ, FNA

Cyperus fendlerianus Boeckl. - HDH, FNA, KTZ, E/W

[Chlorocyperus fend lerianus (Boeckeler) Palla] - FNA

[Mariscus fendlerianus (BókL.) T, Koyana] - SFI, WRW

Cyperus lupulinus (Spreng.) Marcks var. lupulinus - E

[Cyperus filiculmis Vahl] - HDH

[Cyperus lupulinus (Spreng, Marcks sabsp, lupulinus] - FNA. GPF, KTZ

[Mariscus filiculmis (Vahl) T. Koyama] - SFE, W\&W

* Cyperus odoratus L. - GPF, KTZ, FNA, SF!, W\&W, E/W

[C) perus odoratus L. ver, squarrosis (Britton) S. D. Jones] - KTZ

Cyperus rivularis Kunth - GPF, HDH, SFE, UTF, W\&W, E

[Cyperus rivularis Kunth var, rivularis] - IMF

[Cyperus bipartitus Tort.] - Dom01, KTZ. FNA

Cyperus schweinitzii Torr- - Dorn01, GPF, FNA. HDH, IMF, KTZ, UTF, E

Snow, N. Janury 2009. Checklist of Vaccular Plants of tho Southom Rodky Momain Region. CVersion $3 x$ 
[Mariscus schweinitzii (Tort.) T. Koyama] - SFE, W\&W

Cyperus strigosus L. -FNA, GPF, HDH exp. IMF, KTZ, UTF

[Cyperus hanscrii Britton] - FNA

[Cyperus strigosus var. hansenii (Brition) Kükenthal] - FNA

[Cyperus strigosus var. stenolepis (Torrey) Kükenthal] - FNA

[Mariscus tenolepis (Torney) C. B. Clarke] - FNA

[Mariscus strigosus (L.) C. B. Clarke] -FNA

Eleocharis

[NS - FNA took the continent-wide perspective but knowledge of our material would bencfit from a dissertation level study of the genus regionsilly?

(Identification sources:

Eleocharis acicularis (L.) Roem. \& Schult, - Dom01, GPF, FNA,HDH, KTZ, SF!, W\&W, E/W

Eleocharis atropurpurea (Retz.) J. Presl \& C. Presl - KTZ, FNA, E

[Eleocharis atropurpurea (Retz.) Kunth] - GPF, HDH, rep.

[Eleocharis atropurpurea (Retz.) C. Presl] - SFE, W\& W

Eleocharis bolanderi A. Gray - HDH, IMF, FNA, KTZ, SF!, UTF, W\&W, E/W

Eleocharis compressa Sull- FNA, GPF, KTZ, E

[Eleocharis elliptica Kunth var. compressa (Sull.) Drapalik \& Mokilenb.] - SFE, Wd W

Eleocharis elliptica Kunth - FNA

[Eleocharis capitara (L.) R. Brown var, borealis Svens.] - FNA, KTZ

[Eleocharis compressa Sullivant var. borealis Drepalik \& Moblentrock] - FNA, KTZ

[Eleocharis temuis var. borealis (Svens.) Gleason] - Dorn01. FNA, KTZ

Eleocharis engelmannii Steud. - FNA, KTZ, E

[Eleocharis engelmannii Steud. yar. deconsa A. Gray], FNA

[Eleocharis engelmannii Stcud, var, monticola (Femald) Svenson] - HDH

[Eleocblaris monticola Femald] - FNA

[Eleocharis monticola Femald var. leviseta Fernald] - FNA

[Eleocharis obtusa (Willd.) Schult var, detonsa (A. Gray) Drapalik \& Moblenb.]-SFE, WRW

[Elcocharis obtusa (Willd.) Schult, var, ovata (Roth) Drapalik \& Mohlenb.] - GPF

[Eleocharis ovata (Roth) Roem, \& Schult] - IMF, UTF

[Eleochars ovsta (Roth) Roem. \& Schult. var, detnoss (A. Gray) Mohlenbrock] - FNA

Eleocharis erythropoda Steud, - FNA, GPF, KTZ $<$ KS, NE, OK>, W\& W ${ }^{\prime}$

[Elescharis palustris (L.) Roem. \& Schalt.] - Dom01, IMF

Eleocharis macrostachya Britt. in J. K. Small - FNA

[Eleocharis perlonga Fernald \& Bracken] - FNA

[Eleocharis xyridiformis Fernald $\&$ Brackett]- FNA

Eleocharis montevidensis Kunth - FNA $\angle \mathrm{NM}>$, GPF, HDH rep., KTZ, SF!, W\&W. E/W

Elcocharis obtusa (Willd) Schult. - HDH. FNA, KTZ

[Elexcharis obtusa (Wild) Schult, var, obtusa] - GPF, W: W. W \& W

[Eleccharis ovata (Roch) Roem. \& Schult.] - Dom01, IMF

Eleocharis palustris (L.) Roem. \& Schult. -FNA. IMF, KTZ, SF!, UTF, W\&W, W\&W ${ }^{1}, E / W$

[Elexcharis macrostachya Brition] - GPF, HDH, KTZ, W\&W

Eleocharis parvula (Roem. \& Schult.) Link ex Bluff \& Fingerh. - UTF, E

[Eleocharis coloradoensis (Brition) Gilley] - FNA

[Eleocharis parvula (Roem, \& Schait,) Link ex Bluff, Nees \& Sehauer] - KTZ, W\&W

[Eleocharis parvula (Roem, \& Schalt,) Link var, anachaeta (Torr.) Svenson] - SFE

[Elexcharis parvula (Roem. \& Schult,) Link ex Bluff \& Fingerh, var, anachaeta (Torr.) Svensoo] - Dono01, IMF

[Eleocharis parvula (Rocm. \& Schalt,) Boff, \& Fingerbr, var, anachacta (Torr,) Svenson] - GPF

[Elexcharis parvula (Roem. \& Schalt, Link ex Bluff var, anachaeta (Torr.) Svenson] - HDH

[Eleocharis parvula (Roem. \& Schalt,) Link var, anachacta (Torr,) Svenson] - W \& W' 
Eleocharis paticiflora (Lightf.) Link - GPF, HDH, IMF, UTF, E/W

[Eleocharis quinqueflora (Hartmann) Schwarz] - Dom01, FNA, KTZ, 5F!, W\& W

Eleocharis rostellata (Torr.) Torr. - Dorn01. FNA, GPF, IMF, KTZ. SF!, UTF, E/W

[Eleocharis rostellata Torr.] - HDH, W\&W

Eleocharis suksolorfiana Beauverd - FNA

Eleocharis wolfii (A. Gray) A. Gray ex Britton - FNA, KTZ, W\&W'

[Eleocharis wolfii (A. Gray) Patt.] - GPF

[Eleocharis wolfii A. Gray] - HDH rep., W\&W

Eleocharis xy ridiformis (Femald) Brackett - GPF, W\& W ${ }^{1}$

[Eleocharis macrostachya Britton] - KTZ, FNA

[Eleocharis palustris (L.) Rocm. \& Schalt.] - IMF

Eriophorum angustifolium Honck, - Dom01, SF!, W\&W, FNA

[Eriopheram angustifolium Roth] - $\mathrm{HDH}$

[Eriophorum angustifotium Honck-subsp. scabriusculum Hultén] - KTZ

[Eriophorum polystachion L. - GPF, ]MF, UTF, E'W; FNA [rejected name]

Eriophorum chamissonis C. A. Mey. - Dorn01, FNA. GPF, HDH, KTZ, W\&W

[Eriophorum altaicum Meinsh. var, neogaeum Raymond sentsa FNA.]

Eriophorum gracile W. D. J. Koch var, gracile - Doen01, KTZ, FNA, E/W

[Eriophorum gracile W, D. J. Koch]-GPF, HDH, SF!, W\&W

Eriophorum scheuchzeri Hoppe - IMF, FNA, KTZ, UTF, W\&W err, rep., E/W

[Eriophorum altaicum Meinsh. var. neogaeum Raymond misapp. in SFW and in 7 specimeers

of $E$. schewchzeri at RM annotated as E. afroic on by W. A. Weber in 1991] - KTZ. SFI. W\&W

Eriophorum viridicarinatum (Engelm.) Fernald - FNA, GPF, KTZ

\section{Hemicarpa (see Lipocarpa)}

Fimbristylis puberula (Michx.) Vahl var. interior (Britton) Kral - FNA $\angle$ NM $>$, GPF, see IMF, KTZ, W\&W, E

[Fimbristylis puberula (Michx.) VahI] - SFE, HDH

[Fimbristylis spadicea (L.) Vahl] - IMF. UTF

Kobresia bellardii (All.) Degland - HDH, IMF, UTF, E/W

[Kobresia myosurioides (ViLL) Ficri] - KTZ, FNA

[Kobresia myosurioldes (VilL) Ficri \& Paoli] - SFI, W\&W

Kobresia sibirica Turez. - (E/W

[Kobresia bellardii (All.) Degland var, macrocarpa (Clokey) Harrington] - HDH

[Kobresia seboenoides [C. A. Mey.) Stend.]-SFT, WR W

[Kobresia sibirica (Turcz, ex Ledeb.) BoeskL] - KTZ, FNA

Kobresia simpliciuscula (Wahlenb.) Mack. - IMF, FNA, KTZ, SF!, UTF, W\&W, E/W

Lipocarpha aristulata (Coville) G. C. Tucker - KTZ, E

[Hemicarpha micrantha (Vahl) Britton] - GPF, IMF

[Hemicarpha micrantha Pax var, aristulara Coville]- HDH. W\&W

[Hemicarpha micrantha (Vahl) Britton var, aristulata Coville]- see IMF, UTF

[Hemicarpha micrantha (Vahl) Pax var, aristulat Coville] - SFE, W\&W'

Lipocarpha drummondii (Nees) G. Tucker - Dom01, KTZ, FNA

[NS: reported by KTZ and WY checklist for WY; for our reeion only in NM fide FNA 23: 196]

[Hemicirpha drummondii Nees] - KTZ, FNA

[Hemicarpha micrantha var, drummondii (Nees) Friedland] - KTZ

[Seirpus mieranthas var, drumenoodii (Nees) Moblenbrock] - KTZ 


\section{Mariscus (see Cyperus)}

Rhynchospora alba (L.) Vahl - J. Lemly ef al. $181 /$ (at COLO)

[NS - collected in fen from La Plata Councy, J. Lemley \{pers. comm. 2008\}]

Schoenoplectus acutus (Muhl, ex Bigelow) A.Lð3ve \& D. Lōe var, acutus - FNA,KTZ, EJW

[Schoenoplectus lacustris (L.) Palla subsp. acutus \{MuhL. ex Bigelowy) A. Lōve \& D. Lōve] - W\&W

[Schoenoplectus lacustris (L.) Palla subsp. acuhus (MuhL) A. Lōve \& D. Lōve] - SF!

[5cirpus acutus Muhl.] - GPF

[Scirpus acutus Muhl. ex Bigelow] - HDH, IMF, UTF

Schoenoplectus acutus (Muhl, ex Bigelow) A. Löve \& D. Löve var, occidentalis (S. Watson) S, G. Sm. - Dorn01, KTZ. FNA

Schoenoplectus americanus (Pers.) Volkart ex Schinz \& R. Keller - FNA, FCF, KTZ

[Schoenoplectus americanus (Pers.) Volkart] + W\&W, misapp.

[Scirpus americamus Pers.] - GPF, IMF, UTF

Schoenoplectus pungens (Vahl) Palla var, pungens - E/W

[Schoenoplectus pungens (Vahl) Palla] - SF!, W\&W, FNA

[Schoenoplectus pungens (Vahl) Pall var. longispicatus (Britton) 5. G. Sm.] - KTZ

[Scirpus americanus. Pers. var. polyphyllus (Bock.) Boctle] - HDH

[Scirpus pungens Vah1] - GPF

[Scirpus pungens Vaht var. longispicatas (Britton) Cronquist] - IMF, UTF

[Scirpus pungens (Vahl) Pall. var, polyphyllus (Boeck.) Dom] - Dorn0]

Schoenoplectus saximontanus (Fernald) J. Raynal - Dorn01, FNA, KTZ, SFE, W\&W, E

[Scirpus saximontamus Fernald] - RMC

[Scirpus saximontanus A. Gray] - GPF

[Scirpus supinus L.] - HDH, UTF

[Scirpus supinus L. var, saximontanus (Fernald) T. Keywma] - IMF

Schoenoplectus tabernaemontani (K, C, Gmel.) Palla - Dorn01, FNA, KTZ, E/W

[Schoenoplectus lacustris L. subsp. validus (Vahl) A. Löve \& D. Lïre] - W\& W, misapp.

[Schoenoplectus kacuatris (L.) Palla subsp. creber (Fernald) A. Love \& D. Love] - SFE. W\&W'

[Schoceoplectus lacustris (L.) Palla subsp. validus (VahI) A. Love \& D. Löve] - SFW

[Scirpus validus Vahi] - GPF, HDH, IMF, UTF

Scirpus (see also Amphiscirpus, Bolboschoenus, Schoenoplectus, Trichophorum)

Scirpus microcarpus J. Presl \& C. Presl - Dorn01, KTZ, FNA, W\&W, E/W

[Scirpus microcarpus C. Prest - GPF, IMF, SFI, UTF

[Scirpus rubrotinctus Fernald] - $\mathrm{HDH}$

Scirpus pallidus (Britton) Femald - Dorn01, GPF, HDH, IMF, KTZ, FNA, SF!, W\&W, E/W

$\div$ Scirpus pendulus Muhl. - KTZ, FNA, W\&W

[Scirpus linemus Michx.] - SFE, W\&W, W\&W', misid

\{Trichophorum caespitosum (L.) Hartman\} - KTZ, W\&W

\{Trichophoroum cespitosum (L.) Schuri) - FNA <UT>

Trichophorum pumilum (Vahl) Schinz \& Thell. - Dorn01, KTZ, FNA CMT>, SF!, W\&W, E/W [Scirpus pumilus Vahi] - HDH rep.

\section{Cypripediaceae (see ORC)}

\section{Dipsacaceae - DPS}

* Dipsacus fullonum L. - Dom01, GPF, KTZ, SF!, W\& W, E/W 
[Dipsacus sylvestris Huds.] - HDH, IMF, UTF

* Dipsacus laciniatus L. - GPF, KTZ, SFE, W\&W, E

[W] - this species appears to hybridize with D. fullonum in parts of Jefferson $\mathrm{Co}, \mathrm{CO}$ ]

* Knautia arvensis (L.) Coult. - GPF, IMF, KTZ, UTF, W\&W. E/W

[Knautia arvensis $\{$ L.) Duby] - SF!

\section{Droseracene - DRS}

Drosera anglica Huds - J. Lemly el al. 1806 (COLO)

[NS - collected in fen in La Plata County, J. Lemly \{pers. comm. 2008?]

Drosera rotundifolia L. var, rotundifolia - KTZ. E/W

[Drosera rotundifolia L.] - GPF, SF!, W\&W

\section{Ehretiaceace (see BOR)}

\section{Elacagnaceae - ELE}

* Elaeagnus angustifolia L. - Dorn01, GPF, HDH, IMF, KTZ, SF!, UTF, W\&W, E/W

- Elaeagnus commutata Bernh ex Rydb, - Dorn01, IMF, KTZ, E [Elaeagnus commutala Bernh.] - GPF. HDH, SFE, UTF, W\& W

Shepherdia argentea (Pursh) Nutt, - Dom01, GPF, HDH, IMF, KTZ, SF!,UTF, W\&W, E/W Shepherdia canadensis (L.) Nutt, - Dom01, GPF, HDH, IMF, KTZ, SF!, UTF, W\&W, E/W

\section{Elatinaceae - ELT}

Bergia texana (Hook.) Seub. ex Walp. - KTZ, W\&W, E

[Bergia texana (Hook.) Seub.] - SFE, UTF

[Bergia texana (Hook.) Walp.] - GPF

\{Elatine americana (Pursh) Am.\} - HDH, KTZ $<$ KS, OK $>$, W\&W, misapp.

Elatine brachysperma A. Gray - WY checklist, GPE, KTZ, UTF

[Elatine obowata (Faseet) Mascon]- KTZ

[Elatinc triandra vat, brachyspentas (Gray) Fassett] - KTZ

Elatine rubella Rydb, - Dom01, KTZ, W\&W, E/W

[Elarine triandra Schkuhr] - GPF, HDH rep., SF!, UTF, W\&W'

\section{Ericaceae - ERI (including Monotropaceae, Pyrolaceae)}

(Identification sources:

- Arctostaphylos X coloradensis Rollins - ENDEMIC $<\mathrm{CO}>, \mathrm{KTZ}$

[Arctostaphylos patula X A. uxz-ursi] - KTZ

[Arctostaphylos aevadensis A. Gray var, coloradensis (Rollins) Harrington] - HDH

[Aretostaphylos patula Greene forma platyphylla (A. Gray) P, V. Wells] - W\&W

Arctostaphylos patula Greene - HDH, KTZ, UTF, W

[Arctostaphylos patula Greene subsp, platyphylla (A. Gray) P. V. Wells] - SFW

[Aretostaphylos patula Greene forma platyphylla (A. Gray) P, V, Wells] - W\&W

Arctostaphylos pungens Kunth - KWA, KTZ, M\&H. W\&S 
[Arctostaphylos chalonecorum ].B. Roof] - KTZ

[Arctostaphylos pseudopungens J.B. Roof] = KTZ

[Aretostaphylos pungens subsp. chaloneorum (J.B. Roof) J.B. Roof] - KTZ

Arctostaphylos uva-ursi (L.) Spreng. var, adenotricha Fernald \& J. F. Macbr, - Dom01, GPF. $\mathrm{HDH}, \mathrm{E} / \mathrm{W}$

[Arctostaphylos uva-ursi L., subsp. adenotricha (Fernald \& J. F. Mactr.) Calder \& R. L. Tilylor - SfW

[Arctostaphylas uva-ursi (L.) Spreng. subsp. adenotricha (Fernald \& J. F. Macbr.) Calder \& R. L Taylor - GPF. HDH, SFE, W\& W

[Arctostaphylos adenotricha (Fernald \& J. F. Macbr.) A. Love, D. Love, \& B. M. Kapoor] - W\&W

[Arctostaphylos uva-ursi (L.) Spreng.] - KTZ UTF

Arctostaphylos uva-ursi (L.) Spreng, var, stipitata (Packer \& Denford) Dorn - Dorn01

Arctostaphylos uva-ursi (L.) Spreng var, uva-ursi - Dorn01, HDH, E

[Arctostaphylos ava-ursi $\{\mathrm{L}$. . Spreng.] - KTZ, UTF

[Arctustaphylas uvá-ursi L. subsp. coactilis (Fernald \& J. F. Macbr.) A. Lõve, D. Lơve, \& B. M. Kapooe] WRW

[Arctostaphylos uvá-ursi (L.) Spreng. subsp. coactilis (Femald \& J. F. Macbr.) A. Löve, D. Löve, \& B. M. Kapoor]-SFE, W\&W

[Arctostaphylos uva-ursi (L.) Spreng. vart, coactilis Fernald \& J. F. Macbr.] - GPF, HDH

\section{Azaleastrum (see Rhododendron)}

Chimaphila umbellata (L.) W.P.C. Barton var, occidentalis (Rydb.) S.F.Blake - Dorn01, HDH, UTF, E/W

[Chimaphila umbellata (L.) W. P. C. Bartob] - GPF

[Chimaphila umbellata (L.) W. P. C. Barton subsp. acuta (Rydb.) Huhen] - KTZ

[Chimaphila umbellata (L.) W. P. C. Bartoe var. acuta (Rydb.) S. F. Blake]- HDH, exp.

[Chimaphila umbellata (L.) W. P. C. Barton subsp. oceidentalis (Rydb.) Hultén] - KTZ. SF!, W\&W

Gaultheria humifusa (Graham) Rydh. - Dorn01, HDH, KTZ, SF!, UTF, W\&W, E/W

Hypopitys (see Monotropa)

Kalmia microphylla (Hook.) A. Heller var. microphylla - E/W

[Kalmia microphy lla (Hook.) A. Hellor] - Dom01, KTZ, SF1, UTF, WSW

[Kalmia politolia Wang, var. microphylla (Hook.) Rehd.] - $\mathrm{HDH}$

Moneses uniflora (1.) A. Gray var, uniflora - E/W

[Moneses uniflora (L.) A. Gray] - Domb1, HDH, KTZ, SFL, W\&W

[Moneses uniflora L.] - UTF

[Pyrola uniflora L.]-GPF

Monotropa hypopithys L. - GPF, KTZ, E/W

[Hypopitys monotropa Crantz] - Dom01, SFI, WeW

[Monocropa hypopicys L. var. latisquama (Kydb,) Keaney \& Peebles] - HDH

\{Monotropa uniflora L.\} - M\&H, exp., KWA (not exp.), JM (not rep.), HDH, exp., GPF, KTZ $<\mathrm{KS}, \mathrm{NE}, \mathrm{OK}>$

Orthilia secunda (L.) House - Dorn01, KTZ, W\&W, E/W

[Orthilia secunda (L.) House subsp. obtusata (Turcz) Böcher] - SF], W\& W'

[Orthilia secunda (L.) House subsp. secuoda] - SF!

[Pyrola secunda L.] - GPF, HDH, UTF

[Ramischia secunda Rydb.] - $\mathrm{HDH}$ 
(Phyllodoce empetriformis (Sm.) D. Don) - HDH rep., KTZ $\angle A Z$, WY $>$, W\&W, misid.

Pterospora andromedea Nutt, - Dom01, GPF, HDH, KTZ, SF!, UTF, W\&W, E/W

Pyrola (see also Moneses, Orthilia)

(Identification sources:

Pyrola asarifolia Michx, var, asarifolia - $\mathrm{HDH}, \mathrm{E} / \mathrm{W}$

[Pyrola asarifolia Michx.] - Dom01, GPF, UTF

[Pyrola asarifolia Michx. subsp. asarifolia] - KTZ

[Pynola asarifolia Miehx. var. purpurea (Bunge) Femald] - HDH

[Py nola notundifolia L. subsp. asarifolia (Michx_) A. Lōve] - SF!

[Py rola rotundifolia L. subsp. asarifolia (Michx_) A. Lòve \& D. Lơve] - W\&W

Pysola chlorantha SW, var, chlorantha - E/W

[Pyrola chlorantha Sw.] - Dorn01. HDH. KTZ, SF!, UTF. W\&W

\{Pyrola elliptica Nutt.\} - KWA, KTZ, M\&H

[W] - a northeastern species that appears to reach the Black Hills; specinens from our arva probably

misidentified and Jikely $\mathrm{P}$. chlorantha)

Pyrola minor L. - Dorn01, HDH, KTZ, SF!, UTF, W\&W, E/W

Pyrola picta Sm, var, picta - Dorno1, E/W

[Prola pieta Sm.] - GPF, HDH. KTL, SF!, UTF, W\&W

- Rbododendron albiflorum Hook. var, warrenii (A. Nelson) M. A. Lane - ENDEMIC $<C O>$, KTZ, E/W

[Azaleasirum albifloum (Hook.) Rydb.] - W\&W

[Azaleastrum albiflocum (Hook.) Rydb, subsp, warrenii (A. Nelson) W. A. Weber] - SF!. W\& W'

[Rhododendron albifkorum Hook.] - $\mathrm{HDH}$

Vaccinium cespitosum Michx. - Dorn(1, SF!, W\&W, E/W

[Vaccinium caespitosum Michx.]-HDH, UTF

[Vaceinium cespitosum Michx, var, cespitosum] - KTZ

Vaccinium globulare Rydb. - W\& W

[Vaceiniam membranaceum Dougl.] - UTF

[Vaccinium membranaceum Dougl. ex Tom.] - KTZ $<A Z$. UT, WY>

Vaccinium myrtillus L. var. oreophilum (Rydb.) Dorn - Dorn01, KTZ, EJW

[Vaccinium myrtillus L.] - HDH, UTF

[Vaccinium myrtillas L. subsp, areophilum (Rydb, A A. Love \& D. Love] - SFI, W\&W

\{Vaccinium oceidentale A. Gray\} -SFW, exp.

Vaccinium scoparium Leiberg ex Coville - Dorn01, KTZ, W\&W, E/W

[Vaccinium scoparium Leiberg] - GPF. HDH. SF! UTF

\section{Euphorbiaceac - EUP (sce also PLL)}

(Identification sources: )

Agaloma (sce Euphorbia)

Argythamnia humilis (Engelm. \& A. Gray) Mōll. Arg- var. humilis - GPF, KTZ, E

Snow, N, Junury 2009. Checklist of Vaccular Plants of the Southom Rocky Momain Region. CVersion $3 x$ 
[Argythamnis humilis (Engelm. \& A. Gray) Mill. Arg.] - SFE, W\&W

[Ditaxis humilis (Engelm. \& A. Gray) Pax] - HDH

Argythamnia mercurialina (Nutt.) Moll. Arg, var, mercurialina - KTZ, E

[Argythamnia mercurialina (Nutt.) Müll. Arg.] - GPF, SFE, W\& W

[Ditaxis mencurialina (Nutt.) J. M. Coult.] - HDH

\section{Chamaesyce}

(Identification sources:

+Chamaesyce albomarginata (Torr. \& A, Gray) Small - IMF, KTZ, W\&W, misid.

[Euphorbia albomarginata Torr. \& A. Gray]-GPF $\triangle C O D$. HDH, exp., UTF

Chamaesyce chaetocaylx (Boiss.) Wooton \& Standl. var, chaetocalyx - KTZ

Chamaesyce fendleri (Torr. \& A. Gray) Small - Dorn01, IMF, KTZ, SF!, W\&W, E/W

[Euphorbia fendleri Torr. \&. A. Gray]-GPF, UTF

[Euphorbia fendleri Torr. \& A. Gray var. fendleri] - HDH

Chamaesyce geyeri (Engelm.) Small - Dorn0I, SFE, W\&W, E

[Chamaesyoe geyeri (Engelm.) Small var. geyeri] - KTZ

[Euphorbia gejeri Engelm.] - GPF, HDH

[Euphorbia geseri Engelm. var, geyeri] - RMC

Chamaesyce glyptosperma (Engelm.) Small - Dorn01, IMF, KTZ, SF!, W\&W, E/W

[Euphorbia glyptospema Engelm.] - GPF, HDH, UTF

Chamaesyce lata (Engelm.) Small - KTZ, SFE, W\&W, E

[Euphorbia lata Engelm.] - GPF, HDH

Chamaesyce maculata (L.) Small - Dorn01, IMF, K1Z, E

[Chamaesyoe supina (Raf.) Moldenke] - SFE, W\& W?

[Euphorbia maculata L.] - GPF, UTF, W\&W, out of range

\{Chamaesyce micromera (Boiss, ex Engelm.) Wooton \& Stand, - - IMF, KTZ

\{Euphorbia microenera Boiss. ex Engelm.\} - HDH, exp.

Chamaesyce missurica (Raf.) Shinners - KTZ, SFE, W\&W, E

[Euphorbia missurica Raf.] - GPF

[Euphorbia missurica Raf. var. inkermedia (Engelm.) Whocler] - HDH

[Euphorbia missurica Raf, var. petakidka (Engelm.) Dorn] - Dons01

Chamaesyce nutans (1.ag.) Small - Dom01, KTZ

[Clumaesyce maculata auct, non (L.) Small] - KTZ

[Chamesyce preshii (Guss.) Arthur] - KTZ

[Euphorbia maculata auct, non L.] - KTZ

[Euphorbia nutans Lag.] - KTZ

[Euphorbia preslii Guss.] - KTZ

Chamaesyce parryi (Engelm.) Rydb. - IMF. KTZ. SFW, W\&W. W

[Euphorbia parryi Engelm.] - HDH rep. UTF

\%Chamaesyce prostrata (Aiton) Small - Dorn01, IMF, KTZ

[Euphorbia prosirata Ait]-GPF, UTF

Chamaesyce revoluta (Engelm.) Small - 1MF. KTZ, SF!, W\&W, E/W

[Euphorbia revoluta Engelm.] - GPF, HDH, UTF

\%Chamaesyoe serpens (Kunth) Small - Dorn01, KTZ, SFE, W\&W, E

[Euphorbia serpens Kunth] - GPF, HDH, RMC

* Chamaesyce serpyllifolia (Pers.) Small - Dorn01, IMF, SF!. W\&W. E/W

[Chamaesyce serpyllifolia (Pers.) Small subsp, serpyllifolia] - KTZ

[Euphorbia serpyllifolia Pers.] - GPF, UTF

[Euphorbia serpyllifolia Pers, ver, serpyllifolia] - HDH

\{Chamaesyce serrula (Engelm.) Wooton \& Standl\} - KTZ 
\{Euphorbia serrula Engelm.\} - HDH, exp.

Chamaesyce stictospora (Engeim.) Small - Dorn01, KTZ, SFE, W\&W, E

[Euphorbia stictospora Engelm.] - GPF, HDH

Croton pottsii (Klotzech) Müll, -Arg, var, pottsii - KWA, KTZ, JM (not rep.)

[Croton corymbulosus Engelm.] - KWA, KTZ

[Crotos eremophilus Wooton \& Standley] - KWA

Croton texensis (Klotzsch) Mall-Arg. - Dorn01, GPF, HDH, SF!, W\&W, E/W

[Crotos tevensis (Klotzsch) Mail. Arg. var. texensis] - IMF, KTZ, UTF

\section{Ditaxis (see Argythamnia)}

Euphorbia

(Identification sources: )

Euphorbia (see also Chamaesyce)

Euphorbia agraia Bieb, - Dom01, KTZ

Euphorbia brachycera Engelm. - IMF, KTZ, UTF, E/W

[Euphorbia beachycera Engelm. var, robusta (Engelm.) Dorn] - DornoI

[Euphorbia robusta (Engelm.) Small] - GPF, HDH, KTZ

[Tithymalus brachyceras (Engelm.) Sinall] - SF!, W\&W

\{Euphorbia crenulata Engelm. - HDH. IMF, KTZ, W

(WJ - This species is native to CA and OR (see Jepson Manual). Presence in CO based on old specimen at COLO from Montezuma Co. thar is $E$. brochycera in my opinion. A disjuction flom 100 years ago is unlikely: species is not mentioned by other floras of the surrounding states?

\{Tithymahus crenulatus (Engelm.) A. Heller\}-SFW, W\&W

* Euphorbia cyparissias L. - Dorn01, GPF, HDH, IMF, KTZ, UTF, E

[Tithymalus cyparissias (L.) Lam.] - SFE, W\&W

Euphorbia dentata Michx. - Dorn01, GPF, UTF, E/W

[Euphorbia dasidif Subils] - IMF, KTZ

[Euphorbia dentata Michx. var, cuphospenna Engeim.] - HDH

[Euphortia dentata Michx. var, dentasa] - HDH, KTZ

[Poinsettis denstata (Michx.) Kiousch \& Garcke] - SF', w\&W

* Euphorbia esula L. var. esula-Dom01, KTZ, E/W

[Euphorbia esula L.] - GPF, HDH, IMF, UTF

[Euphorbia x pseudovirgata (Schur) So0] - GPF

[Euphorbia esula X E virgata] - GPF

[Tithymalus esula (L.) Scopoli] - SFI, W\&W

* Euphorbia esula L. var. uralensis (Fisch. ex Link) Dorn-Dom01, KTZ, E/W

[Euphorbia uralensis Fisch, ex Link] - GPF

[Tithymalus uralensis (Fisch.) Prokhanov] - SFI

[Tithymalus ura lensis (Fisch. ex Link) Probhanov] - w\&W

Euphorbia exstipulata Engelm. - Dorn01, GREE (Harmon $\$ 507$ for CO; Hazleu 12212 for NM), Smith09?, KTZ

[Euphorbia exstipulata Engelm, var. lata Warnock \& M. C. kohnston] - KTZ

Euphorbia hexagona Nutt. ex Spreng. - HDH, KTZ, E

[Euphorbia hexagona Nutt.] - GPF, IMF

[Zygophyllidium hemagom (Nutt.) Small] - SFE, W\&W

Euphorbia helioscopia L. - Dorn01

[Galarhocus helioseopia (t.) Haw, - KTZ

[Tithyenalus helicseopia (L., Hill] - KTZ 
Euphorbia incisa Engelm. var, mollis (Norton) Wheeler - KTZ, W

[Euphorbia incisa Engelm.] - IMF

[Tithymalus incisus (Engelm.) W. A. Weber] - W\&W

[Tithymalus incisus (Engelm.) Norton var. mollis W. A. Weber] - SFW

Euphorbia marginata Pursh - Dom01, GPF, HDH, IMF, KTZ, UTF, EN

[Agaloma marginata (Pursh) A. Love \& D. Luve] - SF!, WA W

* Euphorbia myrsinites L. - IMF, KTZ, UTF, E

[Tithymalus myrsinites (L.) Hill] - SFE, W\&W

\{Euphorbia nephradenia Barneby) - IMF, KTZ $\angle \mathrm{UT}>$, UTF $\angle \mathrm{CO}>$

[W] - Reported presence in $\mathrm{CO}$ bassed entirely on repert in IMF. Ive seen no specimens in $\mathrm{CO}$; its presumed

presence in Mosileruma $\mathrm{Co}$. woukd be a substantial range disjurction from sw UT]

* Euphorbia peplus L, - GPF, IMF, KTZ, UTF, E

[W] - Collected once in $\mathrm{CO}$ in Golden. L. Yeatis has collected Golden area for 30 years and never encountered it. Probably a non-persistent waif]

[Tithymalas peplus (L) Hill] - SFE, W\&W

Euphorbia spathulata Lam, - Dom01, GPF, IMF, KTZ, UTF, E/W

[Euphorbia dictyosperma Fisct. \& C. A. Mey.] - HDH

[Tithymalus spatulatus (L.am.) W. A. Weber]-SF!. W\&W

\section{Poinsettia (see Euphorbia)}

\section{Reverchonia (see PLL)}

Stillingia sylvatica Garden ex L. subsp. sylvatica - KTZ, E

[Stillingis sylvatica L.] - SFE, GPF

[Stillingia sylvatica Garden ex L.] - HDH, WeW

\section{Tithymalus (see Euphorbia)}

\{Tragia nepetifolia Cav \} - HDH, W\&W, misapp.

\{Tragia betonicifolia Nutt.\} - GPF

(Tragia nepetifolia Cav, var, dissecta Müll. Arg, - KTZ

Tragia ramosa Torr, - GPF, IMF, KTZ, SF!, UTF, E/W

[Tragia ramosa (Mill, Arg) Torr.] - W W W

\section{Zygophyllidium (see Euphorbia)}

\section{Fabaceae - FAB (= Leguminosae, including Caesalpintaceae, Mimosaceae)}

(Identification sources:

* Alhagi maurorum Medik. - IMF, KTZ, W\&W ${ }^{1}, \mathrm{NNL}$

[Allogi camelorum Fisch.] - WR.W

[Allogi macrounam Medik.] - UTF

Amorpha canescens Pursh - Dorn01, GPF, HDH, KTZ, SFE, UTF, W\& W, E

[Amorpha canesecns Nut.] - IMF

[Amorpha canesecns Nutr, ex Pursh] - NNL

Amorpha fruticosa L. - Dornol, GPF, IMF, KTZ, NNL, UTF, W\&W, E

[Amorpha fnuticosa L var, angustifolia Pursh] - SFE, $\mathrm{HDH}$

[Amorpha fruticosa L- var, occidentalis (Abrams) Kearney \& Peebles] - HDH, rep. 
Amorpha nana Nutt - GPF, HDH, KTZ, NNL, SFE, W\&W, E

Apios americana Medik, - GPF, KTZ, NNL, SFE, W\&W, E

Astragalus

(Identification sources:

Astragalus adsurgens Pall. var, robustior Hook, - GPF, IMF, NNL, SFE, W\&W, EW

[Astragalus adsurgens Pall.] - SFW

[Astragalus adsurgens Pall, subsp. nobustior (Hook.) S. L. Welsh] - UTF

[Astragalus laxmannii Lam.] - W\& W'

[Astragalus laxmannii Jacq. var. robustior (Hook.) S. L. Welsh \& Barneby] - Dorn01, K1Z

[Astragalus striatus Nutt. ex Torr. \& A. Gray] - HDH

[Astragalus selphurescens Rydb.] - $\mathrm{HDH}$

Astragalus agrestis Douglas ex G. Don - Dorn01, GPF, HDH, IMF, KTZ, NNL, W\&W, E/W

[Astragalus agrestis Douglas] - SF!

Astragalus albulus Woot. \& Standl. - FCF, NNI

Astragalus alpinus L, var. alpinus - KTZ, NNL, E/W

[Astragalus alpinus L] - Dorn01, GPE, HDH, IMF, SFI, UTF, W\&W

Astragalus americanus (Hook.) M. E. Jones - GPF, KTZ, NNL, SFE, W\&W, E

[Astragalus frigidus (L.) A. Gray var. americanus (Hook.) S. Watson] - W\& W'

Astragalus amphioxys A. Gray var, amphioxys - FCF, NNL

[Astragalus amphioxys var, me hanocalyx (Rydb.j Tidestrom] - KTZ

Astragalus amphioxys A. Gray var, vespertinus (E. Sheld.) M. E. Jones - HDH, IMF, KTZ,

NNL, SFW, UTF, W\&W, W

Astragalus anisus M. E, Jones - ENDEMIC $<\mathrm{CO}>$, HDH, KTZ, NNL, SFW, W\&W, W

Astragalus aretioides (M. E. Jones) Barneby - IMF, KTZ, UTF, W

[Astragalus sericoleucus A. Gray var, aretioides M. E. Jones] - HDH

[Orophaca aretioides (M. E. Jones) Rydb.] - NNL, SFW, W. W

Astragalus argophyllus Nutt. var. argophyllus - Dorn01

[Astragalus argophyllus Nutt. ex T. \& G, var argophyllus] - NNL.

[Xylophacos argophyllus (Nutt.) Rydo,] - KTZ

Astragalus argophyllus Nutt. var. martinii M. E. Jones - IMF, KTZ, SFW. W

[Astragalus argophyllus Nutr. var. pephragmenoides Barneby]

[Astragalus argophyllus Nutt, ex Torr. \& A. Gray var, martinii M. E. Jones] - NNL.UTF, W\& W

[Astragalus argophyllus Nutt, ex Torr. \& A. Gray var, pephragmenoides Barneby] - HDH. W\&W

Astragalus asclepiadoides M. E. Jones - HDH, IMF, KTZ. NNL, SFW, UTF, W\&W, W

Astragalus australis (L.) Lam. var- glabriusculus (Hook_) lsely - Dorn01, IMF, NNL, W\&W'. E/W

[Astragalus aberipiaum Richandson] - GPF

[Astragalus aborigiaum Richandson var, aboriginum] - HDH

[Astragalus aboriginum Richardson var, fostigionum M. E, Jones] - SF!. W \& W

[Astragalus aboriginum Richardson var, glabriusculus (Hook.) Rydb.] - HDH, SFE, W\&W

[Astragalus australis (L) Lam.] - KTZ

[Astragalus australis (L.) Lam. subsp. '] - W W W'

[Astragalus australis Fisch, var, glabeiusculus (Hook.) Isely] - UTF

Astragalus barrii Barneby - Dom01

[Orophaca barrii (Barneby) Iskey] - KTZ, NNL.

Astragalus bisulcatus (Hook.) A. Gray var. bisulcatus - Dorn01, IMF, KTZ. NNL. UTF, E/W

[Astragalus bisukatus A. Gray] - W\&W

[Astragalus bisulcatus (Hook.) A. Gray] - GPF, HDH, WRW'

Snow, N. Janury 2009. Checklist of Vascular Plants of tho Southom Rocky Momain Region. CVersion 31 
[Astragalus diholeos Tidestr.] - HDH

Astragalus bisulcatus (Hook.) A.Gray var, haydenianus (A.Gray) Barneby - Dom01, KTZ, E/W

[Astragalus bisulcatus (Hook.) A. Gray var. haydenianus (A. Gray ex Brandegee) Barneby] - [MF, NNL.

[Astragalus haydenianus A. Gray] - HDH, SF!, WE W

Astragalus bisulcatus (Hook.) A. Gray var, major (M. E. Jones) S. L. Welsh - Dorn01, KTZ, NNL

Astragalus bodinii E. Sheld. - Dorn01, GPF, HDH, IMF, KTZ, NNL. SFE, UTF, W\&W, E Astragalus brandegeei Porter - HDH, IMF, KTZ, NNL, SF!, UTF, W\&W, E/W

Astragalus calycosus Torr. ex S, Watson var, ealycosus - IMF, KTZ, NNL, UTF, W\&W

[Astragalus calycesus Torr.] - Dom01. HDH

Astragalus calycosus Torr, ex S, Watson var, seaposus (A, Gray) M. E. Jones - IMF, KTZ, NNL, UTF, W\&W, W

[Astragalus calycosus Torr.] - HDH

[Astragalus calycosus A. Gray var. scaposus M. E. Jones] - SFW

[Astragalus calycosus Torr. ex S. Watson var, scaposus M. E. Jones] - W \& W

Astragalus canadensis L. var, brevidens (Gand.) Barneby - Dorn01. IMF, KTZ, NNL, SFW, W

Astragalus canadensis L, var, canadensis - Dorn01, HDH, IMF, KTZ, NNL, UTF, W\&W, E

[Astragalus canadensis L.] - GPF, SFE

Astragalus canadensis L, var, mortonii (Nutt.) S. Watson - HDH, IMF, KTZ, NNL, W\&W

[Astragalus canadensis L. var. brevidens (Gand.) Bankeby] - RMC

Astragalus ceramicus E. Sheld, var, ceramicus - HDH, IMF, KTZ, NNL, SFW, UTF, W\&W, W

[Astragalus ceramicus E. Sheld. var, imperfoctus E. Sheld.] - $\mathrm{HDH}$

Astragalus ceramicus E. Sheld, var, filifolius (A. Gray) F. J. Hem. - Dorn01, GPF, IMF, KTZ, NNL, SF!, W\&W, E/W

- Astragalus cerussatus E. Sheld. - ENDEMIC $<C O>, N N L, H D H, K T Z, ~ S F$ !, W\&W, E/W

Astragalus chamaeleuce A. Gray var. chamaleuce - IMF, KTZ, NNL, UTF, W

[Astragalus chambeleuce A. Gray] - Dorn01, HDH, SFW, W\&W

Astragalus chlosdes Barneby - IMF, NNL

[Astragalus chloodes Bameby] - KTZ -UT?, UTF

Astragalus cibarius E. Sheld. - Dom01, IMF, KTZ, NNL, SFW, UTF, W\&W, W

* Astragalus cicer L. - Dorn01, IMF, KTZ, NNL, SF!, UTF, W\&W, E/W

Astragalus coltonii M. E. Jones var. moabensis M. E. Jones - Dom01, HDH, IMF, KTZ, NNL.

SFW, UTF, W\&W, W

Astragalus convallarius Greene var. convallarius - IMF, KTZ, NNL. UTF, W\&W, W

[Astragalus convallarius Greene] - Dem01, SFW

[Astragalus diversifolius A. Gray] - Donol (uncertain for WY as taxon separate from A, c, va, c.), HDH

Astragalus convallarius Greene var- scopulorum Barneby - IMF, KTZ,NNL, SFE, W\&W, E

Astragalus crassicarpus Nutt. var. cavus Barneby -NNL. KWA. KTZ, M\&H, JM, not rep.

Astragalus crassicarpus Nutt. var. crassicarpus - Dorn01, GPF, KTZ, NNL, W\&W, E

[Astragalus crassicarpas Nutt,] - HDH, SFE

Astragalus crassicarpus Nutt. var. paysonii (Kelso) Barncby - Dorn01, GPF. KTZ, NNL. W\&W.

$\underline{E}$

[Astragalus crassicarpus Nutt,] - SFE

[Astragalus secculentus Richardson] - HDH

Astragalus cronquistii Barneby - IMF, KTZ. NNL, SFW, UTF, W\&W, W

- Astragalus debequacus S. L. Welsh - ENDEMIC <CO>, KTZ, SFW, W\& W, W

[Astragalus eastwoodac M. E. Jones] - W\&W

[Astragalus eastwoodac M. E. Jones var, dehequacus (S. L. Welsh) lsely] - NNL.

Astragalus desperatus M. E. Jones var. desperatus - IMF. KTZ. NNL. UTF. W

[Nstragalus desperatus M. E. Jones] - HDH, SFW. W\&W

[Astragalus desperatus M. E. Jones var. neeseas Barneby] - IMF, KTZ, W\&W' 
[Astragsalus equisolensis Neese \& S. L. Welsh] - UTF

- Astragalus deterior (Barneby) Bameby - ENDEMIC $<C O>$, KTZ, NNL, SFW, W\&W, W

[Astragalus naturitensis Payson var. deterior Barneby] - $\mathrm{HDH}$

Astragalus detritalis M. E. Jones - HDH, IMF, KTZ, NNL, UTF, W\&W, W

[Astragalus detrilalis Bameby] - SFW

Astragalus drummondii Douglas ex Hook, - Dorn01, NNL, GPF, IMF, KTZ, UTF, W\&W, EW

[Astragalus drummondii Douglis] - HDH, SF!

Astragalus duchesnensis M. E. Jones - HDH rep., IMF, KTZ, NNL, SFW, UTF, W\&W, W

Astragalus eastwoodiae M. E. Jones - IMF, KTZ, SFW, UTF, W\&W, W

[Astragalus enstwoodac M. E. Jones var, eastwoodae] - NNL.

[Astragalus preussii A. Gray var, eastwoodiae (M. E. Jones) M. E. Jonies] - HDH

Astragalus eucosmus B. L. Rob, - Dorn01, HDH, IMF, KTZ, NNL, SF!, UTF, W\&W, ENW

* Astragalus falcatus Lam, - IMF, KTZ, NNL, SF! UTF, W\&W, E/W

Astragalus flavus Nutt. - Dorn01, IMF, KTZ, SFW, W\&W, W

[Astragalus confertiflorus A. Gray var. confertiflorus] - $\mathrm{HDH}$

[Astragalus confertiflorus A. Gray var. flaviflorts (Kuntze) M. E. Jones] - HDH

[Astragalus flavus Nutt, var, flavus] - NNL

[Astragalus flaves Nutt, ex Torr. \& A. Gray var, flaws] - UTF

Astragalus flexuosus (Hook.) Douglas ex G. Don var. diehlii (M. E. Jones) Barneby - KTZ, E/W

[Astragalus flexwosus (Hook.) G. Don] - GPF, SFE

[Astragalus flexuosus Douglas ex Hook. var. dichlii (M. E. Jones) Barneby]- HDH

[Astragalus flexwosus (Hook.) G. Don var. dichlii (M. E. Jones) Bameby]-IMF, NNL, UTF, W\&W

Astragalus flexuosus (Hook.) Douglas ex G. Don var, flexuosus - KTZ, E/W

[Astragalus flexuosas (Hook.) Douglas] - SFW

[Astragalus flexnosus (Hook.) G. Don] - SFE, GPF

[Astragalus flexuosas (Hook.) G. Don var. flexaosus] - IMF, NNL, UTF, W\& W

[Astragalus flexuosas Douglas ex Hook. var. flexoosus] - $\mathrm{HDH}$

Astragalus flexuosus (Hook.) Dougl, ex G. Don vas, greenei (A. Gray) Barneby - KTZ

[Astragalus flexuosus (Hook.) Doegli] - SF!

[Astragalus flexuosus (Hook.) G. Don] - GPF

[Astragalus flexwosus (Hook.) G. Don var, greenei (A. Gray) Barneby] - KTZ. NNL

Astragalus fucatus Bameby - IMF $\angle C O \geqslant$, KTZ, NNL, SFW, W\&W, W

Astragalus geyeri A. Gray var. geyeri - IMF, KTZ. NNL, W

[Astragalus geyeri A. Gray] - Dom01, SFW, UTF, W\&W

Astragalus gilviflorus E. Sheld. var. gilviflorus - Dom01, KTZ, E

[Astragalus gilviflones E. Sheid.] -GPF, IMP, UTF

[Orophaca triphylla Britton] - SFE, WdW

[Orophaca triphylla (Eat. \& J. Wright) Britton] - NNL

Astragalus gracilis Nutt. - Dom01, GPF, HDH, KTZ, NNL, SFE, W\&W, E

[Astragalus parviflonas (Pursh) Nutt.] - $\mathrm{HDH}$

Astragalus grayi Parry ex S. Wats. - Dom01, KTZ

Astragalus hallii A. Gray var. hallii - IMF, KTZ, NNL, E/W

[Astragsalus hallii A. Gray] - HDH, SFt, W\&W

Astragalus hamiltonii Ced. Porter - IMF. KTZ. UTF

[Astragalus lonchocarpas Torr, var, hamiltonii (Ced. Porter) Isely] - NNL, W\&W

Astragalus humillimus A. Gray - KTZ, SFW, W\&W, W

[Astragalus humillimus A. Gray ex Brandeges] - IMF, NNL.

Astragalus humistratus A. Gray - HDH, KTZ $<\mathrm{AZ}$, NM, UT>, W\&W, misid.

[Astragalus humistratus A, Gray var hamistratus] - IMF $\angle C D \geqslant, N N L$.

Astragalus hyalinus M. E. Jones - Dorn01, GPF, HDH, KTZ, E

[Orophaca hyalina M. E. Jones] - SFE

[Orophasa hyalina (M, E. Jones) ]sely] - NNL., WR W

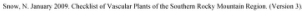


- Astragalus iodopetalus (Rydb.) Bameby - ENDE,MIC $<\mathrm{CO}, \mathrm{NM}>$, KTZ, NNL, W

[Astragalus iodopetales Greene ex Bameby]- HDH

[Astragalus iodopetalus (Greene) Bameby] - SFW

[Astragalus iodopetalus (Greene ex Rydb.) Bamoby] - Ws:W

Astragalus jejunus S. Watson var. jejunus - Dorn01, KTZ, W

[ sstragalus jejunes S. Watson] - IMF, NNL, SFW, UTF, W\&W

Astragalus kentrophyta A, Gray var, elatus S, Watson - IMF, KTZ, NNL, UTF, W

[Astragalus kentrophyta A. Gray]-HDH

[Astragalus kentrophyta A. Gray subsp. elatus (S. Watson) W. A. Weber] - SFW, W\&W

Astragalus kentrophyta A. Gray var. jessiae (Peck) Bameby - WY checklist

[Astragalus jessiae M. E. Peck] - KTZ

[Astragalus kentrophyta A. Gray suhsp. jessiae (M.E. Peck) W.A. Weber] - KTZ, NNL.

Astragalus kentrophyta A. Gray var, kentrophyta - NNL, IMF, KTZ, E

[Astragalus kentrophyta A. Gray] - GPF, HDH

[Astragalus kentrophyta A. Gray subsp. kentrophyta] - SFE, W\&W

Astragalus kentrophyta A. Gray var, tegetarius (S. Watson) Dorn - IMF, KTZ, UTF, E/W

[Astragalus kentrophyla A. Gray subsp. implexus (Canby ex Porter \& J. M. CoulL) W. A. Weber] - W\&W

[Astragalus kentroptyta A. Gray subsp. implexus (Canby) W. A. Weber]-SF!

[Astragalus kentrophyta A. Gray vat, implexus (Porter \& J. M. Coult.) Barneby] - NNL.

[Astragalus kentrophyta A. Gray var, implexus (Canby ex Porter \& J. M. Coult.) Barneby]-KTZ

[Astragalus tegetarius S. Watson] - $\mathrm{HDH}$

Astragalus lentiginosus Douglas ex Hook, var. chartaceus M. E. Jones - Dom01, IMF, KTZ

[Astragalus lentiginosus Douglas ex Hook. var. platyphyllidius (Rydb.) Bameby] - UTF

[Astragalus lentiginosus Douglis ex Hook, var, platyplaylidius (Rydb,) M, Pock] - NNL, W\&W

[Astragalus lentiginosus Douglas ex Hook. var. aranosus (E. Sheld.) Bamesby] - UTF

Astragalus lentiginosus Douglas ex Hook, var, diphysus (A. Gray) M. E. Jones - IMF, KTZ, UTF, W\&W

[Astragalus lemiginosus Douglas ex Hook. var-allbiflerus (A.Gray) Schoence] - NNL. W\& W"

[Astragalus lentiginosus Douglas var. diphysus (A. Gray) M. E. Jones] - HDH

Astragalus lentiginosus Douglas ex Hook, var, palans (M. E. Jones) M. E. Jones - IMF, KTZ,

UTF, W\&W, W

[Astragalus leniginosus Douglas ex Hook, var. palans (A. Gray) M. E. Jones]

[Astragalus lensiginosus Doughas var. palans (A. Gray) M. E. Jones] - SFW

[Astragalus lestiginosus Doughas var, palans (M. E. Jones) M. E. Jooes] - HDH, NNL.

Astragalus leptaleus A. Gray - Dorn01, HDH, KTZ, NN1, SF!, W\&W, EW

- Astragalus linifolius Osterh. - E.NDEMIC $<C O>$, IMF, KTZ, NNL, SFW, W\&W, W

[Astragalus tooms M. E. Jones] - HDH

Astragalus lonchocarpus Torr. - HDH, IMF, KTZ, SF!. UTF, E/W

[Astragalus lonchocarpus Torr, var. lonchocarpus] - NNL, WEW

Astragalus lotiflorus Hook. - Dorn01, GPF, HDH, K1Z, NNL, SFE, W\&W, E

Astragalus lutosus M. E. Jones - HDH, IMF, KTZ, NNL, SFW, UTF. W\&W. W

Astragalus megacarpus (Nutt.) A. Gray var- megacarpus - UT3

[Astragalus megacarpus (Nutt.) A. Gray]- Dorn01, HDH, exp., IMF, KTZ, NNL, SFW, W\&W, W

[Astragalus megacarpus (Nuts.) A. Gray var, megacarpus] - UTF

- Astragalus megacarpus (Nutt) A. Gray var. neeseae S. Welsh - ENDEMIC $<\mathrm{CO}>$. UT3

- Astragalus microcymbus Barneby - ENDEMIC <CO>, KTZ, NNL, SFW, W\&W, W

Astragalus miser Douglas var. oblongifolius (Rydb.) Cronquist - Dom01, KTZ, NNL, SF!,

W\&W, E/W

[Astragalus miser Douglas ex Hook. var. decumbens (Nuit.) Croncuist] - Darn0]

[Astragalus miser Douglas var. decurnbers (Nutt.) Cronq.] - W\& W' ${ }^{1}$, err. rep.

[Astragalus miser Douglas ex Hook. var. oblongifolius (Rydb.) Cronquist] - IMF, UTF

[Astragalus decumbers (Nutt. ex Torr. \& A. Gray) A. Gray var. decumbens]-HDH 
[Astragalus decumbers (Nutt ex Torr. \& A. Gray) A. Gray var. decurrens (Rydb.) Cronquist] - HDH

[Astragalus decumbens (Nutt. ex Torr. \& A. Gray) A. Gray var. oblangifolius (Rydlo.) Cronquist] - HDH

Astragalus miser Douglas var. proeteritus Bameby - Dom01, KTZ

[Astragalus miser Douglas var. tenuifolius (Nutt.) Bameby] - NNL.

Astragalus miser Douglas var. tenuifolius (Nutt.) Barneby - Dorn01, KTZ, NNL

Astragalus missouriensis Nutt, var, amphibolus Barneby - HDH, IMF, KTZ, NNL, SFW, UTF, W\&W, W

Astragalus missouriensis Nutt, var, humistratus Isely - KTZ, NNL, W\&W

Astragalus missouriensis Nutt, var, missouriensis - HDH, IMF, KTZ, NNL, SFW, UTF, W\&W, E/W

[Astragalus missouriensis Nutt.] - GPF, SFE

Astragalus mollissimus Torr. var, mollissimus - GPF, IMF, KTZ, NNL, SFE, UTF, W\&W, E

[Astragalus mollissimus Torr.] - Dorna1, HDH

Astragalus mollissimus Torr. var, thompsoniae (S. Watson) Barneby -IMF, KTZ, SFW, UTF, W\&W, W

[Astragalus bigelovii A. Gray var, thompscaiae (S. Watson) M. E. Jonies] - HDH

[Astragalus thompsonae S. Wacson] - NNL

- Astragalus molybdenus Barneby - ENDEMIC <CO>, HDH, corr, \& add, KTZ, NNL, SF!. W\&W, Lavin97, ENW

[Astragalus plunbeus Bemeby] - HDH

Astragalus monumentalis Barneby var, cottamii (S. L. Welsh) Isely -NNL.IMF, KTZ, W\&W, W

[Astragalus cottamii S. L. Welsh] - UTF

[Astragalus monamestal is Barneby] - SFW

Astragalus musiniensis M. E. Jones - IMF, KTZ, NNL, SFW, UTF, W\&W, W

Astragalus naturitensis Payson - IMF, KTZ, NNL, SFW, UTF, W\&W, w

[Astragalus naturitensig Payson var, naturiteasis] - $\mathrm{HDH}$

Astragalus nelsonianus Barneby - Dorn01, IMF, KTZ, NNL., SFW, UTF, W\&W, W

[NS - a "regional endemic' of CO and $\mathrm{WY}$, including parts of UT]

Astragalus newberryi A. Gray var. newberryi - IMF, KTZ, NNL, UTF, W

[Astragalus newbempi A. Gray]-SFW, W\&W

Astragalus nuttallianus DC. var. austrinus (Small) Barneby - KWA, M\&H, KTZ

[Astragalus austrinus (Small) E.D. Schulz] - KTZ

[Austsragalus nuttallianus DC: var, austrimus (Smali) Shreve \& Wiggins] - NNL

[Hamosa austrinus Small] - KWA

Astragalus nuttallianus DC. var. micranthiformis Barneby -IMF, KTZ, NNL, SF!, UTF, E/W

[Astragalus nutrallianus DC.] - $\mathrm{HDH}$

[Astragalus numalliasus DC, var, mikranheniformis Barnebs] - W\&W

\{Astragalus nuttallianus DC. var. trichocarpus Torr. \& A. Gray\} - HDH, KTZ $\angle O K>, N N L$, W\& $W^{\prime}$

Astragalus ō̃calycis M. E. Jones - HDH, KTZ, NNL., W

[Astragalus oocalycis M. E. Jones] - SFW, W\&W

Astragalus oophorus S. Watson var. caulescens (M. E. Jones) M. E. Jones - IMF, KTZ, NNL, UTF, W

[Astragalus owphorus S. Watson] - HDH

[Astragalus oôphonus M. E. Jones var, cauleseens (M. E. Jones) M. E. Jones] - SFW. W\&W

Astragalus oreganus Nutt. - Dorn01, KTZ

- Astragalus asterhoutii M. E. Jones - ENDEMIC $<\mathrm{CO}>, \mathrm{HDH}, \mathrm{KTZ}, \mathrm{NNL}, \mathrm{SFW}$, W\&W, W Astragalus parryi A. Gray - Dorn01, HDH, KTZ, NNL, SF!, W\&W, E/W

Astragalus pattersonii A. Gray - KTZ. NNL. SFW. W\&W. W

[Astragalus patterscoii A. Gray ex Brandegee] - IMF, NNL, UTF 
[Astragalus patterscoii A. Gray var. pattersonii] - HDH

Astragalus pectinatus (Hook.) Douglas ex G. Don - Dorn01, KTZ, E

[Astragalus pectinatus Douglas ex G. Don]-GPF, IMF, UTF

[Astragalus pectinatus (Hook-) G. Dan] - NNL.

[Astragalus pectinatus Douglas] - SFE

[Astragalus pectinatus (Hook-) Douglas] - HDH, W\&W

Astragalus piscator Barneby \& S. L. Welsh - IMF, KTZ, NNL, SFW, UTF, W\&W, W

Astragalus plattensis Nutt. - Dorn01, HDH, KTZ, NNL, SFE, E

[Astragalus plattensis Nurt, ex Torr. \& A. Gray] - GPF, W\&W

Astragalus praelongus E. Sheld. var, ellisiae (Rydb.) Bameby - GPF, IMF, KTZ, UTF, W\&W. W

[Astragalus praelongus E. Sheld.] - SFW

[Astragalus praelongus E. Sheld. var. praclongus] - NNL

Astragalus praelongus E.Sheld, var. lonchopus Barneby - IMF $<\mathrm{CO}>$, KTZ, NNL, UTF $\langle$ AZ, UT $>$

Astragalus praelongus E. Sheld, var, praelongus - IMF, KTZ, NNL, UTF, W\&W, W

[Astragalus patiersenii A. Gray var. praclongus (E. Sheld.) M. E. Jones] - HDH

[Astragalus praelongus E. Sheld.] - SFW

[Astragalus reoedicns (Greene ex Rydlo.) Ced. Porter] - HDH

- Astragalus proimanthus Barneby - ENDEMIC <WY>, Dorn01, KTZ

[Orophasa proimanhiua (Bameby) Isley] - KTZ NNL

-Astragalus proximus (Rydb.) Wooton \& Standl. - ENDEMIC <CO, NM>, HDH, KTZ, NNL, SFW, W\&W, W

Astragalus pubentissimus Torr. \& A. Gray - Dorn01, IMF, KTZ, SFW, W\&W, w

[Astragalus pubentissimus Tort. \& A. Gray var. pabentissimus] - NNL, UTF

Astragalus puniceus Osterh, var, puniceus - GPF, KTZ, NNL, E

[Astragalus puniceus Ostefh.] - HDH, SFE, W\&W

Astragalus purshii Douglas ex Hook. var, purshii - IMF, KTZ, NNL. UTF, E/W

[Astragalus purshii Doughas] - SF!

[Astragalus purshii Douglas ex Hook.] - Dom01, GPF, HDH, W\&W

Astragalus racemosus Pursh var. longigetus M. E. Jones - Dom01, GPF, KTZ, W\&W, E

[Astragalus racemosus Pursh] - HDH, NNL, SFE

Astragalus racemosus Pursh var, racemosus - Dom01, GPF, IMF, KTZ, NNL, UTF, W\&W, E

[Astragalus racemosus Pursh] - HDH. SFE

Astragalus rafaelensis M. E. Jones - HDH, IMF, KTZ, NNL, SFW, UTF, W\&W, W

Astragalus ripleyi Barneby - $\mathrm{HDH}, \mathrm{KTZ}$ NNL, SFE, W\&W, E

Astragalus robbinsii (Oakes) A. Gray var. minor (Hook.) Barneby - IMF, KTZ, NNL, SFE,

W\&W, ENW

[Astragalus occidentalis (S. Watson) M. E. Jones] - HDH

[Astragalus roboinsii A. Gray var, minor (Hook.) Bumeby] - SFW

[Astragalus robbinsii (Oakes) A. Gray] - UTF

Astragalus saurinus Bameby - IMF, KTZ <UT>, NNL, SFW, UTF, W

- Astragalus schmolliac Ced. Porter - ENDEMIC <CO>, HDH, KTZ, NNL, SFW. W\&W. W

Astragalus scopulorum Porter -HDH, IMF, KTZ, NNL, SF!, UTF.W\&W, E/W

Astragalus sericoleucus A. Gray var. aretioides M. E. Jones - Dorn01

[Astragalus aresoidoides (M. E. Jones) Barneby]-KTZ

Astragalus sericoleucus A. Gray var. sericoleucus - Dorn01, HDH

[Astragalus sericoleucus A. Giray] - GPF, KTZ, E

[Orophasa sericea Nutt] - SFE

[Orophaca serices (Nutt. $\}$ Britton] - NNL, W\&W

Astragalus sesquiflorus S. Watson-IMF, KTZ, NNL. SFW, UTF, W\&W, W

Snow, N, Junury 2009. Checklist of Vaccular Plants of the Southom Rocky Momain Region. CVersion $3 x$ 
Astragalus shortianus Nutt, - Dom01, KTZ, NNL, SF!, W\&W, E/W

[Astragalus shortianus Nutt. ex Torr. \& A. Gray] - GPF, HDH

- Astragalus simplicifolius (Nutt.) A, Gray - ENDEMIC <WY>, Dorn01, HDH, IMF, KTZ. W\&W $W^{1}$ misid.

\{Astragalus simplicifolius (NurL) Rydb.\} - NNL, W\&W, misid.

- Astragalus sparsiflorus A. Gray var. majusculus A. Gray - ENDEMIC $\angle C O$. HDH. KTZ.

NNL, E

[NS - 2 varieties recognized by some, e.g. KTZ]

[Astragalus sparsillorus A. Gray] - SFE, W\&W

Astragalus sparsiflorus A. Gray var, sparsiflorus - HDH, KTZ, NNL, E

[Astragalus sparsiflorus A. Gray] - SFE, W\&W

Astragalus spatulatus E, Sheld. - Dorn01, GPF, HDH, IMF, KTZ, NNL, SF!, UTF, W\&W, ENW

Astragalus tenellus Pursh - Dorn01, GPF, HDH, IMF, KTZ, NNL, SF!, UTF, W\&W, E/W

[Reveak04 recommend that this species now should be called $A$. muldifloris (Pursh) A. Gray]

Astragalus tephrodes Gray var, brachylobus (Gray) Barneby - NNL, KTZ, M\&H, KWA

[Astragalus arrectus var. peptragnenus (M.E. Jones) M.E. Jones] - KT2

[Astragalus curtilobus Tidestrom] - KWA. M\&H

[Astragalus remulcus M. E. Jones] - KWA, M\&H

[Astragalus shortianus Nuth. \&x T. \& G. var. bractiylobus Gray] - KWA, M\&H

[Xylophacos brachylobus (Gray) Rydberg] - KWA, M\&H

- Astragalus wortipes J. L. Anderson \& J. M. Porter - ENDEMIC $<$ CO $>$ KTZ, NNL, SFW, W\& $W^{\prime}, W$

Astragalus tridactylicus A. Gray - KTZ, E

[Astragalus sericoleucus A. Gray var, tridactylicus (A, Gray) M. E. lones] - $\mathrm{HDH}$

[Orophaca tridactylica (A, Gray) Rydb.] - NNL., SFE, WdW

Astragalus vexilliflexus Sheld. var. vexilliflexus - KTZ, NNL

[Homalobus vexilliflexus (Sheld.) Rydb.] - Dom01, KTZ

Astragalus wetherillii M. E. Jones - HDH, IMF, KTZ, NNL, SFW, UTF, W\&W, W

Astragalus wingatanus S. Watson - HDH, IMF, KTZ, NNL, SFW, UTF, W\& W, W

Astragalus wittmannii Barneby - GREE (Hazlet /1700 [Harding Co., NM])

Astragalus wootonii E. Sheld. var. wootonii - W\&W. E

[Astragalus allochrous A. Gray var. playanus Isely] - KTZ.

[Astragalus allochroes A. Gray var. playanus (M. E. Joses) Isely] - NNL., W\&W'

[Astragalus wootonii E. Sheld] - SFE

\section{Caesalpinia (see also Hoffmanseggia, Pomaria)}

\section{Cassia (sec Chamaecrista)}

* Caragana arborescens Lam - Dorn01, GPF, IMF, KTZ, SFE, UTF, W\&W, E

[Caragana artorescens (L.) Lam.] - NNL.

* Caragana aurantiaca Kochne - KTZ, NNL, SFE, W\&W, E

\{Chamaecrista fasciculata (Michx.) Greene\} - NNL, W\&W, rep.

(Cassia chamaecrista L.\} - GPF $<\mathrm{CO}$ >

[Cassia fesciculata Michx.\} - HDH, exp.

\{Chamsecrista fasciculat (Michx.) Greene var. fasciculata\} - KTZ $<$ KS, NE, NM, OK $>$

* Colutea arborescens L. - IMF, KTZ, NNL, SFE, UTF, W\&W, E

* Coronilla varia L. - Dorn01. GPF, IMF. KTZ. UTF. E/W

Snow, N, Junury 2009. Checklast of Vaccular Plants of the Southom Rocky Momain Region. CVersion $3 x$ 
[Securigera varia (L.) L.assen]] - NNL., SF!, W\&W

Dalea

(Identification sources:

Dalea albiflora Gray - NNL, M\&H, KTZ, KWA. JM, not rep.

[Dalea crdiae Gray] - KTZ

[Parosela albiflora [Gray) Vail] - KWA

[Petalostemon pilosulus Rydb.] - KTZ

[Thombera albiflora (Gray) Rydb.] - M\&H

Dalea aurea Nutt, ex Pursh - Dorn01, GPF, KTZ, NNL, W\&W, E

[Dalea aurea NunL] - HDH. SFE

Dalea candida Michx, var. oligophylla (Torr.) Shinners - Dorn01, SF!, ENW

[Dalea candida Willd. var. oligophylla (Torr.] Shinners] - W \& W

[Dalea candida Michx, ex Willd. var, oligophylla (Torr.) Shinners] - GPF, IMF, KTZ

[Dalea occidentalis (Britton \& Keamey) Isely] - NNL

[Dalea oligophylla (Torr.) Shinners] - UTF

[Petalostemon candidus Michx_ var. oligophyllus (Torr.) Hermann] - HDH

Dalea cylindriceps Bameby - Dom01, GPF, KTZ, NNL, SFE, W\&W, E

[Petalostemon compactus iSpreng-) Sweney]- $\mathrm{HDH}$

Dalea enneandra Nutt, - Dorn01, GPF, HDH, KTZ, NNL, SFE, W\&W, E

Dalea formosa Torr. - GPF, HDH, KTZ, NNL, SFE, W\&W, E

Dalea jamesii (Torr.) Torr. \& A. Gray - GPF, HDH, KTZ, NNL, SFE, W\&W, E

Dalea lanata Spreng. var. lanata - IMF, KTZ, NNL, UTF, E

[Dalea lansta Spreng.] - GPF, HDH, SFE, W\&W

Dalea lanata Spreng. var, terminalis (M. E. Jones) Bameby - IMF, KTZ, NNL, UTF, E [Dalea lanata Spreng.] - SFE, W\& W'

Dalea leporina (Aiton) Bullock - GPF, KTZ, NNL, SFE, W\&W, E

[Dalea leporisa (Aiton) Keamcy \& Pocbles var. alba (Michx.) Harrington] - HDH

Dalea multiflora (Nutt.) Shinners - GPF, KTZ, NNL, SFE, W\&W, E

Dalea nana Torr. var. carnescens (Rydb.) Kearney \& Peebles - HDH, W\&W, Hazlen 10763

(COLO)

[Dalea nana Tor, ex A. Gray var, carnescens (Rydb.) Kearney \& Pecbles] - KTZ <AZ, NM $>$, NNL.

Dalea nana Torr. \& A. Gray var. nana - KTZ. E.

[Dalea nana Torr.] - SFE

[Dalea nana Tor, ex A, Gray] - GPF

[Dalea nana Tor, var, nana] - HDH, W\&W

[Dalea naina Tor, ex A. Gray var, Bana] - NNL

Dalea polygonoides A. Gray - Kartesz (pers.comm. 2008), Legler (pers. comm. 2009)

[NS - confirmed now for Mora and Colfax cos., NM]

Dalea purpurea Vent, var, arenicola (Wemple) Burneby - GPF $\angle C O>$, KTZ, NNL

Dalea purpurea Vent, var, purpurea - GPF, KTZ, NNL, E

[Dalea parpurea Vent.] - Dorn01, SFE, WRW

[Petalostemon purpareum (Ventenat) Rydb, var, pubescens (A. Nelsco) Harrington] - HDH

[Petalostemon purpareum (Ventenat) Rydb, var, purpureum] - HDH

Dalea tenuifolia (A. Gray) Shinners - GPF, KTZ, NNL, SFE. W\&W, E

Dalea villosa (Nutt.) Spreng. var. villosa - KTZ, NNL, E

[Dalea villosa Spreng,] - Wew

[Dalea villosa (Nutt.) Spreng.] - Domb1, GPF, SFE

[Petalostemon villosus Nutt.] - HDH 
Desmanthus cooleyi (Eat) Trel, - GPF, HDH, KTZ, NNL, SFE, W\&W, E

* Desmanthus illinoensis (Michx.) MacMill, ex B. L. Rob, \& Fernald - KTZ, NNL. W\&W ${ }^{\prime}$, E

[W] - seen in roadside ditches, Bent and Otero cos, apparently introduced]

[Desmanthus illinoensis (Michx.) MacMill.] - GPF, HDH, SFE, UTF, W\&W

Desmodium obtusum (Muhl. ex Willd.) DC, - GPF, KTZ

[Desmodium oblusum (Willd.) DC] - NNL

[Desmodium rigidum (Elliott) DC.] - W\&W

Galega officinalis L, - IMF, KTZ, NNL, UTF

\% Gleditsia triacanthos L. - Dorn01, GPF, IMF, KTZ, NNL

Glycyrthiza lepidota Pursh - Dorn01, HDH, KTZ, SF!, UTF, W\&W

[Glycyrrhiza kepidota Nutt. ex Pursh] - E/W

[Glycyrrhiza lepidota Nutt. ex. Pursh var. glutinosa fNunt) S. Watson] - RMC, NNL

[Glycyrrhiza lepidota Nut. ex Pursh yar. glutinosa [NutL ex Torr. \& A. GraysS. Waison] - IMF

[Glycyrthiza lepidola Pursh var. glutinosa (Nutl.) S. Watson] - GPF

[Glycyrthiza kpidota Nut. ex Pursh var. lepidota] - IMF, RMC

[Glycyrrhiza lepidota Pursh var. lepidota] - GPF, NNL.

Hedysarum alpinum L. var, philoscia (A. Nels, Rollins - NNL

[Hedysarum alpinum L. var, americanum Michx_] - Dorn01, KTZ

Hedysarum boreale Nutt, var. boreale - Dorn01, GPF, IMF, NNL, UTF, E/N

[Hedysarum boreale Nutt.] - HDH, SF!, W\&W

[Hedysarum bereale Nuth. vart. borcale] - IMF, UTF

[Hodysarum borealo Nutt. subsp. boreale var. boreale] - KTZ

Hedysarum boreale Nutt, var, cinerascens (Rydb.) Rollins - NNL.

[Hedysaram boreale Nut, subsp. boreale var. boreale] - KTL

[Hedyskrum boreale Nuth.] - GPF. SF

[Hedysmum boreale Nut, var, boceale] - IMF, UTF

Hedysarum boreale Nutt. var. pabulare (A. Nels.) Dorn - Dorn01

Hedysarum occidentale Greene - Dom01, HDH, IMF, NNL, SF!, W\&W, E/W

[Hedysarum occidentale Greene var, canone S.1. Welsh] - KTZ

[Hedysarum occideatale Greene var. oceidentale] - KTZ, UTF

Hoffimanseggia drepanocarpa A. Gray - GPF, HDH, NNL, SFE, W\&W, Simpson99, E [Caesalpinia drepunocarpa (A. Gray) E. Fisher] - [MF, KTZ

Hoffmanseggia glauca (Ortega) Eifert - GPF, KTZ, NNL, SFE, W\&W, Simpson99, E [Hoffminseggia densithora Benthe] - HDH

Hoftmanseggia repens (Eastw_) Cockerell - NNL. Simpson99

[Caesalpinia repents Easiw.] - [MF, KTZ. UTF $=\mathrm{CO}$ -

Lathyrus

(Identification sources:

Lathyrus brachycalyx Rydb. var. zionensis (C. L. Hitche.) S. L. Welsh - IMF, NNL, SFW, UTF, W\&W,W

[Lathyrus brachycalyx Rydb, subsp. zionis (C. L. Hitche) S. L. Welsh] - KTZ.

Snow, N. Junury 2009. Checklist of Vascular Plants of the Southom Bocky Mosmain Region. CVersion 3 . 
Lathyrus eucosmus Butters \& H. St. John - Dorn01, HDH, IMF, KTZ, NNL, SF!, W\&W, E/W

Lathyrus laetivirens Greene ex Rydb, - IMF, KTZ, E/W

[Lathyrus lanscowertii Kellogg var. laetivirens (Groene) S. L. Welsh] - UTF

[Lathyrus lanscovertii Kellagg var. laetivirens (Rydb.) S. L. Welsh] - NNL.

[Lathyrus leucanthus Greene] - SFW

[Lathyrus leucianthus Rydb.] - SEE, W\&W

Lathyrus lanszwertii Kellogg var. lanszwertii - Dorn01, IMF, KTZ, NNL, UTF

Lathyns lanszwertii Kellogg var, leucanthus (Rydb.) Dorn - Dorn01, IMF, KTZ, UTF, E/W

[Lathyrus arizonicus Britton] - HDH

[Lathyrus lanscrwertii Kellagg var, arizonicus (Britton) S, L. Welsh] - NNL

[Lathy rus leucanthus Greene] - SFW

[Lathyrus leucanthus Ryib.] - HDH, SFE, W\&W

* Lathyrus latifolius L. - GPF, IMF, KTZ, NNL, SF!, UTF, W\&W, E/W

Lathyrus pauciflorus Fernald var, utahensis (M. E. Jones) R. Davis - IMF, W

[Lathyrus pauciflorus Femald] - HDH, SFW, W\&W

[Lathy rus pauciforus Femald var. utahensis (M. E. Jones) Piper ex M. Peck] - KTZ

[Lahyrus pauciflorus Femald var. utahensis (M. E. Jones) Piper ex R. J. Davis] - NNL

[Lathy rus pauciflorus Femald var. utahensis (M. E. Jones) M. Peck] - UTF

Lathyrus polymorphus Nutt. var, incanus (J, G. Sm, \& Rydb, ex Rydb.) Dorn - Dorn01, E W\&W

[Liathyrus polymorpbus Nutt. subsp. incanus (J.G. Sm, \& Rydb.) C. L. Hitche] - GPF, HDH, KTZ, SFE.

[Lifhyrus polythorptous Nutt. vart. irxanus (J. G. Sm. \& Rydb.) bely] - NNL

Lathyrus polymorphus Nutt. var, polymorphus - Dorn01, NNL

[Latby rus pelymarpbus Nutt. subsp. polymorphus] - GPF, HDH, W\&W

[Lathyrus pelymorphus Nutt. subsp. polymorphus var. polymorphus] - KTZ

*Lathyrus sylvestris L. - KTZ, UTF3

[NS - one naturalized collection at GREE from Beulder CO; nacural bod in several other western scanes (MT.

WY, UT, ID, WA, OR)]

坣 Lotus comiculatus L. - Dom01, GPF, IMF, KTZ, NNL, UTF

\{Lotus purshianus (Benth.) Clem. \& E, G. Clem.\} - HDH, rep.

\{Loxus purshianus Ckm, \& E. G. Clem - GPF

\{Loxus purshiaews (Benth. ex Lindi.) Clem. \& E. G. Clem.\} - IMF

\{Loxus purshianus (Benth. ex Lindi.) Clem. \& E. G. Clem. ex Otley\} - NNL

\{Lotus unifoliolatus (Hook.) Benth, var, unifoliolatus) - KTZ $<$ AZ, KS, NE, OK, WY

* Lotus tenuis Waldst. \& Kit. ex Willd. - GPF, IMF, KTZ, E/W

[Lotus comiculatus L.] - NNL.

[Lotus glaber Mill.] - Kirkbridoo6

[Lotus tenuis Kit] - UTF

[Lotus tenuis Waldst. \& Kit] - SFI, W\&W

Lotus wrightii (A. Gray) Greene - HDH, IMF, KTZ, NNL, SF!, UTF, W\&W, E/W

Lupinus

(Identification sources: )

Lupinus argenteus Pursh var. argenteus - Dorn01, GPF, IMF, NNL, UTF, EW

[Lupinus argenceus Pursh] - HDH, SFW, W\&W

[Lupinus argenteus Pursh subsp. argenteus] - SFE

[Lupinus argenteus Pursh subsp. argenteus var. argenteus] - KTZ

[Lupinus argenteus Pursh subsp. spatulacus (Rydb.) L. W. Hess \& D. B. Dunn] - KTZ

Lupinus argenteus Pursh yar. argophyllus (A. Gray) S. Watson-Dorn01, IMF. E/W

[Lupinus adancus Greene] - HDH 
[Lupinus caudatus Kellogg] - SF!, W\&W

[Lupinus caudatus Kellogg subsp. argophyllus (A. Gray) L. L. Phillips] - KTZ, SFW

[Lupinus caudatus Kellogg var, argophyllus (A. Gray) S. L. Welsh] - NNI, UTH

Lupinus argenteus Pursh var. fulvomaculatus (Payson) Barneby - IMF, NNL, UTF, E

[Lupinus argerteus Pursh] - W\& W

[Lupinus argenteus Pursh subsp. ingratus (Greene) Harman] - KTZ, SFE

\{Lupinus argenteus Pursh var, heteranthus (S. Watson) Barneby\} - IMF, E

[Lupinus caudatus Kellogg] - GPF, SFE, W\&W

\{Lupinus caudatus Kellogg subsp. caudatus\} - KTZ

[Lupinus caudatus Kellogg var. caudatus\} - NNL

\{Lupinus eaudalus Kellogg var-cutleri (Eastw.) S. L. Welshi - UTF

\{Lupinas greenei A. Nelson\} - $\mathrm{HDH}$

Lupinus argenteus Pursh var, holosericeus (Nutt. ex Tort, \& A. Gray) Barneby - IMF

[Lupinus argenteus Pursh var. holosericeus (Nutt.) Barneby] - NNL

[Lupinus holosericeus Nutt.] - KTZ

Lupinus argenteus. Pursh var, laxiflorus (Douglas ex Lindl.) Dom - Dom01

[Lupinus argenteus Pursh subsp. argenteus var. laxiflorus (Douglas ex Lindl.) Dom] - KTZ

Lupinus argenteus Pursh var, moabensis S. L. Welsh - IMF, UTF

[Lupinus argenceus Purab] - W\& W

[Lupinus argeneus Pursh subsp. moabensis D. B. Dunn \& Harmon] - KTZ

[Lupinus argenseus Pursh var, moaberisis Harmon \& D. B. Dunn ex S. L. Welsh] - NNL.

Lupinus argenteus Pursh var, parviflorus (Nutt. ex Hook. \& Am.) C. L. Hitchc. - IMF, E/W

[Lupinus argetneus Pursh] - W\& W

[Lupinus argenseus Pursh var, nyrianthus (Greene) Isely] - NNL

[Lupinus argeneus Pursh var, parviflonis (Hook: \& Am.) C. L. Hirche.] - NNL

[Lupinus argeneus Pursh var, parviflons (Nutt.) C. L. Hische.] - GPF, UTF

[Lupinus parviflorus Nut.] - SFW

[Lupinus parviflorus Nutt، ex Hook. \& Am.]-HDH

[Lupinus parviflorus Nutt, ex Hook, \& Am, subsp, floribundus (Gresne) Harmon] - KTZ

[Lupinus parviflorus Nutt. subsp. myrianthus (Greene) Harmon var. myrianthus] - KTZ

[Lupinus parviflorus Nutt. var. myrianthus (Greene) Harmon] - SFE

[Lupinus parviflorus Nutt, ex Hook. \& Am, subsp, myrisathus \{Grecne) Harmon var. myrianthus] - KTZ

[Lupinus parviflorus Nutt، subsp. parviflorus] - W\& W ${ }^{1}$

[Lupinus parviflorus Nutt, ex Hook. \& Am, subsp; parviflorus] - KTZ

Lupinus argenteus Pursh var, rubricaulis (Greene) S. L. Welsh - Dorn01, IMF, NNL, UTF

[Lupinus X alpestris A. Nelson] - KTZ

[Lupinus argenceus X L. easidatus] - KTZ

[Lupinus argenteus Pursb] - HDH, W\& W

[Lupinus argenteus Pursh subsp. rubricaulis (Greene) L. W. Hess \& D. B. Dunn] - KTZ

[Lupinus depressus Ryob.] - KTZ

Lupinus argenteus Pursh var, utahensis (S. Watson) Barneby - IMF $<\mathrm{CO}>$

[Lupinus caudacus Kelloge] - W\& W ${ }^{1}$

[Lupinus caudacus Kellogg subsp. caudarus] - KTZ

[Lupinus caudacus Kellogg var, uahensis (S. Watsen) S. L. Welsh] - NNL. UTF

\{Lupinus barbiger S. Watson\} - HDH, KTZ <AZ, UT>, W\&W, misapp.

\{Lupinus sericeus Pursh\} - IMF

\{Lepinas sericeus Pursh var, barbiger (S. Warspe) S. L. Welsh\} - NNL, UTH

Lupinus brevicaulis S. Watson - HDH, IMF, KTZ, NNL, SFW, UTF, W\&W, W

Lupinus kingii S. Watson - HDH, IMF, NNL, SF!, W\&W, E/W

[Lupinus kingii S, Watson var, kingii] - KTZ, UTF

[Lupinus kingii S. Watson var, argillaceus (Wooton \& Standl.) C. P. Sm.] - HDH rep., KTZ

Lupinus lepidus Douglas ex Lindl var. utahensis (S. Watson) C. L. Hitche. - Dorn01, NNL, E/W

[Lupinus caespitorus Nutt.] - HDH, W\&W

[Lupinus caespitosus Nutt. var, utahensis (S. Watson) Cos] - KTZ 
[Lupinus lepidus Douglas subsp. caespitosus (Nutt) Detling] - SF!, W\& W'

[Lupinus lepidus Douglas var. utahensis (S. Watson) C. L. Hitchc.] - IMF, UTF

Lupinus neomexicanus Greene - NNL, KWA, M\&H, KTZ, JM, not rep.

[Lupinus blumeri Greene] - KTZ

Lupinus plattensis S. Watson - Dom01, GPF, HDH. IMF, KTZ, NNL, SFE. W\&W, E

Lupinus polyphyllus Lindl, var. ammophilus (Greene) Barneby - IMF, UTF, W

[Lupinus ammophilus Greene] - HDH, KTZ, SFW, w\& W

[Lupinus ammophilus Greene var. ammophilus] - NNL

[Lupinus ammophilus Greene var. crassus (Payson) lsely] - NNL.

[Lupinus crassus Payson]- KTZ, SFW, W\&W

[Lupinus lasalensis D. B. Dunn, unpubl.] - WRW, possibly a variety of this taxion

Lupinus polyphyllus Lindl, var, humicola (A. Nelson) Barneby - Dorn01, IMF, NNL, UTF, W

[Lupinus humicols A. Nelson] - HDH

[Lupinus polyphyllas Lindl.] - WE: $W^{\prime}$

[Lupinus wyethii S. Watson]-SFW, W\&W, vet of range

[Lupinus wyethii S. Wasson subsp. wyethii] - KTZ

Lupinus polyphyllus Lindl, var, prunophilus (M. E. Jones) L. LI. Phillips = Dorn01, IMF, NNL. UTF, E.WW

[Lupinus bakeri Greene subsp. amplus (Greene) Fleak \& D. B. Dunn] - KTZ, SFW, W\& W

[Lupinus polyplyyllas Lindl.] - W\&: $W^{\prime}$

[Lupinus prunephilus M. E. Jones] - KTZ, SF!. W\&W

Lupinus pusillus Pursh var, intermontanus (A. Heller) C. P. Sm, - Dorn01, IMF, NNI, UTF, $\mathrm{E} / \mathrm{W}$

[Lupinus pusillus Pursh] - HDH, SF!

[Lupinus pusillas Pursh subsp. intermicetarus (A. Heller) D. B. Dung] - KTZ

Lupinus pusillus Pursh var. pusillus - Dom0l, IMF, NNI, UTF, ENW

[Lupinus pusillas Pursh] - GPF, HDH, SF!

[Lupinus pusillas Pursh subsp. pusillus] - KTZ, W\&W

[Lupinus pusillus Pursh subsp. rubens (Rydb.) D. B. Duna] - KTZ, W\& W'

[Lupinus pusillus Pursh var, rubents (Rydb.) S. L. Welsh] - UTF

Lupinus sericeus Pursh var, sericeus - NNL, UTF, E/W

[Lupinus bakeri Groene subsp. bakeri] - KTZ, SFW, W \& W

[Lupinus sericesus Pursh] - Dom01, GPF, IMF, SFE, WR W'

[Lupinus sericeus. Pursh subsp. sericeus var, seriosus] - KTZ

[Lupinus sericeus Pursh subsp. sericeus var, egglestonianus C. P. Sm.] - KTZ

[Lupinus sericets Pursh subsp. sericeus var. flexuosus (Lindl. ex J. Agardh) C. P. Sra.] - KTZ

[Lupinus sericeus Pursh va. seticeus] - SFW, UTF

[Lupinus sericeus. Pursh var. egglestonianus C. P. Sm.] - SFW, WaW

[Lupinus sericeus Pursh var. flexuoses (Lindl.) C. P. Sm.] - SFW, W \&W

Medicago falcata L. - NNI.

[Medicago sativn L subsp. falcnta (L.) Areang.] - KTZ

[Medicago sativa L var, falcata (L.) Doell] - Dorn01

* Medicago lupulina L. - Dorn01, GPF, HDH, IMF, KTZ, NNL, SF!, UTF, W\&W, E/W

* Mediago polymorpha L. - Dorn01

* Medicago sativa L. - Dorn01, HDH, IMF, NNL, SF!, UTF, W\&W, E/W

[Mediciggo sativa L subsp sativa] - GPF, KTZ

* Melilotus albus Medik. - Dom01, NNL. SF!. W\&W. E/W

[Melilotus allba Desr.] - $\mathrm{HDH}$

[Metilotus albe Desr, ex Lam.] - UTF

[Melilotus albe Medik.] - GPF, IMF

[Melilotus officinalis (L.) Lam.] - KTZ 
* Melilotus officinalis (L, ) Pall, - Dom01, GPF, IMF, NNL, UTF, W\&W, ENW

[Melilotus officinalis (L.) Lam.] - HDH, KTZ

[Melilotus officinalis (L.) Pall-] - SF]

Mimosa aculeaticarpa Ortega var, biuncifera (Bentham) Barneby - KWA, NNL. KTZ

[Mimosa biuncifera Benth.]-KWA, KTZ

[Mimosa biuncifera var. glabrescens Gray] - KWA, KTZ

[Mimosa biuncifera var, lindheimeri (Gray) B.L. Robins. ] - KWA, KTZ

[Mimosa flexuosa Bentham] - KWA

[Mimosa lindheimeri Gray ] - KWA. KTZ

[Mimosa warnockii B.L. Turner] - KWA, KTZ

[Mimosopsis biuncifera (Benth.) Britt. \& Rose] - KWA, KTZ

[Mimoscpsis biuscifera (Benth_) var. tlexuoss (Bentham) Robinson]-KWA

[Mimosopsis flexuosa (Benth.) Britt. \& Rose]-KWA

Mimosa borealis A. Gray - GPF, HDH, KTZ, NNL, SFE, W\&W, E

Mimosa quadrivalvis L, var. angustata (Torr. \& A. Gray) Barneby - E

[Mimosa nuttallii (DC.) B. L. Tumer] - KTZ

[Mimosa quadrivalvis L. var, nuttalii (DC.) L. S. Beard ex Barneby] - NNL

[Schrankia nutallii (DC.) Slandl.] - GPF, HDH

[Sclirankia uncinala Willd.] - SFE, W\&W

Mimosa rupertiana B. L. Turner - KTZ, E

[Mimosa quadrivalvis L. var. occidentalis (Wooton \& Slandl.) Barneby] - NNL

[Schirankia occidentalis (Wooton \& StandI.) Standi.] - GPF, HDH, SFE, W\&W

* Onobrychis viciifolia Scop, - Dom01, GPF, HDH, IMF, KTZ, NNL, UTF, W\&W

Orophaca (see Astragalus)

Oxytropis

(Identification sources:

Oxytropis besseyi (Rydb.) Blank. var. besseyi - Dorn01, NNI.

[Aragallas besseyi Rydb.] - KTZ

[Astragalus besseyi Rydb.] - KTZ

[Oxytropis nana var, besseyi (Rydb.) Isley] - KTZ

Oxytropis besseyi (Rydb.) Blank. var. fallax Barneby - Dom01, NNI.

[Oxytropis nana var, fallax (Barneby) bley] - KTZ

Oxytropis besseyi (Rydb.) Blank. var- obnapiformis (Ced. Porter) S. L. Welsh - Dorn01, KTZ.

NNL, SFW, UTF, W\&W, W

[Oxytropis obnapiformis Ced. Ponter] - $\mathrm{HDH}$

Oxytropis besseyi (Rydb.) Blank. var. ventosa (Greene) Barneby - Dorn01, NNL.

[Aragallas ventosis Greene] - KTZ

[Oxytropis nana var, ventosa (Gecene) Isely] - KT2

[Oxytropis nana var, ventosus (Greene) lskey] - KTZ

Oxytropis campestris (L.) DC. var, cusickii (Greenm.) Barneby - Dorn01, IMF $\angle C O>$, KTZ,

NNL, SFE, UTF, W\&W, E

[Oxyıropis susickii Groenm.] - W\& $W^{1}$, err, rep.

\{Oxytropis campestris (L.) DC. var. glabrata Hook.\} - NNL, HDH, W\&W, err. rep.

\{Oxytrogis maydelliana Trauty, - KTZ, WRW', err, rep.

Oxytropis campestris (L.) DC. var. gracilis (A. Nelson) Bameby - GPF, NNL, SF!, W\&W, ENW

[Oxytropis monticola A. Gray]-KTZ, W\& W'

Snow, N, Junury 2009. Checklist of Vaccular Plants of the Southom Rocky Momain Region. CVersion $3 x$ 
Oxytropis deflexa (Pall.) DC, var, deflexa - HDH, IMF, NNL, SF!, UTF, E/W

[Oxytropis deflexa (Pall.) DC. subsp. deflexa] - W\&W

Oxytropis deflexa (Pall.) DC, var, foliolosa (Hook.) Bameby - Dom01. IMF, E/W

[Oxytropis deflexa (Pall.) DC. subsp. deflexa] - W\&W

[Oxytropis deflexa (Pall.) DC. var. deflexa] - NNL., SF!

Oxytropis deflexa (Pall, DC. var, sericea Tor, \& A. Gray - Dom01, GPF, HDH, KTZ, NNL. $\mathrm{SF} ! \mathrm{W} \& \mathrm{~W}, \mathrm{E} / \mathrm{W}$

Oxytropis lagopus Nutt, var, atropurpunea (Rydb, Barneby - Dom01, KTZ

Oxytropis lagopus Nutt, var, lagopus - Dorn0l, KTZ

[Oxytropis lagepus Nutt.] - HDH. exp... NNL

Oxytropis lambertii Pursh var, bigelovii A. Gray - IMF, KTZ. NNL, UTF, ENW

[Oxytropis lambertii Pursh] - HDH, SF!

[Oxyaropis lambertii Pursh subsp. bigelovii (A.Gray) W. A. Weber] - W \& W

Oxytropis lambertii Pursh var, lambertii - GPF, IMF, KTZ, NNL, UTF, E/W

[Oxytropis lambertii Pursh] - Dom0L, HDH, SF!

[Oxytropis lambertii Pursh subso. lambertia] - WEW

Oxytropis multiceps Nutt. - Dom01, HDH, KTZ, NNL, SFE, W\&W, E

[Oxytropis multiosps Torr. \& A. Gray]-GPF, IMF, UTF

- Oxytropis nana Nutt, - ENDEMIC <WY>, Dom01, KTZ

[Oxyltopis mana NutL is Torr. \& A. Gray] - NNL

Oxytropis paryi A. Gray - Dorn01, HDH, IMF, KTZ, NNL, SF!, UTF, W\&W, EW

Oxytropis podocarpa A. Gray - Dom01, HDH, KTZ, NNL, SF!, W\&W, E/W

Oxytropis riparia Litv, - Dorn01, KTZ, NNL

Oxytropis sericea Nut, var, sericea - Dorn01, GPF, IMF, KTZ, NNL, UTF, E/W

[Oxytropis sericeat Nutt]- HDH. SF!, W\& W

Oxytropis sericea Nutt. var. speciosa (Torr. \& A. Gray) S. L. Welsh - Dorn01

Oxytropis sericea Nutt. var. spicata (Hook.) Barneby - NNL.

[Oxyaropis campestris (L.) DC. var, spicata Hook.] - Dornll

Oxytropis splendens Douglas ex Hook, - Dom01, GPF, KTZ, NNL, W\&W, E/W

[Oxytropis splendens Douglas] - HDH, SF!

Oxytropis viscida Nutt. var. viscida - E/W

[Oxytropis borealis DC, var, viscida (Nurt.) S. L. Weish] - Dom01, KTZ NNL, UTF, W\& W

[Oxytropis viscida Nutt.] - IMF, SF!

[Oxytropis viscida Nutt. ex Torr. \& A. Gray] - HDH, W\& W

Parryella filifolia Torr. \& A. Gray ex A. Gray - IMF, KTZ, NNI. UTF. W

[Parryella filifolia Torr, \& A. Gray] - HDH, SFW, W\&W

Pediomelum

(Identification sources:

\section{(1)}

Pediomelum argophyllum (Pursh) J. W. Grimes - Dorn01, KTZ, NNL. W\& W'. E [Psoralea argophylla Pursh] - GPF, HDH

[Psoralidiam argophyllum (Pursh) Rydo,] - SFE, W\&W

Pediomelum aromaticum (Payson) W. A. Weber-IMF, KTZ, SFW, W\&W. W

[Pediomelum aromaticum (Payson) W. A. Weber var, aromaticum) - NNL, UTF

[Psoralea aromatica Pays.] - HDH

Pediomelum cuspidatum (Pursh) Rydb. - KTZ, NNL, SFE. W\&W, E

[Psoralea cuspidata Pursh]-GPF, HDH

Pediomelum digitatum (Nutt. ex Torr. \& A. Gray) lsely - Dom01, KTZ, E

Snow, N, January 2009. Checklist of Vacculer Plants of the Southum Rodky Momain Regioe. CVersion $3 x$ 
[Pedionelum digitatum (Nutt.) Isely] - NNL, W: W ${ }^{1}$

[Psoralea digitata Nutt.] - GPF, HDH

[Psoralidium digitatum (Nutt.) Rydb.] - SFE, W\&W

Pediomelum esculentum (Pursh) Rydb, - Dorn01, KTZ, NNL, SFE, W\&W, E

[Psoralea esculenta Pursh] - GPF, HDH

Pediomelum hypogaeum (Nutt. ex Torr. \& A, Gray) Rydb, var, hypogaeum - Dorn01, KTZ, E

[Pediomelum hypogaeum (Nutt.) Rydb] - SFE

[Pediomelum hypogaeum (Nutt. ex Torr. \& A. Gray) Rydb.] - W\&W

[Pediomelum hypogaeum (Nutt.) Rydb. var. hypogaeum] - NNL.

[Psoralea hypogaes Nutt.] - GPF, HDH

[Psoralea hypogaea Nutl. var. hy pogasa] - GPF

Pediomelum linearifolium (Torr. \& A. Gray) J, W, Grimes - Dom01, KTZ, NNL, E

[Psoralea linearifolia Torr. \& A. Gray]-GPF, HDH

[Psoralidium linearifolium (Torr. \& A. Gray) Rydb.] - SFE, W\& W

Pediomelum megalanthum (Wooton \& Standl.) Rydb, var, megalanthum - IMF, KTZ, NNL, W

[Pediomelum megalanthum (Wooton \& StandL) Rydb.] - SFW, UTF, W\& W

[Psoralea megalantha Wootan \& Standl.] - HDH

\section{Petalostemon (see Dalea)}

Peteria seoparia A. Gray - FCF

* Phaseolus vulgaris L. - NNL

[Phaseolus vulagris L. var. humilis Alef.] - Donz01, KTZ

Pomaria jamesii (Torr. \& A. Gray) Walp. - Simpson98, E

[Caesalpinia jamesii (Torr. \& A. Gray) E Fisher] - GPF, KTZ, NNL, SFE, W \& W

[Hoffmanseggia jamesii Torr. \& A. Gray] - HDH

Prosopis glandulosa Torr. var, glandulosa - IMF, KTZ, NNL, UTF, E

[Prosopis glandulosa Torr.]-GPF, SFE, W\&W

\section{Psoralea (see Pediomelum, Psoralidium)}

\section{Psoralidium (see also Pediomelum)}

Psoralidium lanceolatum (Pursh) Rydb. - Dorn01, IMF, KTZ, NNL, SF!, W\&W, E/W

[Psoralea lancoolata Pursh] - GPF. HDH

[Psoralidium lanceolanum (Pursh) Rydb، var, lanceodatum] - UTF

Psoralidium tenuiflorum (Pursh) Rydb. - Dorn01, IMF, KTZ, NNL., SF!, UTF, W\&W, E/W

[Psoralea renuiflora Pursh var, bigelowii (Rydb), J, F, Macbe.] - HDH

[Psoralea tenuifloca Pursh var, tenuiflora] - GPF, HDH

Psorothamnus scoparius Rydb, - (Vheent 9956+, GREE)

[Dalea scoporius A. Gray]

Psorothamnus thompsoniac (Vail) S. L. Welsh \& Atwood var. whitingii (Kearney \& Pecbles)

Barneby - IMF, KTZ

[Psorothamnus thompsonac (Vail) S, L Welsh \& Arwood vas, whitingii (Kearney \& Peebles) Barneby] - NNL. UTF

* Robinia hispida L. var. hispida - KTZ. NNL

[Robinis hispida L.] - GPF, UTF 
Robinia X holdtii Beissn, - KTZ, NNL

[Robinia neomexicana X R. pseudoacacia] - KTZ

* Robinia neomexicana A. Gray var. neomexicana- IMF, KTZ, NNL, ENW

[Robinia neomexicana A. Gray] - Dorn01, HDH, SF!, UTF, W\&W

* Robinia pseudocacia L. - Dom01, GPF, HDH, IMF, KTZ, NNL, SF!,UTF, W\&W, E/W

[Robinia pseudo-acacia L.] - W\& W W $^{1}$

\section{Schrankia (see Mimosa)}

\section{Securigera (see Coronilla)}

Senna roemeriana (Scheele) Irwin - KWA, KTZ

[Cassia rocmeriana Schoele] - KTZ, M\&H

[Earleocassia nemeriana (Scheele) Britt.] - M\&H

[Senna roemeriana (Scheele) Irwin \& Barneby] - NNL.

Sophora nuttalliana B. L. Tumer - Dorn01, GPF, IMF, KTZ, NNL, UTF, E/W

[Sophora sericea NuIL] - HDH

[Vexibia nutcalliana (B. L. Tumer) W. A. Weber] - SF!, W\& W

* Sphaerophysa salsula (Pall) DC, - Dorn01, GPF, IMF, KTZ, NNL, SF!,UTF, W\&W, E/W

[Swainsona salsula (PalL) Taubert] - HDH

Strophostyles leiosperma (Torr. \& A. Gray) Piper - GPF, HDH, KTZ, NNL, SFE, W\&W, E

\section{Swainsona (see Sphaerophysa)}

Thermopsis montana Nutt. var, divaricarpa (A. Nelson) Dorn - EWW

[Thermopsis divaricarpa A. Nelson] - Dom01. HDH. KTZ SF!. W\& W

[Thermopsis fhombitolia (Nuel. ex Pursh) Richards.] - W\& W'

[Thermopsis nhombifolia Nutt, ex Richardson var, divaricarpa (A. Nelson) Isely] - GPF

[Thermopsis rhombifolia (Pursh) Richardson var. divaricarpa (A. Nelson) Jsely] - NNL

Thermopsis montana Nutt. var. montana - KTZ, E/W

[Thermopsis montana Nurt.] - Dom01, SF?, UTF

[Thermopsis montzon Nurt. ex Torr, \& A. Gray]-HDH, W\&W

[Thermopsis mombifolia (Nutt. ex Pursh) Richardson var, montans (Nurt. ex Torr. \& A. Gary) Isely] - IMF

[Thermopsis thombifolia (Pursh) Richardson var. montans (Nutt) lsely] - IMF, NNL.

Thermopsis rhombifolia (Nutt. ex Pursh) Nutt. ex Richardson var, annulocarpa (A. Nelson) L. O. Williams - Dorn01

[Thermopsis rhombifot ia (Nurt. ex Pursh) Richardson] - WeW

[Thermopsis rhombifolia (Nutt. ex Pursh) Nutt ex Richandson] - KTZ

Thermopsis thombifolia (Nutt. ex Pursh) Nutt. ex Richardson var. ovata (B. L. Rob. ex Piper) Iscly

[Thermopsis gracilis T. J. Howell var, ovata (B, L. Rob, ex Piper) Mendenhall] - KTZ

[Thermopsis thembifolia (Pursh) Richardson var. ovata (Piper) Isely] - NNL.

[Thermopsis thombifotia (Nutt, ex Pursh) Richardson var, ovata (B, L. Rob, ex Piper) Isely] - IMF <CO.

Thermopsis rhombifolia (Nutt. ex Pursh) Nutt. ex Richardson var. rhombifolia - Dorn01, E/W

[Thermopsis arenosa A. Nelsco] - $\mathrm{HDH}$

[Thernopsis rthombifolia (Nutt.) Richardson] - SP:

[Thermopsis rhombifolia (Nutt. ex Pursh) Richardson] - W\&W

[Thermopsis rhombifolia Nutt ex Richardson] - HDH, UTF

[Thermopsis rhombifolia (Nutt. ex Pursh) Nutt ex Richandson] - KTZ 
[Thermopsis rhembifolia Nutt, ex Richardson var. rtsomhifolia] - GPF

[Thermopsis rhembifolia (Nutt. ex Pursh) Richardson var. rhombifolia] - IMF

[Thermopsis rhombifolia (Pursh) Richardson var- rhembifolia] - IMF, NNL.

\section{Trifolium}

(Identification sources:

Trifolium andinum Nutt, var, andinum - IMF, KTZ, NNL, W

[Trifolium andinum NutL] - Dom01, SFW, UTF, W\&W

Trifolium attenuatum Greene - KTZ, NNL, SF!, W\&W, EW

[Trifolium dasyphyllum Torr. \& A. Gray]-HDH

- Trifolium brandegeei S, Watson - ENDFMIC <CO, NM>, HDH, KTZ, NNL, SF!, W\&W, E/W

* Trifolium campestre Schreeber-Dorn01, KWA, KTZ, NNL

[Trifolium procumbens L. 1755, non 1753] - KTZ

Trifolium dasyphyllum Torr, \& A. Gray var. dasy phyllum - NNL.

[Trifolium dasyphyllum Torr. \& A. Gray]-Dorn01, HDH, IMF, SF!, w\&W, E/W

[Trifolium dasyphyllum Tort. \& A. Gray subsp. dasyphyllum] - KTZ

Trifolium dasyphyllum Tor, \& A. Gray var, anemophilum (Greene) J. S. Martin ex Isley Smith09?

[Trifolium ancmophilum Greene] - KTZ

[Trifolium dasyphyllum var. anemophilum [Greene) JS. Matin ex Isely] - KTZ

[Trifolium scariosum A. Nels.] - KTL

* Trifolium fragiferum L. - Dorn01, HDH, IMF, KTZ, NNL, SF!,UTF, W\&W, EW

[Trifolium fragiforum L. subsp. fragiferum] - GPF

Trifolium gymnocarpum Nutt. var, gymnocarpum - Dorn01, HDH, NNL, EW

[Trifolium gymnosarpon Nutt.] - SFI, UTF, W\&W

[Trifolium gymnocarpum Nurt. ex Torf. \& A. Groyl- IMF

[Trifolium gymocsarpun NutL subsp. gymoocarpum] - KTZ

[Trifolium gymmocarpum Nust. var, subacauleseens (A. Gray) A. Nelson]- HDH

\{Trifolium gymnocarpum Nutt. var. plummerae (S. Watson) J. S. Martin\} - NNL, E/W

\{Trifolium gymoocarpon Nutt.\} - SF!, UTF, W\&W

\{Trifolium gymeocarpon Nut. ex Torr. \& A. Gray) - IMF

'Trifolitam gymeocarpum Nutt, sabsp. plumnserac (S, Watson) J. M. Gillett\} - KTZ

* Trifolium hybridum L. - Dorn01, HDH, IMF, KTZ, NNL, SF!, UTF, W\&W, E/W

[Trifolium hybridum L. subsp. elegans (Savi) Asch. \& Graebn.] - GPF

Trifolium kingii S. Watson var. kingii - W, IMF, UTF

[Trifolium kingii S, Watson]- NNL, SFW. WLW

[Trifollum kingi S. Watson subsp, kingii] - KTZ

\{Trifolium kingii S. Watson var. macilentum (Greene) Isely\} -W

\{Trifolium kingii S. Watson\}-SFW, W\&W

\{Trifolitum kingii S. Warson subsp, macilentum (Greene) J, M. Gillett\}-KTZ<AZ,UT>

TTrifolium macilentum Greene\} - $\mathrm{HDH}, \mathrm{IMF}$

[Trifolium macilentum Greene var, macilentum] - NNL, UTF

Trifolium longipes Nutt. var. neurophyllum (Greene) J. S. Martin ex Isley - KWA, NNL

[Trifolium neurophyllum Greete] - KTZ

[Trifolium nasbyi subsp, neurophyllum (Gireetse) Heller \& Zoh.] - KWA, KTZ

Trifolium longipes Nutt. var. reflexum A. Nelson - Dom01, IMF. NNL, UTF, E/W

[Trifolium longipes Nitt.] - SF!

[Trifolium longipes Nott. subsp, reflexum (A. Nelson) J, M. Gillett]- KTZ

[Trifolium rasbyi Greene subsp. reflexum (A. Nelson) A. Heller \& Zchary] - W \&W 
Trifolium longipes Nutt, var, nusbyi (Greene) Harrington - HDH, IMF, NNL, UTF, E/W

[Trifolium longipes Natt.] - SF!

[Trifolium longipes Nutt, var. longipes] - HDH $<n \times . ~ N A>, N N L$

[Trifolium longipes Nutt. subsp. pygmakum (A. Gray) J. M. Gillett] - KTZ.

[Trifolium rusbyi Gircene subsp. rusbyi] - W\&W

Trifolium nanum Torr - Dorn01, HDH, IMF, KTZ, NNL, SF!, UTF, W\&W, E/W

Trifolium parryi A. Gray var. parryi - Dorn01, NNL, E/W

[Trifolium parryi A. Gray]-HDH, IMF, SFI, W\&W

[Trifolium parryi A. Gray subsp. parryi] - KTZ

[Trifolium parryi A. Gray subsp. salictorum (Greene ex Rydb.) J. M. Gillett] - KTZ

[Trifolium salictorum Gireene] - SF]

[Trifolium salistorum Greene ex Rydb., nom. nudum] - W\&W

* Trifolium pratense L. var, pratense-Dorn01, IMF, E/W

[Trifolium praterse L.] - GPF, HDH, KTZ, SF!, UTF, W\&W

* Trifolium repens L. - Dom01, GPF, HDH, IMF, KTZ, NNL, SF!, UTF, W\& W, E/W

Trifolium wormskjoldii Lehm. var, arizonicum (Greene) Bameby - IMF, NNL, UTF $\angle A Z, U T>$

[Trifolium fendleri Greene] - HDH

[Trifolium mecronatum Willd. subsp. lacerum (Greene) J. M. Gillet] - W\& W', misapp.

[Trifolium mecronanum Willd ex Spreng. stubsp. lacerum (Greerse) J. M. Gilket] - KTZ

[Trifolium wormskioldii Letim.] - W\&: W

Trifolium wormskjoldii Lehm. var, wormskjoldii - IMF, NNI, UTF, E/W

[Trifolium fendleri Greene] - $\mathrm{HDH}$

[Trifolium wermskioldei Letm-] - Dorn01, KTZ, SFI, W\&W

\section{Vexibia (see Sophora)}

Vicia

(Identification sources:

Vicia americana Muhl, ex Willd, var, americana - Dorn01, GPF, IMF, NNL, UTF, W\&W, E/W

[Vicia americana Muhl. ex Willd.] - $\mathrm{HDH}$

[Vicia americana Muhi. subsp. americana] - SFE

[Vicis americana Muhl. ex Willd. subsp. americana] - KTZ

[Vicia americana Muhl.] - SFW

Vicia americana Muhl. ex Willd. var. minor Hook. - Dorn01, GPF, IMF, NNL, UTF, W\&W, E

[Vicia americana Muhl. subsp. minoe (Hook.) A. Löve \& D. Love] - SFE

[Vicia americana Muhl. ex Willd. subsp, minor (Hook.) C. R. Gunn] - KTZ

[Vicia lincaris (Nutt) Greene] - w\& w'

[Vicia americana Muhi.] - SFW

* $\{$ Vicia cracea I.. $\}$ - HDH, exp., NNL.

\{Vicis cracca L subsp. cracca\}-KTZ $\leq$ UT, WY>

Vicia ludoviciana Nutt var. ludoviciana - E/W

[Vicia exigua Torr. \& A. Gray] - GPF

[Vicia ludoviciana Nutt.] - IMF, UTF

[Vicia ludoviciana Nutt, subsp, ludoviciana] - KTZ, NNL

[Vicia ludoviciana Natt, var, texana (Torr. \& A. Gray) Shinners] - SF!, W\&W

[Vicia producta Rydb.] - HDH

* Vicia sativa L. var. nigra L. - E

[Vicia angustifolia L.] - W\&W

[Vicia angustifolin (L.) Reichand] - SFE

[Vicia sativa L var, angustifolia L.] - GPF, IMF

[Vicia sativa L- subap, nigra (L.) Ehrh.] - KTZ, NNL 
* Vicia villosa Roth - Dom01, HDH, SF!, W\&W, E/W

[Vicia villosa Roth subsp, villosa] - KTZ

[Vicia villosa Roth var, villosa] - GPF, IMF, NNL, UTF

\section{Fagaceae - FAG}

\section{Quercus}

(Identification sources:

\{Quercus ajoensis C. H. Muller\} - FNA $\angle A Z>$, KTZ, W\&W, W

[WJ - based on misid. of Q. turbinella?

\{Quercus ajotrisis C. H. Muller) - SFW

\{Quercus carmenensis C. H. Muller\} - FNA, KTZ, WJ

Quercus gambelii Nutt. - Dom01, FNA, GPF, HDH, SF!, W\&W, E/W

[Quercus gambelii NutL. var. gambelli] - KTZ, UTF

[Quercus X pauciloba Ryub.]-KTZ, UTF

[Quercus gambellii X Q. Lurbinella] - KTZ

[Quercus vreelandii Rydb.] - $\mathrm{HDH}$

[Quercus x pauciloba Rydb.] - FCF

Quercus grisea Liebm. - FNA, KTZ, SFE, W\&W, E

[W]-oscurs in Baca and Las Animas cos, CO, despite map in FNA]

Quercus bavardii Rydb, - FCF, FNA, GPF, W

[W] - plants from the Four Corners area are hybrids of Q. gambellii and Q. turbinella; NS - close to entering our area from the southenst ats well]

[Quercus X eastwoodlac Rydb.]] - Tucker 70 and UTF are in error

[Quercus havardii Rydb. var tackeri S. L. Welsh] - KTZ SFW, UTF, W\&W

Quercus pungens Liebm. - FNA, HDH rep., KTZ, Hazlett 1178 / (Harding Co., NM, [GREE])

[Records apply to hybrids betweco Qucreus grisea X Q. gambelit, WE W]

[Quercus venustula Greene] - $\mathrm{HDH}$, rep.

Quercus turbinella Greene - FNA, HDH, KTZ, SF!, UTF, W\&W, E/W

Quercus X undulata Tort, - FNA; Tucker 71, E

[Quercus gambelii $X Q$. grisca] - wJ, w\&W

[Quercus gambelii X Q turbinella] - KTZ

[Quercus fendleri Lict.] - HDH

[Quercus undulara Tort.] - GPF, GPF, HDH, SFE

\section{Frankeniaceae - FNK}

Frankenia jamesii Toer. ex A. Gray - KTZ, W\&W, E/W

[Frankenia jamesii Tort.] - HDH, SFI, UTF

\section{Fumariaceac (see PAP)}

\section{Gentianaceate-GEN}

Centaurium calycosum (Buckley) Fernald - KWA, KTZ, M\&H

[Centaurian calycosum (Buckley) Fernald var, arizonicuen (A, Gray) Tidestr.] - HDH

[Centauriun cklycosum var, anum (Gray) B.L., Pobins.] - KTZ

[Zeltnera calyca (Buckley) Mansion] 
Centaurium exahtatum (Griseb.) W, Wight ex Piper - Dom01, GPF, IMF, KTZ, UTF, W\&W, W [Centaurium exaltakum (A. Gray) Tidestr.]-SFW

[Centaurium exaltatum (Griseb-) W. Wight]-HDH, exp.

Centauriunt pulchellum (Sw, Druce - GPF, IMF, KTZ 4 NE>, SFE, W\&W $W^{1}$. E

[Centaurium pulchellum (Sw.) Druce ex B. L. Rob. \& Fernald] - Dorno]

Chondrophylla (see Gentiana)

Comastoma (see Gentianella)

Eustoma grandiflorum (Raf,) Shinners - Dorn01, GPF, SFE, W\&W, E

[Eustoma exaltatum (L.) Salisb. ex G. Don subsp. russellianum (Hook.) Kartesz] - KTZ

[Eustona russellianum (Hook.) Griseb.] - HDH

Frasera albomarginata S, Watson var, albomarginata - IMF, W

[Frascra albumarginata S. Watson] - KTZ, SFW, W\&W

[Swrtia albenarginata (S. Watsen) Kuntze] - HDH, UTF

- Frasera coloradensis (C. M. Rogers) D. M. Post - ENDEMIC $<\mathrm{CO}>$, KTZ, SFE, W\&W, E

[Swertia coloradensis C. M. Rogers] - HDH

Frasera paniculata Torr. - IMF, KTZ, W\&W, W

[Frassra paniculata S. Watson] - SEW

[Swertia usahensis (M. E. Jones) H. H. St. John] - UTF

Frasera speciosa Douglas ex Griscb. - Dorn01, IMF, KTZ. W\&W, E/W

[Frastra speciosa Dougkas] - SF!

[Suertia radiata (Kelloge) Kuntwe] - GPF. UTF

[Swertia radiana (Kelloge) Kuntze var, radiata] - $\mathrm{HDH}$

[Swertia radiuta (Kellogeg) Kuntre var. macrophylla (Greene) H. St. John] - HDH

Gentiana

(Identification sources:

Gentiana (see also Gentianella, Gentianopsis; Yuan96)

Gentiana affinis Griseb, - GPF, HDH, IMF, KTZ, UTF, E/W

[Gentiana affinis Griseb, var, affinis] - RMC

[Gentiana forwoodii A. Gray] - HDH

[Pnecumonanthe affinis (Griscb, Greene] - SF1, W\& W

Gentiana algida Pall. - Dorn01, IMF, KTZ, UTF, E/W

[Gentiana romanzovii Ledeb_] - $\mathrm{HDH}$

[Gentiancodes algida (Pall.) A. Löve \& D, Love] - SF1, Waw

Gentiana andrewsii Griseb. var andrewsii - PC, KTZ

[Gentiana andrewsii Griseb.] - GPF, HDH

[Pneumonanthe andrewsii (Griseb.) W.A. Weber] - WaW

Gentiana aquatica L. - Dorn01. E/W

[Chondrophylla aquatice (L.) W. A. Weber] - SF!, W\&W

[Gentiana fremontil Torr.] - HDH, KTZ

Gentiana bigelovii A. Gray - HDH. E

[Gentiana affinis Griseb.] - KTZ

[Gentiana affinis Griseb, var, bigelovii (A, Gray) Kusn,] - Dorn0]

[Pneumonanthe bigelovii (A, Gray) Greene] - SFE, W\&W'

\{Gentiana calycosa Griseb.\} - FCF 
$\{$ WJ - does not occur in our area; nearest ares is Lintah Range in UT. Ours all are G. parryi. Allred trealment in Great Easin Naturalist lumped species too aggressively]

Gentiana parryi Engelm, - Dom01, HDH, IMF, KTZ, UTF, E/W

[Pneumonanthe parry i (Engelm.) Groeno] - SF!, W\&W

Gentiana prostrata Haenke - Dorn01, IMF, KTZ, CC \& WJ, E/W

[Chondrophylla nutans (Burge) W. A. Weber] - SF!, W\&W

[Chondrophylla prostrata (Hzerike) J. P. Anderson] - SF!

[Chondrophylla prositrata (Haenke ex Jacq.) J. P. Andersen] - WaW

[Gentiana nutans Bunge] - KTZ

[Gentiana prostrata Hacnke ex Jacq. - UTF

[Gentiana prostrata Haenke var. americana Engelm.] - HDH

Gentianella amarella (L.) Börner var, acuta (Michx.) Herder - E/W

[Gentiana plebeia Chamisso var. bolmii Wettst.] - HDH

[Gentiana plebeia Chamisso var. plebeia] - $\mathrm{HDH}$

[Gentiana strictiflora (Rydb.) A. Nelson] - HDH

[Gentianella acuta (Michx.) Hiitonen] - SF!, W.W

[Gentianella amarella (L.) Bẻmer] - IMF, UTF

[Gentianella amarella (L.) Bỏmor subsp. scuta (Michx.) J. M. Gillett] - GPF. KTZ

[Gentianella amarella (L.) Bỏmor var. amarella\} - Ws W', Eurasian

[Gentianella strictiflora (Rydb.) W. A. Weber] - SFE, W\&W

Gentianella amarella (L.) Börner var, heterosepala (Engelm.) Dorn - Dorn01, EW

[Gentiana heterosepala Engelm.] - HDH

[Gentiana scopulorum TIdestr.]-KTZ

[Gentianella amarelia (L.) Bëmor subsp. heterosepala (Engelm.) J. M. Gilleti] - KTZ

[Gentianella heterosepala (Engelm.) Holub] - IMF, SF!, UTF, W\&W

Gentianella tenella (Rottb.) Bōrner - Dorn01, GPF, IMF, UTF, EW

[Comastoma tovella (Routb.) Toyokuni] - SFI, W\& W

[Gentiana tenella Rostb.] - HDH

[Gentianella tenella (Rotib.) Bōmer subsp. tescella] - KTZ

Gentianella tortuosa (M. E. Jones) J. M, Gillett - IMF, KTZ, SFW, UTF, W\&W, W

Gentianodes (see Gentiana)

Gentianopsis barbellata (Engelm.) H. H. Iltis - Dom01, IMF, KTZ, SF!, UTF, W\&W, E/W

[Gentiana barbellata Engelm.] - $\mathrm{HDH}$

Gentianopsis detonsa (Rottb.) Ma var. elegans (A. Nelson) N. H. Holmgren - Dorn01, IMF, UTF, E/W

[Gentiana thermalis Kunze] - $\mathrm{HDH}$

[Gentiancpsls thermalls (Kuntze) H. H. fltis] - KTZ SFI, WAW

Gentianopsis procera (Holm) Ma - WJ (Kelso et at 0]-187 [COCO])

Lomatogonium rotatum (L..) Fr. - Dorn01, UTF, E/W

[Lomatogonium rotatum (L.) Fr. ex Fernald] - KTZ

[Lomatogonium notatum (L.) Fr, subsp, tenuifolium (Griscb.) Porsild] - SF!

[Lomatogonium notatum (L.) Griseb, subsp, tenuifolium (Griseb.) Porsild] - W\& W

[Pleurogyne fontana A. Nelson] - IPNI

[Pleurogyne recta (L.) Griseb.] - HDH

Pleurogyne (see Lomatogonium)

Pneumonanthe (sce Gentiana)

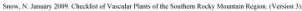


Swertia (see also Frasera)

Swertia perennis L. - Dorn01, HDH, IMF, KTZ, SF!, UTF, W\&W, E/W

\section{Geraniaceae - GFR}

*Erodium cicutarium (L.) L'Hér. ex Aiton - Dorn01, HDH, IMF, E/W

[Enodium cicutarium (L.) L'Her.] - GPF, SF!, UTF, W\&W

[Erodium cicutarium (L.) L'Hér. ex Aiton subsp. cicutarium] - KTZ

Geranium

(Identification sources:

\{Geranium atropurpureum A. Heller var, atropurpureum\} - KTZ, E/W

Geranium stropurpureum A. Heller\} - see IMF

\{Geranium casspitosum E. James subsp. atropurpureum (A. Heller) W. A. Webere\} - SF!

(Geranium casspitosum E. James ex Torr. subsp. atropurpureum (A. Heller) W. A. Weber) - W\& W

(Geranium marginale Rydb) - W\& W

\{Geranivm marginale Rydb. ex Hanks \& Suall; - HDH, rep.

Geranium atropurpureum A. Heller var, cowenii (Rydb,) Dorn - Dorn01, KTZ

[Geranium caespitosum E. James] - IMF

[Geranium caespitosum E. James ex Torr. subsp. cacspitosum] - W\&W

[Geranium frementii Tort. ex A. Gray] - soe IMF

[Geranium fremontii Torr. ex A. Gray var. cowenii (Rydb.) Harrington] - HDH

[Geranium intermedium E. James] - Goodmans5

*Geranium bicknellii Britton var, longipes (S. Wat.) Femald - SF!, W\&W, E/W

[Geranium bicknellit Britton] - Dorn01, GPF, IMF, KTZ, UTF

[Geranium carolinianum L. var. longipes S. Was.] - HDH

Geranium caespitosum E. James var. caespitosum - Dorn01, KTZ, E/W

[NS - Soe UT3 p. 324 regarding taxonom ic work needed in G. caespitosum complex]

[Geranium caespitosum E, James] - HDH. IMF, UTF

[Geranium caespitosum E. James subsp. caespinosum] - SF ]

[Geranium caespitosum E. James ex Torr. subsp. cacspitosum] - W\&W

[Geranium caespitosum E. James var, parryi (Eggelm.] W. A. Weber] - KTZ. UTF

[Geranium parryi (Engelm.) A. Heller] - HDH

Geranium caespitosum E. James var. fremontii (Torr. ex A. Gray) Dorn - Dorn01, KTZ, E/W

[Geranium caespitosum E. James] - see IMIF, UTF

[Geranium caespitosum E. James subsp. caesphosum] - SF!

[Geranium caespitosum E. James ex Torr. subsp. chespitosum] - W\& W

[Geranium fremontii Torr. ex A. Gray]-sev IMF

[Geranium fremonsii Toer. ex A. Gray var, frementii] - HDH

*Geranium carolinianum L. - Dom01, GPF, IMF, UTF

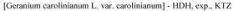

*Geranium columbinum L. - KTZ, SFE, W\&W. E

*Geranium ihericum Cav. - KTZ, SFE, W\& W', E

\{Geranium pusillum Burm. f.\} - HDH, SFE, not persisting, W\&W, misapp., E

*Geranium pusillum L. - Dorn(1, GPF, IMF, KTZ, UTF

Geranium richardsonii Fisch. \& Trauty. - Dom01, GPF, HDH, IMF, KTZ, SF!, W\&W, E/W

Geranium viscosissimum Fisch. \& C. A. Mey. ex C. A. Mey. var. viscossimum - Dom01

Geranium viscosissimum Fisch. \& C. A. Mey. ex C. A. Mey. var. incisum (Torr. \& A. Gray) N.

H. Holmgren - Dorn01, IMF, KTZ.

[Geranium nervosum Rydb,] - $\mathrm{HDH}$

Snow, N, Junury 2009. Checklist of Vaccular Plants of the Southom Rocky Momain Region. CVersion $3 x$ 
[Geranium viscosissimum Fisch. \& C. A. Mey. subsp. nervosum (Rydb.) W. A. Weher] - SF!. W\&W

[Geraniurn viscosissimum Fisch. \& C. A. Mey. ex C. A. Mey. var, nervosum (Rydb.) C. L. Hitehe.] - EW

[Geranium viscasissimum Fisch. \& C. A. Mey. var, nervassum (Rydb.) C L. Hitche.] - GP. UTF

\section{Gramineae (see POA)}

\section{Grossulariaceae - GRS}

(Ideritification sources:

)

Ribes americanum Mill, - Dorn01, GPF, HDH, KTZ, SFE, W\&W, E

Ribes aureum Pursh var. aureum - Dorn01, KTZ, E/W

[Ribes aureum Pursh] - HDH, [MF, SF!, UTF, W\&W

ฯRibes aureum Pursh var, villosum DC. - Dorn01, KTZ, E

[Ribes odoratum H. Wendl.] - GPF, IMF, SFE, UTF, W\&W

Ribes cereum Douglas - HDH, IMF, SF!, W\&W, E/W

[Rabes cereum Douglas var. comum] - KTZ

[Ribes cereum Douglas var. pedicellare Brewer \& 5. Watson] - Dorn01. KTZ

[Ribes cercum Douglas var. inetorians (Lindl.) C. L. Hitche.]-GPF, UTF

Ribes coloradense Coville - HDH, SF!, UTF, W\&W, E/W

[Ribes laxiflorum Pursh] - IMF, KIZ, UTF

\{Ribes divaricatum Douglas\} - IMF, KTZ, SF!, W\&W, EW

\{WJ - A Pacific Coast species. Anocations by Q. Sirmoxt in error. T. Hogan agrees that all are R. inorme. Sinnotr's own paper (Rhodora 87,1985 ) nakes no mention of this taxon in our area)

Ribes inerme Rydb, var, inerme - HDH, IMF, KTZ, E/W

[Ribes inenthe Rydb. var. pubescens $A$. Berger] - $\mathrm{HDH}$

[Ribes inerrne Rydb.] - Dorn01. SFI. UTF, WEW

[Ribes inerne Rydb, torian incisam W. A. Weber]- SFE, WkW

Ribes lacustre (Pers.) Poir- - Dorn01, GPF, HDH, IMF, KTZ, SF!, UTF, W\&W, E/W

Ribes leptanthum A. Gray - HDH, IMF, KTZ, SF!, UTF, W\&W, ENW

Ribes montigenum McClat, - Dorn01, HDH, IMF, KTZ, SF!, UTF, W\&W, E/W

Ribes niveum Lindl. - [MF, KTZ, Sinnott85

Ribes oxyacanthoides L. var. oxyacanthoides - Dorn01, IMF

[Ribes oxyacanthoides L.] - GPF $<\mathrm{CO}$ ?

[Ribes axyacanchoides L. subsp. oxyacanthoides] - KTZ $\triangle N E$, WY $>$-Sinnonss

Ribes oxyacanthoides L, var. setosum (Lindl.) Dom - Dorn01, TMF

[Ribes oxyacanthoides L. subsp. setosum (Lindi.) Q. P. Sinnot]] - KTZ $<$ NE, UT, WY $>$, Sinnon8s

[Röbes sctosum Lindl.] - GPF $<\mathrm{CO}$ ?, HDH, exp_UTF

Ribes viscosissimum Pursh - HDH, IMF, KTZ, SF!, W\&W, E/W

[Ribes viseosissimum Pursh var, viscosissimum] - UTF

Ribes wolfii Rothrock - HDH, IMF, KIZ. SF!, UTF, W\&W, E/W

Haloragacene - HAL (= Haloragidaceac; sce also PTG)

\{Myriophyllum heterophyllum Michx.\}-M\&H, exp.

Myriophyllum sibiricum Kom. - Dom01, IMF, KTZ, SF!, W\&W, E/W

[Myriophy llum exalbescens Fernald] - GPF, HDH, UTF

Myriophyllum spicatum L - IMF, KTZ

[Andy Kratz, USFS, pers, comm.]

Myriophyllum verticillatum L. - GPF, HDH, IMF, KTZ, SFW, UTF, W\&W, W 


\section{Haloragidaceae (see HAL)}

\section{Helleboraceae (see RAN)}

\section{Hemerocallidaceae (see $\mathrm{XAN}$ )}

\section{Hippocastanaceae (see SAP)}

\section{Hippuridaceae - HPU}

Hippuris vulgaris L. - Dorn01, GPF, HDH, IMF, KTZ, SF!, UTF, W\&W, E/W

\section{Hydrangeaceae - HDR}

Fendlera rupicola A. Gray

[W] - sou noces in IMF ngarding rationale for not recognizing varieties]

[Fendlera rupicola A. Gray var, fakata] - KWA. M\&H

[Fendlera rupicola A. Gray var, rupicola] - W

[Fendlera rupicola A. Gray] - HDH, KTZ. SFW. UTF, W\&W

[Fendlera rupicola Engelm. \& A. Gray] - IMF

[Fendlera rupicola A. Gray var. wrightia A. Gray] - KWA, M\&H

[Fendlera wrightii (A. Gray) Heller] - KTZ

Fendlerella utahensis (S, Watson) A. Heller - HDH, SFW, UTF, W\&W, W

[Fendlerella utahens is (S. Wason) A. Helke var, utahensis] - IMF, KTZ

Jamesia americana Torr, \& A. Gray var, americana - GPF, IMF, KTZ, E/W

[Aamesia americans Tort.] - SFW

[Aamesia americans Torf, \& A. Gray]-Dom0I, HDH, SFE, W\&W

[Damesia americana Torf. \& A. Gray var. macrocalyx (Small) Engler] - UTF

Philadelphus microphyllus A. Gray - IMF, KTZ, SF!, UTF, W\&W, E/W

[Philadelpbus arzenteus Rydb.] - KTZ

[Philadelphus microphyllus A. Gray subsp. microphyllus] - HDH

[Philadelphus microphyllus A. Gray subsp. occidentalis (A. Netsen) Hitche.] - HDH

[Philadelpbus microphyllus A. Gray var, occidentalis (A. Nelson) Dorn] - Dorn01

[Philadelpbus microplyyllus A. Gray var, ovaurs $\mathrm{Hu}$ - KTZ

[Philadelpbus oceidental is A. Nelson var, minutus (Rydb.) Hu] - KIZ

[Philadelptous oecidental is A. Nelson var, occidentalis] - KTZ

\section{Hydrocharitaceae - HDC (see also NAJ)}

(Identification sources:

*Egeria densa Planch. - FNA, KTZ <AZ, KS, NE, NM, OK, UT>

[Eloden densa (Planch) Caspary] - GPF, HDH, possible escape

Elodea (sec also Egeria)

Eloden bifoliata H. St. John - Dom01, FNA, KT乙, SF!, W\&W ${ }^{1}$, E/W

[Elodea loogivaginata H. St. John]-GPF, IMF, UTF, W\&W

Elodea canadensis Michx. - Dom01, FNA, GPF, HDH, IMF, KTZ, SF!, UTF, E/W

[Elodea canadensis Richards.] - WEW 
Elodea nuttallii (Planch.) H. St. John - Dorn01, FNA, GPF, HDH, IMF, KTZ, SF!, W\&W, E/W

[Vallisneria americana Michx, - FNA, GPF, KTZ $<\mathrm{AZ}, \mathrm{NM}, \mathrm{OK}>$

\{Vallisneria spiralis L.\} - HDH, exp., WAW, err. rep.

\section{Hypoxidaceac - HPX}

Hypoxis hirsuta (L.) Coville - FNA, GPF, HDH, KTZ, SFE, W\&W, E

[W] - nat collected in $\mathrm{CO}$ since 1959; possibly extirpoted in $\mathrm{CO}$ ]

[Ornithogalum hirsulum L.] - FNA

[Hypoxis carolinensis Michx.] - FNA

[Hypoxis graminea Pursh] - FNA

[Hypoxis grandis Pollard] - FNA

[Hypoxis micrantha Pollard] - FNA

[Hypoxis pallida Salisbury] - FNA

[Hypoxis villosa Raf.] - FNA

\section{Iridaceae - IRI}

Iris missouriensis Nut, - Dorn01, FNA.GPF, HDH, IMF, KTZ, SF!, UTF, W\&W, E/W

[Iris arizonica Dykes] - FNA

[Iris longipetala Herbert var. montana Baker] - FNA

[Iris montanla Nutt. ex Dykes] - FNA

[Iris periensis S. L. Welsh] - FNA

[Iris pelogonus Goodding] - FNA

[Iris tolmeiana Herbert] - FNA

Sisyrinchium angustifolium Mill. - Dorn01

[S isyrinchum bermudiana aset. nom L.] - KTZ

[S isyrinchium graninoides Bickn.] - KTZ

Sisyrinchium demissum Greene - FNA, GPF, HDH,IMF, KTZ, SF!, UTF, W\& W, E/W

[S isyrinchium anscthystinum E. P. Bicknel1] - FNA

[S isyrinchium demisstaum var. amethystimum (E. P. Bicknell) Kearney \& Peebles] - FNA

[Sisyrinchim longipedanculatum E. P. Bickneli] - FNA

\{Sisyrinchium halophilum Greene\}- HDH, IMF, KTZ UT?, W\&W

Sisyrinchium idahoense E. P. Bicknell var. occidentale (E. P. Bicknell) D. M. Hend.- Dorn01,

FNA, KTZ, SF!, W\&W, E/W

[Sisyrinchum idahocrise E. P. Bickncil] - UTF, IMF

[S isyrinchium juncellum Greene] - FNA

[S isyrinchium occidentak E. P. Bicknell] - HDH

Sisyrinchium montanum Greene var, montanum - FNA, KTZ

[Sisyrinchium alpestre E. P. Bicknell] - FNA

[Sisyrinchium heterocamum E. P. Bicknell] - HDH. FNA

[Sisyrinchium montanum Grecne] - Dom01, GPF, HDH, IMF, SFI, W\&W, EW

- Sisyrinchium pallidum Cholewa \& D. M. Hend. - ENDEMIC $<C O$, WY $>$, Dom01, FNA.

KTZ, SFE, W\&W, E

\section{Juglandaceae - JUG}

Juglans nigra L. - Hartman06

\{Juglans rupestris Engelm. var. major Torr.\} - HDH rep., W\& W

(Juglans majee (Torr.) A. Heller) - KTZ $<$ AZ, NM, OK, UT?, UTF 
\{Juglans major (Torr.) A. Heller var. major\} - FNA $<$ AZ, NM, OK>

\section{Juncaceae - JUN}

(Identification sources:

Juncus acuminatus Michx, - FNA, GPF, HDH, KTZ, SFE, W\&W, E

Juncus alpinoarticulatus Chaix - FNA. W\&W, EW

[Juncus alpino-articulatus Chaix]-SF!, W\& W'

[Juncas alpinoarticulatus Chaix subsp. nodulosus (Wahlenb.) Hămet-Ahti] - KTZ

[Juncus alpinus Villars] - GPF. HDH, IMF, UTF

Juncus articulatus L. - Dorn01, FNA, GPF, HDH, IMF, KTZ, SF!, UTF, W\&W, ENW

Juncus arcticus Willd. var, balticus (Willd.) Trautv, - FNA, E/W

[Juncus arcticus Willd.] - UTF

[Juncus arcticus. Willd. subsp. alter (Rydb.) Hultén] - SFE, W\& W'

[Juncus ater Rydb] - W.W

[Juncus balticus Willd. var. montanus Engelm.] - Dome1, GPF, HDH, IMF, KTZ

[Juncus balticus Willd. var. vallicola Rydh.] - Dorno], HDH, KTZ

Juncus arcticus Willd. var, mexicanus (Willd, ex Roem. \& Schult.) Balslev - FNA

[Juscus balticus Willd. var. mexicanus (Willd.) Kuntoo] - [MF

[Juncus mexicanus Willd. ex J. A. Schult. \& J. H. Schalt.] - KTZ $\triangle A Z, ~ N M$.

Juncus biglumis L. - Dom01, FNA, KTZ, SF!, W\&W, E/W

Juncus brachycephalus (Engelm.) Buchenau - FNA, GPF, KTZ, SFE, W\&W, E

Juncus brachyphyllus Wieg, - Legler 5083 [voucher at RM]

[NS - Specimen identification confimed by specialist P. Zika at U of WA [B. Legke, pers. comm. 2008\}]

Juncus brevicaudatus (Engelm.) Fernald - FNA, KTZ, SFE, W\&W, E

[Juncus tweedyi Rydb.]-IMF, KTZ, SFE, UTF, W\&W

Juncus bryoides F, J. Herm. - FNA, KTZ, SFW, UTF, W\&W, W

[hunsus kelloggii Engelm.] - IMF

Juncus bufonius L. - Dom01, FNA, GPF, HDH, IMF, SF!, UTF, E/W

[Jurnos ambiguus Guss.] - KTZ

[Jurcus bufonius L. var, bufonius] - KTZ, W\&W

[Juncus bufonius L. var. occidentalis F. I. HermL] - KTZ, SF!, W及 W

[Juncus sphacrocarpes Nees]- HDH

$\leftarrow$ Juncus castancus J. E. Sm. - Dom0I, FNA, HDH, IMF, SF!, UTF, W\&W, E/W

[Juncus castancus J. E. Sm. subsp. castaneus var, castancus] - KTZ

*Juncus compressus Jacq, - Dom01, FNA, KTZ, SF!, UTF, W\&W, ENW

Juncus confusus Coville - Dorn01, FNA, HDH, IMF, KTZ, SF!, UTF, W\&W, E/W

๘Juncus dichotomus Elliott - FNA, KTZ, E

[Juseus platyphyllus (Wiegand) Fernald] - SFE, W \& W

Juncus drummondii E. Mey. - Dom01, FNA, HDH, IMF, SF!, UTF, W\&W, E/W

[Juncas drummondii E. Mey. var, drummondii] - KTZ

[Juncus drummondii E. Mey, var, subtriflones (E. Mey.) C. L. Hirche.] - KTZ

Juncus dudleyi Wiegand - FNA, GPF, HDH, KTZ, SF!, W\&W, E/W

[Duness tenuis Willd var, dudleyi (Wiegand) F. J. Herm. - Dom01, IMF, UTF

ษJuncus effusus L. - FNA, GPF, SFE, W\&W, E

[Juncus effusus L. var, solutus Fermald \& Wiegand] - KTZ

Juncus ensifolius Wikstr. var. ensifolius - Dom01, FNA, IMF, UTF, E/W

[Juneus, ensifolius Wikstr.] - KTZ, SFt, W\&W

Juncus ensifolius Wikstr. var, montanus (Engelm.) C. L. Hitchc. - Dorn01, FNA, GPF, IMF, E/W

[Juncus ensifolius Wikstr, var, brunnescens (Rydb.) Crong, - IMF, UTF

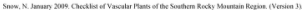


[Juncus saximontanus A. Nelson] - HDH, KTZ, SF!, W\& W

[Juncus saximontanus A. Nelson forma brumescens (Rydb.) F. J. Hermann] - SFE

[Juncus tracyi Rydh.] - KTZ, SF!, W\&: W

Juncus filiformis L. - Dorn01, FNA, HDH, IMF, KTZ, SF1, UTF, W\&W, EW

† Juncus gerardii Loisel, - Dom01, FNA, GPF, SFE, UTF, W\&W, E

[Juncus gerardii Loisel. var. gerardii] - KTZ

Juncus hallii Engelm. - Dorn01, FNA, HDH, IMF, KTZ, SF!, UTF, W\&W, EW

Juncus interior Wiegand - Dom01, FNA, GPF, HDH, SFE, W\&W, E/W

[Juncus interiar Vasey] - SFW

[Juncus interior Wiegand var. interior] - KTZ, RMC

[Juncus interior Wiegand var. neomexicanus (Wiegand) F. J. Herm.] - KTZ

[Juncess tenuis Willd.]-IMF, UTF

Juncus longistylis Torr. - Dorn01, FNA, GPF, HDH, IMF, SF!, UTF, W\&W, E/W

[Juncus kngistylis Torr, var. longistylis] - KTZ

\{Juncus macrandrus Coville\} - FNA, IMF, KTZ

Juncus marginatus Rostk, - FNA, GPF, HDH, KTZ, SFE, W\&W, E

Juncus mertensianus Bong, - Dom01, FNA, HDH, IMF, KTZ, SF!, UTF, W\&W, E/W

Juncus nevadensis S. Watson - Dorn0I. FNA. IMF, SF!, UTF, W\&W, E/W

[Juncus badius Sukad.] - HDH

[Juncus nevadensis S. Watson] - IMF, SF!, UTF, W\&W

[Juncus nevadensis S. Watson var. badius (Suksd.) C. L. Hitche]- K.TZ

[Juncas nevadensis S. Watson var, nevadensis] - KTZ

Juncus nodosus L, - Dom01, FNA, GPF, HDH, IMF, SF!, UTF, W\&W, E/W

[Juncus nodosus L. var. nodesus] - KTZ

Juncus parryi Engelm. - Dom01, FNA, HDH, IMF, KTZ, SF!, UTF, W\&W, E/W

Juncus tenuis Willd. - FNA, KTZ, SFE, W\&W $W^{1}$, E

[Juncus tenuis Wilk. var, multicumis E. Mey.] - HDH, W\&.W

Juncus torreyi Coville - Dorn01, FNA, GPF, HDH, IMF, KTZ, SF!, W\&W, E/W

Juncus trighumis L. var, albescens Lange - Dorn01, FNA, IMF, E/W

[Juncus albescens (Lango) Fernald] - HDH, KTZ SF!, W \& W

[Jubcus triglumis L. vat. albescens (Fernald) Lange]- UTF

Juncus triglumis L. var. triglumis - Dom01, FNA, IMF at high latitudes, E/W

[Juncus triglumis L.] - KTZ, SFL, W\&W

Juncus tweedyi Rydb. - IMF, KTZ, SFE, UTF. W\&W

[Juncas brevicaudatus (Engelm.) Fern.] - FNA

Juncus vaseyi Engelm. - Dorn01, FNA, GPF, HDH, IMF, KTZ, SF!, W\&W, E/W

Luzula comosa E. Mey. - FNA, KTZ, SF!, W\&W, E/W

[Luzula campestris (L) DC.] - IMF, WS W', misapp.

[Luzula campestris (L.) DC. var. multiflora (Ehrh.) Celak.] - IMF, UTF

[Luzula multiflora (Ehrth.) Lej]- - Domol. FNA

[Luzula multiflora (Retz, Lej.] - GPF, HDH, W\&W, misid.

[Luzula multiflora (Ehrt.) Lej. subsp. multiflora var, multiflora] - KTZ

Luzula fastigiata E. Mey. - KTZ

[Luzula parvifloen (Ehrb.) Desv.] - IMF

Luzula parvifhora (Ehrh.) Desy, - Dom01, FNA, GPF, HDH, IMF, KTZ, SF!, UTF, W\&W, E/W

Luzula spicata (L.) DC. - Dom01, FNA, HDH, IMF, KTZ, SF!. UTF. W\&W. E/W

- Luzula subcapitata (Rydb.) Harrington - ENDEMIC <CO>, FNA, HDH, KTZ, SF!, W\&W, E/W

\{Luzula sudetica (Willd.) DC. \} - FNA, HDH

\{Lusula multiflora (E.hrh.) Lej. subsp. frigida (Buchenau) Krecz, - KTZ

Snou, N, Janury 2009. Checklist of Vacculer Plants of tho Southom Rodky Mosmain Region. CVersion $3 x$ 


\section{Juncaginacene - JCG}

Triglochin maritima L. - FNA, HDH, KTZ, SF!, UTF, W\&W, E/W

[Trigloch in concinana Burtt-Davy] - SF!

[Trigloch in concinma Burts-Davy var, debilis (M. E. Jones) J. T. Howell] - GPF, HDH rep., IMF, UTF

[Triglochin dehilis (M. E. Jones) A. LAve \& D. Löve] - W'\&W

[Triglochin maritima L. var. elatz (Nutt.) A. Gray] - Darn0], GPF, IMF

Triglochin palustre L. - FNA, E/W

[Triglochin palustris L.] - Dom01, GPF, HDH, IMF, KTZ, SF!, UTF, W\&W

\section{Krameriacene - KRM}

\{Krameria erecta Willd, ex Schult, \& Schult. f.\} - IMF

[WJ - Aven Neivan 8034 erroneously labeled Colorado\}

\{Krameria parviflora Benth. var. glandulosa (Rose \& Painter) J. F. Macbr.\} - HDH, W \& W

\{Krameria paryillora Benth.\} - UTF

\{Krameria erecta Willd ex Schult, - KTZ <AZ, NM, UT>

Krameria lanceolata Tor, - GPF, HDH, KTZ, SFE, W\&W, E

Lamiacene - L.AM (= Labiatae)

(Identification sources:

Agastache foeniculum (Pursh) Kuntze - Dorn01, GPF, KTZ, SFE, E

[Agastakte foeniculum Kuntze]- HDH, W\&W

Agastache pallidiflora (A. Heller) Rydb. subsp, neomexicana (Briq.) Lint \& Epling - KWA.

$\mathrm{HDH}, \mathrm{KTZ}$ [also recogn, 2 vars.], W\&W, err. rep.

[Agastache neomexicana (Briq.) Standl.] - KWA

R.

[Agastache pallidiflora (A. Heller) Rydb, subsp. neomexicana (Brie.) Lint \& Epling var, neomexicana (Briq.)

W. Sanders] $-\mathrm{KTZ} \times \mathrm{NM}$ ?

Agastache pallidiflora (A. Heller) Rydb. var, pallidiflora-IMF, ENW

[Agastache pallidiflora (A. Heller) Rydb.] - SFt, UTF

[Agastache pallidiflora (A. Heller) Rydb, subsp, pallidiflora] - HDH

[Agastache pallidiflora (A. Heller) Rydb، subsp, pallidiflora var, groseci (Brig,) R. W. Sanders] - KTZ, W\&W

Agastache urticifolia (Benth.) Kuntze var. urticifolia - IMF, KTZ, E/W

[Agastuche urticifolia Kuntze] - $\mathrm{HDH}$

[Agastache urticifolia (Benth.) Kantre] - Dom01, SF!, UTF, W\&W

$\div$ Clinopodium vulgare L. - HDH, KTZ, SF!, W\&W, E/W

[Satureja valgaris (L.) Fritseh] - Dom01, IMF

Dracocephalum (sec also Physostegia)

Dracocephalum parviflorum Nutt. - Dorn01, GPF, IMF, KTZ, SF!, UTF, W\&W, E/W

[Moldavica parviflora (Nutt.) Britton] - $\mathrm{HDH}$

Dracosephalum thymiflorum L, - Dom01

*Galeopsis bifida Boenn, - GPF, KTZ, SF!, W\&W, E/W

[Galeopsis tetrahit L.] - $\mathrm{HDH}$ 
*Glecoma hederacea L, - Dom01, GPF, HDH, IMF, KTZ, SF!, UTF, W\&W, ENW

Hedeoma drummondii Benth - Dorn01, GPF, HDH, IMF, KTZ, SF!, UTF, W\&W, E/W

Hedeoma hispidum Pursh - Dom01, GPF, SFE, W\&W, E

[Hedesma hispida Pursh] - HDH, KTZ

Hedeoma oblongifolia (Gray) Heller - KWA, M\&H, KTZ

[Hedeoma piperita var. oblongifolia Gray ] - KTZ

[Hedeoma thymoides var. oblongifolia (Gray) Gray ] - KTZ

*Hyssopus officinalis L. - KTZ, W\&W

*Lamium amplexicaule L. - Dorn01, GPF, HDH, IMF, KTZ, SF!. UTF, W\&W, E/W

*Lamium purpureum L, var, purpureum - KTZ, E/W

[Lamium purpureum L.] - GPF, HDH rep. IMF, SF!, UTF, W\&W

*Leonurus cardiaca L. - Dom01, GPF, HDH, IMF, SFE, UTF, W\&W, E

[Leonurus cardiaca L. subsp. cardiaa] - KTZ

Lycopus americanus Muhl, ex W. P. C. Barton - Dorn01, GPF, HDH, KTZ, UTF, W\&W, E/W

[Lycopus americanas Muhl.] - SF!

Lycopus asper Greene - Dorn01, GPF, IMF, KTZ, SF!, UTF, W\&W, E/W

[Lycopus lucidus Turczan inov ex Bench.] - $\mathrm{HDH}$

Lycopus uniflorus Michx, - Dom01, GPF, HDH, SFE, W\&W, E

[Lycopus aniflorus Michs, var, uniflons] - KTZ

*Marrubium vulgare L. - Dorn01, GPF, HDH, IMF, KTZ, SF!, UTF, W\&W, E/W

Mentha (see also Monarda)

Mentha arvensis L. - Dornol, GPF, HDH, [MF, KTZ, SF!, W\&W, E/W

[Mentha arvensis L. var, glabrata (Benth.) Fernald] - UTF

Mentha X citrata Ehrh. - IMF

[Mencha acuatica X. M. spicata]-IMF

[Mentha agantica L.] - KTZ SUT>

[Mencha citrata Ehrh.]- $\mathrm{HDH}, \mathrm{UTF}$

*Mentha X piperita L. - GPF, IMF, KTZ, UTF, E

[Mencha aguatica X M. spicata]- IMF

[Mencha piperita L.] - HDH. SFE, W\&W

*Mentha spicata L. - Dorn01, GPF, HDH, IMF, KTZ, SFE, UTF, W\&W, E

\section{Moldavica (see Dracocephalum)}

Monarda fistulosa L. var. menthifolia (Graham) Fernald - Dom01, GPF, HDH, IMF, SFE, UTF, W\&W, E/W

[Monarda fistulosa L.] - SFW

[Monarda fistulosa L. subsp, fistulosa var, menthifolia (Graham) Fenald] - K.TZ

Monarda pectinata Nutt. - Dorn01, GPF, HDH, IMF, KTZ, SF!, UTF, W\&W, EW

Monarda punctata L. var. occidentalis (Epling) Palmer \& Steyerm.

[Mencha pinctata $\left.L_{1}\right]$ - W\& W', err. rep.

[Mencha punctats L. subsp. oceidentalis Epling] - GPF $\angle \mathrm{CO}>\mathrm{HDH}$, W\&W. err. rep,

[Mencha punctata L. subsp. punctata var. oecidentalis (Epling) Palmer \& Steycrm.] - KTZ <KS, OK, NM.

Snow, N, Junury 2009. Checklist of Vaccular Plants of the Southom Rocky Momain Region. CVersion $3 x$ 
Monardella odoratissima Benth. var. glauca (Greene) H. St. John - Dorn01. IMF, W

[Monardella glawea Grecne] - KTZ

[Mconardella odoratissima Benth.] - HDH, KTZ, SFW, UTF, W\&W

*Nepeta cataria L. - Dom01, GPF, HDH, IMF, KTZ, SF!, UTF, W\&W, EW

*Nepeta racemosa Lam. - Dorn01

*Nepeta grandiflora M.-Bieb, - Dorn0!

Physostegia parviflora Nutt. ex A, Gray - Dom01, KTZ GPF, IMF, KTZ,UTF, W\&W, exp.

[Dracosephalum nuttallii Britton] - HDH, rep.

Poliomintha incana (Torr, A. Gray - HDH, exp., IMF, KTZ, SFW, UTF, W\&W, W

↔Prunella vulgaris L, var. lanceolata (W, P. C. Barton) Femald - GPF, IMF, UTF, EW

[Prunella vulgaris L.] - Dorn01, HDH, SF!. W\&W

[Prunella vulgaris L. subsp. lancedala (W. P. C. Barton) Hultén] - KTZ

Prunella vulgaris L, var, vulgaris - KTZ

Pycnanthemum virginianum (L.) T. Durand \& Jackson ex B. L. Robins, \& Fernald - GPF, HDH, KTZ <KS, NE, OK>, W\&W, err, rep.

[Pycnanthentum virginianum (L.) T. Durand \& Jackson] - W\& W'

*Salvia aethiopis L. - IMF, KTZ, SFE, UTF, W\&W, E

\% Salvia azurea Michx. ex Lam, var. grandiflora Benth. - HDH, KTZ, E

[Salvia azurva Lam.] - UTF

[Salvia azurva Michx. ex Lam. subsp. pitcheri (Torr.) Epling] - HDH

[Salvia azurea Lam. var. grandiflora Benth.] - SFE

[Salvia azurca Michx. \& Lam. var, grandiflora Benth.] - W\&W

[Salvia azurea Lam. var. grandiflora Benth.] - GPF

Salvia subincisa Bentham - KWA, M\&H, KTZ

*Salvia pratensis L. - GPF, KTZ, SFE, W\&W', E

Salvia reflexa Hornem. - Dom01, GPF, HDH, IMF, KTZ, SF!, UTF, W\&W, E/W

*Salvia sclarea L, - GPF, IMF, KTZ, UTF, W\&W

*Salvia $\mathrm{X}$ sylvestris L. - KTZ. W\&W', misapp., E/W

[Salvia pratensis X S, nemorosa] - KTZ

[Salvia nemorosa L.] - Dom01, GPF, KTZ, SFl, W\&W'

[Salvia sylvestris L.]-HDH, W\&W

Saturcja (sec Clinopodium)

Scutellaria brittonii Porter - Dorn01, GPF, HDH, KTZ, SF!, W\&W, E/W

Scutellaria galericulata L. - Dorn01, GPF, HDH, IMF, KTZ, UTF, E/W

[Scutellaria galericulata L, var, epilobuifolia (Hamition) Jordal] - SF], W\&W

Scutellaria lateriflora L. var. lateriflora - KTZ, E

[Scutellaria lateriflora L] - GPF, HDH, SFE, W\&W

Scutellaria wrightii A. Gray - KTZ $<\mathrm{OK}>$, W

[Scutellacin resinosa Torr.]-GPF, KTZ, SFW, W\&W

Stachys palustris L. var. pilosa (Nutt.) Fernald - Dorn01, IMF, UTF, E/W

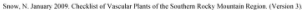


[Stachys palustris L. subsp. pilosa (Nutt) Epling] - GPF, HDH, SF!. W\& W

[Stachys pilosa Nutt. var. pilosa] - KTZ

Teucrium canadense L. var, occidentale (A. Gray) E. M. MeClint. \& Epling - Dorn01, HDH, IMF, KTZ, UTF, E/W

[Teucrium canadense L. subsp. occidentale (A. Gray) W. A. Weber] - W\& W

[Teucrium canadense L. subsp. occidentale (A. Gray) E. M. McClint. \& Epling] - SF!

[Teucrium cansdense L. var, boreale (E. P. Bickneli) Shimners] - GPF

Teucrium laciniatum Tor. - GPF, HDH, KTZ, SFE, W\&W, E

\section{Leguminosae (see FAB)}

\section{Lemnaceae (sce ARA)}

\section{Lentibulariaceae - LNT}

Utricularia minor L. - Dom01, GPF, HDH, IMF, KTZ, SF!, UTF, W\&W, E/W

Utricularia ochroleuca R. W. Hartm. - KTZ, SF!, W\&W, E/W

Utricularia macrorhiza Leconte - Dom01, KTZ, SF!, W\&W

[Utricularia vulgaris L.]-GPF, HDH, IMF, UTF, W\&W', misapp. EW

\section{Liliacene - LIL (see also ALL, ASG, CVL, HPX, MLN, SML, CHC, XAN)}

(Identification sources:

Allium (see ALL)

Androstephium (see ALL)

Anticlea (see Zigadenus [MLN])

\{Calochortus aureus S. Watson\} - IMF, KTZ, UTF

[W] - erroneous report, no specimens seen at ARIZ, BRY, COLO, CS, KHD, RM]

[NS - FNA (vol. 26) indicates out of our range]

\{Calochortus neattallii Torr, var, aurvus (S. Wassonj Owabey\} - FNA, HDH. W \& W

fCalochortus nuttallii Torr. \& A. Gray var, aureus (S. Watson) Ownbey?

Calochortus flexuosus S. Watson - FNA, HDH, IMF, KTZ, SFW, UTF, W\&W, W

Calochortus gunnisonii S. Watson var, gunnisonii - FNA, IMF, KTZ, E/W

[Caloctortus gunnisonii S. Watson] - Dondl, GPF, HDH, SF!, UTF, W\&W'

Calochorthus gunnisonii S. Watson var, perpulcher Cockerell - FNA

\{Calochortus macrocarpus Douglas\} - HDH rep., IMF, W\&W, err. rep.

\{Calochortus macrocarpus Douglas var, macrocarpus; - KTZ

Calochortus nuttallii Torr. \& A. Gray - Dorn01, FNA, GPF, IMF, KTZ, SFW, UTF, W

[Calochertus nutralli Tom, var, nuttallii] - HDH, W\&W

\section{Disporum (sec CHC)}

Erythronium grandiflorum Pursh subsp. chrysandrum Applegate - HDH, E/W

[Erythronium giganteum Lindky] - FNA 
[Erythronium grandiflorum Pursh] - IMF, SF!, UTF, W\&W

[Erythronium grandiflorum Pursh subsp. chrysandrum (Piper) Abrams] - FNA

[Erythronium grandiflorum Pursh var. grandiflorum] = Dorno1

[Erythronium grandiflorum Pursh subsp. grandiflorum] = FNA, KTZ

[Erythronium grandiflorum Pursh var. nudipetalum [as "nodepetalum"] (Applegate) C. L.. Hitche.] - FN.A

[Erythronium grandiflorum Pursh var. pallidum H. St. John] - FNA

[Erythronium parviflorum (S. Watson) Goodding] - FNA

Fritillaria atropurpurea Nutt, - Dom01, FNA, GPF, HDH, IMF, KTZ, SF! UTF, W\&W, ENW

[Fritillaria adamantina M. Peck] - FNA

[Frilillaria gracillima Smiley] - FNA

[Fritillaria linearis J. M. Couluter \& Fisher] - FNA

Fritillaria pudica (Pursh) Spreng, - Dom01, GPF, HDH, IMF, KTZ, SFW, UTF, W\&W, W

Lilium philadelphicum L. var, andinum (Nutt.) Ker Gawler - Dorn01, GPF, KTZ, E/W

[Liliuim andinum Nuttall] - FNA

[Lilium muntanum A.Nelsoa] - FNA

[Lilium philadelphicum L.] - FNA, SF!, W\& W

[Lilium umbellarum Pursh] - HDH, FNA

Lloydia serotina (L.) Rehb, var, serotina - FNA, E/W

[Bulbosodium serotinum L.] - FNA

[Lloydis serotina Rehb.] - Dorn01, SFE

[Lloydis serotina (L.) Sweet] - HDH

[Lloydis serotina (L.) Salisb.] - SFW

[Lloydis serotina (L.) Salisb. ex Rshb.] - IMF, W\&W

[Lloydis serotina (L.) S. Watson] - UTF

[Lloydia serotina (L.) Rchb. subsp. serotina] - KTZ

Prosartes (see Disporum $[\mathrm{CHC}]$ )

Smilacina (see Maianthemum [RUS])

Toxicoscordion (see Zigadenus [MLN])

\section{Limnanthaceae - LIM}

Floerkea proserpinacoides Willd. - Dorn01, HDH, IMF, KTZ, SF!, UTF, W\&W, E/W

Limoniaceae (see PLB)

Linaceae - LIN

(Identification sources:

Adenolinum (sec Linum)

Linum aristatum Engelm. - GPF, IMF, KTZ, UTF, W

[Mesyniam aristatum (Engelm.) W. A. Weber] - SFW, W\&W

Linum australe A. Heller var, australe - KTZ, E/W

[Limum australe A. Heller] - Domo1, HDH, [MF, UTF 
[Mesyniam australe (A. Heller) W. A. Weber] - SF!, W\&W

Linum berlandieri Hook, var, berlandieri - KTZ

[Linum berlandieri Hook.] - HDH

[Linum rigidum Pursh var, berlandieri (Hook.) Torr. \& A. Gray] - GPF

[Mesyniam rigidum (Pursh) A. Löve \& D. Löve] - W\&W

*Linum grandiflorum Desf. - IMF, KTZ, UTF, E

[Adenolinum grandiflorum (Desf) W. $A$ Weber] - SFE, W\&W

Linum kingii S. Watson - Dorn01, HDH, IMF, KTZ, UTF, W

[Mesyniopsis kingii (S. Watsoa) W. A. Weber] - SFW, W\&W

Linum lewisii Pursh var. lewisii - IMF, KTZ, E/W

[Adenolinum leswisii (Pursh) A. Lồve \& D. Löve] - SF!, W\& W

[Linum lexisii Pursh] - Dom0I, HDH

[Liram perenne L. subsp. Kcwisii (Parsh) Hullén] - UTF

[Limum perenne L. var. lewisi (Pursh) Eat. \& J. Wrigha] - GPF

*Linum perenne $L$.

[W] - $A$ ubiquitous weed in $\mathrm{CO}$. It is the plant sold in highway/pipeline revegetation mixtures as $L$ lewisii, which is seen widely along highways. $L$ perenete is very erect. producing a very attractive bouquet shaped plant with copious flowers. It has pin and thrum morphs. L. kewisii has fewer flowers and cones out of the ground nearly horizontally: ics style exceods amthers on all plants|

Linum pratense (Norton) Small - GPF, KTZ, E

[Adenolinum pratense (Norice) W. A. Weber] - SFE, W\&W

Linum puberulum (Engelm,) A. Heller - Dorn01, GPF, HDH, IMF, KTZ, UTF, EW

[Mesyniam puberulum (Engelm.) W.A. Weber]-SF, W\&W

Linum rigidum Pursh var, compactum (A. Nelson) C. M. Rogers - Dorn01, GPF, E

[Liram ompactum A. Nelson] - HDH, KTZ

[Mesyniam rigidum (Pursh) A. Love \& D. Löve] - SFE, W\&W

Linum rigidum Pursh var, rigidum - Dom01, GPF, KTZ, E

[Libum rigidum Pursh] - HDH

[Mesyniam rigidum (Tursh) A. Lôve \& D. Lowe] - SFE, W\&W

Linum rigidum Pursh var. simulans C. M, Rogers - KTZ

*Linum usitatissimum L. - Dorn01, GPF, HDH, KTZ, SF!, W\&W, E/W

[NS - evidently only 2 collections from $C O$ and none from wild: one from 1890 at CS (W), pers. comm.) and one at GREE from 1905]

Mesyniopsis (see Linum)

Mesynium (see Linum)

\section{Linnaeaceae - LNA}

Linnaca borealis L. var. longiflora Torr. - Dom0I, IMF, UTF, E/W

[Linnaea bocealis L.] - GPF

[Limaea bocealis L. sabsp, americana (Forbes) Hulteñ] - W\& W

[Linnaea borealis L. subsp, americana (Forbes) Huhên ex Clausen] - KTZ, W\&W

[Linnaea borealis L, var, americana (Forbes) Rehder] - HDH

[Linnaea bocealis L. subsp, longiflora (Torr. Hulten] - KTZ, SFt

\section{Loasaceae - LOA}

(Identification sources: )

\section{Acrolasia (see Mentzelia)}


Cevallia sinuata Lag. - KWA. M\&H, KTZ

Mentzelia

[NS - existing keys do not work well for same material in the southern part of our range [Schiebout08\}]

Menizelia albicaulis (Douglas ex Hook.) Douglas ex Torr. \& A. Gray - KTZ, EJW

[Aerolasia albicaulis (Douglas) Rydb.] - SF?

[Acrolasia albicaul is (Douglas ex Hook.) Rydb.] - W\&W

[Aerolasia gracilis Rydb.]-SFE, W\&W

[Mentrelia albicaulis (l look.) Torr. \& A. Gray]-GPF, UTF

[Mentrelia albicaulis (Dougl, ex Hook.) Dougl. ex Torr. \& A. Gray]- Dom01

[Mentzelia albicaulis Douglas ex Hook. var. albicaulis] - HDH

Mentzelia argillosa J. Darl. - KTZ, UTF, W

[Nuttalia argillosa (J. DarL.) W. A. Weber] - SFW. W\&W

- Mentzelia chrysantha Engelm, ex Brandegee - ENDEMIC <CO>, HDH, KTZ, E

[Mencaelia lutea Gresne] - HDH, exp.

[Nuttallia chrysantha Greene] - SFE

[Nutallia chrysantha (Engelm. ex Brandegee) Greene] - W\& W

Mentzelia cronquistii H. J. Thomps. \& Prigge - KTZ, W

[Menczelia marginata (Osterh.) H. J. Thomsps. \& Prigge] - UTF

[Nuttallia cronquistii (H. J. Thomps. \& Prigge) W. A. Weber] - SFW. W\&W

Mentzelia decapetala (Pursh ex Sims) Urb. \& Gilg ex Gilg - Dorn01, KTZ, E

[Menczelia decapetala (Pursh) Urb. \& Gilg] - GPF, HDH

[Nuttallia decaperala (Pursh) Greene] - SFE

[Nuttallia decapetala (Pursh ex Sims) Greene) - WAW

- Mentzelia densa Greene - ENDEMIC $\langle\mathrm{CO}>, \mathrm{HDH}, \mathrm{KTZ}, \mathrm{E}$

[Mentzelia speciosa (Osterh.) Greene]

[Nuttallia dersa (Greene) Greene] - W\& W

[Nuttallia speciosm (Osterh.) Geeene] - SFE

Mentrelia dispersa S. Watson

[NS - following Docm01 in not recognizing variecties in our region]

[Acrolasia dispersa (S. Watson) A. Davids.] - SFI, WA W

[Mentzelia dispersa $\mathrm{S}$. Watson]-GPF, UTF

[Menczelia disperso S. Warson var, compacta (A. Nelson) J. F, Macbr. - HDH, KTZ, E/W

[Mentzelia dispersa S, Warson var, dispersa] - HDH, KTZ, EW

[Mentzelia dispersa S. Warson var, latifolia (Rydb., J, F, Macbr.] - HDH, KTZ, E/W

[Mentzelia dispersa $\mathrm{S}$. Watson] - GPF, UTF

Mentzelia humilis (A, Gray) J. Darl. - KWA, M\&H, HDH, KTZ, W

[Acrolasia hamilis Ostertiout ] - KTZ

[Acrolasia thompsonii (Glad.) W.A. Weber ] - KTZ

[Mentrelia thompsonii Glad,] - KTZ

[Nuttalia gypsea Woot \& Standi,] - M\&H

[Nuttallia humilis (A. Gray) Rydb.] - SFW, W\&W, err, rep.

Mentzelia laciniata (Rydb.) J. Darl. - HDH. KTZ. W

[Nuttallia laciniata (Rydb.) Wocton \& Standl.] - SIW. W\&W

Mentzelia laevicaulis (Douglas ex Hook.) Torr. \& A. Gray var. laevicaulis - KTZ, W

[Mentzelia lacvicaulis (Douglas) Torr. \& A. Gray] - Dorn01, GPF, UTF

[Nuttallia laevicaulis (Dougglas) Greene] - SFW

Mentzelia marginata (Osterh.) H. J. Thomps, \& Prigge - KTZ. UTE, W

[Nuttallia marginata Osterh.] - SFW, W\&W

Mentzelia montana (Davids.) Davids, - Dorn01. KTZ

[Acrolasia dispersa (S. Waison) Davids.] - W\&W

[Mentzelia albicaulis (Hook.) Torr. \& A. Gray] - UTF 
Mentzelia multicaulis (Osterh.) A. Nelson ex J. Darl, var, multicaulis - KTZ, W

[Mentrelia multicaulis (Osterh.) ). Darl.] - HDH

[Mentrelia multicaulis (Osterh.) ). Darl. var. multicaulis] - UTF

[Nuttalia multicaulis Osterh.] - SFW

[Nuttallia multicaulis fOsterh.) Osterh.] - W\&W

Mentzelia multicaulis (Osterh.) A. Nelson ex J. Darl, var, uintahensis N. H. Homgren \& P. K.

Holmgren - Holmgren02

\{Mentzelia multiflora (Nutt.) A. Gray var integra M. E. Jones\} - KTZ $<$ AZ, NM, UT>

[Mentrelia integra (M. E. Jones) Tidestr.] - HDH, exp., UTF

\{Nuttallia pleresperma (Eastw.) Greene\} - W\&W

Mentzelia multiflora (Nutt.) A. Gray var. multiflora - KTZ, E/W

[Mentzelia multiflora (Nutt.) A. Gray]-GPF, HDH, UTF

[Nuttallia multiflora (Nutt) Greene] - SF!, W\&W

Mentzelia nuda (Pursh) Torr. \& A. Gray

[Menczelia nuda (Pursh) Tort. \& A. Gray var, nuda] - HDH, KTZ, E

[Nuttallia nuda (Pursh) Greene] - SFE, W $\&$ W

[Mencrelia nuda (Pursh) Torr. \& A. Gray var. stricta (Oslerh.) Harringon] - HDH. KTZ, E

Mentzelia oligosperma Nutt, ex Sims - Dorn01, KTZ, W\&W, E

[Mentselia oligosperma Nutt.] - GPF, HDH, SFE

Mentrelia pterosperma Eastw, - HDH, KTZ, UTF, w

[Nuttallia perosperma (Eastw.) Gretne] - SFW, W\& W

Mentzelia pumila Tort, \& A. Gray - Dom01, GPF, HDH.

[NS - we follow Dom01 and IMF in not recognizing varieties]

[Mentzelia integra (M.E. Jones) Tides.] - M\&H

[Menczelia multifloea var, integra M.E. Jones] - KTZ, M\&H

[Nurtalliana integra (M.E. Jones) Rydb.] - M\&H

[Menczelia pumila Torr. \& A. Gray var. integra M.E. Jones] - KWA, M\&H, JM, not rep.

[Menczelia purmila Torr. \& A. Gray var. prosera (WooL \& Standl.) J. DarL] - KWA, M\&H

[Menczelia pumila Torr. \& A. Gray var. pumila] - KTZ. Darlington34, UTF

[Nuttaliana springeri Standl.] - M\&H

[Nuttalliana procira Woot. \& Standl.] - M\& H

[Nuttallia pamilia (NutL.) Greene] - W\&W, out of range

Mentzelia reverchonii (Urb. \& Gilg) H. J. Thomps. \& Zavort. - GPF, KTZ, E

[Nuttalia reverchonil (Urb. \& Gilg) W, A. Weber] - SFE, W\& W

Mentzelia rhizomata Reveal - Reveal

Mentzelia rusbyi Wooton - Dorn01, KTZ, UTF, E/W

[Menczelia muda (Pursh) Torr. \& A. Gray var, nusbyi (Wooton) Harrington] - HDH

[Nuttallia rusbyi (Wooton) Rydb.] - SF!, W\&W

Mentzelia sinuata (Rydb.) R. J. Hill - Dom01,KTZ, E

[Nurtallia sinuata (Rydb.) Daniels] - SFE, W\&W

Mentzelia speciosa Osterh. - Dom01, KTZ, E

[Menczelia multiflora (Nuts.) A. Gray] - UTF

[Nuttallia speciosa (Ostert.) Greene] - SFE, WRW

Mentzelia thompsonii Glad. - UTF, W

[Acrolasia hamilis Osterh.] - SFW, WRW

[Menczelia humilis \{A. Gray) J, Darl.] - KTZ

$\{$ Mentzelia veatchiana Kellogg\} - KTZ $<A Z>$

[Acrolasia vearchiana Kellogg\} - W\& W, misapp.

(Mentzelia albicsalis Douglas ex Hook.)

\{Mentzelia albicaulis Douglas ex Hook, var, veatchiana (Kelloge) Urban \& Gilg? - $\mathrm{HDH}$

Nuttallia (see Mentzelia)

Snow, N, January 2009. Checklist of Vacculer Plants of the Southum Rodky Momain Region. CVersion $3 x$ 


\section{Lobeliaceae (see CAM)}

Loranthaceae - (see $\mathbf{S A N}$; family boundaries with $\mathbf{S A N}$ and VIS still ururesolved)

\section{Lythraceae - LYT}

\{Ammannia coccinea Rottb. - GPF, HDH, IMF, KTZ, W\&W, misapp. Ammannia robusta Heer \& Regel - Dorn01,GPF, IMF, KTZ, SF!, W\&W, E/W

[Ammannia coscinea Rottb. subsp. robusta (Hecr \& Regel) Kuehne] - UTF

Lythrum alatum Pursh var, alatum - GPF, KTZ, E

[Lythrum alatum Pursh] - Dona1,HDH, SFE, W\&W

*Lythrum salicaria L,-Dorn01, GPF, IMF, KTZ, SF!, UTF, W\&W, E/W

Rotala ramosior (L.) Koehne - GPF, IMF, KTZ, SF!, W\&W, E/W

[Rotala ramosior (L.) Koehne var, interior Fernald \& Grise.]

\section{Malvaceae - MLV}

(Identification sources:

Abutilon incanum (Link) Sweet subsp, incanum - E

[Abutilon ineanum (Link) Sweet] - GPF, HDH, KTZ, SFE, W\&W

Abutilon parvulum A. Gitay - GPF, HDH rep., KTZ, SFE, UTF, W\&W, E

*Abutilon theophrasti Medik, - Dom01,GPF, HDH, exp.KTZ, SF!, UTF, W\&W, E/W

*Alcea rosea L. - Dom01, FCF, KTZ, SFE, W\&W', E

[Althaea rosea Cav.]-HDH, corr, \& add, GPF

[Althaes rosea L.] - W\& W

[Althace rosea (L.) Cav.] - UTF

\section{Althaca (sec Alcea)}

๑Anoda cristata (L.) Schltdl, - GPF, KTZ, SFE, W\&W, E

[W] - collected once in onion field at CSU Experiment Station. Rocky Ford in 1966]

Callirhoc alcacoides (Michx.) A. Gray - KWA, KTZ, M\&H, JM, not rep.

[Sida alcaeoides Michx.] - M\&H

Callirhos involucrata (Torr. \& A. Gray) A. Gray var, involucrata - KTZ, E

[Callirboę involucrata (Nutt. ex Torr.) A. Gray] - Darne]

[Callirboe involucrata (Torr. \& A. Gray) A. Gray] - GPF, HDH

[Callirboę involucrata (Nutt. ex Torr, A Gray var, involacrata]

[Callirboę involucrata (Torr, \& A. Gray) A. Gray]-SFE, W\&W

Callirhot involucrata (Torr. \& A. Gray) A. Gray var, lineariloba (Torr. \& A. Gray) A. Gray

- KTZ, Dorr90

[Callirboe involucrata (Torr. \& A. Gray) A. Gray - GPF

[Calliftoe involucrata (Nutt. ex Torf, A. Gray var. lineariloba (Torr. \& A. Gray) A. Gray

*Hibiscus trionum L. - Dom01, GPF, HDH, KTZ, SF!, UTF, W\&W, E'W 
Iliamna

[NS - Tuxonomy follows revision of entird genus (Bodo Slocta01) based primarily on IIS but also morphology]

(Identification sources:

- lliamna crandallii (Rydb.) Wiggins - KTZ, SE!, W\&W, Bodo Slotta01

[Sphaeralcea srandalii Rydb.] - Bodo Slottal

[Phymosisa crandalii (Rydb.) Rydb.] - Bodo Slottand

Iliamna grandiflora (Rydb) Wiggins - KTZ, SFW, W\&W, E/W, Fryxell94, Bodo Slotta01

[liamana angulata Greene] - Booki Slotta01

[Phymosia grandiflora (Rydb.) Rydb.] - Bodo Slotta0]

[Sphacraloea grandiflora Ryd.] - Bodo Skotta01

Iliamna rivularis (Douglas ex Hook.) Greene var, rivularis - KTZ, E/W

[Hiamna rivularis (Douglas) Greetse] - HDH. SF!, UTF, W\&W

[Uliamna rivularis (Douglas ex Hook.) Greene] - Dom01

*Malva neglecta Wallr. - Dorn01,GPF, HDH, KTZ, SF!, UTF, W\&W, EW

*Malva parviflora L. - Dom01, GPF, HDH, KTZ, SFE, UTF, W\&W, E

*Malva pusilla $\mathrm{Sm}$ - Dorno1

[Malva rotundifolia L.] - KTZ

*Malva sylvestris L. - Dorn01

*Malva verticillata L. - Dom01,GPF, KTZ, E

[Malva crispa L.] - HDH

[Malva crispa (L.) L.] - SFE, W\&W

[Malva verticillata L. var, crispa L.] - UTF

Malvella lepidota (A. Gray) Fryxell - W\&W, err, rep., KTZ $<A Z$, NM>, [Voucher of D. Clark \& C. Crawford an COLO]

\&Malvella leprosa (Ortega) Krapov, - GPF, KTZ, SFE, UTF, W\&W, E

[Sida hederacea (Douglas) Torr.] - HDH

Malvella sagittifolia (A. Gray) Fryxell - GPF, KTZ, SFE, W\&W, E

[WJ - one collection. 1939, major disjuct from TX, likely a wait]

[Sida lepidota A. Gray var, sagittifolia A. Gray] - HDH

Sida (see also Malvella)

Sida neomexicana A. Gray - FCF

[NS - otherwise reported for Con:[WJ - ] know of no Colonde eollections]

Sidalcea candida A. Gray var. candida - KTZ, UTF, E/W

[Sidalcea candida A, Gray] - DornO1, HDH, SF1, W\&W

Sidaloca candida A. Gray var. glabrata C. L. Hitchc. - KTZ. UTF, E/W

[Sidalcea candida A, Gray] - SF?, W\&W

Sidalcea neomexicana A. Gray var. neomexicana - UTF, E/W

[Sidalcea neomexicana A. Gray] - Dorne], HDH, SP!, Wa W

[Sidalcea neomexicana A. Gray subsp, noomexicana] - KTZ

Sphaeralcea

(Identification sources:

\section{)}

Sphacralcea angustifolia (Cay.) G. Don - KTZ, E

Snow, N. Junury 2009. Checklist of Vaccular Plants of the Southom Rocky Momain Region. CVersion $3 x$ 
[Sphaeralcea angustifolia (Cav.) G. Don var. cuspidata A. Gray] - GPF, HDH, SFE, W\&W

Sphaeralcea coccinea (NutL) Rydb, - Dorn01. SFE, UTF

[Sphaeralces eoceinea (Nutt.) Rydb, subsp. ceccines] - KTZ

[Sphaeralces coccinea (Pursh) Rydb.] - GPF

[Sphaeralcea coccinea (Pursh) Rydb, subsp. coccinea] - HDH, W\&W

[Sphaeralcea coccinea (Nutt.) Rydb. var. coccinea] - E/W

[Sphaeralces coecinea (Nutt.) Rydb, subsp. dissecta (Nutt.) Kesancy] - SFW

[Sphaeralcea coccinea (Pursh) Rydb, subsp. dissecta (Nutt) Kearney] - HDH, W\& W'

[Sphaeralcea coccinea (Nutt.) Rydb, subsp. elata (Baker f.) Kearney] - KTZ

[Sphaeralcea coccinea (Pursh) Rydh. subsp. elata (Baker f.) Kearney]-HDH, W\&W

\{Sphaeralcea digitata (Greene) Rydb.\} - HDH, exp.

\{Sphaeralcea digitala (Greene) Rydb. subsp. digicata\} - KTZ $\sim$ AZ, NM, UTs

iSphaeralcea đigitata (Greetre) Rydb. subsp. Ienuipes (Woovon \& Standl.) Kearney) - KTZ CNM

Sphaeralcea fendleri A. Gray var, elongata Kearney - KWA, M\&H, KTZ

[Sphaeralcea fendleri A. Gray sabsp. elongata Kearney ]- KTZ

Sphaeralcea fendleri A. Gray var, fendleri - W

[Sphaeralcen fendleri A. Gray] - GPF, HDH, SFW, W\&W

[Sphaeralces fendleri A. Gray sabsp. fendleri] - KTZ

Sphaeralcea grossularifolia (Hook. \& Am.) Rydb. var, grossularifolia - FCF

$\{$ Sphaeralcea hastulata A, Gray\} $-\mathrm{KTZ}<\mathrm{AZ}, \mathrm{NM}>$

[Sphaeralcea subhastata J. M. Coult. subsp. connata Kearney] - HDH, exp.

Sphaerolcea incana Torr. ex A. Gray var, cuneata (Kearney) Kearney - KWA, M\&H, JM, not rep.

[Sphaeralcea incana Torr. ex A. Gray subsp. cuncata Kearney] - KTZ

Sphaeralcea incana Torr, ex A. Gray var, incana - KWA, M\&H, JM, not rep.

[Sphaeralcea incana Tort. ex A. Gray subsp, incana] - KTZ $<$ AZ, NM?

Sphaeralcea leptophylla (A. Gray) Rydb, - HDH, KTZ, SFW, UTF, W\&W, W

Sphaeralcea munroana (Douglas ex Lindl.) Spach. ex A. Gray - Dom01

[W] - intertionally introduced at CSU Piceance Basin Oil Shale Study Site]

[Sphaeralcen mumroara (Douglas) Spach.] - HDH, exp., UTF

[Sphacralcen munreara (Douglas ex Lindi.) Spach. ex A. Gray subsp. munroana] - KTZ

[Sphacralcen munroansa (Douglas ex Lindl.) Spach. ex A. Gray subsp. subrhomboidea (Rydb.) Kearney] - KTZ

Sphaeralcea parvifolia A. Nelson - HDH, KTZ, SFW, UTF, W\&W, W

\section{Martyniaceae - MAR}

\section{Martynia (sce Proboscidea)}

Proboscidea louisianica (Mill.) Thell, subsp. louisianica - KTZ, E/W

[Martynia louisianica MilL] - Dom01, HDH

[Proboscides boulsianica (Mill.) Thell.] - GPF, IMF, SFI, UTF, W\&W

Proboscidea parviflora (Woot.) Woot. \& Standl. - KWA, M\&H, KTZ, JM, not rep.

[Martynia parviflora Woot] - M\&.H

\section{Melanthiaceat - MLN}

(Identification sources:

Anticlea clegans (Pursh) Rydb. - SF!, W\&W, FNA, Zomlefer01, Zomlefer02, FNA

[Anticlea alpina A. Heller]-FNA

[Anticlea chiorantha (Richardson) Rydb] - FNA

[Anticlea coloradensis (Rydb.) Rydb.] - FNA 
[Anticlea glauca (Nutall) Kunth] - FNA

[Anticlea gracilenta (Greene) R. R. Gates] - FNA

[Anticlea longa (Greene) A. Heller] - FNA

[Anticlea mobinorensis (Greenman) R. R. Gates] - FNA.

[Melarthium glaucum Nutt.] - FNA

[Zigadenus alpinus Blankinship] - FNA

[Zigadenus chloranthus Richardson] - FNA

[Zigadenus coloradensis Rydb.] - FNA

[Zigadenus dilatatus Greene] - FNA

[Zigadenus elegans Pursh] - Dorn01. FNA. GPF, HDH, IMF, UTF, E/W

[Zigadenus elegans Pursh subsp. elegans] - KTZ

[Zigadenus elegans var. glaucus (Nutt.) Procece ex Cronquist] - FNA

[Zigadenus elegans subsp. glaucus (Nuat.) Hultén] - FNA

[Zigadenus glaveus (Nutt.) Nett.] - FNA

[Zigadenus gracilintus Greene] - FNA

[Zigadenus longus Gineene] - FNA

[Zigadenus mahincensis Greeneman] - FNA

[Zigadenus washakie $A$. Nelson] - FNA

Anticlea vaginata Rydb, - Zomlefer01, Zomlefer 02 , SFW, W\&W

[Zigadenus vaginatus (Rydb.) J. F. Macbr.] - FNA, KTZ, UTE, W

[Zigadenus elegans Pursh] - IMF

Anticlea virescens (Kunth) Rydb.] - Zomlefer01, Zomlefer02, SF!, W\& W'

[Anticlea mexicans Kunth] - FNA

[Helcaias virescens Kunth in A. von Humboldi] - FNA

[Zigadenus porrifolius Greene] - FNA

[Zigadenus viresectrs (Kunth) J. F. Macbr.]-FNA, KTZ, EW

[Zigadenus virescens var- porrifolus (Govene) O.S. Walsh ex Espejo \& López-Ferrari] - FNA

Toxicoscordion paniculatum (Nutt.) Rydb, - W\&W, Zomlefer01, Zomlefer02, SFW

[Helonias paniculata Nuttall] - FNA

[Zigadenus panicularus (NuI.) S. Watson] - Dorn0I, FNA. HDH, IMF, KTZ, UTF, W

Toxicoscordion venenosum (S. Watson) Rydb, "var, gramineus" (Rydb.) Brasber comb, nov, ined.

[Toxicoscordion verenosum (S. Watson) Rydb.] - SFI, W\&W

[Toxicossordion acutum (Rydo.) Rydo.] - FNA

[Toxicoscordion falcatum (Rydb, Rydb.] - FNA

[Toxicoscordion intermediam (Rydb.) Rydb.] - FNA

[Zigadenus nesutas Rydb,] - FNA

[Zigadenus falcatus Ryob.] - FNA

[Zigadenus gramincus Rydb.] - HDH

[Zigadenus imtermodius Rydb.] - FNA

[Zigadenus venctostas S. Wanson var, gramincus (Rydb,) Walsh ex M. Pesk]- Dom01, FNA, GPF, IMF, KTZ. UTF, EW

Trillium ovatum Pursh subsp. ovatum - FNA, KTZ, E/W

[Trillium ovatum Pursh] - Domo1, HDH, SF!, W\&W

Veratrum califomicum T. Durand - Dorn01, HDH, W\&W, misapp.. E/W

[Veratrum californicum T, Durnd var, californicum] - IMF, FNA

[Verarium tenuipetalum A. Heller] - KTZ, SFL, W\& W

\section{Menyanthaceae - MNY}

Menyanthes trifoliata L. - Dorn01, GPF, HDH, IMF, KT/, SF!, UTF, W\&W, ENW

Snou, N, Janury 2009. Checklist of Vaccular Plants of tho Southom Rocky Moanain Region. CVersion $3 x$ 
Mimosacene (see FAB)

Molluginaceae - MOL

+Mollugo verticillata L, - Dom01, GPF, HDH, KTZ, SFE, W\&W, E

Monotropaceac (see ERI)

Moracene - MOR (see also CAN)

\$Maclura pomifera (Raf, ) C, K. Schneid, - FNA, GPF, KTZ, SFE, exp, SFW, UTF, W\&W, W

*Morus alba L, - Dorn01, FNA, GPF, KTZ, SF!, UTF, W\&W, EWW

Myrsinaceae - MRS (some formerly in PRM)

(Identification sources:

*Anagallis arvensis L, - GPF, HDH, exp., UTF

[Anagallis arvensis L. forma coerulea (Sctrob.) Baumg.] - W\& W'

[Anagallis arvensis L. subsp. foemina (Mill.) Schinz \& Tholl_] - KTZ

Anagallis minima (L.) E, H. L, Krause - KTZ, SFE, W\&W, E

[Centueculus minimus L.] - $\mathrm{HDH}_{\text {, exp., Domol }}$

Glaux maritima L. - Dorn01, GPF, HDH, KTZ, SF!, UTF, E/W

[Glaux maritima L. var. angustifolia B. Boivin] - W: W

[Glaux maritima L. sabsp. obtusifolia (Femald) B. Boivin]

Lysimachia ciliata L. - Dorn01, GPF, HDH, KTZ, SF!, UTF, W\&W, E/W

*Lysimachia nummularia L, - GPF, KTZ, SFE, UTF, W\&W, E.

Lysimachia thyrsiflora L. - Dorn01, GPF, HDH, KTZ, UTF, E

[Naumburgia thyrsiflora (L.) Rchb.] - SFE, W\& W

*Lysimachia vulgaris L. - KTZ, SFE, W\&W, E

Naumburgia (see Lysimachia)

Najadaceae (see also HDC, POT, RUP)

\{Najas flexilis (Willd.) Rostk. \& W. L. E. Schmidt\}- FNA, HDH rep., KTZ <AZ, NE, UT>

\&Najas guadalupensis (Spreng.) Magnus subsp. guadalupensis - FNA, KTZ, E/W

[Najas guada hupensis (Spreng.) Magnus] - GPF, SFE, WEW

[Najas guadalupensis (Spreng.) Morong] - Dond01, HDH, IMF, SFW, UTF

[Najas guadalupensis (Spreng.) Morong var, guadalupensis] - RMC

Nolinaceae (see RUS)

Nyctaginaceac - NYC

Snow, N, January 2009. Checklist of Vacculer Plants of the Southum Rodky Momain Region. CVersion $3 x$ 
(Identification sources:

Abronia elliptica A. Nelson - KTZ, SFW, W\&W, Ackerfieldo8

[Abronia fragrans Nutt. ex Hook_] - UTT

[Ábonia fragrans Nutt. ex Hook, var. elliptica (A. Nelson) Heimerl] - HDH

[Abronia fragrans Nutt, ex Hook. var, elliptica (A. Nelson) M. E. Janes] - Dom01, W

[A bronia salsa Rydb.] - HDH

Abronia fragrans Nut, ex Hook. - Ackerfieldo8, GPF, KTZ, UTF, W\&W

[Abronia carletonii J. M. Coult. \& Fisher] - HDH, KTZ, SFE, W\&W, E

[A bronia fragrans Nutt.] - SF?

[A bronia fragrans Nutt. ex Hook. var, fragrans] - Dom01, HDH, E/W

[Abronia fragrans Nutt. ex Hook. var. glaucescens A. Nels.] - HDH

Abronia glabrifolia Standl, - Ackerfield08

[Abronia argillosa S. L. Welsh \& Goedrich] - KTZ, SFW, UTF, W\&W, W

Abronia nana S. Watson var- nana - KTZ, UTF, W, Ackerfieldos

[Abronia nasas. Watson]-SFW, W\&W

Allionia choisyi Standl, - HDH, KTZ, SFE, W\&W, E

Allionia incarnata L. - GPF, HDH, SF!, UTF, W\&W, E/W

[Allionia incarnata L. var. incarnata] - KTZ

Mirabilis albida (Walter) Heimerl - GPF, KTZ, W\&W

[Mirabilis oblongifolia]-KT2, FCF

Mirabilis alipes (S, Watson) Pilz - KTZ, SFW, UTF, W\&W, W

Mirabilis comata (Small) Standl, - HDH, KTZ, W

[Oxy baphus comatus (Small) Weatherby] - SFW, W\&W

Mirabilis decipens (Standl.) Standl, - HDH rep, KTZ

[Mirabilis linearis (Pursh) Heimerl var, decipiens (StandL.) S. L. Welsh] - UTH

[Oxybaphus linearis (Pursh) B. L Rob.] - WAW

Mirabilis glabra (S. Watson) Standl. - GPF, HDH, exp., KTZ, E.

[Mirabilis carletonii (Standl.) Slandl.] - GPF, HDH

[Oxybaphus carletonii (StandL) Wentherby] - SFE, W\&W

[Oxybaphers glater S. Watson] - SFE, W\&W

Mirabilis hirsuta (Pursh) MacMill. - Dorn01, GPF, HDH, KTZ, E

[Oxybaphes hirsutus (Pursh) Sweet] - SFE, W\& W

Mirabilis linearis (Pursb) Heimer - Dorn01, GPF, HDH, KTZ, EW

[Mirabilis gausapoldes (Standi.) Standl.] - GPF

[Mirabilis lanecolata (Rydb.) Standl.] - $\mathrm{HDH}$

[Mirabilis linearls (Pursh) Heimerl var, linearis] - UTF

[Oxybaphus documbens (Nutt,) Sweet] - SFE, WRW

[Oxybaphus exaltatus (Standl.) Weadierby] - SFE, W\&W

[Oxybaphus lincarb (Pursh) B. L Rob.] - SF1, W\&W

Mirabilis melanotricha (Standl.) Spellenb. - specimens at GREE

Mirabilis multiflora (Torr.) A. Gray var. glandulosa (Standl.) J. F. Macbr. - KTZ, W

[Mirabilis glandulosa (StandL.) W, A. Weber]-SFW, WeW

[Mirabilis multiflora (Torr) A. Gray]-UTF

Mirabilis multiflora (Torr.) A. Gray var, multiflora - KTZ, E/W

[Mirabilis multiflora (Torr.) A Gray] - HDH, SF1, UTF, w\&W

Mirabilis nyctaginea (Michx.) MacMill. - Dorn0I, GPF, HDH, KTZ, UTF, E

[Oxybaphus ayctagineus (Michx.) Porter] - W\&W

[Oxybaghus ayctagineus (Michx.) Porter \& Coult] - SFE 
Mirabilis oxybaphoides (A. Gray) A. Gray - GPF, KTZ,UTF, W\&W, EW

[Mirabilis oxy baphoides A. Gray] - HDH, SF:

- Mirabilis rotundifolia (Greene) Standl. - ENDEMIC $<\mathrm{CO}>$, HDH. KTZ, E

[Oxybaphys rotundifolius (Gireere) StandL.] - SFE, W\&W

\section{Oxybaphus (see Mirabilis)}

Tripterocalyx carnea (Greene) L. A. Galloway var, wootonii (Standl.) L. A. Gallaway -KTZ, W\&W, W

[Tripterocalyx caneus (Greone) L. A. Galloway var, woctorti (Standl.) L. A. Gallaway] - SFW. UTF. WR.'

[Tripterocalyx wootoni Standl.] - $\mathrm{HDH}$

Tripterocalyx micranthus (Torr.) Hook. - Dom01, GPF, HDH, KTZ, SF!, UTF, W\&W, ENW

\section{Nymphaeaceae - NYM}

Nuphar polysepala Engelm. - Dorn0I, FNA, E/W

[Nuphar lutea (L.) Sm. subsp. polysepala (Engelm.) E. O. Besl] - KTZ

[Nuphar lutea Sibch. \& Sm. subsp. polysepala (Engelm.) E. O. Beal] - SF!, W\&W

[Nuphar polysepalum Engelm_] - HDH, UTF

\$Nymphaea odorata Aiton var. odorata

[Nymphaea odorata Aiton] - GPF, UTF

[Nymphaca odorata Aitoll subsp, odorata] - FNA, KT2

[Nymphaea odonata Sol.] - WeW

\section{Oleaceac - OL.E.}

Forestiera puhescens Nutt, var- pubescens - KTZ, W

[Forestiera neomexicana A. Gray] - W], HDH, IMI

[Forestiera pubescens Nutt.] - GPF, SFW, UTF, W\&W

\{Fraxinus americana L. - GPF, IMF, KTZ, UTF, W\& W

[W] - no evidenee that it occurs out of cultivation?

\{Fraxinus americanus L. - $-\mathrm{HDH}$

Fraxinus anomala Torr. ex S. Watson - HDH. IMF, UTF, W\&W, W

[Fraxinus anomala Toxr.] - SFW

[Fraxinus anomala Toct, ex S. Watson var, anomala] - KTZ

\$Fraxinus pennsylvanica Marshall - Dorn01, GPF, HDH, IMF, KTZ, E

[Fraxinus pernsylvanica Marshall var, lanceclata Sarg.] - HDH

[Fraxinus pennsylvanica Marshall var, lanceolata (Borkh.) Sarg.] - SFE, UTF, W\&W

*Ligustrum vulgare L. - GPF, IMF, KIZ $<$ NE, UT>, UTF

[W] - cultivated and escoping]

Menodora scabra A. Gray - IMF, KTZ. UTF, E

[Menodora scabra (Engelm.) A. Gray] - HDH, SFE, W\&W

*Syringa vulgaris L. - GPF, Hartman06, IMF, KTZ $<$ KS, UT>, UTF

\section{Onagraceac - ONA}


[NS - Includes changes from Wagner07; primarily the merging of Calyiophus, Gmara, and Stenosiphow into Denothera, and the splitting of Camissonia into several segregate genera.]

(Identification sources:

Calylophus (see Oenothera)

\{Calylophus berlandieri Spach subsp. berlandieri\} - GPF, KTZ, E

¿Calylophus berlandieri Spach) - SFE, W\&W

iWJ-presence in CO based on report by Towner (Ann. Mo. Bot. Gard. 64: 48-120 [1977]), who cited a collection of Brenckle at SMU (Brenckle 18124$) 12 \mathrm{mi}$ S of Triniesd. This is a specics of the Texas plains and athervise oceurs no closer than Hartey Co., TX at ca. 3900 f elevation. The cited locality 12 mi S of Trindad (CO) would be near the summit of Raton Pass at $7000 \mathrm{~A}$. This site is out of range, habitat, and elevation. There is no evidence Brenckle ever collected in CO. The specimen probably was taken near Trinidad, TX, ca $50 \mathrm{mi}$ $5 E$ of Dallas?

Camissonia (see also Holmgrenia, Chylisimia, Eremothera, Taraxia)

\{Camissonia chamaenerioides (A. Gray) P. H. Raven\} - IMF,KTZ $\angle A Z, N M$, UT>. UTF

\{Oenothera chamaenerioides A. Gray\} - HDH, exp.

\{Camissonia multijuga (S, Watson) P. H. Raven\}- IMF, KTZ $\angle A Z, U T$, UTF, W\&W, out of range

\{Oenothera mulijuga S. Watson var. multijuga\} - $\mathrm{HDH}$

\{Oenothera mulhijuga S. Watson var, orientalis Munz) - $\mathrm{HDH}$

Camissonia parvula (Nutt. ex Torr, \& A. Gray) P. H. Raven-Dorn01, IMF, KTZ, W\&W, W, Wagner07

[Camissunia parvula (Nutt.) P. H, Raven] - SFW, UTF

Chamerion angustifolium (L.) Holub var, canescens (A. W. Wood) N. H. Holmgren \& P. K. Holmgren - Dorn01. IMF, E/W

[Chamerion angustifolium (L.) Holub subsp, circum vagum (Mosu in) Kartesz] - KTZ

[Chsmerion dasielsii D. LOve] - SFW, W \& W

[Chamerion danielsit (Dankels) D. Lōve] - SFE

[Epilobium angustifolium L. subsp. cireunvagum Mosquin] - GPF

[Epilobium angustifolium L. vaf, canescens A. W. Wood] - UTF

[Epilobium angustifolium L. var, platyphyllum (Deniels) Fernald - $\mathrm{HDH}$

Chamerion latifolium (L.) Holub - Dorn01, IMF, KTZ, Raven72, E/W

[Clumerion subdentarum (Rydb.) A. Lave \& D. Love] - SFT, W\& W

[Epilobium latifoliam L.] - HDH, UTF

Chylismia eastwoodiae (Munz) W. L. Wagner \& Hoch - Wagner07

[Camissonia easrweodiae (Munz) P. H. Raven - IMF, KTZ, SFW, UTF, W\&W, W

[Oenodhera eastwocdiae (Munz) P. H. Raven - KTZ

[Oenochera scopoidea Nurt, ex Torr. \& A. Gray var, easrwoodice Munz] - HDH

Chylismia scapoidea (Torr. \& A. Gray) Small subsp. seapoidea - Wagner07

[Camissonia scapoidea (Tor, \& A. Gray) P. H. Raven] - Dornol, SFW, W\& W

[Canaissonia scapoidea (Nutt. ex Toxr, \& A. Gray) P. H. Raven subsp, scapoidea] - KTZ

[Camissonia scapoidea (Tor, \& \&. Gray) P. H. Raven var, scapoidea - IMF, UTF, W

[Oenechera scopoidea Nurt, ex Torr, \& A. Gray var, seopoidea] - HDH

Chylisimia walkeri (A. Nelson) - Wagner07

[Camissonia walkeri (A. Netson) P. H. Raven subsp, walkeri] - KTZ, W

[Camissonia walkeri (A. Nelson) P. H. Raven] - IMF, SFW, UTF, WRW

Circaca alpina L. var. alpina - Dom01 
[Circaea alpina L.] - HDH

[Circasa alpina L. subsp. alpina] - GPF, KTZ, W\&W, W\&W ${ }^{4}$, European

Circaea alpina L. var. pacifica (Asch, \& Magnus) M. E. Jones - Dorn01, IMF, UTF, EW

[Circava alpina L. subsp. pacifica (Asch. \& Magnus) P. H. Raven] - KTZ, SF!, W\&W

[Cirsaea pocifica Asch. \& Magners] - HDH

Epilobium

(Identification sources:

Epilobium (see also Chamerion)

Epilobium anagallidifolium Lam, - Dom01, KTZ, SF!, W\&W, E/W

[Epilobium alpinum L.] - $\mathrm{HDH}$

[Epilobium alpinum L. var. alpinam] - [MF, UTF

Epilobium brachycarpum C. Prest - Dom01, IMF, KTZ, SF!, UTF, W\&W, E/W

[Epilobium paniculatum Nutt. ex Tort. \& A. Gray] - GPF, HDH

(Epilobium canum (Greene) P. H. Raven var, garrettii (A. Nelson) N. H. Holmgren \& P. K. Holmgren? - IMF

\{Epilobiam canum (Greene) P. H. Raven subsp. garrettii (A. Nelson) P. H. Raven\} - KTZ

\{Zauschneria garretii A. Nelson\}- $\mathrm{HDH}$, exp.

[Zauschneria latifolia (Hook.] Greene var. garrettii (A. Nelson) Hilend\} - UTF

Epilobium ciliatum Raf, var. ciliatum - Dorn01, IMF, E/W

[Epilobium brevistylum Barbey] - HDH, W\&W

[Epilobium ciliatum Raf.]- $\mathrm{HDH}$, exp., SFI, UTF

[Epilobium ciliatum Raf. subsp, ciliatum] - GPF, KTZ,

Epilobium ciliatum Raf, var. glandulosum (Lehm.) Dom - Dom01, IMF, E/W

[Epilobium adenocaulon Haussk. var. adenocauloa] - HDH

[Epilobium adenocaulon Haussk. var. occidentale Trel.] - HDH

[Epilobium ciliarum Raf.] - SF!

[Epilobium ciliarum Raf. subsp. glandalosum (Lehm.) Hoch \& P. H. Raven] - GPF, KTZ. W\&W

[Epilobium glandulasum Lehmann]- $\mathrm{HDH}$, exp., UTF

[Epilobium occidentale (Trel.) Rydb.]

Epilobium clavatum Trel. - Dorn01, HDH, KTZ, SF!, W\&W, E/W

[Epilobium alpinum L var, clasaum (Trel.) C. L. Hitche.] - IMF, UTF

Epilobium halleanum Haussk. - Dom01, GPF, HDH, IMF, KTZ SF!, UTF. W\&W, E/W

Epilobium hornemannii Rchb. subsp. hornemannii - KTZ, E/W

[Epilobium bomemannii Haussk.] - SFW

[Epilobium Bornemannii Rchb.] - Dom01, GPF, HDH, SFE, W\&W

[Epilobium bornemannii Rchb, var, hornemennii] - IMF, UTF

Epilobium lactiflorum Haussk, - Dom01, HDH, KTZ, SF!, W\&W, E/W

[Epilobium bornemannii Rechb, var. lactiflorum (Hachsk,) D. LWve] - IMF, UTF

Epilobium leptocarpum Haussk. - KTZ, SF!, E/W

[Epilobium leptocarpum Haussk, var, macounil TreL] - W \&W, HDH

Epilobium leptophyllum Raf. - GPF, HDH, IMF, KTZ, SF!, UTF, W\&W, E/W

[Epilobium palusire L var, gracile (Fanw, Dona] - RMC

\{Epilobium minutum Lindl. ex Lehm.\} - KTZ

\{Epilobiam minutum Lindl, ex Hook, - $\mathrm{HDH}_{\text {, exp. }}$

\{Epilobiam minutum Lindl.; - IMF

Epilobium oregonense Haussk. - Dorn01, IMF, KTZ

Epilobium palustre L. var. gracile (Fanw.) Dorn - Dorn01

Epilobium palustre L. var. palustre - Dorn01, E

[Epilobium palustre L] - GPF, IMF, KTZ

Snow, N, Janury 2009. Checklist of Vacculer Plants of the Southum Rodky Momain Region. CVersion $3 x$ 
[Epilobium palustre L var, grammadophyllum Haussk.] - HDH, SFE, W\&W

Epilobium pygmaeum (Spreg.) Hoch \& Raven - Dom01

[Boisduvalia glabella (Nutt.) Walp.] - KTZ

[Boisduvalia glabella (Nutt.) var. campestris (Jepson) Jepson] -KTZ

[Boisduvalia pygmes (Spreg.) Munz] - K.TZ

[Oenothera glabella Nutt.] - KTZ

Epilobium saximontanum Haussk, - Dorn01, GPF, HDH, IMF, KTZ, SF!, UTF, W\&W, ENW

Eremothera minor (A. Nelson) W. L. Wagner \& Hoch - Wagner07

[Camissonia minor (A. Nelson) P. H. Raven] = Dorn01, IMF, KTZ, SFW, UTF, W\&W, W

[Oenothera minor (A. Nelson) Munz] - HDH, exp.

\section{Gaura (see Oenothera)}

Gayophytum decipiens F. H. Lewis \& Szweyk - Dorn01, IMF, KTZ, SF!, rep, UTF, W\&W

Gayophytum diffusum Torr. \& A. Gray var, strictipes (Hook.) Dorn - Dorn01, IMF, E/W

[Gayophytum diffusum Torr. \& A. Gray] - UTF

[Gayophytum diffusum Torr. \& A. Gray subsp. parviflorum F. H. Lewis \& Sxweyk.] - GPF. KTZ, SF!, W\& W

[Gayophytum lasiospermum Greene] - UTF

[Gayephytum lasiospermum Greene var. lasiospermum] - HDH, UTF

[Gayophytum nuttallii Torr. \& A. Grsy]-HDH

Gayophytum racemosum Torr, \& A. Gray - Dom01, GPF, IMF, KTZ, SF!, UTF, W\&W, ENW

[Gayephytum helleri Rydb. var. glabrum Munc] - HDH

[Gayophytum raccmosum Torr. \& A. Gray var. racemosum] - HDH

Gayophytum ramosissimum Torr, \& A. Gray - Dorn01, HDH, IMF, KTZ, SF!, UTF, W\&W, E)W

Holmgrenia andina (Nutt.) - Wagner07

[Camissonia andina (Nutt.) P. H. Ravea] - Dorn01, IMF, KTZ, SFW, UTF, W \& W, W

[Oenothera andina Nutt. ex Torr. \& A. Gray]- HDH, exp.

Oenothera (see also Calylophus, Camissonia)

(Identification sources:

Oenothea acutissima W. L. Wagner-IMF, KTZ,SFW, W\&W, W

[Oencthera flava (A. Nelson) Garrent var. asatissima (W. L. Wagner) S. L. Welsh] - UTF

Oenothera albicaulis Pursh - Dorn01, GPF, HDH, IMF, KTZ, SF!, UTF, W\&W, E'W

Oenothera canescens Torr. \& Frém. GPF, HDH, KTZ, SFE, W\&W, E

\{Oenothera cavemae Munz\} -IMF, KTZ $<A Z$, UT>, UTF; Wagner85, err. rep.

Oenothera cespitosa Nutt. var. cespitosa - Dorn01, E/W

[Oenothera chespinosa Nutt. subsp. Cespitosa] - GPF, SF!

[Oenochera cuespinosa Nutt, ex Fraser subsp, caespitosa] - W\& W

[Oenothera chespitosa Nutt. var, caspitosa] - IMF, UTF

[Oenothera chespitosa Nutt. ex Fraser var, caespitosa] - HDH, exp.

[Oenothera caespitosa Nutt. ex Fraser var, montana (Nutt.) T. Durand] - HDH

[Oenothera cespitosa Nutt. subsp. ocspitosa] - KTZ

\{Oenothera cespitosa Nutt. var. crinita (Rydb.) Munz\}

\{Oenotherz caespitosn Nutt, var, crinita (Rydb.) Munz\} - IMF, UTF

\{Oenothera caespitosa Nutt, ex Fraser var, jonesii Munz\} - HDH, exp.

[Oenothera cespitosa Nutt. sabsp, crinita (Kydb.) Munz) - KTZ $\angle A Z$, UT $>$

Snou, N, Janury 2009. Checklist of Vascular Plants of tho Southom Rocky Moamain Region. CVersion $3 x$ 
Oenothera cespitosa Nutt, var, macroglottis (Rydb.) Cronquist - Dom01, E/W

[Oenothera cacspitosa Nutt. subsp. macroglotis (Rydb.) W. L. Wagner] - SFE

[Oenothera casspitosa Nutt. subsp. macroglott is (Rydb.) W. L. Wagner, Stockh., \& W. M. Klein] - GPF, SFW

[Oenethera casspitesa Nutt. var. masroglottis (Rydb.) Cronquist] - IMF, UTF

[Oenothera cacspitosa Nutt. ex Fraser subsp. masioglottis (Rydb.) W. L. Wagner, Stockh. \& W. M. Klein] WAW

[Oenothera vespitusa NutL. subsp. macroglottis (Rydb.) W. L. Wagner] - KTZ

Oenothera cespitosa Nutt, var, marginata (Nutt. ex Hook. \& Am.) Munz - Dorn01, W

[Oenothera casspitesa Nutt. var, marginata (NutL) Munz] - UTF

[Oenochera casspitosa NutL. var, marginata (Nutt. ex Hook \& Am.) Murz] - IMF

[Oenethera casspitosa Nutt. subsp. marginata (Nutt.) W. L. Wagner, Stockh_, \& W. M. Klein] - SFW W\&W

[Oenethera casspitesa Nutt. ex Fraser subsp. marginata (Nutt.) W. L. Wagner, Stockh., \& W. M. Kle in] -

[Oenochera casspitosa Nutt. ex Freser yar. eximia (A. Gray) Muna] - HDH

[Oenechera casspitosa Nutt. ex F faser var. marginata (NuIL) Munz] - HDH

[Oenothera cespitosa Nutt. subsp. marginata (Nutt. ex Hook \& Am.) Munz] - KTZ

Oenothera cespitosa Nutt, var, navajoensis (W. L. Wagner, Stockh., \& W. M. Klein) Cronquist - W

[Oenethera casspitesa Nutt. var. navajoensis (W. L. Wagner, S4ockh., \& W. M. Klein) Cronquist] - IMF, UTF [Oenothera casspitosa Nutt. subsp. navajoensis W. L. Wagner, Stockh., \& W. M. Klein] - SFW

[Oenethera cacspitosa Nutt ex Fraser subsp. navajoensis W. L. Wagner, Stockh., \& W. M. Klein] - W\& W

[Oenothera cespitosa Nurt subsp. navajoensis W. L. Wagner, Stockh., \& W. M. Klein] - KTZ

Oenothera cinerea (Woot. \& Standley) W. L. Wagner \& Hoch susp. cinerea - Wagner07

[Gaura villosa Torr.]-SFE, W\&: W

[Gaura villosa Torr. subsp. villosa] - GPF, KTZ

[Gaura villosa Torr, var, villesa - $\mathrm{HDH}, \mathrm{E}$

Oenothera cinerea (Woot, \& Standley) W. L. Wagner \& Hoch susp. parskii - Wagner07

[Guara villosa Torr. subps, parksii (Munz) P. H. Raven \& Gregory] - KTZ

[Guara villosa Torr, var; parskii Munz] - KTZ

Oeonthera coloradoensis Rydb. subsp, colodoensis - Wagner07

[Gaura neoxnexicana Wooton] - SFW

[Gaura ncomexicana Wooton subsp. scomexicana] - KTZ, SFW, WRW

[Gaum neomexicana Woosn var, neomexicana] - HDH, W

Oeonthera coloradoensis Rydb. subsp. neomexicana (Woot.) W. L. Wagner \& Hoch - Wagner07 [Gaura ncomexicana Wooton] - SFW

[Gaura neomexicana Wooton subsp, coloradensis (Rydb.) P. H. Raven \& D. P. Gree]-GPF, KTZ, SFE, W\&W

[Gaura nenuexicana Wooson var. coloradensis (Rydb) Manz] - Dornt1, HDH, E

Oenothera coronopifolia Torr, \& A. Gray - Dom01, GPF, HDH, IMF, KTZ, SF!, W\&W, E/W

Oenothera curtiflora W, L. Wagner \& Hoch - Wagner07

[Gaura mollis E. James] - KTZ, SF!, W\& W

[Gaura parvifloea Douglas] - GPF

[Gaura parviflora Douglas ex Lehm.] - Dom01, IMF, UTF, W\&W, EW

[Gaura parvifloea Douglas ex Hook. var, lachnocarpa Weatherby] - $\mathrm{HDH}$

[Gaura parviflora Douglas ex Hook, var, parviflora] - $\mathrm{HDH}$

[Gaura parviflon Douglas ex Hook, forma glabra Munz] - $\mathrm{HDH}$

Oenothera clata Kunth var. hirsutissima (A. Gray ex S. Watson) Cronquist - IMF. E/W

[Oenochera clata Kunth] - UTF

[Oenothera elata Kunth subsp, hirsutissima (A. Gray) W, Dietr. \& W. L.. Wasner] - SF!

[Oenothera elata Kunth subsp, hirsutissima (A. Gray ex S, Wassoe) W. Dietr.] - GPF, KTZ

[Oenothera elata Kunth subsp, hirsutissima (A. Gray ex S. Watson) W. Dietr. \& W. L. Wagner] - W. W

[Oenochera hookeri Tor. \& A. Gray subsp, angustifolia (Gates) Manz]- HDH

[Oenothera hookeri Tor. \& A. Gray subsp hewettii Cockereli] - HDH

[Oenothera hookeri Tor, \& A. Gray subsp. hirsutissinea (A. Gray) Manz] - HDH

Oenothera engelmannii (Small) Munz - KWA, M\&H, KTZ, GPF, HDH 
[Anogra engelmannii (Small) Woot. \& Stand.] = KWA, MEH, JM, not rep.

Oenothera filiformis (Small) W. L. Wagner \& Hoch - Wagner0?

[Gaura longiflera Spach] - GPE, KTZ

[Gaura filiformis Small] - KTZ

[Gaura pitcheri (Torr. \& A. Gray) Small]- KTZ

Oenothera flava (A. Nelson) Garrett - Dom01, GPF, HDH, IMF, SFE. W\&W, E/W

[Oenothera Gava (A. Nelson) Garrett subsp. flava] - KTZ

[Oenothera flava ( $A$. Nelson) Garrett var- flava] - UTF

[Oenothera tlava (A. Nelson) Munz] - SFW

Oenothea glaucifolia W. L. Wagner \& Hoch - Wagner07

[Stenusiphon linifolius (Nutt, ex E. James) Hoynh.] - KTZ, SFE, W\& W ${ }^{1}, E$

[Stenosiphon linifolium (Nutt.) Brittoa) - HDH, exp.

[Stenosiphon linifolius (Nutt.) Heynh.] - GPF, W\&W

Oenothera glazoviana Micheli - FCF

[Oenothera erythrosepata Borbas] - KTZ

Oenothera grandis (Britton) Smyth - GPF, KTZ, SFE, W\&W, E

$\bullet$ Oenothera harringtonii W. L. Wagner-ENDEMIC $<C O>$. KTZ, E

[Oenothera harringtonii W. L. Wagner, Stockh. \& W. M. Klein] - GPF. SFE, W\&W

Oenothera hartwegii (Benth.) P. H. Raven subsp. fendleri (A. Gray) Towner \& P H. Raven Wagner07

[Calylophus bartwegii (Benth.) P. H. Raven subsp. fendleri (A. Gray) Terener \& P. H. Ravea] - GPF, KTZ

Oenothera hartwegii (Benth.) P. H. Raven subsp. pubescens (A. Gray) Towner \& P. H. RavenWagner07

[Calylophus bartwegi (Benth.) P. H. Raven subsp. pubescens (A. Gray) Towner \& P. H. Raven] - GPF. KTZW\&W, E, SFE

[Oenothera greggii A. Gray var. hampasana (Bockll.) Munz] - HDH

Oenothera howardii (A. Nelson) W, L. Wagner - Dorn01, GPF, IMF, KTZ, SFE, UTF, W\&W, E [Oenothera brachycarpa A. Gray var, wrightii (A. Gray) Léveillk]-HDH. WeW

\{Oenothera jamesii Torr. \& A. Gray\} - GPF, HDH rep.. KTZ $\angle \mathrm{KS}, \mathrm{OK}>$, W\&W

$\div$ \{Oenothera kleinii W. L. Wagner \& S. W. Mill.\} -SFW, W\&W, W

[NS - To be reduced to synonymy of Oenothera delhoides Torr. \& Frem, var, deltoides by

W. L. Wagner (pers, comm., Nov, 2003)]

Oenothera laciniata Hill - KWA, KIZ

[Oenochera laciniata Hill var, mexicana Smalt] - KWA, M\&H

[Oenothera mexicana Spach] - KWA, M\&KH

[Raimannia laciniata (Hill) Rose] - KTZ

[Raimannia mexicana (Spach) Woot. \& Standl.] - KWA, M\&H

Oenothera latifolia (Rydb.) Munz - Dorn01, GPF, HDH, KTZ, SFE, W\&W, E

[Oenothera pallida Lindl, subsp. pallida var, lanifolla Rydb.] - IMF

Oenothera lavandulifolia (Torr. \& A. Gray) P. H. Raven - Wagner07

[Calylophas lavandalifolius (Torr, \& A. Gray) P. H. Raven]-Dorno1, KTZ, SF1, WRW, EN

[Oenechera lavandulifolia Torr, \& A. Gray var, glasdulosa Munz] - HDH

[Oenothera lavandulifolia Torr, \& A. Gray var. lavandulifolia] - HDH

Oenothera longissima Rydb. - HDH, IMF, KTZ, SFW, UTF, W\&W, W

Oenothera nuttallii Sweet - Dorn01. GPF, HDH, KTZ, SFE, W\&W, E

Oenothera pallida Lindl. var. pallida - Dom01. HDH, UTF, W

[Oenothera pallida L.indl.] - SFW

[Oenechera pallida Lindt, suhsp, pallida] - KTZ, W\&W

[Oenochera pallida Lindt, subsp, pallida var, pallida] - IMF

Oenothera pallida Lindl. var. runcinata (Engelm.) Cronquist - E

[Oenothera pallida Lindt, subsp, pallida var, nancinata (Engelm.) Cronq.] - IMF

[Oenethera pallida Lindl. suhsp. runcinata (Engelm.) Munz \& W. Klein] - KTZ, SFE, W\&W

[Oenochera pallida Lindt, var, pallida] - UTF

Snow, N. Junury 2009. Checklist of Vascular Plants of the Southom Rocky Mounain Region. CVersion $3 x$ 
Oenothera pallida Lindl, var, trichocalyx (Nutt.) Dom - Dorn01, UTF, W

[Oenethera pallida Lindt. subsp. trichocalyx (Nutt.) Munz \& W. KIein] - IMF, KTZ, SFW

[Oenothera pallida Lindl. subsp. trichocalyx (Nutt. ex Torr. \& A. Gray) Munz \& W. Klein] - W\&W

[Oenethera trichocalx Nutt. ex Torr. \& A. Gray] - HDH

\{Oenothera primiveris A. Gray\} - HDH, exp.

\{Oenothera primiveris A. Gray subsp. bufonis (M. E. Jones) Munz\}, KTZ $\subset A Z$, UT:

[Oenothera primiveris A. Gray subsp. primiveris\} - KTZ $\subset A Z$. NM

Oenothera pubescens Willd, ex Sprengel - KWA, KTZ

[Angora amplexicsalis (Woot. \& Standi.) Tidestr.] - KWA, M\&H

[Oenothera amplexicaulis (Wout. \& Standl.) Tidestr.] - M\&H

[Oenothera laxiniata subsp. pubsscens (Willd. ex Sprengel) Munz] - KWA, M\&H. KTZ

[Oenothera laciniata var. pubescens (Willd. ex Sprengel) Munz] - KWA, M\&H, KTZ

Oenothera rosea L'Her. - KWA, M\&H

[Hartmannia fosea (Ailon) G. Don] - KWA, JM, KTZ

Oenothera serrulata Nutt, var, serrulata - HDH, Wagner0?

[Calylophus australis Tawner \& P. H. Raven] - K.TZ

[Calylophus serrulatus (Nutt.) P. H. Raven] - Dorn01, GPF, KTZ, SFE, W\& W, E

[Meriolix oblanceolata Rydb.] - KTZ

[Meriolix serrulata (NutL.) Walp.] - KTZ

Oenothera suffretescens (Ser.) W. L. Wagner \& Hoch - Wagner07

[Gaura coccibsa (Nutt) Pursh - Dom01, E/W

[Gaura coccinea Pursh] - GPF, IMF, UTF

[Gaura coccinca Nutt.] - SF!

[Gaura coccinsa Nutt, ex Pursh] - KTZ, WEW

[Gaura coccibsa Nutt, ex Pursh var, coccines] - HDH

[Gaura coccibsa Nutt, ex Pursh var, glabra (Lehmarin) Torr. \& A. Gray] - HDH

[Gaura coccioca Nuth. ex. Pursh var. parvitolia (Torr.) Torr, \& A. Gray]-HDH

Oenothera villosa Thunb, var, strigosa (Rydb.) Dom - Dom01, IMF, KTZ, E/W

[Oenochera bientis L. var, strigosa Rydb.] - UTF

[Oenothera strigosa (Rydo.) Mack. \& Bush] - HDH

[Oenothera villosa Thumb.] - SF]

[Oenothera villosa Thunb, subsp. strigosa (Rydb.) W. Dietr. \& P. H. Raven - GPF, KTZ, W\& W

[Oenochera villosa Thunb. subsp. villosa] - KTZ

Oenothera villosa Thunb, var, villosa - Dorn01, KTZ

\section{Stenosiphon (see Oenothera)}

Taraxia breviflora (Torr. \& A. Gray) Nutt, ex Small - Wagner07

[Camissonia breviflora (Torr. \& A. Gray) P. H. Raven] - Dom01, IMF, KTZ. SFE, UTF, W\&W, E

[Oenochera contorta Douglas ex Hook.]-HDH

Taraxia subacaulis (Pursh) Rydb. - Wagner07

[Camissonia subacautis (Pursh) P. H. Ravell - Dom01, IMF, KTZ, SF], UTF, W\&W, E/W

[Jussiaea subacaulis Pursh] - KTZ

[Oenothera heterancha Nutt.]-HDH, KTZ

[Oenothera stbacnulis (Pursh) Garrett] - KT7

\section{Zauschneria (seeEpilobium)}

\section{Orchidaceae - ORC (including Cypripediaceae)}

(ldentification sources: 


\section{Amesia (see Epipactis)}

\section{Bifolium (see Listera)}

Calypso bulbosa (L.) Oakes var, americana (R. Br.) Luer - FNA, GPF, E/W

[Calypso amerieana R. Br.] - FNA

[Calypso balbosa (L.) Oakes] - Dorn01, HDH, [MF, SF!, UTF, W\&W

[Calypso bulbosa (L.) Oakes var. americana (R. Br. ex W. T. Aiton) Luer] - KTZ

Coeloglossum viride (L.) Hartm. - FNA

[Coeloglossum viride (L.) Hartm. var. virescens fMull. ex Willd.) Luer] - KTZ, E/W

[Coeloglossum viride (L.) Hartm. subsp. bracteatum (MuhL) Hultén] - FNA. SF!

[Coeloglossum viride (L.) Hartm. subsp. bracteatum (MuhL. ex Willd.) Hultéa] - W\& W

[Coeloglossum viride (L.) Hartm. var. islardicum (Lindl.) M. Schulze] - FNA

[Coeloglossum viride (L.) Hartm. var. virescens (Multib.) Luer] - Dorn01. FNA

[Dactylorhiza viridis (L.) R. M Bateman, Pridgeon \& M. W. Chase] - FNA

[Habenaria brasteata (Willd.) R. Br.] - FNA, HDH

[Habenaria viridis (L.) R. Br.] - FNA.

[Habenaria viridis (L.) R. Br. subsp. bracteata (Muhl.) Clausen] - FNA

[Habenaria viridis (L.) R. Br, var, bracteata (MuhL.) A. Gray]-FNA, GPF

[Habenaria viridis (L.) R. Be, var, bracteala (Mubl.) Rehb. ex. A. Gray] - UTF

[Habenaria viridis (L.) R. Br. var. bracteata (Muhl. ex Willd.) Rehb. ex A. Gray] - IMF

[Habenaria viridis (L.) R. Br, var, inerjecta Fenald] - FNA

[Satyrium vinide L.] - FNA

Corallorhiza maculata (Raf.) Raf. - E/W

[Corallorhiza maculata Raf.]-HDH. IMF, SF!, UTF, W\& W

[Corallorhiza maculata (Raf.) Raf.] - GPF

[Corallorhiza maculata (Raf.) Raf. var. maculala] - Dorn01, FNA. KTZ

[Corallorhiza maculata (Raf.) Raf. var. oceidentalis (Lindl.) Ames] - Donr01. FNA. KTZ

Corallorhiza striata Lindl, - FNA. E/W

[Corallonhiza striata LindL] - IMF, SF1, UTF, w\&w

[Corallochiza striata Liedl. var. ochroleuca (Rydb.) Magrath] - GPF

[Corallorhiza striata Liedl. var, siriata] - Dom01, KTZ

[Corallonhiza striata Lindl. var, vreelandia (Rydo.) L. O. Williants] - Dorn01, GPF, HDH

Corallorhiza trifida Châtel - Dorn01, FNA, KTZ, E/W

[Coratlorhiza corallorhiza (L.) MacMillan] - FNA

[Corallorhiza coral lorhiza (L.) MacMillan var. coloradens is Cockereli] - FNA

[Corallorhiza innata R. Br.] - FNA

[Corallothiza innata var, virescens Farr] - FNA

[Corallorhiza trifida (L.) Chătel.|]-GPF, HDH, IMF, UTE, W\&.W

[Corallorhiza tritida Chatel.] - SF:

[Corallowhiza trifida Chatrel. var. verna (Nutt.] Fernald] - FNA

[Corallothiza vema Nut.] - FNA

Corallorhiza wisteriana Conrad - Dorn01, FNA, KTZ, E/W

[Corallochiza bortensis Sacksodor] - FNA

[Corallorhiza wisteriana Conrad] - GPF, HDH, IMF, SFI, UTF, WRW

Cypripedium fasciculatum Kellogg ex S. Watson - Dorn01, FNA, HDH, IMF, KTZ, W\&W, EW

[Cypripedium fasciculatum Kellogg] - SFt

[Cypripediam knightiac A. Nels..] - FNA

Cypripedium parviflorum Salisb. var, pubeseens O. W. Knight - Dorn01, FNA, E/W

[Cypripedium calceolus L] - UTF

[Cypripediam calceolus L. subsp. parviflorum (Salisb.) Hultén] - SF!, WEW 
[Cypripedium calceolus L. var. planipetalum (Fernald) Victwrin \& J. Rousseau] - FNA

[Cypripedium calceolus L. var. pubescens (Willd.) Correll] - FNA, GPF, HDH, IMF

[Cypripedium flavescens DC.] - FNA

[Cypripedium parviflorum Salisb, var. makasin (Farw.) Sheviak] - FNA, KTZ

[Cypripedium parviflorum Salisb. var. planipetalum Femald] - FNA

[Cypripedium parviflorum Salisb var. pubscens (Willd.) O. W. Knight] - KTZ

[Cypripedium pubescers Willd_] - FNA

[Cypripedium veganum Cockerell, P. Barker \& M. Barker] - FNA

\section{Dactylorhiza (see Coeloglossum)}

\section{Diphryllum (see Listera)}

\section{Distomaea (see Listera)}

\section{Epipactis (see also Listera)}

Epipactis gigantea Douglas ex Hook, - Dorn0I, FNA, GPF, HDH, IMF, KTZ, UTF, W\&W, E/W

[Amesia gigantea (Douglas ex Hook.) A. Nelson \& J.F. MacBride] - FNA

[Epipactis gigantea Douglas] - SF!

[Helliborine gigantea (Douglas ex Hook.) Druce] - FNA

*Epipactis helleborine L. - W\&W', E

[W] - appeared sponcaneously in flower beds in fronc yard on Table Mesa. No evidence of spreading]

[Epipactis helleborine (L.) Crantz] - KTZ, SFE

[Epipactis latifolia (L.) Allioni] - FNA

[Serapias helleborine L.] - FNA

Goodyera oblongifolia Raf, - Dom01, FNA, GPF, HDH, IMF, KTZ, SF!, UTF, W\&W, E/W

[Goodyera decipiens (Hook.) F. T. Hubbard] - FNA

[Goodyera oblongifolis var. reticulata B. Boivin] - FNA

[Peramium decipiens (Hook.) Piper] - FNA

Goodyera repens (L.) R. Br. ex W. T. Aiton - KT7., E/W

[Goodyera repens (L.) R. Br. in W. Aiton and W. T. Aison - FNA

[Goodyera repens (L.) R. Br.] - GPF. HDH. W\&W

[Goodyera repens (L.) R. Br. subsp. fides (Ferraild) A Löw \& simon] - SF:

[Goodyera repens (L.) R. Br. var. ophioides Femald] - FNA

[Peramium ophioides (Fernalid) Rydb.] - FNA

[Saryrium repens L.] - FNA

Gyrostachys (see Spiranthes)

Habenaria (see Coeloglossum, Piperia, Platanthera)

Helleborine (sec Epipactis, Listera)

Ibidium (see Spiranthes)

\section{Limnorchis (sec Platanthera)}

Listera borealis Morong - Dorn01, FNA, HDH rep., IMF, KTZ, SF!, UTF, W\&W, ENW

[Neottia borealis (Morong) Szlachetko] - FNA

[Ophrys borealis (Morong) Rydh] - FNA

Listera convallarioides (Sw.) Elliot - FNA 
[Bifolium convallarioides (Sw.) Nieunsland] - FNA

[Diphryllum convallarioides (Sw.) Kuntze] - FNA

[Disiomeaca avata (L.) Spenner] - FNA

[Epipactis conval larioides Sw.] - FNA

[Listera convallarioides (Sw.) Nutt. ex Elliott] - Dorn01, KTZ. EW

[Listera convallarioides Torr-] - SFW

[Listera convallarioides (Sw.) Nutt.] - GPF, HDH, SFE, W\& W

[Listera convallarioides (Sw.) Torr.] - IMF, UTF

[Listera eschscholziana Chamisse]-FNA

[Ophrys convallarioides (Sw.) W. Wight ex House] - FNA

Listera cordata (L.) R. Br, ex W. T. Aiton var, nephrophylla (Rydb.) Hultén - FNA, KTZ

[Listera cordata (L.) R. Br.] - Dorn0I, HDH, IMF, UTF, EW

[Listera cordata (L.) R. Br. subsp, ncphrophylla (Rydb.) A. Love \& D. Lave] - SF!. W\&W

[Listera nephroptrylla Rydb.] - FNA

[Neotia nephrophylla (Rydb.) Szlachecko] - FNA

[Ophirys nophrophylla (Rydb.) Rydb.] - FNA

\section{Lysiella (see Platanthera)}

Malaxis brachypoda (A. Gray) Fernald - KTZ. E

[Malaxis monophyllos (A. Gray) Sol. ex Sw. subsp, brachypoda (A. Gray) A. Lôve \& D. Lôve] - w\& W

[Malaxis monophyllos (L.) Sol. subsp. brachypoda (A. Gray) A. LOVe \& D. Löw] - SFE, W\&W

[Malaxis monophyllos (L.) Sw. var. brachiypoda (A. Gray) Mortis \& Eames] - HDH

Malaxis porphyrea (Ridley) Kuntze - Coleman02

[NS - Ben Leglec fpers, comm. 2009] reornly collected this in Colfax Co, NM]

[Malaxis chrenbergit (Reichenbach f.) Kuncze] - W]

Malaxis soulei L. O. Williams - WJ

[Malaxis macrostachya (Lexarza) Kuntze] - W]; Coleman02

[WJ - oceurs in Colfax, Los Alamos, and Ssndoval cos., NM]

\section{Neottia (see Listera)}

\section{Ophrys (see Listera)}

\section{Orchis (see Platanthera)}

\section{Peramium (see Goodyera)}

Piperia unalascensis (Spreng.) Rydb, - Dom01, GPF, KTZ, SF!, W\&W, E/W

[Habenaria unalaseensis (Spreng) S. Watson] - HDH, IMF, UTF

Platanthera aquilonis Sheviak - FNA, Sheviak99, E/W

[Habenaria hyperborea (L.) R, Br.] - GPF, HDH, IMF, UTF

[Limnorchis hyperborea (L.) Fydb] - SF], WRW

[Platanthera gracilis Lindl.] - K.TZ

[Platanthera huronensis (Nutt,) Lindl.] - KTZ

[Platanthera hyperborea (L.) Lindi. var, ynacilis (Lindl.) Luer] - RMC

[Platanthera hyperborea (L.) Lisdl var, lyperborea] - KTZ

Platanthera dilatata (Pursh) Lindl. ex L. C. Beck var. albiflora (Cham.) Ledeb. - Dorn01, KTZ. E/V

[Habenaria dilatata (Pursh) Hook.] - FNA, HDH

[Habenaria dilarata (Pursb) Hook. var, albiflora (Cham.) Comell] - GPF, IMF, UTF

[Limnorchis dilatata (Pursh) kydb, subsp, albiflora (Cham,) A. Live \& Simon] - SF!, WEW 
[Orchis dilatata Pursti] - FNA

Platanthera dilatata (Pursh) Lindl. ex L. C. Beck var, dilatata - Dorn01, FNA, KTZ

[Habenaria dilatata (Pursh) Hook. var. dilatata] - GPF, IMF, UTF $\angle C O O$ -

Platanthera X estesii Schrenk - KTZ

[Platanthera dilatata X P. stricta] - KTZ

Platanthera huronensis (Nutt.) Lindley - Dorn01, FNA

[Orchis huronensis Nutt.] - FNA

[Habenaria hyperborea (L.). R. Br. var. huronensis] - FNA

[Habenaria X media (Ryob.) Niles] - FNA

[Limnorchis media Rydb.] - FNA

[Platanthera hyperborea (L.) Libdley var. huronensis (Nuat.) Luer] - FNA

[Platanthera X media (Rydb.) Luer] - FNA

Platanthera obtusata (Banks ex Pursh) Lindl. - Dorn01, FNA, KTZ. E/W

[Habenaria obtusata (Banks) Richards] - UTF

[Habenaria obtusata (Banks ex Pursh) Richards] - FNA, HDH, IMF

[Habenaria obtusata var, collectanea Fernald]-FNA

[Lysiella obtusata (Banks ex Pursh) Britton \& Rydb_] - W\&W

[Lysiella obtusata (Banks) Britton \& Rydh.] - SF!

[Orchis obtusata Ranks ex Pursh] - FNA

Platanthera purpurascens (Rydb.) Sheviak \& W. F. Jenn. - Dorn01, FNA, KTZ, Sheviak97, E/W

[Habenaria sactara Greene] - GPF, HDH, IMF, UTF

[Limnorchis stricta (Lindl.) Ryob.] - SF!, W\&W

[Limnorchis purpurascens Rydb.]-FNA

[Platanthera hyperborea (L.) Lindley var. purpurasesens (Rydb.) Luer) - FNA

[Platanthera stricta Lindl.] - KTZ

[Platunthera saccata (Greene) Hulten] - FCF

Platanthera sparsiflora (S. Watson) Schltr. var. ensifolia (Rydb.) Luer - KTZ, ENW

[Limnorchis ensifolia Rydb.] - SFL, W\&W

Platanthera sparsiflora (S, Watson) Schltr. - FNA

[Habenaria sparsiflora S. Watson var. laxiflora (Rydb.) Correli] - FNA, IMF, UTF

[Habenaria sparsiflora S. Watson] - FNA $<$ HDH. rep.

[Habenaria sparsiflora S. Watson var, sparsiflora] - MMF, UTF

[Limnorchis laxiflora Rydb.] - FNA

[Limnorchis sparsiffora (S. Wotson) Rydb.] - WE W, misapp.

[Plananthera sparsifora (S. Watsoa) var. ensifolia (Rydb.) Luer - FNA

[Platanthera sparsiflora (S. Watson) Schltr, var, sparsiflora] - KTZ $\langle A Z, N M$, UT $>$

Platanthera zothecina (L. C. Higgins \& S. I. Welsh) Kartesz \& Gandhi - FNA, KTZ, W

[Habenaria zothecina L. C. Higgins \& S. L. Wetsh] - FNA, UTF

[Limnorchis zothecina (L. C. Higgins \& S. L. Welsh) W. A. Weber]-SFW, W\&W

Satyrium (see Coeloglossum, Goodyera)

\{Spiranthes cernua (I.) Rich.\} - GPF, HDH, exp., IMF, KTZ <KS, NE, OK>

Spiranthes diluvialis Sheviak - Dorn01, FNA, KTZ, SF!, W\&W, E/W

[Spiranthes romanzofmana Cham, var, diluvialis (Slieviak) S, L. Welsh] - FNA, UTF

Spiranthes magnicamporum Sheviak - FNA, GPF, KTZ $<$ KS, NE, NM, OK $>$

[Spiranthes porrifolia Lindl.\} - HDH rep., KTZ, W\&W, misapp.

\{Spiranthes fomanzoffiana Cham. var, porrifolia (Lindl.) Ames \& Correll\} -IMF (UT and w.)

Spiranthes romanzoffiana Cham. - Dorn01, FNA, GPF, KTZ, SFE, W\&W, E/W

[Gyrostachys stricta Rydb] - FNA

[lbidium strictum (Rydb.) House] - FNA

[Spiranthes stricta (Rydb,) A. Nels] - FNA

[Spiranthes romanzoffiana Cham. \& Schl, - HDH, SFW

[Spiranthes romancoffiana Cham, var, romunzoffiana] - IMF, UTF 
\{Spiranthes vernalis Engelm. \& A. Gray\} - GPF, KTZ <KS, NE, OK>

[WJ - based an a Fendler specimen from e. Kansas]

\{Spiranthes vernalis Engelm.; - HDH, exp.

\section{Orobanchaceae - ORO}

(Identification sources:

\{Agalinis aspera (Douglas ex Benth.) Britton\}-WJ, GPF, exp., KTZ $<$ KS, NE, OK>, W\&W

\{Gerardia aspera Douglas ex Benth.\} - HDH, exp.

Agalinis tenuifolia (Vahl) Raf, var, parviflora (Nutt) Pennell - Dom01, GPF, KTZ, E

[Agalinis tenuifolia (Vahl) Raf.] - SFE, W\&W

[Gerardia tesuifolla Vahl] - $\mathrm{HDH}$

\section{Aphyllon (see Orobanche)}

Castilleja

[NS - comments incorporated from Mark Egger [WTU], pers. comm. 2007]

(Identification sources:

Castilleja angustifolia (Nutt.) G. Don var, angustifolia - Dorn01, KTZ

[Castilleja angustifolia (Nutt.) G. Don var. bradburii (Nutt.) Fern.] - KTZ

Castilleja angustifolia (Nutt.) G. Don var, dubia A. Nelson - Dorn01, E/W

[Castilleja angustifolia (Nutt.) G. Don var, flavescens (Penneli ex Edwin). N. Holmgren] - Egger, pers. comm..

[Castilleja flavescens Pennell] - Eger, pes. eomm.

Castilleja chromosa A. Nelson - GPF, HDH, IMF, SF!, W\&W, Egger pers, comm.

Castilleja flava S. Watson var. flava - IMF, KTZ, UTF, E/W

[Castilleja flava S. Watsoo]-Dom01, GPF, HDH, SF!, W\&W

Castilleja haydenii (A. Gray) Cockerell - HDH, KTZ, SF!, W\&W, E/W

Castilleja integra A. Gray - GPF, HDH, KTZ, ENV, SF!, IMF, W\&W

Castilleja linariifolia Benth. - Dorn01, GPF, IMF, KTZ, SF!, UTF, W\&W, EW

[Castilleja linariaefolia Bewh.] - $\mathrm{HDH}$

Castilleja lineata Greene-HDH, KTZ.SF!, W\&W, EJW

Castilleja miniata Douglas ex Hook. var. miniata - E/W

[Castilleja miniann Douglas] - HDH, SF!

[Castilleja miniaca Douglas ex Hook.] - Dom0t, GPF, IMF, UTF, W\&W

[Castilleja miniaca Douglas ex Hook. subsp. miniata] - KTZ

Castilleja minor (A. Gray) A. Gray var, minor

[Castilleja minor (A, Gray) A, Gray)] -W

Castilleja minor (A. Gray) A. Gray var. "ined.", M. Egger, comb. nov. - Egger, pers, comm.

[Castilleja exilis A. Nets.] - Dorne], GPF, IMF, UTF

[Castilleja minor A. Gray] - HDH, SFW, WaW

[Castilleja minor A. Gray subsp, minof] - KTZ

Castilleja occidentalis Torr- - HDH, IMF, KTZ, SF!, UTF, W\&W, E/W

Castilleja pallescens (Gray) Greenm. var. pallescens - KTZ

[Castilleja pallescens (Gray) Greenm.] - Dorn01

Castilleja puberula Rydb. - HDH, KTZ, SF!, W\&W, E/W

Castilleja rhexifolia Rydb. - Dorn01, HDH, IMF, KTZ, SF!, W\&W, E/W

[Castilleja rhexifolia Rydb, var, inexifolia] - UTF

Castilleja scabrida Eastw. var. scabrida - IMF, KTZ, UTF, w 
[Castilleja lineata Greene] - HDH

[Castilleja scabrida Eastw.] - SFW, W\&W

Castilleja sessiliflora Pursh - Dorn01, GPF, HDH, KTZ, SFE, W\&W, E

Castilleja septentrionalis Lindl.] - HDH, W\&W, Egger, pers, comm.

[Castillejat rhexifolia Rydb. var, salphurea (Rydb.) Atwood] - UTF

[Castilleja su]phurea Rydb.] - Dosn01, GPF, IMF, KTZ, SF!, W\& W, EW, Egger, pers. comm.

Conopholis alpina Liebm. var, mexicana (A, Gray ex S. Watson) Haynes - KTZ, W\&W, E

[Conepholis alpina Liehn, var. mexicana (A.Gray) Haynes] - SFE

\{Cordylanthus kingii S. Watson subsp. densiflorus Chuang \& Heckard\}

\{WJ - Specimens known from UT within 18 mi of $\mathrm{CO}$ (Rio Blanco $\mathrm{Co}$.) and to be expested in $\mathrm{CO}$;

\{Cordylanthus kingii S. Watson\} - HDH, W\&W, misid,

\{Cordylanthus kingii S. Warson sabsp. kingii\} - KTZ CUT>, UTF

\{Cordylanthus kingii S. Watson var. Kingii\} - IMF

Cordylanthus ramosus Nutt, ex Benth, - Dom01, HDH, IMF, KTZ, UTF, E/W

[Condylarthus ramesus Nutt.] - SF!, W\&W

Condylanthus wrightii A, Gray subsp, wrightii - KTZ, W

[Cordylanthus wrightii A. Gray] - HDH, IMF, SFW, UTF, ws W

Orobanche cooperi (A. Gray) A. Heller subsp, cooperi - KWA. KTZ

[Orobanche ledovicianta Nutl. var. cooperi (A. Gray) G. Beck] - KWA. M\&H. HDH, IMF

[Orobanche ledoviciana Nutt. var. latiloba Munz] - KWA

[Aphyllon cooperi Gray] - KTZ

[Myzorrhiza cooperi (Gray) Rydb.)]-KTZ

Orobanche fasciculata Nutt. - Dorn01, GPF, IMF, KTZ, UTF, E/W

[Aphylken fasciculatum (NutL) Torr. \& A. Gray] - SF!, W\&W

[Orobanche fasciculata Nutt, var. fasciculata] - HDH

[Orobanche fasciculata Nutt. var. Iutea (Parry) Achcy] - HDH, rep.

Orobanche ludoviciana Nutt. var. ludoviciana - Dorn01, HDH, IMF, E

[W] - I fail to see the difference between the typical varioty and var. antenosa]

[Orobanche ledoviciana Nutt.] - GPF, SFE, W\&W

[Orobanche ledoviciana Nutt. var. aretosa (Suksd.) Cronquist] - UTF

[Orobanche lodoviciana Nutr. subsp. ludoviciana] - KTZ

[Orobanche multiflora Nurt. var. anenosa (Suksd.) Munz] - HDH, W \& W

Orobanche ludoviciana Nutt, var. multiflora (Nutt.) Beck - E/W

[WJ - if present in Colorado, then aloag New Mexico line, both slopes]

[Orobanche ledoviciana Nutt. subsp, multiflora (Nuts, Collins] - KT2

[Orobanche multiflora Nunt.] - GPE. SFI, UTF

[Orobanche multiflora Nunt. var, multiflora] - $\mathrm{HDH}$, w\&W

Orobanche uniflora L. var. occidentalis (Greene) Roy L. Taylor \& MacBryde - Dom01, IMF, E/W

[Aphylkon uniflorum (L.) Torr. \& A. Giray] - SF!, W\&W

[Orobanche uniflora L.] - GPF, KTZ, UTH

[Orobunche uniflora L, var, sedi (Suksd.) Achey] - HDH

Orthocarpus luteus Nutt. - Dom01, GPF, HDH, IMF, KTZ, SF!, UTF, W\&W, E/W

Orthocarpus purpureoalbus A. Gray ex S. Watson - KTZ, E/W

[Orthocarpus purpurcoalbus A. Gray] - SFI, W\&W

[Orthocarpus purpureo-albas A, Gray] - HDH, UTF

[Orthocamus purpureo-albas A. Gray ex S. Warsoo] - IMF, W \& W

Pedicularis

Snow, N. Janury 2009. Checklist of Vaccular Plants of tho Southum Rocky Momain Region. CVersion 31 
(Identification sources:

Pedicularis bracteosa Benth. var, paysoniana (Pennell) Cronquist - Dorn01, IMF, KTZ, E/W

[Pedicularis bracteosa Benth. subsp. paysonisna (Pennell) W. A. Weber - SF!, W\&W

[Pedicularis pasysoniana PennelI] - HDH

\{Pedicularis canadensis L. var. canadensis\} - GPF

(Podicularis canadensis L.\} - HDH

[Pedicularis cansdensis L. subsp. canadensis]- KTZ CKS, NE, NM, OK $>$, W\& W

- Pedicularis canadensis L. var, fuviatilis (A. Heller) Macbr. - ENDEMIC $\angle C O>$, GPF, E

[Pedicularis canadens is $\mathrm{L}$ subsp. fleviatilis (A. Heller) W. A. Weber] - KTZ, SFE, W\&W

Pedicularis centranthera A. Gray - HDH, IMF, KTZ, SFW, UTF, W\&W, W

Pedicularis crenulata Benth. - Dorn01, HDH, IMF, KTZ, SF!, UTF, W\&W, E/W

Pedicularis groenlandica Retz - Dorn01, HDH, IMF, KTZ, SF!, UTF, W\&W, E/W

[Pedicularis groentandica Retz subsp. surrecta (Benth.) Piper] - KTZ

Pedicularis parryi A. Gray var, mogollonica (Greene) Dom - E/W

[Pedicularis parryi A. Gray]-SF:

[Pedicularis pamyi A. Gray subsp. mogolloeica (Greene) G. D. Carr] - KTZ, Ws W

[Pedicularis paryi A. Gray var, parryi] - IMF

Pedicularis parryi A. Gray var. parryi - Dom0I, IMF, UTF, E/W

[Pedicularis parryi A. Gray] - HDH, SF]

[Pedicularis pamyi A. Gray subsp. parryi] - KTZ, W\& W

Pedicularis procera A. Gray - Dorn01, GPF, IMF, KTZ, SF!, UTF, W\&W, E/W

[Pedicularis grasi A. Nelson] - HDH

Pedicularis racemosa Douglas ex Benth. var. alba (Pennell) Cronquist - Dorn01, IMF, UTF, E/W

[Pedicularis racemosa Douglas ex Hook.] - $\mathrm{HDH}$

[Pedicularis racemosa Douglas sulsap. alba Pennell] - SF1

[Pedicularis racemosa Douglas ex Bestbs subsp, allea Pernell] - HDH, KTZ

[Pedicularis racemosa Douglas ex Hook, subsp. alba Pentill] - W\&W

Pedicularis sudetica Willd. subsp. scopulorum (A. Gray) Hultén - KTZ, E/W

[Pedicularis scopulonum A. Gray]-HDH. SF!, W\&W

\section{Oxalidaceae - OXL}

Oxalis albicans Kunth subsp. albicans - KTZ

Oxalis comiculata 1., - WY checklist, M\&H, KTZ

[Aectosella comiculata (L.) Kuntze] - KTZ

[Oxalis albicans HBK] - KWA

[Oxalis comiculata L. var, atropurpurea Planch.]- KTZ

[Oxalis coniculata L. var, langloisii (Small) Wieg.] - KT2

[Oxalis comiculata L. var, lupalina (R. Knath) Zueg. ] - KT2

[Oxalis coniculata L. var, macrophylla Arsene ex R, Kauth] - KTZ

[Oxalis comiculata L. var, minor Laing] - KTZ

[Oxalis comiculata L. var, leptans Laing] - KTZ

[Oxalis comiculata L. var, villosa (Bieh,) Hohen.] - KTZ

[Oxalis coniculata L. var, viscidu la Wieg.] - KT2

[Oxalis langloisii (Small) Fedde] - KTZ

[Oxalis pusilla Salisb,] - KTZ

[Oxalis repens Thunb.] - KTZ

[Oxalis villosa Bieb,] - KTZ

[Oxalis wrightii Gray] - KWA

[Xanthoxalis albicans (HBK) Small] - KWA

[Xanthoxalis corniculata (L.) Small] - KTZ 
[Xanthoxalis corniculata var. atropurpurea (Planch). Moldenke] - KTZ.

[Xanthoxalis langloisii Small] - KTZ

[Xamthoxalis repens (Thunb.) Moldenke] - KTZ

[Xanthoxalis wrightii (Gray) Abrahams] - KW'A

Oxalis dillenii Jacq, - Dorn01, GPF, IMF, KTZ, SF1, UTF, W\&W

[Oxalis dillenii Jace. subsp. dillenii] - EWW

† Oxalis stricta L. - Dom01, GPF, HDH, KTZ, SFI, W\&W, E/W

[Oxalis europaea Jordan var. bushii (Small) Wiegand] - HDH

[Oxalis europaea Jordan var. europaea] - HDH

Oxalis violacea L. - Dom01, GPF, HDH, KTZ, SF!, W\&W, E/W

\section{Paeoniaceae - PAE}

\{Paeonia brownii Douglas ex Hook \}- HDH, exp, KTZ $\langle\mathrm{UT}$, WY $>$, UTF

\section{Papaveraceae - PAP (including Fumariaceae)}

(Identification sources:

Argemone corymbosa Greene - RM (Lyon 5048; Mesa County, CO)

Argemone hispida A. Gray - Dom01, FNA, GPF, KTZ, SF!, W\&W, EJW

[Argemone platyceras Link \& Otto var, hispide \{A. Gray) Prain] - HDH

[Argemone platyceras Link \& Otto var. platyceras] - $\mathrm{HDH}$

\{Argemone mexicana L.\} - HDH rep., FNA, GPF, W\&W', misapp.

Argemone polyanthemos (Fedde) G, B, Ownbey - Dorn01, FNA, GPF, KTZ, SF!, W\&W, E/W

[Argemone intermedia Sweet]- $\mathrm{HDH}$

Argemone squarrosa Greene var, squarrosa - E

[Argemone squarrosa Greene] - GPF, SFE. WR W

[Argenone squarrosa Greene subsp, squarrosa] - FNA, KTZ

Corydalis aurea Willd, var, aurea - Dorn01, $\mathrm{HDH}, \mathrm{E} / \mathrm{W}$

[Corydalis aurea Willd.] - KTZ, SF!. UTF, WEW'

[Corydalis aurea Willd. subsp. aurea] - FNA, GPF, W\&W

Corydalis aurea Willd. var. occidentalis Engelm. ex A. Gray - Dorn01, E/W

[Corydalis aurea Willd. subsp. occidontalis (Engelm.) G. B. Ownbey] - GPF

[Corydalis aurea Willd. subsp. occidental is (Engelm. ex. A. Gray\} G. B. Ownbey] - FNA

[Corydalis aures Willd. var. occidentalis Engelm.] - HDH

[Corydalis curvisiliqua Engelm, subsp, occidentalis (Engeln,) W. A. Weber] - SF1

[Corydalis curvisiliqua Engelm, subsp, oceidentalis (Engelm, ex A, Gray) W, A. Weber] - KTZ, W\&W

Corydalis caseana A. Gray subsp, brandegeei (S, Watson) G. B. Ownbey - FNA, HDH, KTZ, SF!, W\&W, E/W

\{Corydalis curvisiliqua Engelm. var. grandibracteata Fedde\}

\{Corydalis curvisilicat Engelm. subsp, grandibracteata (Fedde) G. B. Ownbey\}-GPF, KTZ ert, artibution to WEW

\{Corydalis curvisilicata (A. Gray) A. Gray subsp, grandibracteas (Fodde) G. B. Ownbey\} - FNA

(Dicentra uniflora Kellogg\} - FNA, KTZ, SFW, UTF, W\&W, W

[W] - no voushers from Colorado; reported from three sites by M. L. Peterson in Garfield and Mesa cos. All searches of these sites have been fruitless?

↔schscholzia californica Cham. subsp. californica - FNA, KTZ, ENW 
[Eschscholria californica Cham.] - Dorn01, HDH, SF!, UTF, W\&W

*Fumaria officinalis L. - Dorn01, FNA, HDH, exp, UTF, W\&W, misapp.

[Fumaria officinalis $L_{\text {. subsp, officinalis] - KTZ UT, WY }}$

[Fumaria officinalis L subsp. wirtgenii (W. D. J. Koch) Arcang.] - KTZ NEE, WY

*\{Fumaria vaillantii Loisel. - FNA, KTZ, SFE, W\&W, E)

[Fumaria vaillenti Loisel.]-GPF [misspelled]

*Glaucium comiculatum (L.) Rudolph - FNA, GPF, KTZ, SFE, W\&W ${ }^{1}$, E

[W] - one specimen from near Beulder, not persisting]

*\{Glaucium flavum Crantz\} - FNA, KTZ, SFE, W\&W, E

[W] - from yard in Boulder, not known outside cultivation]

*Papaver orientale L. - FNA, KTZ, SFE, UTF, W\&W, E

*Papaver nudicaule L, - FNA, EW

[Papenver croceum Ledeb, cultivar of above] - FNA, KTZ, SF!, W\&W

[Papaver pygmaeum Rydb.]

[WJ - endemic to Montana, British Columbia, and Alberta [fide FNA] so the name is misapplied to our material:

Papaver radicatum Rotrb, subsp, kluanensis (D. Löve) D. F. Murray - FNA, KTZ, E/W

[Papener kluanense D. Love] - SF!, W\&W

[Papaner lapponicum (Tolm.) Nordh. subsp. occidencale (Lundstr.) Knaben] - R.MC

[Paponer nudicaule L. var, radicatum (Roitb.) DC.] - HDH. W\& W', misid.

[Papener pygmacum Rydb.] - W\& W'

[Papener radicatum Rottb, var. pygmaeun (Rydb.) S. L. Welsh] - UTF

* Papaver somniferum L. - Dorn01

[Papener somniferum var. setigerum suct. non (DC.) Corb.] - KTZ

\section{Parnassiaceae - PAR}

Parnassia fimbriata Kōnig var. fimbriata - KTZ, UTF, E/W

[Parnassia fimbriata König] - Dorn01, HDH, IMF, W\&W

[Pansassia fimbriata Banks] - Sf]

Parnassia kotzebuei Cham. ex Spreng. - Dom01, IMF, KTZ

[Pamassia kocrebuei Cham.] - SF!

[Pamassia kotrebuei Cham. \& Schlecht] - W\& W

[Parnassia kotadouei Clam. \& Spreng.] - W $\&$ W

[Parnassia kotrebuei Cham. ex Spreng, var, kotrebuei] - E/W

Parnassia palustris L. var. montanensis (Fernald \& Rydb, ex Rydb.) C. L. Hitchc, - Dorn01. KTZ, E/W

[Pamassia palustris L. var, montanensis (Femald \& Rydb.) C. L. Hitche] - UTF

[Pamassia palustris L. var, palustris] - IMF

[Paniassia palustris L. var, parviflora (DC) B, Boivin] - IMF, KTZ $<W Y>$

[Panassia parviflora DC.] - GPF, HDH, KTZ $<$ WY, SFt, WEW

\section{Pedaliaceae (sec MAR)}

\section{Peganaceae - PEG}

* Peganum harmala L.

[NS - repertedly weedy now in Colorado by KHD] 
Phrymacene - PHR (formerly in SCR)

Mimulus

(Identification sources:

Mimulus breweri (Greene) Coville - Dorn01, IMF, KTZ, SF!, UTF, W\&W, ENW

Mimulus eastwoodiae Rydb. - HDH, exp., IMF, KTZ, SFW, UTF, W\&W, W

Mimulus floribundus Lindl, - Dorn01, KTZ, ENW

[Mimalus floribundus Douglas] - HDH, SF!, W\&W

[Mimalus floribundus Douglas ex Lindl.] - GPF, IMF, UTF

[Mimulus floribundus LindL. var. floribundus] - RMC

$\bullet$ Mimulus gemmiparus W. A. Weber- ENDEMIC $<C O>$, KTZ, SF!, W\&W, ENW

Mimulus glabratus Kunth var, glabratus - KTZ, E/W

[Mimulus glabrates Kunth] - SF!

[Mimalus glabratus Kunth subsp. utahensis Pennel]] - [MF, UTF

[Mimalus glabratus Kunth var, utahensis Pennell] - HDH, W\& W

Mimulus glabratus Kunth var, jamesii (Torr. \& A. Gray ex Benth.) A. Gray - Dorn01, KTZ, E/W

[Mimalus fremontii (Benth.) A. Gray] - KTZ

[Mimulus glabratus Kunth] - SF!

[Mimalus glabratus Kunth subsp. fremonti (Benth.) A. L. Grant] - IMF

[Mimalus glabratus Kanth subsp. fremontii (Benth.) Pentnel]] - UTF

[Mimulus glabratus Kunth var, fremontii (Benth.) A. L. Grant] - HDH, W\&W, GPF

Mimulus guttatus DC. - Dorn01, GPF, HDH, IMF, KTZ, SF!, UTF, W\&W

[NS - nearly 70 synonyms recognized in KTZ]

[Mimalus gutakus DC. var, gutiatus] - EW

Mimulus lewisii Pursh - Dorn01, HDH, IMF, KTZ, SF!, UTF, W\&W, E/W

Mimulus moschatus Douglas ex Lindl. var. moschatus - Dorn01, KTZ, E/W

[Mimalus moschatus Douglas] - HDH, SF!, W\& W

[Mimalus moschatus Douglas ex LindL.] - IMF, UTF

Mimulus nanus Hook. \& Arn. subsp. nanus - KTZ

[Mimalus nanus Hook, \& Am.]-HDH, exp.. IMF

Mimulus ringens L. var, ringens - KTZ

[Mimalus riagens L.] - GPF, HDH, IMF, W\&W

Mimulus rubellus A. Gray - Dorn01, HDH, IMF, KTZ, SF!, UTF, W\&W, E/W

Mimulus suksdorfii A. Gray - Dom01

[NS - cited but not recognized or incorrectly resognized previously in HDH, IMF, KTZ $\angle A Z$. NM. UT, WY $>$ UTF, W\&W, misapp.]

Mimulus tilingii Regel var. tilingii - KTZ, E/W

[Mimalus tilingii Regel] - IMF, SFI. UTF, W\&W

[Mimalus gutratus DC.] - HDH

Phyllanthaceac - PLL (formerly in EUP)

Reverchonia arenaria A. Gray - GPF, IMF, KTZ, UTF, W\&W'

Plantaginaceac - PTG (including much of former Serophulariaceac)

(Identification sources:

Snow, N, January 2009. Checklist of Vacculer Plants of the Southom Rocky Momain Region. CVersion $3 x$ 
Bacopa rotundifolia (Michx.) Wettst, - Dorn01, GPF, HDH. IMF, KTZ, SF!, W\&W, E/W

Besseya alpina (A. Gray) Rydb. - Dom01, HDH, IMF, KTZ, SF!, UTF, W\&W, EW

Besseya arizonica Pennell - KTZ, KWA, M\&H

[Synthyris aplina Gray] - M\&H

Besseya plantaginea (E, James) Rydb, - Dorn01, HDH, KTZ, E/W

[Besseya plantaginea (Benth.) Rydb.] - SF!, W\&W

$\bullet$ Besseya ritteriana (Eastw.) Rydb, - ENDEMIC <CO>, HDH, KTZ, SFW, W\&W, W, Hufford04

[Synthyris ritieriana Eastw.] - KTZ

Besseya wyomingensis (A. Nelson) Rydb, - Dorn01, GPF, IMF, KTZ, SFE, UTF, W\&W, E [Besseya cinerea (Raf,) Pennell] - HDH

*Chaenorrhinum minus (L.) Lange - GPF, KTZ, SFE, W\&W, E

Chionophila jamesii Benth. - Dorn01, HDH, KTZ, SF!, W\&W, E/W

Collinsia parviflora Lindl, - Dorn01, KTZ, SF!, E/W

[Collinsia parviflora Douglas] - HDH, UTF, WNW

[Collinsia parviflora Douglas ex Lindl.] - GPF, IMF

*Cymbalaria muralis P. Gaertn., B. Mey, \& Scherb, - GPF, KTZ <UT>, W\& W'

*Digitalis purpurea L, var, purpurea - KTZ, E

[Digitalis purpurea L.] - Dorn01, SFE, UTF, WRW

Gerardia (sec Agalinis)

Gratiola neglecta Torr. - Dorn01, GPF, HDH, IMF, KTZ, SF!, UTF, W\& W

[Giratiola teglecta Torr, var, neglecta] - E.W

Leiostemon (sec Penstemon)

Limosella aquatica L. - Dom01, GPF, HDH, IMF, KTZ, SF!, UTF, W\&W, E/W

Linaria canadensis (L.) Dum. Cours, var. canadensis - Dom01, GPF. IMF

\{Linaria canadensis (L.) Dum. Cours.\} - HDH, exp, UTF

¿Nurtallanthus eanadensis (L.) D. A. Sutton) - KTZ K KS, OK>

Linaria canadensis (L.) Dum. Cours, var, texana (Scheele) Pennell-GPF, IMF. W\&W, E [L.inaria canadensis (L.) Dum. Cours,] - UTF

[Linaria canadensis Dum, Cours, var, texana (Scheele) Pennel]] - SFE, W\&W'

[Linaria texana Schesle] - HDH

[Nuttallanthus texants (Scheele) D. A. Sutton] - KTZ

*L.inaria dalmatica (L.) Mill subsp. dalmatica - KTZ. E/W

[Linaria dalmatica (L.) Mill.] - Dorn01, GPF, IMF, UTF

[Linaria dalmatica (L.) Mill, var, macedonica Fenzl] - HDH

[Linaria genistifolia (L.) Mill. suhsp. dalmatica (L.) Maire et al.] - SF!, WE W

*Linaria vulgaris Mill. - Dom01, HDH, KTZ, SF!, W\&W, E/W

[L,inaria vulgaris Hill.] - GPF, IMF, UTT 
Lindernia dubia (L.) Pennell var, anagallidea (Michx.) Cooperr. - KTZ, SFE, W\&W, E [Lindernia anagallideu (Michx.) Pennell] - HDH

[Lindernia dubia (L.) Pennell] - GPF, IMF

Maurandya antirhiniflora Humb, \& Bonpl. ex Willd. - M\&H, KWA

[Antirrhinum antirthiniflora (Humb. \& Eonpl. ex Willd.) Hitchcock] - KWA

[Antirrhinum maurandioides Gray ] - KTZ.

[Asarina antirrhiniflora (Humb. \& Bonpl. ex Willd_) Pennel] - KTZ

[Maurandella antirnhiniflora (Humb. \& Bompl, ex Willd.) Tothmaler - KWA, KTZ

Maurandya wislizeni Engelm. ex Gray - KWA, M\&H

[Antirnhimum wislizeni (Engelm. ex Gray) Tidestrom ] - KTZ

[Asarina wislizenti (Engelm. ex Gray) PennelI] - KTZ

[Epixiphium wislizeni (Engelm. ex Gray) Munz ] - KTZ

Nuttallanthus (see Linaria)

Penstemon

(Identification sources:

Penstemon acaulis L. Williams - ENDEMIC $<$ WY $>$, Dom01

Penstemon albidus Nutt, - Dorn01, GPF, HDH, KTZ, SFE, W\&W, E

Penstemon ambiguus Torr. - $\mathrm{HDH}, \mathrm{E}$

[Leiostemon ambigaus (Torr.) Greve] - SFE, W\&W

[Penstemon ambiguus Tor. var. ambiguus] - GPF, IMF

[Penslemen ambiguus Tor, var. laevissimus (D. D. Keck) N. H. Holmgrea] - IMF, GPF, KTZ, UTF

Penstemon angustifolius Nutt, ex Pursh var. angustifolius - Dom01, GPF, KTZ, W\&W, E

[Penstemon angustifolius Nutt] - SFE

[Pentatemon angustifolius Nut ex Pursh sabsp. angustifolius] - HDH, W\& W'

[Penstemen angustifolius Pursh var, angustifolius]- IMF, UTF

Penstemon angustifolius Nutt, ex Pursh var. caudatus ( $\mathrm{A}$. Heller) Rydb, - Dorn01, GPF, KTZ, E [Penstemen angustifolius Nuat subsp. caudatus (A. Heller) D. D. Keek] - SFE

[Penstemen angustifolius Nuit ex Pursh subsp, eaudatus (A. Hellet) D. D. Keck] - HDH, W\& W'

[Penstemen angustifolius Pursh var, caudaus (A. Heller) Rydb.] - IMF, UTF

[Penstemee angustifollus Nunt ex Pursh var, angustifolius] - WR W

Penstemon angustifolius Nutt. ex Pursh var, venosus (D. D. Keck) N. H. Holmgren - KTZ, WJ [Penstemen angastifolius Pursh var, venosas (D. D. Keck) N. H. Holengren] - [MF, UTF

Penstemon angustifolius Nutt. ex Pursh var, vernalensis N. H. Holmgren - KTZ, W\&W. W [Penstemon angustifolius Nutt var. venakensis N. H. Holmgrea] - SWF, W W $^{\prime}$

[Penstemon angustifolius Pursh var, vemalensis N. H. Holmgren] - IMF, UTF

Penstemon arenicola A. Nelson - Dorn01, HDH, IMF, KTZ, SFW, UTF, W\&W, W

Penstemon aridus Rydb. - Dorn01

Penstemon auriberbis Pennell - GPF, HDH, KTZ, SFE, W\&W, E

Penstemon barbatus (Cav.) Roth var. barbatus - IMF, UTF

[Chelone barbata Cav.] - M\&H

[Pesistemen barbanus (Cav.) Roth subsp. barbatis] - KWA, HDH, exp. KTZ, W\& W

Penstemon barbatus (Cav.) Roth var. torreyi (Benth.) A. Gray - IMF, UTF, E/W

[Penstemen barbanus (Cav,) Roth subsp, torryi (Benth.) D. D, Keck] - HDH, KTZ, W\& W'

[Penstemon barbatus Tor, subsp, torreyi (Benth.) D, D. Keck] - WSW

[Penstemon barbanus (Cav) Roth var, torreyi (Benth.) D, D, Keek] - SFI

Penstemon barbatus (Cav.) Roth var. trichander A. Gray - IMF, UTF, W

Snou, N, Janury 2009. Checklast of Vaccular Plants of tho Southom Rocky Momain Region. CVersion 3 . 
[Penstemen barbatus (Cav.) Roth subsp, trichander (A. Gray) D. D. Keck] - HDH, KTZ

[Penstemon barbatus (Cay.) Roth. var. trichander (A. Gray) D. D. Keck] - SFW

[Penstemen harbatus Torr. subsp. trichander (A. Gray) D. D. Keck] - W\&W

Penstemon brandegeei (Porter) Porter ex Rydb, - WJ, W\&W'. E/W

[Penstemon alpinus Torr. subsp. brandegei (Porter) Penland] - HDH

[Penstemen brandegei Porter] - SF!

[Penstemen glaber Pursh var, brandegeei (Porter) C. C. Frceman] - KTZ

[Penstemen glaber Parsh var. brandegei (Porter ex Rydh.) C. C. Freeman] - GPF

[Penstemen gleber Pursh var, brandegei (Porter ex Rydb.) Umber \& Latady, unpubl.] - W\&W

Penstemon bridgesii A. Gray - HDH. SFW, W\&W, W

[Pentstemen rostriflorus Kellogg] - IMF, KTZ UTF, W\& W'

Penstemon buckleyi Pennell - GPF, HDH, KTZ, SFE, W\&W, E

Penstemon caespitosus NutL ex A. Gray var, caespitosus - TMF, KTZ, UTF, E/W

[Penstemen cacspilosus Nutt.] - Dorn01, SF!

[Penstemen cacspitosus Nutt. ex A. Gray]-HDH, W\& W

Penstemon caespitosus Nutt. ex A. Gray var, perbrevis (Penneli) N. H. Holmgren - Dorn01, IMF,

KTZ, UTF

\$Penstemon cobaea Nutt. - GPF, HDH, KTZ, SFE, W\&W, E

[W] - a waif]

Penstemon comarthenus A. Gray - HDH, IMF, KTZ, SFW, UTF, W\&W, W

Penstemon crandallii A. Nelson subsp, atratus D. D. Keck - KTZ, W\&W, EW [Penstemon cacspitosus Nutt. ex A. Gray] - SF!, w\& W

[Penstemen crandallii A. Nelson var, atratus (D. D. Keck) N. H. Holmgren] - IMF, UTF

Penstemon crandallii A. Nelson subsp, crandallii - HDH, KTZ, W\&W, E/W

[Petistemen chespitosus Nutt. ex A. Gray] - SF!, W\& W ${ }^{1}$

[Penstemen crandallii A. Nelson] - WJ

[Penstemon crandallii A. Nelson var. crandallii] - [MF, UTF

[Penstemen teucrioides Greene] - HDH, IMF, KTZ, W\&W

Penstemon crandallii A. Nelson subsp. glabrescens (Pennell) D. D. Keck - HDH, KTZ, E/W

[Penstemon carspitosus Nutt. ex A. Gray]-SFI, W\&W

[Penstemon erandallii A. Nelson subsp. erandallii] - W\&W

[Penstemon glabrescens Penneli] - W]

Penstemon crandallii A. Nelson subsp, procumbens (Greene) D. D. Keck - HDH, KTZ, EJW [Penstemen cakspitosus Nutt. ex A. Gray]-SF!, we W'

[Penstemen crandaliii A, Nelson subsp. crandallii] - W\& W

[Penstemes procumbens Greetic] - W]

Penstemon cyaneus Penn. - KTZ

Penstemon cyanocaulis Payson - HDH, IMF, KTZ, SFW, UTF, W\& W, W

Penstemon cyathophorus Rydb. - Dorn01, HDH, IMF, KTZ, SF!, W\&W, E/W

- Penstemon debilis $O$ Kane \& J. L. Anderson - ENDEMIC $<\mathrm{CO}>, \mathrm{KTZ}, \mathrm{SFW}, \mathrm{W} \& W, \mathrm{~W}$

- Penstemon degeneri Crosswhite - ENDEMIC $<\mathrm{CO}>, \mathrm{KTZ}$, SFE. W\&W, E

Penstemon eatonii A. Gray var. eatonii - IMF, SFW. UTF. W

[Peratemon eatcaii A. Gray suhsp. eatcaii] - HDH, KTZ, W\&W

Penstemon eatonii A. Gray var. undosus M. E. Jones - IMF. UTF. W

Penstemon extonii A. Gray suhsp. undosus (M. E. Jones) D. D. Keck] - HDH, KTZ, W\&W

[Penstemon eatsoii A. Gray var, undosus (M. E. Jones) D. D. Keck] - SFW

Penstemon eriantherus Pursh var. cleburnei (M. E. Jones) Dom - Dom01

[Penstemon cleburnei M. E. Jones] - KTZ

Penstemon eriantherus Pursh var. eriantherus - Dorn01, GPF, IMF, KTZ, E

[Penstemon eriantherus Pursh] - HDH, SFE, W\&W

\{Penstemon fendleri A. Gray\} - FCF. SFW, W\&W' ${ }^{1}$. W

$[W]$ - Not present in $\mathrm{CO}$; specimen referenced ty WRW is P. lentus?

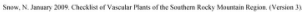


\{Penstemon fendleri Torr. \& A. Gray\} - GPF, KTZ CAZ, KS, NM, OK>

Penstemon fremontii Torr. \& A. Gray ex A. Gray var. fremontii - KTZ, RM, w

[Penstemen frumontii Tarr. \& A. Gray]-Dorn01, HDH, IMF, SFW, UTF

[Penstemen fremontii Torr. \& A.Gray var. fremostii] - W\&W

- Penstemon fremontii Torr. \& A. Gray ex A. Gray var. glabrescens Dom \& Lichvar - ENDEMIC $<\mathrm{CO}>$, KTZ. W

[Penstemen fremontii Torr. \& A. Gray var. glabrescens Dorn \& Lichvar] - SFW, W\&W

Penstemon gibbensii Dorn - Dorn01, KTZ, UTF, W\&W

Penstemon glaber Pursh var. alpinus (Torr.) A. Gray - Dom01, Hartman06, GPF, KTZ, EWW

[Penstemen alpines Torr, forma alpinus] - HDH

[Penstemon alpinus Torr. forma riparius (A. Nels.) Pensell] - HDH

[Penstemon glaber Pursh] - SF?

[Penstemen glaber Pursh var, glaber] - W\&W

Penstemon glaber Pursh var-glaber - Dorn01, GPF, KTZ, W\&W, EW

[Penstemon alpinus Torf. subsp. magnes (Penisell) Penland] - HDH

[Penstemen glaber Parsh] - SF!, HDH, exp.

Penstemon gracilis Nutt, - Dom01, GPF, HDH, SFE, W\&W, E

[Petsatemen gracilis Nutt. var. gracilis] - KTZ

Penstemon grahamii D. D. Keck - IMF, KTZ, SFW, UTF, W

[Penstemee grahamii D. D. Keck ex E. H. Grakam] - Waw

Penstemon grandiflorus Nutt. - Dom01, GPF, HDH, KTZ, SFE, W\&W, E

Penstemon griffinii A. Nelson - KTZ, SFE, W\&W, E

[Penstemon ofiganthes Wooton \& StandL] - $\mathrm{HDH}$

$\bullet$ Penstemon ballii A. Gray - ENDEMIC $<\mathrm{CO}>\mathrm{HDH}, \mathrm{KTZ}, \mathrm{SF}$ !, W\&W, E/W

$\downarrow$ Penstemon harbourii A. Gray - ENDEMIC $<\mathrm{CO}>$, HDH, KTZ, SF!, W\&W, E/W

- Penstemon harringtonii Penland - ENDEMIC <CO>, IMF, KTZ, SFW. W\&W. W

Penstemon haydenii S. Wats. - Dom01

Penstemon humilis Nutt. ex A. Gray var. humilis - IMF, UTF, W

[Penstemen humilis Nutt] - SFW

[Penstemen humilis Nutt. ex A. Gray] - Dorno1, HDH. W\&W

[Penstemen humilis Nutt, ex A. Gray subsp, humilis] - KTZ.

Penstemon jamesii Benth- GPF. IMF, KTZ, SFE, W\&W. E

[Penstemen janesii Benth. subsp. jamesii] - HDH

Penstemon laricifolius Hook. \& Arn. var. exilifolius (A. Nelson) Payson - Dorn01, E

[Penstemon laricifolius Hook. \& Am.] - IMF

[Penstemon laricifolius Hook. \& Am, subsp. exilifolius (A. Nelson) D. D. Keck] - HDH, KTZ, SFE, W\&W

Penstemon laricifolius Hook. \& Arn. var. laricifolius - Dorn01

[Penstemon laricifolius Hook \& Am. subsp. laricifolius] - KTZ

Penstemon lentus Pennell var. lentus - IMF, KTZ, UTF

[Penstemen lentus Penneli] - HDH, W\&W

Penstemon linarioides A. Gray subsp, coloradoensis (A. Nelson) D. D. Keck - HDH, IMF, KTZ, ENW

[Penstemen linarioides A. Gray] - W\&W

[Penstemon linarioides A. Gray subsp. coloradoûnsis (A. Nelson) D. D. Keck] - SF!

[Penstemen linarioides A. Gray var. viridis D. D. Keek] - HDH

\{Penstemon linarioides A. Gray subsp. linarioides\} - HDH, IMF, KTZ

\{Penstemon linarioides A. Gray\} - W\&W

- Penstemon mensarum Pennell - ENDEMIC $<\mathrm{CO}>$. HDH, KTZ, SFW, W\&W, W

[Penstemen gratus Umber \& Moorman, unpubl.] - W\& W

Penstemon moffatii Eastw, - IMF, SFW, UTF, W\&W, W

[Penstemon moftati Eastw. subsp. moffatii]- $\mathrm{HDH}, \mathrm{KTZ}$

[Penstemen moffanti Eastw, subsp. paysonii (Penesil] D. D. Keck] - HDH, KTZ

Snow, N, Janury 2009. Checklist of Vaccular Plants of the Southom Rocky Momain Region. CVersion 3 . 
Penstemon nitidus Dougl, ex Benth. var nitidus - Dorn01, KTZ

Penstemon ophianthus Pennell - IMF, KTZ, SFW, UTF, W\&W, W

[Penstemen breviculus (D. D. Keck) Nishet \& R. C. Jackson] - DMF, KTZ, SFW, UTF, W\&W

[Penstemen jamesii Benth. subsp, ophianthus (Penneli) D. D. Keck] - HDH

[Penstemen parviflons Pennell] - HDH, KTZ SFW, W\&W

Penstemon osterhoutii Pennell - HDH, IMF, KTZ, W\&W, W

[Penstemon ousterhoutii PennelI] - SFW

Penstemon pachyphyllus A. Gray ex Rydb. var, mucronatus (N. H. Holmgren) Neese - Dom01, KTZ, UTF, W\&W, W

[Penstemen pachyphyllus A. Gray var. mucronatus (N. H. Holmgren) Neese] - SFW

[Penstemen mucronatus N. H. Holmgren] - IMF, KTZ

[Penstemon pachyphyllus A. Gray ex Rydb, var, pachyphyllus] - IMF, KTZ $<U T>$, UTF, W\&W

Penstemon palmeri subsp. typicus Keck - KTZ

[WJ - widely cultivated and escaping in CO, e.g. Louisville Open Space]

[Penstemen palmeri A. Gray] - Dorno1

Penstemon paysoniorum Keck - Dorn01, KTZ

- Penstemon penlandii W. A, Weber - ENDEMIC $<C O>$, KTZ, SFW, W\&W, W

Penstemon procerus Douglas ex Graham var, procerus - IMF, KTZ, UTF, E/W

[Penstemon confertas Douglas subsp. procerus (Dovglas) D. V. Clark] - SF!

[Penstetnoe confernis Douglas subsp. procenus (Douglas ex Graham) D. V. Clark, unpubl.]

- WRW

[Penstemon procerus Doinglas ex Graham] - Dom01, HDH

[Penstemen procerus Donglas ex Graham subsp. procerus] - GPF

Penstemon radicosus A. Nelson - Dorn01, HDH, IMF, KTZ, SF!, UTF, W\&W, EWW

- Penstemon retrorsus Payson ex Pennell - ENDEMIC <CO >, HDH, KTZ. W\&W, W

[Penstemon coespitosus Nutt, ex A, Gray] - SFW, W\&W

Penstemon ramaleyi A. Nelson

[W] - Occurs in Saguache Co,; stands erect with long linear leaves, somewbat differerent from other taxa in the cespitosi complex]

Penstemon rydbergii A. Nelson var. aggregatus (Pennell) N. H. Holmgren - Dorn01. IMF. KTZ, E/W

[Penatemon rydbergis A. Nelson] - SFL, UTF, W\&W

[Penstemon rydbergi A. Nelson subap, aggregatus (Pennell) D. D. Keck] - HDH

Penstemon rydbergii A. Nelson var. rydbergii - Dom01, IMF, KTZ, E/W

[Penstemon rydbergii A. Nelson] - SF!, UTF, W\&W

[Penstemon rydbergi A. Nelson subsp. nydbergii] - $\mathrm{HDH}$

Penstemon saxosorum Pennell - Dorn01, HDH, KTZ, SFt, W\&W, E/W

Penstemon scariosus Pernell var. albifluvis (England) N. H. Holmgren - IMF, KTZ,

SFW, UTF, W\&W, W

[Penatemes albifluvis England] - KTZ

Penstemon scariosus Pennell var. cyanomontanus Neese - KTZ, UTF. W\&W

[Penstemon lasiostemon Umber \& Monrman, unpahlished] - W\&W

Penstemon scariosus Pennell var. garrettii (Pennell) Holmgren - Dorn01

[Penstemen garrettii Pennell] - KTZ

Penstemon secundiflorus Benth. subsp. lavendulus Pennell - W\& W'

Penstemon secundiflorus Benth subsp. secundiflorus - W\&W W $^{\prime}$ W

[Penstemen secundiflorus Benth,] - GPF, HDH, KTZ, SF!, W\&W

Penstemon strictiformis Rydb. - IMF. KTZ UTF, E/W

[Penstemen strictus Benth.] - SF!, W\&W

[Penstemen strictus Benth. subsp, strictiformis (Rydh,) D. D. Keck] - HDH

Penstemon strictus Benth. - Dorn01, IMF, KTZ. SF!. UTF, W\&W, E/W

[Penstemen strictus Benth. subsp. angustus PennelI] - HDH 
[Penstemen strictus Benth. subsp. strictus] - HDH

Penstemon subglaber Rydb, - Dom01, KTZ

Penstemon utahensis Eastw, - IMF, KTZ, SFW, UTF, W\&W, W

Penstemon versicolor Pennell - SFE, W\&W, $\mathrm{E}$

$\bullet$ Penstemon virens Pennell ex Rydb. - ENDEMIC $<\mathrm{CO}, \mathrm{WV}>$, Dorn01, HDH, KTZ, W\&W, E/W

[Penstemon virens Penuell] - GPF, SF!

Penstemon virgatus A. Gray var, asa-grayi (Crosswh.) Dom-Dorn01, E

[W] - Var. virgurus in MS so simtilar to Colorado plants variety may not be defensible]

[Penstemen unilateral is Rydb.] - HDH, KTZ

[Penstemon virgatus A. Gray subsp. asa-grayi Crosswh.]-SFE, WsW

Penstemon watsonii A. Gray - HDH, IMF, KTZ, SFW, UTF, W\&W, W

Penstemon whippleanus A. Gray - Dorm01, HDH, IMF, KTZ, SF!, UTF, W\& W, E/W

Penstemon yampaensis Penland $-\mathrm{KTZ}$, W

[Penstemoe acaulis L. O. Willians] - $\mathrm{HDH}$

[Penstemen acsulis L. O. Williams var yampaensis [Pentand) Nerse] - UTF

[Penstemon acaulis L. O. Williams var. yampaünsis (Penland) Nesse) - SFW, W\& W

[Penstemon yampaënsis Penland] - IMF

Plantago

(Identification sources:

\{Plantago aristata Michx.\} - GPF, HDH, exp., IMF, KTZ <KS, NE, NM, OK>

Plantago elongata Pursh var. elongata - IMF, E/W

[Plantago clongata Pursh] - GPF, HDH, SFI, UTF, W\&W

[Plantago elongata Pursh subsp. elongata] - KTZ

Plantago criopoda Torr - Dorn01, GPF, HDH, IMF, KTZ, SF!, UTF, W\&W, E/W

* Plantago lanceolata L. - Dom01, GPF, HDH, IMF, KTZ, SF!, UTF, W\&W, E/W

* Plantago major L. - Dom01, GPF, HDH, IMF, KTZ, SF!, UTF, W\&W

[Plantago asiatica L.] - $\mathrm{HDH}$

[Plantago major L, var, major] - EW

[Plantago major L, var, pachyphylla Pilg] -IMF

[Plantago major L.] - GPF, KTZ, UTF, W\&W'

Plantago patagonica Jacq. - KTZ, SF!, W\&W

[NS - variation associated with "varieties" \{e.g. GPF\} can appear on a single plant and both varietics can occur in same population]

[Plantago paragooica Jace_] - KTZ, SF!, W\& W

[Plantago parngonica Jacq, var, gnaphalioides (Nutt,) A. Gray] - IMF, UTF

[Plantago paragonica Jacq, var, patagonica] - GPF, E/W

[Plantago paragonica Jacq, var, spinulosa (Decne.) A. Gray] - GPF, IMF, UTF, EJW

[Plantago purshii Roem. \& Schult,] - HDH

[Plantago spisulosa Decaisne ex DC.] - HDH

Plantago tweedyi A. Gray - Dorn01, HDH, IMF, KTZ, SF!. UTF, W\&W, EW

\section{Pocilla (see Veronica)}

Rhinanthus minor L. subsp. minor - KTZ, E/W

[Rhinanthus minor L. suhsp. boreslis (Sterneck) A. Live] - SF], WRW

[Rhinanthus rigidus Chabert] - HDH 
Veronica

(Identification sources:

Veronica americana Schwein. ex Benth, - Dorn01, HDH, IMF, KTZ, UTF, W\&W, E/W

[Venonica americana (Raf.) Sehwein.] - SF!

[Veronica americana (Raf.) Schwein. ex Benth.] - GPF

\% Veronica anagallis-aquatica L. - Dom01, GPF, HDH, IMF, KTZ, UTF, W\&W

*Veronica arvensis $\mathrm{L}_{\text {. }}$ - Dorn01, GPF, HDH rep., IMF, KTZ?, UTF, W\&W, no specimen

*Veronica biloba L. - Dom01, GPF, IMF, KTZ, UTF, E/W

[Pocilla biloba \{L.) W. A. Weber]-SF!, W\&W

†Veronica catenata Pennell - Dorn01. IMF, SF. UTF, W\&W, E/W

[Veronica anagallis-aquatica L.] - KTZ

[Veronica catcriata Pennell var- catenata] - GPF

[Veronica salina Schur.] - HDH

Veronica nutans Bongard - DA

[DA - this diploid Westem N. Amer. species is clearly distinct from the tetraploid V. wormskkjoldii]

Veronica peregrina L, var. xalapensis (Kunth) H. St. John \& F. W, Warren - Dorn01, GPF, IMF, UTF, E/W

[Veronika peregrina L. subsp. xalapensis (Kunth) Pennel]] - KTZ, SF!, W\&W

[Veronica peregrima L. var. xalapensis (Kunth) Pennel]- HDH

*Veronica persica Poir. - Dorn01, GPF, HDH, IMF, KTZ, UTF, W\& W', misid.

[Pocilla persical (Poir.) Fourr.] - W\&W, W\& W', misid.

*Veronica polita Fr. - GPF, KTZ, EJW

[Pocilla polita (Fr.) Fourt.]-SFL, w\&W

Veronica seutellata L, - GPF, HDH, IMF, KTZ, SF!, W\&W, E/W

Veronica serpyllifolia L. var. humifusa (Dickson) Vahl - Dom01, GPF, HDH, IMF, E/W

[Veronixa serpyllifolia L.] - UTF

[Veronica serpy llifolia L. subsp. bumifursa (Dickson) Syme] - XTZ

[Veronikasirum serpyllifolium (L.) Fourr. subsp. humifusum (Dickson) W. A. Weber] - W\&W

[Veronicastrum serpyllifoliam (L.) Fourr, subsp, humifusum (Dickson) W. A. Weber]-SFL, W\&W

Veronica wormskjoldii Roem. \& Sehult. - Dorn0I, HDH, IMF, UTF, EWW

[Veronica nutans Boagard] - SF!, W\& W

[Veronica wormakkiohdi Roem. \& Schult. var, wormskjoldii] - KT/

Veronicastrum (sec Veronica)

Plumbaginaceac - PLB (including Limeniaceac)

Armeria scabra Pall. subsp. sibirica (Turez. ex Boiss.) Hylander - FNA, W\&W, EW

[Armeria labeadorica Walli.]-HDH

[Armeria maritima (Mill.) Willd.] - UTF

[Armeria mariaima (Mill.) Willd. subsp. sibirica (Turcz, ex Boiss.) Nyman] - KTZ

[Armeria scabra Pall. subsp, sibirica (Turcz.) Hylander] - SF!

\section{Poaceac - POA (= Gramineac)}

(Identification sources:

Achnatherum (see also Jarava)

Snow, N, January 2009. Checklist of Vacculer Plants of the Southum Rodky Momain Regioe. CVersion $3 x$ 
(Identification sources:

Achnatherum aridum (M. E. Jones) Barkworth - RBS, KTZ, SFW, W\&W'

[Stipa arida M. E. Jones] - HDH, MMF, UTE, W\&W, W

Achnatherum arnowiae (S. Welsh \& N. D. Atwood) Barkworth - FNA

[NS - reported by UT3 in Mesa Co.. CO, voucher not yet seen]

[Stipa amowise S. Welsh \& N. D. Atwood] - UTJ

Achnatherum contractum (B. L. Johnson) Barkworth - Dorn01. FNA. KTZ, W\&W'

[Oryzopsis contracta (B. L. Jobnson) Shechter - RMC

[Stipe hymenoides Roem. \& Schulh.] - W\&W

Achnatherum hymenoides (Roem. \& Schult.) Barkworth - RBS, Dorn01. FNA, KTZ, SF!. W\&W

[Oryzopsis hymenoides (Roem. \& Schult.) Ricker] - GPF, HDH, IMF

[Oryzopsis hymetoides (Roem. \& Schult.) Ricker ex Pipef] - E/W

[Stipa hymenoides Roem. \& Schult.] - UTF, W\& W

Achnatherum leftermanii (Vasey) Barkworth - RBS, KTZ, SF!, W\&W'

[Stipa lettermanii Vasey]- Dorn01, HDH, IMF, UTF, W\&W, E/W

Achnatherum lobatum (Swallen) Barkworth - FNA

[Stipa lobata Swallen] - FNA

Achnatherum nelsonii (Scribn_) Barkworth subsp, dorei (Barkworth \& J. Maze) Barkworth] FNA, RBS

[Achnatherum ne konii (Scribn.) Barkworth] - SF!. W\&W', KTZ

[Stipa columbiana Macoun var, columbiana] - HDH, IMF

[Stipa nelsonii Seribn, var, dorei (Barkworth \& J. Maze) Dorn] - EW

Achnatherum nelsonii (Scribn.) Barkworth subsp. nelsonii - FNA. RBS

[Achuatherum nelsonii (Scribn.) Barkworth] - SFI, W \& W'

[Achnatherum nelsonii (Scribn.) Barkworth var. nelsonii] - Dom0!

[Stipa columbiana Macoun var. nelsonii (Scribn.) Hillche.] - HDH

[Stipa columbiana Macoun var. nelsonii (Stribn.) S1. Joha] - IMF

[Stipa nelsoniii Scribn.] - WEW

[Stipa nelsoniii Scribn. subap. nelsontii] - UTF

[Stipa nelsonii Seribn. var, nelsonii] - Hurtman06. E/W

[Stipa williamsii Scribn.] - HDH. WNW

Achnatherum nevadense (B. L. Johnson) Barkworth - Dorn01, KT7

[Stipa nevadensis B. L. Joknson] - IMF, UTF

Achnatherum occidentale (Thurb,) Barkworth - RBS, Dom01, FNA

[Achnatherum occidentale (Thurb, ex S. Wason) Barkwonh subsp. pubescens (Vasey) Barkwortb] - KTZ, FNA

[Stipa occidentalis Thurb.] - GPF, IMF, UTF

[Stipa oecidetalits Thurb, ex S. Wasson] -W\&W, err. rep.

Achnatherum perplexum Hoge \& Barkworth - RBS, FNA

[Stipa perplera (Hoge \& Barkworth) J, Wipff \& S.D. Jenes]

Achnatherum pinetorum (M. E. Jones) Barkworth - RBS, Dorn01, KTZ, SFW, W\&W

[Stipa pinctorum M. E. Jones] - HDH, IMF, UTF, W\& w, w

Achnatherum richardsonii (Link) Barkworth - RBS. Dorn01. KTZ. SF!, W\&W'

[Stipa richardsonii Link] - GPF, HDH, W\&W, E/W

Achnatherum robustum (Vasey) Barkworth - RBS, Dorn01, KTZ, SFE, W\& W'

[Stipa robusta (Vasey) Scribo,]-GPF, HDH, WRW, E

Achnatherum seribneri (Vasey) Barkworth - RBS. Dorn01, KTZ, SF!, W\& W ${ }^{1}$

[Stipa scriberi Vascy] - GPF, HDH, IMF, W\&W, EW

Achnatherum X bloomeri (Bol.) Barkworth - FNA, KTZ, W\&W'

[Oryzopsis bloomeri (Bol.) Ricker] - GPF, HDH

[Oryzopsis hymenoides X Stipa occidentalis] - KTZ 
[Stipe X bloomeri Bol.] - UTF, EW

[Stipa bloomeri Bol.] - WaW

[X Stiporyzopsis bloomeri (BoL) B. L. Johnson] - IMF, SF!

Achnatherum thurberianum (Piper) Barkworth - Dorn01, FNA, KTZ

[Stipa thurberiana Piper] - IMF, UTF

Achnatherum webberi (Thurb.) Barkworth - RBS, KTZ, W\&W', err. rep.

[Oryzspsis webberi (Thurber) Benth.] - HDH

[Stipa webberi (Thurb.) B. L. Johnsan] - IMF, W\&W

*Aegilops cylindrica Hos - RBS, Dorn01, GPF, HDH, IMF, KTZ, UTF, EW

[Cylindropyrum cylindricum (Hos) A. Löve] - SF!, W\&W

\section{$\mathrm{X}$ Agrohordeum (see Elymus)}

\section{Agropyron (see also Elymus)}

*Agropyron cristatum (L.) Gaertn, var, cristatum - Dom01, E/W

[Agropyron cristatum (L.) Gaertn.] - GPF, IMF, UTF, W\& $W^{t}$

[Agropyren cristatum (L.) Gaertn. subsp. eristatum] - SF!, W\&W

*Agropyron cristatum (L.) Gaertn, var, desertorum (Fisch, ex Link) Dorn - Dorn01, E/

[Agropyron cristatum (L.) Gaerth] - GPF, RBS

[Agropyren cristatum (L.) Gaerth subsp. descrtorum (Fisch.) A. Löve - SF!. W\&W

[Agropyren cristatum (L.) Gaerth subsp. pectinatam (M. Bieb.) Tzvelev] - KTZ

[Agropyron desertorum (Fisch.) Schult.] - HDH

[Agropyren descrtorum Fisch. ex Link] - W\& W'

[Agropyren desertorum (Fisch. ex Lieks) Schult.] - KTZ

[Agropyron desertorum (Link) Schult.] - UTF

[Agropyton sibiricum Willd.]-see IMF

*Agropyron cristatum (L.) Gaertn, var, fragile (Roth) Dom - Dom01, E/W

[Agropyron cristalum (L) Gaerin] - GPF, UTF

[Agropyron cristalum (L., Gaerin. subsp. fragile (Roth) A. Love] - SFt, W\&W

[Agropyren cristatum (L.) Gaerin. subsp. pectinatum (M. Bieb.) Tzwelev] - KTZ

[Agropyron fragik (Roth) P. Candargy] - RBS, KTZ

[Agropyron mongolicum Keng] - W\&W'

[Agropyron pectiniforme Rosm. \& Schult.]- IMF, W\&W

*Agropyron triticeum (iacth. - Domd)1, HDH, E/W

[Eremopyrum triticeum (Gaerth.) Nerski] - RBS, IMF, SF, UTF, W\&W, KTZ

\section{Agrositanion (sec Elymus)}

Agrostis

(Identification sources:

Agrostis (sec also Polypogon)

Agrostis exarata Trin. - Dorn01, RBS, HDH, KTZ, SF!, UTF, W\&W, E/W

[Agrostis exarata Trin. var, exarata] - IMF

[Agrostis exarata Trin. subsp, minor (Hook,) C. L. Hitche,] - GPF

[Agrostis exarata Trin. var, minor Hook.] - IMF

*Agrostis gigantea Roth - RBS. KTZ, SF!, W\&W, E/W

[Agrostlis alba Li] - $\mathrm{HDH}$

[Agrostis stelonifera L.] - GPF, UTF

\{Agrostis hyemalis (Walter) Britton, Stems, \& Poggenb. - GPF $<\mathrm{CO}>$. KTZ $<\mathrm{KS}, \mathrm{NE}$, 
$\mathrm{OK}>$ W\&W, misapp.

[NS - to be expected in southeastern WY based on FNA ]

Agrostis idahoensis Nash - Dorn01. RBS, HDH, IMF, KTZ, SF! W\&W, E/W

[Agrostis bakeri Ryusto.]

[Agrostis X idahoensis Nash] - UTF

[Agrostis idahoensis Nash var. bakeri (Rydb.) W. A. Weber] - W\&W

Agrostis mertensii Trin. - Dorn01, RBS, KTZ, SFI, W\&W, EN

[Agrostis borealis C. J. Harman] - HDH, IMF, UTF

Agrostis scabra Willd - Dom01, RBS, FNA, GPF, IMF, KTZ, SF1, UTF, W\&W

[Agrostis geminata Trin.] - KTZ

[Agrostis scabra Willd.] - Dom01, GPF, IMF, KTZ, SF!, UTF, W\&W

[Agrostis seabra Willd. var. verminata [Trin.) Swallen] - HDH

[Agrestis scabra Willd. var. scabra] - HDH, E/W, RMC

*Agrostis stolonifera L. - Dorn01, RBS, GPF, KTZ, SF!, UTF, W\&W, ENW

[Agrustis pelustris Hudson] - HDH

[Agrostis stolonifera L. var. palustris (Hudson) Farw.] - IMF

[Agrustis stolonifera L. var, stolonifera] - [MF

Agrostis variabilis Rydb, - Dorn01, RBS, HDH, IMF, KTZ, SF!, UTF, W\&W, E/W

Alopecurus aequalis Sobol. var, aequalis - KTZ, E/W

[Alopecurus acqualis Sobol.] - Dom01, RBS, GPF, HDH, IMF, SF!, UTF, W\& W

*Alopecurus arundinaceus Poir, - Dorn01, RBS, KTZ

[Alopercurus vetricosus Pers.] - UTF

Alopecurus carolinianus Walter - Dorn01, RBS, GPF, IMF, KTZ, SFE, UTF, W\&W, E

*Alopecurus geniculatus L. var, geniculatus - KTZ, E/W

[Alopecurus geniculatus L.] - RBS, GPF, HDH, IMF, SF!, UTF, W\&W

Alopecurus magellenicus Lam. - RBS, FNA

[Álopecurus alpines J. E. Sm.] - HDH, IMF, KTZ, SF, UTF

[Alopecurus alpinus J. E. Sm, subsp.] - Ws $W^{1}$, Eurasian

[Alopecurus alpinus J. E. Sm, var. alpinus] - RMC

[Alopecurus alpinus L. subsp. glaocus (Less) Huhen] - W\& W

[Alopecurus boecalis Trint]- Doma1. EWW

*Alopecurus pratensis L. - GPF, RBS, IMF, KTZ, SF!, UTF, W\&W, EJW

Andropogon (see also Bothriochloa, Schizachyrium)

Andropogon gerardii Vitman - Dorn01, RBS, FNA, GPF, HDH, IMF, KTZ, SF!, W\&W, E/W

[Andropogon gerardii Vitman var. gerardii] - UTT

Andropogon hallii Hack, - Dom0I, RBS, FNA, GPF, HDH, IMF, KTZ, SFE, W\& W, E

[Andropogon gerardit Vitman var. chrysosomus (Nash) Fernald] - UTF

Anisantha (sec Bromus)

Anthoxanthum hirtum (Schrank) Y. Schouten \& Veldkamp

[Hierochloc hirta (Schrank) Borbas subsp. arctica (J. Presi) G. Weim.] - RBSS, KTZ, SF", W\&W, E/W

*Anthoxanthum odoratum L. subsp. odoratum - RBS, FNA, KTZ

[Anthoxanthum odoratum L.] - $\mathrm{HDH}$, W\&W

[Hierochloe odorata (L.) P. Beauv.] - Dom01, GPF, HDH, IMF, KTZ, UTF, W\&W, er, rep. based on A.

hirtum

*Apera interrupta (L.) P. Beauv. - Dorn01. RBS, IMF, KTZ, SFE. UTF. W\&W. E

Argillochloa (see Festuca)

Snow, N, January 2009. Checklist of Vaccular Plants of the Southom Rocky Momain Region. CVersion $3 x$ 
Aristida

(Identification sources:

*Aristida adscensionis L. - FNA, RBS, GPF, HDH, IMF, KTZ, SFE, UTF, W\&W, E Aristida arizonica Vasey - FNA, RBS, GPF, HDH. IMF, KTZ, SFE, UTF, W\&W, E

Aristida basiramea Engelm. ex Vasey - FNA, RBS, KTZ, E

[Aristida basiramea Engelm.] - HDH, SFE, W\&W

[Aristida basiramea Engelm. ex Vasey var. basiramea] - GPF

Aristida curtissii (A. Gray) Nash - HDH. E

[Arissida basiramea Engelm.] - WEW, SFE

[Aristida basiramea Engelm. ex Vasey var. curtissii (A. Gray) Shimners] - GPF

[Aristida dichotoma Michx. var. curtissii A. Gray ex S. Watson \& J. M. Coult.] - KTZ

Aristida dichotoma Michx, - FNA, RBS

[NS - It is uncertain which variety in our area]

Aristida divaricata Humb, \& Bonpl, ex Willd. FNA, RBS, GPF, HDH, KTZ, W\&W, E

[Arissida divaricala Humb. \& BonpL] - SFE

Aristida havardii Vasey - FNA, RBS, KTZ, W\&W, E

[Aristida purpurea Nutt.]-SFE

Aristida oligantha Michx. - RBS

[Reported for Boulder County; voucher locality uncertain]

Aristida pansa Wooton \& Standl. - FNA

Aristida purpurea Nutt, var. fendleriana (Steud.) Vasey - Dom01, RBS, FNA, KTZ, EW

[Aristida fendleriana Steud.] - HDH

[Aristida purpurea Nutt.] - SF!. UTF

[Aristida purpurea Nutt. var. longiseta (Stend.) Vasey] - GPF, IMF, WAW

Aristida purpurea Nutt, var. longiseta (Steud.) Vasey - Dorn01, FNA, RBS, GPF, IMF, KTZ. W\&W, E/W

[Aristida longiscta Sread, var, longiseta] - HDH

[Aristida longiseta Steud. var, fariflora Hitchc.] - HDH

[Aristida loegiseta Steud. var. fobusta Merrill] - HDH

[Aristida purpurea Nutt.] - SF!, UTF

Aristida purpurea Nutt, var. nealleyi (Vasey) Allred - RBS, FNA, FCF

Aristida purpurea Nutt. var. purpurea - FNA, RBS, PC, GPF, IMF, KTZ, W\&W, E/W

[Arissida purpuren Nuet.] - HDH, SF1, UTF

Aristida purpurea Nutt. var. wrightii (Nash) Allred - RBS, KTZ, E/W

[Aristida purpurea Nurt.] - SF!, UTF

[Aristida purpurea Nurt. var, glawen (Nees) A. Holmgren \& N. Holmgren] - GPF, IMF

[Aristida wrightii Nash] - HDH, W\&W

Aristida temipes Cav. - FNA

*Arrhenatherum elatius (L.) J. Presl \& C. Presl var. elatius - E/W

[Arrhenatherum elatius (L.) Presi] - GPF, UTF

[Arrhenatherum elatius (L.) J. \& K. Pres]]-Dorn01

[Arrhenatherum elatius (L.) P. Beauy.] - SFE

[Arrhenatherum elatius (L.) P. Benuy, ex J. PresI \& C. Pres]] - RBS, IMF, W\&W

[Arrhenatherum elatius (L.) Mert, \& W. D. J, Koch] - SFW

[Arrhenatherum elatius (L.) Presl var, elatius] - HDH

[Arrhenatherum elatius (L.) P. Beauy, ex J. Presi \& C. Presl var, elatias] - KTZ

*Avena fatua L. - Dorn01, RBS, HDH, IMF, KTZ, SF!, E/W

Snow, N, January 2009. Checklist of Vacculer Plants of the Southam Rocky Momain Regioe. CVersion $3 x$ 
[Avena fatua L var. fatua] = GPF, UTF, W\&W

*Avena sativa L. - Dorn01, RBS, HDH. IMF, KTZ, E.W

[Avena fatua L. var. sativa (L.) Haussk.]-GPF, SF!, UTF, WEW

Avenula hookeri (Scribn.) Holub - RBS, SF!, W\&W, FNA

[Helictotrichon hookeri (Seribn.) Henrard]- Dorn01, GPF, HDH, IMF, KTZ. EW

Beckmannia syzigachne (Steud.) Fernald - Dorn01, RBS, GPF, HDH, IMF, KTZ, SFW, ENW

[Beckmannia syzigachne (Steud.) Fimald subsp. baicalensis (Kusn.) T. Koyama \& Kuwano] - SFE, W. W

Blepharoneuron tricholepis (Torr.) Nash - RBS, HDH, IMF, SF!, UTF, W\&W, E/W

[NS - P. Peterson indicates that this tavon falls into Mnhenbergia basad on TS and chloroplast sequences]

Bothriochloa

(Idertification sources:

Bothriochloa barbinodis (Lag, ) Herter - FNA, RBS, IMF, KTZ, SFW, UTF, W\&W, W

[Andropogon barbinodis Lag.] - GPF

*Bothriochloa bladhii (Retz.) S, T. Blake - FNA, RBS, KTZ, SFE, W\&W, E

[Andropogon blachii Retz.] - GPF

*Bothriochloa ischaemum (L.) Keng var. songarica (Rubr. ex Fisch. \& C. A. Mey.) Celarier \& Harlan - KTZ, W\&W, E

[Bothriochloa ischaemum (L.) Keng var, songarica (Rubr.) Celarier \& Harlan] - SFE

[Andropogon ischasmum L. var. songaricus Rupe, ex Fisch, \& C. A. Mey.]-GPF

[Bothriochloa ischaemum (L.) Keng] - FNA, RBS, UTF

Bothriochloa laguroides (DC.) Herter subsp, torreyana (Steud.) Allred \& Gould - FNA, KTZ,

SFE, UTF, W\&W, E

[Bothriochlos laguroides (DC.) Herter] - RBS

\{Bothriochloa saccharoides (Sw.) Rydb.\} - HDH (as Andropogon s.)

[NS - Our spocimens likely confused with B. laguroides fide FNA]

\{Andropogoo saccharoides Sw, - - HDH

\{Andropogoo saccharoides Sw, var, vorreyanus (Steud.) Hack\} - GPF

\{Bothriochloa laguroides (DC.) Herter subsp. torreyana (Steud.) Allred \& Gould\} - W\&W

*Bothriochloa springfieldii (Gould) Parodi - FNA, RBS, KTZ, UTF, E

[Andropogon springfieldi Gould] - GPF $\angle \mathrm{AZ}$, NM $>$

[Bothriochlos springlieldit Goald] - SFE, W' W'

Bouteloua Lag.

(Identification sources:

[NS - Bowefouc and Choudrasmo in their traditional circumscriptions are denonswably polyphyletic and the contimued recognition in the narrower sense of either genas is no konger justifiable (Columbus98; Columbas99)]

Bouteloua barbata Lag. - FNA, GPF. HDH, IMF, KTZ, E/W

[Bouteloua barbata Log, var, barbata] - UTF

[Chondrosum barbatum (Lag, Claytoo] - SF!. RBS, W\&W

Boutelou curtipendula (Michx.) Torr. var. cacspitosa Gould \& Kapadia - FNA, RBS, GPF, KTZ. W\&W. ENW

[Bouteloua curtipendula (Michx.) Ton-] - SF!

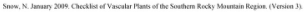


[Bouteloua curtipendula (Michx.) A. Gray var. caespitasa Gould \& Kapadia] - IMF

Bouteloua curtipendula (Michx.) Torr. var. curtipendula - RBS, GPF, KTZ, W\&W, E/W

[Bouteloua curtipendula (Michx.) Torr.] - HDH, SF!, UTF

[Boutekua curtipendula (Michx.) A. Gray var. curtipendula] - IMF

Bouteloua dactyloides (Nutt.) J. T. Columbus - Columbus98; Columbus99

[Buchlot dactyloides (Nutt.) Engelm, - Dorn01, FNA, RBS, GPF, IMF, W\&W, E

[Buchlod dactyloides Engelm.] - SFE

[Buchloe dactyloides (Nutt.) Engelm.] - HDH, KTZ, UTF

[Bulbiles dactyloides Raf.]- TROPICOS

[Calanihera dactyloides Kunth] - TROPICOS

[Lasiostega humilis Rups. ex Benth.] - TROPICOS

Bouteloua eriopoda (Torr) Torr, - Dorn01, FNA, GPF, HDH, IMF, KTZ, UTF, E

[Chondrosum eriopodum Torr.] - SFE, RBS, W\&W

Bouteloua gracilis (Kunth) Lag. = HDH

[Boutekua gracilis (Kunth) Lag ex Griffitho] - Dom01, FNA, GPF

[Bouteloua grasilis (Kunth) Lag ex Steud.] - IMF, UTF

[Boutekua gracilis (Kunth) Lag. var. gracilis] - E/W

[Bouteloua grasilis (Willd. ex Kunh) Lag. ex Griffiths] - KTZ

[Chondrosum gracile Kunth] - SF!, RBS, W\&W

Bouteloua hirsuta Lag, var, hirsuta - FNA, KTZ, E

[Bouteloua hirsula Lag.] - Dorn0I, GPF, HDH. IMF, UTF

[Chondrosum hirsutum (Lag.) Swee - SFE, RBS, W\& W

Bouteloua simplex Lag, - FNA, GPF, HDH, IMF, KTZ, UTF, ENW

[Chondrostam prostratum (Lag.) Sweet] - SF?, W\& W

[Chondrosum simplex (Lag.) Kuntb] - RBS

[Bouteloua prostratum Lag.] - W\&W

*Brachypodium distachyon (L.) P. Beauv, - HDH, RBS, KTZ, W\&W

Briza

[NS - No carrent evidence suggests that these two species are established in our region]

*\{Briza maxima L, \} - FNA, RBS, IMF, KTZ, SFW, W\&W, w

*BBriza media L.\}-FNA, RBS, KTZ, SFE, W\&W, E

Bromelica (see Melica)

Bromopsis (sce Bromus)

Bromus

[NS - See Snow07d for a recen getseric key for SRMR species]

(Identification sources:

\{Bromus anomalus Rupe. ex E. Foum.\}-Dom01, HDH, IMF, KTZ <AZ $>$, UTF, E/W

[NS - segregated from Bromus porteri in FNA Vol. 24)

$*\left\{\right.$ Bromus berteroanus Colla\} $-\mathrm{KTZ}<\mathrm{AZ}, \mathrm{UT}>$, W\& $\mathrm{W}^{\prime}$, err. rep.

\{Bromas trinii Desvatx\} - HDH, IMF, UTF, W\&W

*Bromus briziformis Fisch. \& C. A. Mey. - Dom01, RBS, FNA, KTZ, SF!, UTF, W\&W, E/W

[Bromes brizaeformis Fisch. \& C. A. Mcy.]-HDH, IMF

Bromus carinatus Hook. \& Arn. - Dom01. GPF, HDH, IMF. KTZ, UTF, E/W

[Bromus marginatus Nees] - HDH

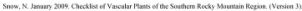


[Bromes marginatus Nees ex Steud.] - KTZ

[Bromes maryinatus Nees ex Steud. var, breviarislatus (Buckley) Beetle] - KTZ

[Ceratochlon carinata (Hook. \& AmL) Tutin] - RBS, SFE, W\& W

*Bromus catharticus Vahl - HDH, KTZ. UTF

[Bromus unioloides Kunth] - GPF

[Bromes willdenowi Kunth] - IMF

[Ceratoshlos unioloides (Willd.) P. Besuy.] - W\&W

Bromus ciliatus L. - Dorn01, GPF, IMF, UTF, E/, Peterson07

[Bcomus canadensis Michx.] - E/W

[Bromopsis eanadensis (Michx.) Holub] - SF!

[Bcomopsis canadensis (Michx.) Holub subsp. canadensis] - WE W

[Bromus ciliatus L. var. ciliatus] - $\mathrm{HDH}$

[Bcomus ciliatus L. var, richardsonii (Link) Boivin] - KTZ

[Bromes frondosus (Shear) Wooton \& Standl.] - UTF

*Bromus commutatus Schrad. - Dorn01, RBS, GPF, HDH, IMF, KTZ, SF!, W\&W, E/W

*Bromus diandrus Roth - FNA, KTZ, E/W, UTF

[Anisantha disndra (Roth) Tutin] - SF!, W\& W

[Anisantha diandra (Roth) Tutin ex Tzvelev] - RBS

[Bromes rigidus Roth] - KTZ, EW

*Bromus erectus Huds, - FNA

Bromus frondosus (Shear) Wooton \& Standl, - FNA. FCF, HDH, IMF, KTZ

[Bromopsis ciliarus complex] - WEW

[Bcomopsis frondosa (Shear) Holub] - RBS, W\& w

*Bromus hordeaceus L. subsp. hordeaceus - KTZ, E/W

[Bromers hordeaceus L.] - RBS, SFI, UTF, W\&W

[Bromes mollis L.] - IMI

*BBromus inermis Leyss, - FNA, HDH, UTF

[Bromepsis inermis (Leyss.) Holub] - RBS, SF!, W\& W

[Bromes inermis Leyss. subsp. inermis] - GPF

[Bromus inermis Leyss, subsp. inermis var, inermis] - KTZ

[Bromes inermis Leyss,var, inermis] - Dorno1, IMF, E/W

*Bromus japonicus Thunb. ex Muray - Dorn01, GPF, KTZ, UTF, E/W

[Bcomus keonicus Thunberg] - RBS, HDH, IMF, SF:, W\&W

Bromus lanatipes (Shear) Rydb. - GPF, KTZ, E/W

[Bromopsis inatipes (Shear) Holub] - RBS, SFI. W \& W

[Bcomus anomalus Ruprecht ex E. Fourn. var, kanatipes (Shear) Hitchc] - $\mathrm{HOH}$

[Bromes porteri (Coult.) Nash var, lanatipes Stosa] - Gould75

\{Bromus latiglumis (Scribn. ex Shear) Hitche.\} - to be expected in e, Wyoming

Bromus mucroglumis Wagnon - FNA, RBS

Bromus polyanthus Scribn, - FNA, HDH

[Bcomus polyanthus Seribn. var. paniculatus Shear] - KT2

[Beomes polyantlus Seribn. var, polyanthas] - KTZ

[Bromus porteri (Coult.) Nash]- KTZ

Bromus porteri (J. M. Coult.) Nash - FNA, SF!, W\&W

[Bromes porteri Rydb] - GPF

[Bromopsis pocteri (Rydb) Holub] - RBS, SFI, W\&W

Bromus pubescens Muhl. ex Willd. - Dom01. GPF. KTZ, E

[Bromopsis pulseseens (Mubl.) Holub] - SFE

[Bromopsis pubescens (Mubl. ex Willd.) Hodub] - RBS, W\& W

[Bromes ciliatus L, var, laeviglumis Scribn, ex Shear - HDH

[Bromes kalmii A. Gray] - GPF

[Bromes lariglumis Hitchc] - $\mathrm{HOH}$

[Bromes lariglumis (Scribn, ex Shear) Hitche.] - GPF

[Bromes pabescens var, laeviglumis (Scribe, ex Shear) Swallen] - Gould75 
[Bromes purgans L] = HDH, WE W'

Bromus pumpellianus Scribn. - FNA

[Bcomopsis pumpelliana (Scribn.) Holub] - RBBS, SF!, W\& W

[Bromes inermis Leyss.] - UTF

[Bromus inermis Leyss, subsp. pumpellianus (Seriben.) Wagnon] - GPF

[Bromes inermis Leyss. subsp. pumpellianes (Seribn.) Wagnon var. pumpellianus] - KTZ

[Bromus inermis Leyss. var. pumpellianus (Scribn.) C. L. Hitche.] - EWW

[Bromus pumpellianus Scribn. var- pumpcllianus] - HDH

[Bromus pumpellianus Scribn. var. tweedyi Scribn.] - HDH

[Bromes inermis Leyss. subsp. pumpellianus (Seribn.) Wagnon var. pumpellianes] - KTZ

[Bcomus inermis Leyss. var. purpurascens (Hook.) Wagnon] - Dern01. IMF

*Bromus racemosus L. - RBS, HDH, KTZ,SF1, W\&W, EW

[Bcomus bordeaceus L.] - UTF

[Bromus mollis L.] - GPF, IMF

Bromus richardsonii Link - FNA, Peterson07

[Bromopsis rikhardsonii (Link) Holub] - RBS

*Bromus rubens L. - FNA, FCF

*Bromus secalinus L. - Dom01, RBS, GPF, HDH, IMF, KTZ, SF!, UTF, W\&W

[Bromus scealinus L. var. secalinas] - EN

*Bromus squarrosus L. - Dorn01, RBS, GPF, KTZ, SF!, W\&W, EJW

*Bromus sterilis L. - HDH, IMF, KTZ, UTF, E/W

[Anisantha sterilis (L.) Nevski] - RBS, SFI, W\&W

*Bromus tectorum L. - Dom01, GPF, KTZ, UTF, E/W

[Anisantha tectorun (L.) Nevski] - RBS, SF!, W\&W

[Bcomes tectorum L. var. glabratus Spcrner] - HDH, IMF

[Bromes tecrorum L. var, toctonem] - HDH, IMF

\section{Buchloè (see Bouteloua)}

Calamagrostis

(Identification sources:

Calamagrostis canadensis (Michx.) P. Beauy, var, canadensis - FNA, KTZ, E/W

[Calamagrostis canadensig (Michx.) P, Benuv] - Dom01, RBS, GPF, HDH, IMF, SF!, UTF, W\&W

[Calamagrossis seribneri Beal]- HDH, EN

Calamagrostis canadensis (Michx.) P. Beauy, var. langsdorfii (Link) Inman - FNA, KTZ, W\&W, Calamagrostis cansdensis (Michx.) P. Beauv, - GPF, UTF

Calsmagrostis langsdoefii (Link) Trin. - w\& $w^{1}$, misapp.

Calamagrostis montanensis (Scribn.) Scribn- Dorn01, RBS, FNA. GPF. HDH, IMF. W\&W

[Calamagrostis montantensis (Seribo.) Seribn. ex Yasey] - EW

[Calamagnostis montanensis Seribn.]-SFI

[Calamagrost ts montanetris Seribn, ex Vusey] - KT2

Calamagrostis purpuraseens R. Br. - Dom01, RBS, FNA, GPF, HDH, IMF, SF!, UTF, W\&W [Calamagrostis purpuraseens R. Br. var, purpurascens] - KTZ. E W

Calamagrostis rubescens Buckley - Dorn01. RBS, FNA, HDH, IMF, KTZ, SFW, UTF, W\&W. W

Calamagrostis scopulorum M. E. Jones - Dorn01, RBS, FNA, HDH, KTZ, SFW, W\&W, W

Calamagrostis stricta (Timm) Koeler subsp, inexpansa (A. Gray) C. W. Greene - FNA, KTZ

[Calamagrostis inexpansa A, Gray] - Dorno1, HDH, EW

[Calamagrostis neglecta (Erhr,) P. Gaetn., B. Mey, \& Serib.] - HDH, IMF

[Calamagostis stricta (Timm) Koeler] - GPF, SFI, UTF, WEW

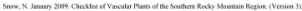


Calamagrostis stricta (Timm) Koeler subsp, stricta - FNA

[Calamagrostis stricta (Timm) Koeler var. stricta] - E/W

[Calamagrostis neglecta (Ehrh.) P. Gaerin., B. Mcy., \& Seherb.] - IMF

[Calamagrostis stricta (Timm) Koeler] - Dorn0I, GPF, SF!, UTF, W\&W

[Calamagrastis stricta (Timm) Koeler subsp. stricta var. stricta] - KTZ

Calamovilfa gigantea (Nutt.) Scribn. \& Merr. - FNA, RBS, GPF, HDH, IMF, KTZ, SF!, UTF, W\&W, E/W

Calamovilfa longifolia (Hook.) Scribn. var, longifolia - KTZ, E

[Calamovilfa lengifolia (Hook.) Seribn.] - Dorn01, FNA, RBS, GPF, HDH, IMF, SFE, W\&W

Catabrosa aquatica (L.) P. Beauv, var, aquatica - KTZ, E/W

[Catabrosa aquatica (L.) P. Beaur.] - Dom01, GPE, HDH, IMF, SF!. UTF, W\& W

*Cenchrus echinatus L. - FNA

Cenchrus longispinus (Hack.) Femald - Dom01, RBS, FNA, GPF, IMF, KTZ, SF!, UTF, W\&W, E/W

Cenchrus spinifex Cav, - FNA, RBS, KT7

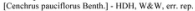

Ceratochloa (see Bromus)

Chloris verticillata Nutt, - Dom01, FNA, RBS, GPF, HDH, KTZ, SF!, UTF, W\&W, ENW

Chloris virgata Sw. - Dornol, FNA, RBS, GPF, HDH, IMF, KTZ, SF!, UTF, W\&W, E/W

Chondrosum (see Bouteloua)

Cinna latifolia (Trev. ex Goepp.) Griseb. - Dorn01, GPF, IMF, KTZ, E/W

[Cinna latifolia (Trev,) Griseb.]-HDH, SF!, W\&W

Critesion (sce Hordeum)

*Crypsis alopecuroides (Pill. \& Mitterp.) Schrad. - Dorn01, FNA, RBS, KTZ, SFE, UTF, W\&W, E

Cylindropyrum (see Aegilops)

*Cynodon dactylon (L.) Pers. var. dactylon - FNA, E/W

[Cynodon dactylon (L.) Pers.] - GPF, RBS, HDH, IMF, KTZ, SF, UTF, W\&W

*Cynosurus cristatus L. - HDH, KTZ, W\&W

wCynosurus echinatus L. - Hartman06

*Dactylis glomerata L. - Dorn01, GPF, HDH, IMF, SF!, UTF, W\&W, E/W

[Dactylis glomerata L. subsp. glomerata] - KTZ

* Dactyloctenium acgyptium (L.) Willd, - KTZ, FNA, RBS

[Dactyloctenium aegyptiuns (L) K, Richs.] - HDH

[Dactyloctenium aegyptium (L.) P. Beauv.] - UTF

Snow, N. Janury 2009. Checklist of Vaccular Plants of the Southom Rocky Momain Region. CVersion $3 x$ 
Danthonia californica Bol, - Dom01, RBS, FNA. HDH, IMF, KTZ, SF!, UTF, W\&W, E/W Danthonia intermedia Vasey - Dorn01, RBS, FNA, GPF, HDH, IMF, KTZ, SFI, UTF, W\&W, E/W

Danthonia parryi Scribn. - Dom01, RBS, FNA, HDH, KTZ, SF!, W\&W, E/W

Danthonia spicata (L.) P. Beauv, ex Roem, \& Schult, - Dorn01, RBS, FNA, GPF, KTZ, E [Danthonia spicata (L.) P. Bcauv.] - SFE

[Danthonia spicata (L.) P. Beauv. ex Roem. \& Schult. var. pinetorum Piper] - HDH, W\&W

Danthonia unispicata (Thurb.) Munro ex Macoun - FNA, IMF, KTZ, UTF, W

[Danthonia unispicata (Thurb.) Macoun] - GPF

[Danthonia unispicata (Thurb.) Munro] - HDH, SFW, W\&W

[Danthonia unispicata (Thurb.) Munre ex Vasey] - RBS

Dasyochloa pulchella (Kunth) Willd, ex Rydb, - FNA, RBS, KTZ, W\&W

[Dasyochloa pukchella (Kunth) Rydb.] - SFW

[Erioneursen pelchellum (Kumth) Tateoka] - IMF, UTF, W

[Tridons pulchellus (Kunth) Hitche.] - HDH

Deschampsia (see also Vahlodea)

Deschampsia brevifolia $\mathrm{R}$. Br, - FNA

Deschampsia caespitosa (L.) P. Beauv, - Dorn01, KTZ, UTF

[Deschampsia cespitosa (L.) P. Beaur.] - IMF, SF!

[Deschampsia cacspitosa (L.) P. Beauv, subsp. caespitosa] - HDH

[Deschampsia cespitosa (L.) P. Beavy. var. cospitosa] - GPF. EIW

[Deschampsia cespitosa (L.) P. Beauv, subsp. alpicola (Rydlo.) A. Löve, D. Love, \& B. M. Kapoor] - W\&W. SFE

[Deschampsia cospitosa (L.) P. Beaur. subsp. cespirosa] - W\&W

[Deschampsia cospitosa (L.) P. Beauv. subsp. glauca (Hartman) Hartman] - KTZ

$\div$ Deschampsia elongata (Hook.) Munfo-Dom0l, FNA

$\div$ Deschampsia flexuosa (L) Trin. - FNA

[Avenetla flexuosa (L.) Orejer] - Clsiapelato

Dichanthelium

(Identification sources:

Dichanthelium acuminatum (Sw,) Gould \& C. A. Clark var-acuminatum - GPF, KTZ, W\&W, E

[NS - infraspecific taxa likely artificial; doctoral studies in progeress by Rick Hammer an Tevas A \& M and others ar North Carol ina State University]

[Dichanthelium acuminatum (Sw.) Gould]-Domol

[Dichanthelium acum inatum (Sw.) Freckmann var, sericeum (Schmol]) Frockmann] - w\&w'

[Dichanthelium acuminatum (Sw) Gould \& C. A. Clark] - RBS, SFE, W\&W]

[Pasicum acumitutum Sw.] - UTF

Dichanthelium acuminatum (Sw.) Gould \& C. A. Clark var. fasciculatum (Torr.) Freckmann - KTZ, W

[Dichanthelium acuminatum (Sw.) Freclsmann] - SFW

[Dichanthelium acuminatum (Sw.) Goeld \& C. A. Clark var, acuminatum] - GPF. W\&W

[Dicanthelium acuminatum (Sw, ) Frechmann var, fascicalatum (Torr, Frechmann] - SFW

[Dicanthelium lanuginosun (Elliott) Gould var, fasciculatum (Torr, Spellenb.] - IMF

[Pasicum haikhucae Ashe] - $\mathrm{HDH}$

[Panicum teanesseense Ashe] - HDH

Dichanthelium acuminatum (Sw.) Gould \& C. A. Clark var. sericeum (Schmoll) Freckmann 
-KTZ, W\&W, W

[Dichanthelium acuminatum (Sw.) Gould \& C. A. Clark var. seriocum (Schmoll) Freckmann \& Lelong] - FNA

[Dichanthelium acuminatum (Sw.) Frechmann] - FNA, KTZ

[Dichanthelium acuminatum (Sw.) Frechmann var. sericeum (Schmoli) Frochmann] - SFW

[Dichanthelium lanuginosum (Elliott) Gould var. sericeum (Schmoll) Freckmann] - IMF

[Dichanthelium lanuginosum (Elliot) Gould var, sericeum (Schmoll) Spellenb.] - IMF

Dichanthelium acuminatum (Sw.) Gould \& C. A. Clark var. thermale (Bol.) Freckmann - KTZ

Dichanthelium depauperatum (Muhl,) Gould - Dorn01, FNA, GPF, KTZ

Dichanthelium linearifolium (Scribn.) Gould - Dorn01, RBS, FNA, GPF, SFE, W\&W, E

[Dichanthelium linearifolium (Scribn. ex Nash) Gould] - KTZ

[Panicum perlongum Nash] - HDH

Dichanthelium oligosanthes (Schult.) Gould var. scribnerianum (Nash) Gould - Dorn01, GPF,

[MF, KTZ, SFE, W\&W, E

[Dichanthelium oligosanthes (Schull.) Gould] - RBS

(Panicum oligosanthes Sctrult. var. scribnerianum (Nash) Freckmann \& Lelong - FNA

[Panicum oligosanthes Schult. var. scribnerianum (Nash) Gould] - UTF

[Panicur scribnerianum Nash] - $\mathrm{HDH}$

Dichanthelium wilcoxianum (Vasey) Freckmann - Dorn01, FNA, KTZ, SFE, W\&W ${ }^{1}$, E

[Dichanthelium oligosanthes (Sthult.) Gould var, scribnerianum (Nash) Gould] - W\& W

[Dichanthelium oligosanthes (Schult.) Goukd var. scribnerianum (Nush) Gould] - KTZ

[Dichanthelium wikcoxianum (Vasey) Freckmann] - RBS, GPF

[Panicun wilcoxianum Vasey]- $\mathrm{HDH}$

Digitaria ealifornica (Benth.) Henrard - FNA, RBS, KTZ, SFE, W\&W, E

[Trichachse califomica (Benth.) Chase] - GPF, HDH

* Digitaria ischaemum (Schreb.) Schreb. ex Muhl, - Dom01, IMF, KTZ, UTF, W\&W

[Digitaria ischsemum (Schreb.) MuhL] - FNA. RBS. HDH

[Digitaria ischocenum (Schrob.) Schreb.]- SFE

[Digitariat ischocmum (Schreb) MuhL. var, ischaemum] - E

[Digitaria ischacmum (Schreb, ex Schweigg.) Schreb ex Mulal.] - GPF

Digitaria pubiflora (Vasey) Wipff - FNA

Digitaria sanguinalis (L.) Scop, - Dorn0I, RBS, FNA, GPF, HDH, IMF, KTZ, SF!, UTF, W\&W, ENW

\section{Diplachne (sec Leptochloa)}

Distichlis stricta (Torr.) Rydb. - Dorn01, HDH, SF!, W\&W

[Distichlis spicata (L.) Greene] - KTZ, RBS,UTF, FNA

[Distichlis spicata (L.) Greene var. stricta (Torr.) Seribn.] - 1MF, E/W

[Distichlis spicata (L.) Greene var, stricta (Torr, Beetle] - GPF

*Echinochloa colona (L.) Link - FNA, RBS

[Panicun colonam L.] - RBS

*Echinochloa crus-galli (L.) P. Beauv. var. crus-galli - FNA, HDH, E/W

[Echisochloa crus-galli (L) P. Beauv, - Dorn01, RBS, GPF, IMF, KTZ, SF, UTF, WaW

*Echinochloa crus-pavonis (Kunth) Schult. var. macra (Wiegand) Gould - FNA

[Echinochloa crus-galli fL-) P, Bcaurv, - IMF, UTF, W\&W

[Echinochloa crus-galli (L.) P. Beaur, var. zelayensis (Kunth) Hitche] - $\mathrm{HDH}$

[Echinochloa crus-pavcois (Kueth) Scheilt, var, macera (Wiegand) Gould - GPF, KTZ

*Echinochloa frumentacea Link - FNA, RBS, KTZ, W\&W

[Echisochloa crus-galli (L)) P. Beauv.] - UTF, W\&W

[Echinochloa crus-galli (L), P. Beauv, ver, frumentacea (Roxburgh) W, F, Wight] - HDH

Snow, N, Junury 2009. Checklist of Vaccular Plants of the Southom Rocky Momain Region. CVersion $3 x$ 
Echinochloa muricata (P. Beauv, Fernald var, microstachya Wiegand - Dorn01, FNA, GPF, KTZ

[Echinuchloa crus-galli (L.) P. Bcauv.] - IMF, UTF, W\&W

[Echinochloa crus-gallii (L.) P. Beauv. var, mitis (Pursh) Peterm.] - HDH

[Echinochloa muricata (P. Beauv.) Fernald.] - RBS

*Eleusine indica (L.) Gaertn, - FNA, RBS, GPF, HDH, IMF, KTZ, SF!, UTF, W\&W, EW

X Elyhordeum (see Elymus)

X Elyleymus (see Elymus)

Elymus L.

(Identification sources:

INS - While many recent authors recognize generic segregates (Kellogg89, Masont-Gamer01, FNA Vol. 24). Seberg98 questions clissical genome analysis on which some generd are based. In my vicw a less subjostive division of Elywas into demonstrably monophyletic gencra awails further data]

Elymus albicans (Scribn. \& J, G. Sm.) A. Löve var, albicans - Dorn01, E/W

[Agropyron albicans Scribn. \& J.G. Sm. - $\mathrm{HDH}$

[Agropyren albicans Scriton. \& J, G. Sm. var. albicans] - IMF

[Elymus albicans (Scribn. \& J. G. Sm.) A. Löwe] - RBS, KTZ

[Elymeas lanceolatus (Seriben. \& J. G. Sm.) Gould] - SF!, UTF, w\& W

[Elymes lanceolatus (Scribn. \& J. G. Sm.) Gould subsp. albicans (Scribn, \& J. G. Sm.] Barkworth \& D. R. Deweyl-FNA

Elymus albicans (Scribn. \& J. G. Sm.) A. Löve var, griffithsii (Scribn. \& J, G, Sm, ex Piper)

Dorn - Dorn01, E/W

[Agropyron albicans Scrion. \& J. G. Sm. var, griflithsii (Scribn, \& J. G. Sm.) A. A. Beetle]- IMF

[Agropyren griffithsii Scribn. \& J. O. Sm.]-HDH

[Elymus albicans (Scribn. \& J. G. Smi) A. Libve] - KT2

[Elymus lanceolatus (Seribn. \& J, G. Sm.) Gould] - SFI, UTF, W\&W

[Elymus lanceolatus (Seribn. \& J. G. Sm. Gould subsp. albicans (Seribe, \& J. G. Sm.) Barkworth \& D. R. Dewey] - FNA

Elymus ambiguus Vasey \& Scribn. - IMF

[Elymes ambigurs Vascy \& Scribn, var.ambiguns] - HDH, E/W

[Elymas ambiguas Vasey \& Seribn, var. strigosus (Rydb.) Hitche.] - HDH, rep.

[Elymes salinus M. E. Jones] - UTF

[Leymus ambiguus (Vasey \& Scribn.) D. R. Dewey]-RBS, FNA, KTZ, SF!, W\&W

Elymus X aristatus Merr.

[Elymus elymoides X E. riticoides] - KTZ

[X Elyloymus aristatus (Merr.) Barkworth \& D. R. Dewesy - KTZ, FNA

[X Elysitanion aristatum (Merr.) Bow den] - IMF

Elymus arizonicus (Scribn. \& J. G. Smith) Gould - RBS, FNA, FCF

Elymus bakeri (E. Nels.) A. LBve - RBS, KTZ

[Agropyron bakeri E. Nelson] - HDH

[Elymus trachycaulas (Link) Gould ex Shinners subsp. bakeri (E. Nelson) A. Lowe] - WAW

Elymus canadensis L. - RBS, GPF, IMF, KTZ, SF!, UTF, W\&W

[Elymus canadensis L. var, canadensis] - Dorn01, FNA, HDH, E/W

[Elymus canadensis L. var, brachystachys (Serihn. \& C. R. Ball) Farw.] - FNA, HDH

[Elymus canadensis L. var, hirsutus (Farw, Doce] - Domol

[Elymus canadensis L. var, robustus (Seribn. \& J, G. Sm.) Mack, \& Bush] - FNA. HDH

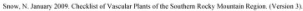


Elymus cinereus Seribn. \& Merr. - Dorn01, GPF, HDH, UTF, EW

[Elymus cinenus Seribn. \& Merr. var. cinereus] - IMF

[Elymus cinereus Seribn. \& Merr, var. pubens (Piper) C. L. Hitche.] - IMF

[Leymus cinereus (Scribn. \& Merr.) A. Löve] - RBS, KTZ SF!, W\& W

*Elymus elongatus (Host) Runem. var, elongatus - RMC

[Agropyron elongatum (Host) P. Beauv.] - GPF, HDH, IMF

[Elymes elongatus (Host) Runern.] - UTF

[Elytrigia elongata (Host) Nevski] - FNA

[Thinopyrum ponticum (Podp.) Barkworth \& D. R. Dewey] - W \& W

[Thinopyrum ponticum (Podp.) Z. W. Liu \& R. C. Wang] - KTZ

*Elymus elongatus (Host) Runem. var, ponticus (Podp.) Dorn - E/W

[Agropyron elongatum (Host) P. Beauv.]-HDH

[Elymus elongatus (Host) Runem. - - UTF

[Elytrigia pontica (Podp.) Holub] - FNA

[Thinopyrum ponticum (Podp.) Barkworth \& D. R. Dewey]- SF!, W \&W

[Thinopyrum ponticum (Podp.) Z W. Liu \& R. C. Wang] - KTZ

Elymus elymoides (Raf.) Swezey var, brevifolius (J. G. Sm.) Dom - Dorn01

[Elymus elymoides (Raf.) Swezoy] - UTF, W\&W

[Elymes elymoides (Raf) Swerey sabsp. brevifolius (J.G. Sm.) Barkworth] - RBS, KTZ

[Silanion hystrix (Nutt.) J. G. Sm.] - HDH

[Sitanion hystrix (Nutt.) J. G. 5 m. var. brevifolium (J. G. Sm_) C. L. Hitche.] - GPF, IMF

[S itanion longifolium J.G. Sm.] - E, KTZ

Elymus elymoides (Raf.) Swezey var. elymoides - Dorn01, E/W

[Elymes elymoides (Raf.) Swezey] - SF!, UTF, W\&W

[Elymus elymoides (Raf.) Swexey sabsp. elymoides] - RBS, KTZ

[Elymus longifolius (J. G. Sm.) Gould] - SF!. W\&W

[S itanion hystrix (Nuet) J. G. Sm.] - HDH

[Sitanion hystrix (Nutr. J. G. Sm. var. hystrix] - IMF

Elymus giganteus Vahl,- Schiebout08

[Elymus arenarius var. gignnteus (Vahl) Schmalh. - KTZ

[Elymus racemosus Lam.] - KTZ

[Leymus giganteus (Vahl) Pilger] - KTZ

[Leymus racemosus (Lam.) Tzvelev] - RBS, FNA

\{Elymus glabriflonus Vasey ex L. H. Dewey) Scribn. \& C. R. Ball\} - FNA

[NS - to be expected entering $\mathrm{e}$. CO from westem KS; as synonym of $\mathrm{E}$. virginicus var, virginces in KTZ)

Elymus glaucus Buckley var. glaucus - IMF, UTF, EJW

[Elymus glaucus Buckley] - Dorn01, GPF, HDH, SF], W\& w

[Elymus glaucus Buckley sabsp, glaucus] - KTZ

Elymus X hansenii Seribn. - KTZ, UTF

[Elymus glaucus X E. elymoides] - KTZ

[X Elysitanion hansenii (Scribn.) Bowden] - IMF

$\mathrm{X}$ Elymus hirtiflorus Hitche.

[Elymes trachyceusis X E. inovatus] - KTZ

[Elyleymus hintiflorus (Hitche,) Barkworth] - FNA, KTZ $<$ WY $>$

[Elymes innovatus Beal var. innovarus] - RMC

*Elymus hispidus (Opiz) Melderis var hispidus - Dorn01, E/W

[Agropyron intermedium (Host) P. Beauv.] - HDH

[A eropyron intermedium (Host) P, Beauv, var, intermedium] - GPF, IMF

[Elymus hisoidas (Opiz) Melderis] - UTF

[Elytrigia intermodia (Host) Nevski] - FNA

[Thinopyrum intermediam (Host) Barkworth \& D, R. Deacy] - RBS, FNA, KTZ, SFI

[Thinopyrum intermedium (Host) Barkworth \& D, R. Dewey subsp, intermedium] - W \&W

*Elymus hispidus (Opiz) Melderis var, ruthenicus (Griseb.) Dom - Dorn01, E/W

[Agropyroe intermedium (Host) P. Beast, var, trichephorem (Link) Halac,] - GPF, IMF 
[Agropyren trichophorum (Link) K. Kicht.] - HDH

[Elymus hispidus (Opiz) Melderis] - UTF

[Elytrigia intermedia (Hast) Nevski] - FNA

[Thinopyrum intermedium (Host) Barkworth \& D. R. Dewey] - FNA. KTZ

[Thinspyrum intennediam (Host) Barkworth \& D. R. Dewey subsp. Barbulata (Schur) Barkworth \& D. R. Dewey] - SFW

[Thinopyrum intermedium (Host) Barkworth \& D. R. Dewey subsp. barbulatum (Schur) Barkworth \& D. R. Dewey] - SFE, W\&W

*Elymus junceus Fisch, - Dorn01, GPF, IMF, UTF, E/W

[NS - the inclusion of Agropyron riparium Scribn. \& J. G. Sm. in synonymy for HDH in Snow04a cannot be correct give number of florests per spikelet indicaled in HDH]

[Psathyrostachys juncea (Fisch.) Nevski] - RBS, FNA, KTZ, SF!, W\&W

\{Elymus interruptus Buckley\} - FNA

(NS - recognitico being withheld given uncerlainty of species status: see FNA 24: 306)

Elymus lanceolatus (Scribn. \& J. G. Sm.) Gould var, lanceolatus - Dorn01, E/W

[Agropyron dasystachyum (Hook.) Scribn.]- HDH

[Agropyron dasystachyum (Hook.) Scribnt. var. dasysachaum] - GPF, IMF

[Elymes lanceolatus (Scribn. \& J. G. Sm.) Gould] - SF:, UTF, W\& W

[Elymus lanceolatus (Scribn, \& J. G. Sm.) Gould subsp. lanceolatus] - RBS, KTZ

Elymus lanceolatus (Scribn. \& J. G. Sm.) Gould var, riparius (Scribn. \& J. G. Sm.) Dom

- Dorn01

[Agropyoe dasystactiyum (Hook.) Scribn. var, riparium (Scribn. \& J. G. Sm.) Bowden] - GPF, IMF

[Agropyon riptrium Seribn. \& J. G. Sm.] - HDH

[Elymus lanceolarus (Scribn. \& J. G. Sm.) Gould] - UTE, W\& W

[Elymers lanceolatus (Scribn. \& J. G. Sm.) Gould subsp. lanceolanus] - KTZ

Elymus X macounii Vasey - Dorn01, E

[Elymus tracbycaulas X Hondcum jubatum] - KTZ

[X Agrohordeum msicounii (Vascy) Lepage] - GPF, IMF, SFE

[Elymus macounii Vasey] - FCF, HDH, W\&W

[X Elyhordeum macounti (Vasey) Barkworth \& D. R. Dewey] - Dom01, KTZ, UTF

Elymus multisetus M. E. Jones - FNA, KTZ, W

[Elymes multisctus (J. G. Sm. Davy] - RBS. SFW, W\& W

[S itanion jubutum J.G. Sm.] - IMF

* Elymus ponticus (Podp.) N. Snow - Snow07e

[Agropyron elongatum (Host) Benuv_] - IMF, KTZ

[Agropyron vamense (Velen.) Hayek] - KTZ

[Elymus elongatus (Host) Runemark] - KTZ

[Elymus elongatus (Host) var, ponticus (Podp) Dom] - Dom01, KT2

[Elymus vamense (Velen.) Runemark] - KTZ

[Elyurigia elongata (Host) Nevski] - KTZ

[Elytrigia pontica (Podp.) Holub] - KTZ

[Lophopyrum slongatyum (Host) A. LWve] - KTZ

[Thinopynam ponticum Barkworth \& D. R. Dewey] - RBS

[Thinopyrum ponticum (Podp.) Z.-W, Liu \& R-C. Wang] - KTZ

[Triticum elongaum Podp.] - IMF

Elymus X pseudorepens (Scribn \& J. G. Sm.) Barkworth \& D. R. Dewey - FNA, KTZ, UTF

[Elymess lanceolatus X E. trachycaulus] - KTZ

[Aeropyron X pseudorepens Scribn, \& J, G. Sin.] - IMF

[A gropyron psetudorepeas Scribn. \& J. G. Sm. var. magnum Scribn. \& J, G. Sm.] - HDH

[Agropyrou pseudorepeos Seribn. \& J, G. Sm. var, pseudorepens]- HDH

[Elytrigia repents (L.) Nevski] - W\&W

* Elymus repens (L.) Gould - RBS, Dom01, HDH, KTZ, UTF, E/W

[Agropyron repens (L.) P, Beauv] - GPF, HDH, IMF

[Elytrigia repens (L.) Nevski] - SFt, W\&W

[Elytrigia repens (L.) Desy, ex B, D. Jackson var, repens] - FNA 
Elymus salinus M. E. Jones - Dorn01, HDH, UTF, W

[Elymus salina M. E. Jones] - IMF

[Leymus salina (M. E. Jones) A. Löve] - RBS, SFW, W\& W

[Leymus salina (M. E. Jones) Barkworth sbusp. salina] - FNA

[Leymus salinus (M. E. Jones) A. Löve subsp. salinus] - FNA, KTZ

Elymus X saundersii Vasey - Donr01, FNA, KTZ, UTF

[Elymes elymoides X E. trachycaulus] - KTZ

[Aeropyron saundersii (Vasey) Hitche.] - HDH

[X Agrositan ion saundersii (Vasey) Bowden] - IMF

[Elymus saundersii Vasey] - W\&W

Elymus X saxicolus Scribn, \& J, G, Sm, ex Seribn. - Dom01

Elymus elymoides X E. spicatus - KTZ

[X Agrositanion saxicola (Seriba. \& J. G. Sm.) Bewden] - IMF

[Elymus saxicola Scribn. \& J. G. Sm.] - KTZ, W\& W

[Elymus X saxicolus Scribn. \& J. G. Sm.]-UTF

[X Pseudelymus saxicola (Scribn. \& J. G. Sm.) Barkworth \& D. R. Dewey] - FNA, KTZ

Elymus scribneri (Vasey) M. E. Jones - RBS, Dorn01, KTZ, SF!, UTF, W\&W, E/W

[Agropyron scribneri Vascy] - HDH, IMF

Elymus scribneri X E. trachycaulus - FNA

[Agropyron subsecundum (Link) Hitche, var. andinum (Scriba. \& J. G. Sm.) Hitche.]- HDH

[Agropyren trachycaulum (Link) Malte var. glaucum (Pease \& Moore) Malte] - IMF

[Elymus tractycaulus (Link) Gould subap. andinus (Scribn. \& J. G. Sm.) A. Lowe \& D. Love] - SF!, W\& W

[Elymus trachy caulas (Link) Gould ex Shinners var. andinus (Scribn. \& J. G. Sm.) Dorn] - Dorno1, E/W

[Elymes trachy caulus (Link) Gould ex Shinners] - UTF

[Elymus trachycaulus (Link) Gould ex Shinners subsp. andinus (Scribn. \& J. G. Sm.) A. Löve \& D. Läve] WEW

[Elymus trachycaulas (Link) Gould ex Shimers subsp. Irachycaulus] - KTZ

Elymus simplex Scribn. \& T. A. Williams ex Seribn. var. luxurians Scribn. \& T. A. Williams ex Scribn, - Dorn01

[Elymus simplex Scribn. \& T. A. Williams] - IMF

[Elymes triticoides Buckley var. simplex (Scribn. \& T. A. Williams) C. L. Hitelx.]-HDH

[Leymus simplex (Scribn, \& T. A. Williams) D. R. Dewey] - KTZ

Elymus simplex Scribn. \& T. A. Williams ex Scribn. var. simplex - Dorn01, FNA

[Elymus simplex Scribe. \& T. A. Williams] - GPF, 1MF, UTF

[Elymus triticoides Buckley var. simplex (Scribn. \& T. A. Williams) Hitchc.] - HDH

[Leymus simplex (Seribn. \& T. A. Williams) D. R. Dewey] - KTZ

[Leymus triticoides (Buckley) Pilg.] - RBS, W\&W

Elymus smithii (Rydb.) Gould - Dorn01, UTF, E/W

[Agropyrou smithii Rydb.] - GPF

[Agropyron smithii Rydb, var. molle (Scribn. \& J. G. Sm.) M. E. Jons] - HDH

[Agropyren smithii Ryob, var, palmeri (Scribn. \& J. G. Sm.) A. Heller] - HDH, IMF

[Agropyron smithii Rydb, var, smithii] - HDH, IMF

[Pascopyram smithii (Rydb.) A. Löre]-KTZ. W\&W

[Pascopyram smithis (Rydb.) A. Live var, smithii] - SFE

[Pascopyram smithii (Rydb.) A. Love var, molle (Seribn, \& J, G, Sim.) Beetle] - SFE

[Pascopyram smithii (Rydb.) A. Live forma smithii] - SFW

[Pascopyram smithii (Rydb.) A. Love forma molle (Scribn, \& ), G. Sm.) Gillett \& Senn] - SFW, WRW

[Pascopyram smithis (Rydb.) Barkworth \& D. R. Dewy] - RBS

Elymus spicatus (Pursh) Gould - Dorn01, UTF, E/W

[Agropyron iscrme (Scribe, \& J.G. Sm.) Rydb.] - HDH

[Agropyron spicatum (Pursh) Scribn, \& J, G. Sm.] - GPF, HDH, IMF

[Psendoroegneria spicata (Pursh) A. Love] - RBS, SFW

[Psendoroegneria spicata (Pursh) A*. Löve subsp, inermis (Seribn, \& J. G. Sm.) A. LOVe] - KTZ, SFE, W\&W

[Pscudoroegneria spicsta (Pursh) A. Löve subsp. spicara]- KTZ, W\&W

Elymus trachycaulus (Link) Gould ex Shinners subsp. subsecundus (Link) A. Love \& D. Love

Snow, N, Janury 2009. Checklist of Vaccular Plants of tho Southom Rocky Momain Region. CVersion $3 x$ 
- KTZ, W\&W

[Agropyron caninum (L.) Beauv, subsp. majus (Vasey) C. L. Hitchc. var. uniliterale Vascy] - GPF

[Agropyron subsecundum (Link) Hithe. var. subsecundum] - HDH

[Agropyron trachycaulum (Link) Malte var. unilaterale (Cassidy) Malte] - IMF

[Elymus trachycaulus (Link) Geuld ex Shinners] - UTF

[Elymus trachy caulus (Link) Gould ex Shinners var. andinus (Seribn. \& J. G. Sm.) Dorn] - RMC

Elymus trachycaulus (Link) Gould ex Shinners var. trachycaulus - Dorn01. FNA, E/W

[Agropyron caninum (L.) Beauv. subsp. majus (Vasey) C. L. Hitche. var. majus] - GPF

|Agrepyren latiglume (Scribn. \& J. G. Sm.) Rydb.] - HDH

[Agropyren trachycaulum (Link) Malie] - HDH

[Agropyton trachycaulum (Link) Malte yar. trachycaalum] - IMF

[Elymus trachy caulas (Link) Gould - SF!

[Elymus trachy caulas (Link) Gould ex Shinners] - UTF

[Elymus trachycaulas (Link) Gould ex Shinners subsp. Irachycaulus] - RBS. KTZ W\&W

[Elymes violaceus (E. Nels.) Á. Löve] - RBS

Elymus triticoides Buckley var, triticoides - HDH, IMF, E/W

[Elymes triticoides Buckley] - Dorn0], UTF

[Elymus triticoides Buckley var, pubescens Hinchc.] - HDH rep., IMF

[Leymus triticoides (Buckley) Pilg.]- KTZ, SF!, W\& W

Elymus villosus Muhl, ex Willd. - Dorn01, GPF, KTZ

Elymus violaceus (Hornem,) Feilberg - FNA

[Elymus alaskanus (Scribn. \& Merr.) A. Lowve subsp. latiglumis (Scribn. \& J. G. Sm.) A. Lōve - KTZ

[Agropyron latiglume (Scribn. \& 3. G. Sm.) Rydo.]-HDH

[Elymes trachy caulus (Link) Gould ex Shinners subsp. andinus (Scribn. \& J. G. Sm.) A. \& D. Love] - W\& W

[Elymus trachycaulas (Link) Gould ex Shinners var. inachycaulus] - RMC

[Elymus violaceus (E. Nels.) A. Löve] - RBS

\{Elymus virginicus L, var. jejunus (Ramaley) Bush\} - FNA

INS - As of July 2007 I have bese unable to confirm this taxce for our regions stanements about distributions for the SRMR in FNA and GPF are vague. Insdequate time was available to exhaustively search larger collections in our region, e.g. COLO and RM:

Elymus virginicus\} - Dorno1

Elymus virginicus L, var, submuticus Hook, - GPF, IMF, UTF, E

[Elymes curvatus Piper] -RBS, FNA

[Elymes submuticus (Hook.) Smyth \& L. C. K. Smyth?] - KTZ

[Elymers virginicus L.] - SFE, W\&W

[Elymes virginicus L. var, australis (Scribn. \& Ball) Hitchc.]- HDH

[Elymes virginicus L. var. virginicus] - RBS. HDH

\section{Elysitanion (sec Elymus)}

\section{Elytrigia (see Elymus)}

Enneapogon desvauxii P. Beauy. - FNA, RBS, GPF, IMF, SFW, UTF, W\&W, W

[Ennsapogon desvauxil Desv. ex P. Beauv.] - KTZ

\section{Eragrostis}

[NS - For Eragrostis I recommend consalting Peterson in FNA Vol 25]

(ldentification sources:

\section{)}

*Eragrostis barrelier Daveau - FNA, RBS, GPF, HDH, KTZ, SFE, UTF, W\&W, E *Eragrostis cilianensis (All.) Vignolo ex Janch. - Dom01. FNA, RBS, KTZ 
[Eragrostis cilianensis (All.) Lutati ex Janch.] - EW

[Eragrostis cilianensis (All.) F. T. Hubbard]-SFE, W\&W

[Eragrostis cilianensis (All.) Link.] - HDH

[Eragrostis cilianensis (All.) Janch.] - SFW

[Eragrostis cilianensis (All.) E. Mosher] - GPF, IMF, UTF

Eragrostis curtipedicellata Buckley - FNA, RBS, GPF, HDH, KTZ, SFE, W\&W, E

*Eragrostis curvula (Schrad.) Nees var, conferta Stapf. - E

[Eragrostis curvula (Schrad.) Nes] - FNA, RBS, GPF, ]MF, KTZ, SFE, UTF, W\&W

Eragrostis erosa Scribn. ex Beal - FNA

Eragrostis hypnoides (Lam.) Britton, Sterns, \& Poggenb, - Dorn01, FNA, RBS, GPF, HDH,

IMF, KTZ, SFE, UTF, W\&W, E

Eragrostis intermdia Hitche, - FNA

Eragrostis lehmanniana Nees - FNA

Eragrostis lutescens Scribn. - FNA, RBS, HDH rep, IMF, KTZ, SFE, W\&W ${ }^{1}$, E

[Eragrostis cilianensis (All.) Vignolo ex Jaxkh.] - UTF, W:W

(Eragrostis mexicana (Hornem,) Link subsp. mexicana\} - KTZ $\angle A Z, N M>$

\{Eragrostis mexicana (Lag.) Link\} - GPF

[Eragrostis ncomexicana Vasey ex L. H . Dewey] - FNA, KT/2

Eragrostis mexicana (Hornem,) Link subsp, virescens (J. Presl) S. D. Koch \& Sanchez - KTZ, RBS, UTF, W\&W, EWW

[Eragrostis mexicana (Honnem.) Link subsp. virescems L. Presl] - FNA, SF!

[Eragrostis orcutiana Vasey] - HDH, IMF

*Eragrostis minor Host - Dorn01, FNA, RBS, GPF, KTZ, SF!, UTF, W\&W, E/W

Eragrostis palmeri S, Watson - FNA

Eragrostis pectinacea (Michx.) Nees ex Steud. var. miserrima (E. Fourn.) Reeder - FNA, RBS, KTZ

[Eragrostis pectinacea (Michx.) Ness] - HDH, W\&W

[Eragrostis pectinacea (Michx.) Ness var. miserrima (E. Foum.) Reeder] - KTZ

[Eragrosis tephrosanthos I. E. Shult.] - IMF

Eragrostis pectinacea (Michx.) Nees ex Steud. var, pectinacea - KTZ, RBS, E/W

[Eragrostis diflusa Buckley] - HDH, W\&W

[Eragrostis pectinacea (Michx.) Nees] - GPF, HDH, IMF, UTE, W\&W

[Eragrostis pectinacen (Mishx.) Nees ex Steud. var pectinacea (Michx.) Nees] - KTZ

[Eragrostis pectinacea (Michx.) Nees var, pectinacea] - FNA

[Eragerostis pilosa (L.) Beauv.]-SF!, W\&W"

*Eragrostis pilosa (L.) P. Beauv, var, perplexa (L. H. Harv.) S. D. Koch - FNA, RBS, GPF, E/W

[Efagrostis perploxa L. H. Han.]-HDH

[Eragrostis pilosa (L.) Beanv.]-KTZ, SF!, W\& W

Eragrostis pilosa (L.) P. Beauv, var, pilosa - FNA, RBS, GPF, E/W

[Eregrostis pilosa (L.) P. Beauv.] - HDH, KTZ, SF1, W\&W

Eragrostis sceundiflora J. Presl subsp. oxylepis (Torr.) S. D. Koch - FNA, RBS, GPF, KTZ, SFE, W\&W, E

[Eragerostis oxylepis (Torr,) Torr.] - $\mathrm{HDH}$

[Eragrostis secundiflora C. Pres] subsp. oxylepis (Torr.] S, D, Koch] - KTZ

Eragrostis sessilispica Buckley - FNA

Eragrostis spectabilis (Pursh) Steud. - FNA, RBS, GPF, HDH, KTZ, SF!, W\&W, E/W

Eragrostis tef (Zucc.) Trotter - RBS

[Reported by RBS for Larimer County]

Eragrostis trichodes (Nutt.) A. W. Wood - Dom01, FNA, RBS, GPF, HDH, KTZ, SF!, W\&W, E/W 


\section{Eremopyrum (see Agropyron)}

\section{Erianthus (see Saccharum)}

Eriochloa contracta Hitchc. - FNA, RBS, GPF, HDH, KTZ, SFE, UTF, W\&W, E

Eriochloa sericea (Scheele) Munro ex Vasey - FNA

*Eriochloa villosa (Thunb.) Kunth - FNA, KTZ

Erioneuron pilosum (Buckley) Nash - FNA, RBS, IMF, KTZ, SF!, UTF, W\&W, E/W

[Tridens pileses (Bucklcy) Hitchc.] - GPF, HDH

Festuca (see also Leucopoa, Schedonorus, Vulpia)

[NS - Soe Snow08b for recent kery to SRMR species]

(Identification sources:

Festuca arizonica Vasey - HDH. IMF, KTZ, SF!, W\&W, Aiken95, EW

[Festuca ovina L. var. arizonica Hackel $\mathrm{ex}$ Beal] - UTF

Festuca baffinensis Polunin - Dorn01, KTZ, SF!, W\&W, Aiken95, E/W

Festuca brachyphylla Schult, ex Sehult, \& Schult. f. subsp. brachyphylla

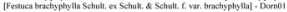

Festuca brachyphylla Schult, ex Schult, \& Schult. f. subsp. coloradensis Frederiksen - KTZ, Aiken95, ENW

[Festuca beactiy phylia Schult, subsp. coloradensis Frederiksen] - SFL, W\& W

[Festuca beachyphylla Schult. \& Schult. f. subsp. coloradensis Froderiksan] - Dom01

[Festuca ovina L. var. brachyphylla (SchulL) Piper] - HDH

[Festuca ovina L. var. brevifolia (R. Br.) S. Watson] - IMF, UTF

Festuca calligera (Piper) Rydb, - FNA

[Festuca ovina L. subsp. calligera Piper]

\{Festuca campestris Rydb.\} - KTZ, SFE, W\&W, E

\{Festuka scabrella Torr.;-GPF

\{Festuaca seabrella Torr, ex Hook, - IMF

Festuca dasyclada Hack. ex Beal - IMF, KTZ, UTF; Aiken95, W

[Argillochloa dasyclada (Hackel) W. A. Weber] - SFW, W\&W

[Argillochloa dasyclade (Hackel ex Beal) W. A. Weber] - RBS, W\&W

Festuca earlei Rydb, - FNA, KTZ, Aiken95

[Festuca rubra L.] - W\&W'

Festuca hallii (Vascy) Piper - Dom01, FNA, KTZ, SF!, W\&W, Aiken95, E/W [Festuca seabrella Torr, ex Hook.] - IMF

Festuca idahoensis Elmer - Dorn01, FNA, GPF, HDH, IMF, KTZ, SF!, W\&W

[Festuca idahocnsis Elmer var, idahoensis] - Aiken9s, EW

[Festuca ovina L. var, ingrata Hackel ex Beal] - UTF

Festuca minutiflora Rydb. - Dom01, KTZ, SF!, W\&W, Aiken95, E/W

[Festuca ovina L. var, brevifolia (R. Br.) S, Watson] - IMF

[Festuca ovina L. var, ovina] - HDH

\{Festuca ovina L. var. ovina\} - HDH, IMF, UTF

[NS - sec discussion in FNA Vol. 24:422; in brief, F, ovias is nos found in our area.?

\{Festuka ovina L. - KTZ CUT?, W\&W

* Festuca rubra L. subsp. rubra - Dorn01. FNA, KTZ.

[Festuca rubra L. vad, rubra] - FNA, HDH, IMF, ENW

[Festuca rubra L.] -GPF, SF1, UTF, W\&W 
(Festuca rubra L. var. lanuginosa Mert, \& W. D. J. Koch\} - HDH, IMF, W\&W, err. rep.

\{Festuca rubra L subsp, anenaria (Osbeck) Syme\} - FNA

\{Festuca rubra L. subsp. arenaria (Osbeck) F. Anesch.\} - KTZ

Festuca saximontana Rydb, var, purpusiana (Saint-Yves) Frederiksen \& Pavlick - FNA. KTZ, Aiken95

[Festuca ovina L. var. brevifolia (R. Br.) S. Watson] - IMF

[Festuca saximontana Rydb. subsp. purpusiana (Saint-Yves) Frederiksen \& Pavlick] - W\& W ${ }^{1}$

Festuca saximontana Rydb, var saximontana - FNA, KTZ, Aiken95, E/W

[Festuca ovina L. var. rydbergii Saint-Yves] - GPF, IMF, UTF

[Festuca saximontana Rydb.] - Dorn01, SF!, Wd W

\{Festuca scabrella Torr.\} - GPF, HDH, W\&W, misid.

\{Festuca altaica Trin.; - KTZ

\{Festuca scabrella Torr. ex Hook.\} - DMF

Festuca sorotia Piper - FNA, HDH, IMF, KTZ, SF!. UTF, W\&W, E/W

Festuca subulata Trin - FNA, GPF, IMF, KTZ, SFW, UTF, W\&W, err. rep., W\&W', W

\{Festuca subverticillata (Pers.) E. B. Alexeev - FNA

\{NS - To be expected to enter WY and CO from adjacent Nebraska?

Festuca thurberi Vasey - Dom01, HDH, IMF, KTZ, SF!, UTF, W\&W, Aiken95, E/W

Festuca trachyphylla (Hackel) Krajina - FNA, FCF

[Festuca brovipila Tracey] - KTZ

[Festuca cinerza auct. non Vill.] - KTZ

[Festuca duriescula auct. non L.] - KTZ

[Festuca duriescula var, cinorea aust, non (Vill.) Krajina] - KTZ

[Festuca bngifolia auct. non Thuill.] - KTZ

[Fesluca ovitha var. Gariuscula auet, non (L.) W.D.J. Koch ] - KTZ

[Festuca ovina aucL. non L.] - KTZ

Glyceria (see also Torreyochloa)

Glyceria borealis (Nash) Batch, - Dorn01, RBS, GPF, HDH, IMF, KTZ, SF!, UTF, W\&W, EW

Glyeeria elata (Nash ex Rydb.) M. E. Jones - Dorn01, RBS, W\&W, E/W

[Glyocria elata (Nash) M. E. Jones] - IME, SF!

[Glyosria elata (Nash) Hitche.] - $\mathrm{HDH}$

[Glyceria strima (Lam.) Hichis.] - KTZ UTF

Glyeeria grandis S. Watson - Dorn01. RBS, HDH, IMF, SF!, UTF, W\&W, E/W

[Glyoeria grandis S. Wasson ex A. Gray] - GPF

[Glyceria grandis S. Watson var, grandis] - KTZ

Glyeeria striata (Lam.) Hitche.] - Dom01, RBS, FNA, GPF, IMF, KTZ, SF!

[Glyceria striata (Lam.) Hitelicock var, stricea (Scribn_) Fernald] - HDH, IMF, W\&W, EW

Graphephorum wolfii (Vascy) Vascy ex Coult. - Finot05. RBS

[Graphephorum mucicuen (Bol.) A. Heller] - Finow05, RBS

[Trisetum subspicatum P. Bcsuv, var, muticum BoL.] - Finot0s, RBS

[Triselum wolfii Vasey] - Dorne1, FNA, HDH, IMF, KTZ, SFI, UTF, W\&W, EN

Helictrotrichon (see also Avenula)

Helictotrichon mortonianum (Seribn.) Henrard - Dorn01. FNA, HDH, KTZ, SF!, W\&W, E/W

[Helictotrichoo mortoniana (Scribn.) Henrand] - UTF

Hesperochloa (sec Leucopoa)

Hesperostipa comata (Trin. \& Rupr.) Barkworth - SF!, W\&W'

[Hesperostipe comata (Trin. \& Rupr.) Barkworth subsp. comats-KTZ, RBS

Snow, N, January 2009. Checklist of Vecculer Plants of the Southum Rodky Momain Regioe. CVersion $3 x$ 
[Hesperostipa comala (Trin. \& Rupr.) Barkworth subsp. intennedia (Scribn. \& Tweedy) Barkworth]-KTZ, RBS [Stipa comata Trin. \& Rupr.] - GPF, W\&W

[Stipa comata Trin. \& Rupr. var. comata] - HDH, IMF, UTF, EW

[Stipa comata Trin. \& Rupr. var, istermedia Scribn. \& Tweody - HDH, IMF, UTF, E/W

Hesperostipa neomexicana (Thurb.) Barkworth - RBS, FNA, SF!, W\&W'

[Hesperostipa neomexicana (Thurb. ex J. M. Coult.) Barkworth] - KTZ

[Stipa nesmexicana (Thurb. ex J. M. Coult.) Scribn.] - W \&W, EW

[Stipa nesmexicana (Thurb.) Scribn.] - GPF, HDH. IMF, UTF

Hesperostipa spartea (Trin.) Barkworth - RBS, FNA, KTZ, SFE, W\&W'

[Stipa spartea Trin_] - GPF, HDH, IMF, W\&W

[Stipas spartea Trin, yar, spartea] - E

\section{Hierochloẽ (see Anthoxanthum)}

Hilaria jamesii (Torr, ) Benth, - Dorn01, FNA, GPF, HDH, IMF, SF!, UTF, W\&W, E/W

[Pleuraphis jamesii Torr.] - KTZ, RBS

* Holcus lanatus L. - RBS, GPF, HDH, IMF, KTZ, SF!, UTF, W\&W, EW

Hordeum

(Identification sources:

Hordeum brachyantherum Nevski - Dom01, GPF, HDH, IMF, UTF, E/W

[Critesion brachyantherum (Nevski) Barkworth \& D. R. Dewey] - RBS, W\&W

[Critesion brachyantherum (Nevski) W. A. Weber] - SF!

[Hordeum brachyancherum Nevski subsp، brachywntherum] - KTZ

[Hordeum jubesum Lu subsp. broviaristatum Bowden] - KTZ

Hordeum distichon L. - Dom01

Hordeum jubatum subsp. jubatum - RBS, FNA, KTZ

[Critesion jubatum (L.) Nevski] - SF!. W\& W

[Hordeam jubetum L.] - IMF, UTF, E/W

[Hordeam jubenum L. var. jubatum] - $\mathrm{HDH}$

[Hordeam jubetum L. subsp, jubatam] - KTZ

Hordeum jubatum L. subsp, intermedium Bowden - RBS, KTZ, FNA

[Critesion juhatum (L) Nevski] - W \&W

[Hordeum brachyantherum $\mathrm{X}$ H. Jubatum] - IMF

[Hordeum X cassphosum Scriba, ex Panamel] - Dorn01

[Hordeum $X$ cassphiosum Scribe.] - UTF

[Hordeum esespitosum Seribn]-GPF

[Hordsum jubsium L.] - [MF

[Hordeum jubsnum L. var, caespitosum (Scribn.] Hitche] - HDH

[Hordsum intennedium Hausskn.] - FNA

*Hordeum murinum L. subsp, glaucum (Steud.) Tzvelev - RBS, FNA, KTZ, E/W

[Critesion glascum (Stend) A. Live] - SF!. W\&W

[Hordeum glaucum Steud.] - [MF

[Hordeum murinum L.] - UTF

\{Hordeum murinum L. subsp. murinum\} - KTZ

[Hoodesm murinam Li] - IMF, VTF

Hordeum pusillum Nutt. - RBS, Dorn01, GPF, HDH, KTZ. UTF. E/W

[Critesion pusillum (Nutt) A L Love] - SF', W\&W

[Hordeam pusillum Nutt, var, pusillum] - [MF 
*Hordeum vulgare L, - RBS, GPF, KTZ, SF!, UTF, EW

[Hordeum distichon L.] - IMF

[Hordeum vulgare L. var. vulgare] - Dorn01, HDH, IMF, W\& W

[Horfeum vulgare L. var, trifurcatum (Schltdl.) Alef.] - Dern01, HDH, IMF, W\&W

\section{Jarava (see Pappostipa)}

Koeleria macrantha (Ledeb.) Schult, - RBS, Dom01, KTZ, SF!, UTF, W\&W, E/W

[Koeleria cristata (L.) Pers.] - HDH

[Koeleria nitida Nutt.] - TMF

[Koeleria pyramidata (Lam.) P. Beauv_] - GPF

ฯLeersia oryzoides (L, ) Sw, - Dorn01, RBS, GPF, HDH, IMF, KTZ, SF!, UTF, W\&W, E/W

Leptochloa dubia (Kunth) Nees - FNA, RBS, GPF, KTZ

[Diplachne dubia (Kunth) Scribn.] - SFE, W\& W

Leptochloa fusca (L.) Kunth subsp, fascicularis (Lam.) N. Snow - FNA, KTZ, Snow98, E/W

[Diplachne fasicularis (Lam.) A. Gray]-SFE

[Diplachne fasicularis (Lam.) P. Beauv.] - SFW, w\& w'

[Leptochloa fascicular is (Lam.) A. Gray]-GPF, HDH, IMF, UTF. WEW

[Leptochloa fusca (L.) Kunth var. fascicularis (Lam.) Dom] - Dorm01. RBS

\{Leptochloa fusca (L.) Kunth subsp, uninervia (J. Presl) N. Snow\} - KTZ; Snow98, FNA

INS - no specimen exists at COLO. HDH personal notes evidently indicabed the CO specimen was at US (T.

Hogan, pers. coemm. Nov 2003), which does bot have a record from CO (P. Peterson, pers, comm. Nov 2003).

however. may eventually be found in playas or edges of reservoirs in se CO of ne NM below 8,000 ft.\}

\{Diplache uninervia (3. Presl) Parodi) - W: W', err. rep.

\{Leptochloa uninervia (J. Presl) Hitche. \& Chase\} - $\mathrm{HDH}$, IMF, UTH

\{Leptochlos uninsrvia (J. Presl) Hitche.) - W\&W, doubtful rep.

Leptochloa panicea (Retz) Owhi - FCF, Snow98

[NS - reported by FCF for Montrose County; woucher not seen, most likely subsp. brachiana]

Leucopoa kingii (S. Watson) W. A. Weber - RBS, Dorn01, GPF, IMF, KTZ, SF!, UTF, W\&W, $\mathrm{E} / \mathrm{W}$

[Festuca kingii (S. Watson) Cassidy] - Aikengs

[Hesperosthloa kingli (S. Wasson) Rydb.] - $\mathrm{HDH}$

Leymus (see Elymus)

Lolium (see also Schedonorus)

*Lolium multiflorum Lam_- RBS, Dorn01, HDH, IMF, E/W

[Lolium perenene L.] - SF-

[Lolium peresne L. subsp, italicum (A. Braun) Syme] - W\& W

[Lolium pereene L. subsp. maitiflorum (Lam.) Hesnot] - KT2

[Lolium peresne L-var, aristatum Willd] - GPF, UTF

*Lolium perenne L. - RBS, Dorn01, HDH, IMF, SF!, E/W

[Lolium perenne L. subsp. perenne] - KTZ

[Lolium perenne L. var, perenne] - GPF, UTF

Lycurus

[NS - Paul Peterson \{US; informs me (Aug, 2007) that this genas falls into Muhientergia based on ITS and

chloroplast sequences \{see also Schodomordus?]

Lycurus phleoides Kunth - FNA, GPF, HDH, IMF, KTZ, RBS, UTF, W\&W, rep., W\&W', rep.

Snow, N, Junury 2009. Checklist of Vaccular Plants of the Southom Rocky Mombin Region. CVersion $3 x$ 
Lycurus setosus (Nutt.) C. Reeder - FNA, RBS, KTZ, SF!, W\&W', E/W

[NS - this taxon will soon be transferred into as Mrbilenbergia alopecuroider (Griseb.) P. M. Peterson \&

Columbus, see Msdroño [in pressi] ]

Melica bulbosa Geyer ex Porter \& J. M. Coult. var, bulbosa - IMF. W

[Bromelica bulbosa (Geyer) W. A. Weber]-SFW, RBS

[Bromelica bulbosa (Geyer ex Porter \& J. M. Coult) W. A. Weber] - W\&W

[Meliea bulbosa Geyer] - GPF, $\mathrm{HDH}$

[Melica bulbosa Geyer ex Porter \& J. M. Coult.] - Dom01, KTZ, UTF

Melica nitens (Scribn.) Nutt. ex Piper - FNA

Meliea porteri Seribn, - RBS, GPF, HDH, SF!, UTF, W\&W, E/W

[Melica porteri Seriben. var-porteri]- KTZ.

Melica spectabilis Scribn, - Dorn01, HDH, IMF, KTZ, UTF, EW

[Bromelica spoctabilis (Scribn.) W. A. Weber] - SF!, RBS, W\&W

Melica subulata (Griseb, ) Scribn, var, subulata - KTZ, W

[Bromelica subulata (Griseb.) Farw.] - SFW, RBS, W\&W'

[Melica subulata (Griseb.) Scribn.] - Dorn0I, GPF

*Miscanthus sinensis Andersson - FNA, HDH, KTZ, W\&W

Monroa (see Munroa)

Muhlenbergia

[NS - I fecommend Petersoo's treatment in FNA Vol. 25 for additional information]

(Identification sources:

Muhlenbergia andina (Nutt.) Hitche. - Dorn01, RBS, FNA, HDH, IMF, KTZ, SF!, UTF, W\&W, E/W

Muhlenbergia arenacea (Buckley) Hitche, - FNA, RBS, HDH, KTZ, SFE, W\&W, E

Muhlenbergia arenicola Buckley - GPF, RBS, HDH, KTZ, SFE, W\&W, E

Muhlenbergia arsenei Hitche, - FNA

Muhlenbergia asperifolia (Nees \& Meyen ex Trin.) Parodi - Dorn01, RBS, FNA, KTZ. W\&W, ENW

[Muhlenbergha asperifotia (Nees \& Meyen) Parodi - GPF, HDH, IMF, SFI, UTF

Muhlenbergia brevis C. O. Goodding - FNA, RBS, HDH, KTZ, SFE, W\&W, E

Muhlenbergia cuspidata (Torr. ex Hook.) Rydb. - Dom01, RBS, FNA, KTZ. E

[Muhlenbergla cuspidata (Torr.) Rydb, -GPF, HDH, SFE, w\& W

Muhlenbergia depauperata Scribn. - FNA, RBS, KTZ, SFW, UTF, W\& W, W

Muhlenbergia dubia E. Fourn. - FNA

Muhlenbergia emersleyi Vasey - FNA

Muhlenbergia filiculmis Vascy - Dom01, FNA, RBS, GPF, HDH, IMF, KTZ, UTF, W\&W, E/W

Muhlenbergia filiformis (Thurb. ex S. Watson) Rydb. - Dom01, RBS, FNA, KTZ W\&W, E/W

[Muhleubergla filiformis (Thurb.) Rydb, - GPF, HDH, IMF, SF!, UTF

Muhlenbergia glomerata (Willd.) Trin, - Dom01, RBS, FNA, GPF, KTZ, UTF, W\&W

Muhlenbergia longiligula Hitche. - FNA

Muhlenbergia mexicana (L.) Trin. - Dom01, FNA, RBS, GPF, HDH, IMF, KTZ, SFE, UTF, W\&W,E 
Muhlenbergia minutissima (Steud.) Swallen - Dorn01, RBS, FNA, GPF, HDH, KTZ, SF!, W\&W, EW

Muhlenbergia montana (Nutt.) Hitche, - Dom01, RBS, FNA, HDH, IMF, KTZ, SF!, UTF, W\&W, EN

Muhlenbergia pauciflora Buckley - FNA, RBS, HDH, IMF, KTZ, SFW, UTF, W\&W, W

Mublenbergia porteri Scribn. ex Beal - FNA, RBS, IMF, KTZ, UTF, ENW

[Muhlenbergia porteri Scribn.] - GPF, HDH, SF', W\&W

Mublenbergia pungens Thurb, - Dorn01, RBS, GPF, HDH, IMF, KTZ, SF!, UTF, E/W

[Muhlenbergia pungens Thurb. ex A. Gray]-FNA. W\&W

Muhlenbergia racemosa (Michx,) Britton, Sterns, \& Poggenb, - Dorn01, RBS, FNA, GPF, HDH, IMF, KTZ, SF!, UTF, W\&W, E/W

Mublenbergia ramulosa (Kunth) Swallen - KTZ, RBS, W\&W, misapp, E [Muhlenbergia uolfii (Vascy) Rydb.] - HDH, SFE, W\&W

Muhlenbergia repens (J. Presl) Hitche, - FNA, KTZ, UTF

Muhlenbergia richardsonis (Trin.) Rydb, - Dorn01, RBS, FNA, GPF, HDH, KTZ, SF!, W\&W, E/W

Muhlenbergia rigens (Benth.) Hitchc, - FNA

Mublenbergia rigida (Kunth) Trin. - FNA

\% Muhlenbergia schreberi J. F, Gmel. - FNA, RBS, GPF, HDH, KTZ, SFE, UTF, W\&W, E

Mublenbergia tenuiflora (Willd.) Britton, Stems \& Poggenb. - FNA

Muhlenbergia texana Buckley - FNA

Muhlenbergia thurberi Rydb, - IMF, RBS, KTZ, SFW, UTF, W

[Munlenbergla thurberi (Scribn.) Rydo.] - FNA. W\&W

Muhlenbergia torreyi (Kunth) Hitchc. ex Bush - Dorn01, RBS, FNA, GPF, KTZ, W\&W, E [Muhlenbergia torreyi (Kusth) Hitche,]-HDH, SFE

Muhlenbergia wrightii Vasey ex J. M. Coult. - FNA, RBS, IMF, KTZ, UTF, W\&W, E/W [Muhlenbergia wrightii Vascy] - HDH, SF!

Munroa squarrosa (Nutt.) Torr, - Dorn01, RBS, FNA, GPF, HDH, IMF, UTF

[Monroa squarrosa (Nutt.) Torr.] - KTZ, SFt, Ws W. E/W

Nassella tenuissima (Trin.) Barkworth - RBS, FNA

[Stipar tenuissima Trin.] - FNA

Nassella viridula (Trin.) Barkworth - RBS, Dorn01, FNA, KT7, SF!, W\&W

[5tipa viridula Trin.]-GPF, HDH, UTF, W\&W, E/W

Oryzopsis (see also Achnatherium, Pipatherum)

[NS - the genus is polyphyletic; but best waty to split still not evident]

Oryzopsis asperifolia Michx. - RBS, Dorn01, FNA, GPF, HDH, IMF, KTZ, SF!, W\&W, E'W

Panicum (see also Dichanthelium)

[NS - the gesus is polyphyletic and is being split actively, primarily by Morone, Zukaga, and colleagues working cat of Argentina. I am w ithholding some of the generic splits pending more data]

(Identification sources:

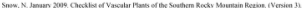


Panicum antidotale Retz. - FNA

Panicum capillare L. - Dorn01, RBS, FNA, GPF, IMF, KTZ, SFE, UTF, W\&W, E/W [Panicum capillare L. var. capillare] - HDH

[Panicum capillare L. var, occidentale Rydb.] - HDH, SFW

+ Panicum dichotomiflorum Michx. var. dichotomiflorum - FNA, KTZ, E

[Panicum dichotomiflorum Michx.] - GPF, RBS, HDH, IMF, SFE, UTF, W\&W

Panicum hallii Vasey var, hallii - FNA, GPF, KTZ, E

[Panicum hallii Vasey] - RBS, HDH, IMF, SFE, UTF, W\& W

Panicum hillmanii Chase - GPF, KTZ, SFE, W\&W, E

* Panicum miliaceum L, - Dorn01, RBS, FNA, GPF, HDH, IMF, SFE, UTF, W\&W, E [Panicum miliaceum L. subsp. milisoeum] - KTZ

Panicum obtusum Kunth - FNA, RBS, GPF, HDH, IMF, KTZ. SFE, UTF, W\&W. E

[NS - Zuloaga 07 described the monotypic genus Hopur for this species, but until the boundaries of the wildly polyphyletic Pumicum are more fully resolved [see their Fig. 2] the recognition of this genus is being withheld] [Hopia obtusa (Kunch) Zuloaga \& Morrone] - Zuloaga07

Panicum virgatum L. - Dorn01, RBS, GPF, HDH, IMF, SF!, UTF, W\&W, E/W

[Panicun virgatum L. var. virgatum] - KTZ

Pappostipa speciosa (Trin \& Rupr.) Romeschenko - Romeschenko08

[Achnatherum speciosum (Tria. \& Rupe.) Barkworth] - KTZ, SFW, W\&W'

[Jarava speciosa (Trin \& Rupr.) Pentail.] - RBS. FNA

[Stipa speciosa Trin. \& Rupr.]-HDH, IMF, UTF, W\& W, W

\section{Pascopyrum (see Elymus)}

* Paspalum dilatatum Poir, - RBS, GPF, HDH, KTZ, SFW, W\& W, w

* Paspalum pubiflorum Rupr. ex E. Fourn, var, glabrum Vasey ex Scribn, - GPF, E [Paspalum pubiflorum Rupr. ex E. Fourn.] - FNA, RBS, KTZ

[Paspalum pubiflorsm Rupr. vaf, glabrum Vasey] - SFE

[Paspalum pubiflorum Rupr. ex E. Fourn. var, glabrum Vssey \& Seribe.] - W\& W

* Paspalum racemosum Lam. - FNA, RBS, HDH, KTZ, W\&W

$\div$ Paspalum setaceum Michx. - FNA, RBS, KTZ

[Paspalum setaceum Michx. var. stramincam (Nash) D, J. Banks] - GPF, SFE, W\& W, E

[Paspalum stramineum Nasb] - HDH

Pennisetum (see also Setaria)

* Pennisetum alopecuroides (L.) Spreng. - RBS

[Panicum abopecuroides L.]

* Pennisetum glaucum (L.) R. Br. - FNA, KTZ.

[Setaria glanca (L.) P. Beauv- GPF, IMF, SF!, UTF, W\&W, E/W

[Setaria lutescens (Weigel) F. T. Hubbard] - HDH

* Pennisetum setaceum (Forsk.) Chiov, - RBS, HDH, IMF, KTZ, W\&W

[NS - Not listed by FNA for our region]

* Pennisetum villosum $\mathrm{R}$. Br. ex Fresen. - KTZ

[Pennisetum villosum R. Br.] - HDH, W\&W

Phalaris arundinacea L. var, arundinacea - HDH, E/W

[Phalaris arundinacen L.] - Dornof1, GPF, IMF, KTZ

[Phalaris anundinacen L. var, picta L.] - $\mathrm{HDH}$

[Phalaris anundinacen L. forma picta (L.) Asch. \& Graebn.] - IMF

[Phalaris amundinacen L. forma variegata (Parnell) Druce] - UTF

[Phalaroides anundinacea (L.) Rauschert] - RBS, SFI. W\&W 
Phalaris canariensis L. - RBS, Dom01, GPF, HDH, IMF, KTZ, SFE, UTF, W\&W, E

Phalaris caroliniana Walter - RBS, GPF, HDH, IMF, KTZ, SFE, W\&W, E

Phalaris minor Retz - RBS, HDH, KTZ, SFE, W\&W, E

Phalaroides (see Phalaris)

Phippsia algida (Phipps) R. Br, - RBS, Dom01, HDH, KTZ, SF!, W\&W, E/W

Phleum alpinum L. - RBS, Dorn01, GPF, HDH, IMF, KTZ, UTF, W\&W ${ }^{1}$, misapp.

[Phleum alpimum L var. alpinum] - E/W

[Phleum commutatum Gaudin]- SF!, W\& W

Phleum pratense L. - RBS, Dorn01, GPF, HDH, IMF, KTZ, SF!, UTF, W\&W

[Phleum pratense L. var. pratense] - E/W

Phragmites australis (Cav, Trin. ex Steud, - Dom01, FNA, RBS, GPF, IMF, KTZ, UTF, W\&W, E/W

[Phragmiles australis (Cav.) Trin.] - SF!

[Phragmites communis Trin.] - $\mathrm{HDH}$

Piptatherum exiguum (Thurb.) Barkworth - RBS, Dom01, KTZ, FNA

[Oryzopsis exigua Thurb.] - GPF, HDH, IMF, SF!, UTF, W\&W, E/W

Piptatherum micranthum (Trin, \& Rupr.) Barkworth - RBS, Dorn01, FNA, KTZ, SF!, W\& W

[Oryzops is micrantha (Trie, \& Rupr.) Thurb.] - GPF, HDH, IMF, UTF, W\& W, E/W

Piptatherum pungens (Torr.) Barkworth - RBS, Dom01, FNA. KTZ

[Oryzopsis pungens (Torr.) Hikche.]-GPF, HDH, SF!

[Oryzopsis pungens (Tort-ex Spreng.) Hitchc. - Hariman06, W\&W, EW

Piptochaetium fimbriatum (Kunth) Hitchc. - FNA

Pleuraphis (see Hilaria)

Poa

[NS - See Snow0sa for a recont key to species from the SRMR]

(Identification sources:

Poa abbreviata R. Br. subsp. pattersonii (Vasey) A. Lõve, D. I.̋ve, \& B. M. Kapoor - FNA. KTZ, SF!, W\&W

[Poa ubbervata R, Br.] - RBS

[Poa pattersonii Vasey] - Doon01, HDH, IMF, UTF, E/W

Poa alpina L. - RBS, Dom01, FNA, HDH, IMF, KTZSF!, UTF, W\&W, E/W

Poa annua L. - RBS, Dorn01, FNA, GPF, HDH, IMF, KTZ, SF!, UTF, W\&W, E/W

\{Poa arachnifera Torr.\}

(NS - approaches our area closely in southeost CO from southwest KS)

Poa arctica R. Br. subsp. aperta (Scribn. \& Merr.) Soreng - FNA, KTZ. SFW, W\&W', E/W

[Pon speria Scriben, \& Merrill] - W\&W

[Pos anctica R. Br.] - IMF

[Poa arctica R, Br, subsp, aperta (Vasey) Soreng] - SFE

Poa arctica R. Br. var. arctica - Dom01, FNA. E 
[Poa arctica R. Br.]-HDH, IMF, SFE, UTF, W\&W

[Pos arctica R. Br. subsp. arctica] - KTZ

Poa arctica R. Br. var- grayana (Vasey) Dorn - Dorn01, FNA, E/W

[Pos anctica R. Br.] - HDH, IMF, UTF, W\&W

[Poa arctica R. Br. subsp. grayana (Vasey) Löwe] - SF!

[Poa arctica R. Br. subsp. grayana (Vasey) A. Love, D. Love, \& B. M. Kapeor] - KTZ, SFE

Poa arida Vasey - RBS, Dom01. FNA, GPF, HDH, MMF, KTZ, SF1, UTF, W\&W, ENW

[Poa glaucifolia Seribn. \& T. A. Williams] - GPF, HDH. IMF, SF!. W\&W

Poa bigelovii Vasey \& Scribn. - RBS, FNA, HDH, IMF, KTZ, SFE, UTF, W\&W, E

[Poa bolanderi Vasey - FNA]

\{NS - to be expected in southwestem CO from UT?

Poa bulbosa L. - RBS, HDH, IMF, KTZ, SF1, UTF, W\&W, E/W

[Poa bulbosa L. subsp. vivipara (Koel.) Arcang.] - FNA

* Poa compressa L, - RBS, Dom01, FNA, GPF, HDH, IMF, KTZ, SF!, UTF, W\&W, E/W

\{Poa cusickii Vasey var, cusickii\} - E/W

\{Poa cusickii Vasey\} - GPF, HDH, IMF

Poa cusickii Vascy subsp. cusickii;-FNA, KTZ, W\&W, W\&W', err. rep.

[Poa cusickii Vascy subsp. pallida Soreng] - Dorn01, SF!, W\& W. W\&W $W^{1}$, putative hy brid of Pca cusickii X P. fendleriana

\{Poa fendleriana (Steud.) Vasey\} - UTF

Poa cusickii Vasey var, epilis (Scribn.) C. L. Hitchc. - Dorn01, E/W

[Pos cusickii Vasey subsp. epilis (Scribn.) W. A. Weber]-RBS, FNA. KTZ, SF!, Ws W

[Poa cpilis Scrihn.]- HDH, IMF

[Poon fendleriana (Steud.) Vasey] - UTF

Poa cusickii Vasey var, pallida (Soreng) Dom

[Pes caskckii Vasey sobsp. pallida Soreng] - RBS, FNA, W\& W', W\&E

Poa fendleriana (Steud.) Vasey subsp, fendleriana - RBS, FNA, KTZ

[NS - synonymized under P. cuskckil in previous checklist]

[Pos fendloriana (Steud.) Vasey] - Donw0, GPF, HDH, IMF, UTF, WeW, EW

Poa fendleriana (Steud.) Vasey subsp. longiligula (Scribner \& Williams) Soreng] - RBS, FNA, KTZ, SF!, W\&W ${ }^{1}$

[Poa loagilizula Scribn. \& T. A. Willams] - HDH

Poa glauca Vahl subsp. glauca - FNA, KTZ

[Pos glauca Vahl var. glauca] - UTF

Poa glauca Vahl subsp, rupicola (Nash) W. A. Weber - FNA, SF!, W\&W

[Poan glauca Vahl var, rupicola (Nash ex Ryob,) B, Boivin] - Dorn01, E/W

[Poa glauca Vahl subsp. nupieola (Nash ex Rydb.) W. A. Weber] - KTZ

[Pos glauca Vahl var, rupicola (Nash) J) Bovinin] - UTE

[Poa rupicola Nash] - $\mathrm{HDH}, \mathrm{IMF}$

[Poa rupicola Nash ex Rydb.] - GPF

Poa interior Rydb. - RBS, Dom01, FNA,GPF, HDH, IMF, E/W

[Poos glauca Vahl var. glouca] - UTF

[Pop nemoralis L. subsp. interior (Rydb.) Batters \& Abbe] - SF]

[Poc nemoralis L- subsp, interior (Rydb.) W, A. Weber] - KTZ, W\&W

Poa laxa Haenke subsp, banffiana Soreng - RBS, FNA, KTZ

[NS - location of a voucher uncertain]

Poa leptocoma Trin. - RBS, Dom01, FNA, HDH, IMF, KTZ, SF!, UTF, W\&W, E/W

Poa lettermanii Vasey - RBS, Dom01, FNA, HDH, IMF, KTZ, SF!, UTF, W\& W, E/W

Poa X nematophylla Rydb. - FNA, KTZ

[Pos cusickii Vasey] - IMF

[Pen cusicki Vasey subsp. pallida Soreng] - W \& W'

Poa occidentalis Vasey - RBS, FNA, HDH, IMF, KTZ, SFW, W\&W, w

Poa palustris L. - RBS. Dom01. FNA. GPF, HDH, IMF. KTZ. SF!. UTF. W\&W. E/W 
Poa pratensis L, subsp, alpigena (Lindm.) Hiitonen - FNA

Poa pratensis L. subsp, agassizensis (B. Boivin \& D. Löve) Roy L. Taylor \& MacBryde - FNA. RBS

*Poa pratensis L. subsp. pratensis - FNA, KTZ, RBS

[Poa agassizensis B. Boivin \& D. L.öve]-SF!, W\&W

Poa reflexa Vasey \& Scribn. - RBS, Dom01, FNA, HDH, IMF, SF!, UTF, W\&W, E/W

[Poa reflexa Vasey \& Scribn. ex Vasey] - KTZ

Poa secunda J. Presl subsp. juncifolia (Scribn.) Soreng - RBS, FNA

[NS - consulk Kellogg85 as well]

[Poa juncifolia Scribn.] - GPF, HDH, IMF, SF!, W\&W

[Poa juncifolia Scribn. var. ampla (Morr.) Dorn] - RMC

[Poa juncifolia Seriben. var. juncifolia] - RMC

[Pos nevadonsis Vassy] - HDH

[Poa nevadensis Vassy ex Scribn.] - JMF

[Pos secunda J. Presl subsp. newadensis]

Poa secunda J. Presl subsp, secunda J. Presl - RBS, FNA

[Poa secunda 1. Presl var. secunda] - Dorn01

[Poa secunda J. Prsel var. elongata (Vasey) Dorn] - RMC

[Poa secunda J. Presl var. incurva (Scribn. \& T. A. Williams ex Scribn.) Beetle] - RMC

Poa stenantha Trin, var. stentantha - FNA, RBS

[Posa macroclada Rydb.] - FNA, KTZ

[Pos sternantha Trin.] - KTZ

Poa tracyi Vasey - RBS, FNA, KTZ, SF!, W\&W, E/W

[Pod occidentalis Rydb, not Vasey] - $\mathrm{HDH}$

Poa trivialis L. - RBS, Dorn01, FNA. HDH, IMF, KTZ, SFE, UTF, W\&W, E

Poa wheeleri Vasey - RBS, Dom01, FNA, KTZ, FNA

[Pou nervosa (Hook.) Vasey] - HDH, SFs, W\& W

[Pos nervosa (Hook.) Vasey var, wheeleri (Vasey) C. L. Hitebs] - IMF, UTF, EW

Podagrostis humilis (Vasey) Björkman - RBS, FNA, SF!, W\& W'

[Agrostis humilis Vasey] - Dorn01, HDH, IMF, KTZ, UTF, W\&W, EW

[Agrostis thurberiana Hitche.] - HDH, IMF, UTF, WRW, E/W

[Podagrostis thurberiana (Hitchc.) Hulton] - SF!, W\& W

Polypogon elongatus Kunth - RBS, FNA, KTZ, W\&W

Polypogon interruptus Kunth - RBS, Dorn01, GPF, IMF, KTZ, SFE, UTF, W\&W', E

Polypogon monspeliensis (L.) Desf, - RBS, Dorn0I, GPF, HDH, IMF, KTZ, SF!, W\&W, E/W

Polypogon viridis (Gouan) Breistr. - RBS, KTZ, SF!, W\&W, E/W

[Agrostis sethiverticillata (Forskali) C. Christensen] - HDH, IMF

[Polypogon semiverticillata (Forskch) Hylander] - UTF

Psathyrostachys (sec Elymus)

X Pseudelymus (sec Elymus)

Pseudorocgneria (see Elymus)

- Ptilagrostis porteri (Rydb.) W. A. Weber - ENDEMIC $<$ CO $>-$ RBS, FNA, SF!, W\&W

[Ptilagrostis mongholica (Turcz, ex Trin.) Griseb. subsp pocteri (Rydh.) Barkworth - KT? E/W

[Stipa porteri Rydb.] - HDH

Snow, N. Junury 2009. Checklist of Vascular Plants of the Southom Rocky Mounain Region. CVersion $3 x$ 
Puccinellia (see also Torreyochloa)

* Puccinellia distans (L.) Parl, - RBS, Dorn0], GPF, HDH, IMF, UTF, W\&W

[Puocinellia distans (Jacq.) Parl. subsp. distars] - KTZ

Puccinellia nuttalliana (Schult.) Hitche, - RBS, Dorn01, GPF, IMF, KTZ. UTF, EW

[NS - Previous chacklist split out P. cusickii as distinct species]

[Pucsinel lia airoides S. Watson \& J. M. Coult.] - W\&. W

[Puccinel lia airoides (Nutt) S. Watson \& J. M. Coult.] - HDH, SF!

[Pucsinellia cusickii Weath.] - Dom01, GPF <WY

Redfieldia flexuosa (Thurb.) Vasey - Dom01, FNA, GPF, HDH, IMF, KTZ, SFE, UTF, E

[NS - Dr. Paul Peterson fUS\} informs that this genas falls into Mulwenfergrio based on ITS and chlocoplast sequences]

[Redfieldia flexuosa (Thurb, ex A. Gray) Vasey] - W\&W. RBS

Saccharum raventae (L.) L, - KTZ

[Erianthus ravennas (L.) P. Beauy.] - GPF, HDH, IMF

[Saccharum raveranac (L.) Murray] - UTF

Schedonnardus paniculatus (Nutt,) Trel, - RBS, Dorn01, FNA, RBS, GPF, HDH, KTZ, SF!, W\&W, EN

[NS - Paul Peterson \{US\} ind icanes that this taxon falls into Mwhlenbergia basod on ITS and chloroplast sequances; no combination is yet available in that Muhlenbergia]

*Schedonorus arundinaceus (Schreb.) Dumort. - RBS, FNA

[NS - This name was proposed for conservation by Soreng et al. (2001) in Tavon (50: 915-917), which was approwed and incorperated into the Vienns Code]

[Festuca arundinacea Schreb.] - Dong01, GPF, IMF, SF!, UTF, W\&W, EW, Darby shire93, Snow03

[Festuca elatior L. var- anundinacea (Schreb.) Celak.] - HDH

[Lolium aruadinaceum (Schreb.) Darbysh.] - KTZ

*Schedonorus pratensis (Huds.) P. Beauv, - RBS, FNA

[Festuca pratensis Huds, - Dorn01, GPF, JMF, SF!, UTF, W\& W, E/W, Seow03

[Festuca elatior L. var. elatior] - $\mathrm{HDH}$

[Lotium pratense (Huds.) Darbysh.] - KTZ

Schizachne purpurascens (Torr.) Swallen - RBS, Dom01, GPF, HDH, KTZ, SF!, W\&W, E/W

Schizachyrium scoparium (Michx, ) Nash var. scoparium - FNA, IMF, KTZ, E/W

[Andropogon scoparius Michx.] - $\mathrm{HDH}$

[Andropogon seoparius Michx. var, neomexicanus (Nash) Hinchc.] - GPF

[Schizachyrimm secparium (Michx.) Nash] - Dom01, RBS, SF1, UTF, W\&W

[Schizachyrium scoparium (Michx.) Nash var, neomexicanam (Nash) Gould] - IMF

Sclerochloa dura (L) P. Beauy. - RBS, FNA, GPF, HDH, IMF, KTZ, SFW, UTF, W\&W, W

Scleropogon brevifolius Phil. - FNA, RBS, GPF, HDH, IMF, KTZ, SFE, UTF, W\&W, E

Secale cereale L. - RBS, Dom01, GPF, HDH, IMF, KTZ, SF!, UTF, W\& W, E/W

Seteria

[NS - Setaria is demonstrably polyphyletic and members in our region fall out in separate clades; Doust07 summarize these findings but do not yet suggest nomenclatural changes] 
(Identification sources:

*Setaria adhaerans (Forssk.) Chiov, - FNA

Setaria faberi R.A.W. Hem. - FNA

Setaria grisebachit E. Fourn. - FNA

* Setaria italica (L.) P. Beauv, - RBS, Dorn01, RBS, FNA, GPF, HDH, KTZ, W\&W

Setaria leucopila (Scribn. \& Merr.) K. Schum, - RBS, FNA, RBS, GPF, KTZ, SFE, W\&W, E

[Secaria miscrostachya Kunth] - HDH

Setaria pumila (Poir) Roem, \& Schult, subsp, pumila - RBS, FNA

Setaria pumila (Poir.) Roem. \& Schult, subsp, pallidefusca (Sehumacker) B. K. Simon FNA, KTZ

[Setaria glauca (L.) P. Beauv.] - Domol, W\&W'?

Setaria verticillata (L.) P. Beauv, var, verticillata - FNA, E/W

[Secaria verticillata (L.) P. Beauv.] - RBS, Dom01, RBS, GPF. HDH, IMF, KTZ, SF!, UTF, W\&W

Setaria viridis (L.) P. Beatv, - RBS, Dorn01, RBS, FNA, GPF, HDH, IMF, SF!, W\&W, E/W

[Setaria virid is (L.) P. Beauv. var. viridis] - KTZ

Setaria vulpiseta (Lam.) Roem. \& Scbult. - KTZ

Sitanion (see Elymus).

Sorghastrum nutans (L.) Nash - Dorn01, RBS, FNA, GPF, HDH, IMF, KTZ, UTF, E/W

[Sorghastrum averiaceum (Michx.) Nash] - SFL, W\&W

Sorghum bicolor (L.) Moench subsp, bicolor - GPF, KTZ

[Sorgham bicolor (L.) Mosnch] - FNA, RBS, IMF, UTF

[Sorghum vulgare Pres.] - HDH, W\&W

Sorghum halepense (L.) Pers, - Dom01, RBS, FNA, GPF, HDH, IMF, KTZ, SFE, W\&W, E

Spartina gracilis Trin. - Dorn01, FNA, RBS, GPF, HDH, IMF, KTZ, SF!, UTF, W\&W, E/W

Spartina pectinata Link - Dorn01, FNA, RBS, GPF, HDH, IMF, SFE, UTF, W\&W, E

[Spsintina pectinata Bose ex Link]-KIZ

Sphenopholis obtusata (Michx.) Scribn. var. major (Torr.) K. S. Erdm. - Dorn01, GPF, IMF, $\mathrm{E} / \mathrm{W}$

[Sphenopholis internedia (Rydb.] Rydb.] - FNA, HDH, KTZ

[Sphenopholis obtusota (Michx.) Scribe.] - SF!, UTF, WR W

Sphenopholis obtusata (Michx.) Scribn. var. obtusata - Dom01, GPF, IMF, E/W

[Sphenopholis obtusata (Michx, Seribe]- HDH, KTZ, SF!, UTF, W\&W

Sporobolus

(Identification sources:

Sporobolus airoides (Torr.) Torr. - Dorn01, FNA, RBS, GPF, HDH, KTZ, SF!, W\&W

[Sporobolus airoides (Tor.) Tor, var, airoides] - IMF, UTF, EN

Sporobolus compositus (Poir.) Merr. var. compositus - FNA, KTZ, E

[Sporobolus asper (Michx, Kusth] - HDH, IMF, SFE, UTF, WRW

[Sporobolas asper (Michx.) Kusth var, asper] - GPF

[Sporobolas compositus (Poir.) Mer.] - Dorno1, RBS 
Sporobolus contractus Hitche, - FNA, RBS, GPF, HDH, IMF, KTZ, SFI, UTF, W\&W, EN

Sporobolus cryptandrus (Torr.) A.Gray - Dom01, RBS, FNA, GPF, HDH, KTZ. SF!,UTF, W\&W, E/W

Sporobolus flexuosus (Thurb. ex Vasey) Rydb, - FNA, RBS, KTZ, W\&W, W

[Sporobolus fexuosus (Thurb.) Rydh.] - GPF, IMF, SFW, UTF, W\&W'

Sporobolus giganteus Nash - FNA, RBS, GPF, HDH, IMF, KTZ, SFI, UTF, W\&W, E/W

Sporobolus heterolepis (A. Gray) A, Gray - Dorn01, RBS, FNA, GPF, HDH, KTZ, SFE, W\&W. E

Sporobolus nealleyi Vasey - FNA, RBS, HDH rep, misid, KTZ, SFE, W\&W, misid, W\& W', E Sporobolus neglectus Nash - FNA, RBS, GPF, HDH, KTZ, SFE, W\&W, E

Sporobolus pyramidatus (Lam.) Hitche, - FNA, RBS, GPF, HDH, W\&W (mislabeled)

[Sparobolas coromandelianus (Retz.) Kunth] - KT/Z

[Sporobolus pulvinatus Swallen] - [MF, UTF

Sporobolus texanus Vasey - RBS, GPF, HDH, KTZ, SFE, UTF, W\&W, E

Sporobolus wrightii Munro ex Seribn, - FNA

Stipa (see also Achnatherum, Nasella, Oryzopsis, Pappostipa, Piptatherum, Ptilugrostis)

[NS - The generic boundaries used in FNA largely are supported by several molecular data sets, eg.

Romaschenko08 and Barkwortho8. Some aspects of the phylogeny of Stiptae are unresolved. Stipa s.s. is restricted to Eurasia. Achnatherum, Jarava and Oryzopsis fas circumsribed traditionally and here for now) are polyphyletic. Cialdella07 also summarize recent insights for Piptochastium and portions of Stipeae. It now seems more appropriare to follow the treacmest in FNA, understonding that mofe transfers will be forthooming.J

\section{Stiporyzopsis (see Achnatherum)}

\section{Thinopyrum (see Elymus)}

Torteyochloa pallida (Torr.) G. L. Church var, pauciflora (J. Presl) J, I. Davis - RBS, Dorn01, KTZ, E/W

[Glyceria pauciflora J. Presl] - HDH

[Pucsinellia pwaciflora (3. Pros]) Munz] - IME, UTF

[Torreyoshloa pallida (Torr.) Church var, fernaldii (Hitche) Dore ex Koyama \& Kawano] - Dorn01

[Torreyochloa pauciflora (J. Presi) G. L. Church]-SFI, WRW

\section{Trichachne (see Digitaria)}

\section{Tridens (see also Erioneuron)}

[Tridens albescens (Vasey) Wooton \& Standl.] - FNA, GPF, HDH, KTZ, W\&W, label:

"Colorado"; needs confirmation

(NS - FNA indicates adjacent county in KS)

\{Tridens muticus (Torr.) Nash var. muticus\} - HDH, misid., IMF, KTZ <AZ, NM, OK,

$\mathrm{UT}>$, UTF, W\&W

Tridens muticus (Torr.) Nash var. elongatus (Buckley) Shinners - GPF,KTZ, SFE, W\&W, E

[Triderts elongatus (Backley) Nash] - HDH

[Tridens, musicus [Torr.) Nash] - FNA, RBS

\{Tridens strictus (Nutt.) Nash\} - FNA

[NS - present in adjacent county in OK fide FNA: probably in 00 or NM:

Triplasis purpurea (Walter) Chapman - FNA, RBS, GPF. HDH, KTZ, SFE, W\&W, E 
Trisetum

(Identification sources:

Trisetum canescens Buckley - RBS, Dorn01, FNA, KTZ

[Trisctum cernum Trin.] - KTZ

[Trisctum cernum spp. canescens (Buckl.) Calder \& Taylor] - KTZ

[Trisetum cernum var. canescens (BuckL.) Beal] - KTZ.

[Trisetum cernuum var. projectum (Lou is-Marie) Boctle] - KTZ

[Trisetum spicatum var. projectum (Louis-Marie) 1. T. Howell] - KTZ

*Trisetum flavescens (L.) P. Beauv, - RBS, FNA, GPF, HDH

[Avena flavescens L.] - KTZ

Trisetum interruptum Buckley - RBS, FNA, GPF, HDH, KTZ, W\&W

Trisetum spicatum (L,) K. Richt, - RBS, Dom01, FNA, GPF, HDH, IMF, KTZ, UTF, E/W

[Trissum montanum Vasey] - HDH, IMF,SFE, UTF, W\& W'

[Trisetum mostanum Vasey var. shearii Louis-Maric]

[Trisetum spicatum (L.) K. Richt. subsp. alaskanum (Nash) Hulten] - SF!, W\& W'

[Trisetum spicatum (L.) K. Richt. subsp. alaskanum Hultén] - KTZ

[Trisetum spicatum (L.) K. Richt, subsp. congdonii (Scribn. \& Merrill) Hulken]- SF!, W \& W

[Trisetum spicatum (L.) K. Richt. suhsp. molle (Michs.) Hultén] - W\&W, W\&W', err. rep.

Triticum aestivum L. - RBS, Dorn01, GPF, HDH, IMF, KTZ, SF!, UTF, W\&W, E/W

\section{Vahlodea (see also Deschampsia)}

Vahlodea atropurpurea (Wahlenb,) Fr, ex Hartman - RBS, FNA, KTZ

[Deschampsia atropurpurea (Wahlenb.) Scheele var, latifolia (Hook.) Scribn. ex Macoun] - EW

[Deschampsia atropurpurea (Wshlenb.) Scheele] - HDH

[Vahlodea atropurparea (Wahlenb.) Ff. subsp. atropurpurea] - W\&W , err, rep.

[Vahlodea atropurpurea (Wahlenb.) Fr, ex Hartman subsp. latifolia (Hook.) Porsild- W\& W'

[Vahlodea atropurparea (Wahlenb.) Fr, subsp, paramushirensis (Kudo) Helren] -W\&W, W\& W', err. rep.

Vulpia myuros (L.L) C. C. Gmel. - FNA

Vulpia octoflora (Walter) Rydb. - RBS, Dom01, SF!, W\&W, E/W

[Festuca octoflora Walter] - GPF, UTF

[Festuca cetoflora Walter var, glauen Femald] - HDH

[Festuca cetofloea Walter var, hirtella Piper] - HDH

[Festuca octoflora Walter vaf. tenella (Willi.y Fernald] - HDH

[Vulpia octoflora (Walter) Rydb, var. giauca (Nurt) Fernakd] - IMF, KTZ

[Vulpia octoflora (Walter) Rydb yar, Lirtella (Piper) Henard] - IMF, KTZ

[Vulpia octoflora (Walter) Rydb, var, octoflora] - IMF, KTZ

ษ Zea mays L. - HDH, RBS, IMF, UTF

[Zea mays L. subsp, mays] - KTZ

\{Zizania aquatica L. var. angustifloia Hitch.\} - HDH, planted, not persisted, W\& W, planted. deceased

\{Zizania polustris L. var. palustris\} - KTZ

\section{Polemoniaceae - PLM}

[NS - See Porter)8, Porter00, and Geant04, whose taxoncenies differ considerably]

Snow, N, January 2009. Checklist of Vacculer Plants of the Southom Rocky Momain Region. CVersion $3 x$ 
Aliciella

(Identification sources:

Aliciella haydenii (A. Gray) J. M. Porter subsp. crandallii (Rydb.) J. M. Porter - W\&W ${ }^{1}$, W. Porter 98,00

[Gilia haydenii $A$. Gray] - H[DH, IMF, KTZ, SFW, W\&W

Alicjella haydenii (A. Gray) J. M. Porter subsp. haydenii - Porter98, 00, W

[Aliciella haydenii (A. Gray) J. M. Porter] - W\& W

[Gilia haydenii A. Gray]-HDH, IMF, KTZ, SFW, UTF, W\&W

[Gilia montezumise Tidestr. \& Dayon] - $\mathrm{HDH}$

Aliciella leptomeria (A. Gray) J. M. Porter-Dorn01, W\& W', Porter98, 00

[Gilia keptomeria A. Gray] - HDH, KTZ, W\&W

[Gilia leptomeria A. Gray var. leptomeria] - IMF, UTF

Aliciella mevickerae (M. E. Jones) J. M. Porter - Dorn0I

Aliciella micromeria (A, Gray) J. M. Porter - W\&W'; Porter98, 00, W

[Gilia kptomeria A. Gray var. micremeria (A. Gray) Cronqist] - IMF, UTF

[Gilia micromeria A. Gray] - KTZ SFW, W\& W

- Aliciella penstemonoides (M. E. Jones) J. M. Porter - ENDEMIC $<C O>$, W\& W'; Porter98, Porter00, w

[Gillia penstemonoides M. E. Jones] - KTZ. SFW. W\&W

[Gillia sodifolla Brandeges] - WRW

Aliciella pinnatifida (A. Gray) J. M. Porter - Dorn01, Porter00. E/W

[Aliciella pianatifida (Nutt. ex A. Gray) J. M. Portef] - We W': Porter98

[Gilia calearea M. E. Jones] - HDH

[Gilis pinitacifica Nutt.] - SF!, UTF, W\&W

[Gillia pintasifida Nuat ex A. Gray] - GPF, IMF, KTZ

- Aliciella sedifolia (Brandegee) J. M. Porter- ENDEMIC $<\mathrm{CO}>$, W\&W'; Porter98, 00, W [Gilia sedifolia Brandegee] - KTZ, SFW

Aliciella stenothyrsa (A. Gray) J. M. Porter - W\&W' ${ }^{1}$; Porter98, 00, W

[Gilia stenothyrsa A. Gray] - IMF, KTZ, UTF, WRW

[Gilia stenothyrsa M. E. Jones] - SFW

Aliciella subacaulis (Rydb.) J. M. Porter \& L. A. Johnson - Porter00

[Aliciella leptomeria (A. Gray) J. M. Porter] - W\& W'

[Gillin ineonspicun (Sm.) Dougl.] - W\&W

[Gillia leptomeria A. Gray var, leptoeneria] - IMF

[Gilin subacaulis Rydb.] - KTZ, UTF

Aliciella subnuda (A. Gray) J. M. Porter - Porter00. Smith09?

[Aliciella subnuda (Tort, ex A. Gray) J. M. Porier] - W\& $W^{1}$; Porter $98<A Z$, NM. UT>

[Gilia subneda Torr.] - WEW

[Gilia subnuda Torr, ex A. Gray] - IMF, KTZ, UTP

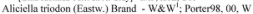

[Gilia leptomeria A. Gray var. leptomeria] - IMF, UTF

[Gilia triodon Fastw.] - KTZ, SFW, W\&W

Collomia grandiflora Douglas ex Lindl. -HDH, IMF, KTZ, UTF, W\&W, Porter00, W

[Collomia grandiflora Douglas] - SFW

Collomia linearis Nutt. - Dom01. GPF, HDH. IMF. KTZ, SF!, UTF. W\&W. Porter00, E/W

Eriastrum wilcoxii (A. Nels.) Nason - Dorn01, KTZ

[Efiastrum sparsiflorum var. wilooxii (A. Nels.) Crone.]- KTZ

Eriastrum diffusum (A. Gray) H. Mason-IMF. KTZ. SFW. UTF. W\&W. Porter00. W 
Gilia

(Identification sources:

Gilia (see also Aliciella, Ipomopsis, Giliastrum, Lathrocasis, Navarretia)

Gilia clokeyi H. Mason - KTZ. W\&W, Porter00, W

[Gilia clokeyi Rydb.] - SFW

[Gilia inconspicua (Sm.) Sweet var. incornspicua] - IMF. UTF

Gilia inconspicua (Sm.) Sweet - KTZ, Porter00

[Gilia inconspicua (Sm.) Dougl.] - W\&W

[Gilia inconspicua (Sm.) Sweet var. incenspicua] - IMF, UTF

Gilia ophthalmoides Brand - Dom01, KTZ, SF!, W\&W, Porter00, E/W

[Gilia inconspicua (Sm.) Swect var. inconspicua] - IMF, UTF

Gilia sinuata Douglas ex Benth. - HDH. KTZ. W\& W, Porter00, W

[Gilia inconspicua (Sm.) Sweet var, sinuata (Dougl.) A. Gray] - IMF, UTF

[Gilia sinuata Douglas] - SFW

Gilia tweedyi Rydb, - Dorn01, KTZ, SFW, W\&W, Porter00, W

[Gilia inconspicua (5m.) Sweet var. tweedyi (Rydb.) Crong.] - ]MF

Giliastrum acerosum (A. Gray) Rydb, - Porter00, E

[Gilia acerosa (A. Gray) Britton] - HDH

[Gilia rigidula Benth] - GPF

[Gilia rigidala Benth. subsp. acerosa [A. Giray) Wherry] - KTZ

[Giliastrum rigidulum (Benth.) Rydb. subsp, acerosum (A. Gray) W. A. Weler] - SFE, W\& W

Gymnosteris parvula (Rydb,) A. Heller - Dorn01, HDH, SWF, UTF; Porter00, E/W

[Gymnosteris parnula A. Heller] - Donn0I, IMF, KTZSFE, W\& W

Ipomopsis

(Identification sources:

Ipomopsis aggregata (Pursh) V. E. Grant subsp, aggregata - KTZ, SFW, W\& W, Porter00, W

[Gilia aggregata (Pursh) Spreng subsp. aggecgata] - HOH

[Gilia aggregnta (Pursh) Spreng var. aggregnta] - IMF, UTF

[Ipomopsis aggregata (Pursh) V. E. Grant var. aggregata] - Dorn0]

Ipomopsis aggregata (Pursh) V. E. Grant subsp, attenuata (A. Gray) V. E. Grant \& A. D. Grant

- KTZ, SF!, W\&W, Porter00, E/W

[Gilia ageregnta (Pursh) Spreng, var, attenuaba A. Gras] - IMF

[Gilia attenuata (A. Gray) A. Nel5.] - HDH

[Ipomopsis ageregaca (Pursh) V. E. Grant var, anenuata (A. Gray) Dons] - Dorn01

Ipomopsis aggregata (Pursh) V. E. Grant subsp. candida (Rydb.) V. E. Grant \& A. D. Grant

- KTZ, SFE, W\&W, Porter00, E

[Gilia aggregata (Pursd) Spreng, vaf, candida (Rydb.) Crone, ] - IMF

[Gilia candida Rydb, subsp, candida] - $\mathrm{HDH}$

[Gilie candida Rydb. subsp. vera Wherry] - HDH

Ipomopsis aggregata (Pursh) V. E. Grant subsp. collina (Greene) Wilken \& Allard - KTZ, SFE, W\&W, Porter00, E

[Gilia aggregata (Pursh) Spreng, intermediate between var, candida (Rydh,) Crong, and var, anaculata M. E. Jones] - IMF 
[Gilia candida Rydb. subsp. collina (Greene) Wherry] - HDH

Ipomopsis aggregata (Pursh) V. E. Grant subsp, formosissima (Greene) Wherry - KTZ, SFW, W\&W, Porter00, W

[W] - taxen is same as subsp. aggregata with stamens slightly exserted]

[Gilia aggregata (Pursh) Spreng- subsp. formosissima (Greene) Wberry] - HDH

[Gilia augregata (Pursh) Spreng, var. maculata M. E., Jones] - IMF

[Gilia texana (Greene) Wooton \& Standl.] - HDH

[lpomopsis aggregata (Pursh) V. E. Grant var. maculata (M. E. Jones) S. Welsh] - UT3

Ipomopsis aggregata (Pursh) V. E. Grant var, tenuituba (Rydb, Dorn - Dorn0]

Ipomopsis aggregata (Pursh) V. E. Grant subsp, weberi V. E. Grant \& Wilken - KTZ,

SF!, W\&W, Porter00, E/W

[W] - not separable from subsp. attenuati]

[Ipomopsis aggregata (Pursh) V. E. Grant var. weberi (V. E. Grant \& Wilken) Dom] - Dom01

\{Ipomopsis arizonica (Greene) Wherry\} - KTZ $<\mathrm{AZ}, \mathrm{UT}>$, W\&W, err, rep., Porter00

\{Gilia aggregala (Pursh) Spreng, var, arizonica (Greete) Fosberg, - IMF. UTF

\{Gilia arizonica (Greente) Rydb.\} - HDH, exp.

Ipomepsis aygregata (Pursh) V. E. Grant subsp. arizonica (Greene) V. E. Grant \& A. E. Grant\} - W\& W', err. rep.

Ipomopsis congesta (Hook.) V. E. Grant subsp. congesta-GPF, KTZ, W\&W, Porter00, EN

[Gilis congesta Hook, var. congesta] - HDH, IMF, UTF

[iponopsis congesta (Hook.) V. E. Gram var. songesta] - Dom01, SEI

Ipomopsis congesta (Hook.) V. E. Grant subsp, frutescens (Rydb.) A. G. Day - KTZ, SF!, W\&W, Porter0O, E,W

[Gilia congesta Hook, var. burleyana (A. Nelson) Constance \& Rollins] - HDH

[Gilia congesta Hook. var, frutescens (Rydb.) Crong.] - IMF, UTF

[Ipomopsis congesta (Hook.) V. E. Grant var. tnitescens (Rydb.) S. Welsh] - UT3

Ipomopsis crebrifolia (Nutt.) Dom - Dorn0I

[Gilia congesta Hook. var. crebrifolia (Nunt) A, Gray] - IMF. UTF

[Iponopsis congesta (Hook.) V. E. Grant subsp. crebifolia (Nuti.) A. G. Day] - KTZ, W\&W, Portero0

[lpomopsis congesta (Hook,) V. E. Gram subsp. crebifolia (Rydb.) A. G. Day] - W \& W'

[Ipomopsis congesta (Hook.) V. E. Grant var, crebifolia (Nuti.) S. Welsh] - UT3

Ipomopsis gunnisonii (Torr. \& A. Gray) V. E. Grant - KTZ, SFW, W\&W, Porter00, W

[Gilia gumnisonii Torr. \& A. Gray] - HDH, IMF, UTF

Ipomopsis laxiflora (J. M. Coult.) V. E. Grant-GPF, KTZ, SFE, W\&W, Porter00, E

[Gilia laxifolia (J. M. Coult.) Osterh.] - HDH

Ipomopsis longiflora (Torr.) V. E. Grant subsp. longiflora - KTZ, Porter00, E/W

[Gilia longiflora (Torr, G. Dob] - HDH, IMF, UTF

[Ipomopsis longiflera (Torr.) V. E. Grant] - Dorn01, SF!, W\& W'

Ipomopsis multiflora (Nutt.) V. E. Grant - KTZ, SFE, W\&W, Porter00, E

Ipomopsis polyantha (Rydb.) V. E. Grant - KTZ, SFW, W\&W, Porter00, W

[Gilia polyastha Rudb] - HOH, IMF, UTF

[Gilia polyantha Rydb, vat, polyantha] - IMF

Ipomopsis polycladon (Torr.) V, E. Grant - Dorn01, KTZ, SFW, Porter00, W

[Gilie polycladoen Tor.] - HDH

[ipomopsis polycladon Tor.] - W\&W

Ipomopsis pumila (Nutt.) V. E. Grant - Dorn01, GPF, KTZ, SF!, W\&W, Porter00, E/W

[Gilia pumila Nutt.]- HDH, IMF, UTH

Ipomopsis roseata (Rydb.) V. E. Grant - KTZ, SFW, W\&W, Porter00, W

[Gilia roseata Rydb,]- HDH, IMF, UTF

- Ipomopsis spicata (Nutt.) V. E. Grant subsp. capitata (A. Gray) V. E. Grant - ENDEMIC

$\langle\mathrm{CO}\rangle, \mathrm{KTZ}$, Porter 90 , E/W

[Gilia spicata Nuts. var. capitata A. Gray] - HDH, IMF

[lpomopsis globularis (Brand) W. A, Weber] - KTZ, SF!, WJ, W\&W

Snow, N. Junury 2009. Checklist of Vascular Plants of the Southom Rocky Mounain Region. CVersion $3 x$ 
Ipomopsis spicata (Nutt.) V. E. Grant subsp. spicata - Dorn01, KTZ, PL, Porter00, E/W

[Gilia spicata Nutt. var. spicata] - HDH, IMF, UTF

[lpomopsis spicata (Nutt.) V. E. Gran] - GPF, SF!, W\& W

[lpomopsis spicata (Nutt.) V. E. Grant var. spicata] - RMC

Ipomopsis tenuituba (Rydb,) V. E. Grant subsp. tenuituba - KTZ. Porter00, W

[Gilia tenuituba Rydb.] - HDH

[lpomopsis tenaituba (Rydb.Y V. E. Grant] - SFW, W\&W

[lpomopsis temuituba (Rydb.) V. E. Grant var. tenuituba] - RMC

Lathrocasis tenerrima (A. Gray) L. A. Johnson - Dom01

[Gilia testerrima A. Gray] - KTZ

Leptodactylon (see Linanthus)

\{Leptosiphon floribundus (A. Gray) J. M. Porter \& L. A. Johnson subsp, floribundus\} Porter00

\{Linanthus floribundus (A. Gray) Greene ex Milliken subsp. floribundus\} - KTZ CAZ, NM:

Linanthastrum floribundum (A.Gray) Wherry\} - HDH, W\&W, misapp.

ILinanthastrum nuttallii (A. Gray) Exan subsp. floribundum (A. Gray) Ewan\} - IMF

Leptosiphon nuttallii (A. Gray) J. M. Porter \& L. A. Johnson subsp. nuttallii - Porter00, E/W

[Leptosiphon nuttallii (A. Gray) J. M. Porter \& L. A. Johnson]

[Linanthus nutallii (A. Gray) Grome ex Milliken subsp. sutrallii] - KTZ

[Liraanthasirum nuttallii (A. Gray) Ewan] - HDH, SF!, UTF, W\&W

[Liranthastrum nuttallit (A. Gray) Ewan var. nuttallii] - IMF

Leptosiphon septentrionalis (H. Mason) J. M. Porter \& I. A. Johnson - Dorn01, Porter00, E/W

[Linanthus harknessi (Curran) Greene var. sepeentrional is (H. Mason) Jeps, \& V. L. Bailey] - HDH, SFE, WEW

[Liranthus harknessii (Curran) Greene var, septontrionalis (Jepson) V. L. Bailey] - SFW

[Linsothes septentrionalis H. Mason] - GPF, IMF, KTZ, UTF

\section{Linanthastrum (see Leptosiphon)}

\section{Linanthus (sec also Leptosiphon)}

Linanthus cespitosus (Nutt.) J. M. Porter \& L. A. Johnson - Dorn01, Porter00, E/W

[Leptodactylon cespitosum Nutt] - GPF, HDH, IMF, KTZ, SF, UTF, W\&W

Linanthus pungens (Torr.) J. M. Porter \& L. A. Johnson - Dorn01, Porter(K), E/W

[Leptodactylon pengens (Torr.) Nutt.] - GPF, SF!, UTF

[Leptodactylon pengens (Torr) Rydb.] - W\& W

[Leptodactylon pengents (Torr.) TorT. ex Nutt] - KT/

[Leptodactylon pungens (Torr) Rydb, sulosp, bresifolium (Rydb.) Wherry] - HDH

[Leptodactylon pungens (Tom,) Rydb, subsp, pangens] - HDH

[Leptodactylon pungens (Torr) Nuth, var, pungens] - IMF

Linanthus watsonii (A. Gray) Wherry - Dorn01, Porter00, W

[Leptodactylon watsonil (A, Gray) Rydb.] - HDH, IMF, KTZ SFW, UTF, W\&W

Microsteris gracilis (Hook.) Greene var. gracilis - Dom01

[Gilia gracilis Hook.] - KT2

[Phlox gracilis (Hook.) Greente subsp. gracilis] - KTZ

Microsteris gracilis (Dougl. ex Hook.) Greene subsp. humilior (Greene) H. Mason -Porter00. E/W

[Microsteris gracilis (Hook.) Greene] - SF!, GPF

[Microsteris gracilis (Hook.) Gresne var, hamilior (Hook.) Cronquist] - Dorn01, IMF, UTF 
[Microsteris gracilis (Dougl. ex Hook.) Greene subsp. humilis (Greene) V. Grant] - W\&W

[Microsteris gracilis (Hook.) Greene var, humilis (Hook.) Croncuist] - KTZ

[Microsteris humilis (Douglas) Greene] - $\mathrm{HDH}$

[Phlox gracilis (Hook.) Greene subsp. humilis (Greene) H. Mason] - KTZ

Navarretia breweri (A. Gray) Greene - Dorn01, HDH, IMF, KTZ, SFW, W\&W, Porter00. W

Navarretia intertexta (Benth.) Hook, subsp propinqua (Suksd.) A, G. Day - KTZ, Porter00. E/W

[Nayarretia intertexta (Benth.) Hook.] - UTF

[Nayarretia leucocephala Benth. subsp. minima (Nutt.) A. G. Day]- KTZ

[Navarretia intertexta (Benth.) Hook. var. propinqua (Suksd.) Brand] - Dom01, GPF, [MF

[Nayarretia minima Nutt.] - GPF, HDH, SF!, W\&W

Navarettia saximontana Stanley C. Spencer-Spencer03

[NS - L. Johnson \{BRY, pers. comm. 2007\} indicates that genetically this is likely 3 distinct taxa]

Navarretia sinistra (M. E. Jones) L. A. Johnson subsp, sinistra - Porter00, E/W

[Gilia capillaris Kellogg] - IMF

[Gilia sinistra M. E. Jones] - SF!, WA. W

[Gilia sinistra M. E. Jones subsp- sinistra] - KTZ

\section{Phlox (see also Mierosteris)}

(Identification sources:

Phlox alyssifolia Greene - Dorn01

[Phlox alyssifolia Greene subsp, alyssifolia] - KTZ

[Phlor alyssifolia subsp, vera Wherry] - KTZ

Phlox andicola E. E. Nelson subsp, andicola - KTZ, Porter00, E

[Phlox andicola E. E. Nelson] - Dorm01

[Phlox andicola Nutt.] - SFE

[Phlox andicola Nutt. ex A. Gray]-GPF

[Phlor andicolar (Nutt. ex Torr. \& A. Gray) E. E. Nelson] - W W W

[Pblox andicola (Britton) E. E. Nelson subsp, andicola] - HDH

[Phlox andicols (Beiton) E. E. Nelson subsp. plantiairam (A. Nelsoa) Wherry] - HOH

Phlox austromontana Coville subsp. austromontana - KTZ, Porter00, E/W

[Phlox austromontana Coville] - IMF. SF!, WS W

[Phlox austromootana Coville subsp. wra wherry] - $\mathrm{HDH}$

[Phlox austromoetana Coville var, austromontana] - UTF

Phlox austromontana Coville subsp. densa (Brand) Wherry - KTZ. Porter00

[Phlox austromontana Coville] - IMF, W\&W

[Phlox austromontana Coville var, austromontana] - UTF

\{Phlox cespitosa Nutt.\} - Porter00

(Phlox caespitesa Nott.) - IMF, KTZ, W\& W, misapp

\{Phlox caespitosa Nutt, subsp, caespitosa\} - HDH

Phlox caryophylla Wherry - HDH, KTZ, SFW, W\&W, Porter00, W

Phlox condensata (A. Gray) E. E. Nelson - IMF, KTZ, SF!, W\&W, Porter00, E/W

[W] - P, pulvinats is a subalpine ecotype of P, condensata]

[Phlox chespitosa Nurt. subsp. condensata (A. Gray) Wherry]- HDH

\{Phlox diffusa Benth. subsp. diffusa\} - HDH, W\&W, misid., Porter00

\{Phlox diffusa Benth. - W\&W'

\{Phlox diffusa Benth. subsp, difflasa\} - KTZ

\{Phlox diffusa Benth var, diffusa) - IMF

\{Phlox diffusa Benth. subsp, longistylis Whens\} - KTZ

\{Phlox diffusa Benth. var, longissylis (Wherry) M. E. Peck\} - IMF

\{Phlox diffusa Benth. subsp. subcarinata Wherry\} - KTZ, Porter00

Snow, N, January 2009. Checklist of Vaculer Plants of the Southom Rocky Momain Regioe. CVersion $3 x$ 
\{WJ - not in Colorado, a taxion from the northwest;

Phlox austromontana Coville; - DMF

Phlox hoodii Richardson - Dorn01, GPF, W\&W, E/W

[Phlox hoodii Richardson subsp. canescens (Torr. \& A. Gray) Wherry] - HDH, KTZ, SF!, Porter00

[Phlox hoodii Richardson subsp. glabrata (E. E. Nelson) Wherry]- HDH, KTZ, Porter00

[Phlox hoodii Richardson subsp. hoodii] - HDH, KTZ, Purter00

[Phlox hoodii Richardson var, hoodii] - IMF

[Phlox hoodii Richardson var. cancseens (Torr. \& A. Gray) M. E. Peck] - IMF, UTF

Phlox kelseyi Britton - Dom01. IMF, E

[WJ - W \& W have identificed speciments of this taxon as P. pulvinata)

\{Phlox kelseyi Britton subsp. kelseyi\} - KTZ $<$ WY $>$ Porter00

\{Phlox kelseyi Britton subsp. salina (M. E. Jones) Wherry\} - KTZ SFE. W\& W misid.

Phlox longifolia Nutt, subsp, longifolia - HDH, KTZ, Porter00, ENW

[Phlox longitolia Nutt] - Dorn01. SF!, UTF, W\& w

[Phlox longifolia Nutt. subsp, calva Wherry]- HDH

[Phlox longitolia Nuat subsp, cortezana (A. Nelson) Wherry]- HDH

[Phlos longifolia Nutt subsp. humiles (Douglas) Wherry] - HDH

[Phlox longifolia Nutt var. kongifolia] - IMF

Phlox multiflora A. Nelson - Dorn01, IMF, SF!, UTF, W\&W, EW

[Phlox multiflora A. Nelson subsp. costata (Rydb.) Wherry] - HDH

[Phlox multiflora A. Nelson subsp. depressa (E. E. Nelsan) Wherry] - HDH, KTZ, Porter00

[Phlox multiflora A. Nelson subsp. maltiflora] - HDH, KTZ, Porter0o

[Phlox mutriflora A. Nelson subsp. patula (A, Nelson) Whony] - KTZ, Porteroo

[Phlox panila A. Nelsos] - HDH

Phlox muscoides Nutt, - Dom01, IMF, SF!, UTF, W\&W", Porter00, E/W

[Phlox bryoides Nuit] - GPF, HDH, w\&W

[Phlox hoodii Rictardson subsp، mussoides (NutL) Wherry] - HDH, KTZ

Phlox nana Nutt. - KTZ

Phlox opalensis Dom - Dom01, KTZ

Phlox pulvinata (Wherry) Cronquist - Dom01, IMF, KTZ, UTF, Porter00, E/W

[W] - P. pulvinata is a subalpine ecotype of P, condensant]

[Phlox caespitosa Nurt. subsp, pulvinata Wherry] - HDH

[Phlox sibifica L. subsp. pulvisata (Wherry) W.A. Weber] - SF!. W\& W

\{Phlox stansburyi (Torr.) A. Heller subsp, stansburyi\} - HDH, KTZ<AZ,NM,UT>, Porter0O

\{Phlox longifolia Nutti\} - UTF

\{Phlox longifolia Nun, var, stansburyi (Torr.) A. Gray\} - IMF

\{Phlox stansburyi (Torr.) A. Hellec\} - W\&W, misapp.

Phlox "sp. nov." - B. Legler, pers. comm. 2008

[NS - evidently local endemic from northern NM]

Phlox variabilis Brand suhsp, variabilis - KTZ, Porter(0)

[W] - likely synonymons with $\mathrm{P}$. coodensata or $\mathrm{P}$. pulvinata. If $\mathrm{P}$. pulvinata, then $\mathrm{P}$. variabilis has priority]

[Phlox sibirica L. subsp. pulvinata (Wherry) W. A. Weber] - W\& W

[Phlox variabilis Brand] - $\mathrm{HDH}$

Phlox viridis E. E. Nelson subsp. longipes (M. E. Jones) Wherry - KTZ, WJ, Porter00

[Phlox longifolia Nunt] - W\&W

[Phlox longifolia Nuat subsp. longipes (M. E. Jones) Wherry] - HDH

[Phlox longifolia Nunt. var, longifolia] - IMF

Polemonium brandegei (A. Gray) Greene - Dorn01, IMF, KTZ, SFE, W\&W, Porter00, E/W

[Polemonium brandegei Greene] - GPF, SFW

[Polemonium viscosum $\mathrm{Natt}$.] - $\mathrm{HDH}$

- Polemonium confertum A. Gray - ENDEMIC <CO>, IMF, KTZ, SF!, W\&W, Porter(X), E/W

[Polemonium visccoum Nutt.] - $\mathrm{HDH}$

Snow, N, Junury 2009. Checklist of Vaccular Plants of the Southom Rocky Momain Region. CVersion $3 x$ 
Polemonium foliosissimum A, Gray - HDH, SF!, W\&W, Porter00, E/W

[Polemonium foliosissimum (A. Gray) A. Gray] - W \& W

[Polemonium foliosissimum A. Gray var. foliosissimum] - IMF, KTZ, UTF

[Polemonium foliosissimum A. Gray var. molle (Greene) Anway] - KTZ

Polemonium occidentale Greene subsp, occidentale - HDH. KTZ, Porter00, E/W

[Polemonium caeruleum L. subsp. anygdalinum (Whemr) Manz] - SF!, W\&W

[Polemonium caeruleum L. var. pterssperma Benth.] - UTF

[Polemonium occidentale Greene] - Dorn01, IMF

Polemonium pulcherrimum Hook. subsp. delicatum (Rydb.) Brand - KTZ, SF!, W\&W, Porter00, $\mathrm{E} / \mathrm{W}$

[Polemonium delicatum Rydb.] - $\mathrm{HDH}$

[Polemonium pulcherrimum Hook. var. delicatum (Ryibo.) Cronquist] - IMF, UTF

[Polemonium pulcherrimum Hook.] - Dorn01

Polemonium viscosum Nutt. - Dorn01, HDH, IMF, KTZ, SF!, UTF, W\&W, Porter00, ENW

\section{Polygalaceae - PGL}

(Identification sources:

\{Polygala acanthoclada A. Gray\} - HDH rep, KTZ $\langle A Z$, UT $>$, W\&W, misapp.

(Polygala acanthoctsda A. Gray var. acanthoclada) - IMF, UTF

Polygala albe Nutt, - Dorn01, GPF, HDH, KTZ, SFE, W\&W

[Polygala alba Nutt. var albo] - E

Polygala sanguinea L. - KWA

[Polygala viridesoeas L.] - MAH, KTZ

Polygala subspinosa S, Watson - HDH, KTZ, SFW, UTF, W\&W, W

[Polygala subspinosa S. Watson var- subspinosa] - IMF

\{Polygala verticillata L.\} - GPF, HDH rep., IMF, UTF, W\&W, misapp.

\{Polygala verticillata L. var. isocycla Fernald\} - KTZ - KS. NE, OK, UT, WY?

\section{Polygonaceae - PLG}

(Identification sources:

Acetosella (see Rumex)

Bistorta bistortoides (Pursh) Small - FNA, SF!, W\&W

[Polygonam bistontoides Pursh] - KTZ UTF, EW

[Polysonum blstontoides Pursh var, bistonoides] - $\mathrm{HDH}$

[Polygovam bistortoides Pursh var. linearifolium (S. Wasson) Small] - HDH

Bistorta vivipara (L.) S. Gray - SF!, W\&W

[Perisicaria viviparum (L.) Ronse Decraene] - FNA

[Polygonam viviparum L.] - GPF, HDH, KTZ, UTF, EW

\section{Eriogonum (see also Stenogonum)}

(Identification sources:

Eriogonum acaule Nutt. - FNA, KTZ, SFW, W\&W, W

[Eriogonum cacspotisum Nutt subsp, acuale (Nuit.) S. Stokes] - FNA

[Eriogonum caespotisum Nutt. var, acuale (Nutt.) R. J. Davis] - FNA 
Eriogonum alatum Torr. var, alatum - FNA, GPF, KTZ, E/W

[Eriogonum alatum Torr.] - UTF

[Eriogonum alatum Torr. subsp. alatum] - HDH

[Eriogonum alatum Torr. subsp. triste (S. Watsoo) S. Stokes] - HDH

[Plerogonum alatum (Tom.) H. Gross]-SF!, W\&W

Eriogonum annum Nutt, - FNA, GPF, HDH, KTZ, SFE, W\&W, E

Eriogonum arcuatum Greene var. arcuatum - FNA

[Erivgonsum arcuatum Gireene] - HDH

[Eriogonum bakeri Greene] - FNA

[Eriogonum flavum Nutt.] - W\& W $^{1}$

[Erivgonum flavum Nutt. var. tectum (A. Nels.) S. Stokes] - FNA

[Eriogonsum jamesii Benth. subsp, bekeri (Greene) S. Stokes] - FNA

[Eriogonum jamesii Benth. var. flavesxens S. Watson] - KTZ, UTF, W\& W

[Eriogonum jamesii Benth. var. higginsii S. L. Welsh] - FNA

- Eriogonum arcuatum Greene var, xanthum (Smal]) Reveal - ENDEMIC $\angle C O$. FNA

Eriogonum chloranthum Greene] - FNA

[Eriogonum flavam Nutt.] - HDH

[Eriogonsum flavum Nuth, subsp, shloranthum (Grecne) S. Siokes] - SF', W\& W'

[Eriogonsum jamesii Benth. var, xamthum (Small) Reveal] - KIZ, W\&W, EW

[Eriogonum xanthum Small] - FNA

Eriogonum batemanii M. E. Jones - FNA, HDH, KTZ, SFW, W\&W, W

Eriogonum bicolor M. E. Jones - FNA, HDH, KTZ, SFW, UTF, W\&W, W

[Efiogonum microthesum Nurt. subsp. bicolor (M. E. Jones) S. Stokes] - FNA

- Eriogonam brandegei Rydb, - ENDEMIC $<\mathrm{CO}>$, KTZ, SFE, W\&W, E

[Eriogonum spathulatum A, Gray] - HDH, rep.

Eriogonum brevicaule Nutt. var. brevicaule - FNA, KTZ, UTF, E/W

[Eriogonum brisvicaule Nutt.] - GPF. SFI, W\&W

[Efiogonim bricvicaule Nun, var, grangerense (M. E. Joess) S. Stokes]- FNA

[Eriosonsm brievicaule Nutt, wasatchense (M. E. Jones) Reveal] - FNA

[Eriogonum campanu latum Nutt.] - HDH

[Eriogonum grangerense M. E. Jones] - FNA

[Eriogonum wasatchense M. E, Jones] - FNA

\{Eriogonum brevicaule Nutt. var. laxifolium (Torr. \& A. Gray) Reveal\} - KTZ $<$ UT, WY>

\{Eriogonum chrysocephalum A. Gray\} - HDH rep. W\&W, rep.

Eriogonum brevicaule Nutt. var. micranthum (Nutt) Reveal - FNA

[Eriogonum brevicaule subsp. orendense (A. Nels.) S. Stokes] - KTZ.

\{Eriogonum caespitosum Nutt.\} - HDH, doubtful, KTZ $\angle A Z, U T$, WY $>$. UTF, W\&W, err. rep.

Eriogonum cernuum Nutt, - FNA, GPF, HDH, SF!, W\&W

[Eriogonum cernuum Nutt, var, cervuum] - KTZ, UTF, E/W

[Eriogonum cernuum Nutt, var, psamnophilums S. L. Welsh] - FNA

[Eriogonum cernuum Nutt, subsp, tenue (Torr, \& A. Gray) S, Stokes] - FNA

[Eriogonum cerbuum Nutt, var, tenue Tor, \& A. Gray] - FNA

[Eriogonum cernulm Nutt, var, unbraticum Eastwood] - FNA

[Eriogonum cernumun Nutt. subsp. viminale S. Stokes] - FNA

[Eriogonum cernuum Nutt. var. viminale (S. Stokes] Reveal] - FNA

Eriogonum clavellatum Small - FNA. KTZ, UTF, W\&W, W. UT3

[Eriogonum clavellatum Eastw.] - SFW

[Eriogonum pelinophilum Reveal] - SfW, W\&W

- Eriogonum coloradense Small - ENDEMIC $<\mathrm{CO}>$. HDH, KTZ, SF!, W\&W, E/W

[NS - See comments by Reveal in FNA 5:262 regarding questionable distinctness of this taxon under $\mathrm{E}$. lenchephyllum]

[Eriogonsum multiceps Ness var. coloradense (Small) S. Stokes] - FNA

Eriogonum contortum Small - FNA, HDH, KTZ, SFW, W\&W, W 
[Eriogonum contortum Small ex Rydb.] - UTF

[Eriogonum effusum Nutt. subsp. contortum (Small ex Rydb.) S. Stokes] - FNA

Eriogonum corymbosum Benth. var, corymbosum - KTZ, SFW, UTF, W\&W, W

[Eriogonim corymbosum Benth.] - HDH

[Eriogonum corymbosum Benth. var. divaricatum Torr. \& A. Gray] - FNA

[Eriogonum carymbosum Benth. var. ercetum Reveal \& Brotherson] - FNA

[Eriegonum divergens Small] - FNA

[Eriogonum effusum Nutt. subsp. divaricatum (Torr. \& A. Gray) S. Stobes] - FNA

[Eriogonum effusum Nutt. subsp. durum S. Stokes] - FNA

[Eriogonum effusum Nutt. subsp. saliaum (A. Nels.) S. Stokes] - FNA

[Eriogonum salinum $A$. Nelsoa] - HDH, rep.

Eriogonum corymbosum Benth, var, orbiculatum (S. Stokes) Reveal \& Brotherson - FNA, KTZ, SFW, UTF, W\&W, W

[Eriogonum divergens Small] - HDH, exp.

[Eriogonum effusum Nutt. subsp. orbiculatum S. Stokes] - FNA

Eriogonum corymbosum Benth, var, velutinum Reveal - FNA, KTZ, SFW, W

[Eriogonum corymbosum Benth. var, orbiculatum (S. Stokes) Reveal \& Brotherson] - UTF

Eriogonum davidsonii Greene $-K T Z \angle A Z$. UT $>$, UTF

\{Eriogonum densum Greene\} - HDH, exp., W\&W

\{Eriogonum polycladen Benth\} - KTZ $\subset$ AZ, NM, UT>

Eriogonum divaricatum Hook, - FNA, HDH, KTZ, SFW, UTF, W\&W, w

Eriogonum ephedroides Reveal - FNA, KTZ, SFW, W\&W, W

[Eriogonum brevicaule Nutt. ex ephedroides (Reveal) 5. L. Welsh] - UTF

Eriogonum effusum Nutt. - FNA, HDH, SFE, W\&W

[Efiogonum effusum Nutt, var, effusum] - GPF, KTZ

[Efiogonum nudicaule (Torr.] Smail] - $\mathrm{HDH}$

[Eriogonum microthecum Nutt. var. effusam (Nutr.) Torr. \& A. Gray] - E

- Eriogonum exilifolium Reveal - ENDFMIC $<C O, W Y>$, FNA, KTZ, SF!, W\&W, E/W

Eriogonum flavum Nutt, var. flavum - FNA, KTZ, E/W

[Eriogonum flavum Nutt.] - GPF, HDH, SFE

[Eriogossim flavum Nutt, subsp. flavum] - SFW

[Eriogonam flavum Nuth, ex Benth. subsp. flavum] - We Wt

Eriogonum fusiforme Small - FNA, HDH, KTZ

[Eriogonum inflatum Torr. \& Frémont var, fusifonme (Small) Reveal] - UTF, W\&W

Eriogonum gordonii Benth. - GPF, HDH, KTZ, SFW, UTF, W\&W, W

Eriogonum heracleoides Nutt var. heracleoides - FNA, KTZ, W

[Eriogonum heracleoides Nutt.] - HDH, SPW, UTF, W\&W

Eriogonum hookeri S. Watson - FNA. HDH, KTZ, SFW, UTF, W\&W, W

[Eriogonum deflexum Tor, var, gilvum $\mathrm{S}$, Stokes] - FNA

[Eriogonum deflexum Tor, subsp, hookeri (S, Wats.) S, Stokes] - FNA

Eriogonum inflatum Torr. \& Frémont - FNA

[Eriogonum fusiforme Small] - HDH, KTZ

[Eriogonem glascum Small] - FNA

[Eriogonum inflatum Torr, \& Frémont var, deflatum I. M. Johnston] - FNA

[Eriogonum inflatum Torr, \& Frémont var, fusiforme (Small) Reveal] - UTF, W\&W

[Eriogonum trichopes Torr, subsp, gluocum (Small) S. Stokes] - FNA

[Eriogonum inflatum Torr. \& Frémont var, inflatum - KTZ,UTF, W\&W, w

[Eriogonum inflatum Torr. \& Frémont] - HDH, SFW

Eriogonum jamesii Benth. var. jamesii - FNA, KTZ, W\&W, E/W

[Eriogonum jamesii Benth.] - GPF, HDH, SF, W\& W'

Eriogonum lachnogynum Torr. ex Benth, var. colobum Reveall \& A. Clifford

Eriogonum lachnogynum Torr. ex Benth. var. lachnogynum - FNA

[Eriogonum lachoogynum Torr, ex Benth.] - HDH, KTZ, W\&W, E

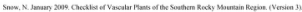


[Eriogonum lachıogynum Torr.] - GPF, SFE

[Eriogonum tetraneuris Small] - HDH, rep.

\{Eriogonum leptocladon Tort, \& A. Gray var- leptocladon\} - SFW, UTF, W \&W, W

\{Eriogonum leptockdon Torr. \& A. Gray\} - HDH, exp.

Eriogonum leptocladon Torr, \& A. Gray var, ramosissimum (Eastw.) Reveal - FNA, KTZ. SFW, UTF, W\&W, W

[Eriogonum effusum Nutt. subsp. pallidum (Small) S. Stokes] - FNA

[Eriugonsum leptocladon Torr. \& A. Gray]- HDH, exp.

[Eriogonum ramosissimum Eastw.] - FNA

Eriogonum leptophyllum (Torr \& A. Gray) Wooton \& Standl. - FNA, KTZ, W

[Eriogonum effusum Nutt. var. leptophyllum Torr.] - FNA

[Eriogonum leptophyllum (Torr.) Wooton \& Standl.] - HDH, SFW, UTF, W\& W

Eriogonum lonchophyllum Torr, \& A. Gray

[Eriogonsum ainslici Standl.] - HDH, exp.

[Eriogonum effusum Nutt. subsp. fendlerianum S. Stokes] - GPF

[Eriogonum fendlefianum (Benth.) Small] - GPF $\infty$ CO- HDH, SFE, W\&W

[Erivgenum fenderlianum (Benth.) Small var. fendlerianum (Benth.) Reveal] - KTZ, E

[Eriugonum lonchophyllum Torr. \& A. Gray]- HDH, SF!, W\&W

[Eriogontum lonchcoly llem Torr. \& A. Gray var, intermontanum (Reveal) Reveal] - FNA

[Eriogorium lonchophyllem Torr. \& A. Gray var. lonchophyllicum] - KTZ. UTF. E'W

[Eriogonum lonchcphyllem Torr. \& A. Gray var. nudicaule (Torr.) Reveal] - FNA

[Eriogonum lonchophyllum Torr. \& A. Gray var. saurinum (Reveal) S. L. Welsh] - KTZ :UT>, UTF

[Eriogonum salicinam Greene] - $\mathrm{HDH}$

[Eriogonum saurinum Reveal] - W\&W

[Eriogonim scoparium Small] - HDH

[Eriogonum tristichum Small] - HDH

\{Eriogonum longifolium Nutt, var, lindheimeri Gand.\} - GPF $\angle \mathrm{CO}$ >

\{Eriogonum longifolium Nuts, var. longifolium\} - KTZ $<\mathrm{KS}, \mathrm{NM}, \mathrm{OK}$ ?

Eriogonum microthecum Nutt. var, laxiflorum Hook, - FNA, KTZ, SFW, UTF, W\&W, W

[Eriogonsum microthesum NutL] - HDH

[Eriogonum microthesum Nurt, var. foliosum (Torr, \& A. Gray) Reveal] - FNA. FCF

Eriogonum microthecum Nutt. var. simpsonii (Benth.) Reveal - FNA, KTZ, SF!, W\&W, E/W

[Eriogonum simpsonii Benth.] - HDH

Eriogonum X nebraskense Rydb, - FNA

\{Eriogonum ovalifolium Nutt. var. ochroleucum (Small ex Rydb.) M. Peck\} - KTZ

\{Eriogonum ochroleucum Small\} - $\mathrm{HDH}$

\{Eriogonum ovalifoliam Nutt. - UTF, WeW

Eriogonum ovalifolium Nutt, var, ovalifolium - FNA, HDH, KTZ, SFW, W

[Eriogonum ovalifotium Nutt.] - w\& w

Eriogonum ovalifolium Nutt. var. purpureum (Nutt.) T. Durand - FNA, KTZ, W

[Eriogonum ovalifolium Nutt.] - W\&W

[Eriogonum ovalifolium Nutt, var, nevadensis Gand.] - SFW

[Eriogonum ovalifolium Nutt, var, purpureum (Nutt.) A. Nels.] - HDH

Eriogonum palmerianum Reveal - FNA $<$ KTZ, SFW, W\&W ${ }^{-1}$, W

[Eriogonum baileyi var. tomentosum S. Wars, non E. nomentosum Mikhx.] - KTZ

[Eriogonsm plumasella Durand \& Hilgard var, palmeri Tor. \& A. Gray]-FNA

Eriogonum pauciflorum Pursh var. pauciflorum - FNA

[Eriogonum effusum XE. pauciflorum] - W\&W

[Eriogonsm nebraskense Rydb.] - HDH

[Eriggonum X nebraskense Rydb.]-KTZ

[Eriogonum pauciflorum Pursh var, ghaphalodes (Benth.) Reveal - GPF, KTZ, SFE, W\&W, E

[Eriogonsum pauciflorum Pursh var. nebraskense (Rydb,) Reveal] - GPF

Eriogonum pauciflorum Pursh var, pauciflorum - GPF, KTZ $\angle N E$, WY $>$, W\&W

[Eriogonum mulriceps Nees] - HDH

Snow, N, January 2009. Checklist of Vacculer Plants of the Southom Rocky Momain Region. CVersion $3 x$ 
[Eriogonum pauciflorum Pursh] - HDH

\{Eriogonum pharnaceoides Torr.\} - HDH, exp., W\&W

\{Eriogonum pharnacooises Torr. var. cervinum Reveal\} - KTZ $<A Z, U T>$

\{Eriogonum pharnaceoides Jorr. var, phamaceoides? - KTZ $\angle A Z, N M \geqslant$, UTF

- Eriogonum pelinophilum Reveal - FNA

[NS - Of grave conservation concern]

Eriogonum racemosum Nutt. - FNA, HDH, KTZ, SF!, W\&W

[Efiogonum raccenosum NutL. var, racemosum] - UTF, E/W

Eriogonum rotundifolium Benth. - HDH, KTZ $<\mathrm{AZ}, \mathrm{NM}>$, W\&W, W\&W', err. rep.

Eriogonum seabrellum Reveal - FNA, KTZ, SFW, UTF, W\&W, W

Eriogonum shockleyi S, Watson - FNA, UTF

[Eriogonum shockleyi S. Watson var. longilobum (S. Sickes) Reveal] - SFW. W\&, W. W

[Eriogonum shockleyi S. Watson var. shockleyi] - KTZ

Eriogonum subreniforme S. Watson - FFC, HDH, KTZ \&AZ, NM, UT>, UTF, W\&W misid. [Eriogonum filicaule S. Stokes] - KTZ

Eriogonum tenellum Torr, var, tenellum - FNA, KTZ, E

[Eriogonum tenellum Torr.]-GPF, HDH. SFE, WQW

Eriogonum trichopes Torr. var. trichopes - KTZ $<$ AZ, NM, UT $>$

[Eriogonum trichopes Torr.] - UTF

Eriogonum tumulosum (Bameby) Reveal - FNA, KTZ, SFW, UTF, W\&W, W

[Eriogonsm villiflorum A. Gray]- $\mathrm{HDH}$, exp.

[Eriogonum villiflonm A. Gray var, tumulosum Bameby] - FNA.

Eriogonum umbellatum Torr, var, aureum (Gand.) Reveal - FNA, KTZ, SF!, W\&W, E/W

[Eriogonsm umbel latum Torr, var. umbellatum] - UTF

Eriogonum umbellatum Torr. var. desereticum Reveal - FNA

\{Eriogonum umbellatum Torr. var. dichrocephalum Gand, - KTZ $<\mathrm{UT}$, WY $>$

\{Eriogonum umbellatum Torr. var, majus Hook, - UTF

Eriogonum umbellatum Torr. var. majus Hook. - FNA, KTZ, UTF, E/W

[Eriogonsim subalpinum Gresne] - HDH, SF!, W\& W

Eriogonum umbellatum Torr. var, porteri (Small) Stokes - FNA, SFW, UTF, W\&W, E/W

[Eriogonem umbellatum Tor, var, aurcum (Gand, Reveal] - KTZ, SFE

- Eriogonum umbellatum Torr. var. ramulosum Reveal - FNA

[NS - endemic to Jefferson and Larimer counties, CO]

Eriogonum umbellatum Torr. var. subaridum S. Stokes - FNA, KTZ, SFW, UTF, W

[Eriogonum umbellatum Torr, subsp, subaridum (S, Stokes) Munz] - W\&W

Eriogonum umbellatum Torr, var, umbellatum - FNA, KIZ, SF!, UTF, W\&W, E/W

[Eriogonum umbellatum Tor.] - $\mathrm{HDH}$

\{Eriogonum viridulum Reveal\}-KTZ, W\&W. W\&W

\{Eriogonum brevicaule Nuts.\} - W\&W

\{Eriogonum brevicaule Nutt. vaz, viridulum (Reveal) S. L. Welsh\} - UTF

Eriogonum wetherillii Eustw. - FNA. HDH, exp.. KTZ. SFW. UTF. W\&W. W

[Eriogonum filiforme L. O. Williams] - FNA

[Eriogonum sessile S. Stokes ex M. E. Jones] - FNA

\{Eriogonum wrightii Torr. ex Benth.\} - HDH rep., W\&W

[Eriogonum wrightii Tor.; - UTF

[Eriogonum wrightii Tor, ex Benth. var, wrightii) - KTZ $\angle A Z$, NM, UT?

* Fagopyrum esculentum Moench - GPF, KTZ, W\&W

[Fagopynum esculentum (L.) Moench] - $\mathrm{HDH}$

[Fagopyrum sagittatum Gilibert] - FNA

[Polygonym fagopyrum L.] - FNA

Snow, N. Junury 2009. Checklist of Vascular Plants of the Southom Rocky Mounain Region. CVersion $3 x$ 
* Fallopia baldschuanica (Regel) Holub - FNA

[Polygonum baldschusinicum Regel] - FNA

* Fallopia convolvulus (L.) A. Löve-FNA, SF!, W\&W

[Polygonum eonvolvulus L.] - GPF, HDH, UTF

[Polyganum oomvolvulus L. var. convolvulus] - KTZ, EW

* Fallopia japonica (Houttuyn) Ronse Decraene var japonica - FNA

[Polygonum cuspidatum Siebold \&. Zuce.] - GPF, KTZ, UTF, E'W

[Reynoutria japonica Houtt.]-SF!, W\&W

Fallopia scandens (L.) Holub - FNA, SFE, W\&W

[Polygonum scandans L var. scandens] - GPF, KTZ. E

[Polygonum scanderis L.] - HDH

Koenigia islandica L, - FNA, KTZ, SF!, W\&W, EJW

Oxyria digyna (L.) Hill - FNA, HDH, KTZ, SF!, UTF, W\&W, E/W

Oxytheca dendroidea Nutt subsp, dendroidea - FNA

[Eriogontum dendroidcum (Nutr.) S. Stokes] - KTZ

[Eriogorium dendroidcum (Nuct.) var. foliscosa M. E. Jones] - KTZ

[Eriogonum dendroidoum (Nutt.) var. hillmannii S. Stokes] - KTZ

[Oxylheca foliesa Nutt.] - KTZ

\section{Periscaria}

(Identification sources:

Persicaria amphibia (L.) A. Gray - FNA

[NS - additional synooyms in FNA $5: 583$ ]

[Polygonam amphibium L.] - FNA, UTF

[Polygonam amphibium L. var. eanersum Michx_] - GPF, KTZ, EW

[Persicaria coecinea (Mual.) Greseo] - SF1

[Persicaria coecinea (Muhl. ex Willd.) Groene] - Ws: W'

[Persicaria coecineum (Muhl. ex Wilh.) Greve] - WeW

[Polygonam eoceinea Mihl. ex Willd, forma terrestre (Willd.) Stanford] - HDH

Persicaria bicomis (Raf.) Nieuwl. - FNA, SFE, W\&W

[Periscario fongistyla (Small) Small] - FNA

[Polygonum bicorne Raf.] - GPF, E

[Polygonum loogistylum Small] - FNA

[Polygonam pensyhanicum L.] - KTZ

* Persicaria hydropiper (L.) Opiz-FNA, SF!, W\&W

[Polygonum hydropiper L.] - GPF, HDH, KTZ, UTF, EW

* Periscaria hydropiperoides (Michx.) Sm. var. opelousanum (Ridd, ex J. K. Small) Ridd. ex W. Stone - Smithog?

ฯ Persicaria lapathifolia (L.) S. Gray - FNA, SF!, W\&W

[Polygonam incarum F. W. Schmidt] - HDH

[Polygonam incarnatum Elliot:] - HDH

[Polygonam lapathifolium L.] - GPF, KTZ, UTF

[Polygonum lapathifolium L. var, laparhifolium]- HDH, EN

[Polygonum laparhifolium L, var, nodosum (Pers,) Small] - $\mathrm{HDH}$

[Polygonum lapathifolium L. var, salicifolium Sibth.] - E/W

[Polygonam scabnum Mocnch] - FNA

[Polygonem tomentosum Willd.] - FNA 
* Periscaria maculosa A. Gray - FNA

[Perisearia fusiformis (Greene) Greene] - FNA

[Periscaria vulgaris Webb \& Moquin-Tandon] - FNA

[Polygonum persicaria L.] - GPF, HDH, KTZ, UTF, E/W

Persicaria pensylvanica (L.) M. Gomez - SF!. W\&W

[Polygonum longistylum Small var. omissum (Greene) Stanford] - HDH

[Polygonum anissum Greene]- FN $A$

[Polygonum pensylvanicum L.] - GPF. KTZ. E/W

[Polygonum pensylvanicum L. var. laevigatum Fernald] - HDH

[Polygonum persicarioides Kunth]- HDH

* Persicaria punctata (Elliott) Small - FNA, SF!, W\&W

[NS - additional synonymy in FNA 5: 586] - FNA

[Polygonum punclatum Elliott] - OPF

[Polygonum punctatum Elliott var. confertiflorum (Meisn.) Fassett] - HDH, KTZ, E/W

[Polygonum punctatum Elliott var. parviflorum Fassett] - HDH

Persicaria sagittatum (L.) H. Gross - FNA

[Polygonum sagittatum L. - GPF, HDH, KTZ, E

[Truellum sagittatum (L.) Sojakk] - SFE, W\&W

Polygonum

(Identification sources:

\$Polygonum achoreum S. F. Blake - FNA, GPF, KTZ, E

[Polygonum erectum L. subsp. achoreum (S. F. Blake) A. Love \& D. LÖve] - SFE, W\&W

* Polygonum arenastrum Jord, ex Boreau - GPF, KTZ, E/W

[Polygonam arvintstrum Borsau] - SFI, W\&W

[Polygonam aviculare L.] - RMC

*Polygonum argyrocoleon Steud. ex Kunze-FNA, KTZ, W\&W, E/W

[Polygonum argyrocolcon Steud.] - SF!, UTH

* Polygonum aubertii L. Henry - KTZ, E

[Fallopia aubertii (L. Henry) Holub] - SFE, W\&W

[Polygonum aubertit (L.) L. Henry] - UTF

* Polygonum aviculare L. - GPF, KTZ, UTF

[NS - FNA 5: $556-557$ rocogniasd several varieties, in my view of questionable taconomic value]

[Polygonum arenasirum Boreau] - SF!

[Polygonem aviculare $\mathrm{L}$. var, aviculare] - $\mathrm{HDH}$, W\&W

索 Polygonum bellardii. All. - KTZ

[Polygonam aviculare L, var, angustissimum Meisn.] - HDH, W\&W

ฯ Polygonum buxiforme Small - GPF, KTZ

[Polygonum arentastram Boreau] - W\& W'

Polygonum douglasii Greene var, douglasii

[Polygonam asstiniae Greene] - KTZ

[Polygonum douglasii Greene sar, latifoliam (Engelma) Gnene] - FNA

[Polygonam doxiglasii Greene sabsp, austiniae (Greene) E. Murr.] - KTZ

[Polygonam douglasii Greene var, austinise (Greene) Jones - WY checklist, KTZ

[Polygonum emaciatum A. Nels.] - FNA

[Polygonum montanum (Small) Greene] - FNA

[Polygonum tenue Michx, var, commuse Engelm.] - FNA

[Polygobum tene Michx, var, laifolium Eneeln.] - FNA

Polygonum douglasii Greene var, microspermum (Engelm.) Dorn - E/W

[Polygonam dexulasii Green] - SFt, W\& W'

[Polygonam dexuglasii Greene sabsp, engelmannii (Greene) Kantesz \& Gandhi] - KTZ

Snow, N. Junury 2009. Checklist of Vascular Plants of the Southom Rocky Mounain Region. CVersion $3 x$ 
[Polygonum engelmannii Greene] - HDH, W\&W

Polygonum engelmannii Greene - FNA

[Polygonum douglassii Greene subsp. engelmannii (Grecne) Kartresz \& Gandhi] - FNA

Polygonum erectum L. - FNA, GPF, HDH, KTZ, W\&W, app, uncertain

[Polyganum aviculare var. erestum (L) Roth ex Meisn.] - KTZ

Polygonum kelloggii Greene var, confertiflorum (Nutt. ex Piper) Dorn - RMC

[Polygonum poly galoides Meisn. subsp. confertiflorum (Nutt. ex Piper) J. C. Hickman] - KTZ

[Polygonum polygaloides. Wall. ex Meisa, subsp. confertiflorum (Nutt. ex Piper) J. C. Hickman] - KTZ

[Polygonum watsonii Small] - HDH

Polygonum kelloggii Greene var, kelloggii - E/W

[Polygonum kelloggii Greene] - HDH, UTF

[Polygonum polygaloides Meisn. subsp. kelloggii (Grecne) J. C. Hickman]-SF!, W\&.W

[Polygonum polygaloides Wall. ex Meisn. subsp. kelloggii (Greene) J. C. Hicknan]- KTZ

[Polygonum unifolium Smal]] - HDH

Polygonum majus (Meisner) Piper - FNA

[Polygonum coarctatum Dougl ex Mcisner var. majus Meisner] - FNA

[Polygonum deuglasii Greene sabsp. majus (Meisoer) J. C. Hickman] - FNA

Polygonum minimum S. Watson - FNA, HDH, KTZ, SF!, UTF, W\&W, E/W

[Polygonum lorrey is. Warson] - FNA

\{Polygonum oxyspermum C. A. Mey, \& Bunge ex Ledeb. \} - KTZ

Polygonum polygaloides Meisn. - FNA, HDH

[NS - 2-3 varieties recognized for our area by FNA]

[Polyzonum polygaloides Meisn. subsp. polygaloides] - W\&W.err. rep.

[Polygonum polygaloides Wall. ex Meisn. subsp, polygaloides] - KTZ

\{Polygonum ramosissimum Michx, subsp, prolificum (Small) Costea \& Tardif - FNA?

\{NS - to be expected given distributions in FNA 5:552\}

Polygonum ramosissimum Michx. subsp. ramosissimum - FNA

[Polygonum ramosissimum Michx, var, ramosissinume] - KTZ, EN

[Polyzobam exsertum Small] - $\mathrm{HDH}$

[Polygonam ramosissimum Michx.] - GPE, HDH, SFL, UTF, W \& W

Polygonum sawatchense Small subsp. sawatchense - FNA, GPF, HDH

[Polygonam doxuglasii Greene sabsp, johnstonii (Munz) J. C. Hickman] - KTZ

[Polygonam deuglasii Greene var, johnstonii Munz] - FNA

[Polygonam exile Eastw.] - FNA

[Polygonum triandrum Coolidec] - FNA

\{Polygonum spergulariaeforme Meisn. ex Small\} - HDH

\{Polygoeum douglasii Greene subsp. spergulariforme (Meisn. ex Small) J. C. Hickman?; - KTZ $\angle W Y>$

\{Polygoeum spergulariacforme Meisn.\} - W\&W, err. rep.

Polygonam tenue Michx. - FNA

Pterogonum (see Eriogonum)

Reynoutria (see Polygonum and Fallopia)

* Rheum rhabarbarum L, - GPF, KTZ

[Rlseum thubarbarum L.] - UTF

$\{$ * Rheum rhaponticum L.\} - HDH, W\&W

\{Rheum rhabarbaram L.\} - KTZ

Rumex

(Identification sources: 
* Rumex acetosella L. - FNA, GPF, HDH, KTZ, UTF, E/W

[Acetosetla vulgaris (K. Koch) Fourr.]-SFI, W\&W

Rumex altissimus A. W. Wood - FNA, GPF, HDH, KTZ, SFE, W\&W, E

Rumex aquaticus L. var, fenestratus (Greene) Dorn - KTZ, E/W

[Rumex aquaticus L. subsp. occiderstalis (S. Watson) Hultén] - SF!, W\& W

[Rumex occidentalis S. Watson] - HDH

* Rumex crispus L, - FNA, GPF, HDH, SF!, UTF, W\&W, EW

[Rumex crispus subsp. crispus] - KTZ

Rumex fueginus Philippi - FNA, HDH

[Rumex maritimus L]- KTZ

[Rumex maritimus L subsp. fueginus (Philippi) Hultén] - SF!, W\& W

[Rumex maritimus L var, fucginus (Phil.) Duskn] - GPF, UTF, E/W

Rumex hymenosepalus Tor, - FNA, GPF, KTZ, SFW, UTF, W\&W, W

[Rumes arizonicus Britton] - FNA

[Rumer hymenosepalus Torr. var. hymenosepalus] - HDH

[Rumer hymenosepalus Torr. var- salinus (A. Nelson) Rechinger f.] - HDH

[Rumex saxei Kellogg] - FNA

Rumex occidentalis S. Watson - FNA

[Rumex aquaticus L. subsp. fencstratus (Greeno) Hultén] - FNA

[Rumex aquaticus L. vaf, fonestratus (Greede) Dom] - FNA

[Rumex aquaticus L. subsp. oecidentalis (S. Wats.) Hultén] - FNA

[Rumex bakeri Greene] - FNA

[Rumex fenestrarus Greene] - FNA

[Rumer gracilipes Grecte] - FNA

* Rumex obtusifolius L. - FNA, GPF, HDH, KTZ, SFE, UTF, W\&W, E

Rumex patientia L. - FNA, GPF, HDH, exp., KTZ <KS,NE,OK,UT, WY>, W\&W doubtfu]

Rumex paucifolius Nutt, - FNA, HDH, UTF

[Acelosella paucifolia (Nalt.) A. Löve] - SF!, WE W

[Rumex paucifolius Nutt. var. paucifolius] - E/W

[Rumex pascifolius Nutt. subsp. paucifolius] - KTZ

Rumex praecox Rydb. - FNA

Rumex pycnanthus Rechinger f. - FNA, HDH

[Rumex densiflorus Osterth.] - FNA. HDH, KTZ, SFt, W\&W, E/W

[Rumex praceox Rydb.] - HDH

[Rumex pycounthus Rechinger f. subsp. pycaanthus (Rechinger f.) A. Lôve] - FNA

Rumex salicifolius Weinm. var. denticulatus Torr. - KTZ, E/W

[Rumex utahersis Rech. f] - HDH, SF!, W\& W

[Rumer mexicants Meisn.] - GPF

[Rumex salicifolius Weinm, var, mexicanus (Meisn.) C. L. Hitche.] - KTZ, UTF

* Rumex stenophyllus Ledeb, - FNA, GPF, KTZ, SFE, UTF, W\&W, E

Rumex triangulivalvis (Danser) Rechinger $\mathrm{f}$ - FNA, HDH, SF!, W\&W

[Rumer salicifoliug Weinm, var, triangulivalvis (Danser) J. C. Hickman] - E/W

Rumex utahensis Rechinger - FNA

Rumex venosus Pursh - FNA, GPF, HDH, KTZ, SF!, UTF, W\&W, E/W

\{Rumex verticillatus $L$. $\}$ - O'Kane88

Stenogonum flexum (M. E. Jones) Reveal \& J. T. Howell - FNA, KTZ. SFW, W\&W

[Eriogonsum glandulosum (Nuct) Nutt, ex Benth.] - HDH. KTZ, W\& W, miskd.

[Eriogonum flexum M. E. Jones] - UTF

Stenogonum salsuginosum Nutt. - Dom01, FNA. KTZ, SFW, W\&W. W

[Eriggonsum salsuginosum (Nutt.) Hook.] - HDH, UTF

Snow, N, Janury 2009. Checklist of Vacculer Plants of the Southom Rocky Momain Regioe. CVersion $3 x$ 
Truellum (see Polygonum)

\section{Pontederiaceac - PON}

* Eichhornia crassipes (Mart.) Solms - Snow04b

Heteranthera dubia (Jacq,) MacMill, - FNA, IDII, exp, KTZ, E [Zosterells dubia (Jacq.) Small] - GPF, SFE, W\& W

Heteranthera limosa (Sw.) Willd. - FNA, GPF, HDH, KTZ, SFE, W\&W, E

Heteranthera rotundifolia (Kunth) Griseb, - FNA, HDH, KTZ, W\&W

\section{Zosterella (see Heteranthera)}

\section{Portulacaceae - POR}

(Identification sources:

[NS - Studies by Hershkovizz Zimmer and others suggest the possibility of splitting POR into Montiaceae (including Clsytonis. Lewisia, Montia, Cistanthe) and POR s.s. (Portulaca)]

\{Cistanthe umbellata (Torr.) Hershkovitz\} - KTZ

\{NS - absent from our area in WY tide Dorn01; not confinmed for our area south from WY\}

\{Calyptridium umbellarum (Tom.) Gresne var. caudicifera A. Gray\} - UTF

Cistanthe rosea (S. Wats.) Hershk. - Dom0l

[Calypcridium rossum S. Wats.] - KTZ

Claytonia lanceolata Pursh var. lanceolata - KTZ, E/W

[Claylonia lanceolata Pursh] - Does01, HDH, SFI, UTF, W\&W

Claytonia lanceolata Pursh var, multiscapa (Rydb.) C. L. Hitche, - KTZ. E/W

[Claytonia lanceolara Pursb] - HDH, SF!, UTF

[Claytonia multiscapa Fydb.] - Dorno]

Claytonia megarhiza (A. Gray) Parry ex S. Watson var. megarhiza - KTZ, E/W

[Claytonia megarhiza (A. Gray) Pany]- Dom011. HOH, SF!. UTF

[Claytonia megarhiza (Pany ex A. Gray) S, Watson] - W\&W, misid.

Claytonia perfoliata Donn ex Willd. subsp. perfoliata - KTZ.

[Claytonia perfoliata Doon] - RMC

[Montia perfoliats (Donn) Howell] - W\& W. W\& W', misid.

[Montia perfoliats (Donn) Howell var. perfoliats] - UTF

Claytonia rosea Rydb. - KTZ, SF!, W\&W, E/W

[Claytonia lanceolata Pursh] - $\mathrm{HDH}$, UTF

[Claytonia perfoliata Donn] - RMC

Claytonia rubra (Howell) Tidestr. subsp. depressa (A. Gray) J. M. Mill. \& K. L. Chambers

- KTZ

[Claytonia nabra] - Dornol

[Montis perfoliata Donn var, depressa (A, Gray) Jeps.] - HDH, exp.

Claytonia rubra (Howell) Tidestr. subsp. rubra - KTZ, E/W

[Claytonia perfoliata Doon] - RMC

[Claytonia rubra (Howeili) Tidestr.] - SF", W\& W'

\{Claytonia umbellata S. Wats.\} - Dorn()I 
[NS - collected anly once in sur region fide Dornol in 1876 [Alhany Co., WY]; thus probably a waif subsequently extirpated given its typical range in CA, OR, and NV;

Crunocallis (see Montia)

\section{Erocallis (see Lewisia)}

Lewisia nevadensis (A, Gray) B, L. Rob, - KTZ, W

[Lewisia pygmaes (A. Gray) B. L.. Rob.] - UTF

[Lewisia pygmaea (A. Gray) B. L. Rob. var. nevadensis (A. Gray) Fosberg] - HDH

[Lewisia pyemaes (A. Gray) B. L. Rob. var. pygmaca] - RMC

[Oreobroma nevadensis ( $A$. Gray) Howell] - SFW, W\&W

Lewisia pygmaea (A. Gray) B. L. Rob, var pygmaea - HDH, E/W

[Lewisia pygmaea (A. Gray) B. L. Rob.] - Dorn01, KTZ. UTF

[Oreobroma pygmaea (A. Gray) Howell] - SF!, W\&W

Lewisia rediviva Pursh var, rediviva - KTZ, E/W

[Lewisia rediviva Pursh] - Domb01, HDH, SF!, UTF, W\&W

Lewisia triphylla (S. Watson) B. L. Rob, - Dom01, HDH, KTZ, UTF, EW

[Erocallis triphylla (S. Watson) Rydb.] - SF!, W\&W

\section{Montia (see also Claytonia)}

Montia chamissoi (Ledeb. ex Spreng.) Greene - Dorn01, KTZ, E/W

[Crunocallis chamissoi (Ledeb.) Cockersll] - SFE

[Crunocallis chamissoi (Ledeb.) Rydb.] - SFW

[Crunocallis chamissoi (Ledeb ox Spreng.) Rydo.] - W\&W

[Montia chamissoi (Ledeb.) T. Darand \& Jaxkson] - HDH

[Montia chamissoi (Ledeb.) B. L. Rob. \& Femald] - UTF

\section{Oreobroma (see Lewisia)}

Phemeranthus breviolius (Torr.) Kiger - KTZ

[Talinum brevifolium Torr.] - HDH, exp, UTF, W\&W

Phemeranthus calycinus (Engelm.) Kiger - KTZ.

[Talinum calycinum Engelm.]-GPF, HDH, KTZ, SFE, W\& W', E

Phemeranthus parviflorus (Nutt.) Kiger

[Talinum parviflorum Nutt.] - Dom01, GPF, KTZ, SFI, UTF, EW

[Talinum parviflorum Nutr. ex Torr, \& A. Gray]- $\mathrm{HDH}$, W\&W

Portulaca halimoides L. - KTZ, SF!, UTF, W\&W', E/W

[Ponulaca parvula A. Gray] - GPF $\triangle \mathrm{CO}>$, HDH, W\& W

Portulaca oleracea L. - Dorn01, GPF, HDH, KTZ, SF!, UTF, W\&W, E/W

[Portulaca retusa Engelm.] - W\&W, HDH, exp.

Portulaca pilosa L. - KTZ, W\&W

Potamogetonaceae - POT (including Zannichelliaceae, Najadaceae)

Potamogeton (sec also Stuckenia)

(ldentification sources:

Potamogeton alpinus Balb. - Dom01, FNA. IMF, KTZ, SFW. UTF, W\&W. E/W 
[Potamogetan alpinus Balb. var. subellipxicus (Fernald) Ogden] - HDH, exp.

[Polamogeton alpinus, Balb. var. tenuifolius (Raf.) Ogden] = GPF, HDH, SFE

Potamogeton amplifolius Tuck, - Dom01, FNA,GPF, HDH, IMF, KTZ, SFI, W\&W, E/W

* Potamogeton crispus L. - Dom01. FNA,GPF, HDH. IMF, KTZ, SFE, UTF, W\&W, E

Polamogeton diversifolius Raf, - FNA, GPF, HDH, IMF, KTZ, SFE, W\&W, E

Potamogeton epihydrus Raf, - Dom01. FNA, GPF, IMF, KTZ, SF!, UTF, E/W

[Potamogeton epihydrus Raf. var. nuttallii (Cham. \& Schltdl.) Fernald] - HDH, W\&W

Potamogeton foliosus Raf. var. foliosus - HDH, IMF, UTF, E/W

[Potamogeton foliosus Raf.] - Dom01, GPF, SF!, W\&W

[Potamogeton foliasus Raf. subsp. foliosus] - FNA, KTZ

[Potamogeton foliosus Raf. var, macellus Fernald] - HDH

Potamogeton friesii Rupr, - Dorn01

Potamogeton gramineus L, - Dom01, FNA, GPF, IMF, KTZ, SF!, UTF, W\&W, EW

[Polamogeton gramineus L. yar. gramintus] - $\mathrm{HDH}$

[Potamogecon gramineus L. var, maximus Morong ex A. Benn.] - HDH

Potamogeton X hagstroemii A. Benn. - FNA, KTZ

[Potamogeton gramineus X P. richardsonii] - KTZ

Potamogeton illinoensis Morong - Dom01, FNA, GPF, HDH, IMF, KTZ, SF!, UTF, W\&W, E/W

[Potamogeton lateralis Morong] - HDH?, W\&w'

Potamogeton natans L, - Dom01, FNA, GPF, HDH, IMF, KTZ, SF!, UTF, W\& W, EW

Potamogeton nodosus Poir - Dom01, FNA, GPF, HDH, IMF, KTZ, SFE, UTF, W\&W, E/W [Pramegeson wodesus L.] - SEW

Potamogeton praelongus Wulfen - Dorn01, FNA, GPF, HDH, IMF, KTZ, SF!, UTF, W\&W, ENW

Potamogeton pusillus L. var, pusillus - Dorn01, GPF, IMF, W\&W, E/W

[Potamogeton pusillus L.] - SF!, HDH

[Potamogeton pusillus L. subsp. pusillus] - FNA, KTZ

Potamogeton pusillus L. var. tenuissimus Mett. \& W. D. J. Koch - Dorn01, IMF, W\&W, E/W

[Potamogenon berehtoldii Finber] - $\mathrm{HDH}$

[Potamogeton pusillus L.] - HDH, SF], UTF

[Potamogeton pusillus L. subsp. tenuissimus (Mert. \& W. D. J. Koch) R. R. Haynes \& Helle.] -FNA, KTZ

Potamogeton richardsonii (A. Benn.) Rydb, - Dorn01, FNA,GPF, HDH, JMF, KTZ, UTF, E/W

[Potamogeton perfolianus L. subsp. richardsonii (A. Bcenn.) Hulnén] - SFt, W\& W

Potamogeton robbinsii Oakes - Dom01, FNA, HDH, exp., IMF, KTZ, UTF

Potamogeton strictifolius A, Benn. - Dom01, FNA, GPF, IMF, KTZ

[Potamogeton strictifolius A. Bent. vaf, rutiloides Fernald] - HDH, exp, UTF

[Pramogeton longiligulatus Fern.] - KTZ

[Potamogeton pusillus var, rutiloides (Fern.) Boivin] - KTZ

[Potamogeton rutilus auct. non Wolfgang] - KTZ

[Potamogeton striktifolies var, futiloides Fem.] - KTZ

[Potanogeton stricuifolius var. typicus Fem.] - KTZ

\{Potamogeton zosteriformis Fernald\} - FNA, GPF, HDH, exp., IMF, KTZ, UTF

Stuckenia

(1dentification sources:

Stuckenia filiformis (Pers.) Bömer subsp, alpinus (Blytt) R. R. Hayes, Les, \& M. Král - FNA. KTZ, E/W

[Potanogeton filiformis Pers.]-GPF, SFI, WiE W

Snow, N. Junury 2009. Checklist of Vascular Plants of the Southom Rocky Mounain Region. CVersion $3 x$ 
[Potamogetan filiformis Pers. var. alpinus (Blytt) Asch. \& Graebn.] - IMF, UTF

[Polamogeton filiformis. Pers, var. alpinus (J. W. Robbins) Dorn] - Dorv0!

[Potamogeton filifarmis. Pers. var. borealis (Raf.) H. St. John] - HDH

[Potamageton filiform is. Pers. var, macounii Morong] - HDH

Stuckenia filiformis (Pers.) Bömer subsp, occidentalis (J. W. Robbins) R. R. Haynes, Les, \& M.

Král-FNA, KTZ, E/W

[Potamogeton filiformis Pers.] - GPF, SFL, W\&W

[Potamageton filiformis Pers. var. occidentalis (J. W. Robbins) Morong] - IMF, UTF

[Potamogecon filiformis Pers. yar. oscidentalis [J. W. Robbins) Dorn] - Dorn0I

Stuckenia pectinata (L.) Börner - Dorn01, FNA, KTZ, E/W

[Potamogeton pectinatus L.] - GPF, HDH, IMF, SF!, UTF, W\&W

Stuckenia striata (Ruiz \& Pay.) Holub - FNA, KTZ

[Potamogeton filiformis Pers.]-GPF, SF!. W\& W ${ }^{1}$

[Potamogeton filiformis Pers, var, latifolius (J.W. Robbins) Reveal] - IMF?, UTF

[Stuckenia striatus (Ruiz \& Pav.) Holub] - K] Z

Stuckenia vaginata (Turcz) Holub - Dorn01, FNA, KTZ, E

[Polamageton vaginatus Turc ] - GPF, HDH, IMF, SEE, UTF, W\&W

Zannichellia palustris L. - Dom01, FNA, GPF, HDH, IMF, KTZ, SF!, UTF, W\&W, EW

Primulaceae - PRM (see also MRS)

(Identification sources:

Anagallis (see MRS)

Androsace chamaejasme Host. subsp, lehmanniana Spreng. - E/W

[TK - pers. comm.]

[Androsace carinata Tor.] - $\mathrm{HDH}$

[Androsace chamsixjasme Wulf: subsp, carinena (Torr.) Hulnen] - KTZ

[Androsace chamaciasme Wult, var, carinaa (Torr.) Knuth] - Domo1

[Androsace chamaxiasme Host subsp. carinata (Torr.) Huheen] - SF], WA W

[Androsace chamaxjasme Host vaf, carinata (Torr.) Kunth] - UTF

Androsace filiformis Retz. - Dom01, HDH, KTZ, SF!, UTF, W\&W, E/W

Androsace occidentalis Pursh - Dorn01, GPF, KTZ, SF!, UTF, W\&W, E/W

[Androsace occidentalis Pursh forma oxidentals] - HDH

[Androsace occidentalis Pursh forma simplex (Rydb.) Robbins] - HDH

Androsace septentrionalis L, - SF!, UTF, W\&W, E/W

[T, Kelso, pers somm.]

[Androsace sepxentrionalis L. subsp. glandulosa (Wooton \& StandL) G. T. Robbins] - KTZ

[Androsace sepsentrionalis L. verr. glandulosa H. St. Jokin] - HDH

[Androsace sepxentrionalis L. subsp. pubsrulenta (Rydb.) G. T. Robbins] - KTZ

[Androsace sepxentrionalis L. var. puberulenta (Rydb.) R. Keuth] - HDH

[Androsace sepxentrionalis L. subsp, subulifera (A. Gray) G. T. Robbins] - KT2

[Androsace sepsentrionalis L. subsp. subumbellata (A. Nels.) G. T. Robbins] - KTZ

[Androsace sepxentrionalis L. var, subulifera A. Gray] - Dom01, GPF, HDH

[Androsace sepentrionalis L. var, subumbellata A. Nelson] - HDH

\section{Centunculus (sec Anagallis)}

\section{Dodecatheon (see also Primula)}

[Mast07 summarizes the considerable evidence for merging Dodecantheon into Primula] 
Dodecatheon conjugens Greene - Dorn01

[Dodecatheon conjugens subsp. conjugens] - KTZ

[Dodecatheon conjugens subsp. leptophyllum (Suksdorf) Piper] - KTZ

[Dodecatheon conjugens var, besmishii Boivin] - KTZ

[Dodecatheon cylindrocarpam Rydb.] - KTZ

[Primula conjugens (Groene) A. R. Mast \& Reveal] - Mast07

Dodecatheon deutatum Hooker subsp, ellisaiae (Standl.) H. J. Thompson - KWA, KTZ

[Dodecatheon ellisiae StandL.] - M\&H, KTZ

Dodecatheon pulchellum (Raf.) Merr, var. pulchellum - UTF, E/W

[Dodecatheon pukhellum (Raf.) Merr.] - Dom01, GPF. HDH. SF], W\&W

[Dodecatheon puichellum (Raf.) Merr. subsp. pulchellum] - KTZ

[Dodecatheon radicatum Greese] - HDH

Douglasia montana A. Gray - Dorn01

- Primula angustifolia Torr, - ENDFMIC $<\mathrm{CO}, \mathrm{NM}>$, HDH, KTZ, SF!, W\&W, E/W

Primula egaliksensis Wormsk. ex Hornem, - KTZ, E

[Primula egalikensls Wormsk.] - HDH rep. SFE, W\&W

Primula incana M. E. Jones - Dorn01, GPF, HDH, KTZ, SF!, UTF, W\&W, E/W

Primula parryi A. Gray - Dom01, HDH, KTZ, SF!, UTF, W\&W, EJW

\{Primula specuicola M. E. Jones\} - SFW, exp.

Pyrolaceae (see ERI)

Ranunculaceae - RAN (including Coptaceae, Helleboraceae; see also Paeoniaceac, under Thelypteridaceae in $W_{\&} W^{3}$ )

(Identification sources:

Aconitum columbianum Nutt. var, columbianum - UTF, E/W

[Aconitum columbianum Nutt.] - Dorn01, GPF, SFI

[Aconitum columbianum Nutr, subsp, columbianum] - FNA, KT2

[Aconitum columbianum Nutt, focrna ochroleaciam St, John] - SF

[Aconitum columbianum Nutt, ex Torr. \& A. Gray var, bakeri (Greene) Harrington]-HDH, W\& W

[Aconitum columbianum Nutt, ex Torr. \& A. Gray var, columbianum] - HDH, W\&W

Actaen rubra (Aiton) Willd. - Dom01, FNA, GPF, UTF, E/W

[Actaen anguta Nutt, \& Torr, \& A. Gray]- HDH

[Actaen rubra (Aiton) Willd, sabsp, arguta (Nutt.) Hultén] - KTZ, SF!, W\&W

Anemonastrum (sec Anemone)

Anemone

(Identification sources:

Anemone canadensis L. - Dorn01, FNA, GPF, HDH, KTZ, E

[Anemsonidium canadense (L.) A. Löve \& D. Live] - SFE, W \& W

Anemone cylindrica A. Gray - Dom01, FNA, GPF, HDH, KTZ, SF!, W\&W, E/W

Anemone multifida Poir. var. multífida - FNA, UTF, EW

Snow, N. Janury 2009. Checklist of Vascular Plants of tho Southum Rocky Momale Region. CVersion 31 
[Anemone globesa Nutt. ex Pritz] - HDH

[Anemone multifida Poir.] - Dorn01, GPF

[Anemone multifida Poir. subsp. globosa (Nutt.) Torr. \& A. Gray] - SE!

[Anemone multifida Poir. var- globosa (Nutt) Torr. \& A. Gray] - W\&W

[Anemone multifida Poir. var. hudsoniana DC.] - KTZ

[Anemone multifida Poir. var. sansonii B. Boivin] = KTZ

[Anemone multifida Poir. subsp. saxicola (B. Boivin) W. A. Webcr] - SFI, W\&W

[W] - I doubt that this can be maintained considering the variable size and coloration of the typical varicty]

[Anemone multifida Poir. var. saxicola B. Boivin] - FNA, KTZ

Anemone narcissiflora L. var, zephyra (A. Nelson) Dutton \& Keener - Dorm01. FNA, KTZ, EW

[Anemoriastrum narcissiflerum (L.) Holub sabsp. zephyrum (A. Nelson) W. A. Weber] - SF!. W \& W

[Anemone narcissiflora L.] - RMC

[Anemone zephyra A. Nelson] - HDH

Anemone parviflora Michx, - Dom01, FNA, HDH, SF!, UTF, W\&W, E/W

[Anemonte parviflora Michx. var. parvifhora] - KTZ

Anemone patens L. var, multifida Pritz. - Dorn01, FNA, UTF, ENW

[Anentorie patens L.] - GPF

[Pulsatilla ludoviciana (Nutt.) A. Heller] - HDH, W\& w $^{1}$

[Pulsatilla patens (L.) Mill. subsp. hirsutissima Zlimels] - SFW

[Pulsatilla patens (L.) Mill. subsp. multifida (Pritz.) Zämels] - KTZ, SFE, W\& W

\{Anemone virginiana L. var, riparia (Fernald) B, Boivin\} - WJ, E

\{Anemone riparia Fentald\} - SFE, W\&W

Anemone virgintiana L.) - GPF

\{Arkence virginiana L var, altha (Oakes) A. W. Wood\} - FNA, KTZ

\{Anemone virginiana L, var. virginiana\} - FNA, KTZ

(Ansmone virginiana L. - GPF

Anemonidiun (see Anemone)

Aquilegia

(Identification sources:

Aquilegia barnebyi Munz - FNA, HDH, KTZ, SFW, UTF, W\&W, W

Aquilegia chrysantha A. Gray - FNA, HDH, UTF, E

[Aquilegia chrysantha A. Gray var, rydbergii Munz] - KTZ, SFE, W\&W

Aquilegia coerulea $\mathrm{X}$ A. elegantula

[W] - See Langerwoin 885 [COLO], Wichener $\$ 60$ [COLO], Kelly 635 [KHD]]

Aquilegia coerulea E. James var. coerulea - FNA, E/W

[Aquilegia cnerulea E. Janses var, cacruka] - HDH, KTZ, UTF

[Aquilegia caerulea E. Jamkes var, daileyac Eastw.] - HDH, KTZ

[Aquilegia coeruica E. James] - Dom01, SF!, W\& W'

[Aquilegia coenulea E. James ex Torr.] - W\&W

[Aquilegia coerulca E. Janes var, daileyac Eastw.] - KTZ

[Aquilegia coerulea E. James forma daileyae (Eastw, W. A. Weber] - SF:

Aquilegia coerulea E. James var. ochroleuca Hook. - FNA

[Aquilegia caenules E. James var, ochroleucs Hook.] - HDH, KTZ. UTH

[Aquilegia coerulea E. James] - W\&W

[Aquilegia coerulea E. Jaenes ex Tons.] - WeW

\{Aquilegia desertorum (M. E. Jones) A. Heller\} - FNA

\{Agailegia desertonum (M. E. Jones) Cockerell ex A. Heller\} - KTZ<AZ, NM, UT>

\{Aquilegia tritemata Pagson\} - HDH, KTZ $<A Z, N M$. W \& W, misid

Aquilegia elegantula Greene - FNA, HDH, KTZ, SF!, UTF, W\& W, E/W

Snow, N. Janury 2009. Checklist of Vaccular Plants of tho Southom Rocky Momain Region. CVersion 3 . 
\{Aquilegia flavescens S. Watson var, flavescens\} - FNA, KTZ, UTF

\{Aquilegia micrantha $X$ A. elkgantula\} - W\&W

\{Aguilogia flavescens S. Watson\} - HDH, W\& W'

- Aquilegia laramiensis A. Nelson - ENDEMIC <WY $>$, Dorn01, FNA

- Aquilegia mictantha Eastw, - ENDEMIC $<C O>$, FNA, SFW, UTF, W\&W, W

[Aquilegia ecalcarata Eastw.] -SFW. sparless form

[Aquilegia mancosana (Eastw.) Cockerel]] - SFW, spurkess form

[Aquilegia micrantha Eastw. var. mancosana Eastw.] - HDH, KTZ

[Aquilegia micrantha Eascw. var. micrantha] - HDH, KTZ

\{Aquilegia pubescens Coville\} - FNA, KTZ, SFE not persisting. W\&W, E

- Aquilegia saximontana Rydb, - F.NDF.MIC <CO>, FNA, HDH, KTZ, SFE, E

[Aquilegia saximonuna Rydb, ex B. L. Rob,] - W\&W

Aquilegia scopulorum Tidestr. var. calcarea (M. E. Jones) Munz; - KTZ $<$ UT, WY $>$

[Aquilegia scopulorum Tidesr.] - HDH, exp.. - FNA, UTF

Atragene (sce Clematis)

Batrachium (see Ranunculus)

Caltha leptosepala DC. - Dorn01, FNA, HDH, E/W

[Caitha leptosepala DC, subsp, keptogepala var, leptosepala] - KT2

[Caltha leptosepala DC, var, leptosepala] - UTF

[Psychmophila leptosepala (DC.) W. A. Weber] - SF!, W\&W

Ceratocephala (see Ranunculus)

Clematis

(Identification sources:

Clematis columbiana (Nutt) Torr. \& A. Gray var. columbiana - Dorn01, FNA, KTZ, E/W

[Atragene columbiana Nutt.] - SF!, W\&W

[Clematis columbiana (Nutt.) Torr. \& A. Gray] - UTF

[Clematis psesdoalpina (Kuntze) A, Nelson] - GPF, HDH

Clematis columbiana (Nutt) Torr. \& A. Gray var. tenuiloba (A. Gray) Pringle - Dorn01. FNA.

$\mathrm{KTZ}$, E/W

[W] - I think FNA may have reated this group incorrectly, Clewals columbiano is either $C$. wualloba or a

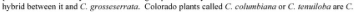

poseadoaipina]

[Atragene columbiana Nutt,] - SF!, W\&W

[Clematis columbiana (Nutt.) Torr. \& A, Gray] - UTF

[Clematis tenuiloba (A. Gray) C. L. Hitche.] - GPF

Clematis drummondii Torr. \& A. Gray - FNA. GPF, KTZ

Clematis hirsutissima Pursh - Dorn01. GPF, UTF

[Clematis hirsutissima Pursh var. hirsutissima - FNA, HDH, KTZ, EW

[Coriflona hirsutissima (Pursh) W. A. Weber] - SF!, W\&W

Clematis ligusticifolia Nutt. - Dorn01. FNA. GPF. SF!. UTF, E/W

[Clematis ligusticifolia Nutt. var. ligusticifolia] - KTZ

[Clematis ligusticifolia Nutt. ex Torr. \& A. Gray] - HDH. W\&W

Clematis oceidentalis (Homem.) DC. var. grosseserrata (Rydb.) Pringle - FNA, KTZ. E/W

[W] $-C$, occifentalis is northeastern. Westem plants should be called $C$. grussesemafa but comb. nov. needed]

Snow, N, Janury 2009. Checklist of Vacculer Plants of the Southom Rocky Momain Region. CVersion $3 x$ 
[Atragene oocidentalis Hornem.] - SF!, W\& W

[Clematis columbiana abct. non (Nutt.) Torr. \& A. Gray] - HDH, fide W\&W

\{Clematis occidentalis (Homem.) DC. var, occidentalis\} - FNA, KTZ

\{Atragene occidentalis Hornem.\} - W\& W

(Clematis oceidentalis (Hornem.) DC. - UTF

* Clematis orientalis L, - FNA. HDH, KTZ, UTF, E/W

[Viticelh arientalis (L) W. A. Weber] - SF!, W\&W

- Clematis seottii Porter

[W] - the reduction of this taxon to variety of $\mathrm{C}$. hirsulissima by some is unjustified. The flowers are very different. It is endemic to se CO and ee NM, where it displaces C. hirsutissima. RH (at RM) agroes with this assessment] [NS - KTZ roports this taxon also from WY, UT, NE, and as rare in SD and OK: further invstigation is warranted]

[Clematis hirsutissinta Pursh var, scottii (Porter) R. O. Erickson] - FNA, HDH, KTZ, E

[Clomatis hirsutissima Pursh] - GPF

[Coriflora scontii (Porter) W. A. Weber] - WEW

[Coriflora scontii (Porter \& Coulc.) W. A. Weber] - SFE

[Vioma scotril (Ponter) Rydb] - KTZ

\{Coptis groenlandica (Oeder) Fernald\}- $\mathrm{HDH}$, W\&W, specimen from MN, FNA

;Copcis trifoliar(L.) Salisb.; - KTZ

\section{Coriflora (see Clematis)}

\section{Cyrtorhyncha (see Ranunculus)}

\section{Delphinium}

(Identification sources:

Delphinium alpestre Rydb, - FNA, HDH, KTZ, E/W

[Delphinium racemosum Rydb. var, alpestre (Rydb,) W, A. Weber] - W\&W

[Delphinium ransosum Rydb.] - SFI

Delphinium barbeyi (Huth) Huth - Dorn01. FNA, HDH, KTZ, W\&W, E/W

[Delphinium barbeyi Huth]-SF:

[Delphinium occidentale (S. Watson) S. Watson var, barbeyi (Huth) S. L. Welsh] - UTF

Delphinium bicolor Nutt. - Dorn01, KTZ

Delphinium geyeri Greene - Dom01, FNA, GPF, HDH, KTZ, SF!, UTF, W\&W, EJW

Delphinium nuttallianum Pritz. - Dorn01, FNA, SF!, UTF, E/W

[Delphinium nelsonii Greene] - HDH, WJ

[Delphinium nuttallianum Pritz, ex Walp.] - KTZ. W\&W

[Delphinium butsallianum Pritz, var, nuttallianmm] - GPF

[Deiphinium nutsallianum Pritz, ex Walp. var, muttallianum] - RMC

Delphinium occidentale (S. Watson) S. Watson - Dorn01, WJ. E/W

[Delphinium glaucum S, Watson] - FNA, KTZ, SF!, W\& W'

[Delphinium X occidentule (S. Watson) S, Watson] - KTZ

[Delphinium oscidentale (S. Watson) S. Watson subsp, cacullatum (A. Nebon) Ewan] - HDH, WAW

[Delphinium occidentale (S. Watson) S. Watson subsp, quercicola Ewao] - HDH, W\&W

[Delphinium occidentale (S. Watson) S. Watson subsp. occidentalis] - HDH, W\&W

Delphinium ramosum Rydb.- Dorn01, FNA, HDH, KTZ, SF!, E/W

[W] - I cannot separate D. robustum from D. ramcsum]

[Delphinium ramosum Rydb, var. ramosum] - W\&W

[Delphinium rohustum Rydh.] - FNA, HDH, KTZ, SFE, W\&W 
Delphinium scaposum Greene - FNA, HDH, KTZ, W

[Delphinium andersonii A. Gray var. scaposum (Grosne) S. L. Welsh] - SFW, UTF, W \& W

Delphinium virescens Nutt, - GPF, WJ, E

[Delphinium carolinianum Walter subsp, vireseens (Nutt.) R. E. Brocks] - FNA, KTZ, W\& W'

[Delphinium carolinianum Walter subsp. vireseens (Nutt.) M. C. Johnst.] - SFE, W\&W

[Delphinium penardii Huth] - GPF, may be called

[Delphinium virescens Nutt. subsp. penardii (Huth) Ewan] - GPF, or may be called, HDH

\{Delphinium wootonii Rydb, -FNA,GPF?, KTZ, SFE, doubtfully distinct, W\&W, E

(WJ - FNA in error, not in Colorado\}

[Delphinium virescens Nutt. subsp. wootonii (Rydb.) Ewan' - GPF, HDH

\section{Halerpestes (see Ranunculus)}

\section{Hecatonia (see Ranunculus)}

\{Myosurus apetalus Gay var, borealis Whittem.\} -FNA, KTZ, E/W

\{Myosurus apetalas Gay\} - SF!, W\&W

Myosurus apetalus Gay var, borealis Whittem. - Dorn01

Myosurus apetalus Gay var, montanus (G. R. Campbell) Whittem, - Dom01, FNA, KTZ, E/W

[Myosunus apetalus Gay] - SF!, UTF. W\&W

[Mysourus aristatus Bendh.] - GPF, HDH rep..

[Myosarus minimus L. subsp. montanus Campbel]] - HDH, W\& W

Myosurus cupulatus S. Watson - FNA, KTZ, LTF, W\&W

Myosurus minimus L. - Dorn01, FNA, GPF, KTZ, SF!, UTF, E/W

[Myosaras minimus L. subsp. minimas] - HDH, W\&W

[Myosunus minimus L var, minimus] - RMC

Myosurus nitidus Eastw, - FNA, HDH rep., KTZ, SFW, UTF, W\&W', W

[Myosurus cupulatus S. Wasson] - W\&W

Psychrophila (see Caltha)

Pulsatilla (see Anemone)

Ranunculus

(Identification sources:

Ranunculus ahortivus L. - Dorn01, FNA, GPF, KTZ, E/W

[Ranumculus abortivus L. var, aborivus] - HDH, W\& W', err, rep.

[Ranunculus abortives L. subsp. acrolasius (Fermald) B. M. Kapoor \& A. Lave]-SFI, WEW

Ranunculus acriformis A. Gray var- acriformis - Dom01. FNA, HDH. KTZ, E/W

[Ranamculus acriformis A. Gray] - SF], UTF, W\&W

Ranunculus acriformis A. Gray var, montanensis (Rydb.) L. D. Benson - Dorn01, HDH exp.,

FNA, KTZ

\{Ranunculus acris L.\} - FNA, GPF, HDH, exp., KTZ, W\&W

\{Ranunculus acris L. var, acris\} - UTF

Ranunculus adoneus A. Gray - Dorn01, FNA, SF!, W\&W, E/W

[Ranunculus adoneus, A. Gray var, adoneus] - HDH, KTZ

[Rananculus adoneas, A. Gray var, alpinas (S, Watson) L. D, Benson] - HDH, KTZ, UTF

Ranunculus alismifolius Geyer ex Benth. var. montanus S. Watson - Dorn01, FNA, HDH. Hartman06, KTZ W\&W. E/W 
[Ranunculus alismifolius Geyer var. montanus S. Watsan] - SF!, UTF

Ranunculus aquatilis L. var diffusa With, - Dom01, FNA, E/W

[Batrachium circinatum (Sibth.) Fr. subsp. subrigidum (E. Drew) A. Löve \& D. Lõve] - SF!, W\&W

[Batrachium kangirostre (Godron) F. Schultz] - SF!, W\&W

[Batrachium trichophyllum (Chaix) Bcsch]-SFL, W\&W

[Ranunculus squatilis L. var. capillaceus (Thuill.) DC.] - HDH, UTF

[Ranunculus aquatilis L. var. eradicatus Laestad.] - HDH

[Ranunculus aquatilis L. var. longirostris (Godr.) C. Lawsca] - UTF

[Ranunculus aquatilis L. var. subrigidus (E. Drew) Breiting] - UTF

[Ranunculus circinatus Sibth. var. subrigidus (E. Drew) L. D. Benson] - HDH

[Ranunculus longinostris Goür.] - GPF, HDH, KTZ

[Ranunculus subrigides W. B. Drew] - GPF

[Ranunculus trichophyllus Chaix var. trichophyllus] - KTZ

\{Ranunculus arvensis L. \} - HDH exp., FNA, GPF, KTZ $<\mathrm{KS}, \mathrm{UT}>$, UTF

Ranunculus cardiophylhus Hook. - Dorn01, FNA, GPF, KTZ, SF!, UTF, W\&W, EW

[Ranunculus cardicohylles Hook, yar. cardiophyllus]- $\mathrm{HDH}$

[Rananculus cardicghylles Hook. var, coloradersis L. D. Benson] - HDH

[Ranunculus colorodensis (L. D. Benson) L. D. Benson] - KTZ

Ranunculus cymbalaria Pursh - Dorn01, FNA, GPF, KTZ, UTF, E/W

[Halerpestes cymbalaria (Pursh) Greene subsp. saximontana (Fernald) Moldenke]-SF!, W\&W

[Ranunculus cymbalaria Pursh var. saximontanus Femald] - Dern01, HDH, SF!

Ranunculus eschscholtzii Schltdl. var, eschscholtzii - Dorn01, FNA, HDH, KTZ, UTF, E/W

[Ranunculus eschscholtzii SchlldL] - SF!, W\& W

Ranunculus eschscholtzii Schltdl. var, eximius (Greene) L. D. Benson - Dorn01, FNA, HDH rep.

[Ranunculus eschscholtzii SshiledL] - W\&W"

[Rananculus eximius Gresene] - KTZ $\leqslant A Z$, UT, WY:

\{Ranunculus fascicularis Muh]. ex Bigelow\} - FNA, GPF, KTZ

\{Rasuncules fascicularis Muhl, ex Bigelow var, apricus (Greene) Fernald\}-HDH rep. W \& W err. rep.

\{Ranunculus flabellaris Raf.\} - FNA, GPF, HDH, KTZ, UTF, W\&W, misid.

Ranunculus flammula L. var, ovalis (Bigelow) L. D. Benson - FNA, GPF, HDH

[Ranunculus tlammula L.] - UTF

[Ranunculus flammula L. var, filiformis (Michx.) Hook.] - HDH, KTZ

[Ranunculus flammula L. var, flemmula] - KTZ

[Ranunculus flammula L. var, reptans (L.) Meyer] - Dom01 (oresumably using different varictal name for same Taxon)]

[Ranunculus reptans L. var, ovalls Torr. A A. Gray] - W\&W

Ranunculus gelidus Karilin \& Karilow - FNA, HDH, SF!, UTF, W\&W", Eurasian, EW

[Ranunculus gelidus Karitin \& Karilow subsp. grayi (Briston) Hultca] - W\& W

[Ranunculus karelinii Crem.] - KTZ

Ranunculus glaberrimus Hook, var, ellipticus (Greene) Greene-Dorn01, FNA, KTZ, E/W

[Rananculus glaberrimus Hook, var, ellipticus Greene] - GPF, HDH, SFI, UTF, W\&W

[Ranunculus oreogenes Greene] - HDH rep, KTZ, UTF

Ranunculus gmelinii DC. - Dom01, FNA, KTZ, UTF, E/W

[Ranamculus gmelinil DC, var, hookeri (D. Don) L. D. Benson] - GPF, HDH, SFI, W\&W

[Rananculus gmelinii DC, var. limosus (Nutt. $H$. Hara] - GPF

[Rasanculus gmelinil DC, var. purshii (Richardscen) H. Hara] - RMC

Ranunculus hyperboreus Rottb. - Dom01, FNA, KTZ, UTF, E/W

[Ranunculus hyperboreus R. Br, subsp, intertextus (Greene) B. M. Kapoos \& A. Love] - SFW

[Rananculus hyperbereus Rothb, subsp. intertextas (Greete) B. M. Kapoor \& A. LËre]-SFE, W\&W

[Ranunculus natans C. A. Mey.] - UTF

[Rananculus natans C. A, Mey, var, intertextus (Greenef L. D. Benson] - HDH

Ranunculus inamoenus Greene var, alpeophilus (A. Nelson) L. D. Benson - Dornd)1

Ranunculus inamoenus Greene var. inamoenus - Dorn01. FNA. HDH. KTZ E/W 
[Ranunculus inambenus Greene] - GPF, SF!, UTF, WEW

[Ranumculus inamoenus Greene var. alpeophilus (A. Nelson) L. D. Benson] - HDH, KTZ

Ranunculus jovis A. Nelson - Dom01, FNA, HDH rep.. KTZ, SFW, UTF, W\&W, W

Ranunculus macauleyi A. Gray - FNA, SF! W\&W, E/W

[Ranunculus macauleyi A. Gray var, brandegei L. D. Benson] - HDH, KTZ

[Ranunculus macauleyi $A$. Gray var. macauleyi] - HDH, KTZ

Ranunculus macounii Britton - Dom01, FNA, GPF, HDH, KTZ, SFI, UTF, W\&W, E/W

\{Ranunculus micranthus Nutr.\} - GPF, FNA, KTZ <KS, OK

\{Ranunculus micranthus Nutt ex Torr. \& A. Gray\} - HDH rep. W\&W, rep.

\{Ranunculus nivalis L. \} - FNA, HDH rep. KTZ. SFW, W

Ranunculus pedatifidus J. E. Sm. var, affinis (R. Br.) L. D. Benson - Dorn0I. FNA. KTZ, E/W [Ranunculus pedatifidus J. E. Sm.] - HDH, SF!, W\&W

[Ranunculus pedarifidus J. E. Sm. ex Rees] - UTF

Ranunculus pensylvanicus L. f, - Dorn01, FNA, GPF, HDH, KTZ, W\&W, no specimens seen

Ranunculus pygmaeus Wahlenb. - Dom01, FNA. HDH, SF!, UTF, W\&W, E'W

[Rananculus pygmacus Wahlenb. var. pygmacus - KTZ

Ranunculus ranunculinus (Nutt,) Rydb, - Dorn01. FNA, HDH, KTZ., UTF, EW

[Cyrtortiyncha ranunculina Nutt.] - SF!

[Cyrtorhyncha ranunculina Nutt. ex Torr. \& A. Gray] - W\&W

* Ranunculus repens L. - Dom01, FNA, Hartman06, KTZ, SF!, W\&W, ENW

[Ranunculus nepens L. var. repens] - HDH, UTF, W\&W'. European

Ranunculus sceleratus L, var, multifidus Nutt. - Dorn01. FNA, GPF, HDH, KTZ, UTF, E/W

[Hecatonia scelerata (L.) Fourr.] - SFI, W\&W

\{Ranunculus sceleratus L. var. sceleratus\} - FNA, GPF, KTZ $\angle K S, N E, O K>, E N$

\{Hectonia scelerata (L.) Fourr.\} - SFt, W\& W

* Ranunculus testiculatus Crantz - Dorn0I, FNA, GPF, HDH, UTF, E/W

[Ceratoosphala orthoceras DC.] - SFI, w\&W

[Ceratoosphala testiculata (Crantz) Besser] - KTZ

Ranunculus uncinatus D. Don - Dorn01. FNA, SF!, E/W

[Ranunculus uncinatus D. Don var. uncinatus] - $\mathrm{HDH}$, rep.

[Ranunculus uncinatus D. Don ex G. Don var. uncinatus] - KTZ

[Ranamculus uncinatus D. Dee var, earlei (Greene) L. D. Benson] - HDH, WeW

[Ranunculus uncinatus D. Doe ex G. Don rar, earlei (Greene) L. D. Benson] - KT2

[Raganculus uncinatus D. Don var. parviforus (Tor.) L. D. Benson] - HDH, W\&W

[Rananculus uncinatus D. Dee ex G. Don var. parviforus (Torr.) L. D. Betriog] - KTZ

Thalictrum

(Identification sources:

Thalictrum alpinum L. - Dorn01, FNA, KTZ, SF!, UTF, W\& W, E/W

[Thalictrum alpienu L. var, heberum B, Boivin] - HDH

Thalictrum dasycarpum Fisch. \& Avé-Lall. - Dom01, FNA, GPF, KTZ, SF!, W\& W, E/W

[Thalictrum dasycarpum Fisch. \& Ave-Lall, ex Fisch., May, \& Ave-Lall.] - HDH

Thalictrum fendleri Engelm. ex A. Gray - Dorn01, FNA, HDH, UTF, W\&W, E/W

[Thalictrum fendleri Engelm.] - SFI

[Thalictrum fendleri Engelm. ex A. Gny var, fendleri] - KTZ

- Thalictrum heliophilum Wilken \& DeMott - ENDEMIC $\langle$ CO $>$, FNA, KTZ, SFW, W\&W, W

Thalictrum occidentale A. Gray - FNA. UTF

[Thalictrum oocidentale A. Gray var, occidentale] - KTZ $<W Y=$

Thalictrum revolutum DC. - FNA, KTZ, W\&W

Snow, N, January 2009. Checklist of Vacculer Plants of the Southom Rodky Momain Region. CVersion $3 x$ 
Thalictrum sparsiflorum Turez. ex Fisch. \& C. A. Mey. - FNA, E/W

[Thalictrum sparsiflorrum Turez.] - SF!

[Thalictrum sparsiflorum Turez. ex Fiseh. \& Avé-L.all.] - W\&W

[Thalictrum sparsiflorum Turez ex Fiseh. \& C. A. Mey. var. saximontanum B. Boivin] - Dorn01, KTZ

[Thalictrum sparsiflorum Turcz ex Fisch., C. A. Mey \& Avé-Lall. var. saximontanum B. Boivin] - HDH

Thalictrum venulosum Trel. - Dorn01, FNA,GPF, HDH, KTZ, W\&W

Trautvetteria caroliniensis (Walter) Vail - FNA, HDH, SF!, UTF, W\&W, EN

[Trautvetteria caroliniensis, Walter) Vail var. accidentalis ( $A$. Gray) C. L. Hitchc.] - KTZ

Trollius albiflorus (A. Gray) Rydb, - Dorn01, FNA, SF!, W\&W, E/W

[Trotlius laxus Salisb.] - UTF

[Trollies laxus Salisb. subsp. albiflorus (A. Gray) A. Lowe, D. Love, \& B. M. Kapoor]-KTZ

[Trollies laxus Salisb. var. albiflorus A. Gray]-HDH

\section{Viorna (see Clematis)}

\section{Viticella (see Clematis)}

\section{Resedaceae - RSD}

* Reseda lutea L. - Dorn01, GPF, HDH, KTZ, SFE, UTF, W\&W, E

* Reseda luteola L- - KTZ, SFE, W\&W, E

[NS - Collected recently in Jefferson County \{fide WJ? in disturbed habicat, and in Weld County on the UNC campas, where it is well established around Ross Hall. Likely to be found elsewhere]

\section{Rhamnaceae - RHM}

Ceanothus fendleri A. Gray - Dorn01, GPF, HDH, IMF, KTZ, SF!, UTF, W\&W, E/W

Ceanothus herbaceus Raf. - KTZ, SFE, W\&W, E

[Ceanothus berbacxus Raf, var. pubescens (Torr. \& A. Gray) Shinners] - GPF

[Ceanothus ovatus Desf.] - $\mathrm{HDH}$

Ceanothus martinii M. E. Jones - Dorn01, HDH, rep. IMF, KTZ, SFW, UTF, W\&W, W

Ceanothus velutinus Douglas ex Hook. var, velutinus - IMF, KTZ, E/W

[Ceanothers velurinus Douglas] - Dorno1, GPF, SF! UTF

\section{Frangula (see Rhamnus)}

Rhamnus betulifolia Greene - FCF, IMF, UTF

[Frangula betulifolla (Greeti) Gruboy subsp, betulifolia] - KTZ <AZ, NM, UT:

[Rhammis betulacfolia Greene] - HDH, exp.

* Rhamnus cathartica L. - Dom01, GPF, HDH, IMF, KTZ, SFE, UTF, W\&W, E

* Rhamnus frangula L. - IMF, E

[Frangula alnus Mill.]-KTZ, SFE, W\&W

Rharmus smithii Greene - KTZ, SFW. W\&W. W

[Rhamsus smithii Greene var, smithi] - $\mathrm{HDH}$

\section{Rosaceae - ROS}

(Identification sources: 
Acomastylis (see Geum)

Agrimonia gryposepala Walle - KWA, M\&H, KTZ, GPF, HDH exp.

Agrimonia striata Michx, - Dom01, GPF, HDH, KTZ, SF!, W\&W, E/W

\{Alchemilla alpina L.\} - HDH rep., KTZ, W\&W labels mixed

Alchemilla vulgaris L. - KWA

[NS - See MaxKay 72; should confirm specimens, as otherwise only reported from New England and NE

Cansida]

[Alchemilla filicaulis subsp. vestita (Buser) M.E. Bradshaw ] - KTZ

[Alchemilla minor Huds.] - M\&H, KTZ

[Alchemilla vulgaris var. vestita (Buser) Murb.] - KTZ

Amelanchier alnifolia (Nutt.) Nutt. ex M. Roem. var, alnifolia - Dorn01, IMF, KTZ, E/W

[Amelanchicr alnifolia Nutt.] - GPF, HDH, SF!, W\&W

[Amslanchicr alnifolia (Nut.) Nutc.] - UTF

Amelanchier alnifolia (Nutt.) Nutt, ex Roem, var, pumila (Tort. \& A. Gray) A. Nelson - Dom01, ENW

[Amelanchict anifolis Nut.] - \$F1, W\&W

[Amelanchict alnifoliat (Nutt.) Natt.] - UTF

[Amelanchicr shifolia (Nutt.) Nutt. ex. Rosen. var, pumils (Natt.) A. Nels.] - IMF

[Amelanchior pumila [Torr. \& A. Gray) Nutt. ex M. Roemer]-KT2

[Anelanchier pumila NutL ex Torr. \& A. Gray]- HDH

Amelanchier utahensis Koehne - Dorn01, HDH, SF!, UTF, W\&W, E'W

[Anclarichicr utalensis Kochne var, utalicnsis] - IMF, KTZ

\section{Argentina (see Potentilla)}

\section{Armeniaca (see Prunus)}

\section{Cerasus (see Prunus)}

Cercocarpus breviflorus Gray - KWA, JM

[Cercocarpus montantes var, paucidentatus (S. Wats.) F.L. Martin ] - KTZ, M.SH

[Cercocarpus paucidentatus (Wats.) Britt.] - M\&H, KWA

Cercocarpus ledifolius Nutt, var. intercedens C. K. Schneid. - Dorn01, KTZ, W

[Cercocarpus ledifolius Nutt.] - SFW

[Cercocarpus ledifolius Nutt, ex Torr. \& A. Grey var, intereedens C. K. Schneid.] - IMF, possible hybrid

[Cercocarpus lodifolius Nutt, var, internonotanus N. H. Holmgren] - KTZ

[Cercocarpus lodifolius Nett, ex Torr. \& A. Gray var. intermontanus N. H. Holmgren] - IMF

Cercocarpus ledifolius Nutt. var. intricatus (S. Watson) M. E. Jones - Dorn01, W

[Cercocarpus intrkarus S. Watson] - HDH. IMF, KTZ SFW, WaW

Cercocarpus ledifolius Nutt. var. ledifolius - Dorn01, KTZ, W

[Cercocarpus ledifolius Nutt.] - SFW, UTF

[Cercocarpus ledifolius Nutt, ex Torr, \& A Gray]-HDH, W\&W

[Cercocarpus ledifolius Nutt, ex Torf, \& A. Gray var, ledifolius] - IMF

\{Cercocarpus montanus Raf, var. argenteus (Rydb.) F. L. Martin\} - GPF, KTZ, E/W

\{Cercocarpar fothergilloides Kunth\} - WRW, misid.

\{Cencocarpus montanas Kaf, - HDH, SF:, WdW

Cercocarpus montanus Raf. var. montanus - IMF. KTZ, E/W

[Cercocarpus montanus Raf.] - Dom01, HOH, SFI, UTF, WEW 
Chamaerhodos erecta (L.) Bunge var, parviflora (Nutt.) C. L. Hitchc. = Dorn01, GPF, see IMF, ENW

[Chamaerhodos erecta (L.) Bunge] - IMF

[Chamaerhodes etecta (L) Bunge subsp. nuttalli (Pickering) Halten] - SF!

[Chamaerhodes erecta (L) Bunge subsp. nuttallia (Pickering ex Rydb.) Hultén] - KTZ W\& W

[Chamaerhodes erecta Bunge var. parviflora (Nutt.) C. L. Hitche.] - UTF

[Chamaerhodos nuttallia (Tor. \& A. Gray) Pickering] - HDH

Coleogyne ramosissima Torr, -HDH, IMF, KTZ, SFW, UTF, W\&W, W

Comarum (see Potentilla)

$\div$ Cotoneaster acutifolia Turcz - Dorm01

Cowania (see Purshia)

Crataegus chrysocarpa Ashe var, chrysocarpa - KTZ, Phipps98

[Crataegus chrysocarpa Ashe] - Demol, HDH, IMF, UTF, W\& W

[Crataegus columbiana Howell var. chirysocarpa (Ashe) Dorn] - RMC

[Crataegus rotundifolia Moench] - GPF

Crataegus douglasii Lindl. var, rivularis (Nutt) Sarg. =IMF, UTF, E/W

[Crategas douglasii LittdL] - Dorno1 [but not for SRMR]

[Crataegus rivularis Nutt.] - Dom01, HDH, KTZ SF!, W\&:W

Crataegus erythropoda Ashe - Dom01, HDH, IMF, KTZ, SF!, W\&W, Phipps98, EJW

Crataegus macracantha Lodd. ex Loud, var-occidentalis (Britton) Eggleston - Dorn01, Phipps98, E/W

[Crataegus columbiana Howell var, oxcidentalis (Britton) Dorn] - RMC

[Crataegus macracantha Lodd, var. oocidentalis (Britton) Egglestoe] - SF!, W \& W

[Cratacgus succuknta Schrad. var. oceidentalis (Britton) E. J. Palm] - GPF

[Cratacgas succukenta Schrad. ex Link] - HDH, IMF, KTZ, UTF, W\& W

Crataegus saligna Greene - HDH, KTZ, SFW, W\&W, W, Phipps99

[NS - Tim Dickinson \{pers. comm., 2007\} indicates this species also occurs in Duscticne Co., UT]: as such it is no longer counted as a regional endemic. See Bearty 04 for its conservarion assesment]

[Crataegus douglasii LithdL var, duschesnesis S. L. Welsh] - UT3

Cylactis (sce Rubus)

Dasiphora (see Potentilla)

Dryas octopetala L. var. hookeriana (Juz.) Breit. - Dorn01, IMF, UTF, E/W

[Dryas octopetala L.] - HDH

[Dryas octupetala L. subsp, hookeriana (Juz,) Hultén] - KTZ, SFI, W \&W

\section{Drymocallis (see Potentilla)}

\section{Erythrocoma (sec Geum)}

Fallugia paradoxa (D. Don) Endl. ex Torr. - KTZ, E

[Fallugia paradoxa (D. Doo) Endl.] - GPF, HDH, IMF, SFE, UTF, WEW

Snow, N, Junary 2009. Checklist of Vacculer Plants of the Southom Rocky Mounain Region. CVersion $3 x$ 
Filipendula ulmaria (L.) Maxim, var- denudata (J. Presl \& C. Presl) Hayne ex Maxim. - E

[Filipendula ulmaria (L.) Maxim. subsp. denudata (J. Presl \& C. Presl) Hayek] - KTZ

[Filipendula ulmaria (L.) Maxim, yar. denudata (Hayno) Maxim.] - SFE

Fragaria vesca $L_{\text {: }}$ - Dorn01, E/W

[Fragaria americana (Porter) Britton] - HDH

[Fragaria vesca L. subsp. americana (Porter) Staudt] - KTZ

[Fragaria vesca L. var, americana Porter] - GPF

[Fragaria vesca L. subsp. bracteata (A. Heller) Staudt] - KTZ, SF!, W\& W

[Fragaria vesca L. var, bracteata (A. Heller) R. J. Davis] - IMF, UTF

Fragaria virginiana Mill. - Dom01, E/W

[Fragaria ovalis (Lehmamn) Rydb.] - HDH

[Fragaria visginiana Duchn.] - GPF

[Fragaria virginiana Mill. subsp, glauca (S. Watson) Staud1] - SF!, W\&W

[Fragaria virginiana Duchn. sutsp. glaoca (S. Watson) Staudt] - KTZ

[Fragaria virginiana Dukhn. var. glauca S. Watson] - UTF

[Fragaria virginiana Mill. var. glauca S. Watson] - IMF

[Fngaria vinginiana Duchn. subsp. platypetala (Rydb.) Staudt] - KTZ

[Fngaria virginiana Mill, var. platypetala (Ryớ.) H. M. Hall] - IMF

\section{Geum}

(Identification sources:

Geum aleppicum Jacq. - Dorn01, GPF, IMF, KTZ, SF!, UTF, EW

[Geum aleppicum Jase, subsp. strictum (Aiton) Clausen] - W\&W

[Geum strictum Aiton var. decurrens (Rydb.) Kearney \& Peebles] - HDH

[Geum strictum Aiton var, strictum] - $\mathrm{HDH}$

Geum macrophyllum Willd. var. perincisum (Rydb.) Raup - Dorn01, GPF, HDH, IMF, KTZ. E/W

[Geum macrophy llam Willd subsp, mikrophyllum] - SF!

[Geum mocrophyllam Willd, subsp. perincisums (Rydb) Huhén] - SF]

[Geums macrophyllum Willd, var, perincisum Raup] - WeW

Geum rivale I.. - Dorn0], GPF, HDH, KTZ, SF!, W\&W, E/W

Geum rossii (R. Br.) Ser, var, turbinatum (Rydb.) C. L. Hitche, - Dorn01, IMF, KTZ, UTF, E/W

[Acomastylis rossit (R, Bri) Greene subsp, turbinata (Rydb.) W. A. Weber] - SFI, W\&W

[Geum turbiasum Rydb.] - $\mathrm{HDH}$

Geum triflorum Pursh var. ciliatum (Pursh) Fass. - Dorn01, GPF, IMF, KTZ, UTF, E/W

[Erythrocoma triflora (Pursh) Greene]-SF, W\&W

[Geum ciliatum Parsh var, ciliatum] - HDH

Geum triflorum Pursh var, triflorum - Dom01, GPF, Hartman06, IMF, KTZ, UTF, EWW

[Erythrocoma triflora (Pursh) Greene] - SF!, W\& W

[Geum ciliatum Pursh var, griseum (Greene) Keames \& Pecbles] - HDH exp.

Holodiscus dumosus (Nutt. ex Hook.) A. Heller var. dumosus - IMF. E/W

[Holodiceus discolor (Pursh) Maxim.] - SFI, W\& W'

[Holodiscus dumosus (Nutt.) A. Heller] - UTF

[Holodiscus dumosus (Nunt. ex Hook.) A. Heller] - Dorn01. KTZ, W\&W

[Holodiscus dumosus (Nutt.) A. Heller var, anstralis (A. Heller) Ley] - HDH

[Holodiscus dumosus (Nutt.) A. Heller var, dumosus] - $\mathrm{HDH}$

[Holodiscus microphyllus Rydb, var, misrophyllus] - HDH

Ivesia gordonii (Hook.) Torr. \& A. Gray - Dom01, HDH, IMF, KTZ, SF!,UTF, W\&W, EW 
* Malus pumila Mill, - Dom01, HDH, IMF, KTZ,UTF

[NS - oceasianally established outside of cultivation, e.g. Poudre Canyon in Lamier Co., CO]

[Malus damestica Borkh.] - WEW

\section{Oreobatus (see Rubus)}

\section{Padus (see Prunus)}

Pentaphylloides (see Potentilla)

Peraphyllum ramosissimum Nutt - HDH, IMF, KTZ, SFW, UTF, W

[Peraphyllum ramosissimum Nett. ex Torr. \& A. Gray] - W\&W

Petrophyton eaespitosum (Nutt.) Rydb, var, caespitosum - KTZ, W

[Petrophyton caespitosum (Nutt.) Rydb.] - Dom0I, IMF, SFW, UTF, W\&W

[Petrophytun caespitosum (Nutt.) Rydb.] - GPF, UTF

[Spiraea caespitosa Nutt.] - HDH

Physocarpus altemans (M. E. Jones) Howell var. altemans - W

[Physocarpus alternans (M. E. Jones) Howell subsp. altemans] - KTZ

[Physocarpus alternans (M, E. Jooes) Howel]] - Dom01, HDH, IMF, SFW, UTF, W\&W

Physocarpus malvaceus (Greene) Kuntze - Dom01, HDH exp., IMF, KTZ, UTF

Physocarpus monogynus (Torr.) J, M. Coult, - Dom01, GPF, HDH, IMF, KTZ, SF!, W\&W, EW

Physocarpus opulifolius (L.) Maxim. var. intermedius (Rydb.) B. L. Rob. - KTZ, E

[Physocarpus intermedius (Rydb.) C. K. Schneid.] - HDH

[Physocarpus opulifolius (L.) Maxim.] - SFE, W. W

[Physocarpus opulifolius (L.) Raf]] - IMF, UTF

[Physocarpus opalifolius (L.) Raf. var, intermedius (Rydb.) B. L. Rob.] - GPF

Potentilla

(Identification sources:

Potentilla ambigens Greene - Dorn01, HDH, KTZ, SFE, W\&W, E

* Potentilla anglica Laicharding - IMF, KTZ, SFE, W\&W, E

Potentilla anserina L. - Dom01, GPF, IMF, LTF, E/W

[Argentina anserina (L.) Rydb.] - KTZ, SF!

[Argentina anserina (L.) Rydb, var, anserina] - W\&W

[Argentina anserina (L.) Rydb, var, eoncolor (Ser,) Rydlo.] - SFE, WAW

[Potentilla anscrina L var, anserina] - HDH

[Potentilla anserina L-var, sericea (L.) Hayne] - $\mathrm{HDH}$

* Potentilla argentea L, var. argentea - KTZ, E

[Potentilla argentea L.] - Dorn01, GPF, SFE, WEW

Potentilla arguta Pursh var. arguta - IMF, UTF. E/W

[Drymocallis arguta (Pursh) Rydb.] - SF4, W\&W

[Potentilla arguta Pursh] - Dom01. GPF

[Potentilla arguta Pursh subsp, argua] - HDH, KTZ

Potentilla arguta Pursh var. convallaria (Rydb.) T. F. Wolf - IMF, UTF, E/W

[Drymocallis arguta (Pursh) Rydb.] - SF-, WAW

[Potentilla arguta Pursb] - GPF 
[Potentilla arguta Pursh subsp. convallaria (Rydb.) D. D. Keck] - HDH, KTZ

Potentilla biennis Greene - Dorn01, GPF, HDH, IMF, KTZ, SF!, UTF, W\&W, E/W

Potentilla bipinnatifida Douglas ex Hook, - Dorn01. IMF, KTZ

[Potentilla pensylvanica L.] - GPF, HDH, W\& W'

[Potentilla pensylvanica L. var. pensylvanica] - W\&W

Potentilla concinna Richardson var, bicrenata (Rydb.) S. L. Welsh \& B. C. Johnst. - Dorn01, KTZ, SF!, UTF, W\&W, EW

[Potentilla bicrenata Rydb.] - IMF

[Potentilla concinna Richardson] - HDH

Polentilla concinna Richardson var, concinna - Dorn01, GPF, KTZ, SF!, UTF, W\&W, E/W

[Potentilla concinna Richardson] - HDH, IMF, SF!

[Potentilla concinna Richardson var. divisa Rydb.] - GPE

[Potentilla quinquefolia Ryob.] - HDH

Potentilla crinita A. Gray var, crinita - KTZ, W

[Potentilla crinita A. Gray] - HDH, [MF, SFW, UTF. W\& W

Potentilla diversifolia Lehm, var, diversifolia - Dorn01, GPF, IMF, KTZ, UTF, E/W

[Potentilla diversifolia Lehm.] - HDH, SFI, W\&W

Potentilla diversifolia Lehm, var. perdissecta (Rydb.) Hitche. - Dom01: Smith09?

Potentilla fissa Nutt, - Dorn01, GPF, HDH, Hartman06, IMF, KTZ, E/W

[Drymocallis fissa (Nutt.) Rydb.] - SF!, W\&W

Potentilla fruticosa 1. - GPF, HDH, UTF, E/W

[Dasiphera floribunda (Pursh) Raf.] - W\& wi

[Dasiphicra fruticosa (L.) Fydb. subsp. floribunda (Pursh) Kartesz] - KTZ

[Pentaphylloides floribundas (Pursh) A. Lovve] - Dorn01, SF!, W\& W

[Pentaphylloides fruticosa (L.) O. Schwarz] - IMF

\{Potentilla glandulosa Lindl. var, glandulosa\} - GPF, E/W

\{Drymocallis glindulosa (LindL) Rydb.\} - SFR, W\&W

Potentilla glandulosa Lindl. var, intermedia (Rydb.) C. L. Hitche.] - Dorn01, GPF, UTF

Potentilla glandulosa Lindl. var, pseudorupestris Rydb,) Breit, - Dorn01, GPF, IMF

Potentilla gracilis Douglas ex Hook, var, brunnescens (Rydb.) C. L. Hitche, - Dom01, KTZ, EJW

[Potentilla gracilis Douglas var. brunbesecns (Rydb.) C. L. Hitchc.] - SF!

[Potentilla brunnescens Rydb.] - HD

[Potentilla gracilis Dowglas] - W\&W

Potentilla gracilis Douglas ex Hook. var. elmeri (Rydb.) Jeps.] - Dorn01, IMF, GREE (Johns/on \& Hedgcock 1072 )

[Potentilla pectinisecta Rydb,] - HDH, KTZ, W\&W, err, rep.

Potentilla gracilis Douglas ex Hook. var, fastigiata (Nutt.) S. Watson - Dom01, IMF, KTZ, E/W

[Potentilla gracilis Douglas var. glabrata (Lehm.) C. L. Hinche.] - SFI

[Potentilla gracilis Douglas ex Hook. var. glabrata (Lehm.) C. L. Hichc.] - UTF, W\& W', ent. rep.

Potentilla gracilis Douglas ex Hook. var. flabelliformis (Lehm.) Nutt. ex Torr. \& A. Gray -

Dorn01. IMF, KTZ, W

[Potentilla flabelliformis Lehmann] - SFW, W\&W

[Potentilla gracilis Douglas ex Hook.] - GPF

[Potentilla gracilis Douglas ex Hook, var, flabelliformis (Lehm.) Nutt, ex Torr. \& A.Gray] - KTZ

Potentilla gracilis Douglas ex Hook. var. nuttallii (Lehm.) E. Sheld. - E/W

[Potentilla gracilis Douglas] - W.\&W

[Potentilla gracilis Douglas var, glabrata (Lehm) C. L. Hitche.] - SFI

[Potentilla gracilis Douglas ex Hook. var. fastigiara (Nutt.) S. Watsoon]- IMF, KTZ

[Potentilla gracilis Douglas ex Hook, subsp, nuttalli (Rydb, D. D. Keck] - HDH

[Potentilla gracilis Douglas ex Hook, var, glabrata (Lehm.) C. L. Hitche.] - GPF, UTT

Potentilla gracilis Douglas ex Hook. var. pulcherrima (Lehm.) Fernald - IMF. UTF, E/W

[Potentilla gracilis Douglas ex Hook.] - GPF

[Potentilla palcherrima Lesm.] - HOH, KTZ, SFI, W\&W 
Potentilla gracilis var. pulcherrima X P,hippiana

[Potentilla hippiana Lehm. var. hippiana X P. gracilis Douglas ex Hook_var. pulcherrima (Lehm.) Fernald -

SF!

E[w]

Potentilla hippiana Lehm, var, effusa (Dougl. ex Lehm.) Dom - Dom01

[Potentilla effusa Douglas] - KTZ SF!

[Potentilla effusa Douglas ex Lehm.] - W\&W

[Potentilla effusa Douglas ex Lehm. var. effuse] - EW

[Potentilla hippiana Letm.] - KWA, M\&H, GPF, HDH

Potentilla hippiana Lehm. var, hippiana - Dorn0I, KTZ, E/W

[Potentilla hippians Lebm_] - GPF, HDH, IMF, SF!, UTF, W\& W

Potentilla hookeriana Lebm. - Dorn01, IMF, SF!, W\&W, EN

[Potentilla hookeriana Lehm. subsp. hookeriana] - KTZ

[Potentilla rubricaulis Lehm.] - UTF

Potentilla multisecta (Wats.) Rydb, - Dom01

Potentilla nivea $\mathrm{L}$. var, nivea - KTZ. E/W

[Potentilla nivea L.] - Dom01. HDH. IMF, SF!, UTF, W\&W

[Potentilla prostrala Rontboel subsp. floceosa Sojak] - W\& W

Potentilla nivea L. var, pentaphylla Lehm. - KTZ

[Potentilla nivea L.] - HDH, UTF, W\&W

Potentilla norvegica L. subsp. monspeliensis (L.) Asch. \& Graebn. - KTZ, E/W

[Potentilla monspeliensis L.] - HDH

[Potentilla norvegica L.] - Dom01, GPF, IMF, SF!, UTF, W\&W

[Potentilla norvegica L. var, hirsuta (Michx.] Lehm.] - see IMF

Potentilla ovina Macoun var, decurrens (S, Watson) S. L. Welsh \& B. C. Johnst. - Dorn01, E/W

[Potentilla ovina Macoun] - SF!

[Potentilla ovina Macoun ex J. M. Macoun var. docurrens (S. Wanson) S. L. Welsh \& B. C. Johnst.] - IMF, KTZ

Potentilla ovina Macoun var, ovina - Dom01, E/W

[Potentilla ovina Maccun] - SFL, W\& W

[Potentilla ovina Macoun ex J. M. Macous var, ovina] - IMF, KTZ

Potentilla palustris (L.) Scop.- Dorn01, IMF, UTF, E/W

[Comarum palusire L.] - KTZ, SFL, W\&W

Potentilla paradoxa Nutt, - Dorn01, GPF, IMF, KTZ, UTF, E

[Potentilla paradosa Nunt ex Torr. \& A. Gray] - HDH

[Potentilla sapian L. subsp. paradoxa (Nutt.) Sojak] - SFE

[Potentilla sapina L. subsp. paradoca (Nutt. ex Toer. \& A. Gray) Soják] - W \& W

Potentilla pensylvanica I. var. pensylvanica - KTZ, UTF, W\&W, E/W

[Potentilla pensylvanica L.] - Dom01, GPF. HDH. IMF. SF!, W\& W

[Potentilla petisylvanica L. var, atrovirens (Rydb.) T. F. Wolf] - KTZ

[Potentilla pensylvanica L. vaf, ovium Jeps.] - KTZ

Potentilla plattensis Nutt. - Dorn01. IMF, KTZ, SF!, UTF, EJW

[Potentilla platensis Nuat ex Torr, \& A. Grsy]- HDH, w\&W

[Potentilla plattensis Rydho,] - GPF

* Potentilla recta L. - Dorn01, GPF, KTZ, SFE, W\&W, E

Potentilla rivalis Nutt. var, millegrana (Engelm. ex Lehm.) S. Watson - E/W

[Potentilla leucocarpa Rydb.] - HDH

[Potentilla rivalis Nunt] - Dom01, KWA, M\&H, GPF, IMF, KTZ, SFI, UTF

[Potentilla rivalis Nunt ex Torr, \& A, Gray] - HDH, W\&W

Potentilla rubricaulis Lehm. - Dom01, HDH, IMF, KTZ, SF!, UTF, W\&W, E/W

[Potentilla rubricaulis Letom, forma nana Clom. \& E. G. Clem.] - HDH

[Potentilla petisylvanica L. var, pauciugga (Rydb.) S. L. Welsh \& B. Johnst.] - SF1, UTF, E/W

- Potentilla rupincola Osterh. - ENDEMIC <CO>, HDH, KTZ, SFE, W\&W. E

[ Potentilla effusa Douglas ex Lehm. var, rupinoola (Osterhout) T. F. Wolf] - KTZ

Snow, N, Junuary 2009. Checklist of Vasculer Plants of the Southom Rocky Moamain Region. CVersion 3 . 
Potentilla subjuga Rydb, - HDH, KTZ, SF1, W\&W, E/W

Potentilla subviscosa Greene - HDH. IMF, SFE, W\&W, E

[Potentilla sabviscesa Greene var. subvisoosa] - KTZ

Potentilla thurberi A. Gray var. atrorubens (Rydb.) Keamy \& Peebles - KWA, M\&H. KTZ

[Potentilla atrorubens Rydb.] - KWA

Potentilla thurberi A. Gray var. thurberi - KWA, M\&H

Potentilla uniflota Ledeb, - KTZ, SF!, W\&W, EN

[Potentilla nivea L.] - HDH

Prunus

(Identification sources:

Prunus americana Marshall - Dorn01, GPF, HDH, IMF, KTZ, SFE, UTF, W\&W, E

Prunus angustifolia Marshall var, angustifolia - KTZ, E

[Prunus angustifolia Marshall] - GPF, SFE, W\&W

* Prunus ammeniaca L. - GPF, IMF, KTZ, UTF

[Armeniaca vulgaris Lam.] - W\&W

Prunus gracilis Engelm. \& A. Gray - GPF, KTZ, SFE, W\&W, E

Prunus pensylvanica L. f. - Dom01, GPF, E

[Cerasus pensylvanica (L. f.) Lobseleur] - SFE, W\&W

[Prunus pensylvanica L. t. subsp. corymbulosa (Rydb.) W. F. Wight] - HDH

[Prumus pensylvanica L. f. var. pensylvanica] - KTZ

[Prunus pensylvanica L. f. var. saximontana Rehd.] - KTZ

*Prunus persica (L.) Batsch - Hartman06

Prunus pumila L. var. besseyi (L. H. Bailey) Gleason - GPF, IMF, KTZ, E

[Cerasers pumila (L.) Michaur subsp. besseyi (L. H. Bailcy) W. A. Weber]-SFE, W\&W

[Prunus besseyi L. H. Bailey]-HDH, UTF

[Prunus pumila L. var, besseyi (L. H. Baiky) Waugh] - Dornol

Prunus rivularis Scheele - GPF, KTZ, SFE, W\&W, E

[Prunus reverehonii Sarg.] - KTZ

Prunus serotina Ehrhart var, rufula (Wooton \& Standley) McVaugh - KWA, KTZ

[Padus rufula Woovon \& Standley] - KWA

[Prunus serocina Ehihart subsp. viretrs var, rufula (Woot, \& StandL) MeVaagh] - M\&H

[Prunus virces vas, fufula (Woos, \& Standl.) Sary.] - KWA. KTZ

Prunus serotina Ehrhart var. virens - KWA

[Padus virens Woot. \& Standi.] - KWA

[Prunus viress (Woot. \& Standl.) Shreve] - KTZ, M\&.H

Prunus virginiana L. var. melanocarpa (A. Nelson) Sarg. - Dorno1, HDH, IMF, KTZ, UTF, E/W

[Padus virginiana (L.) Mill. subsp. melanocarpa (A. Nelson) W. A. Weber] - SF!. W' \& W

[Prunus virgisiana L.] - GPF

Purshia stansburiana (Torr.) Henr. - IMF, KTZ. SFW. W\&W. W

[Cowanla mexicana D. Don] - $\mathrm{HDH}$

[Purshia mexicana (D. Doo) S. L. Welsh var, stansburyana (Torr.) S. L. Welsh] - UTF

Purshia tridentata (Pursh) DC. - Dorn01, HDH. IMF. KTZ, SF!, UTF, W\&W. E/W

Rosa

(ldentification sources:

Rosa acicularis Lindl. subsp. sayi (Schwein.) W. H. Lewis - GPF, KTZ, E/W

Snow, N, Janury 2009. Checklist of Vacculer Plants of the Southom Rocky Momain Region. CVersion $3 x$ 
[Rosa acicularis Lindt] - HDH, IMF

[Rosa engelmannii S. Warson] - HDH

[Rosa sayi Schweinitz] - Dorn0L, SF!, W\&W

Rosa arkansana Porter var, arkansana - KTZ, E

[Rosa arkansana Porter] - Dornel, GPF, HDH, SFE, W\&W

(Rosa arkansana Porter var. suftulta (Greene) Cockerell\} - KTZ, attributed to W\&W

* Rosa blanda Ait, var, blanda - GREE and RM (Reif 3225); Smith09?

* Rosa eglanteria L. - GPF, IMF, KTZ, UTF, E

[Rosa glauca Poum.] - SFE

[Rosa rubiginosa L.] - W\& W'

Rosa foetida Herrm, - FCF

Rosa x barisoni Rivers - Scheibout08

Rosa manca Greene - IMF, KTZ

[Rosa nutkana C. Presl] - $\mathrm{HDH}$

[Rosa woodsii Lindl.] - W \& W

[Rosa woodsii Lindl. var, ultramontana (S. Watson) Jeps.] - UTF

Rosa nutkana C. Presl - HDH, IMF, KTZ, SFW, W\&W misid., W

[Rosa muthana C. Presl var. hispida Fernald] - Dom01. KTZ, UTF, Ws W'

Rosa woodsii Lindl. var, ultramontana (S, Watson) Jeps, - IMF, KTZ, UTF, EJW

[Rosa woodsis Lindl.] - Dom01, GPF, HDH, SF!, W\&W

\section{Rubacer (see Rubus)}

Rubus

(Identification sources:

Rubus acaulis Michx, - Dom01, HDH, E/W

[Cylactis arctica (L.) Raf. subsp. acaulis (Michx.) W. A. Weber] - SF],

[Cylactis arctica (L.) W. A. Weter subsp. acaulis (Michx.) W. A. Weber] - W\& W

[Rubus arcticus L. subsp. ancaul ts (Michx.) Focke] - KTZ

Rubus deliciosus Torr. - Dorn01, GPF, HDH, KTZ, E/W

[Oreobatus deliciosas E. James] - SFE

[Oreobatus deliciosus (E. James) Rlydb.] - SFW

[Oreobatus deliciosus (E. James ex Torr.) Rydb.] - W\&W

[Oreobatus deliciosas (E. James ex Torri) Rydb. subsp. deliciosus] - WeW'

[Oreobatus deliciosus E. James subsp. neconexicana (Rydb.) W. A. Weber] - SFE

* Rubus discolor Weihe \& Nees - IMF, KTZ $\angle$ AZ, NM, UT>. SFW, UTF, W\& W', W

Rubus idaeus L. var. strigosus (Michx.) Maxim. - Dorn01, IMF

[Rubus idacus L, var, aculeatissimus Regel \& TBing ]- E/W

[Rubus idacas 1. subsp, melanolasius (Dieck) Fecke] - SF!, UTF, W\&W

[Rubus idacus L. subsp, sachalinetrsis (H. Lev, focke var, ssehalinensis] - GPF

[Rubus idacas L. subso, strigosus (Michx.) Focke] - KTZ

[Rubus strigosus Michx.] - HDH

Rubus idacus L. var. peramoenus (Greene) Fern. - Dorn01

* Rubus laciniatus Willd. - Dorn01, IMF, KTZ, SFE, W\&W, E

Rubus neomexicanus A. Gray - IMF, KTZ, UTF

[Oreobatus deliciosus (E. James ex Torr.) Rydb, subsp. neomexicanis (A. Gray) W. A. Weber] - W\& W'

[Oreobatus rubicundus Wooton \& StandL] - WRW, misapp

[Rubus [esrubricundus] exrubicundus L. H. Bailey] - HDH

* Rubus occidentalis L. - GPF, HDH, KTZ,SFE, W\&W. E

[Rubus leucodermis Douglas ex Torr, \& A. Gray]- HIDH, exp, IMF 
Rubus parviflorus Nutt, var, parviflorus - KTZ, UTF, E/W

[Rubacer parviflorum (Nutt.) Rydb.] - SF!, W\&W

[Rubus parviflerus Nutt.] - Dorn01, GPF, IDDH, IMF

Rubus pubescens Raf, var, pubescens - KTZ, E

[Cylactis pubescens (Raf.) W. A. Weber]-SFE, W\&W

[Rubus pubescens Raf_] - Domo1, GPF, HDH

* Sanguisorba minor Scop. subsp. muricata Briq. - E/W

[Sanguisorba minor Scop.] - Darn01, IMF, SF!, UTF, W\& W

[Sanguisorba minor Scop, subsp. mericata (Spach) Nordborg] - KTZ.

Sibbaldia procumbens L. - Dorn01, HDH, IMF, KTZ, SF!, UTF, W\&W, E/W

Sorbus dumosa Greene - KWA, M\&H, KTZ

Sorbus scopulina Greene var, scopulina - IMF, KTZ, EW

[Sorbus scopulina Girene] - Dorno I, GPF, HDH, SF!, UTF, WEW

Spiraea (see also Petrophyton)

Spiraea betulifolia Pall. var, lucida (Dougl, ex Hook.) C. L. Hitche, - Dom01

[Spiracs betulifolias subsp. lusida (Dougl, ex Grome) Taylor \& MasBryde] - KTZ

[Spiraca lusida Dougl, ex Greene] - KTZ

Spiraea douglasii Hook, var, menziesii (Hook.) C. Presl - KTZ, SFW, W\&W, W

Spiraea $X$ vanhoutii (Briot) Carriere - KTZ

\section{Rubiaceae - RUB}

(Identification sources:

Asperula (see Galium)

Galium

(Identification sources:

* Galium aparine L. var, aparine-Dom01, HDH, IMF, UTF, E/W

[Galium aparite L.] - GPF, KTZ, SF!, W\&W

* Galium aparine L. var, echinospermum (Wallr.) Farw. - Dorn01, HDH, IMF, UTF, E/W

[Galium aparine L.] - GPF, KTZ

[Galium aparine L, var, echinospennon (Wallr, Farw, - RMC

[Galium spurium L.] - SF!, W\&W

Galium bifolium S. Watson - Dorn01, HDH, IMF, KTZ, SF!, UTF, W\&W, E/W

Galium boreale L. - Dorn0I, GPF, HDH, IMF, KTZ, UTF, E/W

[Galium sepeentrionale Roem. \& Schult.] - SFt, W\&W

Galium fendleri A. Gray - KWA, M\&H, KTZ

Galium mexicanum Kunth var. asperrimum (A. Gray) Higgins \& S. L. Welsh - UTF, E/W

[Galium mexicanum Kunch subsp, asperrimum (A. Gray) Dempster] -KTZ, SF, W\&W

Galium multiflorum Kellogg var, coloradoense (Wight) Cronquist - IMF, UTF, W

[Galium coloradoense W. F. Wight] - Dom01, HDH, KTZ

[Galium coloradoense W. F. Wight] - SFW, W\&W

* Galium odoratum (L.) Scop. - KTZ. W\&W 
Galium proliferum A. Gray - HDH, exp., IMF, KTZ $\angle$ AZ, NM, UT>, UTF, W\&W

[Asperula odarata L.] - HDH]

Galium trifidum L. subsp, columbianum (Rydb, Hultén - KTZ

[Galium trifidum L.] - GPF, UTF

Galium trifidum L, var. subbiflorum Wiegand - IMF, E/W

[Galium brandegei A. Gray] - HDH

[Galium trifidum L.] - GPF

[Galium trifiduen L. subsp. brevipes (Fernald \& Wiegand) A. Löve \& D. Lôve] - SFW

[Galium trifidum L. subsp. subbiflorum (Wiegand) Piper] - KTZ

[Galium trifidum L. subsp. subbiflorum (Wiegand) Puff] - W\&W

[Galium trifidum L. subsp. subbiflorum (Femald \& Wiegand) Puff] - SFE

[Galium subbitlorum (Wiegand) Rydb.] - HDH

Galium trifidum L. var. trifidum - IMF

[Galium trifiduan L.] - Dora01, GPF, HDH, UTF

[Galium trifidum L. subsp. trifidum] - KTZ

Galium triflorum Michx, - GPF, HDH, IMF, KTZ, SF!, UTE, W\&W, E/W

*Galium verum L. - Dorn01, GPF, HDH. IMF, KTZ. SF!, W\&W, EW

Hedyotis nigricans (Lam.) Fosberg var, nigricans - KTZ, E

[Hedyolis nigricans (Lam.) Fosberg] - GPF, SFE, WEW

\section{Ruppiaceae - RUP}

Ruppia cirrhosa (Petagna) Grande - Dorn01, FNA, KTZ, E/W

[Ruppia cirrtosa (Petagna) Grande subsp. oxidentalis (S. Watson) A. Love \& D. Löve] SFI, WeW

[Ruppia maritima L.] - HDH, IMF, UTF, W\&W', etr. rep.

[Ruppia maritima L. var. occidentalis (S. Watson) Graebn_] - GPF

[Ruppia maritima L. var, rostrata 3. Agardh] - GPF

\section{Ruscaceae - RUS}

Maianthemum racemosum (L.) Link var. amplexicaule (Nutt.) Dorn - Dom01, E/W

[Maianthemum anplexicaule (Nutt) W. A. Weber] - SF!, W. W

[Maianthemum racemostem (L.) Link] - WEW', erf, rep.

[Maianthetnum racemosam (L.) Link subsp. amplexicaule (Natt.) Lafrankie] - FNA, KTZ

[Smilacina racemosa (L.) Desf.] - GPF, IMF, UTF

[Smilacina racemosa (L.) Dest. var. amplexicaulis (Nutr.) S. Watson] - HDH

[Smilacina amplexicaulis Nurt] - FNA

[Unifolium amplexicaule (Nutt.) Greene] - FNA

[Vagners anplexicauke is (Nutt.) Greene] - FNA

[Vagnera patlescens Greene] - FNA

Maianthemum stellatum (1., Link] - KT7, SF!, W\&W, E/W

[Smilacina stellata (L.) Desf.] - GPF. HDH. IMF, UTF

\section{Nolina}

[NS - here following focthooning treatment in the next version of the Sywheris by Kantes?]

Nolina greenei S. Watson ex Trel. - FNA, KTZ

Nolina microcarpa S. Watson -KTZ, FNA, HDH rep., IMF, KTZ, UTF, W\&W, misapp.

[W] - The FNA range map for $\mathrm{N}$, microcarpa places it in ne NM; in ny opinion it cecurs no closer than se NM] west of Truth or Consequences, and it is never $\mathrm{e}$ of the Rio Grande. The FNA placement of N. microcarpa in ne NM is probably based on misidentifications at UNM] 
\{Nolina texana S. Watson\}-SFE, WJ, W\&W, E

[W] - I disagree with the treatment in FNA. There is no difference in the se CO plants called N. greenei from those in sw NM, which are N. texana, 1 have seen the type of N. greenei, which is little more than a couple of leaves (collected by Greene at Trinidad, CO, in Jan 1880)]

(Polygonatum biflorum (Walter) Elliott var, commulatum (Schult. \& Schult. f.) Morong\} -

$\mathrm{KTZ}<\mathrm{KS}, \mathrm{NE}, \mathrm{NM}, \mathrm{OK}$, WY>

(Polygonatum biflorum (Walter) Ellivett\} - GPF, RMC

\{Polygosatum commutatum (Schutesf.) A. Dietr. ex. Otto \& A. Dietr.\} - HDH, exp.

\{Polygonatum commutatum (Schutesf.) A. Dietr.\} - WAW

\section{Rutacene - RUT}

Ptelea trifoliata L. - SFE, W\&W, E

[WJ - our plants are distinct and sheuld be called $P$. angiseiforia Benth.]

[Pvelea baldwini Tort. \& A. Gray]- HDH

[Pselea trifoliata L. subsp. pallida (Greene) V. L. Bailey var. confinis (Greene) V. L. Bailey] - [MF. KTZ

[Pvelea trifoliata L. subsp. polyadenia (Girene) V. L. Bailey] - GPF, KTZ

Thamnosma texana (A. Gray) Torr, - GPF, HDH, KTZ, SFE, W\&W, E

[W] - only specimens from $\mathrm{CO}$ are from over 100 years near Canon City; unaware of any populations in northern NM, thas likely extirpared in oor ancal

\section{Salicaceae - SAL.}

(Identification sources:

Populus X acuminata Rydb,- GPF, KTZ, SF!, UTF, E/W

[Populus angustifolia X P. deltoides] - KTZ

[Populus scuminara Rydb.] - Den01, HDH, W\& W

[Populus $\mathrm{X}$ andrewsil Sargent] - $\mathrm{HDH}$

* Populus alba L. - Dom01, GPF, KTZ, UTF

Populus angustifolia E, James - Dorn01, GPF, HDH, KTZ, SF!, UTF, W\&W, E/W

Populus balsamifera L..- Dom01, GPF, HDH, SF!, W\&W

[Populus balsamifera L. var. balsamifera] - EW

[Populus balsamifera L. subsp. balsamifera] - KTZ, UTF

Populus $\mathrm{X}$ brayshawii B. Boivin - GPF $\angle \mathrm{CO}>$, KTZ

[Populus zogustifolia X P. balsamifera] - KTZ

Populus X canadensis Moench - WY checklist, KTZ

[Populus canadensis var, eugesei (Simon-Louis) Schelle] - KTZ

[Populus sanadensis var, serctina (T. Hartig) Rehid.] - KTZ

[Populus X eugenei Simon-Louis] - KT2

[Populus euramericana Guinicr] - KTZ

Populus deltoides W. Bartram ex Marshall var. oceidentalis Rydb. - Dom01, E

[Populus deltoides W. Bartram ex Marshall subsp, monilifera (Aiton) Eckenw.] - KTZ

[Populus deltoides Marshall subsp, monilifera (Aiton) Eckenw.]-GPF, SFE, W\&W

[Populus deltoides Marshail var. occidentalis Rydb.] - UTF

[Populus sargentii Dode] - HDH

Populus deltoides W. Bartram ex Marshall var. wislizenii (S. Watson) Dom - Dorn0I. W

[Populus deltokdes W. Bartram ex Marshall subsp, wislizeni (S. Watson) Eckenw.] - KTZ

[Populus deltokdes Marshall subsp, wislizenii (S. Wasson) Eckeaw.] -SFW, W\&W

[Populus fremontii S. Watson var, wislicenii S. Watson] - UTF

[Populus wislizenii (S. Watson) Sargent] - HDH 
Populus fremontii S. Watson var, fremontii - UTF

[Populus fremoetii S. Watson subsp. fremontii] - KTZ, W\&W

\{Populus X intercurrens S. Goodrich \& S. L. Welsh\} - UT3

\{NS - reparted far CO by UT3, specimens not scen\};

Populus X jackii Sarg, - GPF, KTZ, W\&W

[Populus balsamifera X P. delitoides] - KTZ

* Populus nigra L. - GPF, KTZ, W\&W

Populus tremuloides Michx, - GPF, HDH, KTZ, SF, UTF, W\&W, E/W

Salix

[See Argus07 for distribution maps with caveats regarding tavortomic recognitica]

(Identification sources:

* Salix alba L. var. vitellina (L.) Stokes - GPF, W\&W, EN

[Salix alba L.] -, Argus97, KTZ

[Salix alba L var. vitellina (L.) Koch] - SF!

Salix amygdaloides Andersson - Argus07, Dorn01, GPF, KTZ, SF!, UTF, W\&W, E/W

[Salix amygdabides Andersson forma artydgaloides] - HDH

[Salix amygdaloides Andersson forma angustissima E. C. Sm.] - HDH

[Salix amygdaloides Andersson forma piloseinascula C. K. Schneider] - HDH

Salix arctica Pall. var, petraea (Andersson) Bebb-Dorn01, E/W

[Salix anglorum Chan. var. antiplasta C. K. Schneider] - HDH

[Salix anctica Pall subsp, petraca (Andersson) A. Love, D. Lobve, \& B. M. Kapoor] -SF! W\& W

[Salix anctica Pall. var, petraca Andersson] - UTF

[Salix petrophita Rydb.] - Argus07, KTZ, W\& W'

Salix arizonica Dotn - Argus0?

[NS - absent from provious checklists]

* Salix babylonica L. - SF!, W\&W, E/W

[Salix $X$ pendulina Wender.]-KTZ

[Salix fragilis $X$ ?S. sepulcralls] - KTZ

[Salix X sepulcralis Simonk.]-KTZ

[Salix alba X rS. pendulina] - KTZ

[Salix barclayi Anderss.\} - KTZ, W\&W' err. rep.

Salix bebbiana Sarg. - Argus07, Dorn01, KTZ, SF!, UTF, W\&W, E/W

[Salix bebbiana Sarg. var bebbiana] - GPF. HDH, RMC

[Salix bebbiana Sargent var. pemostrata (Rydb.) C. K. Schneid.] - GPF, HDH

Salix boothii Dorn - Argus07, Dom01, KTZ, SF!, UTF, W\&W, E/W

[Salix pseudocondasa (Andersson) Rydb.] - HDH

Salix brachycarpa Nutt. var- brachycarpa - HDH, KTZ, UTF, E/W

[Salix brachycarpa Nutt] - Dorn01, SFI, WeW

[Salix brachycarpa Nutt, subsp, brachycarpa] - Doris?

[Salix brachycarpa Nutt, var, antimima Kaup] - HDH

[Salix brachycarpa Nutt, var, sansonii C. R. Ball] - HDH

[Salix glauca L. var, glabrescens (Andersson) C. K. Schneid.] - HDH

[Salix pseudolappooum Seem.] - $\mathrm{HDH}$

Salix calcicola Fernald \& Wiegand var. glandulosior B. Boivin-Argus07

[Salix calcicola Femald \& Wicgand] - Snow07b, KTZ, SFE, Dom97, EW

[Salix lanata L. sabsp, calcicola (W iegand) Hultén] - SFW

[Salix lanata L. subsp, calcioola (Fernald \& Wiegand) Hultén] - W\&W

Salix candida Flüeggé ex Willd. - Arguso7, Dorn01, KTZ, E/W

[Salix candida Flieged] - GPF, HDH, SF:, W\&W 
Salix cascadensis Cockerell - Argus07, Dorn01, HDH rep., KTZ, SF! exp., UTF, W\&W err, rep.,

(Salix cordifolia Pursh var, callicarpaea (Trautv.) Fernald\}- HDH, W\&W, err, rep. \{Salix glaca L. subsp. sallicarpaea (Trausy.) Bocher\} - KTZ

Salix discolor Muhl. - Argus07, Dorn01, KT7

[Salix ancorifera Fern.] - KTZ

[Salix conformis Forbex]- KTZ

[Sallix crassa Banatt] = KTZ.

[Salix discolor var. overi BaII] - KTZ

[Salix discolor var. prinoides (Pursh) Andress.] - KTZ

[Salix discolor var, rigidior (Anderss.] Schneid.] - KTZ

[Salix fiscata Pursh]-KTZ

[Salix prinoides Pursh] - KTZ

[Salix sensitiva Barratt] - KT/

[Salix squarnata Rydb_] - KTZ

Salix drummondiana Barr, ex Hook, - Dom01, KTZ, UTF, EJW

[Salix drummondiana Barr.] - SF!, W\&W

[Salix subcoerulea Piper] - HDH

Salix eriocephala Michx, var, famelica (C. R. Ball) Dom - Dom01, E/W

[Salix lutea Nutt.] - Argus07, GPF, KTZ, SF!, W\& W, W\& W'

[Salix lutea Nutc. var. famelica C. R. Ball] - HDH

Salix eriocephala Michx. var, ligulifolia (C. R. Ball) Dorn - Dorn01, Ew

[Salix ligulifolia C. R. Ball] - Argus07, SF!

[Salix ligulitolia (C, R, Ball) C. R, Ball ex C. K. Schneid.] - KTZ

[Salix ligulifolia (C, R. Ball ex C. K. Sehneid.) C. R. Ball ex E. C. Smith] - W\&W'

[Salix lutes Nutt. var. ligulifolia C. R. Bail] - HDH

Salix eriocephala Michx, var, watsonii (Bebb) Dom - Dom01, E

[Salix erioceplata Michx.] - SFE

[Salix ligulifolia (C. R. Ball ex C. K. Schneid.) C. R. Ball ex E. C. Smirh] - W EW $^{\prime}$

[Salix lutea Nutt.] - GPF, KTZ W\&W

[Salix lutea Nutt. var, lutea] - HDH

[Salix lurea Nutt. var. planyphylla C. R. Ball] - HDH

[Salix lurea Nutt. var. watsonii (Bebo) Cronq.] - UTF

[Salix rigkda Muhl. var. watsonii (Bebb) Cronquist] - w\& w

Salix exigua Nutt. - KTZ, SF!

[Salix exigua NurL subsp. exigua] - Argus07, GPF. W\&W

[Salix exigua NerL subsp. exigua var, exigua] - UTF, Done?

[Salix exigua Nust var, exigua] - Dom01, HDH. EW

[Salix exigua Nuts. subsp. exigua var. stenophylla (Rydb.) C. K. Schneid.] - UTF

[Salix exigua Nurt. var, luteosericea (Rydb.) C. K. Schneid.] - $\mathrm{HDH}$

[Salix exigua Nurt, var, senophylla (Rydb.) C. K. Schneid.] - HDH

[Salix Jinearifolia Rydb.] - HDH

[Salix exigua Nuet.] - SF!

[Salix exigua Nust. subsp, interior (Rowlec) Cronquist ] - GPF, W\&W, Dom9?

[Salix exigua Nuat. var, pedicel lata (Andersson) Cronquist] - EFW

[Salix interior Rowlee] - KTZ

[Salix interior Rowlee var, interior] - HDH

[Salix interior Rowlee var, pedicellata (Andersson) C. R. Ball] - HDH

Salix famelica (C. R. Ball) Argus - Argus07

[Salix lurea Nuttall var, familica C. R. Ball] - Argus07

* Salix fragilis L. - Dorno1,GPF, HDH, KTZ, SF!, UTF, W\&W, E/W

[NS - not incladed in our region by Argus07; needs further study]

Salix geyeriana Andersson - Argus07, Dorn01, KTZ, SF!, W\&W, E/W

[Salix gey eriana Andersson var, argeniea (Bebb) C. K. Schneid.] - HDH 
[Salix geyeriara Andersson var. geyeriana] - HDH, UTF

Salix glauca L. var. villosa Andersson - Dom01, E/W

[Salix brachycarpa Nutt.] - W\& $W^{1}$

[Salix glauca L.] - Argus07 [as "s.1."], SF!

[Salix glauca L. subsp. glauca var. villosa (D. Don ex Hook.) Anderssen] - KTZ

[Salix glausa L. var. villosa (Hook.) Andersson] - UTH

Salix gooddingii C. R. Ball-Argus07, KTZ, SFW, UTF, W\&W, Dom97, W

[Salix nigra Marsh.] - GPF

Salix interior Rowlee - Argus07

[NS - relationship to names under S. exigua should be re-examined for our region]

Salix irrorata Andersson - Argus07, Dom01, HDH, KTZ, SFE, W\&W', E

Salix lasiandra Benth, var, caudata (Nutt.) Sudw, - Argus07, Dom01, E/W

[Salix caudata (Nutt.) A. Helier var. caudata] - HDH

[Salix lucida Muhl. subsp. caudata (Nutt) E. Murray] - KTZ, W\&W

[Salix lucida Muhl. subsp. caudata (Nutt) Argus] - SF!, UTF

Salix lasiandra Benth, var. lasiandra-Argus07, Dom 97, E/W

[Salix caudata (NutL.) A. Heller var. bryantiana C. R. Ball \& Bracelin] - HDH

[Salix lasiandra Benth.] - $\mathrm{HDH}$

[Salix lucida Muhl. subsp. Iasiandra (Betith). Argus - SF!

[Salix lucida Multl. subsp. lasiandra (Benth.) E. Murray] - KTZ, WEW

$\{$ Salix lemmonii Bebb\} - Dom01, KTZ $\langle\mathrm{CO}, \mathrm{WY}\rangle$

;NS - voucher for our area not seovi

\{Salix lucida Muhl.\} - GPF, HDH, W\&W, ert, rep.

\{Salix lucida Mubl. subsp. lucista\}- KTZ ×KS>. W\&W', err. rep:

* Salix matsudana Koidz - KTZ, SF!, W\&W, E/W

[Salix matsudana Koidz forma sortuosa Rehd.] - UTF

[Salix marsudana Koidz forma umbraculifera Rehd.] - UTF

Salix melanopsis Nutt. - Argus07, Dom01, KTZ, SF!, W\&W', misid, E/W

Salix monticola Bebb - Argus07, Dorn01, HDH, KTZ,SF!, W\&W, EWW

[Salix barclayi Andersson var, barclayi] - HDH

[Salix barelayi Andersson var. hebecarpa Andersson] - HDH

[Salix monticola Bebb ex I. M. Coult.] - UTF

[Salix preudomonticola C. R. Ball]-GPF, SF!

[Salix pgeudomonticola C. R. Ball var, padophy lla (Rydb.) C. R. Ball]- HDH

[Salix pseudomonticola C, R. Ball var, pseudomonticola] - HDH

Salix myrtillifolia Andersson var. myrtillifolia - Dorn97, E/W

[Salix myrtillifolia Andersson] - Argus07, KTZ, SF, W\& W

Salix nigra Marshall - Argus07, KTZ, SFE, W\&W ${ }^{1}$, Dorn97, E

\{Salix orestera Schneid.\} - KTZ

Salix pentandra L. - Argus07, GPF, KTZ, UTF

Salix petiolaris J. E. Sm. - Argus07, GPF, HDH, KTZ, Dorn97, E

[Salix gracilis Andersson] - SFE, W\& W

Salix planifolia Pursh var, planifolia - Dorn01, E/W

[Salix planifolia Pursh] - Argus07, GPF, SF1, W\&W

[Salix planifolia Pursh subsp. planifolia] - K12

[Salix planifolia Pursh subsp, planifolia (Pursh) Hihenen var, monica (Bebb) Jeps.] - UTF

[Salix planifolia Pursh subsp, planifolia var. planifolia] - UTF

[Salix planifolia Pursh var, monica (Bebb) C. K. Schneid.] - HDH

[Salix planifolia Pursh var, nelsonili (C, R. Ball) C, R. Ball] - HDH

\{Salix X princeps-ourayi L. Kelso\} - KTZ (Argus pers. comm.)

[Salix glaucs X S, monticola] - KTZ

* \{Salix purpurea L.\}-HDH, KTZ,SFE, UTF, W\&W, E

Salix reticulata L. var, reticulate - KTZ 
[Salix nivalis Hook.] - Argus07, W: W $W^{1}$

[Salix reticulata $L$ subsp. reticulata] = KTZ

Salix reticulata L. var, nana Andersson - Dorn01, EW

[Salix nivalis Hook.] - KTZ W\&W

[Salix nivalis Hook. var. nivalis] - HDH

[Salix nivalis Hook. var. saximontana (Rydb.) C. K. Schneid.] - HDH

[Salix reticulata L. subsp, nivalis (Hook.) A. Läve, D. Lôve, \& B. M. Kąpor] - SF!, W \& W, Dorn97

[Salix reticulata L var. nivalis (Hock.) Andersson] - UTF

\{Salix X rubens Schrank\} - KTZ. UTF

[Salix alba $X$ s. fragilist - KTZ

Salix scouleriana Barratt ex Hook. - Argus07, Dorn01, KTZ, W\&W, EWW

[Salix scouleriana Barr.] - GPF. HDH, SF!, UTF

Salix serissima (L. H. Bailey) Fernald - Argus07, Dorn01, GPF, HDH, KTZ, SF!, W\&W, E/W

Salix wolfii Bebb var, wolfii - Argus07, Dom01, HDH, UTF, E/W

[Salix wolfii Bebb]- KTZ, SF!, W\&W

\{Salix wolfii Bebb var, idahoensis C. R. Ball\} - HDH, Dorn9?

\{Salix wolfii Bobb\}- KTZ, W\&W

\section{Sambucacene - SAM}

* Sambucus canadensis L, var, canadensis - E

[Sambocus caradensis L.] - Dom01, GPF, HDH, SFE, UTF, W\&W

[Sambecus nigra L. subsp. canadonsis (L.) R. Bolli] - KTZ

Sambucus cerulea Raf, - Dom01, IMF, E/W

[Sambecus caeruka Raf.] - UTF

[Sambucus cerules Raf. var. cerulea] - KTZ

[Sambocus cosrulea Raf.] - HDH, SFI, W\&W

[Samboxus nigra L, subsp, ceruka (Raf.) R. Bolli] - KTZ

Sambucus racemosa L. var, melanocarpa (A. Gray) McMinn - Dorn0I, KTZ, UTF, E/W

[Sambexus melanocarpa A. Gray] - HDH

[Sambocus microbotrys Rydb.] - W\& W

[Sambecus racemosa L. subsp. pubens (Michx.) Hocise var, melanocarpa (A. Gray) McMinn] - IMF

Sambucus racemosa L. var, microbotrys (Rydb.) Keam. \& Peeb. - Dom01, UTF, E/W

[Sambocus miktobotrys Rydb.] - SFI, wi W

[Sambucus racemosa L. subsp. pubens (Michx.) House] - GPF

[Sambecus racemosa L. subsp. pubens (Michx.) Hocise var, microbotrys (Rydb.) Kearn. \& Peeb.] - IMF, KTZ

[Sambecus macemsasa L, var, racemosa] - KTZ

\{Sambucus racemosa L. var, racemosa\} - KTZ

\{Sambucus pubens Michx. - HDH, W\& W, err.

'Sambucus racemosa L. subsp. pubsens (Michx.) House? - GPF

[Sambucus racemosi L. subsp. pulwris (Michx.) House var, pubens (Michx.) Koehne]

[Sambucus racemosa L. subsp. pubers (Michx.) House var. leusocarpa (Torr, \& A. Gray) Cronquist)

\section{Santalaceae - SAN (including Viscaceae; excluding Loranthaceae)}

Comandra umbellata (L.) Nutt. var. pallida (A. DC.) M. E. Jones - Dorn01, IMF, UTF, E/W

[Comandra umbellata (L.) Nutt.] - HDH, SF!

[Comandra umbellata (L.) Nurt. subsp, pallida (A, DC, Piehl] - GPF, KTZ, W\&W

\section{Sapindaceae - SAP (including Aceraceae, Hippocastanaceae)}

Acer ginnala Maxim. - WY checklist 
Acer glabrum Torr. var, glabrum - HDH, IMF, KTZ, UTF, E/W

[Aoer glabrum Torr.] - GPF, SF!, WEW

[Aoer glaboum Torr. var. neomexicsmum (Greene) Kearney \& Pecbles] - HDH, KTZ

[Aeer glabrum Torr. var. tripartitum (Nutt.) Pax] - HDH

[Acer glabrum Torr, forma trisectum Sarg.] - SF!

Acer grandidentatum Nut, var. grandidentatum - KTZ. W

[Acer grandidentatum Nutt.] - IMF, SFW, UTF

[Acer grandidentatum Nutt. ex Torr. \& A. Gray] - HDH. W\&W

Acer negundo L. var, interius (Britton) Sarg - Dorn01, GPF, HDH, IMF, KTZ, E/W

[Acer negundo L. var. interior (Britton) Sarg.] - UTF

[Negundo aceroides Mosich subsp. interius (Britton \& Shafer) A. Libv \& D. Lōve] - SF!

[Negundo aceroides (L.) Moench subsp. interius (Britton \& Shafer) A. Lōve \& D. Läve] - W \& W

+ Acer negundo L. var, violaceum (Kirchn.) Jaeger - Dom01, GPF, KTZ, EW

[Acer negundo L. var, violaceum Jacger \& Beissi.] - HDH

[Acer negundo L. var. violaceum (Kinchn.) Jacger] - RMC

[Negundo aceroides Moench subsp. violactus (Kirchn.) W. A. Weber]-SFE

[Negundo acervides Mocneh subsp. violaceum (Kirchn.) W. A. Weber] - SFW

[Negundo aceroides (L.) Moench subsp. violaceus (Kirchn.) W. A. Weber] - W\& W

Aesculus glabra Willd, - WY checklist

[Aesculus glabra Willd. var. arguta (BuckL.) B. L. Robinson] - KTZ

Negundo (see Acer)

Sapindus saponaria L. var, drummondii (Hook. \& Am.) L. D. Benson - GPF, IMF, KTZ, SFE, UTF, E

[Sapindus drummondii Hook, \& Am.]-HDH, W\&W

\section{Sarcobataceae - SRB}

Sarcobatus vermiculatus (Hook.) Torr. var, vermiculatus - Dorn01, UTF, EWW

[Sarcobacus vermiculatus (Hook.) Torr.]-GPF, HDH, KTZ SFI, W Q W

\section{Saururacene - SRR}

*Anemopsis californica Hook. \& Arn, - FNA, E

[Anemopsis californica Hook.] - SFE, WEW

[Anemopsis californica (Nutt.) Hook, \& Ara.] - GPF, KTZ, UTT

[Anemsopsis californica Hook. var. subglabrata L. Kelso] - HDH

\section{Saxifragacene - SAX (see also GRS, HDR, PAR)}

(Identification sources:

Boykinia jamesii (Torr.) Engler - E, Dom01 <reportedly in Albany Co, WY>

[Saxifraga jamesii Torr.] - HDH

[Telesonix jamesii (Torr.) Raf.] - GPF, KTZ, SFE, W\&W

[Telesonix jamesii (Torr.) Raf. var. jamesii] - MMF, UTF

Chrysosplenium tetrandrum (Lund ex Malmgr.) Th. Fr, - KTZ, E/W

[Chrysosplenium tetrandrum (N. Lund) Th. Ft.] - W\& W 
[Chrysosplenium tetrandrum Th. Fr.] - HDH, SE!

\section{Ciliaria (see Saxifraga)}

Conimitella williamsii (D. C. Eaton) Rydb. - IMF, KTZ, SF!, W\&W, EW

Heuchera bracteata (Torr.) Ser, - Dom01, HDH, KTZ, SF!, W\&W, E/W

- Heuchera ballii A. Gray - ENDEMIC $<$ CO, NM>, HDH. Hartman06, KTZ, SFE, W\&W, E Heuchera novomexicana Wheelock - KWA, M\&H, KTZ.

Heuchera parvifolia Nur, ex Torr. \& A. Gray - Dom01, HDH, E/W

[Heuchera nival is Rosendahl, Butters, \& Lakela] - HDH

[Heuchera parvifolia NutL] - IMF, SF!

[Heuchera parvifolia NutL. var, utahensis (Rydb.) Garrett] - UTF

[Heuchera pancifolia Nut_ ex Torr. \& A. Gray var. flavescens (Rydb.) Rasendahl, Butsers, \& Labela] - KTZ.

[Heuchera parvifolia Nutt. ex Torr. \& A. Gray var. major Roscndahl, Butters, \& Lakela] - KTZ

[Heuchera parvifolia Nutt. ex Torr. \& A. Gray var, microeame Rosendahl, Butters, \& Lakcla] - KTZ

[Heuchera parvifolia Nutt. var. nivalis (Rosendahl) A. Löve, D. Löve, \&. B. M. Kapoor] - SF!

[Heuchera parvifolia Nutt. ex Torr. \& A. Gray var, nivalis (Rosendahl, Butters, \& Lakela) A. Läve. D. Löve, \& B. M. Kapoer] - KTZ

[Heuchera parvifolia Nutt. ex Tort. \& A. Gray var, nivalis (Rosendahl) A. Lōvê, D. Lōve, \& B. M. Kapoer] W\&W

[Heuchera parvitolia Nutt. ex Torr. \& A. Gray var, parvifolia] - KTZ, W\&W

[Heuchera parvifolia Nutt. ex Torr. \& A. Gray var, utabetsis (Rydo.) Garrett] - KTZ

Heuchera richardsonii R. Br, - Dorn01, HDH, KTZ, SFE, W\&W, E

[Heuchera richardsonii R. Br, yar. richardsonii] - GPF

Heuchera rubescens Torr. var, rubescens - KTZ, UTF, W

[Heuchera rubeseens Torr.]-IMF, SFW, W\&W

Heuchera rubescens Torr. var, versicolor (Greene) M. G. Stewart - KTZ, UTF, E

[Heuchera rubescens Torr.] - IMF

[Heuchera versicolor Greene] - SFE, W\&W

\section{Hirculus (see Saxifraga)}

Lithophragma glabrum Nutt, var. ramulosum (Suksd) B. Boivin - Dorn01, E/W

[Lithophragena bultsifera Rydb.] - GPF, HDH

[Lithophragena glahra Nutr, var, ramulosa (Suksd.) Gocdrich] - UTF

[Lithephragma glabrum Nutt.] - IMF, KTZ, SF!, W\&W

Lithophragma parviflorum (Hook.) Nutt. ex Torr. \& A. Gray - Dom01, IMF, W\&W, E/W

[Lithophragma parsifloea (Hook.) Nurt] - UTF

[Lithophragma parvifloca (Hook.) Nutc ex Torr. \& A, Gray] - HDH

[Lithophragma parviflorum (Hook,) Nutt.] - GPF, SF!

[Lithophragma parsiflocum (Hook,) Nutt, ex Torr. \& A, Gray var, parviflorum] - KTZ

Lithophragma tenellum Nutt. - Dornol, IMF. KTZ, SF!, W\&W, E/W

[Lithophragma tenella Nutt.] - UTF

[Lithophragma tenella Nutr, ex Torr. \& A. Gray] - HDH

\section{Micranthes (see Saxifraga)}

Mitella pentandra Hook. - Dorn01, HDH, IMF, KTZ, SF!, UTF, W\&W. E/W

Mitella stauropetala Piper var. stenopetala (Piper) Rosend, - KTZ, SF!,UTF, W\&W, E/W

[Mitella stauropetala Piper] - Dorno], HDH rep., IMF

[Mitella stenopetala Piper] - HDH 
Muscaria (see Saxifraga)

Parnassia (see PAR)

Saxifraga (see also Boykinia)

(Identification sources:

Saxifraga adscendens L. var, oregonensis (Raf.) Breit, - IMF, UTF, E/W

[Muscaria adsoendesn (L.) Small]-SF!, WE W

[Saxifraga adscendens L.] - HDHI

[Saxifraga adscendens L. subsp. oregonensis (Raf.) Bacig.] - KTZ

Saxifraga bronchialis L. var, austromontana (Wiegand) Piper ex G. N. Jones - Dorn01, EN

[Ciliaria austromontana (Wiegand) W. A. Weber) - SF!, W\& W

[Saxifraga beonchisalis L.] - HDH

[5axifraga bronchialis L. subsp. auscromontana (Wiegand) Piper] - KTZ

[Saxifraga bronchialis L. var. austromontana (Wiegand) G. N. Jones] - IMF. UTF

Saxifraga cernua L. - Dom01, GPF, HDH, IMF, KTZ, SF!, UTF, W\&W, E/W

Saxifraga cespitosa L. subsp. delicatula (Small) Porsild - KTZ, E/W

[Muscaria delicatula Small] - SF!, W\&W

[Saxifraga cacopilosa L.] - HDH

[Saxifraga caespitosa L. subsp. delicakula (SmalI) Porsild] - KTZ

[Saxifraga cespitosa L] - IMF

Saxifraga cespitosa L. subsp. monticola (Small) Porsild - E/W

[Muscaria micropatala Small] - SF!

[Muscaria micropetala (Small) Feddo] - W\& W

[Muscaria monticola Small] - SF!

[Muscaria monticola (Small) Fedde] - W\& W

[Saxifraga caespitosa L. var. minima Blank.] - UTF

[Saxifraga caespitosa L. subsp. monticola (Small) Porsild] - KTZ

[Saxifraga cespitosa L.] - Dorno1, IMF

[Saxifraga cespitosa L- var, minima Blank.] - RMC

Saxifraga chrysantha A. Gray - HDH, KTZ, UTF, E/W

[Hirculas serpyllifolius (Pursh) W. A. Weber subsp, chrysantha (A. Ciray) W. A. Weber] - W\& W'

[Hirculus serpyllifoljus (Pursh) W. A. Weber subsp, chrysanthus (A. Gray) W. A. Weber] - SF!

[Saxifraga serpyllifolia Pursh var, chrysansha (A. Gray) Dorn] - IMF

Saxifraga flagellaris Willd, ex Sternb, var, crandallii (Gand.) Dorn - Dom01, E/W

[Hirculus platysepalus (Traurv.) W. A. Weber subsp. crandallii (Giand.) W. A. Weber]- SF!, W\& W

[Saxifraga flagellaris Willd.] - HDH, UTF

[Saxitraga flagellaris Willd. ex Stemb.] - IMF

[Saxiffaga flagellaris Willd. ex Stemb. subsp. crandallii (Ciand.) Hultén] - KTZ

[Saxifraga platysepala (TrauTV.) Tolm.] - KTZ

Saxifraga foliolosa R. Br. - KTZ, E

[Saxifraga vreelandii (Small) Fedde] - HDH. rep.

[Spentularia foliolosa ( $R$. Br.) Small]- SFE, WEW

Saxifraga hirculus L. subsp. coloradensis Hedd. - EJW

[Hirculus prerepens (Fisch.) A. Liwe \& D, Lave] - SF'

[Hirculus prorepens [Fisch, ex Stemberg) A. Love \& D. Love] - WA W

[Saxifraga hircalus L.] - IMF, UTF

[Saxifraga hircalus L. subsp, hirculas] - KTZ

Saxifraga oceidentalis S. Watson - Dom01, GPF, IMF, KTZ

Saxifraga odontoloma Piper - Dorn01, IMF, KTZ, UTF, E/W

Snow, N, January 2009. Checklist of Vecculer Plants of the Southom Rocky Momain Region. CVersion $3 x$ 
[Micranthes odontoloma (Piper) A. Heller] - W\&W

[Micranthes adontoloma (Piper) W. A. Weber] - SF!

[Saxifraga arguta D. Dan] - HDH

Saxifraga oregana Howell - HDH, IMF, KTZ, E/W

[Micranthes oregana (Howell) Small] - SF!, W\&W

[Saxifraga montanensis Small] - HDH

Saxifraga rhomboidea Greene - Dorn01, HDH, IMF, KTZ, UTF, E/W

[Micranthes rhomboibes (Creene) Small] - SF! W\&W

Saxifraga rivularis L, var. debilis (Engelm, ex A. Gray) Dorn - Dorn01

[NS-Jorgensen06 recognizes S. rivularis, S. debilis, and S. hyperborea all as distinct]

[Saxifraga rivularis L.]- IMF, KTZ, W\&W, EW

[Saxifraga debilis Engelm.] - HDH, UTF

[Saxifraga hyperborea R. Br. subsp. debilis (Engelm.) A. Löve, D. Löwe, \& B. M. Kapont] - SF!

[Saxifraga hyperborea R. Br. subsp. debilis (Engelm, ex A. Gray) A. Love, D. Love, \& B. M. Kapoor] - W \& W

[Saxifraga rivularis L. var. flexuesa (Sternb.) Engl. \& Imsch.] - RMC

Saxifraga serpyllifolia Pursh var, chrysantha (Gray) Dorn - Dorn0I, KTZ

[Hirculus serpyllifolius subsp. chrysanthus (Giray) W.A. Weher] - KTZ

[Saxifraga serpyllifolia subsp. chrysantha (Gray) W.A. Weber] - K.TZ

[Saxifraga chrysantha Gray] - KTZ

\section{Spatularia (see Saxifraga)}

Sullivantia hapemanii (J. M. Coult. \& Fish.) J. M. Coult. var, hapemanii - Dorn01, KTZ

- Sullivantia hapemanii (J. M. Coult. \& Fish.) J. M. Coult, var, purpusii (Brand) Soltis -

ENDEMIC $<\mathrm{CO}>\mathrm{KTZ}, \mathrm{W}$

[Sallivantia hapemanii (3. M. Coult, \& Fish.) J. M. Coult.] - SFW

[Sallivantia hapemanii (J. M. Coulr. \& Fish.] J. M. Coult var. parpusii (Brandegee) Soltis] - W. W

[Sullivantia purpusi (Brandegee) Roscodahi] - HDH

Telesonix (sec Boykinia)

Serophulariaceae - SCR (sce also ORO, PHR, PTG)

(Identification sources:

Scrophularia lanecolata Pursh - Dorn01, GPF, HDH, IMF, KTZ, SF!, UTF, W\&W, E/W

*Verbascum blattaria L. - GPF, HDH, IMF, KTZ, SFE, UTF, W\&W, E

*Verbascum phlomoides L. - HDH, exp., KTZ, SFE, W\&W, E

*Verbascum X pterocaulon Franch. - KTZ, SFE, E

[W] - always sterile]

[Verbascum blattaria $\mathrm{X}$ V. thapsers] - KTZ

[Verbascum pterocaulon Franch.] - W\&W

*Verbascum thapsus L. - Dorn01, GPF, HDH, IMF, KTZ. SF!. UTF. W\&W. E/W

[W] - seems to also hybridize with $\mathrm{V}$. phlomoides but no specimens in berbaria]

\section{Simaroubaceac - SMR}

*Ailanthus altissima (Mill.) Swingle - GPF, IMF, KTZ, SF!, UTF, W\&W, E/W 


\section{Smilacaceae - SML}

Smilax herbacea L. var. lasioneuron (Hook.) A. DC. - Dorn01, HDH

[Coprosmanthus lasionueron (Hook.) Kunth] - FNA

[Nemexia herbacea (L.) Sm. subsp. melica A. Nels.] - FNA

[Nemexia lasioneura (Hook.) Rydb.] - FNA

[Nemexia temuis (Sm.) Sm.] - FNA

[Smilax diversifolia Sm.] - FNA

[Smilax berbaced L. var indora M. E. Jones] - FNA

[Smilax herbaces L. var. lasioneuron (Small) Rydb.] - GPF

[Smilax berbsosed L. subsp. lasioneura (Hook.) A. Lōve \& D. Löve] - FNA

[5milax lasioneura Hook.] - FNA, KTZ, SFE, W\& W, E

[Smilax tenuis Sm.] - FNA

\section{Solanaceae $=$ SOI}

(Identification sources:

$\leftarrow$ Calibrachoa parviflora (Juss,) D $y$ Arcy - KTZ $<A Z, K S, N M>$

Chamaesaracha conioides (Moric.) Britton - HDH, SFE, W\&W, E

[Chamsesaracha conioides (Moric. ex Durtal) Briton] - GPF, KTZ

Chamaesaracha coronopus (Dunal) A. Gray - GPF, HDH, IMF, KTZ, SF!, UTF, W\&W, E/W

\{Datura inoxia Mill.\} - GPF, IMF, a Mexican sp., KTZ, W\&W

\{Datura meteloides Dunal\} - $\mathrm{HDH}$

Datura quercifolia H.B.K. - Hazlen \& Hazlert 12$] 02$; Harding Co., NM [GREE]]

↔Datura stramonium L. - GPF, HDH, IMF, KTZ, SFE, UTF, W\&W, E

$\leftarrow$ Datura wrightii Regel - IMF, KTZ, SFW, UTF, W\&W, W

[Datura inoxia Mill.] - GPF

*Hyoscyamus niger L. - Dom01, GPF, HDH, IMF, KTZ, SF!, UTF, W\&W, ENW

*Lycium barbarum L. - Dom01, IMF, KTZ, SF!, UTF, W\&W, E/W

[Lyciam halimifolium Mill.] - GPF, HDH

Lycium pallidum Miers - GPF, HDH, IMF, SF!, UTF, W\&W, E/W

[Lyciam pallidum Miers var, pallidum] - KTZ

*Nicandra physalodes (L.) Pers. - HDH, W\&W, E

[W] - probably a non-persistent waif. Only CO colkctions are Woren [1914, COCO] and flendricks [1946. CS] from Burlington. Warren collection probably from a ganden. Burlington area recently collecled independently by $\mathrm{C}$. Freeman and D. Clarks neither found this taxoul

[Nicandra physalodes (L.) Gaerin] - GPF, KTZ, SFE

Nicotiana attenuata Torr. ex S. Watson - Dorn01, HDH, IMF, K17, UTF, W\& W, E/W

[Nicctiana artenuata Torr.] - SF!

Nicotiana trigonophylla Dunal - M\&H, KTZ, HDH, exp.. GPF, UTF, W\& W misid.

[Nicctianta obcusifolia Mert, \& Galootti var, obcusifolia] - KTZ

[Nicctiana trigonophylla Dunal var, trigonophylla] - IME

\section{Physalis (see also Quincula)}


(Identification sources:

(Physalis cinerascens (Dunal) Hitche, var, cinerascens\} - KTZ.

(WJ - to be expected in Baca Co.. CO, but no specimens confimed; NS - nearest specimens in NM fide MaEH are SE of our area?

\{Physalis viscosa L. subsp. mollis (Nutt.) Waterf, var. cinerescens (Dunal) Waterf.\} - GPF

Physalis hederifolia A. Gray var, comata (Rydb.) Waterf. - Dom01, GPF, KTZ, SFE, W\&W. E)

[W] - should be recagnized at spccies lovel: has shert, dense, very glandular hairs; native to NE, KS, and CO] [Physalis hederaefolia A. Gray] - HDH, SFW

Physalis hederifolia A. Gray var. fendleri (A. Gray) Cronquist - IMF, KTZ. UTF, E

[W] - differs froen var. comala by different habit, leat shape, and pubescence; bigher elevations in wooded siluations (pinyons and ponderosa pittes)!

[Physaria fendleri A. Gray] - HDH

[Physalis hederifolia A. Gray var. cordifolia (A. Gray) Waterf.]-GPF, SFE, W\&W

Physalis heterophylla Nees var. heterophylla - KTZ, E

[Plyysalis heterophylla Nees] - Dern01, GPF, HDH, IMF, SFE, UTF, W\&W

Physalis hispida (Waterf,) Cronquist $=$ Dorn01, [MF, KTZ, E

[Physalis pumila Nutt] - HDH, rep.

[Physalis pumila Nutt, subsp. hispida (Waterf.) Hinee] - GPF, SFE, W\& W

Physalis longifolia Nutt. - Dom01, HDH, UTF

[Physalis longifolia Nutr. var. longifolia] - GPF, IMF, KTZ, E/W

[Physalis longifolia Nutt. vart. subgiabrata (Mack. \& Bush) Cronequist - KTZ

[Physalis subglabrana Mack. \& Bush] - HDH

[Physalis virginiana Mill.] - SF!, W\&W

$\leftarrow$ Physalis pruinosa L.\} - HDH rep., W\&W

[W] - no specimens sonfinmed yet for $\mathrm{CO}$ at COLO, CS, GREEE or KHD; bot reported from NM by M\&H]

[Physalis pubescens L. var. integrifolia (Dunal) Waterf.] - KTZ

[Physalis pubescens L.] - GPF, HDH, IMF, W\&W ert. nep.

Physalis subulata Rydb, var, neomexicana (Rydb.) Waterf, ex Kartesz \& Gandhi - KTZ, E

[W] - bo confirmed speicmens yet from CO based on COLO, CS, and KHD. Type local ity of this variery from our area in northem NM]

[Physalis foctens Poir. var. neomexicana (Ryob.) Waterf.] - SFE, W\&W

Physalis virginiana Mill, - GPF, IMF, SF!, W\&W, EJW

[Physalis lanceolata Michaux] - HDH

[Physalis virginiana Mill, var. virginiana] - KTZ

[Physalis virginiana Mill. var. polyphylla (Greene) Waserf.] - KTZ

Quincula lobata (Torr.) Raf. - GPF, KTZ, SFE, W\&W, E

[Physalis lobuta Torr.] - HDH, UTF

Solanum

(Identification sources:

Solanum americanum Mill. - KTZ, SF!, W\&W, E/W

[Solanam interius Rydb.]-GPF, KTZ

[Solanum nigrum Li] - IMF, UTF

[Solanum nigrum L. var, virginicum L.] - IMF

ษ Solanum carolinense L. - GPF, HDH, exp., IMF, SEE, UTF, W\&W, E

[Solanum carolinense L. var, carolinense] - KTZ 
*Solanum dulcamara L. - Dorn01, GPF, HDH, exp, IMF, SF!, UTF, W\&W, E/W

[Solanim dukamara L. var. dulcamara] - KTZ

*Solanum elaeagnifolium Cav, - GPF, HDH, IMF, KTZ, SF!. UTF, W\&W, E/W

Solanum fendleri A. Gray ex Torr. var, fendleri - KTZ

[W] - No CO specimens a COLO, CS, GREE. KHD, although type specimen from our area in northern NM]

[Solanum fendleri A. Gray]-HDH, W\&W

†Solanum heterodoxum Dunal - HDH, SFE, W\&W, E

[Solanum hetersdoxum Dunal var. setigeroides Whalen] - GPF. KTZ?

Solanum jamesii Tort - HDH, IMF, KTZ, SF!, UTF, W\&W, E/W

Solanum nigrum L. - Dorn01, HDH, KTZ, UTF, W\&W, misapp.

[Solanum ptycanthum Dunal ex DC.] - GPF

[Solanum ptycanthum Dunal] - KTZ

$\div$ Solanum physalifolium Rusby var, nitidibaccatum (Bitter) Edmonds - Dorn01, SF!, W\&W, E/W

[Solanum physalifolium Rusby] - KTZ

[Solanam sarrachoides Sendin. ex Mart.] - HDH, IMF, UTF

[Solanum sarrachoides Sendin.] - GPF

*Solanum rostratum Dunal - Dom01, GPF, HDH, IMF, KTZ, SF!, UTF, W\&W, ENW

Solanum triflorum Nutt. - Dorn01, GPF, HDH, IMF, KTZ, SF!, UTF, W\&W, E/W

Sparganiaceae - (see TYP)

\section{Tamaricaceae - TAM}

(Identification sources:

[NS - any keal taxonomic etanges should oonsult recent studies by Gaskin et al.]

*Tamarix chinensis Lour, - Dorn01, GPF, KTZ, UTF

[JG- lodistinguishable from $T$. ramossisma on morphological grounds]

* Tamarix gallica L.\}-HDH, KTZ $\langle\mathrm{NM}>$, W\&W, err, rep.

*Tamarix parviflora DC. - GPF, KTZ, SF!, UTF, W\&W, E/W

\{Tamarix pentandra Pallas\}

$\{1 \mathrm{G}$ - an illegitimate nane:

*Tamarix ramosissima Ledeb, - GPF, KTZ, SF!, W\&W, ENW

[Tamarix chinensis Louf.] - UTF

[JG: Ses above re: T, shinensis]

\section{Themidaceae - THF}

Androstephium breviflorum S. Watson - Dom01, FNA, FCF, HDH, IMF, KTZ, SFW, W\&W, W [Bessera breviflora (S. Watson) Jepson] - FNA

[Brodiaca paysonii A. Nels.] - FNA

[Brodiaca breviflora (S. Watson) J. F, MaeBride]

Triteleia grandiflora Lindl. var. grandiflora - [MF, KTZ

[Triteleia grasdiflora Lindl.] - Dorn01, UTF, W\&W

\section{Theophrastaceae - TEO}


\{Samolus floridanus Kunth\} - HDH, exp.

INS - likely to be found in northern NM or $\leq \mathrm{CO}$

[Samolus parviflorus Raf.) - GPF, UTF

[Samolus valerandi L. subsp. parviflorus (Raf.) Helten'] - KTZ CAZ, KS, NM, OK, UT>

\section{Tiliacene- TIL}

*Tilia americana - WY checklist

[NS - many synonyms depending on infraspecific taxon, e.g. see KTZ]

[W] - present by virtue of ornamental plantings]

\section{Trilliaceae (see MLN and LIL)}

\section{Typhaceac - TYP (including Sparganiaceae)}

(Identification sources:

Sparganium angustifolium Michx. - Dorn01, FNA, GPF, HDH, IMF, KTZ, SF!, W\&W, EW

Sparganium emersum Rehmann - Dom01, FNA, SF!, UTF, W\&W, E/W

[Sparganiam angustifolium Michx.] - KTZ

[Spsrganiam emersum Rehmann var. multipedunculata (Morogg) Reveal] - GPF, IMF

[Sparganium multipedunculata (Morong) Rydb.] - HDH

Sparganium eurycarpum Engelm. - Dorn01, FNA, GPF, HDH, IMF, SFE, UTF, E

[Sparganium eurycarpum Engelm, ex A. Gray] - KTZ, W\&W

Sparganium natans L. - Dorn01, FNA, KTZ, E/W

[Sparganiam minimum $\mathrm{Fr}$ ] - HDH, IMF, SFW, UTF

[Sparganium minimum Wallt.] - WE W

[Sparganium minimum (Harimain) Fr.] - SFE

[Sparganiam minimum (Hartntan) Wallr.] - KTZ

Typha angustifolia L. - Dorn01, FNA, GPF, HDH, IMF, KTZ, SF!, W\&W, E/W

Typha domingensis Pers. - FNA, GPF, IMF, KTZ, SF!, UTF, W\&W, E/W

Typha $X$ glauca Godr. - FNA, GPF, IMF, KTZ $<U T$, WY> UTF

[Typha angastifolia XT, Latifolia] - FNA

Typha latifolia L. - Dorn01, FNA, GPF, HDH, IMF, KTZ, SF!, UTF, W\&W, E/W

\section{Ulmaceae - ULM (sec also CAN)}

4 \{Ulmus americana L.\} - GPF,HDH exp.,KTZ, ,RMC,UTF,W\&W cult.

\{WJ - not escaping from cultivation\}

*Ulmus pumila L. - Dorn01, FNA, GPF, HDH, KTZ, SF!, UTF, W\& W, E/W

\section{Umbelliferae (see API)}

\section{Urticaceae - URT}

(Identification sources:

Bochmeria eylindrica (L.) Swartz - FNA

[NS - entering our ares in n.e. NM] 
\{Parietaria floridana Nutt.\} - HDH, KTZ, W\&W err, rep.

Parietaria pensylvanica Muhl, ex Willd. - Dorn01, FNA, HDH, KTZ, UTF, W\&W, EN

[Parictaria pensylvanica MuhL] - GPF, SF!

\{Urtica dioica L. var dioica\} - HDH, W\&W, Eurasian

\{Urtica dioica L.\} - W\& $W^{3}$

IUrtica dioica L subsp. disica\} - GPF, KTZ $<0 \mathrm{OK}$ -

Urtica dioica L, var. occidentalis S, Watson - Dorn01. UTF, W

[Urtica divica L. subsp. holoseriosa (Nult.) Thome] - KTZ

[Urtica gracilis Aiton subsp. holosericea (Nutt.) W. A. Weber] - FNA. SFW, W\&W

Urtica dioica L. var. procera (Muhl ex Willd.) Wedd. - Dorn01, E/W

[Unica dioica L. subsp. graxil is (Aiton) Selander] - FNA, GPF, KTZ

[Urica dioica L. var. procera (Muh1.) Wedd.] - HDH. UTF

[Urtica gracilenta Greene] - SFW, HDH. W\&W'

[Urtica gracilis A iton]-SF!

[Urtica gracilis A iton subsp. gracilis] - W\&W

Urtica gracilenta Greene - FNA

\section{Uvulariaceae (see CHC)}

\section{Valerianacene - VAI}

Valeriana acutiloba Rydb, var, acutiloba - Dorn01, IMF, KTZ, EW

[Valeriana seutiloba Rydb.] - GPF, UTF

[Valerianta capitata Pall. subso, actutiloba (Rydb.) F. G. Mey.] - SF!

[Valeriana capitata Pall. ex Link subsp. acuniloba (Rydb.) F. G. Mey.] - HDH, W\&W

Valeriana acutiloba Rydb, var, pubicarpa (Rydb.) Cronquist - Dom01. IMF, KTZ, E/W

[W] - likely does not occur in Colorado]

[Valeriana scuniboba Rydb] - UTF

[Valeriana capitata Pall. subsp. acutiloba (Rydb.) F. G. Mey.] - SF]

[Valeriana capitata Pall. ex Link subsp. acuiloba (Rydb.) F. G. Mey.]-HDH, WQ W

Valeriana arizonica A. Gray - HDH, IMF, KTZ, SFE, UTF, W\&W, E

Valeriana dioica L. var, sylvatica (Sol. ex Richardson) S. Watson - Dorn01, KTZ

[Valeriana dioica L.] - GPF

[Valeriana dioicn L. subsp sylvatica (Sol, ex Richandson) F, G. Mey.] - W\&W, W\&W'

[Valeriana dioiea L. var, sylvarica S. Watson]-KTZ $<W Y>$ RMC

Valeriana edulis Nutt. ex Torr. \& A. Gray var. edulis - IMF, KTZ, E/W

[Valeriana cdulis Nutt.] - Dorn0I, GPF, SF!, W\&W

[Valeriana edulis Nutt, subsp. edulis] - HDH

[Valeriana edulis Nutt, ex Torr, \& A. Gray]-UTF

Valeriana occidentalis A. Heller - Dorn01, GPF. HDH, IMF. KTZ. SF!. UTF. W\&W. E/W

\section{Verbenaceae - VRB}

(Identification sources:

*Caryopteris clandonensis A. Simmonds ex Rehder - W\&W

[Caryopteris incana X C. mongholica] - KTZ

[Caryopteris X clandonensis hort, ex kehder] - KTZ 
Glandularia bipinnatifida (Nutt.) Nutt, var, bipinnatifida - KTZ, E/w

[Glandularia bipinnatifida (Nutt.) Nutt]. - Dom01, SF!, W\&W

[Verbena ambrosifolia Rydb.] - HDH

[Verbena bipinnatifida Nutt.] - GPF, HDH

[Verbena ciliata Benth.] - HDH

Glandularia bipinnatifida (Nutt.) Nutt. var. ciliata (Benth.) B. L. Tumer - KWA. HDH, KTZ [Glandularia wrightii (A. Gray) Umber] - KWA. W\&W

[Verbena wrightii $\mathrm{A}$. Giray] - HDH

\{Glandularia canadensis (L.) Smal]\} - W\&W

\{Giandularia canadensis (L.) Nutt.\} - KTZ

\{Verbena cansadensis (L.) Britton\}-HDH, GPF

\{Glandularia gooddingii (Briq.) Solbrig\} - KTZ, W\&W

\{Verbena gooddingii Bric.\} - HDH rep, IMF, UTF

Lippia (see P'hyla)

Phyla cuneifolia (Torr.) Greene - Dorn01, HDH, IMF, KTZ, SFE, UTF, W\&W, E

[Lippia ceneifolia (Torr.) Steud.] - GPF

Phyla lanceolata (Michx, Greene - HDH rep., IMF, KTZ, SFE, UTF, W\&W, E

[Lippia lanceolata (Michx_. Greene] - GPF

\{Phyla nodiflora (L.) Greene\} - IMF, KTZ <AZ, KS, NM, OK, UT>, UTF, W\&W

\{Lippia nodiflora (L.) Michx.\} - GPF

Verbena (see Glandularia)

\%Verbena bracteata Lag. \& Rodr, - Dom0I, GPF, HDH, IMF, KTZ,SF!, UTF, W\&W, E/W

\{Verbena X crookshankii Moldenke\} - KTZ, Moldenke80

\{Verbena bracheata X V. hastata\} - KTZ

Verbena hastata L. - Dom01, GPF, HDH, IMF, SFE, UTF, W\&W, E

[Verbena hastata L, var, scabra Moldenke] - KTZ

Verbena macdougalii A. Heller - HDH, IMF, KTZ, SF!, UTF, W\&W, E/W

Verbena officinalis L, var, officinalis - KTZ

Verbena plicata Greene - GPF, KTZ, SFE, W\&W, E

Verbena stricta Vent. - Dom01, GPF, HDH, IMF, KTZ, SFE, W\&W, E

Viburnaceae - VIB

\{Viburnum acerifolium L.\} - KTZ, WY checklist

(Viburnam acerifolium var, densiflovum (Clusman) MeAtec) - KTZ

(Viburmum acsifolium var, glabescens Rehd.) - KTZ

\{Viburnam acerifolium var, ovatum (Rehd.) MeAtee; - KTZ

[Viburnam desiflorum Chapmisa] - KTZ

Viburnum edule (Michx.) Raf, - Dorn01, GPF, KTZ, SF!, W\&W, E/W

[Viburnum parciflorum Pylaic ex Torr. \& A. Gray] - HDH, WY checklist

* Viburnum lantana L. - KTZ, SFE, W\& W, E, WY checklist

[Viburnum lantana var, sphaerocarpum Gray ex Fern.] - KT2

4 Viburnum lentago L. - GPF, HDH, KTZ, SFE, UTF, W\&W, E

[Viburnum $\times$ vteri Zabel] - K.TZ

\section{Violaceae - VIO}

(Identification sources: 
Hybanthus verticillatus (Ortega) Baill. - GPF, HDH, KTZ, SFE, W\&W, E

Viola

[W] \& NS * the genus is badly in noed of revision in North America using well-articulated species criteria. The reworking of accepled taxa shuuld be in context \{minimally\} of all North American material. See also Kelso03 and Mickinney 92 ]

Viola adunca J. E. Sm. - Dorn01, SF!, W\&W, E/W

[Viola adunca J. E. Sm. ex Rees]-UTT

[Viola adunca J. E. Sm. sabsp. adunca] - HDH

[Viola adunca J. E. Sm. sabsp. ashtonac M. S. Baker] - HDH

[Viola adunca J. E. Sm. sabsp. nadicasa M. S. Baker] - HDH

[Viola adunca J. E. Sm. var. adunca] - GPF, KTZ

Viola affinis Leconte $-\mathrm{KTZ}, \mathrm{E}$

[Viola pratincola Greene] - GPF

[Viola sororia Willd. subsp. affinis (Lecone) McKinney] - SFE

[Viola sororia Willd. var, aftinis (Leconte) McKinney]-Dorn01. W\& w'

\%Viola bicolor Pursh = KTZ, E

[Viola kitaibeliana Roem. \& Schulh. var. rafinesquei 〈Greene〉 Fernald] - SFE, W\& W

[Viola rafinesquii Greene] - GPF, HDH

Viola biflora L. - HDH, SF!, W\&W, E/W

[Viola billora L. subsp. biflora] - KTZ

Viola biternata Greene

[W] - seo comments ander V. sheltonii]

Viola canadensis L. - Dorn01, UTF, EJW

[Viola canaderisis L. var. canadensis] - $\mathrm{HDH}$

[Viola canaderisis L. var. rugulosa (Greene) C. L. Hiche.] - GPF, KTZ

[Viola canadensis L. var, scopulorum A. Gray]- HDH, K.TZ

[Viola rugulosa Geene] - $\mathrm{HDH}$, W\&W

[Viola rydbergi Greene] - SFL, w\&W

[Viola scopulorum (A. Gray) Greene] - SF!, W\&W

Viola labradorica Schrank - KTZ, SF!, W\&W, E/W

[W] - probably too many intermediates with V. adunca to recognize at the specific level. Small plants typical

of higher elevations also extend well dow into montane and the big plants extend irso the subalpioe; sev HDH]

[Viola aduncen 1. E. Sm, var, bellidifolia (Greene) Harrington] - HDH

Viola macloskeyi Lloyd var. pallens (Banks ex DC.) C. L. Hitchc. - E/W

[Viola palustris L.] - WJ (pers. comm. 2004, see comments under V. palustris)

[Viola macloskeyi Lloyd var, macloskeyi] - GPF

[Viola macloskeyi Lloyd subsp. pallens (DC.) C. L. Hitche.] - SF]

[Viola macloskeyi Lloyd subsp, pallens (Banks ex DC.) M. S. Baker] - KTZ, W \& W

Viola missouriensis Greene - KTZ, M\&H

[Viola sororia var. missouriensis (Greene) MeKinney] - KWA, KTZ

Viola nephrophylla Greene - GPF. KTZ, UTF, E/W

[Viola sororia Willd.] - SFW

[Viola sororia Willd, subsp, affinis (Leconte) McKinney] - SFE

Viola nuttallii Pursh - Dom01, GPF, WJ, KTZ, SF!, W\&W, E/W

[W] - shoeld include also V. praemersa and V, vallicola. Only one taxon present in CO and oldest name is V. nuctallii. One can find leaves of all three "species" in one population, often on same plant. Type locality of V. pracmorsa is on Pacific coast. V. nuttallii leaves are long and namow, whereas V. pracmorsa are wider. There is a tendency for leaves to widen as one goes west from the Great Plains but where nuttallii ends and praemorsa begins is an unsolvable question. V. vallieola is supposed to have at least one leaf that is somewhat cordote to truncate at the base (fide M. Baker monograph). It is casy to flind such lenves early in the season and they lengthen as the plant matures. I have photographed plants in a narural area near my house and all three "species" are present in that population] 
[Viola nuttallii Pursh var, nuttallii] - HDH

*Viola odorata L. - KTZ, SFE, UTF, W\&W, E

Viola palustris L. - WY checklist, GPF, UTF, W\&W, misid.

[W] - No ecosistent differences between V. palulstris, V. renifolia and V. macloskeyi. The flowers of esch are the same. It is easy to find specimens in a "population" with reniform leaves and an apex on the leaf. Probably none have true rhizomes; the "stolons" become buried in duff and only resemble thizomes. Tavonomy of group problematic due to excessive splitting by Baker in 1940s]

[Viola palustris L. subsp. brevipes M. S. Baker] - HDH

[Viola palustris L. var. brevipes (M. S. Baker) R. J. Davis] - KTZ

[Viola palustris L. var. palustris]. $\mathrm{HDH}$

\{Viola pedata L.) - GPF, HDH labels possibly mixed, KTZ $<\mathrm{KS}, \mathrm{NE}, \mathrm{OK}>$, W\&W

Viola pedatifida G, Don - GPF, HDH, KTZ, SF!, W\&W, E/W

Viola praemorsa Douglas ex Lindl, var, altior - Dorn01

[Viola nuttallii Pursh] - WJ (see comments under V. nuttallii)

[Viola nuttallii Pursh var. linguifolia (Nutt.) Jeps.] - HDH

[Viola nuttallii Pursh var. major Hook.] - UTF

[Viola praemorsa Douglas ex Linutl, subsp. linguifolia (Nutt.) M. S. Baker \& Clausen] - W\&W

[Viola praemorsa Douglas ex Lindl. sabsp. linguifolia (Nutn.) M. S. Baker \& Clausen ex Pock] - KTZ

Viola pratincola Greene - GPF

[Viola nephrophylla Greene] - KTZ

[Viola retusa Greene] - $\mathrm{HDH}$

[Viola sororia Willd. var. sororia] - W\&W

Viola purpurea Kellogg var, venosa (S. Watson) Brainerd - Dorn01, HDH, UTF, EJW

[Viola purpurea Kellogg subsp. venossi (S. Walson) M. S. Baker \& J. C. Clasuen] - KTZ, SF1, W\&W

Viola renifolia A, Gray var, brainerdii (Greene) Fernald - Dorn01, HDH, SFE, W\&W, E/W

[Viola palussris L] - WJ (pers. comm. 2004; see comments under V, palustris)

[Viola renifolia A. Gray]-GPF, KTZ, SFW

Viola selkirkii Pursh ex Goldie - HDH, KTZ, W\&W, E

[Viola selkirkii Pursh] - GPF. SFE

\{Viola sheltonii Torr.\} - HDH, KTZ, W\&W, W\&W', W

[W] - A west coastPaciffe NW species that does not appear to ocsur in the laternountain region. Putative specimens from $\mathrm{CO}$ confincd to Grand Mesa highly disjunct from main range. Unil better studied, should use

V. biternaka as sugecsted by WeW

[Viola biternata Greme] - SFW, W\& W'

Viola sororia Willd. - GPF, KTZ $<$ KS, NE, OK>, SFW, W

[NS - for further discussion of this complex see Kelson 03 and MeKinney92]

[Viola nephrophylla Geeene] - HDH

[Violo papilionacea Pursh]- HDH

[Viola retusa Greene] - HDH

[Viola sororia Willd var sororia] - W\&W

Viola tricolor L. - Dorn0)

Viola utahensis M. S. Baker \& Clausen ex M. S. Baker - Dom01, SFW, W\&W. W

[Viola purpurea Ketloge] - UTF

[Viola ucabensis M. S. Baher \& Clausen ex M. S. Baker] - KTZ

Viola vallicola A. Nelson var. majoe (Hook.) Fabijan - KTZ, E/W

[Viola nuttallii Pursh] - WJ (see comments under V, nuttallii)

[Viola nuitalli Pursh var, major Hook.] - UTF

[Viola praenorsa Douglas subsp. major (Hook.) M. S. Baber] - SFI, W \& W

[Viola praemorsa Douglas ex Lindley subsp, major (Hook.) M. S. Baker] - W\&W

Viola vallicola $\mathrm{A}$. Nelson var, vallicola - KTZ, E/W

[Viola nuttatlii Pursh] - GPF

[Viola nuttalli Pursh var, vallicola (A. Nelson) H. St, John] - HDH, UTF

[Viola vallicola A. Nelson] - SF!, W\&W 


\section{Visenceae - VIS}

Arceuthobium

(Identification sources:

Arceuthobium americanum Nutt. ex Engelm. - Dorn01, HDH, IMF, KTZ, UTF, W\&W, E/W [Arecuthobium americanum Nutt] - SF!

\{Arceuthobium campylopodum Engelm.\} - IMF, KTZ, W\&W

(Arceuthobium campylopodum Engelm. forma campylopedun] - HDH, possibly

Arceuthobium cyanocarpum (A. Nelson ex Rydb.) A. Nelson - Dorn01, IMF, KTZ, E/W

[Arseuthobium campylopodum Engelm.] - KTZ

[Arceuthobium campylopodum Engelm. forma abietinum (Engelm.) Gill] - HDH, possibly

[Arsecuthobium campylopodum Engelm. forma cysnocarpum (A. Nebon) Gill] - HDH

[Arceuthobium campylopodum Engelm. forma microcarpum (Engelm.) Gill] - HDH, possibly

[Arccuthobium cyanocarpum J.M. Coult. \& A. Nelson] - SF!, UTF, W\&.W

Arceuthobium divaricatum Engelm. - IMF, KTZ, SF!, UTF, W\&W, E/W

[Arceuthobium campylopodum Engelm. forma divaricatum (Engelm.) Gill] - HDH

Arceuthobium douglasii Engelm. - Dom01, HDH, IMF, KTZ, SF!. UTF, W\&W, E/W

Arceuthobium vaginatum (Willd.) J. Presl var, cryptopodium (Engelm.) Cronquist - IMF, E/W

[Arceuthobium vaginarum (Willd.) J. Pres]) - $\mathrm{HDH}$

[Arceultobium vaginatum (Willd.) J. Pres] subsp. cryplopodium (Engelm.) Hawksworth \& Wiens] - KTZ. UTF

[Arecuthobium vaginatum (Willd.) C. Presl subsp. sryptopodium (Engelm.) Hawksworth \& Wiens] - SFI, WeW

(Arceuthobium vaginatum (Willd.) C. Presl var. vaginatum\} - IMF

\{Arceuthobium vaginatum (Willd.) C. Preal\} - $\mathrm{HDH}$

\{Arceuthobium vaginatum (Willd.) C. Presl subsp vaginatum\} - W'EW

\{Phoradendron cockerellii Trelease\} - HDH, questionable, W\&W, mixed labels

\{Phoraderdron macrophyllum (Engelm.) Cockerell subsp. cockerellii (Trelease) Wicrsi - KTZ

Phoradendron juniperinum Engelm. - HDH, SFW, W\&W, W

[Phoradendron jusiperinum Engelm. ex A. Gray] - IMF. KTZ

[Phoradendron jusiperinum A. Gray subsp. jusiperinum] - UTF

\section{Vitaceae - VTT}

\section{Ampelopsis (see Parthenocissus)}

* Parthenocissus quinquefolia (L) Planch.\} - GPF, HDH, IMF, KTZ, SF!, UTF, W\&W cult., W\& $W^{\prime}, E / W$

[W]- doubtfully occurs outside of cultivarion]

\{Parthenocissus inserta (Kerner) Fritsch?

Parthenocissus vitacea (Knerr) Hitche. - Dom01, GPF, HDH, IMF, KTZ, SF!, W\& W', E/W

[Parthenocissus inserta (Kerner) Fritseh] - W\&W

Vitis acerifolia Raf. - GPF, KTZ, SFE, W\&W ${ }^{1}$, E

[Vitis longii Prince] - HDH

[Vitis riparia Michx, subsp. loegii (Prince) D. Rogers, in ed] - W\&W

\{Vitis arizonica Engelm.\} - HDH exp., IMF, KTZ <AZ, NM, UT>, UTF, W\& W

\{W] - doubtful for Colorado\}

$\{$ Vitis X doaniana Munson ex Viala\} - KTZ $<\mathrm{NM}, \mathrm{OK}>$ 
[Vitis acerifolia X V: mustangensis\} - KTZ

\{Vitis doaniana Munson ex Vialat - HDH, exp, W\&W

Vitis riparia Michx. - Dorn01, KTZ, SFE, E

[Vitis riparia Michx. subsp. riparia] - W\&W

[Vitis riparia Michx. var. riparia] - GPF

[Vitis vulpina L.] - HDH, W\& $W^{\prime}$

\section{Xanthorrhbocacene - XAN}

*Hemerocallis fulva (L.) L. - Dorn01, KTZ <KS, NE, UT>, UTF

[Hemerocallis fulva L.] - GPE -KS 5 , HDH, corr. \& add.

\section{Zannichelliaceac (see POT)}

\section{Zygophyllaceae - ZYG (including Krameriacene)}

Kallstroemia califomica (S. Watson) Vail - KWA, IMF, KTZ, UTF

[Kallstroemia brachystylis Vail] - KWA, KTZ, HDH, rep.

[Kallstroemia califomica (S. Watson) Vail var. brachystylis (Vail) Kearney \& Peebles] - KTZ, M\& IL, KWA.WEW, err. rep.

Kallstroemia hirsutissima Vail - M\&H, GPF, HDH, W\&W, err. rep.

[Kallstroemia hirsutissima Vail ex Small] - KTZ

Kallstroemia parviflora Norton - GPF, IMF, KTZ, SFE, UTF, W\&W, E

[Kallstrocmia maxima (L.) Hook. \& Ari.] - KWA. M\&.H

[Kallstromia intermedia Rydb.] - M\&H

\{Larrea divaricata Cav, - $\mathrm{HDH}$, exp.

\{Larrea divaricata (DC.) Coville) - UTF

\{Larres tridentata DC. - W\& W, err. fep.

\{Larrea tridentata (Sessé \& Moç. ex DC.) Coville\} - IMF

\{Larrea tridentata (Sesse \& Moç, ex DC, Coville var, tridencata) - KTZ $<$ AZ, NM. UT?

*Tribulus terrestris L. - Dom01, GPF, HDH, IMF, KTZ, SF!, UTF, W\&W, E/W

\{Zygophyllum fabago L.; - HDH, IMF, KTZ $<$ KS, NM>, W\& W 


\section{LITERATURE CITED}

Ackerfield08: J. Ackerfield, W. F, Jennings. 2008. The genus Abronia (Nyctaginaceas) in Colorado, with notes on Ahronia bolackit in New Mexico. J. Bot. Inst. Texas 2: $419-423$.

Aiken95: S. G. Aiken, L. L. Consaul. 1995. Leaf cross sections and phytogeography: A potent combination for identifying members of Festuca subge. Fesfuca and Lezcopora (Poaceae), occurring in North America. Amer. J. Bot. 82: 1287-1299.

Al-Shebbaz07a: I. A. Al-Shehbaz. 2007. Descurainia kenheilit (Brassicaceae), a new species from the San Juan Mountains, Colorado. Harvard Papers in Botany 12:395-396.

Al-Shehbaz07b: I. A. Al-Shehbaz, M. D. Windham. 2007. New or noteworthy North American Draba (Brassicaceae). Harvard Papers in Botany $12 ; 409-419$.

Al-Shebbaz03: I. A. Al-Shehbaz. 2003. Transfer of most North American species of Arabis to Boechera (Brassicaceae). Novon 13; $381-391$.

Al-Shehbaz02: I. A. Al-Shebbaz, S. L, OKane, Jr. 2002. Lesquerella is united with Physaria. Novon 12:319-329.

Anderson96: J. L. Anderson, J. M. Porter, M. K. Debacon. 1996. Is Hymenoms helenioides (Rydb,) Ckll a species or a hybrid? Morphological and molecular evidence. IN: J.

Maschinski, H. David, \& L. Holter (eds.) Southwestem Rare and Endangered Plants: Proceedings of the Second Conference. USDA; Forest Service, General Technical Report RMC-GTR-283, pp. 113-124.

Anderson97; J. L. Anderson, J, L. Reveal, R. C. Rollins. 1997. Lesquerella vicina (Brassicaceae), a new species from the Uncompahgre River Valley in western Colorado. Novon $7: 9-12$.

APG II: Angiosperm Phylogeny Groupll (APG II). 2003. An update of the Angiosperm Phylogeny Group classification for the orders and families of flowering plants: APG II. Bot. J. Linn. Soc. 141:399-436.

Argus07; G. W. Argus. 2007. Salix (Salicaceae) distribution maps and a synopsis of their classification in North America, north of Mexico. Harvard Papers in Botany 12: 335-368.

Bain88: J. F. Bain. 1988. Taxonomy of Senecio streptanthtfolius Greene, Rhodora 90:277-312. Barkley99: T. M. Barkley. 1999. The segregates of Seriecio, s.1, and Cacalia, s.1., in the flora of North America North of Mexico. Sida 18: 661-672.

Barkworth08: M. E. Barkworth, M. O. Arriaga, J. F. Smith, S. W. L. Jacobs, J. Valdes-Reyna, B. S. Bushman. 2008. Molecules and morphology in South American Stipeae (Poaceae). Syst. Bot. 33: 719-731.

Beatty04: B. Beatty, W. Jennings, R. Rawlinson. 2004. Crataegus saligna Greene (willow hawthorn): A technical conservation assessment. USDA Forest Service, Rocky Mountain Region. http//www. fs. fed.us/2/projects/scp/assessments/crataegussaligna.pdf.

Bodo Slotta01: T. A. Bodo Slotta. 2001. Phylogenetic analysis of Iliamna (Malvaceac) using the Intemal Transcribed Spacer Region. M.S. Thesis, Virginia Polytechnic Institute and State University. Blacksburg, VA.

Brasher06: J. W. Brasher. 2006. The Southern Rocky Mountain Interactive Flora (SRMIF) and factors correlated with recognition of plants and mammals. Ph.D. Dissertation, University of Northern Colorado, Greeley.

Brasher04: J. W. Brasher, N. Snow. 2004. Further updates to Weber's three-letter family acronym system. Taxon $53(1): 1-3$ (online version). 
Chiapella07: J. Chiapella, 2007. A molecular phylogenetic study of Deschampsia (Poaceae: Aveneae) inferred fromn nuclear ITS and plastid $\mathrm{hm} /$ sequence data: support for the recognition of Avene $7 / a$ and Vahlodea. Taxon 56; 55-64.

Cialdella07: A. M. Cialdella, L. M. Giussani, L. Aagesen, F, O. Zuloaga, O. Morrone. 2007. A phylogeny of Pipvochaerium (Poaceae: Pooideae: Stipeae) and related genera based on a combined analysis including $t r n L-F, r p l / 6$, and morphology. Syst. Bot. 32: $545-559$.

Clayton09: W.D. Clayton, N. Snow. 2009. A Key to Pacific Grassex. Royal Botanic Gardens, Kew (in press).

Coleman02: R. A. Coleman. 2002. Wild Orchids of Arizona and New Mexico. Comell University Press, NY.

Columbus99; J. T. Columbus, 1999. An expanded circumscription of Boudelona (Gramineae; Chlorideae): New combinations and names. Aliso 18:61-65.

Columbus98: J. T. Columbus, M. S. Kinney, R. Pant, M. E. Siquieros Delagado, 1998. Cladistic parsimony analysis of internal transcribed spacer region (nrDNA) sequences of Boweloza and its relatives (Gramineae: Chloridoideae). Aliso 17:99-130.

Crow 78: G. E, Crow, 1978. A taxonomic revision of Sagina (Caryophyllaceae) in North America. Rhodora 80; 1-91.

Darbyshire93. S. J, Darbyshire. 1993. Realignment of Festaca subgenus Shedonorus with the genus Loliam, Novon 3: 329-243.

Darlington 34: J. Darlington. 1934. A monograph of the genus Mcntzelia. Ann. Missouri Bot. Gard. 21: 103-226.

Davis92: J. I. Davis, K. C. Nixon. 1992. Populations, genetic variation, and the delimitation of phylogenetic species, Syst. Biol. 41:421-435.

Dorn03: R. D. Dorn. 2003. A new species of Boechera (Brassicaceae) from Utah and Colorado. Brittonia 55: 1-3.

Dorn01: R. D. Dorn. 2001. Vascular Plants of Wyoming. Ed. 3. Mountain West Publ, Cheyenne.

Dorn97: R. D. Dorn. 1997. Rocky Mountain Region willow identification field guide, USDA: Forest Service. Renewable Rescurces R2-RR-97-01. $107 \mathrm{pp}$.

Dorr90: L. J, Dorr. 1990. A revision of the North American genus Callirhoe (Malvaceae). Mem. New York Bot. Gard. 56: 1-74.

Doust07: A. N. Doust, A. M. Penly, S. W. L. Jacobs, E. A. Kellogg. 2007. Congruence, conflict, and polyploidization shown by nuclear and chloroplast markers in the monophyletic "bristle clade" (Paniceae, Panicoideae, Poaceae). Syst. Bot. 32:532-544.

Finot05: V. L. Finot, P. M. Peterson, R. J. Soreng. F. O. Zuloaga. 2005. A revision of Trisetum and Graphephorwm (Poaceae: Pooideae: Aveneae) in North America north of Mexico. Sida 21: 1419-1453.

Fryxell94: P. A. Fryxell. 1994. Malvaceae, Mallow Family; Part one. All genera except Sphaeralcea St.-Hil. J. Arizona-Nevada Acad. Sci. 27: 222-236.

Galloway75: L. A. Galloway. 1975 (1976). Systematics of the North American desert species of Abronia and Tripterocalyx (Nyctaginaceac). Brittonia 27: 328-347.

Goffinet, B., R. W. Buck. 2004. Systematics of the Bryophyta (Mosses): from molecules to a new classification. Monographs in Systematic Botany from the Missouri Bot. Garden 98 : 205-239.

Goodman92: G. J. Goodman, C. A. Lawson. 1992. Two new combinations and a name change from the Long Expedition of 1820. Rhodora 94: 381-382.

Goodman95: G. J. Goodman, C. A. Lawson. 1995. Retracing Major Stephen H. Long's 1820 Expedition. Univ. Oklahoma Press, Norman. 
Goodson07: B. E. Goodson, I. A. Al-Shehbre. 2007. Two new combinations in North American Descurainia (Brassicaceae). Harvard Paprs in Bolany 12; 421-423.

Gould75; F. W. Gould. The Grasses of Texas. Texas A \& M University Press, College Station.

Grady07: B. R, Grady, S. L. O'Kane, Jr, 2007. New species and combinations in Physaria (Brassicaceae) from western North America. Novon 17:182-192.

Grant04. V. Grant. 2004. Taxonomy of the Polemoniaceae Gilia and Lathrocasis. Sida 21:531546.

Harrington54: H. D. Harrington. 1954. Manual of the Plants of Colorado. Sage Press, Denver.

Hartman08: R.L. Hartman, R.K. Raebler. 2008. Minuartia macrantha (Alsinoideae: Caryophyllaceae); Morphological circumscription, geographical range, and phylogenetic affinities. Journal Botanical Insititute of Texas 2: 1225-1231.

Hartman06: R. I. Hartman, B. Reif, B. E. Nelson, B. Jacobs. 2006. New vascular plant records for New Mexico. Sida 22: 1225-1233.

Heklau08: H. Heklau, M. Rõser. 2008. Delineation, taxonomy and phylogenetic relationships of the genus Krascheminnikovia (Amaranthatceae subtribe Axyridinae). Taxon 57: 563-576.

Heywood, V. H., R. K. Brummit, A. Culham, O. Seberg. 2007. Flowering Plant Families of the World. Royal Botanic Gandens, Kew.

Holmgren02: N. H. Holmgren, P. K. Holmgren, 2002. New mentzelias (Loasaceae) from the Intermountain region of the western United States, Syst. Bot. 27: 747-762.

Hufford04: L. Hufford, M. MeMahon. 2004. Morphological evolution and systematies of Symhyris and Besseya (Veronicaceae): A phylogenetic analysis, Syst. Bot. 29: 716-736.

Jennings04: W. Jennings. 2004. The status of Physarla vitulifera in Wyoming. Castilleja 23: 5-6.

Jørgensen06; Jergensen, M. E., R. Elven, A. Tribsch, T. G, Gabrielsen, B. Stedje, C. Brochmann. 2006. Taxonomy and evolutionary relationships in the Sacifraga rividaris complex. Syst. Bot. 31:702-728.

Judd07: W. S. Judd, C. S. Campbell, E. A. Kellogg, P. F. Stevens, M. J. Donoghue. 2007. Plant Systematics: A Phylogenetic Approach. Third Ed. Sinauer Assoc., Inc., Sunderland, MA.

Kellogg85: A. E. Kellogg. 1985. A biosystematic study of the Poa secunda compelx. J. Amold Arbor. $66: 201-242$.

Kelso03: T. Kelso, M. Schuman. 2003. The puzzling purples: Viola selkirkit, Vlola cadunca, and the Vlola saroria complex in Colorado. Aquilegia $27(4): 3-4$.

Kirbkbride06: J. H. Kirkbride, Jr. 2006. The scientific name of narrow-leaf trefoil. Crop Science 46: $2169-2170$.

Kirkpatrick07: R. E. B. Kirkpatrick. 2007. Investigating the monophyly of Pellaea (Pteridaceac) in the context of a phylogenetic analysis of Cheilanthoid ferns. Syst. Bot. 32: 504-518.

Koch04: M. Koch, 1. A. Al-Shehbaz. 2004. Taxonomic and phylogenetic evaluation of the American "ThlopsI" species: Identity and relationship to the Eurasian genus Noccaea (Brassicaccac). Syst. Bot. 29: 375-384.

Lane96, M. R. Lane, R. L. Hartman. 1996. Reclassification of North American Haplopappus (Compositae: Astereac) completed: Rayjacksonia Gen. nov. Amer. J. Bot. 83: 356-370.

Lavin97: M. Lavin, H. Marriott. 1997. Astragaliz molybdemes s.1. (Leguminosae): Higher taxonomic relationships and identity of constituent species. Syst. Bot. 22: 199-217.

Liede96: S. Liede. 1996. Sarcostemina (Asclepiadaceac)- a controversial generic circumscription reconsidered: morphological evidence. Syst. Bot. 21: 31-41.

Lowry84: P. P. Lowry II A. G. Jones. 1984 (1985). Systematics of Osmorhiza Raf. (Apiaceae: Apioideac). Ann. Missouri Bot. Gard. 71: 1128-1171. 
MacKay 72: H. A, MacKay, 1972. Alchemilla minor Huds, (Rosaceae) in New Mexico. Southw. Nat. 17; 197.

Mangaly68: J, K. Mangaly. 1968. A cytotaxonomic study of the herbaceous species of Smilax: Section Coprosmanshus, Rhodora 70:55-82, 247-273.

Mason-Gamer01: R. J. Mason-Gamer. 2001. Origin of North American Elymus (Poaceae: Triticeae) allotetraploids based on granule-bound starch synthase gene sequences. Syst. Bot. 26: 757-768.

Mast07: A. R. Mast, J. L. Reveal. 2007. Transfer of Dodecatheor to Primula (Primulaceae). Brittonia 59: 79-82.

M\&H: W, C. Martin, C. R. Hutchins, 1980, 1981. A Flora of New Mexico, Vols, 1 \& 2. Vaduz, West Germany, J. Cramer.

MicKinney 92; L. E. McKinney, 1992. A taxonomic revision of the acaulescent blue violets (Vola) of North America. SIDA, Botanical Miscellaney 7. Botanical Res. Inst, Texas.

Moldenke80: H. N. Moldenke. 1980. A sixth summary of the Verbenaceae, Avicenniaceae, Stilbaceae, Chloanthaceae, Symphoremaceae, Nyctanthaceae, and Eriocaulaceac of the world as to valid taxa, geographic distribution and synonymy. Phytologia Mem. 2: 1-629,

Morton89; J, K. Morton, R. K. Rabeler, 1989. Biosystematic studies on the Stellaria calycantha (Caryophyllaceae) complex. I. Cytology and Cytogeography, Canad. J. Bot. 67:121-127.

Mosyakin95: S. L. Mosyakin. 1995. New taxa of Corispermum L. (Chenopodiaceae), with preliminary comments on the taxonomy of the genus in North America. Novon 5:340-353.

Mulligan80: G. A. Mulligan. 1980, The genus Cicuta in North America. Canad. J. Bot, 58; 1755 1767.

Nesom89; G. L. Nesom. 1989. The separation of Trimorpha (Compositae: Astereae) from Erigeron. Phytologia 67;61-66.

Nesom 91; G. L. Nesom. 1991. Taxonomy of Jsocoma (Compositae: Astereae). Phytologia 70: 69-114.

Noyes99; R. D. Noyes, L. H. Rieseberg. 1999. ITS sequence data support a single origin for North American Astereae (Asteraceae) and reflect deep geographic divisions in Aster s.I. Amer. J. Bot. 86:398-412.

O'Kane07: S. L. O’Kane, Jr. 2007. Physaria scrotiformls (Brassicaceac), a new high-elevation species from southwestern Colorado and new combinations in Physarla. Novon 17: 376382 .

O'Kane06. S. L. O'Kane, Jr., J. L. Reveal. 2006. Physaria pulvinata (Brassicaceae), a new species from southwestern Colorado. Brittonia 58: 74-77.

O'Kane03: S. L. O'Kane, Jr., I. A. Al-Shehbaz. 2003. Phylogenetic position and generic limits of Arabldopsis (Brassicaceae) based on sequences of nuclear ribosomal DNA. Ann. Missouri Bot. Gard. 90: 603-612.

O'Kane88: S. L. O'Kane, Jr., D. H. Wilken, R. L. Hartman. 1988. Noteworthy collections: Colorado. Madrono 35: 72-74.

Pelser07: P. B. Pelser, B. Nordenstam, J. W. Kadereit, L. E. Watson. 2007. An ITS phylogeny of tribe Senecioneae (Asteraceae) and a new delimitation of Seneclo L. Taxon 56: 1077-1104.

Peterson07: P. M. Peterson. J. Cayouette, Y. S. N. Femandez, B. Coulman 2001. Recognition of Bromus richardsomii and $B$. ciliatus: Evidence from morpholog, cytology, and DNA fingerprinting (Poncese, Bromese). Aliso 2: 21-36.

Phipp599; J. B. Phipps. 1999. Introduction to the red-fruited hawthorns (Crataegecs, Rosaceac) of westem North America. Canad. J. Bot. 76: 1863-1899. 
Porter98: J. M. Porter, 1998. Aliciella, a recircumscribed genus of Polemoniaceae. Aliso 17;23. 46.

Porter00; J. M. Porter, L. A. Johnson. 2000. A phylogenetic classification of Polemoniaceae. Aliso 19: 55-91.

Pryer93: K. M. Pryer, C. H. Haufler. 1993. Isozymic and chromosomal evidence for the allotetraploid origin of Gymnocarpizm dryopicris (Dryopteridaceae). Syst. Bot. 18: 150-172.

Raven72; P. H. Raven, D. P. Gregory. 1972. A revision of the genus Gara (Onagraceat). Mem. Torrey Bot. Club 23: 1-96.

Reveal06: J. L. Reveal, C. R. Broome. 2006. Crypranho gypsophila (Boraginaceas: Boraginoideae), a new species from western Colorado. Brittonia 58: 178-181.

Reveal04: J. Reveal, K. N. Gandhi, D. H. Nicolson. 2004. The demise of the name Astragalus tenellars Pursh (Fabaceae). Taxon 53: 1055-1058.

Rollins93: R. L. Rollins. 1993. The Cruciferae of Continental North America: Systematics of the Mustard Family from the Arctic to Panama. Stanford: Stanford University Press.

Romaschenko08: K. Romaschenko, P. M. Peterson, J. Garcia-Jacas, R. J. Soreng, A. Susanna. 2008. Molecular phylogenetic analysis of the American Stipeae (Poaceae) resolves Jarava sensu lato polyphyletic: evidence for a new genus Pappostipa. J. Bot. Res. Inst. Texas 2: $165-192$.

Saarela01 : J. M. Saarela, B. A. Ford. 2001. Taxonomy of the Carex backif complex (Section Phyllostachyae, Cyperaceae). Syst. Bot. 26: 704-721.

Schiebout08; Schiebout, M. H. D. Hazlett, N. Snow. 2008, A floristic survey of vascular plants over parts of northeastern New Mexico. J. Bot. Res. Inst. Texas 2(2): 1407-1447.

Schneider08; A. Schneider, P. Lyon, G. Nesom. 2008. Gutierrezia elegans sp. nov. (Asteraceae: Astereae), a shale barren endemic of southwestern Colorado. J. Bot. Res. Inst. Texas 2(2): $771-774$.

Seberg98: O. Seberg, G. Petersen. 1998. A critical review of concepts and methods used in elassical genome analaysis. The Botanical Review 64: 372-417.

Semple96: J. C. Semple. 1996. A revision of Heterotheca sect. Phyllotheca (Nutt.) Harms (Compositae: Astereae): The prairie and montane goldenasters of North America. Univ. Waterloo Biol. Ser. 37: 1-164.

Semple01: J. C. Semple, C. Xiang, J. Zhang, M. Horsburgh, R. Cook. 2001. Chromosome number determinations in Fam. Compositae, Tribe Astereac, VI. Westem North American taxa and comments on generic treatments of North American asters. Rhodora 103:202-218.

Shaw08: R. B. Shaw. 2008. Grasses of Colorado. University Press of Colorado, Boulder.

Sheviak97: C. J. Sheviak, W. F. Jennings 1997. N. Amer. Native Orchid J. 3: 445-449

Sheviak99: C. J. Sheviak. 1999. The identities of Platanthera hyperborea and P. huronensis, with the description of a new species from North America. Lindleyana 14: 193-203.

Simpson98: B. B. Simpson. 1998. A revision of Pomaria (Fabaceac) in North America. Lundellia $1: 46-71$.

Simpson99: B. B. Simpson. 1999. A revision of Hoffmannseggia (Fahaceae) in North America. Lundellia 2: 14-54.

Simpson06: M. G. Simpson. 2006. Plant Systematics. Elsevier Academic Press.

Sinnott85: Q. P. Sinnott. 1985. A revision of Ribes L. Subg-Grossularia (Mill.) Pers. Sect. Grossularia (Mill.) Nutt. (Grossulariaceae) in North America. Rhodora 87: 189-286.

Smith09?: P. Smith, D. Hazlett, B. Legler, N. Snow. 2009? Noteworthy Collections: Colorado and New Mexico. In review. 
Snow97: N, Snow, 1997, Application of the phylogenetic species concept: a botanical monographic perspective, Ausirobaileya 5; 1-8.

Snow98: N. Snow, 1998. Nomenclatural changes in Leprochloa P. Beauvois sensu lato (Poaceate. Chloridoidene), Novon 8: 77-80.

Snow00: N. Snow. N. Holton. 2000. Additions to Weber's three-letter family acronyms based on results of the Angiosperm Phylogeny Group. Taxon 49; 77-78.

Snow03: N. Snow, G. P. Guymer, G. Sawvel, 2003. Systematics of Austronnyrus, Lenwebbia. and the Australian species of Gossia (Myrtaceae), Syst. Bot. Monogr. 65: 1-95.

Snow04a: N. Snow, J. W. Brasher. February 2004. A provisional checklist of vascular plants for the Southern Rocky Mountain Interactive Flora. (web link defunct).

Snow04b: N, Snow, J. W. Brasher, R. Wallace, J, Grasser, W, F, Jennnings, 2004, Noteworthy Collections: Colorado. Madroño 51:331-332.

Snow07a: N. Snow. Checklist of Vascular Plants of the Southern Rocky Mountain Region. Version 1. September 2007. (web page defunct; see next entry)

Snow07b: N. Snow. Checklist of Vascular Plants of the Southem Rocky Mountain Region. Version 2. October 2007, (www.conps.org/plant-lists,html)

Snow07e:N, Snow, 2008. A new combination in Elymus (Poaceae, Triticieae). Phytologia 89; $223-224$.

Snow07d: N. Snow, 2008. Updated generic keys for Poaceae for the Southem Rocky Mountain Region. 1. Bromers L. Aquilegia 31: 1-4.

Snow08a: N. Snow, 2008. Updated generic keys for Poaceae for the Southern Rocky Mountain

Region. 2. Poo. Aquilegia 32 (1): 1-3.

Snow08b: N. Snow, 2008. Updated generic keys for Poaceae for the Southern Rocky Mountain

Region. 3. Festuca. Aquilegia 32 (2): 1-3.

Snow09: N. Snow. New 3-letter family acronyms for vascular plants with comprehensive listings online. Bishop Museum Occasional Papers 102: In reivew.

Spencer03: S, C. Speneer, A. E. Speneer. 2003, Navarretia willametrensis and Navaretia saximoniana (Polemoniaceae), new species from ephermeral wetlands of western North America. Madrotio 50: 196-199.

Sprichiger04: R.-E. Sprichiger, V. Savolainen, M. Figeat, D. Jeanmonod. 2004. Systematic Botany of Flowering Plants. Seience Publishers, Inc. Enfield (NH), USA \& Plymouth, UK.

Stutz87: H. C. Stutz S. C. Sanderson, E. D. MeArthur, G, L. Chu. 1987. Chromosome races of Grayia brandegel (Chenopodiaceae). Madrono 34: 142-149.

Sun05: F.-J. Sun, G. A. Levin, S. R. Downic. 2005. A multivariate analysis of Cymopterus glomeratus, formerly known as $C$. acaulis (Apiaceae). Rhodora 107: 359-385.

Trock98: D. K. Trock, T. M. Barkley. 1998. Seven new combinations and a new name in Packera (Asteraceae: Senecioneae). Sida 18:385-387.

Tucker70: J. M. Tucker. 1970. Studies in the Quercus undilata complex: IV. The contribution of Quercus havardl. Amer. J. Bot. 57: 71-84.

Tucker71: J. M. Tucker. 1971. Studies in the Qnencus undilata complex: V. The type of Quercus undulata. Amer. J. Bot. 58: 329-341.

Tumer87: B. L. Tumer. 1987. Taxonomic study of Machaeranthera, sections Machaeranthera and Hesperastrom (Asteraceac). Phytologia 62: 207-266.

Veldkamp08: J. F. Veldkamp. 2008 . Senecio cymablarioides, S subnudus, and S. subpelfatusi, history of a mudlle (Asteraceae: Senecioncae). J. Bot. Res. Inst. Texas 2:1215-1218.

Vinnersten07: A. Vinnersten, J. Manning. 2007. A new classification of Colchicaceae. Taxon 56: $171-178$. 
Wagnet85: W. L. Wagner, R. E. Stockhouse, W. M. Klein. 1985. The systematics and evolution of the Ocnothera caespitosa species complex (Onagraceae). Monogr. Syst. Bot. Missouri Bot. Gard. 12:1-103.

Wagner07: W. L. Wagner, P. C. Hoch, P. H. Raven. 2007. Revised classification of the Onagracene. Syst. Bot. Monogr. 83:1-240.

Weber82: W. A. Weber. 1982. Mnemonic three-letter acronyms for the families of vascular plants: a device for more effective herbarium curation. Taxon 31: 74-88.

Welsh03; S. L. Welsh, N. D. Atwood, S, Goodrich, L. C. Higgins (Editors), 2003. A Utah Flora. Third edition, revised. Brigham Young University, Provo, UT.

Wen98: J. Wen, S. Shi, R. K. Jansen, E. A. Zimmer. 1998. Phylogeny and biogeography of Aralia sect. Aralia (Araliaceae), Amer. J, Botany 85; 866-875.

Windham06: M. D. Windham, I. A. Al-Shehbaz, 2006. New and noteworthy species of Boechera (Brassicaceae) I: Sexual diploids. Harvand Papers in Botany 11:61-88.

Windham07a: M. D. Windham, I. A. Al-Shehbaz, 2007. New and noteworthy species of Bowchera (Brassicaceae) II: Apomictic hybrids. Harvard Papers in Botany 11:257-274.

Windham07b; M. D. Windham, I. A. Al-Shehbaz. 2007. New and noteworthy species of Boechera (Brassicaceae) III: Additional sexual diploids and apomictic hybrids. Harvard Papers in Botany 12: 235-257.

Wolf79: S. J. Wolf, J. G. Packer, K. E. Denford. 1979. The taxonomy of Minuarita rassit (Caryophyllaceae). Canad. J. Bot. 57; 1673-1686.

Yuan96; Y,-M.Yuan, P. Küper, J. J. Doyle, 1996. Infrageneric phylogeny of the genus Gentiano (Gentianaceae) inferred from nucleotide sequences of the internal transcribed spacers (ITS) of nuclear ribosomal DNA. Amer. J. Botany 3:641-652.

Zomlefer94: W. B. Zomlefer. 1994. Guide to Flowering Plant Families. The Univ, of North Carolina Press, Chapel Hill \& London.

Zomlefer01: W. B. Zomlefer, N. H. Williams, W. Mark Whitten, W. S. Judd. 2001. Generic circumscription and relationships in the tribe Melanthieae (Lilales, Melanthiaceae), with empahsis on Zigadenus; Evidence from ITS and TRVL-F sequences. Amer. J. Botany 88: 1657-1669.

Zomlefer02: W. B. Zomlefer, W. S. Judd. 2002. Resurrection of segregates of the polyphyletic genus Zigadewass s.l. (Liliales: Melanthaceae) and resulting new combinations. Novon 12: $299-308$.

Zuloaga07: F. O. Zuloaga, L. M. Giussani, O. Morrone. 2007. Hopla, a new monoty pic genus segregated from Panicum (Poaceac). Taxon 56: 145-156. 